\title{
Non-specialist health worker interventions for the care of mental, neurological and substance-abuse disorders in low- and middle-income countries (Review)
}

van Ginneken N, Tharyan P, Lewin S, Rao GN, Meera SM, Pian J, Chandrashekar S, Patel V

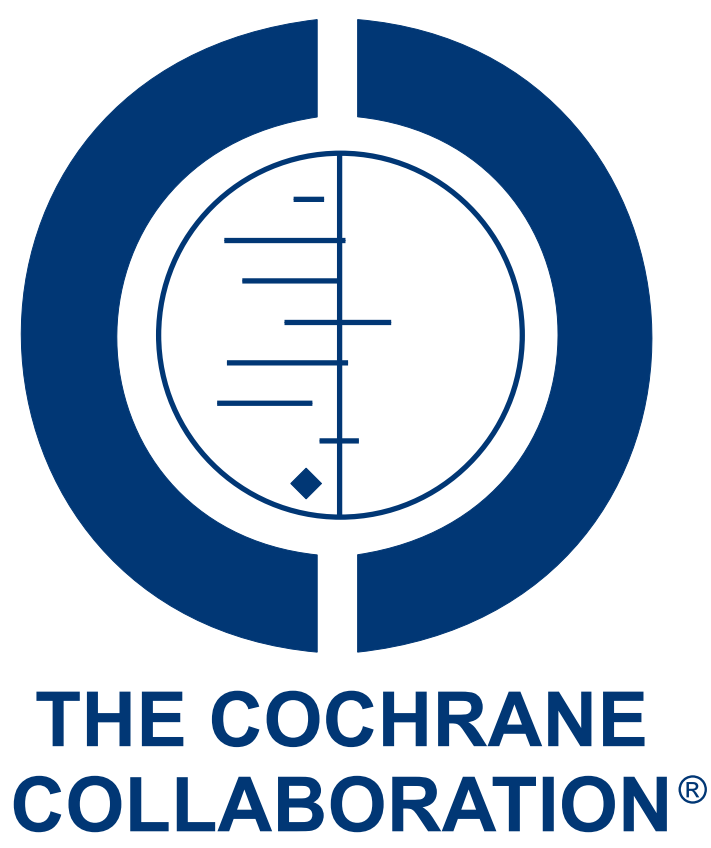

This is a reprint of a Cochrane review, prepared and maintained by The Cochrane Collaboration and published in The Cochrane Library 2013, Issue 11

http://www.thecochranelibrary.com

\section{WILEY}

Non-specialist health worker interventions for the care of mental, neurological and substance-abuse disorders in low- and middleincome countries (Review)

Copyright $\odot 2013$ The Cochrane Collaboration. Published by John Wiley \& Sons, Ltd. 
TABLE OF CONTENTS

HEADER . . . . . . . . . . . . . . . . . . . . . . . . . . . . . . . . . . . . . . . 1

ABSTRACT . . . . . . . . . . . . . . . . . . . . . . . . . . . . . . . . . . . . . . 1

PLAIN LANGUAGE SUMMARY . . . . . . . . . . . . . . . . . . . . . . . . . . . . . . . . . . . . . . . . 2

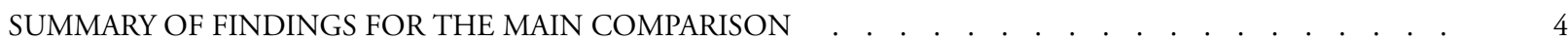

BACKGROUND . . . . . . . . . . . . . . . . . . . . . . . . . . . . . . . . . . . . . 6

OBJECTIVES . . . . . . . . . . . . . . . . . . . . . . . . . . . . . . . . . . . . . . . . . . . . . . .

METHODS . . . . . . . . . . . . . . . . . . . . . . . . . . . . . . . . . . . . . . . . .

RESULTS . . . . . . . . . . . . . . . . . . . . . . . . . . . . . . . . . . 12

Figure 1. . . . . . . . . . . . . . . . . . . . . . . . . . . . . . . . . . . . . . 13

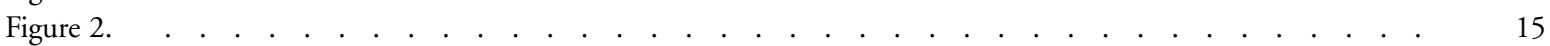

Figure 3. . . . . . . . . . . . . . . . . . . . . . . . . . . . . . . . . . . . . .

Figure 4. . . . . . . . . . . . . . . . . . . . . . . . . . . . . . . . . . . . . . 19

Figure 5. . . . . . . . . . . . . . . . . . . . . . . . . . . . . . . . . . . . . . . 20

Figure 6. . . . . . . . . . . . . . . . . . . . . . . . . . . . . . . . . . . . . . 22

Figure $7 . \quad$. . . . . . . . . . . . . . . . . . . . . . . . . . . . . . . . . . . . 23

Figure 8. . . . . . . . . . . . . . . . . . . . . . . . . . . . . . . . . . . . . . 425

Figure 9. . . . . . . . . . . . . . . . . . . . . . . . . . . . . . . . . . . . . 27

Figure 10. . . . . . . . . . . . . . . . . . . . . . . . . . . . . . . . . . . . . . . . . .

Figure 11. . . . . . . . . . . . . . . . . . . . . . . . . . . . . . . . . . . . . . 29

Figure 12. . . . . . . . . . . . . . . . . . . . . . . . . . . . . . . . . . . . . . 29

Figure 13. . . . . . . . . . . . . . . . . . . . . . . . . . . . . . . . . . . . . . 30

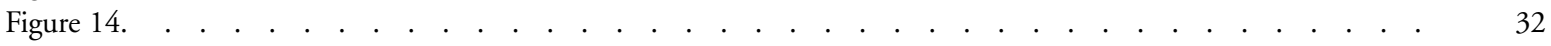

ADDITIONAL SUMMARY OF FINDINGS . . . . . . . . . . . . . . . . . . . . . . . . . . . . . . . . . .

DISCUSSION . . . . . . . . . . . . . . . . . . . . . . . . . . . . . . . . . . . . . . 46

AUTHORS' CONCLUSIONS . . . . . . . . . . . . . . . . . . . . . . . . . . . . . . . . . . . . . . 48

ACKNOWLEDGEMENTS . . . . . . . . . . . . . . . . . . . . . . . . . . . . . . . . . . . . . . 49

REFERENCES . . . . . . . . . . . . . . . . . . . . . . . . . . . . . . . . . . . . . . . . . 49

CHARACTERISTICS OF STUDIES . . . . . . . . . . . . . . . . . . . . . . . . . . . . . . . . . . . . . 71

DATA AND ANALYSES . . . . . . . . . . . . . . . . . . . . . . . . . . . . . . . . . . . . . . . . . . . . . . . . . .

Analysis 1.1. Comparison 1 NSHW-led psychological interventions versus usual care in treating common mental disorders in adults (RCTs), Outcome 1 Prevalence of depression (completers). . . . . . . . . . . . . . . . . . . . . 242

Analysis 1.2. Comparison 1 NSHW-led psychological interventions versus usual care in treating common mental disorders in adults (RCTs), Outcome 2 Prevalence of depression (ITT sensitivity analysis - assumption non-completers depressed).

Analysis 1.3. Comparison 1 NSHW-led psychological interventions versus usual care in treating common mental disorders in adults (RCTs), Outcome 3 Prevalence of depression (ITT sensitivity analysis - assumption non-completers not depressed).

Analysis 1.4. Comparison 1 NSHW-led psychological interventions versus usual care in treating common mental disorders in adults (RCTs), Outcome 4 Prevalence of depression (ITT sensitivity analysis - worse-case scenario intervention group depressed; control group not depressed).

Analysis 1.5. Comparison 1 NSHW-led psychological interventions versus usual care in treating common mental disorders in adults (RCTs), Outcome 5 Prevalence of depression (ITT sensitivity analysis - best-case scenario: intervention group not depressed; control group all depressed).

nalysis 1.6. Comparison 1 NSHW-led psychological interventions versus usual care in treating common mental disorders in adults (RCTs), Outcome 6 Severity of common mental disorder symptoms (includes anxiety and depression). 247

Analysis 1.7. Comparison 1 NSHW-led psychological interventions versus usual care in treating common mental disorders in adults (RCTs), Outcome 7 Functional impairment/disability in common mental disorders.

Analysis 2.1. Comparison 2 Collaborative care model (NSHWs plus specialist) versus usual care in treating common mental disorders (RCTs), Outcome 1 Prevalence of common mental disorders (CMDs - includes anxiety and depression) (completers-combined) all facilities and in public and private facilities.

Non-specialist health worker interventions for the care of mental, neurological and substance-abuse disorders in low- and middle- 
Analysis 2.2. Comparison 2 Collaborative care model (NSHWs plus specialist) versus usual care in treating common mental disorders (RCTs), Outcome 2 Severity of symptoms of CMDs (completers-combined) in all facilities and in public

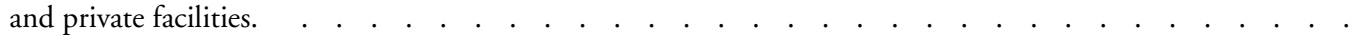

Analysis 2.3. Comparison 2 Collaborative care model (NSHWs plus specialist) versus usual care in treating common mental disorders (RCTs), Outcome 3 Functional impairment/disability in CMD (completers- combined) all facilities and in public and private facilities (SMD)

Analysis 2.4. Comparison 2 Collaborative care model (NSHWs plus specialist) versus usual care in treating common mental disorders (RCTs), Outcome 4 Suicide attempt for those with CMDs all facilities and in public/private facilities (completers). . . . . . . . . . . . . . . . . . . . . . . . . . . . . . . . . . . 256

Analysis 2.5. Comparison 2 Collaborative care model (NSHWs plus specialist) versus usual care in treating common mental disorders (RCTs), Outcome 5 Prevalence of CMDs (only Patel - sensitivity analysis (SA)) (completers) all facilities and in public and private facilities.

Analysis 2.6. Comparison 2 Collaborative care model (NSHWs plus specialist) versus usual care in treating common mental disorders (RCTs), Outcome 6 Severity of symptoms in CMD (only Patel and Jenkins (SA)) in all facilities and in public and private facilities. . . . . . . . . . . . . . . . . . . . . . . . . . . . . . . . . . . . . . . .

Analysis 2.7. Comparison 2 Collaborative care model (NSHWs plus specialist) versus usual care in treating common mental disorders (RCTs), Outcome 7 Prevalence of depression (completers) (SA) all facilities and in public and private facilities.

nalysis 2.8. Comparison 2 Collaborative care model (NSHWs plus specialist) versus usual care in treating common mental disorders (RCTs), Outcome 8 Severity of symptoms of depression (SA) in all facilities and in public and private facilities.

alysis 2.9. Comparison 2 Collaborative care model (NSHWs plus specialist) versus usual care in treating common mental disorders (RCTs), Outcome 9 Functional impairment/disability in CMD (SA) all facilities and in public and private facilities $(\mathrm{SMD})$. . . . . . . . . . . . . . . . . . . . . . . . . . . . . . . . . . . . . . . .

Analysis 2.10. Comparison 2 Collaborative care model (NSHWs plus specialist) versus usual care in treating common mental disorders (RCTs), Outcome 10 Functional impairment/disability in CMD (SA) all facilities and in public and private facilities (MD). . . . . . . . . . . . . . . . . . . . . . . . . . . . . . . .

Analysis 2.11. Comparison 2 Collaborative care model (NSHWs plus specialist) versus usual care in treating common mental disorders (RCTs), Outcome 11 Functional impairment/disability in depression (SA) all facilities and in public and private facilities. . . . . . . . . . . . . . . . . . . . . . . . . . . . . . . . . . . . .

Analysis 3.1. Comparison 3 NSHWs versus usual care in treating maternal depression (RCTs), Outcome 1 Severity of symptoms in treating maternal depression. . . . . . . . . . . . . . . . . . . . . . . . . . . . . .

Analysis 4.1. Comparison 4 NSHWs versus specialists in treating depression in adults (controlled before-and-after studies), Outcome 1 Severity of depression short term (2 months post intervention).

Analysis 4.2. Comparison 4 NSHWs versus specialists in treating depression in adults (controlled before-and-after studies), Outcome 2 Frequency of adverse events. . . . . . . . . . . . . . . . . . . . . . . . . . . . . . . . $\quad 272$

Analysis 4.3. Comparison 4 NSHWs versus specialists in treating depression in adults (controlled before-and-after studies), Outcome 3 Number of days spent in hospital. $\quad . \quad$. . . . . . . . . . . . . . . . . . . . . . . . . . . . $\quad$. 273

Analysis 4.4. Comparison 4 NSHWs versus specialists in treating depression in adults (controlled before-and-after studies), Outcome 4 Number of days spent on sick leave. . . . . . . . . . . . . . . . . . . . . . . . . . . . . . . . . $\quad 274$

Analysis 5.1. Comparison 5 NSHW-led psychological interventions versus usual care in treating adults with post-traumatic stress disorder (RCT and NRCT), Outcome 1 Prevalence of post-traumatic stress disorder (PTSD). . . . . . 275

Analysis 5.2. Comparison 5 NSHW-led psychological interventions versus usual care in treating adults with post-traumatic stress disorder (RCT and NRCT), Outcome 2 Severity of PTSD symptoms. . . . . . . . . . . . . . . . 276

Analysis 5.3. Comparison 5 NSHW-led psychological interventions versus usual care in treating adults with post-traumatic stress disorder (RCT and NRCT), Outcome 3 Severity of depression. . . . . . . . . . . . . . . . . . $\quad 277$

Analysis 6.1. Comparison 6 NSHWs versus usual care in improving dementia patients' and carers' outcomes (RCTs), Outcome 1 Severity of behavioural problem (patient). . . . . . . . . . . . . . . . . . . . . . . . .

Analysis 6.2. Comparison 6 NSHWs versus usual care in improving dementia patients' and carers' outcomes (RCTs), Outcome 2 Patient functional ability. . . . . . . . . . . . . . . . . . . . . . . . . . . . . . . . . . .

Analysis 6.3. Comparison 6 NSHWs versus usual care in improving dementia patients' and carers' outcomes (RCTs),

Outcome 3 Patient quality of life. . . . . . . . . . . . . . . . . . . . . . . . . . . .

Non-specialist health worker interventions for the care of mental, neurological and substance-abuse disorders in low- and middle- 
Analysis 6.4. Comparison 6 NSHWs versus usual care in improving dementia patients' and carers' outcomes (RCTs), Outcome 4 Carer mental health status.

Analysis 6.5. Comparison 6 NSHWs versus usual care in improving dementia patients' and carers' outcomes (RCTs), Outcome 5 Carer burden.

Analysis 6.6. Comparison 6 NSHWs versus usual care in improving dementia patients' and carers' outcomes (RCTs), Outcome 6 Carer distress.

Analysis 6.7. Comparison 6 NSHWs versus usual care in improving dementia patients' and carers' outcomes (RCTs), Outcome 7 Carer quality of life.

Analysis 7.1. Comparison 7 NSHW-led brief alcohol interventions versus usual care for adults with alcohol-use disorders (RCTs), Outcome 1 Amount of alcohol consumed (MD).

Analysis 7.2. Comparison 7 NSHW-led brief alcohol interventions versus usual care for adults with alcohol-use disorders (RCTs), Outcome 2 Frequency of binge drinking.

Analysis 7.3. Comparison 7 NSHW-led brief alcohol interventions versus usual care for adults with alcohol-use disorders (RCTs), Outcome 3 Adverse consequences.

Analysis 8.1. Comparison 8 NSHWs/OPHRs versus usual care in conducting interventions for children with post-traumatic stress and depression (RCTs), Outcome 1 Severity of PTSD symptoms - teacher/LHW-led interventions (SMDs).

Analysis 8.2. Comparison $8 \mathrm{NSHWs/OPHRs} \mathrm{versus} \mathrm{usual} \mathrm{care} \mathrm{in} \mathrm{conducting} \mathrm{interventions} \mathrm{for} \mathrm{children} \mathrm{with} \mathrm{post-traumatic}$ stress and depression (RCTs), Outcome 2 Severity of PTSD symptoms - classroom-based LHW interventions (MCDs)

Analysis 8.3. Comparison $8 \mathrm{NSHWs}$ /OPHRs versus usual care in conducting interventions for children with post-traumatic stress and depression (RCTs), Outcome 3 Severity of PTSD symptoms - classroom-based LHW interventions boys/girls.

Analysis 8.4. Comparison $8 \mathrm{NSHWs}$ /OPHRs versus usual care in conducting interventions for children with posttraumatic stress and depression (RCTs), Outcome 4 Severity of depressive symptoms - teacher/LHW-led interventions (SMDs).

Analysis 8.5. Comparison $8 \mathrm{NSHWs/OPHRs} \mathrm{versus} \mathrm{usual} \mathrm{care} \mathrm{in} \mathrm{conducting} \mathrm{interventions} \mathrm{for} \mathrm{children} \mathrm{with} \mathrm{post-traumatic}$ stress and depression (RCTs), Outcome 5 Severity of depressive symptoms - classroom-based LHW interventions (MCDs).

Analysis 8.6. Comparison 8 NSHWs/OPHRs versus usual care in conducting interventions for children with posttraumatic stress and depression (RCTs), Outcome 6 Severity of depressive symptoms (MCDs) Tol 2012 boys/girls.

Analysis 8.7. Comparison 8 NSHWs/OPHRs versus usual care in conducting interventions for children with posttraumatic stress and depression (RCTs), Outcome 7 Severity of anxiety symptoms - classroom-based intervention (within 6 months post intervention).

Analysis 8.8. Comparison $8 \mathrm{NSHWs}$ /OPHRs versus usual care in conducting interventions for children with posttraumatic stress and depression (RCTs), Outcome 8 Severity of anxiety symptoms - classroom-based intervention boys/girls.

Analysis 8.9. Comparison 8 NSHWs/OPHRs versus usual care in conducting interventions for children with posttraumatic stress and depression (RCTs), Outcome 9 Functional impairment teacher/LHW-led interventions. .

Analysis 8.10. Comparison 8 NSHWs/OPHRs versus usual care in conducting interventions for children with posttraumatic stress and depression (RCTs), Outcome 10 Functional impairment LHW-led - classroom-based intervention.

Analysis 8.11. Comparison 8 NSHWs/OPHRs versus usual care in conducting interventions for children with posttraumatic stress and depression (RCTs), Outcome 11 Functional impairment - classroom-based LHW intervention boys/girls. . . . . . . . . . . . . . . . . . . . . . . . . . . . . . . . . . . .

ADDITIONAL TABLES . . . . . . . . . . . . . . . . . . . . . . . . . . . . . . . . . .

APPENDICES . . . . . . . . . . . . . . . . . . . . . . . . . . . . . . . . . . . . .

WHAT'S NEW . . . . . . . . . . . . . . . . . . . . . . . . . . . . . . . . . . . . .

CONTRIBUTIONS OF AUTHORS . . . . . . . . . . . . . . . . . . . . . . . . . . . . . . . . . . . . . . . . . .

DECLARATIONS OF INTEREST . . . . . . . . . . . . . . . . . . . . . . . . . . . . . . . . . . . . . .

SOURCES OF SUPPORT . . . . . . . . . . . . . . . . . . . . . . . . . . . . . . . . . . 366

DIFFERENCES BETWEEN PROTOCOL AND REVIEW . . . . . . . . . . . . . . . . . . . . . . . . . . . . . . .

Non-specialist health worker interventions for the care of mental, neurological and substance-abuse disorders in low- and middle- 


\title{
Non-specialist health worker interventions for the care of mental, neurological and substance-abuse disorders in low- and middle-income countries
}

\author{
Nadja van Ginneken ${ }^{1,2}$, Prathap Tharyan ${ }^{3}$, Simon Lewin ${ }^{4,5}$, Girish N Rao ${ }^{6}$, SM Meera ${ }^{2}$, Jessica $\operatorname{Pian}^{7}$, Sudha Chandrashekar ${ }^{7,8}$, \\ Vikram Patel ${ }^{1,2}$ \\ ${ }^{1}$ Centre for Global Mental Health, London School of Hygiene \& Tropical Medicine, London, UK. ${ }^{2}$ Sangath, Goa, India. ${ }^{3}$ South Asian \\ Cochrane Network \& Centre, Prof. BV Moses \& ICMR Advanced Centre for Research \& Training in Evidence Informed Health Care, \\ Christian Medical College, Vellore, India. ${ }^{4}$ Global Health Unit, Norwegian Knowledge Centre for the Health Services, Oslo, Norway. \\ ${ }^{5}$ Health Systems Research Unit, Medical Research Council of South Africa, Tygerberg, South Africa. ${ }^{6}$ Department of Epidemiology, \\ National Institute of Mental Health and Neuro Sciences, Bangalore, India. ${ }^{7}$ London School of Hygiene \& Tropical Medicine, London, \\ UK. ${ }^{8}$ St. Johns Research Institute, Bangalore, India \\ Contact address: Nadja van Ginneken, nadja.vanginneken@1shtm.ac.uk.nvanginneken@yahoo.co.uk. \\ Editorial group: Cochrane Effective Practice and Organisation of Care Group. \\ Publication status and date: New, published in Issue 11, 2013. \\ Review content assessed as up-to-date: 2 October 2012.
}

Citation: van Ginneken N, Tharyan P, Lewin S, Rao GN, Meera SM, Pian J, Chandrashekar S, Patel V. Non-specialist health worker interventions for the care of mental, neurological and substance-abuse disorders in low- and middle-income countries. Cochrane Database of Systematic Reviews 2013, Issue 11. Art. No.: CD009149. DOI: 10.1002/14651858.CD009149.pub2.

Copyright (C) 2013 The Cochrane Collaboration. Published by John Wiley \& Sons, Ltd.

\begin{abstract}
A B S T R A C T
Background

Many people with mental, neurological and substance-use disorders (MNS) do not receive health care. Non-specialist health workers (NSHWs) and other professionals with health roles (OPHRs) are a key strategy for closing the treatment gap.

Objectives

To assess the effect of NSHWs and OPHRs delivering MNS interventions in primary and community health care in low- and middleincome countries.

\section{Search methods}

We searched the Cochrane Central Register of Controlled Trials (CENTRAL) (including the Cochrane Effective Practice and Organisation of Care (EPOC) Group Specialised Register) (searched 21 June 2012); MEDLINE, OvidSP; MEDLINE In Process \& Other Non-Indexed Citations, OvidSP; EMBASE, OvidSP (searched 15 June 2012); CINAHL, EBSCOhost; PsycINFO, OvidSP (searched 18 and 19 June 2012); World Health Organization (WHO) Global Health Library (searched 29 June 2012); LILACS; the International Clinical Trials Registry Platform (WHO); OpenGrey; the metaRegister of Controlled Trials (searched 8 and 9 August 2012); Science Citation Index and Social Sciences Citation Index (ISI Web of Knowledge) (searched 2 October 2012) and reference lists, without language or date restrictions. We contacted authors for additional studies.
\end{abstract}

\section{Selection criteria}

Randomised and non-randomised controlled trials, controlled before-and-after studies and interrupted-time-series studies of NSHWs/ OPHR-delivered interventions in primary/community health care in low- and middle-income countries, and intended to improve

Non-specialist health worker interventions for the care of mental, neurological and substance-abuse disorders in low- and middle-

income countries (Review)

Copyright $(2013$ The Cochrane Collaboration. Published by John Wiley \& Sons, Ltd. 
outcomes in people with MNS disorders and in their carers. We defined an NSHW as any professional health worker (e.g. doctors, nurses and social workers) or lay health worker without specialised training in MNS disorders. OPHRs included people outside the health sector (only teachers in this review).

\section{Data collection and analysis}

Review authors double screened, double data-extracted and assessed risk of bias using standard formats. We grouped studies with similar interventions together. Where feasible, we combined data to obtain an overall estimate of effect.

\section{Main results}

The 38 included studies were from seven low- and 15 middle-income countries. Twenty-two studies used lay health workers, and most addressed depression or post-traumatic stress disorder (PTSD). The review shows that the use of NSHWs, compared with usual healthcare services: 1. may increase the number of adults who recover from depression or anxiety, or both, two to six months after treatment (prevalence of depression: risk ratio (RR) $0.30,95 \%$ confidence interval (CI) 0.14 to 0.64 ; low-quality evidence); 2 . may slightly reduce symptoms for mothers with perinatal depression (severity of depressive symptoms: standardised mean difference (SMD) $-0.42,95 \%$ CI -0.58 to -0.26 ; low-quality evidence); 3. may slightly reduce the symptoms of adults with PTSD (severity of PTSD symptoms: SMD -0.36 , 95\% CI -0.67 to -0.05 ; low-quality evidence); 4. probably slightly improves the symptoms of people with dementia (severity of behavioural symptoms: SMD - $0.26,95 \%$ CI - 0.60 to 0.08 ; moderate-quality evidence); 5 . probably improves/slightly improves the mental well-being, burden and distress of carers of people with dementia (carer burden: SMD -0.50, 95\% CI -0.84 to -0.15 ; moderate-quality evidence); 6 . may decrease the amount of alcohol consumed by people with alcohol-use disorders (drinks/drinking day in last 7 to 30 days: mean difference $-1.68,95 \%$ CI -2.79 to -0.57 ); low-quality evidence).

It is uncertain whether lay health workers or teachers reduce PTSD symptoms among children. There were insufficient data to draw conclusions about the cost-effectiveness of using NSHWs or teachers, or about their impact on people with other MNS conditions. In addition, very few studies measured adverse effects of NSHW-led care - such effects could impact on the appropriateness and quality of care.

\section{Authors' conclusions}

Overall, NSHWs and teachers have some promising benefits in improving people's outcomes for general and perinatal depression, PTSD and alcohol-use disorders, and patient- and carer-outcomes for dementia. However, this evidence is mostly low or very low quality, and for some issues no evidence is available. Therefore, we cannot make conclusions about which specific NSHW-led interventions are more effective.

\section{PLAIN LANGUAGE SUMMARY}

The effect of non-specialist health workers on people with mental, neurological and substance-abuse disorders in developing countries

\section{Background}

In developing countries, most people with mental, neurological and substance-abuse (MNS) disorders do not receive adequate care mainly because of a lack of mental health professionals. Non-specialist health workers, but also other professionals with health roles, such as teachers, may therefore have an important role to play in delivering MNS health care.

Researchers in The Cochrane Collaboration carried out a review of the effects of using non-specialist health workers or other professionals with health roles to help people with MNS disorders in developing countries. After searching for all relevant studies in scientific databases, they found 38 studies published before October 2012. Their findings are summarised below.

\section{What is a non-specialist health worker?}

Any type of health worker (like a doctor, nurse or lay health worker) who is not a specialist in mental health or neurology but who may have had some training in these fields. We also looked at teachers, as they can be particularly important in the care of children and youths.

\section{What the research says}

Non-specialist health worker interventions for the care of mental, neurological and substance-abuse disorders in low- and middle- 
The studies in this review were from 22 developing countries. In most studies, lay health workers delivered the mental health care, and addressed depression or anxiety (or both), or post-traumatic stress disorder. The review shows that the use of non-specialist health workers, compared with usual healthcare services:

- may increase the number of adults who recover from depression or anxiety (or both) two to six months after treatment;

- may slightly reduce symptoms formothers with depression;

- may slightly reduce the symptoms of adults with post-traumatic stress disorder (non-specialists and teachers were used in one study);

- probably slightly improves the symptoms of people with dementia;

- probably improves/slightly improves the mental well-being, burden and distress of carers of people with dementia;

- may decrease the quantity of alcohol consumed by problem drinkers.

It is uncertain whether lay health workers or teachers reducepost-traumatic stress disorder symptoms among children. There were too few studies to draw any conclusions about the cost-effectiveness of using non-specialist health workers or teachers, or about their impact on people with other MNS conditions such as epilepsy, schizophrenia, and alcohol and drug abuse problems. In addition, very few studies measured unintended consequences of non-specialist health worker-led care - such effects could impact on the appropriateness and quality of care.

\section{Quality of the evidence}

Overall, non-specialist health workers and teachers have some promising benefits in improving people's outcomes for general and perinatal depression, post-traumatic stress disorder and alcohol-use disorders, and patient and carer outcomes for dementia. However, this evidence is of low or very low quality in some areas, and for some issues no evidence is available. Therefore, we cannot make conclusions about which specific interventions using non-specialist health workers to help people with MNS disorders are more effective.

Non-specialist health worker interventions for the care of mental, neurological and substance-abuse disorders in low- and middle- 
SUMMARY OF FINDINGS FOR THE MAIN COMPARISON [Explanation]

\section{What are the effects of NSHW-led psychological interventions for treating depression in adults in low- and middle-income countries?}

Patient or population: Adults with depression

Settings: Low- and middle-income countries (Taiwan, Pakistan, Uganda)

Intervention: NSHWs conducting psychological interventions

Comparison: Usual care

\begin{tabular}{|c|c|c|c|c|c|c|}
\hline \multirow[t]{3}{*}{ Outcomes } & \multicolumn{2}{|c|}{ Illustrative comparative risks* $(95 \%$ Cl) } & \multirow{3}{*}{$\begin{array}{l}\text { Effect estimate } \\
(95 \% \text { CI })\end{array}$} & \multirow{3}{*}{$\begin{array}{l}\text { No of Participants } \\
\text { (studies) }\end{array}$} & \multirow{3}{*}{$\begin{array}{l}\text { Quality of the evidence } \\
\text { (GRADE) }\end{array}$} & \multirow[t]{3}{*}{ Comments } \\
\hline & Assumed risk & Corresponding risk & & & & \\
\hline & Usual care & NSHWs & & & & \\
\hline $\begin{array}{l}\text { Prevalence of depres- } \\
\text { sion (adults), short term } \\
\text { (0-8 weeks) } \\
\text { measured using various } \\
\text { depression rating scales }{ }^{1}\end{array}$ & 300 per 1000 & 91 per 1000 & $\begin{array}{l}\mathbf{R R} \mathbf{0 . 3 0} \\
(0.14 \text { to } 0.64)\end{array}$ & $\begin{array}{l}1082 \\
\text { (3 studies) }\end{array}$ & $\begin{array}{l}\oplus \oplus \bigcirc \bigcirc \\
\text { low }^{2,3}\end{array}$ & - \\
\hline
\end{tabular}

*The basis for the assumed risk is the mean control group risk across studies for pooled results and the control group risk for single studies. The corresponding risk (and its $95 \% \mathrm{Cl}$ ) is based on the assumed risk in the comparison group and the relative effect of the intervention (and its $95 \% \mathrm{Cl}$ ).

CI: confidence interval; DSM: Diagnostic and Statistical Manual of Mental Disorders; NSHW: non-specialist health worker; RCT: randomised controlled trial; RR: risk ratio .

GRADE Working Group grades of evidence

High quality: Further research is very unlikely to change our confidence in the estimate of effect.

Moderate quality: Further research is likely to have an important impact on our confidence in the estimate of effect and may change the estimate.

Low quality: Further research is very likely to have an important impact on our confidence in the estimate of effect and is likely to change the estimate.

Very low quality: We are very uncertain about the estimate.

${ }^{1}$ Bolton 2003 C-RCT Uganda: DSM-IV criteria A, C and E; Rahman 2008 CRCT Pakistan: Hamilton Depression Rating scale; Chen 2000

RCT Taiwan: Taiwanese Beck Depression Inventory.

2 Serious study limitations: Two of the three studies were at risk of bias. Bolton 2003 C-RCT Uganda was judged unclear for allocation concealment, and quasi-randomisation of individuals within clusters (though randomisation was in clusters) could have introduced bias; Chen 2000 RCT Taiwan was unclear for sequence generation and allocation concealment, all outcomes were self reported, there was 

dropouts. These two studies contributed $62 \%$ of the weight in the pooled analysis. Downgraded by 1 .

${ }^{3}$ Serious inconsistency: $\left.\right|^{2}$ was $81 \%$. However, the inconsistency related to the magnitude of benefit favouring collaborative care rather than in the direction of effect. Downgraded by 1. 


\section{B A C K G R O U N D}

\section{Description of the condition}

The global burden of mental, neurological and substance-abuse (MNS) illnesses is high. The latest global burden of disease estimates have shown that mental, behavioural and neuropsychiatric disorders all feature in the top 30 causes of all years lived with disability, the highest contributors being major depression (ranked second), anxiety (ranked seventh) and substance-use disorders (ranked twelfth) (Vos 2012). The contribution of major depressive disorders to worldwide disability-adjusted life years (DALYs) has increased by $37 \%$ from 1990 to 2010 and is predicted to rise further (Murray 2012; Prince 2007). Furthermore, self inflicted injuries and alcohol-related disorders are likely to increase in the ranking of disease burden due to the decline in communicable diseases and because of a predicted increase in war and violence. The disease burden due to Alzheimer's disease is also increasing, linked to the demographic transition towards an ageing population (Vos 2012).

These illnesses also come with substantial economic costs. One recent report on the global economic burden of non-communicable diseases (NCDs) suggests that by the early 2030s, mental health conditions alone will account for the loss of an additional USD16.1 trillion with dramatic impact on productivity and quality of life (Bloom 2011). Data remain poor on the macro-economic costs for low- and middle-income country (LMIC) settings (Hu 2006). However, the economic and social costs for individuals and families are substantial. High direct costs are incurred in countries where health spending is met largely through private, as opposed to public, spending and where health insurance and employer-met health payments are insubstantial (Patel 2007a). High indirect costs are also incurred due to informal care-giving and lost work opportunities, as well as due to untreated disorders and their associated disability (Chisholm 2000a; WHO 2003a).

The gap between those who could benefit from MNS health interventions and those who receive such care is very large (WHO 2008; WHO 2010); in LMICs up to $90 \%$ of people needing care do not receive it (Demyttenaere 2004; Saxena 2007). This is despite the existence of a range of cost-effective interventions in mental health care (Patel 2007b; WHO 2010). Major barriers to closing the treatment gap are the huge scarcity of skilled human resources, large inequities and inefficiencies in resource distribution and utilisation, and the significant stigma associated with psychiatric illness (Saxena 2007). Some papers have advocated for scaling up evidence-based services and for the task-shifting of mental health interventions to non-specialists as key strategies for closing the treatment gap (Jacob 2007; Lancet 2007; Patel 2007b; Prince 2007; Saraceno 2007; Saxena 2007).

\section{Description of the intervention}

Non-specialist health workers (NSHWs) are first-level providers who have received general rather than specialist mental health training. Cadres included are professionals (doctors, nurses and other general paraprofessionals) and non-professionals (such as lay providers). NSHWs do not include, for example, psychiatrists, neurologists, psychologists, psychiatric nurses or mental health social workers. Other professionals with health roles (OPHRs), such as teachers and community-level workers, are a further human resource used in delivering mental health care and are also included in this review. These OHPRs have an important role, particularly in the promotion of mental health and the detection of mental disorders (Patel 2007b; Patel 2008b; WHO 2003b).

NSHWs and OPHRs have been used in various services, including those delivered by governmental, private and non-governmental organisations (NGOs) in clinics, half-way homes and communities. They have been involved in a variety of activities and roles, including detecting, diagnosing, treating and preventing common and severe mental disorders, epilepsy and mental retardation. Their roles differ according to their level of training. For example, lay health workers (LHW) have been involved in supporting carers, befriending, ensuring adherence and in detection of mental health problems (Chatterjee 2003; Dias 2008 RCT India; Rahman 2008 CRCT Pakistan). Nurses, social workers and lay workers may also take on follow-up or educational/promotional roles (Araya 2003 RCT Chile; Chatterjee 2003; Patel 2008b). In addition, doctors with general mental health training have been involved in the identification, diagnosis, treatment and referral of complex cases (Murthy 1987; Patel 2008b; Saxena 2007).

\section{How the intervention might work}

In many LMICs, training and retaining sufficient numbers of specialists is not feasible in the near future. It is, therefore, important in these settings to consider options for expanding access to mental health services. The use of NSHWs, who are far more numerous and affordable than specialists, is one such option that is of high relevance to LMICs.

Training these NSHWs to deliver MNS interventions may be a way of expanding provision of mental health services as well as making these services more accessible to communities. It has been suggested that interventions that rely on NSHWs could deliver general health and mental health interventions that are at least as effective and acceptable as those delivered by specialist health workers (Chatterjee 2003; Lewin 2008; McKenzie 2004; Thornicroft 2004; WHO 2001; Wiley-Exley 2007). In addition, NSHW interventions often have lower up-front costs compared with reliance on professional specialist health workers. However, it is possible that these savings may be cancelled out by higher downstream resource use (Chisholm 2000a), and this review will, therefore, include data on the costs and cost-effectiveness of NSHW 
interventions.

The review is limited to LMICs where the need for NSHWs is greater than in high-income settings. The prevalence of psychiatrists and psychiatric nurses is much lower in LMICs (the median number of psychiatrists is 172 times lower in low-income countries (LICs) than high-income countries (HICs) (Kakuma 2011; Mental Health Atlas 2011)) and the organisation and resourcing of mental health services is poorer. These differences in the organisation of mental health services between LMICs and HICs, with poorer countries having little or no mental health service structures in primary care or the community, means that the problem of providing mental health care is different in such settings. NSHWs may need to work with little or no support from specialist mental health services and fewer options for referral. Consequently, NSHWs interventions might be expected to function differently in many LMICs compared with HICs.

\section{Why it is important to do this review}

The continuing shortage of specialist human resources for health in LMICs has made the need to involve non-specialists in MNS healthcare provision more urgent. Reliable evidence is needed on the effectiveness of NSHWs and OPHRs in scaling up mental health interventions, including for the detection, treatment and rehabilitation of MNS disorders. This systematic review will provide the evidence needed to inform policy development for the sustainable scaling up of mental health services in LMICs (Cohen 2003; Murthy 2008).

The intention of this review is to examine which non-specialised cadres of healthcare providers can effectively deliver different aspects of treatment interventions.

\section{O B J E C T I V E S}

To assess the effectiveness of the delivery of mental, neurological and substance abuse (MNS) interventions by non-specialist health workers (NSHWs) and other professionals with health roles (OPHRs) in LMICs. This includes the effects on patient and health delivery outcomes of NSHWs and OPHRs:

- delivering acute MNS interventions;

- delivering long-term follow-up and rehabilitation for people with MNS disorders;

- detecting MNS disorders.

For each of these areas, we have also examined the impacts of delivery by NSHWs and OPHRs on the resource use and costs associated with MNS healthcare provision in LMICs.

\section{MET HODS}

\section{Criteria for considering studies for this review}

\section{Types of studies}

We included randomised controlled trials (RCT), non-randomised controlled trials (NRCT), controlled before-and-after (CBA) studies and interrupted time series (ITS) studies. We only included CBAs with at least two control sites and two intervention sites. We included controlled and non-controlled ITS that had at least three time points before the intervention and three time points after the intervention (as per the Cochrane Effective Practice and Organisation of Care (EPOC) review group criteria) (Ballini 2010). We only included studies conducted in LMICs, as defined by the World Bank.

We also included economic studies conducted as part of included effectiveness studies. We considered full economic evaluations (cost-effectiveness analyses, cost-utility analyses or cost-benefit analyses), cost analyses or comparative resource utilisation studies. We extracted and reported only cost and resource usage outcomes from these studies.

\section{Types of participants}

We included children (aged below 18 years) or adults with any MNS seeking first-level care/primary care or who were detected in the community in LMICs. Additionally we included carers of people with MNS disorders (i.e. any relative or friend of any age who defined themselves as a key supporter to a person with an MNS disorder) as some interventions may be directed at the carers rather than at patients themselves - for example interventions to alleviate carer burden.

(See Table 1 for further definitions of participants, 'LMIC' and 'primary care'.)

\section{Types of interventions}

Clinical (medical and psychological) and service interventions delivered in primary care or the community by NSHWs or OPHRs, and intended to improve MNS disorders were included (see Table 1 for definitions of OPHR and NSHW and types of interventions). We did not include social interventions (such as income generation or general social support) if the trial did not also include a specific MNS intervention.

We included interventions delivered for any MNS disorder. Acute interventions delivered by NSHWs/OPHRs could include various forms of psychotherapy or pharmacological treatment. Long-term interventions delivered by NSHWs/OPHRs could include roles in follow-up or rehabilitation of people with chronic severe mental 
disorders, and roles in detecting and dealing with relapse/recurrence, compliance issues, side effects of treatment or psychosocial problems.

We considered the following comparisons:

- provision of MNS care by NSHWs/OPHRs with some

MNS care training compared with usual/no care;

- provision of MNS care by NSHWs/OPHRs trained and supervised in MNS care (i.e. the highest level of training for NSHWs) compared with mental health specialists in primary care and the community;

- provision of MNS care by NSHWs/OPHRs with some MNS care training compared with non-trained NSHWs/ OPHRs.

We included studies where a specialist teaches NSHW/OPHRs about psychiatric illness and its management. The only interventions of this type that we excluded were those where there were no patient outcomes (i.e. where they only assessed knowledge or attitude changes, such as pre-post training interventions).

We included studies that considered the effect of detection, screening or case-finding of MNS disorders by NSHWs or OPHRs on subsequent patient and health provider outcomes, compared with NSHWs/OPHRs not actively detecting cases, or where specialists did the detection.

The identification methods used by NSHWs could include 'naturalistic' detection (i.e. detection in the course of a routine clinical consultation), or detection using a validated screening/detection tool (e.g. in the context of a trial). We did not examine diagnostic accuracy between these NSHWs and specialists, as this was likely to be confounded by the screening/detection tools used. Therefore, it would be difficult to differentiate between the effect of the screening tool and the skills of the health worker (specialist or non-specialist).

\section{Types of outcome measures}

We organised these outcomes into categories drawing on the Cochrane Consumers and Communication Review Group's outcome taxonomy (La Trobe 2008), and consultation with co-reviewers and service users from the Movement for Global Mental Health discussion board. Where studies reported more than one measure for each relevant outcome, we abstracted the primary or main measure (as defined by the study authors). We separately documented the other measures used, as necessary.

We grouped outcomes into two sets of time points:

- up to six months post intervention (to detect illness recovery/symptom reduction);

- six to 12 months post intervention (which indicates medium- to long-term avoidance of recurrence and chronicity).

For depression and other common mental disorders, we did not group results up to three months post intervention. This time point would normally elicit whether the length of a depressive episode would be shortened compared with spontaneous recovery (which occurs for $50 \%$ of people with depression at three months after treatment initiation and for $65 \%$ of people with depression at six months) (Spijker 2002). However, most of these studies had very variable lengths of interventions (zero to 18 months) and it was difficult to ascertain how long the depression had been present when treatment started (we could assume that people who have not recovered naturally within three months seek help). Pooled results up to three months post intervention would, therefore, not reflect whether the intervention shortened recovery from depression to less than or equal to a spontaneous recovery.

\section{Primary outcomes}

1. Improvement of symptoms (e.g. level of anxiety, depression, psychosis).

2. Psychosocial functioning and impairment (e.g. levels of self esteem, perception of coping, level of dependency, self care ability).

3. Quality of life outcomes (including disability).

We changed the definitions of outcomes 2 and 3 during our analysis from those stated in the protocol, as many scales measured both impairment and functioning and were considered part of the same spectrum. Quality of life outcomes were deemed different from outcomes related to psychosocial functioning as the former encompass a summary of many other aspects of life in addition to psychosocial functioning.

For the detection component of the review, we aimed to consider the outcomes for the patient, the carer, the health provider, or a combination of these people, not the accuracy of diagnosis among NSHWs, compared with specialists, as this is likely to be confounded by the screening/detection tools used. Therefore, it would be difficult to differentiate between the effect of the screening tool and the skills of the health worker (specialist or non-specialist). We did not base inclusion decisions on whether a reference or validated standard measure (either a screening instrument or psychiatric assessment) had been used in studies to differentiate between those correctly and incorrectly diagnosed by NSHWs, but this featured as part of the assessment of the quality of evidence (within study limitations).

\section{Secondary outcomes}

\section{For studies evaluating the detection of mental disorders and the delivery of acute and chronic mental health interventions}

\section{Patient/carer-oriented outcomes and societal outcomes}

- Patient or carer satisfaction and involvement in decisionmaking processes. 
- Patient health behaviour outcomes: such as rates of patient adherence or treatment/follow-up compliance, utilisation of primary level services.

- Adverse clinical outcomes: such as adverse effects rates, suicide/deliberate self harm rates, relapse or recurrence, hospital admission/readmission rates.

- Patient social outcomes: return to work, offending rates, perception of social inclusion.

- Carer outcomes: such as mental health outcomes, quality of life and functioning.

\section{Health provider and service delivery related outcomes}

- Measures of changes in management (such as referral rates, prescribing patterns and appropriateness).

- Measures of health worker behaviour (such as improvement in knowledge/skills, attitude/acceptability, retention rates, absenteeism).

- Measures of service delivery change (such as number of supervision sessions, effect on other health services provided).

\section{For studies of costs and resource use}

We considered:

- direct and indirect costs to the patient and health services

(including opportunity costs);

- resource use (such as the patient's lost productivity, and health service personnel's time allocated/number of consultations).

The economic outcome measures considered were informed by the training material of, and discussion with, the Campbell \& Cochrane Economics Methods Group (CCEMG 2010). We included only measures related to resource use and costs in this review. We recognise that costs and resource use are intertwined but divided the outcomes in this way to make it clear which outcomes we intended to assess.

\section{Search methods for identification of studies}

\section{Electronic searches}

We searched the following electronic databases for primary studies:

- the Cochrane Central Register of Controlled Trials

(CENTRAL) 2012, Issue 6 (including the Cochrane EPOC

Group Specialised Register (searched 21 June 2012);

- MEDLINE, 1946 to June week 1 2012, OvidSP (searched

15 June 2012);

- MEDLINE In-Process \& Other Non-Indexed Citations 14

June 2012, OvidSP (searched 15 June 2012);

- EMBASE, 1980 to 2012 week 23, OvidSP (searched 15

June 2012);
- CINAHL (Cumulative Index to Nursing and Allied Health Literature), 1980 to 19 June 2012, EBSCOhost (searched 19 June 12);

- PsycINFO, 1806 to June week 2 2012, OvidSP (searched 18 June 2012);

- Latin American and Caribbean Health Sciences database (LILACS), Virtual Health Library (VHL) (searched 9 August 2012);

- WHO Global Health Library (World Health Organization Library Information System (WHOLIS), AIM (AFRRO), IMEMR (EMRO), IMSEAR (SEARO, WPRIM, WPRO) (searched 29 June 2012);

- Science Citation Index and Social Sciences Citation Index, ISI Web of Knowledge (searched 2 October 2012).

The EPOC Trials Search Co-ordinator (TSC) (Marit Johansen), in consultation with the authors, developed the search strategies. Search strategies were comprised of keywords and controlled vocabulary terms (selected index terms and free-text terms relating to NSHWs and mental health).

We applied no language limits. We searched all databases from database start date to date of search.

We used a combination of two methodology search filters to limit retrieval to appropriate study designs: a modified version of the Cochrane Highly Sensitive Search Strategy (sensitivity- and precision-maximising version - 2008 revision) to identify RCTs (cf. Cochrane Handbook for Systematic Reviews of Interventions Section 6.4d); and an EPOC methodology filter to identify NRCT designs.

\section{Searching other resources}

\section{Grey Literature}

- OpenGrey www.opengrey.eu/ (searched 9 August 2012).

\section{Trial Registries}

- metaRegister of Controlled Trials (mRCT) ( www.controlled-trials.com/mrct/) (searched 8 August 2012).

- International Clinical Trials Registry Platform (ICTRP), WHO (apps.who.int/trialsearch/) (searched 9 August 2012).

We also searched:

- the reference lists of existing reviews (De Vet 2008);

- other grey literature (unpublished material), through contacting experts;

- conducted cited reference searches for all included studies in ISI Web of Knowledge.

We did not search for economic analyses. We retrieved potentially eligible economic analyses when screening records generated from 
the various searches reported above, but only selected those performed alongside identified effects studies. We contacted the authors of all included effects studies for information on any published or unpublished economic studies related to their trials. We also scanned the reference lists of eligible trials and economic analyses (where these were reported separately to the eligible trials), and other related reviews and papers, for further eligible studies. See Appendix 1 for all search strategies used.

\section{Data collection and analysis}

\section{Selection of studies}

Four review authors (NvG, GR, MSM, JP) and a Chinese researcher for the Chinese included study double-screened all records obtained from the searches. We retrieved full-text copies of all articles identified as potentially relevant by at least one review author. Two review authors checked each full paper for inclusion criteria. We resolved disagreements on inclusion by discussion. If no agreement was reached, we asked a third review author to make an independent assessment (SL). Where appropriate, we contacted the study authors for further information.

\section{Data extraction and management}

Five review authors (NvG, GR, MSM, JP, PT) and the Chinese and Spanish researchers independently extracted descriptive and outcome data for each paper using an adapted version of the EPOC data collection checklist. Two review authors together or by one and cross-checked by another (except the Chinese paper, which relied on one researcher's data extraction only) extracted data. Review authors obtained any missing data by contacting trial authors. Review authors entered the final agreed descriptive extracted data into the relevant tables of characteristics in Review Manager 5 (RevMan 2012). One review author (NvG) entered the checked outcome data into Review Manager 5 for meta-analysis and this was checked by PT (RevMan 2012).

We extracted the following information for all included studies, in the form that this was reported in the original text:

- details of the intervention: the type and length of each of the clinical, psychosocial and service interventions; a full description of cadre(s) of NSHW/OPHRs consulting with the patient, including details of their training and supervision/support; and the length, frequency and type of intervention delivered by each NSHW/OPHR; description of the specialist providing care (type, experience, training in using reference standard);

- participants: a full description of the participants (sex, age, socioeconomic status, ethnicity), including details of the MNS condition being treated;

- setting: country; type of health service (e.g. government funded, $\mathrm{NGO}$, etc.), organisation of the primary care and specialist services; specialist outreach or generalist;
- results: organised into patient, provider and process outcomes (see above).

\section{Assessment of risk of bias in included studies}

Five review authors (NvG, GR, MSM, JP, PT) and the Chinese researcher working in pairs independently assessed each study for risk of bias. NvG and PT independently checked assessments for all studies. We followed the Cochrane EPOC group format (Ballini 2010) (which follows the Cochrane Collaboration approach (Higgins 2009)) to assess risk of bias for each of the study designs (RCT, CBA, NRCT, ITS). For two of the EPOC risk of bias criteria, we did the following:

- divided detection bias into two categories, assessing whether subjective (requiring a judgement, such as clinical improvement) and objective outcomes (such as number of hospitalised days, etc.) were assessed blindly;

- assessed attrition bias for two types of outcome: efficacy outcomes and safety outcomes (e.g. adverse events and unintended consequences).

For economic studies, we adapted the Consensus on Health Economic Criteria (CHEC) criteria list (see Appendix 2) to include an extra question on the sources of data used, and we excluded some questions that were already covered as part of the main risk of bias assessment described above.

We incorporated risk of bias assessments by generating 'Risk of bias' summary graphs and figures using Review Manager 5 (RevMan 2012).

\section{Measures of treatment effect}

\section{Measures of intervention effect regarding clinical (medical and psychological) and service interventions}

For dichotomous outcomes, we used risk ratios (RR). For continuous outcomes, we used the mean difference (MD), standardised mean difference (SMD) or mean change difference (MCD). We expressed all effect estimates with their $95 \%$ confidence intervals (CI). For SMDs, we used the Cochrane Handbook for Systematic Reviews of Interventions to interpret their clinical relevance: 0.2 represented a small effect, 0.5 a moderate effect, and 0.8 a large effect (Cohen 1988). We attempted to establish minimally important differences per outcome (as suggested in Guyatt 2013) but this was not possible due to the wide variety of instruments used.

\section{Measures of effect of detection of MNS disorders interventions}

We aimed to report the effects of detection of MNS disorders by NSHWs or OPHRs by assessing patient outcomes, looking at the proportion of patients who recovered or improved over a specific length of time as described in the included studies. We 
aimed to measure health worker outcomes by examining changes in prescribing rates, referral rates and treatment initiation rates.

\section{Unit of analysis issues}

Where possible, we re-analysed studies that randomised or allocated clusters (patients, health professionals, healthcare settings or geographical areas) but did not account for clustering in their analysis (Ukoumunne 1999). We adjusted the results for clustering by multiplying the standard errors of the estimates by the square root of the design effect where the design effect is calculated as DEff $=1+(\mathrm{M}-1)$ ICC, where $\mathrm{M}$ is the mean cluster size and ICC is the intracluster correlation coefficient. All of the included studies reported the ICCs that we needed.

We combined the adjusted measures of effects of cluster-randomised trials with the results of non-cluster trials, if it was possible to adjust adequately the results of the cluster trials. There were too few studies per meta-analysis to perform sensitivity analyses comparing the effects estimates with and without the inclusion of the cluster trials.

We contacted authors when we needed additional information for the analysis.

\section{Dealing with missing data}

For missing or unclear information, we contacted the study investigators for clarification or additional information. We were able to access all required authors for the purpose of statistical information. Some remaining missing information on the qualitative description of the interventions that we did not get despite several attempts at following up with study authors, is highlighted in the Characteristics of included studies tables. To reduce the risk of overly positive answers, we use open-ended questions (as recommended in the Cochrane Handbook for Systematic Reviews of Interventions, Higgins 2009).

Where possible, we extracted data to allow an intention-to-treat (ITT) analysis in which all randomised participants were analysed in the groups to which they were originally assigned. If ITT data were not present, where possible, we did a full ITT analysis where we considered four scenarios in which the people reassigned to the control and intervention groups either had the condition or not. For studies that reported continuous data but did not report standard deviations, we either calculated these from other available data such as standard errors, or imputed these using the methods suggested in Higgins 2009. We did not make any assumptions about loss to follow-up for continuous data and we analysed results for those who completed the trial.

\section{Assessment of heterogeneity}

We first made a qualitative assessment of the extent to which the studies assessing a particular comparison where similar to one another. This included an assessment of the settings, the interventions, the participants and outcomes to determine whether meta- analysis was appropriate. We obtained an initial visual overview of statistical heterogeneity through scrutinising the forest plots, looking at the overlap between CIs around the estimate for each included study. To quantify the inconsistency across studies, and thus the impact of heterogeneity on the meta-analysis, we used the $\mathrm{I}^{2}$ statistic, and defined an $\mathrm{I}^{2}$ greater than $50 \%$ as indicative of substantial heterogeneity. We then considered these assessments when interpreting the results of a pooled analysis: the importance of an observed $\mathrm{I}^{2}$ was interpreted in light of 1 . the magnitude and direction of effects and, 2. the strength of evidence for heterogeneity (e.g. a CI for the $\mathrm{I}^{2}$, or the $\mathrm{P}$ value from the $\mathrm{Chi}^{2}$ test).

\section{Assessment of reporting biases}

To reduce possible publication bias, we employed strategies to search for and include relevant unpublished studies. These strategies included searching the grey literature and prospective trial registration databases to overcome time-lag bias.

We used funnel plots for the outcomes with more than four studies to visualise whether there was asymmetry. None of them showed asymmetry. We performed no statistical testing for funnel plot asymmetry as none of the pooled outcomes included more than 10 studies.

\section{Data synthesis}

We grouped the studies for comparison by type of disorders (common mental disorders, severe mental disorders, neurological and substance-abuse disorders); by mix of healthcare providers (NSHW-led, collaborative, NSHWs and OPHRs); and by types of community intervention (pharmacological, non-pharmacological and mixed approach). We did this as these categories fit with current models of service delivery in LMICs.

The number of comparisons was larger than anticipated at the protocol stage and we have outlined each comparison in the results section below. For each comparison (groups of disorders), we created tables of summary statistics according to study designs (RCTs, NRCTs and CBAs). These tables included study design, baseline and follow-up summary statistics, effect estimates and their statistical significance. We used forest plots to display the data graphically.

Where the outcomes assessed and the settings and interventions were very diverse (as agreed by at least two review authors), we did not consider it appropriate to combine the results quantitatively. For these results, we have presented a descriptive summary of data. For all data syntheses, we used the generic inverse-variance model of analysis as this allows the analysis of continuous and dichotomous data and allows clustered and non-clustered data to be combined. We based the choice of whether to use a fixed-effect or random-effects model on the extent to which studies were similar, or homogeneous, based on their PICOS characteristics (population, intervention, comparators, outcomes and settings). No studies were homogeneous enough to apply the fixed-effect model. 
We reported the results separately for RCTs and for NRCTs. No ITS studies were included in the review. We used effect estimates adjusted for confounding (baseline differences in control and intervention groups) where possible, and used the methods described in Reeves 2009 to guide data synthesis.

\section{Economic data}

We conducted all the elements of the economics component of this review according to current guidance on the use of economics methods in the preparation and maintenance of Cochrane reviews (Shemilt 2009). We classified the included economic evaluations based on an established system (Drummond 2005). We summarised the characteristics and results of included economic evaluations using additional tables, supplemented by a narrative summary that compared and evaluated methods used and principal results between studies.

We displayed resource use and cost data in a table, along with unit cost data (where available). A unit cost was defined as the cost of each specific resource input calculated by multiplying the measured number of units (quantities) of an item of resource use (e.g. the number of hours of time provided by a senior teacher) by an applicable unit cost (e.g. the salary cost of one hour of senior teacher time). We reported the currency and price year applicable to measures of costs and unit costs in each original study. Measures of costs are highly likely to vary across and within study settings, and over time. This is the product of variations in the underlying quantities of resource use and variations in the underlying unit costs.

Because the data on resource use and costs were very heterogeneous, meta-analysis was not appropriate and we presented the findings narratively. We discussed the limitations of this approach below.

\section{Subgroup analysis and investigation of heterogeneity}

Within each comparison, the following subgroups were considered: by category of health worker (professionals: e.g. doctors, nurses), OPHRs and non-professionals (LHWs); by types of community intervention (e.g. collaborative versus psychological interventions in comparison 3); and by setting (government versus non-government). We were not able to perform subgroup analyses to check if the intervention effect varied with different population characteristics as the number of included studies for each comparison was not sufficient. Where applicable, we have described subgroup differences narratively under Main results.

For random-effects meta-analyses, we used the formal $\mathrm{Chi}^{2}$ test and $\mathrm{I}^{2}$ statistic for subgroup differences in RevMan 2012 to detect statistically significant subgroup differences.

\section{Sensitivity analysis}

It was not possible to compare intervention effects according to risk of bias using meta-regression due to insufficient data. We conducted sensitivity analyses based on attempting to reduce clinical heterogeneity.

\section{Summarising and interpreting results}

We used the GRADE approach to assess the quality of evidence related to each of the key outcomes (Schünemann 2009). We used the GRADE profiler (GRADE 2007), to import data from Review Manager 5 (RevMan 2012) and create 'Summary of findings' tables.

For assessments of the overall quality of evidence for each outcome that included pooled data from RCTs only, we downgraded the evidence from 'high quality' by one level for serious (or by two for very serious) study limitations (risk of bias), indirectness of evidence, serious inconsistency, imprecision of effect estimates or potential publication bias. Data from observational studies started at low quality. None were upgraded to moderate or high quality as no pooled estimates revealed a large magnitude of effect, negligible concerns about confounders or a strong dose-response gradient.

We used these assessments, along with the evidence for absolute benefit or harm of the interventions and the sum of available data on all critical and important outcomes from each study included for each comparison, to draw conclusions about the effectiveness of NSHWs in mental healthcare provision in LMICs. 'Summary of findings' tables consisted of critically important clinical and functional outcomes identified in the selected trials.

When judging the importance of SMDs, we acknowledged that 0.2 represents a slight effect, 0.5 a moderate effect, and 0.8 a significant effect; and chose a threshold of 0.5 to indicate a minimum clinically important difference (Guyatt 2008; Higgins 2011).

\section{RES U L T S}

\section{Description of studies}

\section{Results of the search}

We included 38 studies in this review. Including the four consecutive searches performed in January 2011, May 2011, June 2012 and August 2012, we screened 11,825 titles and abstracts (excluding duplicates), of which we sourced 739 full texts to check inclusion criteria and we sourced 90 relevant references to screen their bibliographies (Figure 1). 
Figure I. Study flow diagram.

12,545 records identified through database searching

16 additional records identified through other sources

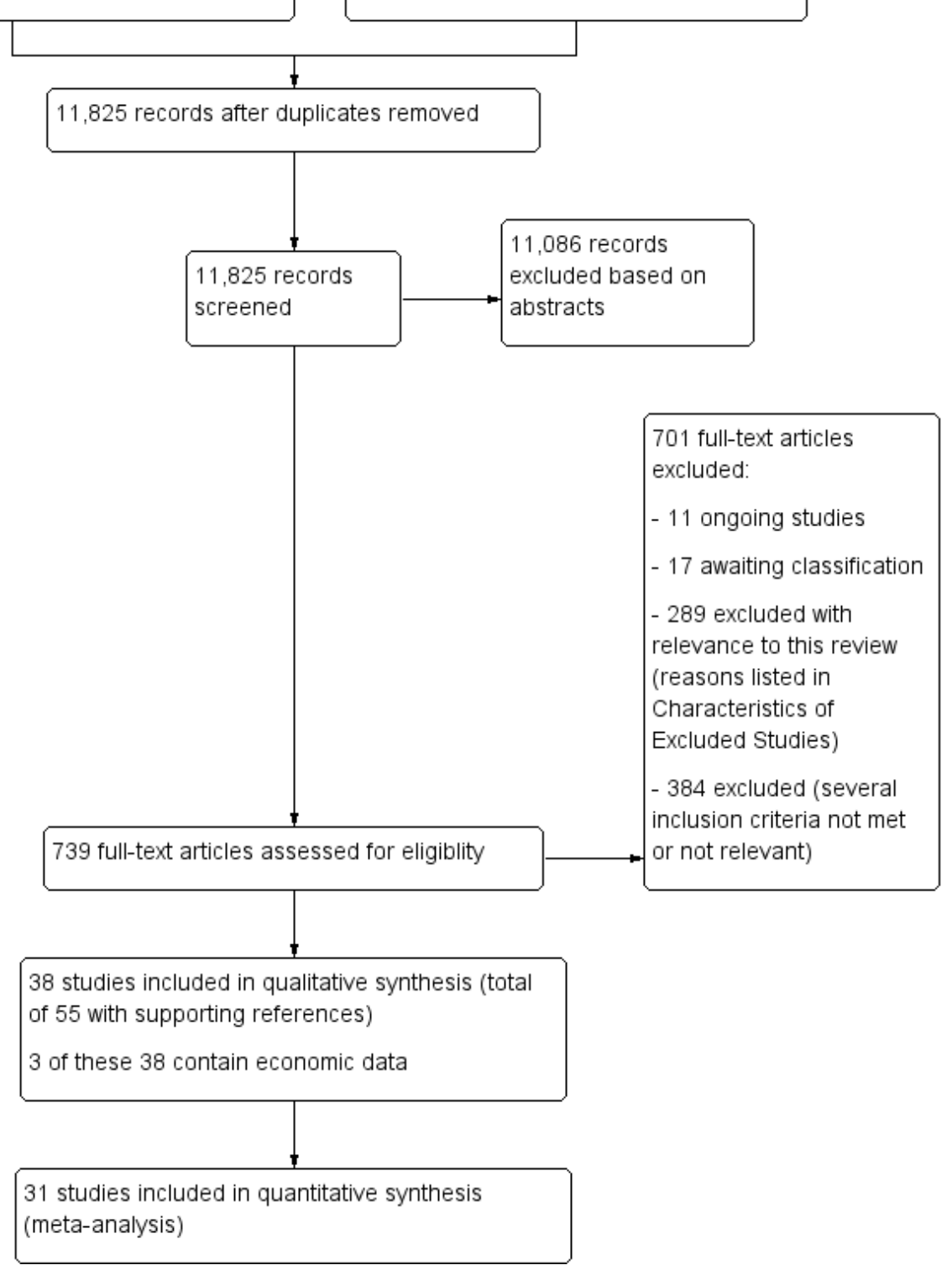

Non-specialist health worker interventions for the care of mental, neurological and substance-abuse disorders in low- and middle- 


\section{Included studies}

\section{Study design}

Of the 38 included studies, 17 were RCTs, 10 were cluster RCTs, nine were CBA studies and two were NRCTs. Analysis was by ITT in eight studies (Bolton 2007 RCT Uganda; Ertl 2011 RCT Uganda; Hirani 2010 CRCT Pakistan; Jenkins 2012 C-RCT Kenya; Jordans 2010 C-RCT Nepal; Tiwari 2010 RCT China; Tol 2008 C-RCT Indonesia; Tol 2012 C-RCT SriLanka), and was unclear in one (Neuner 2008 NRCT Uganda). It was not possible to do an ITT for the remaining studies (see Dealing with missing data).

\section{Setting}

Fifteen included studies were conducted in seven LICs: Burundi (one study), Kenya (two studies), Nepal (one study), Pakistan (three studies), Rwanda (two studies), Sri Lanka (two studies) and Uganda (four studies). Twenty-three studies were from 15 middle-income countries: Argentina (one study), Bosnia (one study), Chile (three studies), China (three studies), Hungary (one study), India (two studies), Indonesia (two studies), Jamaica (one study), Kosovo (one study), Malaysia (one study), Palestinian Territories (two studies), Russia (one study), Thailand (two studies), Turkey (one study) and Vietnam (one study). These LIC and middle-income country assignments are based on the World Bank's classification of countries by gross national income per capita in 2010 . In this section, as well as following sections (participants, interventions, etc.), the numbers when added up may exceed 38 due to double counting. There were 16 studies from rural, 23 from urban and five from refugee camp settings. Most interventions were delivered in community groups/centres (11 studies). Others were delivered at home (nine studies), in primary healthcare (PHC) centres (eight studies), in schools (seven studies) and in other health clinics (three centres).

\section{Participants}

Twenty-seven studies included adults. Of the studies including children, 10 included children up to the age of 12 years, and eight focused on adolescents (aged 12 to 17 years). Most studies covered common mental disorders (18 included depression, anxiety, maternal depression) and PTSD (12 studies). See 'Effects of interventions' for details of these by analysis groups.

\section{Interventions}

NSHWs and OPHRs: various cadres were used: LHWs (22 studies), doctors (nine studies), nurses (six studies), teachers (six studies) and social workers (three studies). The educational level of the LHWs was poorly documented, but of the 15 studies that did specify this, eight selected LHWs with a minimum of secondary school education, three used illiterate LHWs and three included LHWs who had primary school education and who were or were not literate. Remuneration was generally poorly described. The training and supervision of these providers are described in detail under 'Effects of interventions'.

Interventions: many studies combined different types of interventions. The eight interventions providing pharmacotherapy also provided follow-up to check adherence, the effect of medication and side effects (provided by a LHW (four studies), a nurse/clinical officer (one study), a social worker (one study) or a doctor (two studies). Twenty-five studies had some form of psychosocial intervention (which included psycho-education, various support and general counselling/coping skills interventions and stimulation programmes for children). Sixteen studies used specific psychological interventions on their own or as part of a collaborative care model (e.g. cognitive behavioural therapy (CBT), interpersonal therapy (IPT), motivational interviewing). One study evaluated economic skills building as a second arm to the trial, which were expected to have an effect on mental health outcomes. No studies examined detection by NSHWs or OPHRs and none reported health worker outcomes. More details on these are provided under 'Effects of interventions'.

\section{Economic studies}

Three economic studies were conducted alongside included RCTs (Araya 2003 RCT Chile; Jordans 2011 (which is linked to Tol 2008 C-RCT Indonesia; Tol 2012 C-RCT SriLanka) and Zambori 2002 CBA Hungary). One further study noted that the financial burden and severity of schizophrenia decreased marginally for both intervention and control groups, but did not reach statistical significance; however, it did not measure costs (Paranthaman2010CBAMalaysi). In addition, one study mentioned they had collected cost data but results were not yet available before the end of the search period (Patel 2010 C-RCT India). This was subsequently published (Buttorff 2012). We aim to include these data in a future update.

\section{Excluded studies}

We excluded 701 studies, of which 289 were of interest to this area of study but did not fulfill all inclusion criteria. These 289 
studies, together with their reasons for exclusion, are documented in Characteristics of excluded studies.

Thirteen studies that included economic data on MNS conditions, but were not linked to studies included in this review, are reviewed in Appendix 3.

\section{Risk of bias in included studies}

The most often identified biases across studies were allocation concealment, random sequence generation, reliability of primary outcomes and blinding of outcome assessment (Figure 2; Figure 3).

Figure 2. Risk of bias graph: review authors' judgements about each risk of bias item presented as percentages across all included studies.

\begin{tabular}{|r|r|}
\hline Random sequence generation (selection bias) & \\
Allocation concealment (selection bias) & \\
Blinding of participants and personnel (performance bias) & \\
Blinding of outcome assessment (detection bias): objective outcomes & \\
Blinding of outcome assessment (detection bias): subjective outcomes & \\
Baseline outcome measurements similar & \\
Baseline characteristics similar? & \\
Incomplete outcome data (attrition bias): Efficacy data & \\
Incomplete outcome data (attrition bias): Safety data (e.g. adverse events) & \\
Protection against contamination & \\
Reliable primary outcome measures & \\
Selective reporting (reporting bias) & \\
Other bias & \\
\hline Low risk of bias & \\
\hline Unclear risk of bias & \\
\hline High risk of bias
\end{tabular}

Non-specialist health worker interventions for the care of mental, neurological and substance-abuse disorders in low- and middle- 
Figure 3. Risk of bias summary: review authors' judgements about each risk of bias item for each included study.

\begin{tabular}{|c|c|c|c|c|c|c|c|c|c|c|c|c|c|}
\hline & 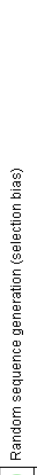 & 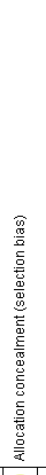 & 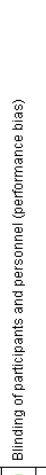 & 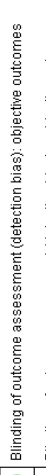 & 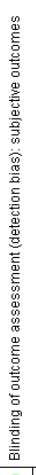 & 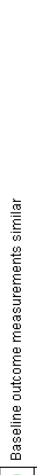 & 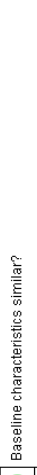 & 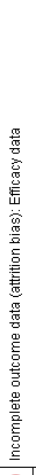 & 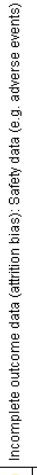 & 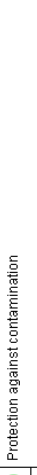 & 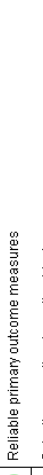 & 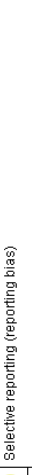 & 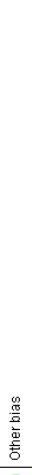 \\
\hline Ali 2003 RCT Pakistan & $\odot$ & $?$ & ๑ & - & ๑ & ๑ & (†) & ? & $?$ & † & ๑) & $?$ & † \\
\hline Araya 2003 RCT Chile & $\oplus$ & $\odot$ & $\odot$ & $\odot$ & 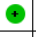 & $\odot$ & $\odot$ & $\odot$ & $?$ & - & ๑ & ๑ & 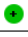 \\
\hline Baker-H 2005 CRCT Jamaica & $\odot$ & ๑ & ๑) & $\odot$ & $\odot$ & ๑ & 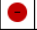 & $\odot$ & ? & † & - & ๑) & ๑ \\
\hline Bass 2012 CBA Indonesia & (2) & - & ๑ & 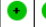 & (†) & - & $\odot$ & (†) & ○ & (†) & ○ & $?$ & - \\
\hline Berger2009 CRCT SriLanka & $\odot$ & ○ & $\odot$ & $\odot$ & $\odot$ & $\odot$ & $\odot$ & $\odot$ & $?$ & ○ & ○ & $?$ & ○ \\
\hline Bolton 2003 C-RCT Uganda & $(+$ & $?$ & $\odot$ & $\odot$ & $\odot$ & $\odot$ & $\odot$ & $\odot$ & $?$ & (†) & $\odot$ & • & ๑ \\
\hline Bolton 2007 RCT Uganda & $\odot$ & $?$ & $\odot$ & + & $\odot$ & $\odot$ & - & $\odot$ & $?$ & ○) & $\odot$ & † & † \\
\hline Brown 2009 CBA Rwanda & - & ○ & $\odot$ & $\odot$ & $?$ & $\odot$ & $\odot$ & ? & $?$ & † & ? & ๑ & ? \\
\hline Chen 2000 RCT Taiwan & $?$ & $?$ & 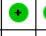 & $\oplus$ & ? & $\odot$ & $\odot$ & ? & $?$ & $?$ & † & $?$ & (†) \\
\hline Dias 2008 RCT India & $\odot$ & $\odot$ & $\odot$ & + & $\odot$ & $\odot$ & $\odot$ & $\odot$ & $?$ & $?$ & ○ & $\odot$ & $\odot$ \\
\hline Dybdahl 2001 RCT Bosnia & $\odot$ & $?$ & $?$ & $\odot$ & $\odot$ & $\odot$ & $?$ & 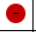 & $?$ & $?$ & ○ & • & ○ \\
\hline Ertt 2011 RCT Uganda & $?$ & $?$ & $?$ & ๑ & ๑ & (†) & ๑ & † & $\odot$ & ๑ & $?$ & † & (†) \\
\hline Fritsch $2007 \mathrm{RCT}$ Chile & $\odot$ & + & $\odot$ & $\odot$ & + & $\odot$ & $\odot$ & $?$ & ? & $?$ & $\odot$ & ๑) & ๑ \\
\hline Gavrilova 2009 RCT Russia & $\odot$ & $?$ & ๑ & 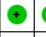 & $\odot$ & $\odot$ & $\odot$ & + & $?$ & ๑ & $?$ & ๑) & ๑ \\
\hline Gordon 2008 RCT Kosovo & $\odot$ & ○ & $\odot$ & $\odot$ & $?$ & ( & $\odot$ & $\odot$ & $\odot$ & $?$ & $\odot$ & + & (†) \\
\hline Hirani 2010 CRCT Pakistan & + & $?$ & $\odot$ & + & $?$ & $?$ & + & $?$ & $?$ & (†) & $?$ & $?$ & ๑ \\
\hline Jenkins 2012 C-RCT Kenya & $\odot$ & $\odot$ & 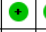 & $\odot$ & $\odot$ & $\odot$ & $\odot$ & $\odot$ & † & (†) & - & (†) & ๑ \\
\hline Jordans 2010 C-RCT Nepal & $\odot$ & ○ & $\odot$ & $\odot$ & ○ & $?$ & $\odot$ & 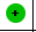 & $\odot$ & ๑) & $\odot$ & $\odot$ & $\odot$ \\
\hline Li 1989 RCT China & $?$ & $?$ & $\odot$ & 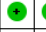 & $\odot$ & $?$ & $?$ & $\odot$ & 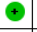 & ๑) & $?$ & † & ๑ \\
\hline Loughny 2006 CBA Palestin & (?) & ○ & $?$ & - & ○ & ○ & - & $?$ & $?$ & ○) & $\odot$ & ๑) & (†) \\
\hline Lyketsos1999CBA Argentina & ? & - & $?$ & $\odot$ & ? & $\odot$ & - & $\odot$ & ๑) & $?$ & $\odot$ & $?$ & $\odot$ \\
\hline Neuner 2008 NRCT Uganda & ○ & ○ & - & $\odot$ & + & $?$ & ○ & $\odot$ & † & - & • & $?$ & + \\
\hline Noknoy 2010 RCT Thailand & (†) & 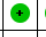 & $\odot$ & $\odot$ & ○ & $\odot$ & ( & $\theta$ & ? & () & ? & (†) & ○ \\
\hline Papas 2011 RCT Kenya & + & + & $\odot$ & $\odot$ & $?$ & $\odot$ & $\odot$ & $\odot$ & $?$ & (†) & ๑ & ๑ & ๑ \\
\hline Paranthaman 2010CBAMalaysi & ○ & 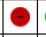 & $\odot$ & $\odot$ & ○ & $\odot$ & - & $\odot$ & 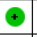 & - & ๑) & + & $\odot$ \\
\hline Patel 2010 C-RCT India & + & + & $\odot$ & + & + & $\odot$ & + & $?$ & (†) & (-) & + & † & $\odot$ \\
\hline Rahman 2008 CRCT Pakistan & + & $\odot$ & $\odot$ & $\odot$ & $?$ & $\odot$ & $\odot$ & $\odot$ & $?$ & (†) & $\odot$ & + & ๑ \\
\hline Rojas 2007 RCT Chile & $\odot$ & 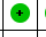 & ๑) & $\odot$ & ( & $\odot$ & (†) & (†) & $?$ & † & ๑ & () & † \\
\hline Scholte 2011 CBA Rwanda & $\ominus$ & ○ & $\odot$ & $\odot$ & + & $\odot$ & $?$ & $?$ & $\odot$ & (†) & $?$ & $?$ & + \\
\hline Shin 2009 RCT Vietnam & $\odot$ & $?$ & $\odot$ & $\odot$ & ○ & $\odot$ & $\odot$ & $\odot$ & $?$ & ๑) & $\odot$ & ๑) & $\odot$ \\
\hline Sutcliffe2009RCT Thailand & ○ & $?$ & $\odot$ & $\odot$ & ○ & ○ & ○ & † & $?$ & ○ & ○ & $?$ & ○ \\
\hline Thabet 2005 CBA Palestine & - & ○ & $\odot$ & $\odot$ & $?$ & ○ & $\odot$ & $\odot$ & $?$ & ๑) & + & ๑) & $\odot$ \\
\hline Tiwari 2010 RCT China & $(+$ & $\odot$ & $\odot$ & $\odot$ & $\odot$ & $\odot$ & $\odot$ & $\odot$ & $?$ & (†) & + & • & $\odot$ \\
\hline Tol $2008 \mathrm{C}-\mathrm{RCT}$ Indonesia & $\oplus$ & + & + & + & ○ & + & $\odot$ & $\odot$ & $?$ & (4) & + & † & (†) \\
\hline Tol 2012 C-RCT SriLanka & $?$ & $?$ & $\odot$ & $\odot$ & $?$ & $\odot$ & $\odot$ & $\odot$ & $\odot$ & ๑) & - & $?$ & (†) \\
\hline Wolmer 2005 CBA Turkey & $\theta$ & ○ & + & $\oplus$ & + & $?$ & $\odot$ & $\Theta$ & (†) & (†) & - & (†) & (†) \\
\hline Yeomans 2010 RCT Burundi & + & $\oplus$ & $\odot$ & $\odot$ & + & $(+$ & (๑) & $\odot$ & $?$ & ๑) & $?$ & $?$ & (†) \\
\hline Zambori 2002 CBA Hungary & $\theta$ & ○ & \begin{tabular}{|c|c|}
+ & \\
\end{tabular} & $\odot$ & $\theta$ & $\theta$ & ○ & $?$ & (†) & (†) & $\odot$ & () & (†) \\
\hline
\end{tabular}

Non-specialist health worker interventions for the care of mental, neurological and substance-abuse disorders in low- and middleincome countries (Review)

Copyright $\odot 2013$ The Cochrane Collaboration. Published by John Wiley \& Sons, Ltd. 


\section{Allocation}

Only 13 of the 38 included studies met the 'low risk of bias' criteria for allocation concealment. Of the remaining studies, 13 explicitly did not conceal allocation (of which 10 were not RCTs). For 12 studies, the risk of bias was unclear due to poor reporting.

Eleven studies did not utilise randomised sequence generation. One RCT was also at high risk of bias with regard to allocation sequence generation because they had a combination of random and non-random sequence generation (Sutcliffe2009RCT Thailand). Several studies did not have similar subjective or objective outcome measurements (such as numbers of days in hospital) at baseline between the two arms (subjective outcomes: seven unclear and 10 not similar; objective outcomes: five unclear and five not similar) or did not have similar baseline characteristics (seven not similar and three unclear). The studies in which two or three of the baseline characteristics were not similar included the following CBA studies (Loughry 2006 CBA Palestin; Lyketsos 1999CBA Argentina; Paranthaman2010CBAMalaysi; Thabet 2005 CBA Palestine; Zambori 2002 CBA Hungary), and RCTs (Li 1989 RCT China; Sutcliffe2009RCT Thailand).

\section{Blinding}

We divided the blinding domain into blinding of participants and personnel, and blinding of outcome assessment. All studies reported blinding of outcome assessment, one study did not blind participants/personnel (Neuner 2008 NRCT Uganda), and for four studies it was unclear if participants/personnel were blinded (Dybdahl 2001 RCT Bosnia; Ertl 2011 RCT Uganda; Loughry 2006 CBA Palestin; Lyketsos1999CBA Argentina).

\section{Incomplete outcome data}

We considered incomplete outcome data separately for efficacy and for adverse outcomes. For most studies, outcome data were complete. However, for six studies, this was unclear and seven had incomplete outcome data. Twenty-two studies did not clearly report whether they had data on adverse outcomes, and an additional four studies stated explicitly that they had not collected adverse outcome data (or we obtained this information from the authors). This made analysis of adverse outcomes difficult for most comparisons.

\section{Selective reporting}

For 26 of the 38 studies, there appeared to be no selective reporting, based on the outcomes listed in the methods section of these papers, and from contacting authors where there was doubt. In only one study was it clear that there had been selective reporting (Dias 2008 RCT India). In 11 studies, this was not clear (see Characteristics of included studies tables).

\section{Other potential sources of bias}

Risk of contamination was quite common among both RCTs and CBA studies. We assessed six studies as unclear because insufficient information was available regarding whether contamination across groups was likely and conclusive information on this from the authors could not be obtained (Chen 2000 RCT Taiwan; Dias 2008 RCT India; Dybdahl 2001 RCT Bosnia; Gavrilova 2009 RCT Russia; Hirani 2010 CRCT Pakistan; Li 1989 RCT China). We assessed an additional six studies as being at high risk of contamination (Araya 2003 RCT Chile; Berger2009 CRCT SriLanka; Bolton 2007 RCT Uganda; Loughry 2006 CBA Palestin; Neuner 2008 NRCT Uganda; Sutcliffe2009RCT Thailand).

For a number of studies, it was not clear whether the primary outcome measures were reliable: in 11 studies, these measures were not validated in the study context; and we assessed an additional six studies as 'unclear' because insufficient information was available on the validity of the measures.

Other sources of bias that were detected included:

- the control and intervention arms potentially delivering interventions that were too similar, as mentioned by the authors (Sutcliffe2009RCT Thailand);

- high likelihood of confounding: for example, due to incentives being provided to patients (Brown 2009 CBA Rwanda), or a teetotal religious festival occurring between baseline and follow-up that may have had a greater impact on alcohol consumption than the motivational interviewing intervention in Noknoy 2010 RCT Thailand.

\section{Economic studies - risk of bias assessment with the adapted} CHEC list criteria

All studies had significant risks of bias (Table 2), although we considered no study at high risk of bias on more than seven of the 23 adapted CHEC list criteria. The risk of biases identified were potentially important for the interpretation of costing, such as not discounting costs (Araya 2003 RCT Chile; Jordans 2011), not including the appropriate costs or outcomes and not valuing some outcomes appropriately.

\section{Effects of interventions}

See: Summary of findings for the main comparison NSHW-led psychological interventions compared with usual care in treating depression in adults in low- and middle-income countries (RCTs); Summary of findings 2 Collaborative care model (NSHWs 
plus specialist) compared with usual care in treating common mental disorders in adults in low- and middle-income countries (RCTs); Summary of findings 3 NSHWs compared with usual care for treating maternal depression (RCTs); Summary of findings 4 NSHWs compared with specialists in treating depression in adults in low- and middle-income countries (CBAs); Summary of findings 5 NSHW-led psychological interventions compared with usual care in treating adults with PTSD (NRCT); Summary of findings $\mathbf{6}$ NSHWs compared with usual care in improving dementia patients' and carers' outcomes in lowand middle-income countries (RCTs); Summary of findings 7 NSHW-led brief alcohol interventions compared with usual care for adults with alcohol-use disorders (RCTs); Summary of findings $8 \mathrm{NSHWs}$ /OPHRs compared with usual care in conducting interventions for children with post-traumatic stress disorder and depression (RCTs)

This review covered a wide range of NSHWs delivering a wide range of healthcare interventions for a variety of MNS disorders. However, no MNS detection studies were found that reported patient outcomes. We grouped studies by MNS disorders as different interventions and roles of NSHWs will in particular differ between severe and common mental disorders. These broad groups have, in turn, been subdivided into types of interventions that made clinical sense to group together (e.g. studies on depression have been divided into those involving collaborative care, where NSHWs are only one aspect of a complex intervention, and those involving psychological interventions provided by just one type of NSHW). We have further grouped studies by study design, and according to their comparator group (usual care or specialist care). We performed meta-analyses for eight groupings covering common mental disorders, PTSD, dementia and alcohol abuse. All analyses include the primary outcomes specified for this review, and some secondary outcomes. Below are the meta-analysis groupings that we have reported:

1. NSHW-led psychological interventions versus usual care in treating common mental disorders in adults (RCTs);

2. collaborative care model (NSHWs plus specialist) versus usual care in treating common mental disorders (RCTs and cluster RCTs);

3. NSHWs versus usual care in treating maternal depression (RCTs);

4. NSHWs versus specialist care in treating common mental disorders (CBA studies);

5. NSHWs versus usual care in delivering PTSD interventions to adults (RCTs);

6. NSHWs versus usual care in improving dementia patients' and carers' outcomes (RCTs);

7. NSHW-led brief alcohol interventions versus usual care in delivering interventions to adults with alcohol-use disorders (RCTs);

8. NSHWs/OPHRs versus usual care in delivering interventions for children with PTSD and depression (RCTs).
We could not pool the remaining studies, as they were individual studies of different disorders (severe mental disorders, epilepsy, drug abuse and child mental disorders other than PTSD and depression). We reported the results of these studies narratively in the text and in Table 3.

\section{Comparison I. Non-specialist health workers-led psychological interventions versus usual care in treating common mental disorders in adults (RCTs)}

Setting: we identified seven studies from four countries: China (two studies) (Chen 2000 RCT Taiwan; Tiwari 2010 RCT China), Jamaica (one study) (Baker-H 2005 CRCT Jamaica), Pakistan (three studies) (Ali 2003 RCT Pakistan; Hirani 2010 CRCT Pakistan; Rahman 2008 CRCT Pakistan), and Uganda (one study) (Bolton 2003 C-RCT Uganda). Interventions were delivered in urban settings (Ali 2003 RCT Pakistan; Baker-H 2005 CRCT Jamaica; Chen 2000 RCT Taiwan; Hirani 2010 CRCT Pakistan), rural settings (Bolton 2003 C-RCT Uganda; Rahman 2008 CRCT Pakistan), and both (Tiwari 2010 RCT China).

Participants: participants were mostly from deprived backgrounds, though those in Ali (2003) were lower middle class and those in Chen (2000) were split equally between high-, middle- and low-income groups. Six studies included only women with depression (Ali 2003 RCT Pakistan; Hirani 2010 CRCT Pakistan; Tiwari 2010 RCT China), or perinatal depression (Baker-H 2005 CRCT Jamaica; Rahman 2008 CRCT Pakistan). Studies including women tended to exclude adult women over the age of 50 years.

Intervention: NSHWs: there were four LHW-led interventions (Ali 2003 RCT Pakistan; Baker-H 2005 CRCT Jamaica; Bolton 2003 C-RCT Uganda; Hirani 2010 CRCT Pakistan). The LHWs in these studies all had primary or no education, and some had high school or further education (Bolton 2003 C-RCT Uganda; Rahman 2008 CRCT Pakistan). The group also includes one nurse-led (Chen 2000 RCT Taiwan), and one social worker-led (Tiwari 2010 RCT China), intervention. Most of the NSHWs were women, though Bolton had sex-specific health workers for sex-specific groups. In two studies, the NSHWs were employed by the government (Baker-H 2005 CRCT Jamaica; Rahman 2008 CRCT Pakistan), and the others were salaried or volunteers within NGOs.

Training duration and intensity very varied from three days (Hirani 2010 CRCT Pakistan), to four weeks (Baker-H 2005 CRCT Jamaica). Though information was often incomplete, most studies that reported the content of the training had a mixture of didactic and practical training.

Supervision was highly varied in terms of organisation and intensity from ad-hoc checking (Ali 2003 RCT Pakistan; Tiwari 2010 RCT China), to structured meetings every two weeks (Baker-H 2005 CRCT Jamaica). All training and supervision was done by the principal investigators or specialists (psychiatrists and psychol- 
ogists), or both.

Description of interventions: LHWs provided psychological interventions: CBT-like problem solving (Ali 2003 RCT Pakistan; Rahman 2008 CRCT Pakistan), and group interpersonal therapy (G-IPT) (Bolton 2003 C-RCT Uganda). LHWs also provided general counselling and economic skills building in one study (Hirani 2010 CRCT Pakistan). In two trials, non-medical professionals delivered psychosocial counselling and problem solving (Chen 2000 RCT Taiwan; Tiwari 2010 RCT China). Interventions were delivered in community centres or groups (Baker-H 2005 CRCT Jamaica; Bolton 2003 C-RCT Uganda; Hirani 2010 CRCT Pakistan; Tiwari 2010 RCT China), in healthcare settings (Chen 2000 RCT Taiwan), and in homes (Ali 2003 RCT Pakistan; Rahman 2008 CRCT Pakistan).

Interventions varied in duration (30 to 120 minutes), in frequency (weekly to monthly, often with increasing intervals between sessions, e.g. Rahman 2008 CRCT Pakistan), and in total time (one month (Chen 2000 RCT Taiwan) to one year (Baker-H 2005 CRCT Jamaica)). Three interventions included manuals for training and for conducting the intervention (Baker-H 2005 CRCT Jamaica; Bolton 2003 C-RCT Uganda; Rahman 2008 CRCT Pakistan).

Comparison groups included usual care without the addition of a NSHW (Ali 2003 RCT Pakistan; Bolton 2003 C-RCT Uganda; Hirani 2010 CRCT Pakistan), or usual care where the NSHW was already present but was not trained to deliver the intervention Baker-H 2005 CRCT Jamaica; Chen 2000 RCT Taiwan; Rahman 2008 CRCT Pakistan; Tiwari 2010 RCT China).

\section{Results}

\section{Prevalence of depression}

LHW-led psychological interventions may reduce depression prevalence within six months (RR $0.30,95 \%$ CI 0.14 to $0.64,3$ studies, 1082 participants) but this evidence was of low quality due to heterogeneity $\left(\mathrm{I}^{2}=81 \%\right.$; $\mathrm{P}$ value $\left.=0.005\right)$ and selection bias (Summary of findings for the main comparison) (Bolton 2003 C-RCT Uganda; Chen 2000 RCT Taiwan; Rahman 2008 CRCT Pakistan). ITT analyses (looking at the four possible scenarios where re-assigned participants are either assigned with improved outcomes or not) showed that these results varied from RR 0.20 (95\% CI 0.09 to 0.45 ) to RR 1.11 (95\% CI 0.56 to 2.21 ) indicating uncertainty of this result. Chen (2000) and Bolton (2003) varied widely through these four scenarios from favouring NSHW to favouring usual care, probably because of their relatively small sample size and large dropout rate. Rahman (2008) was least susceptible to change in figures, indicating possibly more reliable results (Figure 4).

Figure 4. Forest plot of comparison: I NSHW-led psychological interventions versus usual care in treating CMDs in adults (RCTs), outcome: I.I Prevalence of depression (adults) (completers).

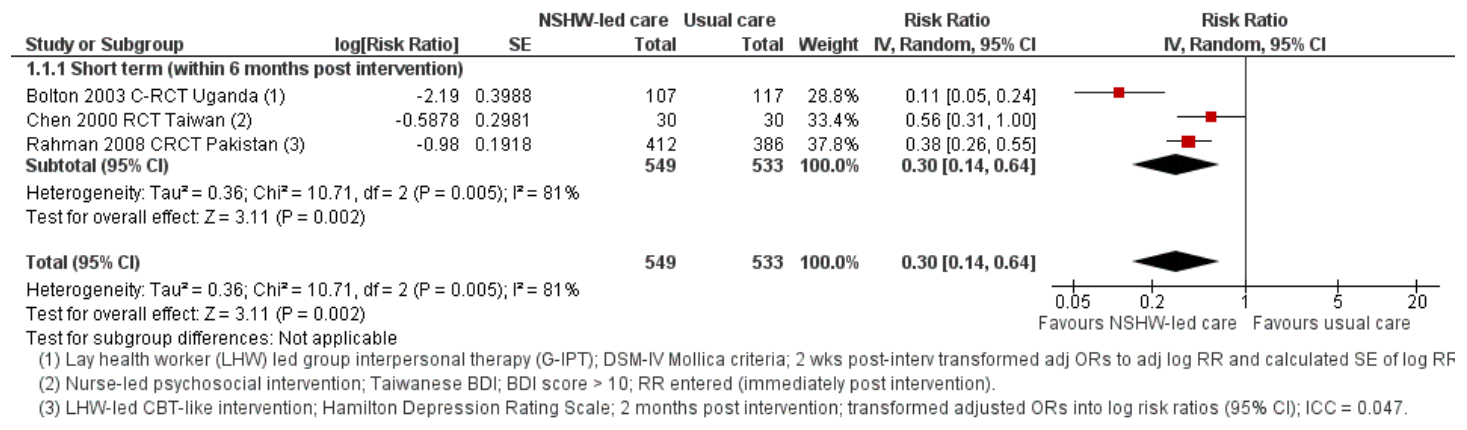

2. Severity of common mental disorder symptoms (including anxiety and depression)

Seven studies reported severity of common mental disorder symptoms (including anxiety and depression). LHW-led psychological interventions (Ali 2003 RCT Pakistan; Bolton 2003 C-RCT Uganda; Hirani 2010 CRCT Pakistan; Rahman 2008 CRCT Pakistan), were pooled with nurse and social worker-led interven- tions (Chen 2000 RCT Taiwan; Tiwari 2010 RCT China). It is uncertain whether these interventions lead to appreciable clinical benefit in common mental disorder symptom severity at six months post-intervention, because despite an apparent clinical appreciable benefit (SMD -0.75, 95\% CI -1.29 to $-0.21,1470$ participants), the evidence was of very low quality due to high 
intervention suggested that it is uncertain whether this reduces functional impairment (Bass 2012 CBA Indonesia).

However, LHW-led interventions probably reduce functional impairment of patients with common mental disorders in the medium term (12 months) (SMD -0.56, 95\% CI -0.70 to -0.42 , 1 study, 798 participants, moderate-quality evidence). The improvement at 12 but not six months may suggest that it takes longer for functional recovery.

\section{Comparison 2. Collaborative care model (non- specialist health workers plus specialist) versus usual care in treating common mental disorders (including depression and anxiety) (RCTs)}

Setting: we identified five studies from Chile (Araya 2003 RCT Chile; Fritsch 2007 RCT Chile; Rojas 2007 RCT Chile), India (Patel 2010 C-RCT India), and Kenya (Jenkins 2012 C-RCT Kenya). Both Patel (2010) and Jenkins (2012) were interventions located in a combination of urban and rural settings. The Chilean trials were conducted in deprived urban areas. All trials were conducted in government-funded PHC facilities. The Patel trial presented combined and separate results for government- and privately funded facilities.

Participants: In all studies, participants were adults (over 16 (Jenkins 2012 C-RCT Kenya) and over 17 (Patel 2010 C-RCT India) years; over 18 years for other studies) with common mental disorders (including anxiety or depression, or both) or just depression. Araya (2003), Fritsch (2007) and Rojas (2007) included only women. Most participants were of low socioeconomic status.

Interventions: Types of NSHWs: these collaborative care models involved existing PHC staff, including private and government PHC doctors (Araya 2003 RCT Chile; Fritsch 2007 RCT Chile; Jenkins 2012 C-RCT Kenya; Patel 2010 C-RCT India; Rojas 2007 RCT Chile), non-medical professional staff (nurses, social workers, midwives) (Araya 2003 RCT Chile; Jenkins 2012 C-RCT Kenya; Rojas 2007 RCT Chile), and LHWs (Fritsch 2007 RCT Chile; Patel 2010 C-RCT India; Rojas 2007 RCT Chile).

Training and supervision of NSHWs: doctors received four to six hours of training in all studies (except for Jenkins (2012) where it was not specified how many hours frontline staff received). LHWs training varied from two hours to two months. Those with longer training (Patel 2010) were expected to deliver a wider range of services. In all studies, NSHWs received some supervision (weekly to monthly/ad hoc) though those in Jenkins (2012) received no supervision and had poor medication supply.

Description of interventions: collaborative care models involved a multidisciplinary team consisting of one or several NSHWs and specialists. Doctors and nurses in Jenkins (2012) diagnosed pa- tients, provided medical treatment and follow-up/referral as per the existing government health delivery model. Araya (2003), Rojas (2007) and Patel (2010) used a stepped care intervention where doctors prescribed antidepressants and provided usual physical care and referred if there was high suicide risk. Jenkins' (2012) PHCs had poor medication supply. LHWs and non-medical professionals provided several services such as psychoeducation, medication adherence/follow-up (in person or by telephone) and IPT (Araya 2003 RCT Chile; Fritsch 2007 RCT Chile; Patel 2010 C-RCT India; Rojas 2007 RCT Chile). The intensity of these interventions varied from ad hoc (Fritsch 2007 RCT Chile; Jenkins 2012 C-RCT Kenya; Patel 2010 C-RCT India) to eight weekly psychoeducation sessions (Rojas 2007 RCT Chile). Comparison groups were the same settings where NSHWs did not receive training/supervision (Araya 2003 RCT Chile; Fritsch 2007 RCT Chile; Jenkins 2012 C-RCT Kenya; Rojas 2007 RCT Chile), and same settings without the addition of a lay counsellor, and where current staff received a training manual (enhanced usual care) (Patel 2010 C-RCT India).

\section{Results}

The primary analysis performed was of prevalence, severity and functional impairment of common mental disorders. Where trials only reported depression scores, these were combined within the common mental disorder analysis (including both anxiety and depression). Data reported at six months post intervention (if available) were chosen to represent the medium-term time point, otherwise an earlier time point (zero to five months) was combined.

\section{Prevalence of common mental disorders}

Three studies reported prevalence of CMDs (CMD scores: Patel 2010 C-RCT India; depression scores: Araya 2003 RCT Chile; Patel 2010 C-RCT India; Rojas 2007 RCT Chile). Across all facilities (private and government), the use of NSHWs may reduce the prevalence of CMDs within two to six months (RR 0.63, 95\% CI 0.44 to $0.90,2380$ participants, low quality of evidence due to serious study limitations and inconsistency $\left(\mathrm{I}^{2}=79 \%\right.$; $\mathrm{P}$ value $=$ 0.001) (Figure 6; Summary of findings 2). For government facilities only (where data from Patel 2010 C-RCT India was substituted for just the government health facilities data), the effect size was similar (RR $0.57,95 \%$ CI 0.42 to $0.78,1528$ participants, low-quality evidence). There is probably no reduction in prevalence at 12 months in 'all facilities' (RR 0.95 , 95\% CI 0.68 to 1.33, 1 study, 2009 participants, moderate-quality evidence due to imprecision) or in government facilities alone (RR 0.72, 95\% CI 0.39 to $1.34,1$ study, 1104 participants; low-quality evidence due to very serious imprecision). 
Figure 6. Forest plot of comparison: 2 Collaborative care model (NSHWs plus specialist) versus usual care in treating common mental disorders (CMD) (RCTs), outcome: 2.I Prevalence of common mental disorders (CMDs - includes anxiety and depression) (completers combined) all facilities and in public and private facilities.

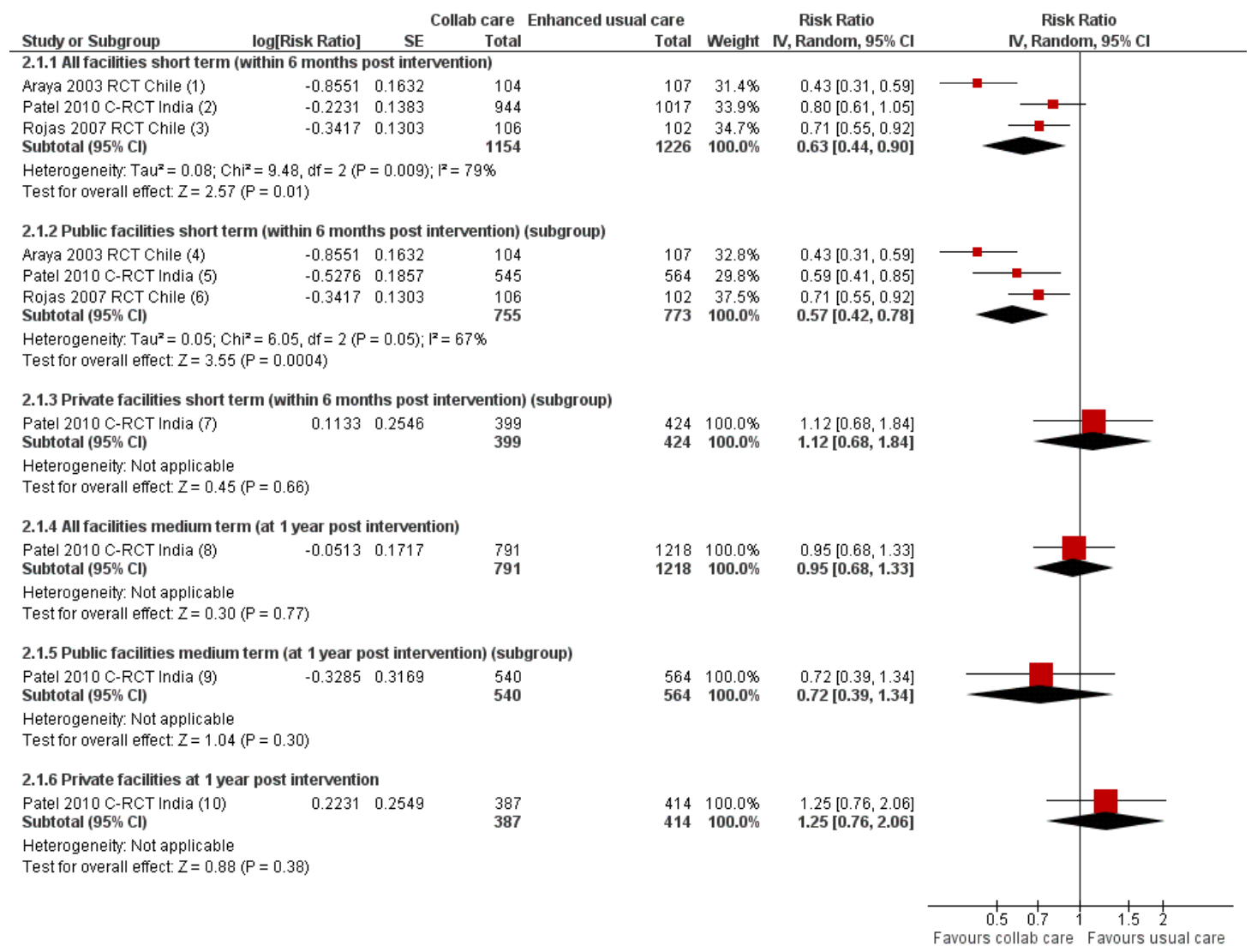

(1) Collab model for persistent recurrent depression;HRDS scores $<8$ (ie recovered) at 6 months; transformed to fit with Patel prevalence=total - nb recovered (2) stepped care for CMDs; CIS-R; 6 month prevalence of CMDs adjusted RRs

(3) collab care for post natal depression; $\mathrm{nb}$ of patients with EPDS 6 point reduction $3 \mathrm{mths}$ post-int ( $=6 \mathrm{mths}$ post baseline).prev of depression (total - $\mathrm{nb}$ recovered) (4) Collab model for persistent recurrent depression;HRDS scores $<8$ (ie recovered) at 6 months; transformed to fit with Patel prevalence by doing total - $\mathrm{nb}$ recovered (5) stepped care for CMDs; CIS-R; 6 month prevalence of CMDs adjusted RRs

(6) collab care post natal depr; nb of patients with EPDS 6 point reduction at 3 mths post-int ( $=6$ months post baseline). prevalence of depression (total - nb recovered)

(7) stepped care for CMDs; CIS-R; 6 month prevalence of CMDs adjusted RRs

(8) stepped care for CMDs; CIS-R; adjusted RR for non-recovered completers

(9) stepped care for CMDs; CIS-R; numbers and totals for CMDs; adjusted RR for non-recovered (completers)

(10) stepped care for CMDs; CIS-R; adjusted RR for non-recovered (completers)

We conducted a sensitivity analysis to analyse CMD scores and depression scores separately. This revealed very similar results (depression: RR $0.61,95 \%$ CI 0.40 to $0.94,3$ studies, 1092 participants, low-quality evidence; CMD: RR $0.80,95 \%$ CI 0.61 to 1.05, 1 study, 1961 participants, moderate-quality evidence).

\section{Severity of common mental disorders}

Severity of CMDs was measured in five studies (CMD scores: Jenkins 2012 C-RCT Kenya; Patel 2010 C-RCT India, depression scores: Araya 2003 RCT Chile; Fritsch 2007 RCT Chile; Patel 2010 C-RCT India; Rojas 2007 RCT Chile). It is uncertain whether collaborative care reduces the severity of CMDs in the short term (two to six months) despite a statistically significant small benefit (SMD -0.31, 95\% CI -0.56 to -0.06, 5 studies, 3604 participants, very-low-quality evidence due to serious study limitations, serious inconsistency $\left(\mathrm{I}^{2}=91 \%\right.$; P value $\left.<0.00001\right)$, and serious indirectness) (note that a small clinically appreciable benefit was set at SMD < 0.2) (Cohen 1988) (Table 6). Government facilities analysis shows a similar magnitude of effect (SMD -0.32, $95 \%$ CI -0.58 to -0.07 , very-low-quality evidence). There is probably no medium term (12 months) reduction in CMD symptom 
severity (SMD -0.03, 95\% CI -0.12 to 0.06, 1 study, 1905 participants, moderate-quality evidence) (Figure 7), possibly due to recurrence of depression at this point in time.

Figure 7. Forest plot of comparison: 2 Collaborative care model (NSHWs plus specialist) versus usual care in treating common mental disorders (RCTs), outcome: 2.2 Severity of symptoms of common mental disorders (completers combined) in all facilities and in public and private facilities.

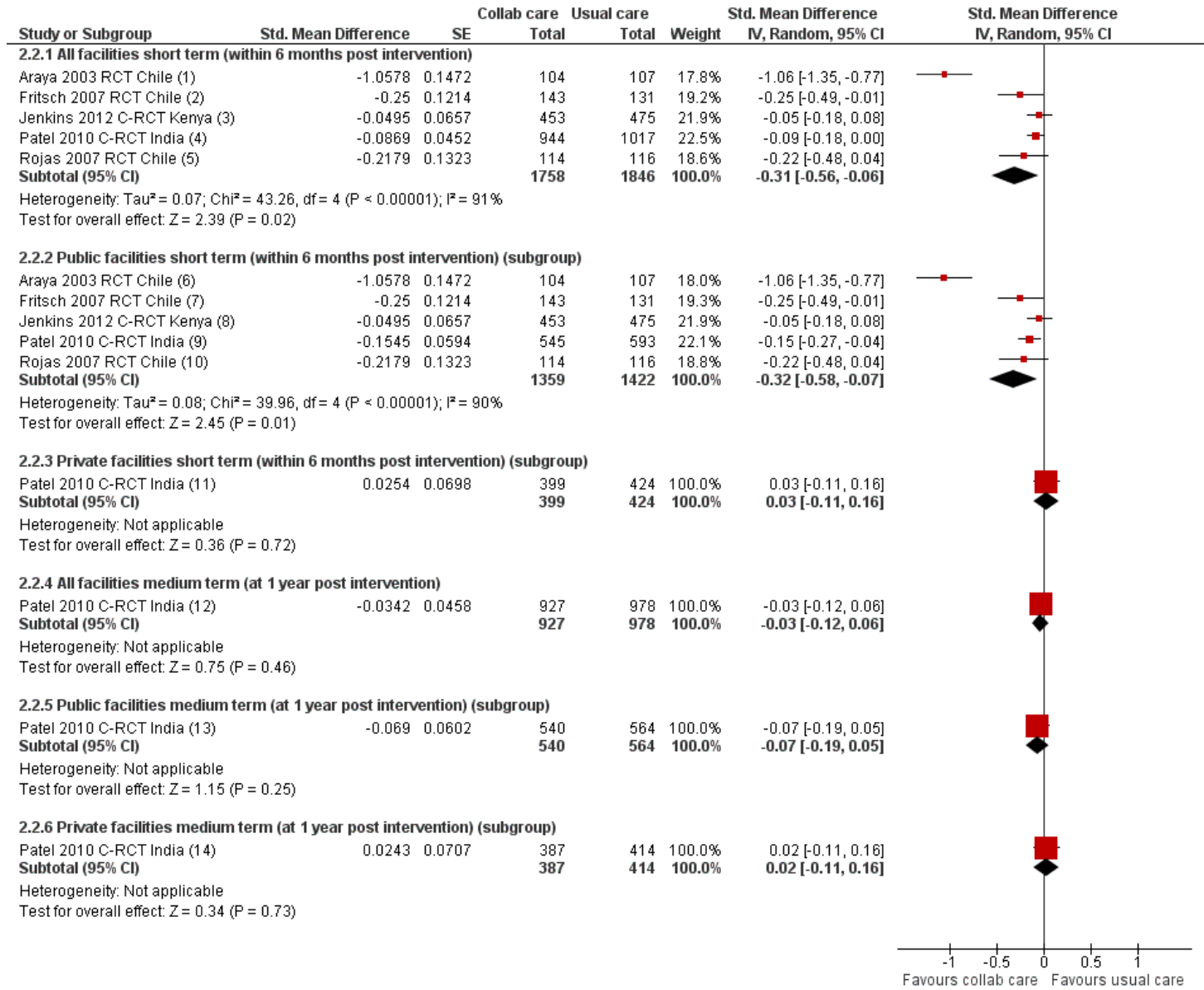

(1) collab care for persistent/recurrent depression; HDRS; adjusted MD (for baseline and clinic) and $95 \% \mathrm{Cl}$ at 6 months. (2) as per Araya;HDRS; 6 month endpoint scores

(3) Collab care in public facilities (doctor/nurse) all mental disorders; $\mathrm{GHQ}-12$; adjusted MD (for baseline scores and clustering) and $95 \% \mathrm{Cl}$ at 3 months post-intervention

(4) collaborative stepped care model for CMDs (lay counsellor, physician); CIS-R; MD and $95 \% \mathrm{Cl}$ at $6 \mathrm{mths}$ (adjusted for clustering); patient numbers (denominator) are frc

(5) physician, nurse, midwife-led multicomponent intervention for post natal depression; Edinburgh Post Natal Depression Score; MD at 3 months post-interv

(6) collab care for persistentrecurrent depression; HDRS; adjusted MD (for baseline and clinic using random effects model) and $95 \% \mathrm{Cl}$

(7) as per Araya; HDRS

(8) Collab care in public facilities (doctor/nurse) all mental disorders; GHQ-12; MD (adjusted for baseline scores and clustering) and $95 \% \mathrm{Cl}$ at 3 months post-intervention (9) Collaborative stepped care model for CMDs (lay counsellor, physician); CIS-R; MD and $95 \% \mathrm{Cl}$ at 6 months (adjusted for clustering, $\mathrm{N}=\mathrm{CMD}$ group.

(10) physician, nurse, midwife-led multicomp interv for post-natal depr; Edinburgh Post-Natal Depr Score; scores entered: MD not adjusted (adj only for MD at $3 \mathrm{mths}$ )

(11) Collaborative stepped care model for CMDs (lay counsellor, physician); CIS-R; MD and 95\% Cl at 6 months (adjusted for clustering, $N=C M D$ group.

(12) Collab stepped care; CIS-R; MD cluster-adjusted entered at 12 months; $N=$ CMD group

(13) Collab stepped care; CIS-R; cluster-adjusted means at 12 months entered; $N=$ CMD group

(14) Collab stepped care; CIS-R; cluster-adjusted means at 12 months entered; $N=$ CMD group

Non-specialist health worker interventions for the care of mental, neurological and substance-abuse disorders in low- and middle- 
The Araya trial results were an outlier for this outcome, with a much larger effect size reported (although with the same direction of effect). This may be because it was the only trial measuring major depression (moderate to severe depression). Other trials included mild depression in their inclusion criteria. This would explain the larger effect size as there is strong evidence that baseline severity of depression is a predictor of the effectiveness of depression treatments (Kirsch 2008). In a sensitivity analysis in which Araya was excluded, the reduction in symptoms no longer showed appreciable benefit (SMD -0.10, 95\% CI -0.17 to $-0.03,3394$ participants, low-quality evidence) and the results were consistent across studies $\left(\mathrm{I}^{2}=0 \%\right.$; P value $\left.=0.39\right)$.

We conducted a sensitivity analysis to analyse CMD scores and depression scores separately. CMD scores suggested collaborative care models probably do not result in a clinically appreciable reduction in the severity of CMDs in either the short term (two to six months) (SMD -0.07, 95\% CI -0.15 to 0, 2 studies, 2889 participants, moderate-quality evidence due to serious indirectness) or the medium term (one year). The short-term findings are inconsistent with the above prevalence findings. Possible explanations may be that the tools used to assess severity, particularly General Health Questionnaire (GHQ)-12 in Jenkins, may not be appropriate for assessing severity, and that the sample size is smaller in this comparison, thereby giving a less precise estimate. In addition, CMDs could include many milder symptoms of anxiety and depression whereas depression scales would identify patients with more moderate to severe symptoms. The effect of the intervention would be expected to have a greater impact on those with more symptoms (Kirsch 2008).

We could not examine the difference between outcomes for government and private facilities for the severity of CMDs due to limited data.

\section{Functional impairment and disability in adults with common mental disorders}

Five studies (CMD scores: Jenkins 2012 C-RCT Kenya; Patel 2010 C-RCT India; depression scores: Araya 2003 RCT Chile; Fritsch 2007 RCT Chile; Rojas 2007 RCT Chile) reported functional impairment and disability in adults with CMD. Collaborative care probably does not reduce functional impairment over 12 months (SMD -0.02, 95\% CI 0.11 to $0.07,1$ study, moderatequality evidence).

It is uncertain whether collaborative care reduces functional impairment in CMDs at six months (SMD - $0.22,95 \%$ CI -0.44 to -0.01 , very-low-quality evidence because of serious risk of bias, serious inconsistency $\left(\mathrm{I}^{2}=87 \%\right.$; $\mathrm{P}$ value $\left.<0.00001\right)$ and serious indirectness).

The Araya trial results were outliers for this outcome, with a much larger effect size reported (although with the same direction of effect). As above, this may because included patients had more severe symptoms and, therefore, more likely to respond to an intervention. In a sensitivity analysis in which Araya was excluded, there was no longer any appreciable clinical benefit for reducing functional impairment (SMD -0.05, 95\% CI -0.12 to -0.02, 3394 participants) but the results were now consistent $\left(\mathrm{I}^{2}=0 \%\right.$; P value $=0.40)$. At 12 months, there was no difference in functional impairment scores with collaborative or with usual care (SMD -0.02, $95 \%$ CI -0.12 to $0.15,1$ study, moderate-quality evidence).

We conducted a sensitivity analysis to analyse CMD scores and depression scores separately. Depression scores were similar or no different but again showed very-low-quality evidence. CMD scores on their own suggested no reduction in functional impairment in people with CMDs at six months (SMD - 0.03 , 95\% CI -0.1 to $0.04,2889$ participants, high-quality evidence) or at 12 months (one study).

Patel's study was the only study to report disability days. This showed that, over 12 months, collaborative care probably reduces the number of days of no or reduced work in the last month by 4.43 days (MD -4.43 days, $95 \%$ CI -8.37 to -0.48 , moderatequality evidence) in government facilities but seems to have no reduction in disability days in private facilities (MD 0.78 days, $95 \%$ CI -2.25 to 3.82 ).

\section{Suicide attempts in adults with common mental disorders}

Only one study reported suicide attempts in adults with CMDs (Patel 2010 C-RCT India). There was no difference in suicide attempts for those diagnosed with CMDs at one year (RR 0.56, 95\% CI 0.24 to $1.32,1905$ participants) and within two to six months. The quality of evidence was low due to very serious imprecision.

\section{Comparison 3. Non-specialist health workers versus usual care in treating maternal depression (RCTs)}

This group of studies combined RCTs that were also included above as part of the 'NSHW-led' and 'collaborative' intervention comparisons and that assessed perinatal depression outcomes.

Setting: we identified four studies, which were conducted in urban settings in Chile (Rojas 2007 RCT Chile), Jamaica (Baker-H 2005 CRCT Jamaica), and Taiwan (Chen 2000 RCT Taiwan), and rural settings in Pakistan (Rahman 2008 CRCT Pakistan).

Participants: the trials recruited mothers at different times from the third trimester of pregnancy (Rahman 2008 CRCT Pakistan), up to 13 months' postpartum (Baker-H 2005 CRCT Jamaica). Participants in all of the trials were generally from lower socioeconomic backgrounds, except for Chen (2000) where there was an equal distribution of participants across all socioeconomic groups. Interventions: $N S H W s$ : these were mainly existing government employees or aides, including doctors, midwives and LHWs (Rojas 2007 RCT Chile), nurses (Chen 2000 RCT Taiwan), and LHWs (Baker-H 2005 CRCT Jamaica; Rahman 2008 CRCT Pakistan). In Baker-Henningham (2005), LHW training was much more intensive than in Rahman (2008) though in both studies LHWs also received refresher training. In Rojas (2007), the midwives only 
were given an eight-hour training session (other cadres' training was not specified). In all of the trials, weekly to monthly supervision was provided, apart from Chen (2000), where this was not specified.

Description of interventions: interventions were delivered at home ( Baker-H 2005 CRCT Jamaica), in the community (Rahman 2008 CRCT Pakistan), in postnatal wards (Chen 2000 RCT Taiwan), and PHC clinics (Rojas 2007 RCT Chile). Interventions ranged from collaborative care (Rojas 2007 RCT Chile), to CBT-like intervention (Rahman 2008 CRCT Pakistan), to general adapted counselling (Baker-H 2005 CRCT Jamaica; Chen 2000 RCT Taiwan). They varied in intensity from four weeks (Chen 2000 RCT Taiwan), to weekly home visits over 12 months (Baker-H 2005 CRCT Jamaica).

Comparison groups from all four studies included usual care (existing NSHWs without training).

Results

\section{Severity of maternal depressive symptoms}

There was high-quality evidence that NSHW interventions improved the severity of perinatal depressive symptoms (SMD within three months: $-0.50,95 \%$ CI -0.63 to $-0.36,2$ studies), and moderate-quality evidence that collaborative interventions slightly improved perinatal depressive symptoms within two to six months (SMD $-0.22,95 \%$ CI -0.48 to $0.04,1$ study). LHW interventions may have slightly improved perinatal depressive symptoms at 12 months (SMD - $0.41,95 \%$ CI -0.76 to $-0.06,1$ study, low-quality evidence) (Table 7). A meta-analysis including all four studies showed that these interventions may have slightly reduced the severity of perinatal depressive symptoms (SMD - $0.42,95 \%$ CI 0.58 to -0.26 , low-quality evidence due to very serious risk of bias). Results were similar if only the three short-term studies were combined (SMD $-0.42,95 \% \mathrm{CI}-0.65$ to -0.20$)$. The statistical heterogeneity was low $\left(\mathrm{I}^{2}=29 \%\right.$; P value $\left.=0.24\right)$ (Figure 8 ; Summary of findings 3).

Figure 8. Forest plot of comparison: 3 NSHWs versus usual care in treating maternal depression (RCTs), outcome: 3.I Severity of symptoms in treating maternal depression.

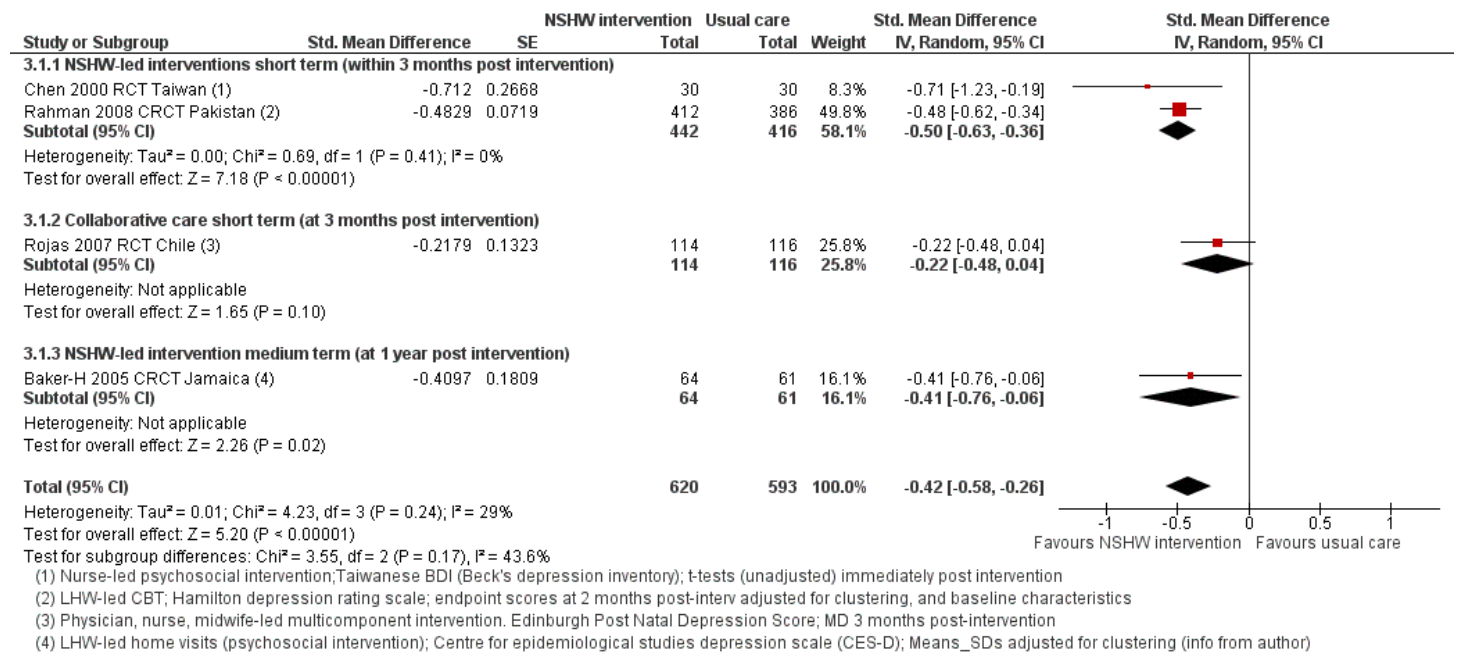

\section{Comparison 4. Non-specialist health workers versus specialist care in treating common mental disorders (controlled before-and-after studies)}

Setting: two CBA studies compared NSHWs (primary care doctors/general practitioners (GPs)) to 'gold standard' care (psychiatrists) for pharmacotherapy. These were designed as equiv- alence studies and were conducted in urban settings in Argentina (Lyketsos1999CBA Argentina) and Hungary (Zambori 2002 CBA Hungary).

Participants: Adults with common mental disorders (anxiety and depression) (Zambori 2002 CBA Hungary), and major depressive disorder (Lyketsos 1999CBA Argentina).

Interventions: NSHWs: GPs in Lyketsos (1999) received half a 
day of training and ad hoc supervision from support staff. GPs in Zambori (2002) did not receive either training or supervision in the context of the trial.

Description of interventions: the GPs provided usual care for depression (prescribing medications, supportive therapy and referring). In Lyketsos (1999), both GPs and control group psychiatrists were given a protocol for prescribing antidepressants.

\section{Results}

We could not combine any outcomes. Below is a summary of the studies.

\section{Severity of depression}

It is uncertain whether GPs are equivalent to specialists in delivering pharmacotherapy for depression (MD -0.90, 95\% CI - 1.20 to $-0.60,1$ study, Lyketsos1999CBA Argentina) as the quality of evidence was very low (CBA study and very serious risk of bias) (Summary of findings 4).

\section{Adverse events}

It is uncertain whether GPs are equivalent to specialists when adverse events get reported (RR 0.85, 95\% CI 0.67 to $1.07,1$ study, Lyketsos 1999CBA Argentina) as the quality of evidence was very low (Table 8).

\section{Number of days spent at hospital and on sick leave}

It is uncertain whether GPs were equivalent to specialists in the number of days spent at hospital (MD -1.79 days, 95\% CI -3.59 to 0.01 in favour of NSHWs) and on sick leave (MD 14.63 days, 95\% CI -0.76 to 30.02 , 1 study, Zambori 2002 CBA Hungary) as the quality of evidence was very low (very serious risk of bias and imprecision).

\section{Comparison 5. Non-specialist health workers/other professionals with health roles-led psychological interventions versus usual care in delivering post- traumatic stress disorder interventions to adults (RCTs and NRCT)}

Setting: we identified three studies, where participants lived in internally displaced camps (Dybdahl 2001 RCT Bosnia; Yeomans 2010 RCT Burundi) and refugee settlements (Neuner 2008 NRCT Uganda).

Participants: adults of both sexes who were diagnosed with PTSD, or with symptoms suggesting PTSD in mothers (Dybdahl 2001 RCT Bosnia).

Interventions: NSHWs/OPHRs: in Neuner (2008), LHWs with secondary school education were trained for six weeks in two counselling techniques (NET - narrative exposure therapy a psychological therapy, and general trauma counselling), which they delivered in different sessions. In Yeomans (2010), the LHWs had experience in trauma workshop facilitation (so only were given one-day training to adapt the workshop delivery) but little formal education. In Dybdahl (2001), preschool teachers were trained during a five-day workshop that used a range of group, role play and lecture teaching methods. There was intensive supervision in Neuner (2008) and Dybdahl (2001) (not specified in Yeomans (2010)).

Description of interventions: duration: Neuner and Yeomans interventions had four to six sessions (but at different intervals) whereas Dybdahl's intervention consisted of weekly sessions for five months (20 sessions). Content: three studies' interventions were manualised (Neuner - NET, Yeomans (both arms), Dybdahl). Neuner's non-manualised trauma counselling, Yeomans workshop with counselling and Dybdahl's interventions were similar (problem solving and coping strategies, interpersonal skills, relaxation techniques and healing through reconciling communities, psychoeducation (and childcare in Dybdahl)). Neuner's first intervention was a psychological therapy NET.

Neuner and Dybdahl's comparison groups were usual care (without any LHWs, and in Dybdahl they received free medical care). Yeomans' comparison group was usual care (with LHWs without training for this intervention).

\section{Results}

\section{Prevalence of post-traumatic stress disorder symptoms}

Neuner's (2008) LHW-led interventions may have reduced the prevalence of PTSD symptoms (NET intervention: RR 0.48, 95\% CI 0.27 to 0.85 ; trauma counselling: RR 0.55 , $95 \%$ CI 0.33 to 0.93; 1 study, low-quality evidence) (Figure 9). 
Figure 9. Forest plot of comparison: 5 NSHW-led psychological interventions versus usual care in treating adults with post-traumatic stress disorder (RCT and NRCT), outcome: 5.I Prevalence of post-traumatic stress disorder (PTSD).

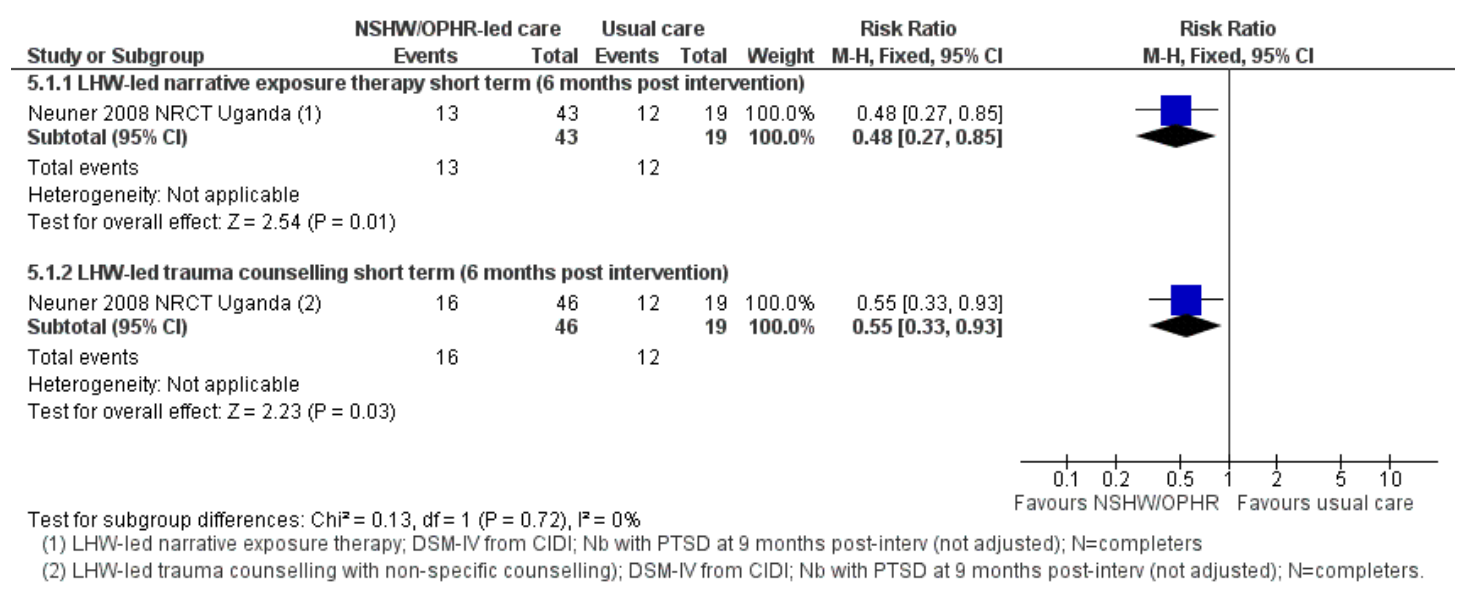

\section{Severity of post-traumatic stress disorder symptoms}

We pooled the three interventions that were most similar to each other (see description above). At assessment between two and six months post-intervention, teacher/LHW interventions may have slightly improved PTSD symptoms (SMD -0.36, 95\% CI -0.67 to $-0.05,3$ studies, 223 participants, $\mathrm{I}^{2}=22 \%$, P value $=0.02$, lowquality evidence) (Summary of findings 5). As Neuner and Yeomans had two intervention arms, we also combined these results in four ways (Neuner NET + Yeomans no psychoeducation; Neuner NET + Yeomans psychoeducation; Neuner - trauma counselling + Yeomans no psychoeducation; Neuner - trauma counselling + Yeomans psychoeducation). The results were very similar, ranging from SMD $-0.31,95 \%$ CI -0.58 to -0.04 (Dybdahl + Neuner $\mathrm{NET}+$ Yeomans psychoeducation) to SMD $-0.41,95 \% \mathrm{CI}-0.72$ to -0.11 (Dybdahl + Neuner NET + Yeomans no psychoeducation) (Figure 10; Table 9). 
Figure 10. Forest plot of comparison: 5 NSHW-led psychological interventions versus usual care in treating adults with PTSD (RCT and NRCT), outcome: 5.2 Severity of PTSD symptoms (N = completers).

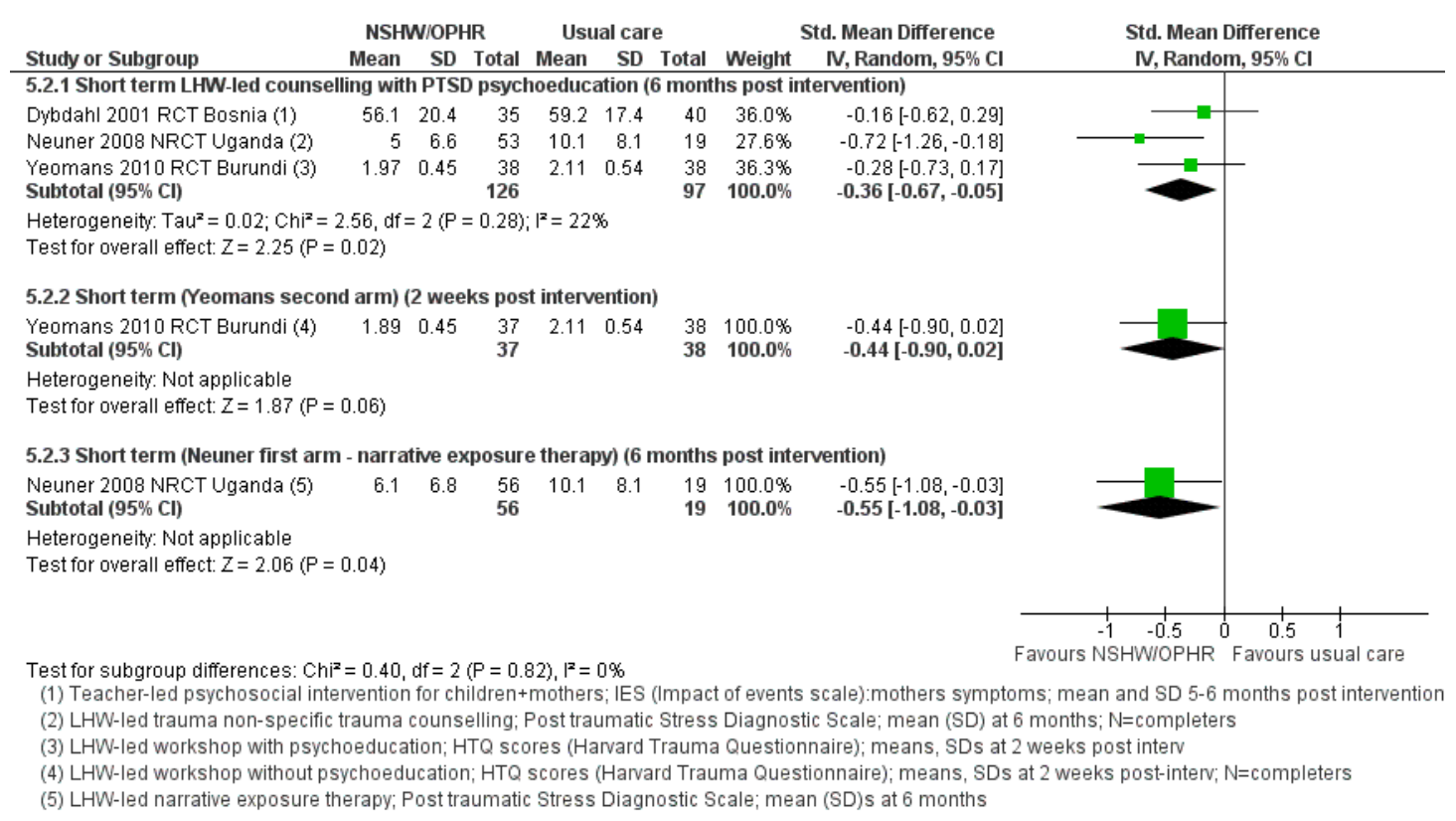

A sensitivity analysis excluding Neuner (2008) (as it uses quasirandomisation) showed a lower effect size and imprecision in the first comparison (SMD -0.22, 95\% CI -0.54 to $0.10,2$ studies, 151 participants, $\mathrm{I}^{2}=0 \%, \mathrm{P}$ value $=0.03$ ), with similar results for the other comparisons using the other intervention arms. A subgroup analysis excluding Dybdahl, which was teacher-led, and therefore retaining only LHWs suggested a slightly higher magnitude of effect (SMD -0.47, 95\% CI -0.90 to -0.05, 2 studies, 148 participants, $\mathrm{I}^{2}=34 \%$, P value $=0.03$ )

\section{Severity of depressive symptoms}

LHW-led psychological interventions may not have reduced depression severity (SMD - $0.07,95 \%$ CI -0.36 to 0.22 , 1 study, both arms had similar results, 76 participants, low-quality evidence due to imprecision and study limitations) (Analysis 5.3).

\section{Comparison 6. Non-specialist health workers versus usual care in improving dementia patients' and carers' outcomes (RCTs)}

Setting: we found two studies, which were conducted in urban areas in India (Dias 2008 RCT India), and Russia (Gavrilova 2009 RCT Russia).

Participants: the interventions were directed at carers of people with dementia. The carers were generally aged between 50 and 60 years and had varying economic backgrounds.
Interventions: NSHWs: Dias 2008 RCT India used two types of LHWs (home care advisors and lay counsellors) trained intensively for one week whereas Gavrilova 2009 RCT Russia used newly qualified doctors trained for two days to deliver the intervention. The LHWs were supervised every two weeks by a specialist. The supervision provided to the doctors was not described.

Description of interventions: in both studies brief carer interventions were conducted, based on a larger 10/66 dementia initiative (Prince 2004). However, Gavrilova (2009) organised a short training package for carers only, whereas Dias (2008) implemented a collaborative care package (LHWs undertook psychoeducation, counselling and followed up on treatment effects during home visits.

\section{Results}

\section{Patient outcomes}

At six months post intervention, NSHW-led carer interventions for dementia probably led to slightly improved patient outcomes (including severity of behavioural symptoms (SMD -0.26, 95\% CI -0.60 to $0.08,2$ studies) (Figure 11; Summary of findings 6), quality of life (MD $-0.43,95 \%$ CI -0.98 to $0.12,1$ study), and functional impairment (MD - $0.24,95 \%$ CI -0.67 to $0.20,1$ study) (moderate-quality evidence) (Table 10)). 
Figure II. Forest plot of comparison: 6 NSHWs versus usual care in improving dementia patients' and carers' outcomes (RCTs), outcome: 6.I Severity of behavioural problem (patient).

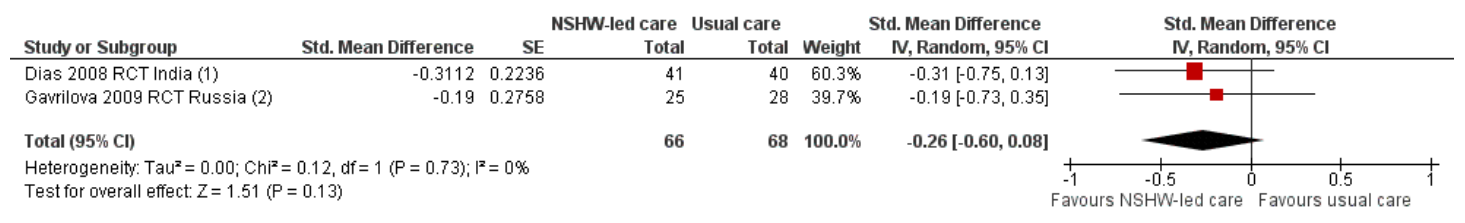

(1) LHW-led brief carer intervention. NPI-S (neuropsychiatric inventory- severity); adjusted effect sizes at 6 months post-interv

(2) Doctor-led brief carer intervention. NPI-S (neuropsychiatric inventory - severity); adjusted effect sizes (standardised MD) and Cls at 6 months post-interv.

\section{Carer outcomes}

NSHWs probably improved/slightly improved carer outcomes, including burden (SMD -0.50, 95\% CI -0.84 to -0.15) (Figure 12 ), mental health status (SMD $-0.42,95 \% \mathrm{CI}-0.76$ to -0.08$)$ and distress (SMD $-0.47,95 \%$ CI -0.82 to -0.13 ) (moderate-quality evidence). NSHWs probably led to little or no difference in carer quality of life. The study authors suggested that this result, which is out of keeping with the other carer outcomes, may be due to a type 2 error because the study was not statistically powered to detect differences of this size in the quality of life outcome.

Figure 12. Forest plot of comparison: 6 NSHWs versus usual care in improving dementia patients' and carers' outcomes (RCTs), outcome: 6.5 Carer burden.

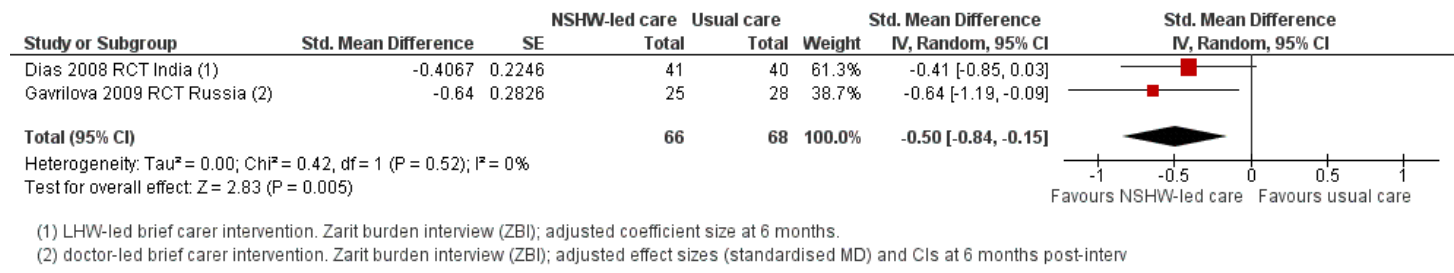

\section{Comparison 7. Non-specialist health worker-led brief alcohol interventions versus usual care for people with alcohol-use disorders}

Setting: we found two studies from rural Thailand (Noknoy 2010 RCT Thailand), and urban Kenya (Papas 2011 RCT Kenya). Participants: adults with hazardous use of alcohol (AUDIT score $\geq 8$ ) from primary care settings (Thailand) and patients (AUDIT score $>3$ ) enrolled at a human immunodeficiency virus (HIV) clinic in Kenya. Patients with alcohol dependency were excluded in Noknoy (2010).

Interventions: NSHWs: nurses in primary care clinics (Noknoy 2010 RCT
Thailand), and LHWs (Papas 2011 RCT Kenya). Training ranged from six hours (Thai nurses) to 175 hours (Kenyan LHWs). Thai nurses received no specific supervision whereas the Kenyan LHWs received 300 hours, weekly monitoring and telephone supervision in the later stages of the trial.

Description of interventions: Noknoy's (2010) intervention was less intensive (three sessions (baseline, two weeks, six weeks) - 15 minutes each) than Papas's (2011) (six sessions, once a week, 90 minutes per session). Noknoy's (2010) intervention was motivational enhancement therapy (MET), Papas's (2011) was a CBT intervention.

The comparison group was usual care. In Noknoy (2010), these were existing nurses without intervention training, and in Papas 
(2011), these were normal staff at the HIV clinic (without the LHW).

\section{Results}

\section{Amount of alcohol consumed and frequency of binge drinking}

At three to six months, NSHW-led interventions for alcohol-use problems may reduce the amount of alcohol consumed (MD 1.68 drinks/day, $95 \%$ CI -2.79 to $-0.57,2$ studies, low-quality evidence) and may reduce the frequency of binge drinking (MD $0.50,95 \%$ CI -1.14 to $0.14,1$ study, low-quality evidence due to risk of bias and imprecision) (Figure 13; Summary of findings 7).

Figure 13. Forest plot of comparison: 7 NSHW-led brief alcohol interventions versus usual care for adults with alcohol-use disorders (RCTs), outcome: 7.I Amount of alcohol consumed (MD).

\begin{tabular}{lrrrrrrr} 
& \multicolumn{3}{c}{ NSHW-led care } & Usual care & \multicolumn{2}{c}{ Mean Difference } \\
Study or Subgroup & Mean Difference & SE & Total & Total & Weight & IV, Random, 95\% Cl & Mean Difference \\
IV, Random, 95\% Cl
\end{tabular}

(1) Nurse-led motivational enhancement therapy (MET); number of drinks/ drinking day in previous week; 6 month outcome scores mean, SD (non adjusted)

(2) LHW-led adapted CBT and education; average drinks/drinking day (past 30 days); 3 month post-interv means and SD (unadjusted)

\section{Adverse consequences}

NSHW interventions for alcohol problems may not reduce road traffic accidents (RR 0.36, 95\% CI 0.12 to $1.08,1$ study, 92 participants, low-quality evidence due to sparse data, study limitations and serious imprecision). It is uncertain whether these interventions increase withdrawal symptoms (RR 2.67, 95\% CI 0.29 to 24.37, 1 study, 68 participants, very-low-quality evidence due to sparse data, study limitations and very serious imprecision) (Table $11)$.

\section{Comparison 8. Non-specialist health workers/other professionals with health roles versus usual care in delivering interventions for children with post- traumatic stress disorder and depression (RCTs)}

Setting: we identified eight studies, which were conducted in internally displaced people camps in Bosnia (Dybdahl 2001 RCT Bosnia), Indonesia (Tol 2008 C-RCT Indonesia), Kosovo (Gordon 2008 RCT Kosovo), Nepal (Jordans 2010 C-RCT Nepal), Sri Lanka (Berger2009 CRCT SriLanka; Tol 2012 C-RCT
SriLanka), and Uganda (Bolton 2007 RCT Uganda; Ertl 2011 RCT Uganda). Most studies were undertaken in post-conflict or peri-conflict settings, except for Berger (2009), which followed a natural disaster. The settings were rural/semi-rural (Bolton 2007 RCT Uganda; Gordon 2008 RCT Kosovo; Jordans 2010 C-RCT Nepal; Tol 2008 C-RCT Indonesia), urban (Berger2009 CRCT SriLanka; Dybdahl 2001 RCT Bosnia), or urban and rural (Ertl 2011 RCT Uganda; Tol 2012 C-RCT SriLanka).

Participants: children with PTSD diagnoses or symptoms were included. Some also had depressive and anxiety symptoms, or conduct problems, or a combination. The ages of the children varied from five to six years (Dybdahl 2001 RCT Bosnia), to adolescents aged 14 to 18 years (Bolton 2007 RCT Uganda; Gordon 2008 RCT Kosovo). One study included child soldiers aged 12 to 25 years (Ertl 2011 RCT Uganda). Most children came from lowresource backgrounds.

Interventions: NSHWs: five studies used LHWs (of both sexes) and had manual-based training for their respective interventions ( Bolton 2007 RCT Uganda; Ertl 2011 RCT Uganda; Jordans 2010 C-RCT Nepal; Tol 2008 C-RCT Indonesia; Tol 2012 C-RCT 
SriLanka). Supervision varied from being regular (Jordans 2010 C-RCT Nepal; Tol 2008 C-RCT Indonesia; Tol 2012 C-RCT SriLanka) to intensive (e.g. case discussions of their treatment sessions and their notes) (Ertl 2011 RCT Uganda).

OPHRs: three studies used existing high school or preschool teachers (Berger2009 CRCT SriLanka; Dybdahl 2001 RCT Bosnia; Gordon 2008 RCT Kosovo), who were given an additional threeday (Berger2009 CRCT SriLanka) to 10-day (Gordon 2008 RCT Kosovo) intensive training by researchers. Supervision was weekly (Berger2009 CRCT SriLanka; Dybdahl 2001 RCT Bosnia), or regularly (Gordon 2008 RCT Kosovo), by mental health professionals. There was no information on training for Dybdahl (2001). Description of interventions: all interventions were delivered to groups in schools except for two in community groups (Bolton 2007 RCT Uganda; Dybdahl 2001 RCT Bosnia), and one in child soldiers in their home (Ertl 2011 RCT Uganda). All interventions were targeted at children except Dybdahl (2001) where the target group was mothers. Group interventions varied from 12 to 20 sessions spread over five weeks to five months. Jordans (2010), Tol (2008) and Tol (2012) had the same manualbased, classroom-room-based intervention (CBI). This intervention included elements of creative-expressive therapy, co-operative play and CBT. Berger (2009), Dybdahl (2001) and Ertl (2000) were similar psychosocial/psychological interventions (psychoeducation, group activities, coping skills training) though Ertl (2000) had two arms: NET and academic catch up. Bolton (2007) was a three-armed trial, comparing two LHW interventions (G-IPT and creative play) delivered to single-sex groups. Gordon (2008) used slightly different psychosocial techniques (imaginative mind- body techniques, meditation, etc.)

\section{Results}

\section{Severity of post-traumatic stress disorder symptoms}

Because of differences in outcome measures for short-term outcomes (MCDs could not be combined with MD), we present these outcomes separately. We followed this approach for all outcomes in this comparison.

In the short term (< six months post intervention), despite a large apparent clinical benefit (SMD -0.89, 95\% CI -1.49 to $-0.30,3$ studies (including Ertl's first intervention arm: NET - a psychological therapy), 298 participants), it is uncertain whether LHWs and teachers reduce the severity of PTSD symptoms due to verylow-quality evidence (very serious study limitations and serious inconsistency $\mathrm{I}^{2}=78 \%$; P value $=0.003$ ) (Figure 14; Summary of findings 8). Results were similar if Ertl's second intervention arm (academic catch-up - assisting children with their academic activities only) was combined (SMD $-0.85,95 \%$ CI -1.52 to $0.19,295$ participants, $\mathrm{I}^{2}=82 \%$; $\mathrm{P}$ value $\left.=0.003\right)$. In a planned subgroup analysis, interventions led by teachers were analysed separately to attempt to reduce heterogeneity (Berger2009 CRCT SriLanka; Dybdahl 2001 RCT Bosnia). However, it was still uncertain whether teacher-led interventions may reduce the severity of PTSD symptoms (SMD -1.20, 95\% CI -1.52 to -0.88, 2 studies, 244 participants, $\left(\mathrm{I}^{2}=0 \%\right.$; $\mathrm{P}$ value $\left.=0.64\right)$ because of verylow-quality evidence (serious study limitations and imprecision due to sparse data). 
Figure 14. Forest plot of comparison: 9 NSHWs/OPHRs versus usual care in conducting interventions for children with PTSD (RCTs), outcome: 9.3 Severity of PTSD symptoms - teacher-led interventions (children) (MDs).

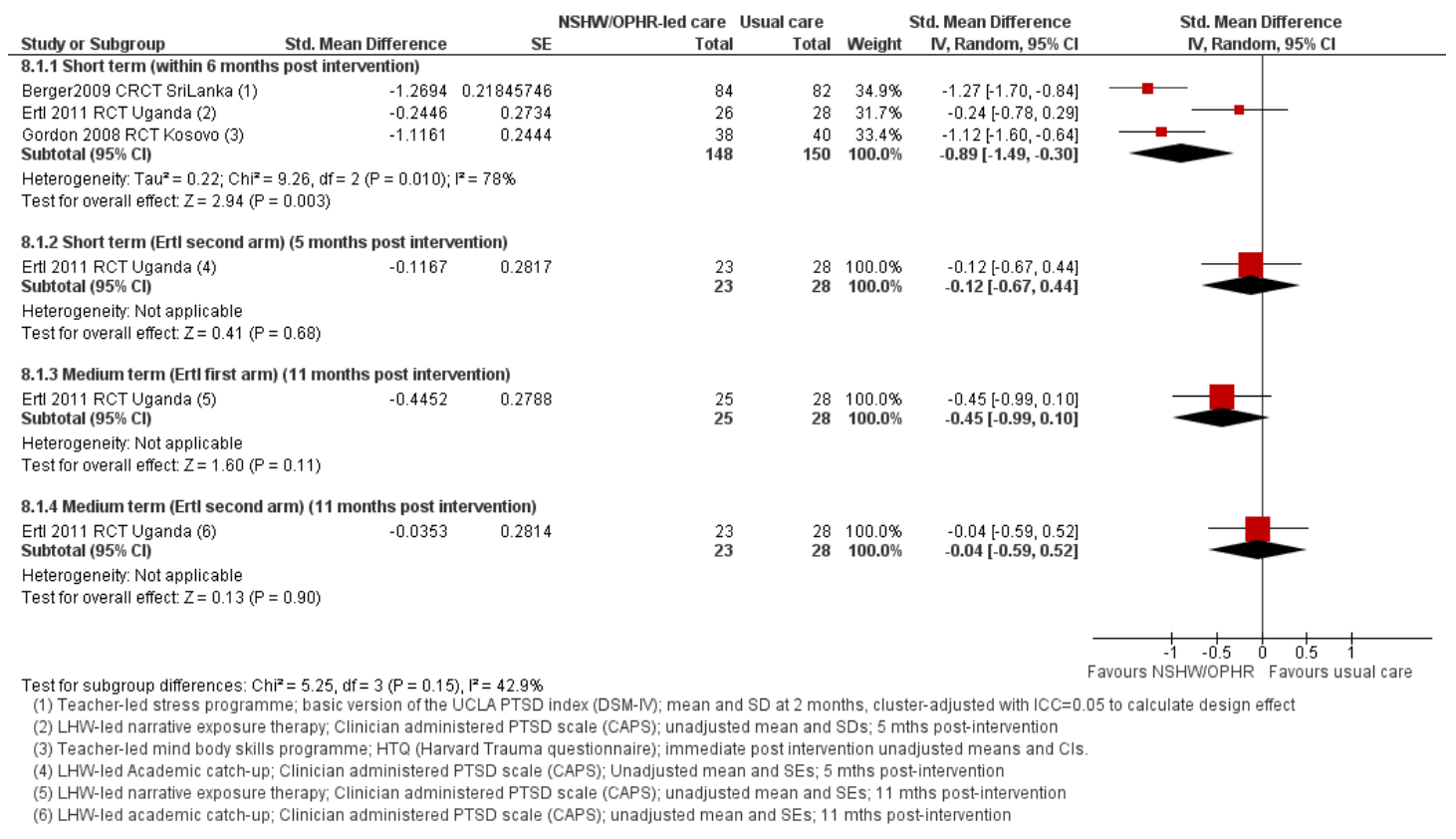

It is uncertain whether LHW-led CBI reduce PTSD symptoms (MCD $-0.56,95 \%$ CI -2.82 to 1.70 , very-low-quality evidence due to very serious risk of bias, heterogeneity $\left(\mathrm{I}^{2}=82 \%\right.$; $\mathrm{P}$ value $=$ 0.004) and serious imprecision). In one study (Tol 2012 C-RCT SriLanka), PTSD symptoms improved in girls in the control group (not in the intervention group), but there was no difference for boys (Analysis 8.3).

At 11 months, one study (Ertl 2000) suggested that NET or academic catch-up interventions probably does not reduce PTSD severity (SMD $-0.45,95 \%$ CI -0.99 to $0.10,1$ study, 53 participants, moderate-quality evidence due to serious imprecision and sparse data) (Figure 14; Table 12).

Two CBA studies also assessed teacher-led interventions for children with PTSD (aged six to 17 years) from displaced populations (Thabet 2005 CBA Palestine (short term - two months); Wolmer 2005 CBA Turkey (long term - three years post intervention). It is uncertain whether these interventions reduced PTSD severity (SMD -0.10, 95\% CI -0.34 to $0.14,329$ participants, very-lowquality evidence) (Table 13).

\section{Severity of depression symptoms}

In the short term (< six months), interventions delivered by either teachers or LHWs may slightly reduce depressive symptoms compared with usual care (SMD $-0.23,95 \%$ CI -0.45 to $-0.22,4$ studies, 504 participants, low-quality evidence due to very serious study limitations) (Table 12). However, LHW-led CBI may have led to little or no difference in the severity of depression symptoms compared with usual care (MCD - $0.18,95 \%$ CI -0.33 to 0.03 , low-quality evidence). In one CBA study, it was uncertain if interventions delivered by teachers reduced depressive symptoms (SMD -0.12, 95\% CI -0.63 to 0.40) (Thabet 2005 CBA Palestine; very-low-quality evidence; Table 13$)$.

In the medium term (11 months post intervention), LHW-led interventions may not have reduced depressive symptoms (SMD $0.02,95 \%$ CI -0.52 to $0.56,1$ study, 53 participants, low-quality evidence due to very serious imprecision). Similarly, Loughry 2006 CBA Palestin's study, a LHW-led intervention for displaced children with PTSD, suggested that the effects are uncertain (SMD $-0.27,95 \%$ CI -0.50 to -0.04 , very-low-quality evidence).

\section{Severity of anxiety symptoms}

It is uncertain whether LHW-led CBI reduced anxiety severity in children compared with usual care (MCD - $0.34,95 \%$ CI -0.75 to $0.07,3$ studies, very-low-quality evidence due to selection bias and imprecision). Tol 2012 C-RCT SriLanka undertook a subgroup analysis by sex that showed there may be little or no difference for boys (MCD - $0.63,95 \%$ CI -1.23 to $-0.03,245$ participants, lowquality evidence). 


\section{Functional impairment}

In the short term (< six months), LHW/teacher-led interventions probably reduce functional impairment (SMD -0.61, 95\% CI 1.13 to $-0.08,2$ studies, 220 participants, moderate-quality evidence due to serious study limitations) (Analysis 8.9) and LHWled CBI (MCD -0.81, 95\% CI -1.48 to -0.13 , 3 studies, 1092 participants) may have reduced functional impairment (low-quality evidence due to very serious study limitations) (Analysis 8.10). At 11 months, Ertl's LHW-led NET group probably also reduced functional impairment (SMD -0.69, 95\% CI -1.25 to $-0.14,1$ study, 53 participants, moderate-quality evidence due to serious imprecision).

\section{Outcomes of studies not assigned to the above comparisons}

The individual studies that could not be pooled are fully described in the Characteristics of included studies tables and their outcomes are summarised in Table 3 and Appendix 4.

These studies included the following comparisons:

1. NSHW versus usual care (life skills training) in improving drug abuse outcomes (RCT);

2. NSHWs versus usual care for treating schizophrenia (CBA study);
3. NSHWs versus specialist care in treating epilepsy (equivalence trial RCT);

4. OPHRs versus usual care in delivering a psychosocial/ activities intervention for parents of children with intellectual disabilities (RCT).

\section{Economic studies}

Although literature is emerging on the effectiveness of NSHWs in delivering mental health services, very limited data are available on the unit costs and resource requirements. This is mainly due to the difficulties associated with conducting economic analyses, time lags from inputs to outcomes and many confounding variables. Table 14 shows the data from the three included studies that reported cost effectiveness or costs in relation to the care of depression in adults and PTSD in children. These studies underline the feasibility and potential cost effectiveness of NSHWs in providing mental health care, and report costs related to absenteeism and healthcare utilisation. However, all of the studies had significant risks of bias that cast doubt on the accuracy and reliability of these data. Not all relevant alternatives and costs (such as productivity loss) were considered or reported, some costs relied on estimates, future costs were not discounted properly and chosen time horizons were less than one year in Araya. 
ADDITIONAL SUMMARY OF FINDINGS [Explanation]

What are the effects of a collaborative care model (NSHW plus specialist supervision) for mental health care in adults with common mental disorders low- and middle-income countries?

Patient or population: Adults ( $\geq 18$ years) with CMDs (includes anxiety or depression, or both)

Settings: Middle-income countries (Chile, India)

Intervention: Collaborative care model (NSHW plus specialist supervision)

Comparison: Enhanced usual care

\begin{tabular}{|c|c|c|c|c|c|c|c|}
\hline \multirow[t]{3}{*}{ Outcomes } & \multicolumn{3}{|c|}{ Illustrative comparative risks* $(95 \%$ Cl) } & \multirow{3}{*}{$\begin{array}{l}\text { Effect estimate } \\
(95 \% \text { Cl) }\end{array}$} & \multirow{3}{*}{$\begin{array}{l}\text { No of Participants } \\
\text { (studies) }\end{array}$} & \multirow{3}{*}{$\begin{array}{l}\text { Quality of the evidence } \\
\text { (GRADE) }\end{array}$} & \multirow[t]{3}{*}{ Comments } \\
\hline & \multirow{2}{*}{$\begin{array}{l}\text { Assumed risk } \\
\text { Usual care }\end{array}$} & \multicolumn{2}{|c|}{ Corresponding risk } & & & & \\
\hline & & $\begin{array}{l}\text { Collaborative } \\
\text { model }\end{array}$ & care & & & & \\
\hline $\begin{array}{l}\text { Prevalence of CMDs, } \\
\text { short term ( } 2-6 \text { months) } \\
\text { measured using vari- } \\
\text { ous CMD/depression rat- } \\
\text { ing scales }{ }^{1}\end{array}$ & 205 per 1000 & 140 per 1000 & & $\begin{array}{l}\text { RR } 0.63 \\
(0.44 \text { to } 0.90)\end{array}$ & $\begin{array}{l}2380 \\
\text { (3 studies) }\end{array}$ & $\begin{array}{l}\oplus \oplus \bigcirc \bigcirc \\
\text { low }^{2,3}\end{array}$ & $\begin{array}{l}\text { In Patel } 2010 \text { C-RCT } \\
\text { India; collaborative care } \\
\text { reduced the prevalence } \\
\text { of CMDs at } 6 \text { months } \\
\text { in a subgroup of people } \\
\text { treated at public health fa- } \\
\text { cilities (RR } 0.57,95 \% \mathrm{Cl} \\
0.42 \text { to } 0.78 ; 1528 \text { par- } \\
\text { ticipants). This effect was } \\
\text { not seen in people treated } \\
\text { at private facilities (RR } 1 \text {. } \\
12,95 \% \text { Cl } 0.68 \text { to } 1.84 \text {; } \\
823 \text { participants) }\end{array}$ \\
\hline
\end{tabular}

*The basis for the assumed risk is the mean control group risk across studies for pooled results and the control group risk for single studies. The corresponding risk (and its $95 \% \mathrm{Cl}$ ) is based on the assumed risk in the comparison group and the relative effect of the intervention (and its $95 \% \mathrm{Cl}$ ).

CI: confidence interval; CIS: Clinical Interview Schedule; CMD: common mental disorder; EPDS: Edinburgh Postnatal Depression Scale; GP: general practitioner; HDRS: Hamilton Depression Rating Scale; ICD: International Classification of Diseases; NSHW: non-specialist health worker; RCT: randomised controlled trial; RR: risk ratio. 


\section{GRADE Working Group grades of evidence}

High quality: Further research is very unlikely to change our confidence in the estimate of effect.

Moderate quality: Further research is likely to have an important impact on our confidence in the estimate of effect and may change the estimate.

Low quality: Further research is very likely to have an important impact on our confidence in the estimate of effect and is likely to change the estimate.

Very low quality: We are very uncertain about the estimate.

${ }^{1}$ Araya 2003 RCT Chile: HDRS; Patel 2010 C-RCT India: CIS-R generated ICD-10 diagnosis for CMD; Rojas 2007 RCT Chile: EPDS with a 6-point reduction in score indicating recovery.

${ }^{2}$ Serious study limitations: In Araya 2003 RCT Chile, GPs provided both intervention and control treatments, so there was a high risk of contamination. Downgraded by 1.

${ }^{3}$ Serious inconsistency: $I^{2}$ was $79 \%$ with Araya 2003 RCT Chile clearly an outlier, contributing to this unexplained inconsistency. However,

the inconsistency related to the magnitude of benefit favouring collaborative care rather than in the direction of effect. Downgraded by 1. 
What are the effects of NSHW-led interventions for treating maternal depression in low- and middle-income countries?

Patient or population: Adult women with maternal depression

Settings: Low- and middle-income countries (Chile, Jamaica, Pakistan, Taiwan)

Intervention: NSHW-led interventions

Comparison: Usual care

\begin{tabular}{|c|c|c|c|c|c|c|}
\hline \multirow[t]{3}{*}{ Outcomes } & \multicolumn{2}{|c|}{ Illustrative comparative risks* $(95 \%$ CI) } & \multirow{3}{*}{$\begin{array}{l}\text { Estimate effect } \\
(95 \% \mathrm{CI})\end{array}$} & \multirow{3}{*}{$\begin{array}{l}\text { No of Participants } \\
\text { (studies) }\end{array}$} & \multirow{3}{*}{$\begin{array}{l}\text { Quality of the evidence } \\
\text { (GRADE) }\end{array}$} & \multirow[t]{3}{*}{ Comments } \\
\hline & Assumed risk & Corresponding risk & & & & \\
\hline & Usual care & NSHWs & & & & \\
\hline $\begin{array}{l}\text { Severity of symptoms } \\
\text { of perinatal depression, } \\
\text { (short and medium term: } \\
0-12 \text { months) } \\
\text { measured using various } \\
\text { depression rating scales }{ }^{1}\end{array}$ & - & $\begin{array}{l}\text { The mean severity of } \\
\text { symptoms of perinatal } \\
\text { depression - medium } \\
\text { term with NSHW-led in- } \\
\text { terventions was } \\
0.42 \text { standard deviations } \\
\text { lower } \\
\text { ( } 0.58 \text { to } 0.26 \text { lower) }\end{array}$ & $\begin{array}{l}\text { SMD }-0.42(-0.58 \text { to }-0 \text {. } \\
\text { 26) }\end{array}$ & $\begin{array}{l}1213 \\
\text { (4 studies) }\end{array}$ & $\begin{array}{l}\oplus \oplus \bigcirc \bigcirc \\
\mathbf{l}_{\mathbf{0}} \mathbf{w}^{2,3}\end{array}$ & $\begin{array}{l}\text { Note that a small clini- } \\
\text { cally appreciable benefit } \\
\text { was set at SMD }<0.2 \text {, } \\
\text { and a moderate benefit at } \\
\text { SMD of } 0.5 \text { to } 0.8 \text { (Cohen } \\
1988 \text { ) }\end{array}$ \\
\hline
\end{tabular}

*The basis for the assumed risk is the mean control group risk across studies for pooled results and the control group risk for single studies. The corresponding risk (and its $95 \% \mathrm{Cl}$ ) is based on the assumed risk in the comparison group and the relative effect of the intervention (and its $95 \% \mathrm{Cl}$ ).

BDI: Becks Depression Inventory; CES-D: Center for Epidemiologic Studies Depression Scale; CI: confidence interval; EPDS: Edinburgh Postnatal Depression Scale; HDRS: Hamilton Depression Rating Scale; NSHW: non-specialist health worker; RCT: randomised controlled trial; SMD: standardised mean difference.

GRADE Working Group grades of evidence

High quality: Further research is very unlikely to change our confidence in the estimate of effect.

Moderate quality: Further research is likely to have an important impact on our confidence in the estimate of effect and may change the estimate.

Low quality: Further research is very likely to have an important impact on our confidence in the estimate of effect and is likely to change the estimate.

Very low quality: We are very uncertain about the estimate.

${ }^{1}$ Baker-H 2005 CRCT Jamaica CES-D; Chen 2000 RCT Taiwan Taiwanese BDI; Rahman 2008 CRCT Pakistan: HDRS; Rojas 2007 RCT

Chile: EPDS. 


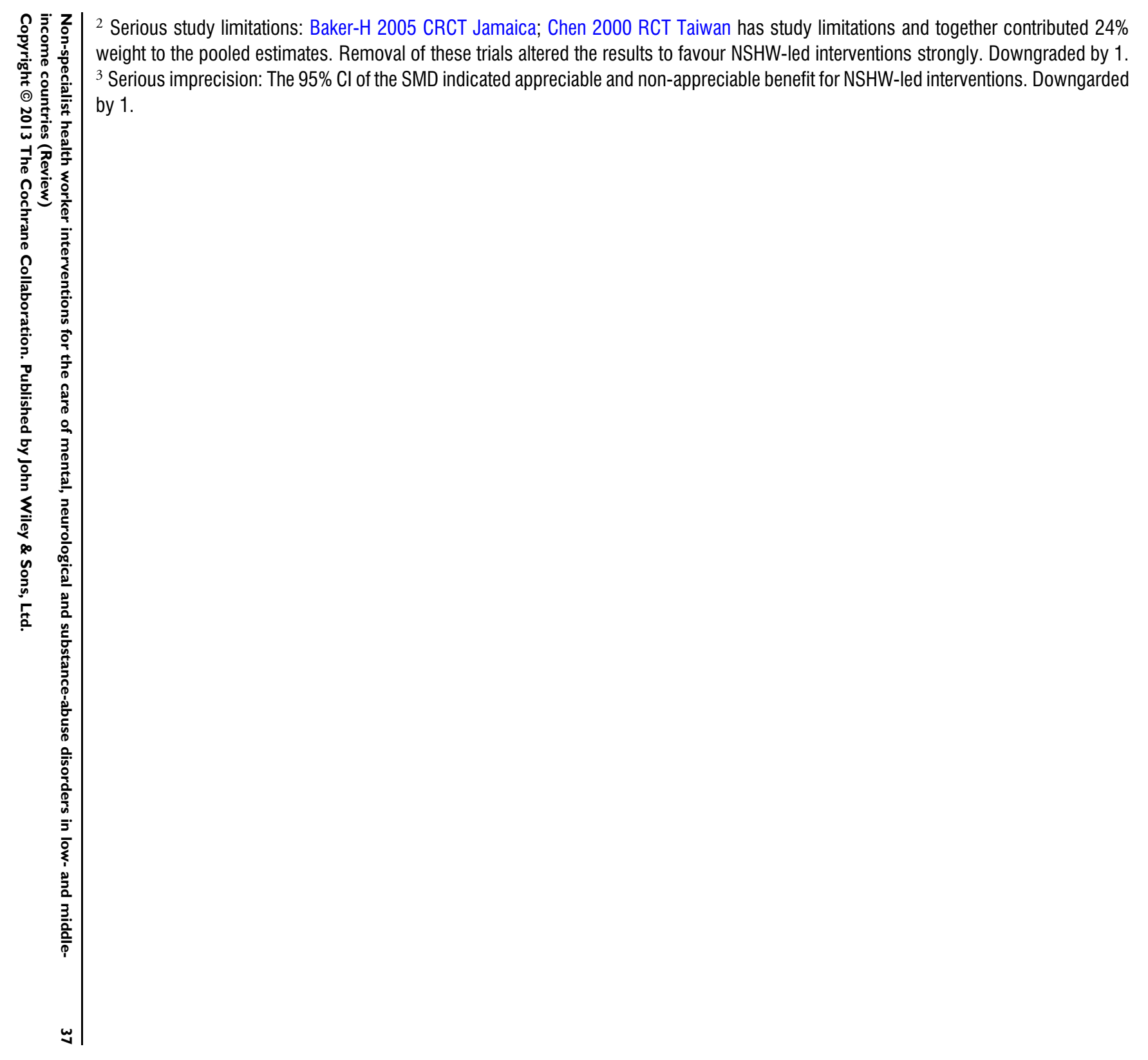


Patient or population: Adults with depression

Settings: Middle-income countries (Hungary and Argentina)

Intervention: NSHWs providing pharmacological intervention

Comparison: Specialists providing pharmacological intervention

\begin{tabular}{|c|c|c|c|c|c|c|}
\hline \multirow[t]{3}{*}{ Outcomes } & \multicolumn{2}{|c|}{ Illustrative comparative risks* (95\% CI) } & \multirow{3}{*}{$\begin{array}{l}\text { Relative effect } \\
(95 \% \mathrm{CI})\end{array}$} & \multirow{3}{*}{$\begin{array}{l}\text { No of Participants } \\
\text { (studies) }\end{array}$} & \multirow{3}{*}{$\begin{array}{l}\text { Quality of the evidence } \\
\text { (GRADE) }\end{array}$} & \multirow[t]{3}{*}{ Comments } \\
\hline & Assumed risk & Corresponding risk & & & & \\
\hline & Specialists & NSHWs & & & & \\
\hline $\begin{array}{l}\text { Severity of depression, } \\
\text { short term ( } 0-56 \text { days) } \\
\text { measured using HDRS } \\
\text { Follow-up: } 56 \text { days }\end{array}$ & $\begin{array}{l}\text { The mean score (SD) on } \\
\text { the HDRS was } 9.6(2.1)\end{array}$ & $\begin{array}{l}\text { The mean severity of de- } \\
\text { pression - short term ( } 2 \\
\text { months post intervention) } \\
\text { in the NSHW group was } \\
\mathbf{0 . 9} \text { lower } \\
\text { (1.2 to } 0.6 \text { lower) }\end{array}$ & MD $-0.90(-1.20$ to -0.60$)$ & $\begin{array}{l}768 \\
\text { (1 study) }\end{array}$ & $\begin{array}{l}\oplus \bigcirc \bigcirc \bigcirc \\
\text { very low }{ }^{1,2}\end{array}$ & $\begin{array}{l}\text { Note that a small clini- } \\
\text { cally appreciable benefit } \\
\text { was set at SMD }<0.2 \text {, } \\
\text { and a moderate benefit at } \\
\text { SMD of } 0.5 \text { to } 0.8 \text { (Cohen } \\
1988 \text { ) }\end{array}$ \\
\hline
\end{tabular}

*The basis for the assumed risk is the risk in the control group. The corresponding risk (and its $95 \% \mathrm{Cl}$ ) is based on the assumed risk in the comparison group and the relative effect of the intervention (and its $95 \% \mathrm{Cl}$ ).

CBA: controlled before-and-after; CI: confidence interval; HDRS: Hamilton Depression Rating Scale; MD: mean difference; NSHW: non-specialist health worker; RR: risk ratio; SD: standard difference; SMD: standardised mean difference.

GRADE Working Group grades of evidence

High quality: Further research is very unlikely to change our confidence in the estimate of effect.

Moderate quality: Further research is likely to have an important impact on our confidence in the estimate of effect and may change the estimate.

Low quality: Further research is very likely to have an important impact on our confidence in the estimate of effect and is likely to change the estimate.

Very low quality: We are very uncertain about the estimate.

${ }^{1}$ Very serious study limitations: Lyketsos1999CBA Argentina was a CBA study so selection bias was likely. There was a risk of contamination and outcome assessments were done by same physicians doing the intervention. Downgraded by 2.

2 Serious imprecision: The MD on the HDRS was $<1$ point and this is not clinically a meaningful difference on the HDRS; and the $95 \% \mathrm{Cl}$

of the MD indicated only non-appreciable benefits with NSHW intervention versus specialist intervention. However, the data came from

only one study, so estimate is imprecise. Downgraded by 1 . 
What are the effects of NSHWs compared with usual mental health care in low- and middle-income countries for data from an NRCT in adults with PTSD?

Patient or population: Adults with PTSD

Settings: Low- and middle-income countries (Bosnia, Burundi, Uganda)

Intervention: NSHWs and OPHRs delivering psychological interventions (narrative exposure therapy, trauma counselling and workshops with psychoeducation)

Comparison: Usual care

\begin{tabular}{|c|c|c|c|c|c|c|}
\hline \multirow[t]{3}{*}{ Outcomes } & \multicolumn{2}{|c|}{ Illustrative comparative risks* $(95 \% \mathrm{Cl})$} & \multirow{3}{*}{$\begin{array}{l}\text { Relative effect } \\
(95 \% \text { CI })\end{array}$} & \multirow{3}{*}{$\begin{array}{l}\text { No of Participants } \\
\text { (studies) }\end{array}$} & \multirow{3}{*}{$\begin{array}{l}\text { Quality of the evidence } \\
\text { (GRADE) }\end{array}$} & \multirow[t]{3}{*}{ Comments } \\
\hline & Assumed risk & Corresponding risk & & & & \\
\hline & Usual care & NSHWs/OPHRs & & & & \\
\hline $\begin{array}{l}\text { Severity of PTSD symp- } \\
\text { toms in LHW/teacher- } \\
\text { led psychological inter- } \\
\text { ventions (trauma coun- } \\
\text { selling, workshop with } \\
\text { psychoedu- } \\
\text { cation, mother interven- } \\
\text { tion) in the short term (2 } \\
\text { weeks to } 6 \text { months) } \\
\text { measured using various } \\
\text { PTSD symptom scales }{ }^{1}\end{array}$ & & $\begin{array}{l}\text { The mean severity of } \\
\text { PTSD with psychological } \\
\text { interventions in the short } \\
\text { term (within } 6 \text { months } \\
\text { post-intervention) was } \\
\mathbf{0 . 3 6} \text { standard deviations } \\
\text { lower } \\
\text { (0.67 to } 0.05 \text { lower) }\end{array}$ & $\begin{array}{l}\text { SMD }-0.36(-0.67 \text { to }-0 . \\
\text { 05) }\end{array}$ & $\begin{array}{l}223 \\
\text { (3 studies) }\end{array}$ & $\begin{array}{l}\oplus \oplus \bigcirc \bigcirc \\
\text { low }^{2,3}\end{array}$ & \\
\hline
\end{tabular}

*The basis for the assumed risk is the median control group risk or mean control group risk across studies for pooled estimates and the control group risk for single studies. The corresponding risk (and its $95 \% \mathrm{Cl}$ ) is based on the assumed risk in the comparison group and the relative effect of the intervention (and its $95 \% \mathrm{Cl}$ ).

CI: confidence interval; LHW: lay health workers; NRCT: non-randomised controlled trial; NSHW: non-specialist health worker; OPHR: other professionals with health roles; PTSD: posttraumatic stress disorder; SMD: standardised mean difference.

GRADE Working Group grades of evidence

High quality: Further research is very unlikely to change our confidence in the estimate of effect.

Moderate quality: Further research is likely to have an important impact on our confidence in the estimate of effect and may change the estimate.

Low quality: Further research is very likely to have an important impact on our confidence in the estimate of effect and is likely to change the estimate.

Very low quality: We are very uncertain about the estimate. 


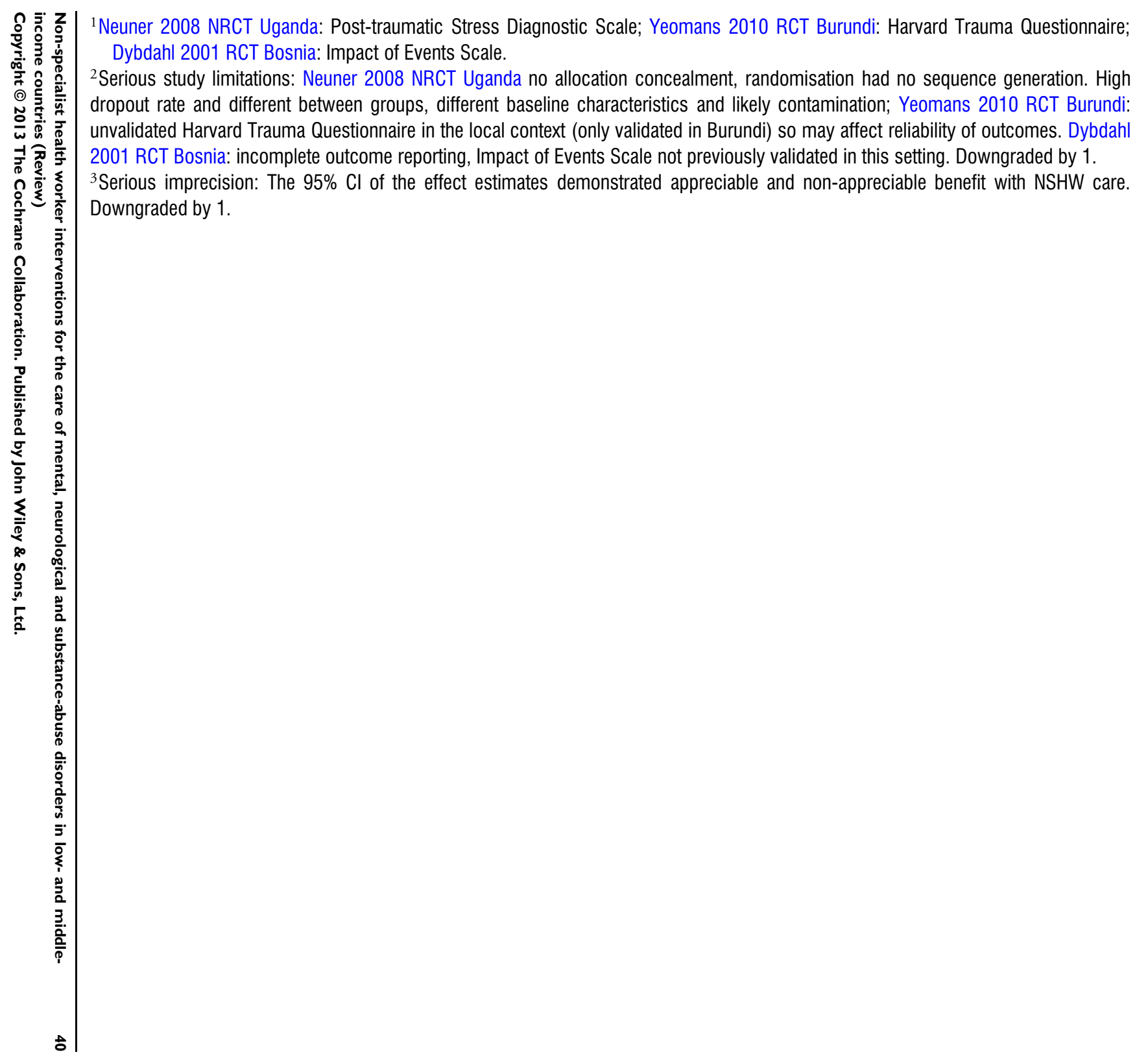


Patient or population: People with dementia and their carers

Settings: Middle-income countries (India, Russia)

Intervention: NSHWs delivering brief intervention

Comparison: Usual care

\begin{tabular}{|c|c|c|c|c|c|c|}
\hline \multirow[t]{3}{*}{ Outcomes } & \multicolumn{2}{|c|}{ Illustrative comparative risks* $(95 \% \mathrm{Cl})$} & \multirow{3}{*}{$\begin{array}{l}\text { Estimate effect } \\
(95 \% \mathrm{Cl})\end{array}$} & \multirow{3}{*}{$\begin{array}{l}\text { No of Participants } \\
\text { (studies) }\end{array}$} & \multirow{3}{*}{$\begin{array}{l}\text { Quality of the evidence } \\
\text { (GRADE) }\end{array}$} & \multirow[t]{3}{*}{ Comments } \\
\hline & Assumed risk & Corresponding risk & & & & \\
\hline & Usual care & NSHWs & & & & \\
\hline $\begin{array}{l}\text { Severity of patient } \\
\text { behavioural problems, } \\
\text { short term ( } 6 \text { months) } \\
\text { measured using the be- } \\
\text { havioural symptom scale } \\
\text { (NPI-S) }\end{array}$ & & $\begin{array}{l}\text { The mean severity of pa- } \\
\text { tient behavioural prob- } \\
\text { lems with this brief carer } \\
\text { intervention was } \\
0.26 \text { standard deviations } \\
\text { lower } \\
(0.60 \text { lower to } 0.08 \\
\text { higher) }\end{array}$ & $\begin{array}{l}\text { SMD }-0.26(-0.60 \text { to } 0 . \\
08)\end{array}$ & $\begin{array}{l}134 \\
\text { (2 studies) }\end{array}$ & $\begin{array}{l}\oplus \oplus \oplus \bigcirc \\
\text { moderate } 1,2\end{array}$ & $\begin{array}{l}\text { Note that a small clini- } \\
\text { cally appreciable benefit } \\
\text { was set at SMD }<0.2 \text {, } \\
\text { and a moderate benefit at } \\
\text { SMD of } 0.5-0.8 \text { (Cohen } \\
1988 \text { ) }\end{array}$ \\
\hline
\end{tabular}

*The basis for the assumed risk is the mean control group risk across studies for pooled results and the control group risk for single studies. The corresponding risk (and its $95 \% \mathrm{Cl}$ ) is based on the assumed risk in the comparison group and the relative effect of the intervention (and its $95 \% \mathrm{Cl}$ ).

CI: confidence interval; NPI-S: Neuropsychiatric Inventory - Severity; NSHW: non-specialist health worker; RCT: randomised controlled trial; SMD: standardised mean difference.

\section{GRADE Working Group grades of evidence}

High quality: Further research is very unlikely to change our confidence in the estimate of effect.

Moderate quality: Further research is likely to have an important impact on our confidence in the estimate of effect and may change the estimate.

Low quality: Further research is very likely to have an important impact on our confidence in the estimate of effect and is likely to change the estimate.

Very low quality: We are very uncertain about the estimate.

${ }^{1}$ No serious study limitations: Gavrilova 2009 RCT Russia was unclear whether allocation concealed. Dias 2008 RCT India was at low risk of bias and contributed $>60 \%$ of the weight to the pooled estimates. Removal of the former study did not alter the results. Not downgraded. 


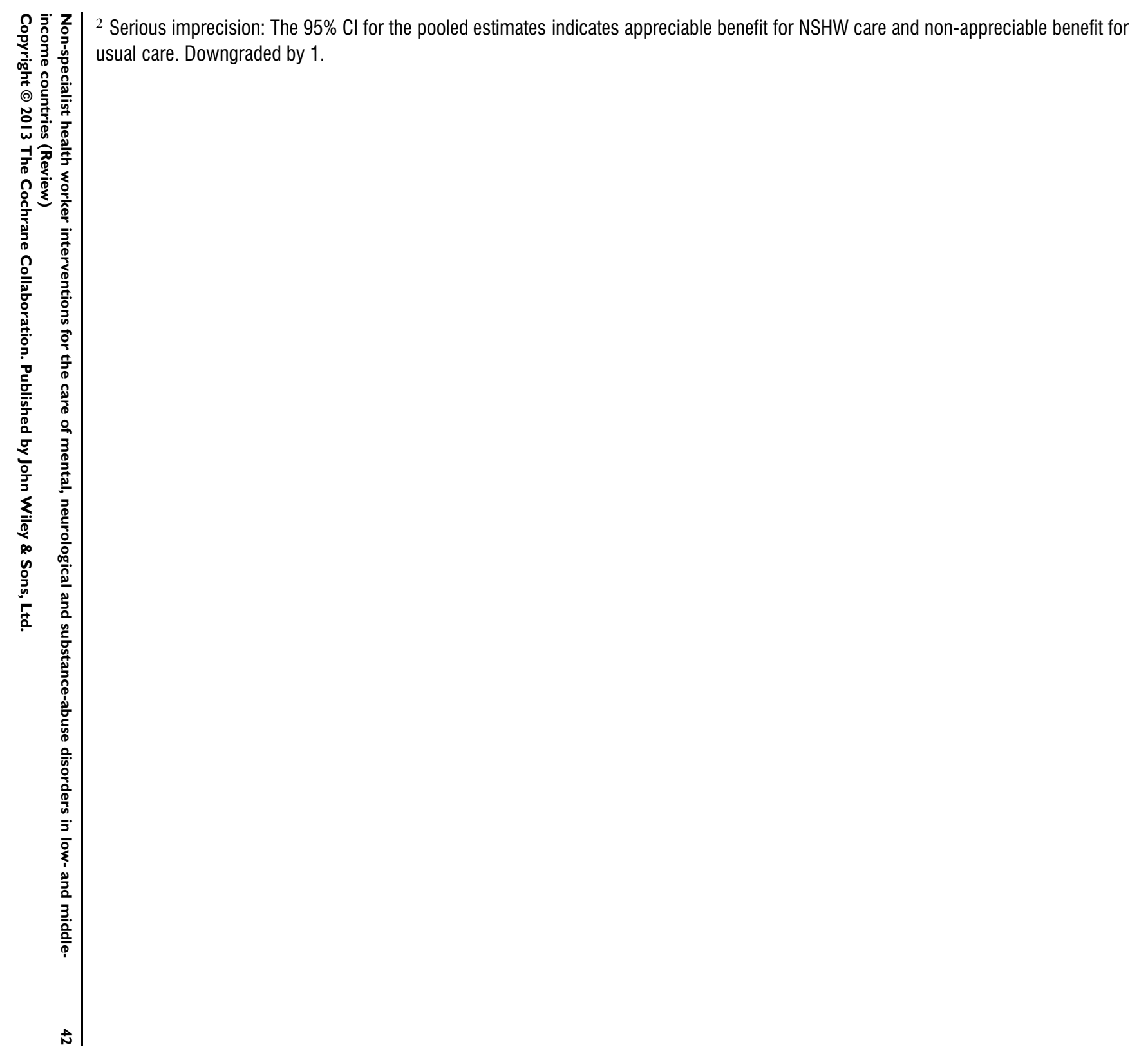


What are the effects of NSHWs in delivering brief alcohol interventions in RCTs for alcohol-use disorders?

Patient or population: People with alcohol-use disorders

Settings: Low- and middle-income countries (Thailand, Kenya)

Intervention: NSHWs in delivering brief alcohol interventions

Comparison: Usual care

\begin{tabular}{|c|c|c|c|c|c|c|}
\hline \multirow[t]{3}{*}{ Outcomes } & \multicolumn{2}{|c|}{ Illustrative comparative risks* $(95 \% \mathrm{CI})$} & \multirow{3}{*}{$\begin{array}{l}\text { Relative effect } \\
(95 \% \mathrm{CI})\end{array}$} & \multirow{3}{*}{$\begin{array}{l}\text { No of Participants } \\
\text { (studies) }\end{array}$} & \multirow{3}{*}{$\begin{array}{l}\text { Quality of the evidence } \\
\text { (GRADE) }\end{array}$} & \multirow[t]{3}{*}{ Comments } \\
\hline & Assumed risk & Corresponding risk & & & & \\
\hline & Usual care & NSHWs & & & & \\
\hline $\begin{array}{l}\text { Amount of alcohol con- } \\
\text { sumed, short term (3-6 } \\
\text { months) } \\
\text { measured using the num- } \\
\text { ber of drinks/drinking day } \\
\text { (in past week to } 30 \text { days) }\end{array}$ & & $\begin{array}{l}\text { The mean amount of al- } \\
\text { cohol consumed in the in- } \\
\text { tervention groups was } 1 . \\
68 \text { lower ( } 2.79 \text { lower to } \\
0.57 \text { lower) }\end{array}$ & MD -1.68 (-2.79 to -0.57) & $\begin{array}{l}167 \\
\text { (2 studies) }\end{array}$ & $\begin{array}{l}\oplus \oplus \bigcirc \bigcirc \\
\mathbf{l}_{\mathbf{0}} \mathbf{w}^{1,2}\end{array}$ & \\
\hline
\end{tabular}

*The basis for the assumed risk is the mean control group risk across studies for pooled data or the control group risk for individual studies. The corresponding risk (and its $95 \% \mathrm{Cl}$ ) is based on the assumed risk in the comparison group and the relative effect of the intervention (and its $95 \% \mathrm{Cl}$ ).

CI: confidence interval; MD: mean difference; NSHW: non-specialist health worker; RCT: randomised controlled trial; RR: risk ratio.

GRADE Working Group grades of evidence

High quality: Further research is very unlikely to change our confidence in the estimate of effect.

Moderate quality: Further research is likely to have an important impact on our confidence in the estimate of effect and may change the estimate.

Low quality: Further research is very likely to have an important impact on our confidence in the estimate of effect and is likely to change the estimate.

Very low quality: We are very uncertain about the estimate.

${ }^{1}$ Serious study limitations: Noknoy 2010 RCT Thailand: high dropout rate with no information on whether they are different to completers,

no validated tools in the setting, so unreliable primary outcomes. Papas 2011 RCT Kenya: unclear about whether the non-blinding of

outcome assessors would have impacted on study. Downgraded by 1.

${ }^{2}$ Serious imprecision: The $95 \% \mathrm{Cl}$ of the MD in number of drinks indicates marginal benefit and no appreciable benefit with interventions

The sample size was also low. Downgraded by 1. 
What are the effects of NSHWs/OPHRs conducting interventions for children with PTSD from RCTs in low- and middle-income countries?

Patient or population: Children/adolescents with PTSD and related depressive/anxiety symptoms

Settings: Low- and middle-income countries (Bosnia, Kosovo, Sri Lanka)

Intervention: NSHWs/OPHRs delivering psychological and psychosocial interventions

Comparison: Usual care

\begin{tabular}{|c|c|c|c|c|c|c|}
\hline \multirow[t]{3}{*}{ Outcomes } & \multicolumn{2}{|c|}{ Illustrative comparative risks* $(95 \% \mathrm{Cl})$} & \multirow{3}{*}{$\begin{array}{l}\text { Estimate effect } \\
(95 \% \text { Cl) }\end{array}$} & \multirow{3}{*}{$\begin{array}{l}\text { No of Participants } \\
\text { (studies) }\end{array}$} & \multirow{3}{*}{$\begin{array}{l}\text { Quality of the evidence } \\
\text { (GRADE) }\end{array}$} & \multirow[t]{3}{*}{ Comments } \\
\hline & Assumed risk & Corresponding risk & & & & \\
\hline & Usual care & NSHWs/OPHRs & & & & \\
\hline $\begin{array}{l}\text { Severity of PTSD symp- } \\
\text { toms in LHW/teacher- } \\
\text { led interventions, short } \\
\text { term (1-6 months) } \\
\text { measured using various } \\
\text { PTSD severity of symp- } \\
\text { tom scales }{ }^{1}\end{array}$ & & $\begin{array}{l}\text { The mean severity of } \\
\text { PTSD symptoms in chil- } \\
\text { dren in teacher-led inter- } \\
\text { vention groups was } \\
\mathbf{1 . 2} \text { standard deviations } \\
\text { lower } \\
\text { (1.52 to } 0.88 \text { lower) }\end{array}$ & $\begin{array}{l}\text { SMD }-\mathbf{0 . 8 9}(-1.49 \text { to }-0 . \\
\text { 30) }\end{array}$ & $\begin{array}{l}298 \\
\text { (3 studies) }\end{array}$ & $\begin{array}{l}\oplus \bigcirc \bigcirc \bigcirc \\
\text { very low } \text { low }^{2,3}\end{array}$ & $\begin{array}{l}\text { Note that a small clinically } \\
\text { appreciable benefit was } \\
\text { set at } S M D<0.2 \text {, a mod- } \\
\text { erate benefit at SMD of } 0 \text {. } \\
5-0.8 \text {, and a large benefit } \\
>0.8 \text { (Cohen 1988) }\end{array}$ \\
\hline
\end{tabular}

*The basis for the assumed risk the mean control group risk across studies for pooled results and the control group risk for single studies. The corresponding risk (and its $95 \% \mathrm{Cl}$ ) is based on the assumed risk in the comparison group and the relative effect of the intervention (and its $95 \% \mathrm{Cl}$ ).

CI: confidence interval; LHW: lay health workers; NSHW: non-specialist health worker; OPHR: other professionals with health roles; PTSD: post-traumatic stress disorder; RCT: randomised controlled trial; SMD: standardised mean difference; UCLA: University of California at Los Angeles.

GRADE Working Group grades of evidence

High quality: Further research is very unlikely to change our confidence in the estimate of effect.

Moderate quality: Further research is likely to have an important impact on our confidence in the estimate of effect and may change the estimate.

Low quality: Further research is very likely to have an important impact on our confidence in the estimate of effect and is likely to change the estimate.

Very low quality: We are very uncertain about the estimate.

${ }^{1}$ Berger2009 CRCT SriLanka: UCLA PTSD scale; Gordon 2008 RCT Kosovo: Harvard Trauma Questionnaire; Ertl 2011 RCT Uganda:

Clinician-administered PTSD scale (CAPS). 


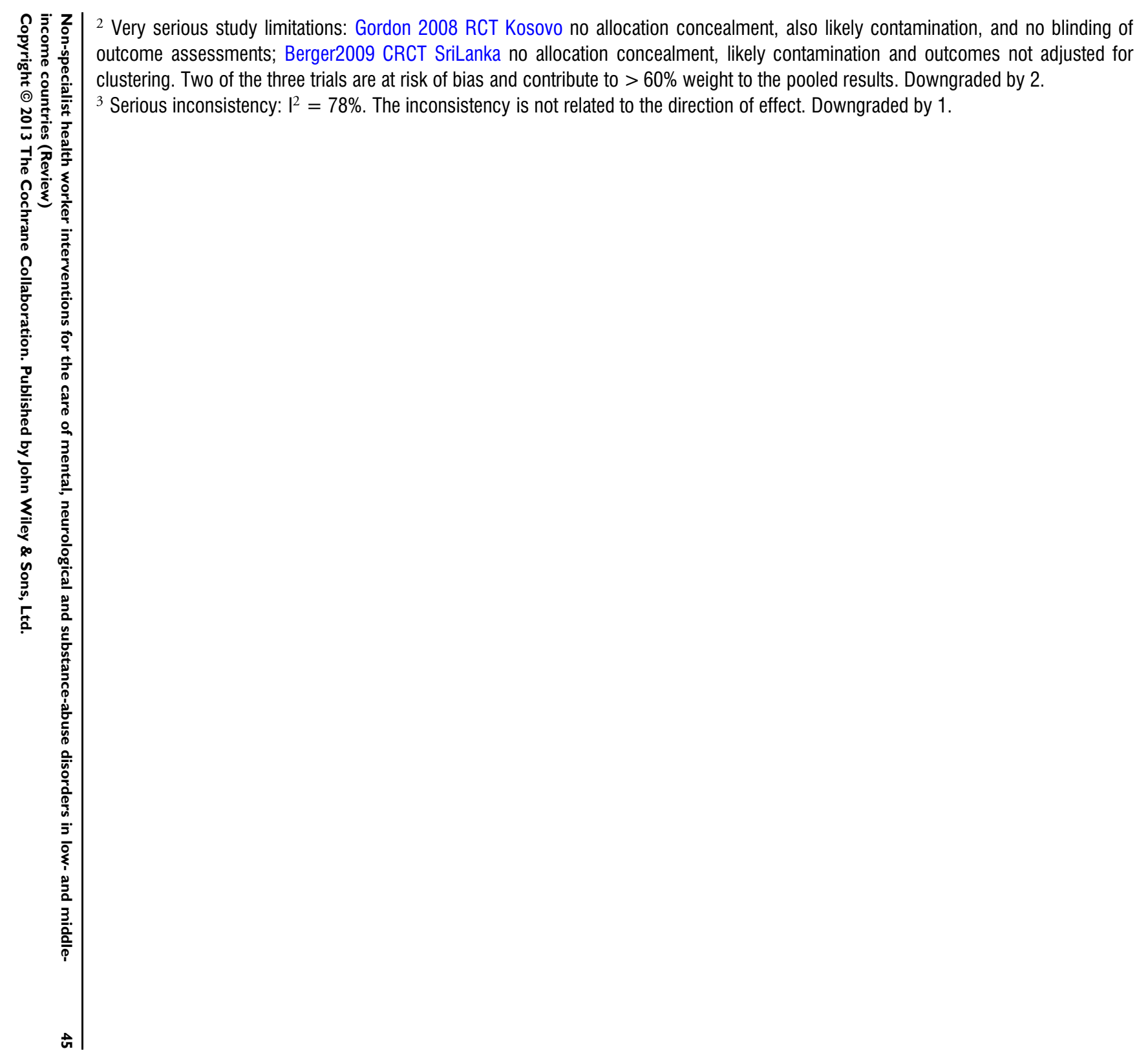




\section{DISCUSSION}

\section{Summary of main results}

This review identified 38 RCTs and NRCTs and CBA studies evaluating the effectiveness of NSHWs delivering care for MNS disorders in seven LICs and 15 middle-income countries. Twenty-two studies used LHWs, and most addressed depression or PTSD. The diversity of included studies limited meta-analysis to outcomes for eight comparisons. All analyses presented below compare interventions versus usual care.

The review showed that the use of NSHWs, compared with usual healthcare services:

- may increase the number of adults who recover from depression or anxiety (or both) two to six months after treatment (low-quality evidence). At seven to 12 months, LHWled psychological interventions probably reduced common mental disorder (anxiety and depression) symptoms and functional impairment, but collaborative care interventions (a multidisciplinary team that included one or several NSHWs and specialists) showed little or no effect over the same time period. It is unclear why this effect was lost by 12 months for collaborative care and this may be because of depression recurrence and because of the relatively short duration of the intervention. The intervention may need to carry on longer, even if just as case management, to detect early signs of relapse. There is also insufficient evidence, due to sparse data, to favour LHW-led psychological interventions over collaborative care at this time;

- may slightly reduce symptoms for mothers with perinatal depression symptoms (low-quality evidence);

- may slightly reduce the prevalence and the symptoms of adults with PTSD over six months (low-quality evidence);

- probably slightly improves the symptoms of people with dementia (moderate-quality evidence);

- probably improves/slightly improves the mental well-being, burden and distress of carers of people with dementia (moderate-quality evidence);

- may decrease the amount of alcohol consumed by people with alcohol-use disorders (low-quality evidence).

In children experiencing PTSD, teachers and LHWs:

- probably reduce functional impairment of PTSD-affected children at six and 12 months following the intervention (moderate-quality evidence);

- may have little or no effect on depressive or conduct symptoms (low-quality evidence);

- it is uncertain whether LHWs or teachers reduce PTSD symptoms over six months among children (very-low-quality evidence).

The three studies measuring costs suggested that NSHW interventions may be cost effective for depression and PTSD, but there is insufficient evidence to draw firm conclusions. For other outcomes (including the equivalence CBA studies for NSHWs versus specialists in treating depression), the evidence is insufficient to draw conclusions regarding the effects of NSHWs. There is also insufficient evidence to determine which NSHW training or intervention strategies are likely to be most effective.

\section{Overall completeness and applicability of evidence}

This review aimed to assess the effectiveness of NSHWs in delivering care to people with MNS disorders in order to provide guidance to health policy makers in LMICs. Several issues need to be considered when making judgements about the applicability of these findings to large-scale programmes.

\section{Factors related to the type and role of non-specialist health workers}

The included studies reported using many different types of NSHWs/OPHRs (some of whom were existing cadres within health services while others were additionally trained resources), particularly for common mental disorders and PTSD. However, there were few studies in each comparison and often information on details of the intervention and training were inadequate. We were, therefore, not able to explore the effects of interventions according to different NSHW characteristics (including selection, training, support, incentives or remuneration). We were also not able to explore the independent effect of NSHWs when they were part of complex interventions (such as collaborative care) or the effect of the intensity of the NSHW-led interventions. This information would help guide policymakers to tailor the type of NSHWs and their roles within scaled up programmes appropriately.

Furthermore, the review provides limited data on the effects of task-shifting to NSHWs. Most studies considered NSHWs or OPHRs as an add-on to usual care. Only three studies (Li 1989 RCT China for epilepsy, and Lyketsos 1999CBA Argentina; Zambori 2002 CBA Hungary for depression) compared these cadres versus specialists, but these studies were of low quality and data for most outcomes could not be pooled. We, therefore, cannot be certain if task-shifting (with appropriate supervision) to nonspecialists leads to equivalent quality of care or results in terms of appropriate care. Furthermore, very few studies measured adverse effects or unintended consequences of NSHW-led care - such effects could impact on the appropriateness and quality of care, and could lead to patient harm.

\section{Interventions}

Comparisons of studies were possible by MNS disorder and by broad types of interventions (such as drug treatment and psychological interventions), as well as who delivered them. However, again there were too few studies and substantial intervention variation within these categories, so it was not possible to draw strong 
conclusions on what type of intervention was most effective in relation to specific mental health disorders.

None of the included studies addressed the impact of delivering mental health care on other elements of NSHWs' healthcare roles (e.g. the impact of a mental health intervention on a PHC doctors' other tasks such as diabetes, or on their working pattern, such as consultation times). One study assessed the impact of a depression intervention on the number of days spent in hospital (i.e. both a patient outcome and a health service outcome) (Zambori 2002 CBA Hungary), but more studies looking at these indirect outcomes or unintended consequences are needed.

\section{Programme delivery}

Several issues need to be considered in applying these findings to healthcare delivery systems.

First, these are interventions delivered in a research setting where NSHWs are more likely to have been carefully selected; project leaders are more motivated; remuneration may be more available because of research funding; and training, supervision and monitoring are generally much more intensive. These conditions may not be replicable at scale or may not be as effective at scale.

Second, the types of study design chosen here were not appropriate or sufficient to inform judgements regarding the sustainability of programmes; alternative study designs, such as longitudinal studies, economic evaluations and qualitative studies, are needed for this.

Third, the elements necessary for assessing the applicability of interventions need to be considered in each setting where decisions on task-sharing or task-shifting are being made (Lavis 2009). These elements include the extent to which these real-life settings resemble those of included studies, such as on-the-ground constraints, health service arrangements, differences in baseline conditions, presence of specific groups who might benefit from the intervention and the availability of routine data.

Fourth, it is important to know the financial burden of such interventions. Few studies reported cost data, which makes it difficult to draw any conclusions on this question.

\section{Quality of the evidence}

The review included 38 studies covering a wide range of interventions and settings. For studies included in meta-analyses, the evidence for most outcomes was of low to moderate quality. Risk of bias assessments highlighted concerns regarding insufficient information on sequence generation and allocation concealment; differences in baseline outcome measurements; the reliability of primary outcome measures; and a failure to address incomplete outcome data, particularly safety data, adequately. Several studies were small and were probably underpowered.

Where meta-analysis was possible, the results were fairly consistent in showing improvements in favour of NSHW interventions, although for some interventions and outcomes there were important variations in the reported effects that could not be explained. Some studies assessed large numbers of outcomes, increasing the probability of finding statistically significant differences for some outcomes by chance. Furthermore, the diversity of the psychometric and other outcome measures used made the interpretation of statistically pooled outcome data difficult.

In the update of this review, we will consider RCTs and cluster RCTs only, as we found few NRCTs and CBA studies and no ITS studies. Those NRCTs and CBA studies that were included did not contribute significant additional data to the review.

\section{Potential biases in the review process}

NSHWs, and in particularly LHWs, are still currently poorly indexed in the literature. Though we tried covering a broad range of different synonyms for these health workers, it is possible that some studies have been missed. In addition, NSHWs and LHWs do not have standard widely accepted definitions, so some readers may disagree with these definitions or how this review has aggregated different health workers together.

There were too few studies for each comparison to assess publication bias through assessment of asymmetry. However, because many studies reported non-statistically significant results, publication bias is probably unlikely.

Many meta-analyses were performed; therefore, some of the findings may be due to chance. Many pooled results were statistically and clinically heterogeneous, mainly because of the small number of studies and the breadth of geographical, health worker and patient characteristics - these results, therefore, need to be interpreted with caution.

Furthermore, we did not record whether, for NRCTs, the study restricted participant selection or demonstrated balance or matching between intervention and control groups on prognostic factors, or a combination of these. An imbalance of these may act as confounders (such as age, sex, socioeconomic status). However, most of the findings were reported from RCTs, so this is unlikely to have a major impact on the interpretation of our findings.

A further limitation was that trials that did not conduct an ITT analysis were generally not re-analysed or their missing data was not imputed (except for one analysis were we were able to source data: NSHW-led psychological interventions for depression - prevalence of depression). Doing so may have impacted on the estimates of effect.

\section{Agreements and disagreements with other studies or reviews}

Several reviews in primary or community mental health care have been conducted but none have focused exclusively on the effectiveness of mental healthcare delivery by a non-specialist workforce. 
Reviews have covered alternatives to inpatient care but with a focus on specialist outreach services such as specialist child community services (Shepperd 2009), or community-based rehabilitation (without specifying the workforce) (Robertson 2012). Other studies addressed resource use and primary care provider behaviour with the addition of a mental health resource at primary care level, but did not assess the effect on patient outcomes (Harkness 2009). Certain reviews compared interventions themselves rather than the provider (Abas 2003; Huntley 2012; Wiley-Exley 2007).

Seven reviews incorporated aspects of interventions that were included in this review (Boer 2005; Bower 2006; Huntley 2012; Parker 2008; Rahman 2013; Tol 2011; Woltmann 2012). Details of agreements and disagreements with these reviews are presented in Table 15.

\section{Economic studies}

Appendix 3 describes other relevant economic studies that were not included in this review. The findings of these studies are similar to those of the three studies (Araya 2003 RCT Chile; Jordans 2011; Zambori 2002 CBA Hungary) included in this review, that is, that NSHW interventions seem cost-effective, and that these findings are difficult to generalise due to the different healthcare systems in various countries.

\section{A U THORS'CONCLUSIONS}

\section{Implications for practice}

Most results from the 38 studies suggest non-specialist health workers (NSHW) delivering mental, neurological and substanceuse disorders (MNS) interventions have some impact on patients' outcomes, though the evidence is overall of low quality. Given the multitude of settings, disorders, interventions and health worker expertise covered in this review, there are still too few studies within each category to draw conclusions on specific intervention characteristics (such as type of health worker, duration of intervention, levels of training and supervision, etc.) that may impact on effectiveness.

The results show that in adults, lay health worker (LHW)-led psychological interventions and collaborative care (a multidisciplinary team with NSHWs and specialists) may increase the number of adults who recover from depression or anxiety, or both, two to six months after treatment (low-quality evidence). At seven to 12 months after treatment, it is uncertain whether the delivery of psychological treatment by LHWs alone is more effective than delivery by non-specialists who are part of a multidisciplinary team (collaborative care). NSHWs may also slightly reduce symptoms for mothers with perinatal depression symptoms (low-quality evidence).
Among the other disorders, NSHWs probably slightly improve the symptoms of people with dementia and the mental well-being, burden and distress of carers of people with dementia (moderatequality evidence). They may also slightly reduce the symptoms of adults with post-traumatic stress disorder (PTSD) and may decrease the amount of alcohol consumed by people with alcoholuse disorders (low-quality evidence).

It is uncertain whether LHWs or teachers reduce PTSD symptoms among children (very-low-quality evidence). There were insufficient data to draw conclusions about the cost-effectiveness of using NSHWs or teachers, or about their impact on people with other MNS conditions such as epilepsy, schizophrenia, and alcohol and drug abuse problems. There is also insufficient evidence to determine which NSHW training or intervention strategies are likely to be most effective.

\section{Implications for research}

While this review has identified a large number of studies conducted in low- and middle-income countries (LMICs), a number of important research questions remain. Research recommendations have been subdivided into those for trialists, systematic reviewers and other researchers.

\section{Trialists}

Trialists need to:

- describe trial interventions better, for example in terms of training, supervision and incentives for NSHWs or other professionals with health roles (OPHRs). This will allow systematic reviewers to identify and compare characteristics that may help to explain the effects of NSHW interventions better;

- conduct trials comparing interventions with different characteristics/types of NSHWs/OPHRs or modes of delivery, to be able to understand the effects of these variations. This is particularly applicable to collaborative care and other complex interventions where there may be several types of specialists and NSHWs, and several types of interventions on offer (such as stepped care);

- compare NSHWs/OPHRs versus specialists to be able to assess the potential for task-shifting;

- include assessments of potential adverse effects or unintended consequences of NSHWs and OPHRs

- design better quality trials, which includes more rigorous local validation of instruments and agreeing on standard instruments for specific outcomes and disorders to facilitate pooling and comparing data;

- focus on clinical issues that have been poorly addressed to date, including epilepsy and other neurological disorders, severe mental disorders and substance abuse;

- include economic data in their trials, as costs and costeffectiveness are important information for health planning. 


\section{Systematic reviewers}

Further systematic reviews, drawing on a range of study designs (such as studies of effects, but also process evaluations, economic evaluations and qualitative work), are needed on:

- factors affecting the sustainability of NSHW/OPHR interventions when scaled up;

- the effectiveness of different approaches to ensure programme sustainability, including the use of different types of incentives and payment systems for NSHWs/OPHRs;

- mechanisms for integrating LHW (subset of NSHW) programmes into the formal health system;

- the equity impacts of these programmes.

\section{Other researchers}

Given the very broad range of NSHWs and OPHRs (with considerable variation in their characteristics (training, supervision, etc.), settings, interventions and delivery mechanisms in mental health care), there is a need to develop a comprehensive typology of NSHWs and OPHRs, as well as of the interventions they provide, which would help health planners and future researchers to have more standardised and comparable interventions and situations.

\section{ACKNOWLEDGEMENTS}

We thank: Jan Odgaard-Jensen from the Cochrane Effective Practice and Organisation of Care (EPOC) Group in Norway for his statistical guidance and support; Ian Shemilt from the Campbell \& Cochrane Economics Methods Group (CCEMG) for extensively reviewing and providing guidance on the development of the economics component of this review; Marit Johansen from the EPOC Norwegian Satellite for her help with compiling the search strategy and to the following people for their help with papers that were not in English: Liu Qin (Chinese), Ji Eun Park (Korean), Oded Horn (Czech), Gerard Urrútia Cuchí (Spanish), Krystyna Hviding (Polish), Claire Glenton (Swedish), Ozren Polasek (Croatian), Ahmet Metin Gulmezoglu (Turkish), Abbas Kasymov (Russian) and Firoze M. (Farsi).

\section{RE F E R E N C E S}

\section{References to studies included in this review}

\section{Ali 2003 RCT Pakistan \{published data only\}}

* Ali BS, Rahbar MH, Naeem S. The effectiveness of counselling on anxiety and depression by minimally trained counsellors: a randomised controlled trial. American Journal of Psychotherapy 2003;57(3):324-36.

Gul A, Ali BS. The onset and duration of benefit from counselling by minimally trained counsellors on anxiety and depression in women. Journal of the Pakistan Medical Association 2004;54(11):549-52.

\section{Araya 2003 RCT Chile \{published data only\}}

Araya R, Flynn T, Rojas G, Fritsch R, Simon G. Costeffectiveness of a primary care treatment program for depression in low-income women in Santiago, Chile. American Journal of Psychiatry 2006;163(8):1379-87. * Araya R, Rojas G, Fritsch R, Gaete J, Rojas M, Simon G, et al.Treating depression in primary care in low-income women in Santiago, Chile: a randomised controlled trial. Lancet 2003;361 (9362):995-1000.

Baker-H 2005 CRCT Jamaica \{published data only\} Baker-Henningham H, Powell C. The effect of early stimulation on maternal depression: a cluster randomised controlled trial. Archives of Disease in Childhood 2005;80: $1230-4$.

Bass 2012 CBA Indonesia \{published data only\} Bass J, Poudyal B, Tol W, Murray L, Nadison M, Bolton P. A controlled trial of problem-solving counselling for waraffected adults in Aceh, Indonesia. Social Psychiatry and Psychiatric Epidemiology 2012;47(2):279-91.
Berger2009 CRCT SriLanka \{published data only\} Berger R, Gelkopf M. School-based intervention for the treatment of tsunami-related distress in children: a quasi-randomized controlled trial. Psychotherapy and Psychosomatics 2009;78:364-71.

Bolton 2003 C-RCT Uganda \{published data only\} Bass J, Neugebauer R. Group interpersonal psychotherapy for depression in rural Uganda: 6-month outcomes randomised controlled trial. British Journal of Psychiatry 2006;188:567-73.

* Bolton P. Group Interpersonal Psychotherapy for Depression in Rural Uganda: a randomized controlled trial. JAMA: the Journal of the American Medical Association 2003; 289(23):3117-24.

Clougherty KF, Verdeli H, Weissman MM. Interpersonal psychotherapy for group in Uganda (IPTG-U). Unpublished Manual 2002.

Verdeli H, Clougherty K, Bolton P, Speelman L, Lincoln N, Bass J, et al.Adapting group interpersonal psychotherapy for a developing country: experience in rural Uganda. World Psychiatry 2003;2(2):114-20.

Bolton 2007 RCT Uganda \{published data only\} Bolton P, Bass J. Interventions for depression symptoms among adolescent survivors of war and displacement in northern Uganda. JAMA: the Journal of American Medical Association 2007;298(5):519-27.

Bolton P, Betancourt T, Bass J, Speelman S, Onyango G. A Randomized Controlled Trial of Group Interpersonal Psychotherapy and Creative Play for Psychosocial Problems in War-Affected Acholi Adolescents. Final Programme 
Report 2006.

Bolton P, Wilk CM, Ndogoni L. Assessment of depression prevalence in rural Uganda using symptom and function criteria. Social Psychiatry and Psychiatric Epidemiology 2004; 38(6):442-7.

Brown 2009 CBA Rwanda \{published data only\}

Brown L, Thurman TR. Impact of a mentoring program on psychosocial wellbeing of youth in Rwanda: results of a quasi-experimental study. Vulnerable Children and Youth Studies 2009;4(4):288-99.

Chen 2000 RCT Taiwan \{published data only\} Chen CH, Tseng YF, Chou F-H, Wang S-Y. Effects of support group intervention in postnatally distressed women: a controlled study in Taiwan. Journal of Psychosomatic Research 2000;49:395-9.

Dias 2008 RCT India \{published data only\} Dias A, Dewey ME, D'Souza J, Dhume R, Motghare DD, Shaji KS, et al.The effectiveness of a home care program for supporting caregivers of persons with dementia in developing countries: a randomised controlled trial from Goa, India. PLoS one 2008;3(6):e2333.

Dybdahl 2001 RCT Bosnia \{published data only\} Dybdahl R. Child Development and Impact of Stress on Young Children. An Intervention Programme for Mothers. Prepared for the UNICEF/Centre for Crisis Psychology Psychosocial Project 1999.

* Dybdahl R. Children and mothers in war. Child Development 2001;72(4):1214-30.

Ertl 2011 RCT Uganda \{published data only\} Ertl V, Pfeiffer A, Schauer A, Elbert T, Neuner F. Community-implemented trauma therapy for former child soldiers in Northern Uganda: a randomised controlled trial. JAMA - Journal of the American Medical Association 2011; 306(5):503-12.

Fritsch 2007 RCT Chile \{published data only\} Fritsch R, Araya R, Solis J, Montt E, Pilowsky D, Rojas G. A randomised trial of pharmacotherapy with telephone monitoring to improve treatment of depression in primary care in Santiago, Chile. Revista Medica de Chile 2007;135 (5):587-95.

Gavrilova 2009 RCT Russia \{published data only\} Gavrilova SI, Ferri CP, Mikhaylova N, Sokolova O, Banerjee $S$, Prince M. Helping carers to care-the $10 / 66$ dementia research group's randomised control trial of a caregiver intervention in Russia. International Journal of Geriatric Psychiatry 2009;24(4):347-54.

Gordon 2008 RCT Kosovo \{published data only\} Gordon JS, Staples JK, Blyta A, Bytyqi M. Treatment of posttraumatic stress disorder in postwar Kosovo high school students using mind-body skills groups: a pilot study. Journal of Traumatic Stress 2004;17(2):143-7. * Gordon JS, Staples JK, Blyta A, Bytyqi M, Wilson AT. Treatment of posttraumatic stress disorder in postwar Kosovar adolescents using mind-body skills groups: a randomised controlled trial. Journal of Clinical Psychiatry 2008;69(9):1469-76.
Hirani 2010 CRCT Pakistan \{published data only\} Hirani SS, Karmaliani R, Mcfarlane J, Asad N, Madhani F, Shehzad S. Testing a community derived intervention to promote women's health: preliminary results of a three-arm randomised controlled trial in Karachi, Pakistan. Southern Online Journal of Nursing Research 2010;10(3):e5.

Jenkins 2012 C-RCT Kenya \{published data only\} * Jenkins R. Assessment of the impact of a Kenya Medical Training College delivered structured five day training programme on mental health core concepts, skills and competencies on mental health for primary care staff in Kenya. ISRCTN53515024 2012:ISRCTN53515024. Jenkins R, Kiima D, Njenga F, Okonji M, Kingora J, Kathuku D, et al.Integration of mental health into primary care in Kenya. World Psychiatry 2010;9(2):118-20. Jenkins R, Kiima D, Okonji M, Njenga F, Kingora J, Lock $\mathrm{S}$. Integration of mental health into primary care and community health working in Kenya: context, rationale, coverage and sustainability. Mental Health in Family Medicine 2010;7(1):37-47.

Jordans 2010 C-RCT Nepal \{published data only\} Jordans MJD, Komproe IH, Tol WA, Kohrt BA, Luitel NP, Macy RD, et al.Evaluation of a classroom-based psychosocial intervention in conflict-affected Nepal: a cluster randomised controlled trial. Journal of Child Psychology and Psychiatry, and Allied Disciplines 2010;51(7): 818-26.

Li 1989 RCT China \{published data only\} Li SC. A report on a feasibility test of "community control of epilepsy" proposed by WHO. Chinese Journal of Neurology and Psychiatry 1989;22(3):144-7.

Loughry 2006 CBA Palestin \{published data only\} Loughry M, Ager A, Flouri E, Khamis V, Afana AH, Qouta S. The impact of structured activities among Palestinian children in a time of conflict. Journal of Child Psychology and Psychiatry, and Allied Disciplines 2006;47(12):1211-8.

Lyketsos1999CBA Argentina \{published data only\} Lyketsos CG, Taragano F, Treisman GJ, Paz J. Major depression and its response to sertraline in primary care vs psychiatric office practice patients: results of an open-label trial in Argentina. Psychosomatics 1999;40(1):70-5.

Neuner 2008 NRCT Uganda \{published data only\} Neuner F, Onyut PL, Ertl V, Odenwald M, Schauer E, Elbert T. Treatment of posttraumatic stress disorder by trained lay counsellors in an African refugee settlement: a randomised controlled trial. Journal of Consulting and Clinical Psychology 2008;76(4):686-94.

Noknoy 2010 RCT Thailand \{published data only\} Noknoy S, Rangsin R, Saengcharnchai P, Tantibhaedhyangkul U, McCambridge J. RCT of effectiveness of motivational enhancement therapy delivered by nurses for hazardous drinkers in primary care units in Thailand. Alcohol and Alcoholism (Oxford, Oxfordshire) 2010;45(3):263-70.

Non-specialist health worker interventions for the care of mental, neurological and substance-abuse disorders in low- and middle- 
Papas 2011 RCT Kenya \{published data only\}

* Papas RK, Sidle JE, Gakinya BN, Baliddawa JB, Martino

$S$, Mwaniki MM, et al.Treatment outcomes of a stage 1 cognitive-behavioral trial to reduce alcohol use among human immunodeficiency virus-infected out-patients in Western Kenya. Addiction 2011; Vol. 106, issue 12: 2156-66.

Papas RK, Sidle JE, Martino S, Baliddawa JB, Songole $\mathrm{R}$, Omolo OE, et al.Systematic cultural adaptation of cognitive-behavioral therapy to reduce alcohol use among HIV-infected outpatients in western Kenya. AIDS and Behavior 2010;14(3):669-78.

Paranthaman2010CBAMalaysi \{published data only\} Paranthaman V, Satnam K, Lim J-L, Amar-Singh HSS, Sararaks S, Nafiza M-N, et al.Effective implementation of a structured psychoeducation programme among caregivers of patients with schizophrenia in the community. Asian Journal of Psychiatry 2010;3(4):206-12.

Patel 2010 C-RCT India \{published data only\} * Patel V, Weiss HA, Chowdhary N, Naik S, Pednekar S, Chatterjee $S$, et al.Effectiveness of an intervention led by lay health counsellors for depressive and anxiety disorders in primary care in Goa, India (MANAS): a cluster randomised controlled trial. Lancet 2010;376(9758):2086-95.

Patel VH, Kirkwood BR, Pednekar S, Araya R, King M, Chisholm D, et al.Improving the outcomes of primary care attenders with common mental disorders in developing countries: a cluster randomised controlled trial of a collaborative stepped care intervention in Goa, India. Trials 2008;9(4):e.

Rahman 2008 CRCT Pakistan \{published data only\} Rahman A. Challenges and opportunities in developing a psychological intervention for perinatal depression in rural Pakistan-a multi-method study. Archives of Women's Mental Health 2007;10(5):211-9.

* Rahman A, Malik A, Sikander S, Roberts C, Creed F. Cognitive behaviour therapy-based intervention by community health workers for mothers with depression and their infants in rural Pakistan: a cluster-randomised controlled trial. Lancet 2008;372(9642):902-9.

Rojas 2007 RCT Chile \{published data only\}

Rojas G, Fritsch R, Solis J, Jadresic E, Castillo C, Gonzalez $\mathrm{M}$, et al.Treatment of postnatal depression in low-income mothers in primary-care clinics in Santiago, Chile: a randomised controlled trial. Lancet 2007;370(9599): 1629-37.

Scholte 2011 CBA Rwanda \{published data only\} Scholte WF, Verduin F, Kamperman AM, Rutayisire T, Aeilko $\mathrm{H}$. The effect on mental health of a large scale psychosocial intervention for survivors of mass violence: a quasi-experimental study in Rwanda. PLoS One 2011;6(8): e21819.

Shin 2009 RCT Vietnam \{published data only\} Shin JY, Nhan NV, Lee S-B, Crittenden KS, Flory M, Hong HTD. The effects of a home-based intervention for young children with intellectual disabilities in Vietnam. Journal of Intellectual Disability Research: JIDR 2009;53(4):339-52.
Sutcliffe2009RCT Thailand \{published data only\}

German D, Sutcliffe CG, Sirirojn B, Sherman SG, Latkin CA, Aramrattana A, et al.Unanticipated effect of a randomised peer network intervention on depressive symptoms among young methamphetamine users in Thailand. Journal of Community Psychology 2012;40(7): 799-813.

* Sutcliffe C, Srirojn B, Latkin CA, Aramratanna A, Celentano DD, Sherman SG. Evaluation of a peer network intervention trial among young methamphetamine users in Chiang Mai, Thailand. Social Science \& Medicine 2009;68 (1):69-79.

Thabet 2005 CBA Palestine \{published data only\} Thabet AA, Vostanis P, Karim K. Group crisis intervention for children during ongoing war conflict. European Child \& Adolescent Psychiatry 2005;14(5):262-9.

Tiwari 2010 RCT China \{published data only\} Tiwari A, Fong DYT, Yuen KH, Yuk H, Pang P, Humphreys J, et al.Effect of an advocacy intervention on mental health in Chinese women survivors of intimate partner violence: a randomised controlled trial. JAMA: the Journal of the American Medical Association 2010;304(5):536-43.

Tol 2008 C-RCT Indonesia \{published data only\} Jordans MJD, Komproe IH, Tol WA, Susanty D, Vallipuram A, Ntamatumba P, et al.Practice-driven evaluation of a multi-layered psychosocial care package for children in areas of armed conflict. Community Mental Health Journal 2011; 47(3):267-77.

Tol WA, Komproe IH, Jordans MJD, Gross AL, Susanty D, Macy RD, et al.Mediators and moderators of a psychosocial intervention for children affected by political violence. Journal of Consulting and Clinical Psychology 2010;78(6): 818-28.

* Tol WA, Komproe IH, Susanty D, Jordans MJD, Macy RD, De Jong Joop TVM. School-based mental health intervention for children affected by political violence in Indonesia: a cluster randomised trial. JAMA: the Journal of the American Medical Association 2008;300(6):655-62.

Tol 2012 C-RCT SriLanka \{published data only\} Jordans MJD, Komproe IH, Tol WA, Susanty D, Vallipuram A, Ntamatumba P, et al.Practice-driven evaluation of a multi-layered psychosocial care package for children in areas of armed conflict. Community Mental Health Journal 2011; 47(3):267-77.

* Tol WA, Komproe IH, Jordans MJD, Vallipuram A, Sipsma H, Sivayokan S, et al.Outcomes and moderators of a preventive school-based mental health intervention for children affected by war in Sri Lanka: a cluster randomised trial. World Psychiatry: Official Journal of the World Psychiatric Association (WPA) 2012;11(2):114-22.

Wolmer 2005 CBA Turkey \{published data only\} Wolmer L, Laor N, Dedeoglu C, Siev J, Yazgan Y. Teachermediated intervention after disaster: a controlled threeyear follow-up of children's functioning. Journal of Child Psychology, Psychiatry and Allied Disciplines 2005;46(11): 1161-8. 
Yeomans 2010 RCT Burundi \{published data only\} Yeomans PD, Forman EM, Herbert James D, Yuen E. A randomized trial of a reconciliation workshop with and without PTSD psychoeducation in Burundian sample. Journal of Traumatic Stress 2010;23(2):305-12.

Zambori 2002 CBA Hungary \{published data only\} Zambori J, Szadoczky E, Rozsa S, Furedi J. Cost-outcome of anxiety treatment intervention in primary care in Hungary. Journal of Mental Health Policy and Economics 2002;5: $115-20$.

\section{References to studies excluded from this review}

Abiodun 1991 \{published data only\}

Abiodun OA. Knowledge and attitude concerning mental health of primary health care workers in Nigeria. International Journal of Social Psychiatry 1991;37(2): 113-20.

Acha 2007 \{published data only\}

Acha J, Sweetland A, Guerra D, Chalco K, Castillo H, Palacios E. Psychosocial support groups for patients with multidrug-resistant tuberculosis: five years of experience. Global Public Health: an International Journal for Research, Policy and Practice 2007;2(4):404-17.

Acuda 1992 \{published data only\}

Acuda W. Nairobi, Kenya. In: Babor TF, Grant M editor(s). Identification and Management of Alcohol-related Problems: Report on Phase II, a Randomized Clinical Trial of Brief Interventions in Primary Health Care. 91. Geneva: World Health Organization, 1992:113-28.

Adamolekun 2000 \{published data only\}

Adamolekun B, Mielke J, Ball D, Mundanda T. An evaluation of the management of epilepsy by primary health care nurses in Chitungwiza, Zimbabwe. Epilepsy Research 2000;39(3):177-81.

Adams 2012 \{published data only\}

Adams JL, Almond MLG. Feasibility of nurse-led antidepressant medication management of depression in an HIV clinic in Tanzania. International Journal of Psychiatry in Medicine 2012;43(2):105-17.

Ahn 2004 \{published data only\}

Ahn Y-M, Kim M-R. The effects of a home-visiting discharge education on maternal self-esteem, maternal attachment, postpartum depression and family function in the mothers of NICU infants. Journal of Korean Academy of Nursing 2004;34(8):1468-76.

Ali 2010 \{published data only\}

Ali NS, Ali BS, Azam IS, Khuwaja AK. Effectiveness of counselling for anxiety and depression in mothers of children ages 0-30 months by community workers in Karachi, Pakistan: a quasi experimental study. $B M C$ Psychiatry 2010;10:57.

Alvarado 2011 \{published data only\}

Alvarado R, Rojas G. Evaluation of the program for detection and treatment of depression in Chilean primary health care centers. Revista Médica de Chile 2011;139(5): $592-9$.
Anand 2005 \{published data only\}

Anand K, Jain S, Paul E. Development of a validated clinical case definition of generalized tonicclonic seizures for use by community-based health care providers. Epilepsia 2005;46 (5):743-50

Apil 2011 \{published data only\}

Apil SRA. A stepped care relapse prevention program for depression in older people: a randomized controlled trial. International Journal of Geriatric Psychiatry 2011;27: 583-91.

Aravena 2011 \{published data only\}

Aravena CP, Ruiz GP. Experience of implementing a mental health first response polyclinic in a primary health centre [Experiencia en la implementacion de un policlinico de Choque de salud mental en un establecimiento de atencion primaria]. Cuadernos médico-sociales 2011;51(1):5-15.

Arcel 1995 \{published data only\} Arcel LT, Folnegovic-Smalc V, Kozaric-Kovacic D, Marusic A. Psycho-Social Help to War Victims: Refugee Women and their Families. International Rehabilitation Council for Torture Victims, 1995, 1995.

Armstrong 2011 \{published data only\} Armstrong G, Kermode M, Raja S, Suja S, Chandra P, Jorm AF. A mental health training program for community health workers in India: impact on knowledge and attitudes. International Journal of Mental Health Systems 2011; Vol. 5 , issue $1: 17$

Babor 1992 \{published data only\} Babor T, Grant M. Identification and Management of Alcohol-related Problems: Report on Phase II, a Randomised Clinical Trial of Brief Interventions in Primary Health Care. Geneva, Switzerland: World Health Organization, 1992: 266.

Babor 1994 \{published data only\}

Babor TF, Grant M. Comments on the WHO report "Brief Interventions for Alcohol Problems": a summary and some international comments. Addicition 1994;89:657-61.

Bae 2009 \{published data only\}

Bae J, Kim K-Y, Panuncio RL, Choi N, Im S-B. Inauguration of the first Psychological Support Center for disaster victims in Korea. Nursing \& Health Sciences 2009; 11(4):351-6.

Baker-Henningham 2009 \{published data only\} Baker-Henningham H, Walker S, Powell C, Gardner J Meeks. A pilot study of the Incredible Years Teacher Training programme and a curriculum unit on social and emotional skills in community pre-schools in Jamaica. Child: Care, Health and Development 2009;35(5):624-31.

Bakran 2001 \{published data only\}

Bakran Z, Bobinac-Georgievski A, Dzidic I, Jelic M, Eldar R. Medical rehabilitation in Croatia - impact of the 19911995 war: past problems, present state, future concerns. Croatian Medical Journal 2001;42(5):556-64.

Balaji 2011 \{published data only\} Balaji M, Andrews T, Andrew G, Patel V. The acceptability, feasibility, and effectiveness of a population-based 
intervention to promote youth health: an exploratory study in Goa, India. The Journal of Adolescent Health: Official Publication of the Society for Adolescent Medicine 2011;48(5): 453-60.

\section{Ball 2000 \{published data only\}}

Ball DE, Mielke J, Adamolekun B. Community leader education to increase epilepsy attendance at clinics in Epworth, Zimbabwe. Epilepsia 2000;41(8):1044-5.

Bangirana 2006 \{published data only\}

Bangirana P, Idro R, Chandy JC, Boivin MJ. Rehabilitation for cognitive impairments after cerebral malaria in African children: strategies and limitations. Tropical Medicine \& International Health 2006;11(9):1341-9.

Barcala 2009 \{published data only\} Barcala A, Torricelli F, Alvarez ZP, Marotta J. Programa de atencion comunitaria a ninos, ninas y adolescentes con trastornos metales severos. Vertex: revista argentina de psiquiatria 2009;XX:282-92.

Becker 2006 \{published data only\}

Becker SM. Psychosocial care for adult and child survivors of the 2004 tsunami disaster in India. American Journal of Public Health 2006;96(8):1397-8.

Becker 2007 \{published data only\}

Becker SM. Psychosocial care for adult and child survivors of the tsunami disaster in India. Journal of Child and Adolescent Psychiatric Nursing 2007;20(3):148-55.

Becker 2009 \{published data only\} Becker SM. Psychosocial care for women survivors of the tsunami disaster in India. American Journal of Public Health 2009;99(4):654-8.

\section{Beckerleg 1996 \{published data only\}}

Beckerleg S. Private struggles, public support: rehabilitating heroin users in Kenya. Drugs: Education, Prevention, and Policy 1996;3(2):159-69.

Bedregal 2010 \{published data only\} Bedregal P, Hernandez V, Prado P, Castanon C, Mingo V, de la Cruz R. Preliminary Report of a Governmental Program on Children Psychosocial Development Care in Chile. Revista Medica Chile 2010;138:791-3.

Bellali 2006 \{published data only\} Bellali Th, Kalafati M. Greek psychiatric care reform: new perspectives and challenges for community mental health nursing. Psychiatric and Mental Health Nursing 2006;13: 33-9.

Berman 1993 \{published data only\} Berman R, Roel G. Encounter with death and destruction: the 1985 Mexico City earthquake. Group Analysis 1993;26: 81-9.

Bichescu 2007 \{published data only\}

Bichescu D, Neuner F, Schauer M, Elbert T. Narrative exposure therapy for political imprisonment-related chronic posttraumatic stress disorder and depression. Behaviour Research and Therapy 2007;45(9):2212-20.
Blair 2006 \{published data only\}

Blair KC, Liaupsin CJ, Umbreit J, Kweon G. Functionbased intervention to support the inclusive placements of young children. Education and Training in Developmental Disabilities 2006;41(1):48-57.

Boavida 2000 \{published data only\}

Boavida J. Community-based early intervention: the Coimbra Project (Portugal). Child: Care, Health and Development 2000;26(5):343-54.

Bochen 2006 \{published data only\}

Bochen $\mathrm{D}$. The role of music therapy in care of terminally ill cancer patients. Onkologia Polska 2006;9(4):133-6.

Bondy 1993 \{published data only\}

Bondy AS, Frost LA. Mands across the water: a report on the application of the picture-exchange communication system in Peru. The Behavior Analyst 1993;16:123-8.

Booth 2011 \{published data only\} Booth RE, Lehman WEK, Latkin CA, Dvoryak S, Brewster JT, Royer MS, et al.Individual and network interventions with injection drug users in 5 Ukraine cities. American Journal of Public Health 2011;101(2):336-43.

Boothby 2011 \{published data only\} Boothby N. Evaluating treatment of Axis I mental health disorders in Aceh, Indonesia. The Psychiatrist 2011;35: 248-55.

Borucka 2003 \{published data only\} Borucka A, Pisarska A, Okulicz-Kozaryn K. Evaluation of a school-based intervention method for drug using students. Medycyna Wieku Rozwojowego 2003;1(2):157-72.

Bower 2001 \{published data only\}

Bower P, Garralda E, Kramer T, Harrington R, Sibbald B. The treatment of child and adolescent mental health problems in primary care: a systematic review. Family Practice 2001;18(4):373-82.

\section{Boyadjieva 1992 \{published data only\}}

Boyadjieva M. Centre report: Pleven, Bulgaria. In: Babor TF, Grant M editor(s). Identification and Management of Alcohol-related Problems: Report on Phase II, a Randomised Clinical Trial of Brief Interventions in Primary Health Care. Geneva: World Health Organization, 1992:91-103.

Bragin 2007 \{published data only\}

Bragin M, Prabhu V, Czarnocha B. Mathematics, psychosocial work and human rights: a unique partnership between technical consultants and community organisers in India. Intervention 2007;5(2):97-108.

Brown 2005 \{published data only\} Brown GK, Ten Have T, Henriques GR, Xie SX, Hollander JE, Beck AT. Cognitive therapy for the prevention of suicide attempts: a randomised controlled trial. JAMA 2005;294 (5):563-70 .

Cabildo 1973 \{published data only\} Cabildo MH. Mental health services at health centres. Acta Psiquiátrica y Psicológica de América Latina 1973;19:226.

Non-specialist health worker interventions for the care of mental, neurological and substance-abuse disorders in low- and middle- 
Caciula 2010 \{published data only\}

Caciula I, Livingston G, Caciula R, Cooper C. Recognition of elder abuse by home care workers and older people in Romania. International Psychogeriatrics 2010;22(3):403-8.

Caciula 2010a \{published data only\}

Caciula I, Livingston G, Caciula R, Cooper Ca. Recognition of elder abuse by home care workers and older people in Romania. International Psychogeriatrics 2010;22(3):403-8.

Calderon 2008 \{published data only\}

Calderon Diana. Programme to improve psychosocial elements with creative techniques (a youth intervention including treatment for drug abuse) [Programa para mejorar las habilidades psicosociales con tecnicas creativas (intervencion para jovenes en tratamiento por drogadependencia)]. Facultad de Ciencias Medicas. Cordoba: Universidad nacional de Cordoba, 2008; Vol. Magister e:176.

Campillo 1992 \{published data only\} Campillo SC, Martinez DRD, MMR Mendoza, Sanchez JC, Velazquez JV. Centre report: Mexico City, Mexico. In: Babor TF, Grant M editor(s). Identification and Management of Alcohol-related Problems: Report on Phase II, a Randomised Clinical Trial of Brief Interventions in Primary Health Care. 91. Geneva: World Health Organization, 1992:129-42.

\section{Carli 2010 \{published data only\}}

Carli V, C Wasserman, C Hoven, M Sarchiapone, D Wasserman. SAYLE health promoting programme adolescents (risk taking and suicide behaviours). Proceedings of the 18th European Congress of Psychiatry; 2010 Feb 27-Mar 2; Munich. 2010.

Castellarin 1985 \{published data only\}

Castellarin C. Evaluation of a rehabilitation service for the psychosocially chronic patient [Avaliacao de um servico de reabilitacao de doentes psicossocialmente cronicos]. Revista de psiquiatria do Rio Grande do Sul 1985;7(3):199-215.

Catani 2009 \{published data only\} Catani C, Kohiladevy M, Ruf M, Schauer E, Elbert T, Neuner F. Treating children traumatized by war and Tsunami: a comparison between exposure therapy and meditation-relaxation in North-East Sri Lanka. BMC Psychiatry 2009;9(1):22.

Cavlek 2006 \{published data only\} Cavlek T, Rezek S, Bartolic A, Dosen D, Jaksic A. Team approach to the treatment of school children with chronic non-communicable diseases: a model and efficiency. Paediatria Croatica 2006;50:79-84.

Cereceda 2011 \{published data only\} Cereceda Pablo Aravena, Gonzalez Patricio Ruiz. Experience of implementing a mental health first response polyclinic in a primary health center [Experiencia en la implementacion de un policlinico de choque de salud mental en un etablecimiento de atencion primaria]. Cuadernos médicosociales 2011;51(1):5-15.

\section{Cerny 1975 \{published data only\}}

Cerny L. Experience in the reeducation of children with dyslexia in Czechoslovakia. International Journal of Mental Health 1975;4:113-22.

Chang 2000 \{published data only\}

Chang T, $\mathrm{Li}$ I. The effect of social support intervention on home-bound stroke patients' physical and mental health in the Ilan area [in Chinese]. The Journal of Nursing (Taiwan) 2000;8(4):423-34.

Chankrachang 2009 \{published data only\}

Chankrachang $S$. The evaluation of the first caregiver training program from patient with dementia in Thailand. Journal of the Neurological Sciences 2009;285(Suppl 1):S130.

Chapman 1988 \{published data only\} Chapman PLH, Huygens I. An Evaluation of Three Treatment Programmes for Alcoholism: an experimental study with 6-and 18-month follow-ups. Brinsh Journal of Addiction 1988;83(1):67-81.

Chatterjee 2003 \{published data only\} Chatterjee S, Patel V, Chatterjee A, Weiss HA. Evaluation of a community-based rehabilitation model for chronic schizophrenia in rural India. British Journal of Psychiatry 2003; 182:57-62.

Chatterjee 2005 \{published data only\} Chatterjee P. Mental health care for India's tsunami survivors. Lancet 2005;365(9462):833-4.

Chatterjee 2009 \{published data only\} Chatterjee S, Pillai A, Jain S, Cohen A, Patel V. Outcomes of people with psychotic disorders in a community-based rehabilitation programme in rural India. British Journal of Psychiatry 2009;195(5):433-9.

Chen 2000a \{published data only\} Chen C-H, Tseng Y-F, Chou F-H, Wang Sg-Y. Effects of support group intervention in postnatally distressed women: a controlled study in Taiwan. Journal of Psychosomatic Research 2000;49(6):395-9.

Chhabra 2010 \{published data only\} Chhabra R, Springer C, Leu C-S, Ghosh S, Sharma SK, Rapkin B. Adaptation of an alcohol and HIV school-based prevention program for teens. AIDS and Behavior 2010;14 (1):S177-84.

\section{Chibanda 2011 \{published data only\}} Chibanda D, Mesu P, Kajawu L, Cowan F, Araya R, Abas MA. Problem-solving therapy for depression and common mental disorders in Zimbabwe: piloting a task-shifting primary mental health care intervention in a population with a high prevalence of people living with HIV. $B M C$ Public Health 2011;11:828.

\section{Chien 2008 \{published data only\}} Chien W-T, Thompson DR, Norman I. Evaluation of a peer-led mutual support group for Chinese families of people with schizophrenia. American Journal of Community Psychology 2008;42(1-2):122-34.

Chisholm 2000 \{published data only\} Chisholm D, Sekar K, Kumar Kishore, Saeed K, James S, Mubbashar M, et al.Integration of mental health care into

Non-specialist health worker interventions for the care of mental, neurological and substance-abuse disorders in low- and middle- 
primary care. Demonstration cost-outcome study in India and Pakistan. British Journal of Psychiatry 2000;176:581-8.

Cho 2011 \{published data only\}

Cho S-C, Kim H-W, Kim B-N, Shin M-S, Yoo HJ, Kim J$\mathrm{W}$, et al.Are teacher ratings and parent ratings differently associated with children's intelligence and cognitive performance?. Psychiatry Investigation 2011;8:15-21.

Chou 2002 \{published data only\} Chou K-R, Liu S-Y, Chu H. The effects of support groups on caregivers of patients with schizophrenia. International Journal of Nursing Studies 2002;39(7):713-22.

Chow 2010 \{published data only\}

Chow W, Law S, Andermann L, Yang J, Leszcz M, Wong $\mathrm{J}$, et al.Multi-family psycho-education group for assertive community treatment clients and families of culturally diverse background: a pilot study. Community Mental Health Journal 2010;46(4):364-71.

Chowdhury 2004 \{published data only\}

Chowdhury AN, Brahma A, Banerjee S. How to operationalise community mental health service at the primary care? Experience of IRMC model from Sundarban, India. International Medical Journal 2004;11(2):105-10.

Chowdhury 2005 \{published data only\}

Chowdhury AN, Brahma A, Banerjee S, Biswas MK. Psychiatric morbidity at primary care: study from a community mental health clinic at Sundarban, India. International Medical Journal 2005;12(1):11-8.

Climent 1981 \{published data only\}

Climent C E, De Arango M V. Primary mental health care in Latin America. The Cali program. Acta Psiquiatricay Psicologica de America Latina 1981;27(4-5):257-74.

Climent 1983 \{published data only\} Climent CE, de Arango MV, Plutchick R. Development of an alternative, efficient, low-cost mental health delivery system in Cali, Colombia. Social Psychiatry 1983;18(2): 95-102.

Colon de Marti 1993 \{published data only\} Colon de Marti Luz N. Day-treatment center: its origin and impact mental health services for children and adolescents [El centro de tratamiento diurno: su origen e impacto en los servicios de salud mental para ninos y adolescentes]. Boletin de la Asociacion Medica de Puerto Rico 1993;85(1/3):27-33.

Cooper 2002 \{published data only\}

Cooper PJ, Landman M, Tomlinson M, Molteno C, Swartz L, Murray L. Impact of a mother-infant intervention in an indigent peri-urban South African context: pilot study. British Journal of Psychiatry 2002;180(1):76-81.

Cooper 2009 \{published data only\}

Cooper PJ, Tomlinson M, Swartz L, Landman M, Molteno C, Stein A, et al.Improving quality of mother-infant relationship and infant attachment in socioeconomically deprived community in South Africa: randomised controlled trial. BMJ (Clinical Research Ed.) 2009;338: b974.
Coyle 1998 \{published data only\}

Coyle SL. Women's drug use and HIV risk: findings from NIDA's Cooperative Agreement for Community-Based Outreach/Intervention Research Program. Women \& Health 1998;27(1-2):1-18.

Crawford 2004 \{published data only\} Crawford MJ, Patton R, Touquet R, Drummond C, Byford S, Barrett B, et al.Screening and referral for brief intervention of alcohol-misusing patients in an emergency department: a pragmatic randomised controlled trial. Lancet 2004;364(9442):1334-39.

Cummings 2008 \{published data only\} Cummings SM, Long JK, Peterson-Hazan S, Harrison J. The efficacy of a group treatment model in helping spouses meet the emotional and practical challenges of early stage caregiving. Journal of Aging \& Mental Health 2008;20(1): 29-45.

Dabrowski 1998 \{published data only\}

Dabrowski S, Brodniak W, Gierlacki J, Welbel S.

Community self-help houses as a form of community social support. Psychiatria Polska 1998;32(4):453-62.

Das 2006 \{published data only\}

Das S, Saravanan B, Karunakaran KP, Manoranjitham S, Ezhilarasu P, Jacob KS. Effect of a structured educational intervention on explanatory models of relatives of patients with schizophrenia: randomised controlled trial. British Journal of Psychiatry 2006;188(3):286-7.

Davis 1988 \{published data only\}

Davis B, Reis J. Implementation and preliminary evaluation of a community-based prenatal health education programme. Family and Community Health 1988;11(1): 8-16.

De Arellano 2005 \{published data only\} De Arellano MA, Waldrop AE, Deblinger E, Cohen JA, Danielson CK, Mannarino AR. Community outreach programme for child victims of traumatic events: a community-based project for underserved populations. Behavior Modification 2005;29(1):130-55.

De Clercq 2001 \{published data only\}

De Clercq L, Lagerkvist B, Kapelanovic T, Puratic V. Assessment of community mental health care in the Federation of Bosnia-Hercegovina (FBH) after the 1992-95 war. Medicinski arhiv 2001;55(2):105-12.

De Jong 1996 \{published data only\}

De Jong JTVM. A comprehensive public mental health programme in Guinea-Bissau: a useful model for African, Asian and Latin-American countries. Psychological Medicine 1996;26(01):97-108.

Dernovsek 2010 \{published data only\} Dernovsek M, Sprah L. Assessment of the preventive and promotion activities in the mental health area in 12 Slovenian regions. Conference: 18th European Congress of Psychiatry Munich Germany.Conference Start: 27 February - 2 March. European Psychiatry. 2010. 
Devaramane 2011 \{published data only\}

Devaramane V, Pai NB, Vella S-L. The effect of a brief family intervention on primary carer' $s$ functioning and their schizophrenic relatives levels of psychopathology in India. Asian Journal of Psychiatry 2011;4(3):183-7.

Devine 2007 \{published data only\}

Devine A, Kermode M, Chandra P, Herrman H. A participatory intervention to improve the mental health of widows of injecting drug users in north-east India as a strategy for HIV prevention. BMC International Health and Human Rights 2007;7(1):3.

Dias 2004 \{published data only\} Dias A, Samuel R, Patel V, Prince M, Parameshwaran R, Krishnamoorthy ES. The impact associated with caring for a person with dementia: a report from the 10/66 Dementia Research Group's Indian network. International Journal of Geriatric Psychiatry 2004;19:182-4.

Diken 2010 \{published data only\}

Diken IH, Cavkaytar A, Sema BE, Bozkurt F, Kurtyilmaz Y. First step to success - a school/home intervention program for preventing problem behaviors in young children: Examining the effectiveness and social validity in Turkey. Emotional \& Behavioural Difficulties 2010;15(3):207-21.

Dorji 2006 \{published data only\}

Dorji C. Mental health and psychosocial aspects of disaster preparedness in Bhutan. International Review of Psychiatry 2006;18(6):537-46.

Dvorin 1989 \{published data only\} Dvorin DV, Kuznet ME. Current approaches to conducting anti-alcohol health education work by paramedical personnel. Feldsher Akush 1989;8:38-42.

Eaton 2008 \{published data only\} Eaton J, Agomoh AO. Developing mental health services in Nigeria: the impact of a community-based mental health awareness programme. Social Psychiatry and Psychiatric Epidemiology 2008;43(7):552-8.

Ehntholt 2005 \{published data only\}

Ehntholt KA. School-based cognitive-behavioural therapy group intervention for refugee children who have experienced war-related trauma. Clinical Child Psychology and Psychiatry 2005;10(2):235-50.

Eickmann 2003 \{published data only\} Eickmann SH, Lima ACV, Guerra MQ, Lima MC, Pedro IC, Huttly SRA, Ashworth A. Improved cognitive and motor development in a community-based intervention of psychosocial stimulation in northeast Brazil. Developmental Medicine \& Child Neurology 2003;45(8):536-41.

El Gaili 2002 \{published data only\}

El Gaili DE, Magzoub MM, Schmidt HG. The Impact of a community-oriented medical school on mental health services. Education for Health 2002;15(2):149-57.

El Sayed 2002 \{published data only\}

El Sayed EM. Evaluation of self care programme of mentally retarded children at Alwafaa volunteering social centre,
Sohar Wilayat, Sultanate of Oman. Alexandria Journal of Pediatrics 2002;20(1):1-7.

Ensink 2007 \{published data only\}

Ensink K, Robertson BA, Zissis C, Leger P, de Jager W. Conduct disorder among children in an informal settlement. South African Medical Journal 2007;87(11):1533-7.

Erbas 2004 \{published data only\}

Erbas D. Comparison of two approaches for identifying reinforcers in teaching figure coloring to students with Down syndrome. Education and Training in Developmental Disabilities 2004;39(3):253-64.

Ezard 2010 \{published data only\}

Ezard N, Annabel D, Catillon R. Screening and brief intervention for high-risk alcohol use in Mae La refugee camp, Thailand: a pilot project on the feasibility of training and implementation. Intervention 2010;8(3):223-32.

Farooq 2011 \{published data only\} Farooq S, Nazar Z, Irfan M, Akhter J, Gul E, Irfan U, et al.Schizophrenia medication adherence in a resource-poor setting: randomised controlled trial of supervised treatment in out-patients for schizophrenia (STOPS). British Journal of Psychiatry 2011;199:467-72.

Fawzy 2012 \{published data only\}

Fawzy FI, Fawzy NW, Hyun CS, ER, Guthrie D, Fahey JL, et al.Malignant melanoma. Effects of an early structured psychiatric intervention, coping, and affective state on recurrence and survival 6 years later. Archives of General Psychiatry 2012;50(9):681-9.

Fayyad 2010 \{published data only\} Fayyad JA, Farah L, Cassir Y, Salamoun MM, Karam EG. Dissemination of an evidence-based intervention to parents of children with behavioral problems in a developing country. European Child \& Adolescent Psychiatry 2010;19 (8):629-36.

\section{Feksi 1991 \{published data only\}}

Feksi AT, Kaamugisha J, Gatiti S, Sander JW, Shorvon SD. A comprehensive community epilepsy programme: the Nakuru project. Epilepsy Research 1991;8(3):252-9.

Feksi 1991a \{published data only\} Feksi AT, Kaamugisha J, Sander JW, Gatiti S, Shorvon SD. Comprehensive primary health care antiepileptic drug treatment programme in rural and semi-urban Kenya. Lancet 1991;337(8738):406-9.

Fernandes 2007 \{published data only\} Fernandes PT, Noronha ALA, Sander JW, Bell GS. Training the trainers and disseminating information: a strategy to educate health professionals on epilepsy. Arquivos de Neuropsiquiatria 2007;65(1):14-22.

Fernandes 2011 \{published data only\} Fernandes PT, Shih L, Hui L, Correa C, Felice N, Min L. Social Interaction Group (GIS): A strategy of empowerment for people with epilepsy [Grupos de Interação Social (GIS): estratégia de empowerment para pessoas com epilepsia]. Journal of Epilepsy and Clinical Neurophysiology 2011;17(2): $70-4$.

Non-specialist health worker interventions for the care of mental, neurological and substance-abuse disorders in low- and middle- 
Ferrinho 1993 \{published data only\}

Ferrinho P, Robb D, Cornielje H, Rex G. Primary health care in support of community development. World Health Forum 1993;14(2):158-62.

Fischman 1990 \{published data only\}

Fischman Y, Ross J. Group treatment of exiled survivors of torture. American Journal of Orthopsychiatry 1990;60(1): $135-42$.

Fleischmann 2008 \{published data only\}

Fleischmann A, Bertolote JM, Wasserman D, De Leo D, Bolhari J, Botega NJ, et al.Effectiveness of brief intervention and contact for suicide attempters: a randomized controlled trial in five countries. Bulletin of the World Health Organization 2008;86(9):703-9.

Fleming 1999 \{published data only\} Fleming MF, Manwell LB, Barry KL, Adams W, Stauffacher EA. Brief physician advice for alcohol problems in older adults: a randomized community-based trial. Journal of Family Practice 1999;48(5):378-84.

Friedlander 1985 \{published data only\} Friedlander SR, Watkins EC. Therapeutic aspects of support groups for parents of the mentally retarded. International Journal of Group Psychotherapy 1985;35(1):64-78.

Futterman 2010 \{published data only\} Futterman D, Shea J, Besser M, Stafford S, Desmond K, Comulada WS, et al.Mamekhaya: a pilot study combining a cognitive-behavioral intervention and mentor mothers with PMTCT services in South Africa. AIDS care 2010;22 (9):1093-100

Gardner 2003 \{published data only\}

Gardner JM, Walker SP, Powell CA, Grantham-McGregor $\mathrm{S}$. A randomized controlled trial of a home-visiting intervention on cognition and behavior in term low birth weight infants. Journal of Pediatrics 2003;143(5):634-9.

Gentilello 1999 \{published data only\}

Gentilello LM, Rivara FP, Donovan DM, Jurkovich GJ, Daranciang E, Dunn CW, et al.Alcohol interventions in a trauma centre as a means of reducing the risk of injury recurrence. Annals of Surgery 1999;230(4):473-83.

Ghasseimi 2005 \{published data only\} Ghasseimi GR, Assadulahi GA, Mallik S. Family education and social adjustment of psychiatric clients. Medical Journal of Islamic World Academy of Sciences 2005;15(12):73-80.

Ghosh 2004 \{published data only\}

Ghosh N. Mental health promotion in post-conflict countries. The Journal of the Royal Society for the Promotion of Health 2004;124(6):268-70.

Giannopoulou 2006 \{published data only\} Giannopoulou I, Dikaiakou A, Yule W. Cognitivebehavioural group intervention for PTSD symptoms in children following the Athens 1999 earthquake: A pilot study. Clinical Child Psychology and Psychiatry 2006;11(4): 543-53.
Goenjian 1997 \{published data only\}

Goenjian AK, Karayan I, Pynoose RS, Minassian D, Najarian LM, Steinberg AM, et al.Outcome of psychotherapy among early adolescents after trauma. American Journal of Psychiatry 1997;154:536-42.

Goldin 2008 \{published data only\} Goldin S, Hagglof B, Levin L, Persson LA. Mental health of Bosnian refugee children: a comparison of clinician appraisal with parent, child and teacher reports. Nordic Journal of Psychiatry 2008;62(3):204-16.

Gondim 2001 \{published data only\}

Gondim DSM. Analysis of the implementation of a service of psychiatric emergency in the Municipal district of Campos: innovation or reproduction of an assistance model? [Análise da implantação de um serviço de emergência psiquiátrica no município de Campos: inovação ou reprodução do modelo assistencial?]. Masters Dissertation. Fundação Oswaldo CruzEscola Nacional de Saúde Pública 2001:p119.

Goodfriend 2004 \{published data only\} Goodfriend M. Psychosocial Care of Severely Malnourished Children. Pediatric Annals 2004;33(10):708-11.

Gordon 2004 \{published data only\} Gordon JS, Staples JK, Blyta A, Bytyqi M. Treatment of posttraumatic stress disorder in post-war Kosovo high school students using mind-body skills groups: A pilot study. Journal of Traumatic Stress 2004;17(2):143-7.

\section{Gruber 2005 \{published data only\}}

Gruber EN, Kajevic M, Bjedov M, Agius M. A combination of psychodynamic, supportive and psycho-educational approaches in parents of patients with schizophrenia [in Croatian]. Soc Psihijat 2005;33:3-9.

\section{Guerra 2011 \{published data only\}}

Guerra M, Ferri CP, Fonseca M, Banerjee S, Prince M. Helping carers to care: the $10 / 66$ dementia research group's randomized control trial of a caregiver intervention in Peru. Revista Brasileira de Psiquiatria 2011;33(1):47.

Guinhouya 2010 \{published data only\} Guinhouya KM, Aboki A, Kombate D, Kumako V, Apetse K, Belo M, et al.The epilepsy treatment gap in six primary care centres in Togo (2007-2009). Cahiers Sante. Guinhouya, Kokou Mensah: Service de Neurologle CHU de Lome-Tokoin, Lome, Togo, BP 57, herve'guinhouya@yahoo.fr: John Libbey Eurotext, 2010; Vol. 20, issue 2:93-7.

Guzman 1985 \{published data only\} Guzman-Baird M, Florenzano-Urzua R. Secondary prevention for alcoholism and drug addiction: a mental health care centre for adolescents in the Comuna de La Reina [Prevencion secundaria de alcoholismo y drogadiccion: un centro de atencion de la salud mental de adolescentes en la Comuna de La Reina]. Revista Brasileira de Psiquiatria 1985;7(24):23-31.

Hamadani 2006 \{published data only\} Hamadani JD, Huda SN, Khatun F, Grantham-McGregor SM. Psychosocial stimulation improves the development of

Non-specialist health worker interventions for the care of mental, neurological and substance-abuse disorders in low- and middle- 
undernourished children in rural Bangladesh. The journal of Nutrition 2006;136(10):2645-52.

Han 2010 \{published data only\}

Han YR, Song MS, Lim JY. The effects of a cognitive enhancement group training program for communitydwelling elders [in Korean]. Journal of Korean Academy of Nursing 2010;40(5):724-35.

Harder 2012 \{published data only\}

Harder VS, Mutiso VN, Khasakhala LI, Burke HM, Ndetei DM. Multiple traumas, post-election violence, and posttraumatic stress among impoverished Kenyan youth. Journal of Traumatic Stress. United States: Africa Mental Health Foundation, Nairobi, Kenya. vharder@uvm.edu, 2012; Vol. 25, issue 1:64-70.

Harding 1983 \{published data only\}

Harding TW, Busnello EA, Climet CE, El-Hakim A, Giel R, Ibrahim HHA, et al.The WHO collaborative study on strategies for extending mental health care. III: evaluative design and illustrative results. The American Journal of Psychiatry 1983;140(11):1481-5.

Harris 1985 \{published data only\}

Harris MJ, Fisher BS. Group therapy in the treatment of female partners of Vietnam veterans. Journal for Specialists in Group Work 1985;10(1):44-50.

Hasanovic 2009 \{published data only\}

Hasanovic M, Srabovic S, Rasidovic M, Sehovic M, Hasanbasic E, Husanovic J, et al.Psychosocial assistance to students with posttraumatic stress disorder in primary and secondary schools in post-war Bosnia Herzegovina. Psychiatria Danubina 2009;21(4):463-73.

Heather 2006 \{published data only\}

Heather N. WHO collaborative project on identification and management of alcohol-related problems in primary health care. Report on Phase IV. Development of countrywide strategies for implementing early identification and brief intervention in primary health care. World Health Organization 2006:41-51 and 173-189.

Hegerl 2009 \{published data only\}

Hegerl U, Wittenburg L, Arensman E, Audenhove CV, Coyne JC, McDaid D, et al.Optimizing suicide prevention programmes and their implementation in Europe (OSPI Europe): an evidence-based multi-level approach. $B M C$ Public Health 2009;9(428):1-8.

Heh 2003 \{published data only\}

Heh S-S, Fu Y-Y. Effectiveness of informational support in reducing the severity of postnatal depression in Taiwan. Journal of Advanced Nursing 2003;42(1):30-6.

Hensel-Dittmann 2011 \{published data only\} Hensel-Dittmann D, Schauer M. Treatment of traumatized victims of war and torture: A randomised controlled comparison of narrative exposure therapy and stress inoculation training. Psychotherapy and Psychosomatics 2011; 80:345-52.
Hernandez 2003 \{published data only\}

Hernandez FLJ. Evaluation of results and impact of the first phase of a community-based mental health model in localities in Bogotá, D.C. [Evaluación de resultados e impactos de un modelo de salud mental basado en la comunidad en localidades de Bogotá, D.C.]. Revista de Salud Publica 2003;5(3):272-83.

Hu 2006a \{published data only\}

Hu HY, Tang M-N, Ma C, Miao G-D, Hu W-S, Zheng H-

B. Evaluation on the effects of community management mode in improving the quality of life in patients with dementia [in Chinese]. Chinese Journal of Clinical Rehabilitation 2006;10(14):3-6.

Idris 2006 \{published data only\}

Idris N. Exploring the effects of TI-84 Plus on achievement and anxiety in mathematics. Eurasia Journal of Mathematics, Science \& Technology Education 2006;2(3):66-78.

Igreja 2004 \{published data only\} Igreja V, Kleijn WC, Schreuder BJN, van Dijk JA, Verschuur M. Testimony method to ameliorate post-traumatic stress symptoms: community-based intervention study with Mozambican civil war survivors. British Journal of Psychiatry 2004;184(3):251-7.

Ivanets 1992 \{published data only\} Ivanets NN, Lukomskaya MI. Moscow, USSR. In: Babor TF, Grant M editor(s). Identification and management of alcohol-related problems: report on phase II, a randomized clinical trial of brief interventions in primary health care. Geneva: World Health Organisation, 1992:157-74.

Jacob 2007a \{published data only\} Jacob ME, Abraham VJ, Abraham S, Jacob KS. The effect of community based daycare on mental health and quality of life of elderly in rural south India: a community intervention study. International Journal of Geriatric Psychiatry 2007;22(5):445-7.

Jacob 2007b \{published data only\} Jacob KS, Senthil KP, Gayathri K, Abraham S, Prince MJ. Can health workers diagnose dementia in the community?. Acta psychiatrica Scandinavica 2007;116(2):125-8.

Jain 2010 \{published data only\} Jain $S$. The role of paraprofessionals in providing treatment for posttraumatic stress disorder in low-resource communities. Journal of the American Medical Association 2010;304(5):571-2.

James 2006 \{published data only\} James S, Reddy P, Ruiter RAC, McCauley A, van den Borne B. The impact of an HIV and AIDS life skills programme on secondary school students in KwaZulu-Natal, South Africa. AIDS Education and Prevention 2006;18(4):281-94.

Jenkins 2007 \{published data only\} Jenkins R, Lancashire S, McDaid D, Samyshkin Y, Green $S$, Watkins J, et al.Mental health reform in the Russian Federation: an integrated approach to achieve social inclusion and recovery. Bulletin of the World Health Organization 2007;85(11):858-66.

Non-specialist health worker interventions for the care of mental, neurological and substance-abuse disorders in low- and middle- 
Johnson 2011 \{published data only\}

Johnson S, Hinton M, Pilling S, Bebbington P, Hobbs L, Cohen S. Strategies for implementation of early intervention for psychosis in a catchment area mental health system: a mixed methods comparison. Psychiatrische Praxis. 2011; Vol. 38, issue S 01 .

\section{Jordan 2006 \{published data only\}}

Jordan K. A case study: how a disaster mental health volunteer provided spiritually, culturally, and historically sensitive trauma training to teacher-counselors and other mental health professionals in Sri Lanka, 4 weeks after the tsunami. Brief Treatment and Crisis Intervention 2006;6(4): 316-25.

Kaaya 1992 \{published data only\} Kaaya S, Goldberg D, Gask L. Management of somatic presentations of psychiatric illness in general medical settings: evaluation of a new training course for general practitioners. Medical Education 1992;25(2):138-44.

Kabura 2005 \{published data only\} Kabura P, Fleming LM, Tobin DJ. Microcounseling skills training for informal helpers in Uganda. International Journal of Social Psychiatry 2005;51:63-70.

Kaiser 1998 \{published data only\}

Kaiser C, Asaba G, Mugisa C, Kipp W, Kasoro S, Rubaale T, et al.Antiepileptic drug treatment in rural Africa: involving the community. Tropical Doctor 1998;28(2):73-7.

Kalichman 2007 \{published data only\} Kalichman SC, Simbayi LC, Phil D, Vermaak R, Cain D, Jooste $S$, et al.HIV/AIDS risk reduction counseling for alcohol using sexually transmitted infections clinic patients in Cape Town, South Africa. Journal of Acquired Immune Deficiency Syndrome 2007;44(5):594-600.

Kalichman 2008 \{published data only\} Kalichman SC, Simbayi LC, Vermaak R, Cain D, Smith G, Mthebu J, et al.Randomized trial of a communitybased alcohol-related HIV risk-reduction intervention for men and women in Cape Town South Africa. Annals of Behavioral Medicine 2008;36:270-9.

Kalichman 2009 \{published data only\} Kalichman SC, Simbayi LC, Cloete A, Clayford M, Arnolds $\mathrm{W}$, Mxoli M, et al.Integrated gender-based violence and HIV risk reduction intervention for South African men: results of a quasi-experimental field trial. Prevention Science 2009;10(3):260-9.

Karnell 2006 \{published data only\}

Karnell AP, Cupp PK, Zimmerman RS, Feist-Price S, Bennie T. Efficacy of an American alcohol and HIV prevention curriculum adapted for use in South Africa: Results of a pilot study in five township schools. AIDS Education and Prevention 2006;18(4):295-310.

Kermode 2008 \{published data only\} Kermode M, Devine A, Chandra P, Dzuvichu B, Gilbert T, Herrman H. Some peace of mind: assessing a pilot intervention to promote mental health among widows of injecting drug users in north-east India. BMC Public Health 2008;8(1):294.
Khamis 2004 \{published data only\}

Khamis V, Macy R. The impact of the classroom / community / camp-based intervention ( CBI ) program on Palestinian children. Save The Children. New York, 2004: 104.

Khan 2009 \{published data only\}

Khan TM, Arif NHB, Tahir H, Anwar M. Role of the husband's knowledge and behaviour in postnatal depression: a case study of an immigrant Pakistani woman. Mental Health in Family Medicine 2009;6:195-201.

Kim 2001 \{published data only\}

Kim S, Kim J-H. The effects of group intervention for battered women in Korea. Archives of Psychiatric Nursing 2001;15(6):257-64.

Kitsumban 2009 \{published data only\} Kitsumban V, Thapinta D, Sirindharo PB, Anders RL. Effect of cognitive mindfulness practice program on depression among elderly Thai women. Thai Journal of Nursing Research 2009;13(2):95-108.

Klein 2012 \{published data only\}

Klein A, Saphonn V, Reid S. Reaching out and reaching up - developing a low cost drug treatment system in Cambodia. Harm Reduction Journal 2012;9:11.

Kozinzky 2012 \{published data only\} Kozinzky Z, Dudas RB, Devosa I, Csatordai S, Toth E, Szabo D, et al.Can a brief antepartum preventive group intervention help reduce postpartum depressive symptomatology?. Psychotherapy and Psychosomatics 2012; 81:98-107.

Kozulin 2010 \{published data only\} Kozulin A, Lebeer J, Madella-Noja A, Gonzalez F, Jeffrey I, Rosenthal N, et al.Cognitive modifiability of children with developmental disabilities: a multicentre study using Feuerstein's instrumental enrichment-basic program. Research in Developmental Disabilities 2010;31(2):551-9.

Kunz 2004 \{published data only\}

Kunz FM, French MT, Bazargan-Hejazi S. Costeffectiveness analysis of a brief intervention delivered to problem drinkers presenting at an inner-city hospital emergency department. Journal of Studies on Alcohol 2004; 65(3):363-70.

Lafalaise 2003 \{published data only\} Lafalaise EM. In Haiti a program linking the educational, research, and professional communities with psychological services, education, and training at the mental health hospitals. Dissertation Abstracts International 2003; Vol. 64 , issue 5.

Lara 2003 \{published data only\} Lara MA, Navarro C, Rubi NA, Mondragon L. Outcome results of two levels of intervention in low-income women with depressive symptoms. American Journal of Orthopsychiatry 2003;73(1):35-43.

Leitch 2009 \{published data only\}

Leitch L, Miller-Karas E. A case for using biologicallybased mental health intervention in post-earthquake china:

Non-specialist health worker interventions for the care of mental, neurological and substance-abuse disorders in low- and middle- 
evaluation of training in the trauma resiliency model. International Journal of Emergency Mental Health 2009;11 (4):221-33.

\section{Leteka 2003 \{published data only\}}

Leteka JMM. Alcohol use / abuse among teenagers in selected high schools in Maseru city: the development of a health education programme. PhD Thesis. Department of Nursing. University of South Africa 2003:234.

Li 2005 \{published data only\}

Li Z, Arthur D. Family education for people with schizophrenia in Beijing, China: randomised controlled trial. British Journal of Psychiatry 2005;187(4):339-45.

Li 2009 \{published data only\}

Li S, Liu M. Influence of community nursing intervention on recurrence rate of schizophrenia patients [in Chinese]. Chinese Nursing Research 2009;23(5B):1308-9.

Liu 2010 \{published data only\} Liu X, Li Z. Practice and effect of carrying out comprehensive nursing intervention for patients with traumatic paraplegia [in Chinese]. Chinese Nursing Research 2010;24(7C):1888-90.

Luengo-Fernandez 2011 \{published data only\} Luengo-Fernandez R, Leal J, Gray AM. Cost of dementia in the pre-enlargement countries of the European Union. Journal of Alzheimers Disease 2011;27:187-96.

Lund 2009 \{published data only\} Lund C, Boyce G, Flisher AJ, Kafaar Z, Dawes A. Scaling up child and adolescent mental health services in South Africa: human resource requirements and costs. Journal of Child Psychology and Psychiatry 2009;50(9):1121-30.

Machona 1992 \{published data only\} Machona AM. Harare Zimbabwe. In: Babor TF, Grant M editor(s). Identification and management of alcohol-related problems: report on phase II, a randomized clinical trial of brief interventions in primary health care. Geneva: World Health Organisation, 1992:211-20.

Macic 2010 \{published data only\} Macic A, Mitric B, Stjepcevic A. Problems and opposition to constitution of mental health centers in Montenegro. Conference: 18th European Congress of Psychiatry Munich Germany. 27 Feb-2 March. European Psychiatry. 2010.

Madianos 1999 \{published data only\}

Madianos MG, Tsiantis J, Zacharakis C. Changing patterns of mental health care in Greece (1984-1996). European Psychiatry 1999;14:462-7.

Maheswaran 1992 \{published data only\} Maheswaran R, Beevers M, Beevers DG. Effectiveness of advice to reduce alcohol consumption in hypertensive patients. Hypertension 1992;19(1):79-84.

Mavrommati 2002 \{published data only\} Mavrommati TD, Miles TR. A pictographic method for teaching spelling to Greek dyslexic children. Dyslexia 2002; 8(2):86-101.
McAuliffe 1985 \{published data only\}

McAuliffe WE, Chien James MN, Launer E, Friedman B. The Harvard group aftercare program: preliminary evaluation results and implementation issues. National Institute of Drug Abuse Research Monograph 1985;58: 147-55.

McCorkle 2000 \{published data only\}

McCorkle R, Strumpf NE, Nuamah IF, Adler DC, Cooley $\mathrm{ME}$, Jepson C, et al.A specialized home care intervention improves survival among older post-surgical cancer patients. Journal of the American Geriatrics Society 2000;48(12): 1707-13.

\section{Merritt 2007 \{published data only\}}

Merritt RK, Price JR, Mollison J, Geddes JR. A cluster randomized controlled trial to assess the effectiveness of an intervention to educate students about depression. Psychological Medicine 2007;37(03):363-72.

Miller 1981 \{published data only\} Miller WR, Gribskov CJ, Mortell RL. Effectiveness of a self-control manual for problem drinkers with and without therapist contact. The International Journal of the Addictions 1981;16(7):1247-54.

Mishara 2006 \{published data only\} Mishara BL, Ystgaard M. Effectiveness of a mental health promotion program to improve coping skills in young children: Zippy's Friends. Early Childhood Research Quarterly 2006;21(1):110-23.

Mohammad-Alizadeh-Charandabi 2011 \{published data only\} Mohammad-Alizadeh CS. Effect of telephone counseling on exclusive breastfeeding and postpartum depression. IRCT201105093706N6 2011.

Montazeri 2001 \{published data only\}

Montazeri A, Jarvandi S, Haghighat S, Vahdani M, Sajadian A, Ebrahimi M, et al.Anxiety and depression in breast cancer patients before and after participation in a cancer support group. Patient Education and Counseling 2001;45 (3):195-8.

Montero 1992 \{published data only\} Montero S. San Jose, Costa Rica. In: Babor TF, Grant M editor(s). Identification and management of alcohol-related problems: report on phase II, a randomized clinical trial of brief interventions in primary health care. 91. Geneva: World Health Organisation, 1992:103-13.

Mooren 2003 \{published data only\} Mooren TTM, de Jong K, Kleber RJ, Ruvic J. The efficacy of a mental health program in Bosnia-Herzegovina: impact on coping and general health. Journal of Clinical Psychology 2003;59(1):57-69.

Moretti-Pires 2011 \{published data only\} Moretti-Pires RO, Corradi-Webster CM. Implementation of brief intervention for problematic alcohol use in primary health in the Amazon context [Implementação de intervenções breves para uso problemático de álcool na atenção primária, em um contexto amazônico]. Revista Latino-Americana de Enfermagem 2011;19(Spe No.): 813-20.

Non-specialist health worker interventions for the care of mental, neurological and substance-abuse disorders in low- and middle- 
Morrell 2009 \{published data only\}

Morrell CJ, Warner R, Slade P, Dixon S. Psychological interventions for postnatal depression: cluster randomised trial and economic evaluation. The PoNDER trial. Health Technology and Assessment 2009;13(30):1-173.

Mueller 2011 \{published data only\} Mueller J, Alie C, Jonas B, Brown E, Sherr L. A quasiexperimental evaluation of a community-based art therapy intervention exploring the psychosocial health of children affected by HIV in South Africa. Tropical Medicine \& International Health 2011;16(1):57-66.

Mufti 1986 \{published data only\}

Mufti KA. Community programme in Pakistan aimed at preventing and reducing drug abuse. Bulletin on Narcotics 1986;38(1-2):121-7.

Murphy 1997 \{published data only\}

Murphy L, Pynoose Robert S, Boyd James C. The trauma/ grief-focused group psychotherapy module of an elementary school-based violence prevention/intervention program. In: Osofsky JD editor(s). Children in a violent society. New York: Guilford Press, 1997:223-55.

Murthy 2005 \{published data only\} Murthy RS, Kumar Kishore KKV, Chisholm D, Thomas T, Sekar K, Chandrashekar CR. Community outreach for untreated schizophrenia in rural India: a follow-up study of symptoms, disability, family burden and costs. Psychological Medicine 2005;35:341-51.

Naeem 2003 \{published data only\}

Naeem S, Ali BS. The transformative effect of training in counselling and its application, on the community counsellors themselves. Journal of the Pakistan Medical Association 2003;53(9):388-90.

Neuner 2004 \{published data only\}

Neuner F, Schauer M, Klaschik C, Karunakara U, Elbert

T. A comparison of narrative exposure therapy, supportive counseling, and psychoeducation for treating posttraumatic stress disorder in an african refugee settlement. Journal of Consulting and Clinical Psychology 2004;72(4):579-87.

Ng 2008 \{published data only\}

Ng RMK, Cheung MSM. Cognitive-behavioural therapy by novices for supervised community hostel residents with treatment-resistant schizophrenia in Hong Kong: a pilot study. Hong Kong Journal of Psychiatry 2008;18(2):49-54.

Ng 2009 \{published data only\}

Ng C, Ma H, Raphael B, Yu X, Fraser J, Tang D. ChinaAustralia training on psychosocial crisis intervention: response to the earthquake disaster in Sichuan. Australasian Psychiatry 2009;17(1):51-5.

Nizamie 2009 \{published data only\} Nizamie SH, Akthar S, Banerjee I, Goyal N. Health care delivery model in epilepsy to reduce treatment gap: World Health Organization study from a rural tribal population of India. Epilepsy Research 2009;84(2-3):146-52.

Ockene 1999 \{published data only\}

Ockene JK, Adams A, Hurley TG, Wheeler EV, Hebert JR. Brief physician- and nurse practitioner-delivered counseling for high-risk drinkers: does it work?. Archives of Internal Medicine 1999;159(18):2198-205.

Okuyemi 2006 \{published data only\}

Okuyemi KS, Thomas JL, Hall S, Nollen NL, Richter KP, Jeffries $S$, et al.Smoking cessation in homeless populations: a pilot clinical trial. Nicotine and Tobacco Research 2006;8 (5):689-99.

Omerov 1999 \{published data only\}

Omerov M, Wistedt B. Management of PTSD among Bosnia refugees. Competent personnel when it comes to language and culture. Laekartidningen 1999;96(21): 2621-2.

Onbun-Uea 2008 \{published data only\}

Onbun-Uea A, Morrison G. Educating young children with autism in inclusive classrooms in Thailand. Kasetsart Journal 2008;29:268-278.

Ooi 2008 \{published data only\} Ooi YP, Lam CM, Sung M, Tan WTS, Goh TJ, Fung DSS, et al.Effects of cognitive-behavioural therapy on anxiety for children with high-functioning autistic spectrum disorders. Singapore Medical Journal 2008;49(3):215-20.

Ould 2009 \{published data only\}

Ould HA, Gerard DA. Impact of the Nouadhibou pilot program on access to care of schizophrenic patients: partnerships between public and private sectors. Conference: 17th European Psychiatric Association, EPA Congress Lisbon, Portugal. 24-28 January. European Psychiatry. 2009:S1141.

Paek 2009 \{published data only\}

Paek MJ, Ahn JK, Lim SY, Kim YR, Park MH, Kim BN, et al.The effectiveness of school-based short-term social skills training in children with attention-deficit / hyperactivity disorder (ADHD). Journal of the Korean Academy of Child and Adolescent Psychiatry 2009;20(2):82-9.

Pai 1985 \{published data only\}

Pai S, Channabasavanna SM, Nagarajaiah, Raghuram R. Home care for chronic mental illness in Bangalore: an experiment in the prevention of repeated hospitalisation. British Journal of Psychiatry 1985;147(2):175-9.

Pal 2007 \{published data only\}

Pal HR, Yadav D, Mehta S, Mohan I. A comparison of brief intervention versus simple advice for alcohol use disorders in a North India community-based sample followed for 3 months. Alcohol and Alcoholism 2007;42(4):328-32.

Palyska 1987 \{published data only\}

Palyska M, Raduj J. The attempt to change the attitude towards mentally disturbed persons through education (experiment in the group of teachers). Psychiatria Polska 1987;21(5):401-6.

Park 2010 \{published data only\} Park JS, Oh YJ. The effects of psychosocial intervention on depression, hope and quality of life of home-based cancer patients. Journal of the Korean Academy of Adult Nursing 2010;22(6):594-605.

Non-specialist health worker interventions for the care of mental, neurological and substance-abuse disorders in low- and middle- 
Patel 2003a \{published data only\}

Patel V, Chisholm D, Rabe-Hesketh S, Dias-Saxena F, Andrew G, Mann A. Efficacy and cost-effectiveness of drug and psychological treatments for common mental disorders in general health care in Goa, India: a randomised, controlled trial. Lancet 2003;361(9351):33-9.

Patel 2003b \{published data only\} Patel V, Thara R. Meeting the mental health needs of developing countries: NGO innovations in India. New Delhi: Sage Publications, 2003:402.

Peltzer 2006 \{published data only\} Peltzer K, Seoka P, Babor T, Tlakula J. Training primary care nurses to conduct alcohol screening and brief interventions in South Africa. Curationis 2006;29(2):16-21.

Perrin 2010 \{published data only\} Perrin PB, Johnston A, Vogel B, Heesacker M, Anderson J, Rittman M. A culturally sensitive transition assistance program for stroke caregivers: examining caregiver mental health and stroke rehabilitation. Journal of Rehabilitation Research and Development 2010;47(7):605-16.

Perry 1989a \{published data only\}

Perry CL, Grant M, Ernberg G, Florenzano-Urzua R, Langdon MC, Myeni AD, et al.WHO collaborative study on alcohol education and young people: outcomes of a four-country pilot study. The International Journal of the Addictions 1989;24(12):1145-71.

Petersen 2012 \{published data only\} Petersen I, Bhana A, Baillie K, Consortium MhaPP Research Programme. The feasibility of adapted groupbased interpersonal therapy (IPT) for the treatment of depression by community health workers within the context of task shifting in South Africa. Community Mental Health Journal. United States: School of Psychology, Howard College, University of KwaZulu-Natal, Durban, 4000, South Africa, peterseni@ukzn.ac.za., 2012; Vol. 48, issue 3: $336-41$.

Placencia 1993 \{published data only\}

Placencia M, Sander JWAS, Shorvon SD, Roman M, Alarcon F, Bimos C, et al.Antiepileptic drug treatment in a community health care setting in northern Ecuador: a prospective 12-month assessment. Epilepsy Research 1993; 14(3):237-44.

Powell 2004 \{published data only\}

Powell C, Baker-Henningham H, Walker S, Gernay J, Grantham-McGregor S. Feasibility of integrating early stimulation into primary care for undernourished Jamaican children: cluster randomised controlled trial. British Medical Journal 2004;329(7457):89.

Prasetiyawan, 2006 \{published data only\} Prasetiyawan, Viora E, Maramis A, Keliat BA. Mental health model of care programmes after the tsunami in Aceh, Indonesia. International Review of Psychiatry 2006;18(6): 559-62.

\section{Qi 2007 \{published data only\}}

Qi BH, Zeng FL. Interventional strategy for a child with learning disability. Clinical Rehabilitative Tissue Engineering Research 2007;11:39.

Qureshi 2001 \{published data only\} Qureshi NA, Al-Ghamdy YS, Al-Haddad NS, Abdelgadir $\mathrm{MH}$, Tawfik MH. Integration of mental health care into primary care. Saudi Medical Journal 2001;22(10):899-906.

Rahman 1998 \{published data only\} Rahman A, Mubbashar MH, Gater R, Goldberg D. Randomised trial of impact of school mental-health programme in rural Rawalpindi, Pakistan. Lancet 1998; 352:1002-25.

Ramos-Cerqueira 2005 \{published data only\} Ramos-Cerqueira AT, Torres AR, Crepaldi AL, Oliveira NI, Scazufca M, Menezes PR, et al.Identification of dementia cases in the community: a Brazilian experience. Journal of the American Geriatrics Society 2005;53(10):1738-42.

Ran 2003 \{published data only\} Ran M-S, Xiang M-Z, Chan CL-W, Leff J, Simpson P, Huang M-S, et al.Effectiveness of psychoeducational intervention for rural Chinese families experiencing schizophrenia-a randomised controlled trial. Social Psychiatry and Psychiatric Epidemiology 2003;38(2):69-75.

Reay 2012 \{published data only\}

Reay RE, Mulcahy R. The development and content of an interpersonal psychotherapy group for postnatal depression. International Journal of Group Psychotherapy 2012;62(2): 221-51.

Reay 2012a \{published data only\} Reay RE, Owen C, Shadbolt B, Raphael B, Mulcahy $\mathrm{R}$, Wilkinson RB. Trajectories of long-term outcomes for postnatally depressed mothers treated with group interpersonal psychotherapy. Archives of Women's Mental Health 2012;15(3):217-28.

Rhyne 2002 \{published data only\} Rhyne RL, Hertzman PA. Pursuing community-oriented primary care in a Russian closed nuclear city: the Sarov-Los Alamos community health partnership. American Journal of Public Health 2002;92(11):1740-2.

Rigotti 2009 \{published data only\}

Rigotti NA, Bitton A, Richards AE, Reyen M, Wassum K, Raw M. An international survey of training programs for treating tobacco dependence. Addiction 2009;104:288-96.

Rotheram-Borus 2011 \{published data only\} Rotheram-Borus MJ, le Roux IM, Tomlinson M, Mbewu N, Comulada WS, le Roux K, et al.Philani Plus (+): A mentor mother community health worker home visiting program to improve maternal and infants' outcomes. Prevention Science. Rotheram-Borus, Mary Jane: Center for Community Health, 10920 Wilshire Blvd., Suite 350, Los Angeles, CA, US, 90024-6521, MRotheram@mednet.ucla.edu: Springer, 2011; Vol. 12, issue 4:372-88.

Non-specialist health worker interventions for the care of mental, neurological and substance-abuse disorders in low- and middle- 
Rowe 2007 \{published data only\}

Rowe M, Bellamy C, Baranoski M, Wieland M, O'Connell MJ, Benedict P, et al.A peer-support, group intervention to reduce substance use and criminality among persons with severe mental illness. Psychiatric Services 2007;58(7): 955-61.

Sadik 2011 \{published data only\}

Sadik S, Abdulrahman S, Bradley M, Jenkins R. Integrating mental health into primary health care in Iraq. Mental Health in Family Medicine 2011;8(1):39-49.

Saltzman 2001 \{published data only\}

Saltzman WR, Pynoos RS, Layne CM, Steinberg AM, Aisenberg E. Trauma / grief-focused intervention for adolescents exposed to community violence: results of a school-based screening and group treatment protocol. Group Dynamics: Theory Research and Practice 2001;5: 277-90.

Schoenmakers 2010 \{published data only\}

Schoenmakers B, Buntinx F, Delepeleire J. Supporting family carers of community-dwelling elder with cognitive decline: a randomized controlled trial. International Journal of Family Medicine. Egypt: Academic Centre of General Practice, Catholic University Leuven, Kapucijnenvoer 33, Blok J, box 7001, 3000 Leuven, Belgium., 2010; Vol. 2010: 10.

Schultz 1995 \{published data only\}

Schultz R, Greenley JR. Innovating in community mental health: international perspectives. Westport, USA: Greenwood Publishing Group, 1995.

Serrano-Garcia 1991 \{published data only\} Serrano-Garcia I, Lopez-Sanchez G. Community interventions in Puerto Rico : the impact of socialcommunity psychology. International Association of Applied Psychology 1991;40(2):201-18.

Skounti 2009 \{published data only\} Skounti M, Mpitzaraki K, Philalithis A, Galanakis E. Clinical evaluation of children testing positive in screening tests for attention-deficit / hyperactivity. European Journal of Psychiatry 2009;23(2):115-20.

Slupczynska-Kossobudzka 1999 \{published data only\} Slupczynska-Kossobudzka E, Boguszewska L. Effects of community mobile team intervention in the Drewnica hospital catchment area. 1. patient outcomes. International Journal of Social Psychiatry 1999;45(3):207-15.

Smith 2008 \{published data only\} Smith EA, Palen L-A, Caldwell LL, Flisher AJ, Graham JW, Mathew C, et al.Substance use and sexual risk prevention in Cape Town, South Africa: An evaluation of the Healthwise program. Prevention Science 2008;9:311-21.

So 2005 \{published data only\}

So $\mathrm{H}$. The effects of environmental enrichment program on cognitive function among institutionalized elderly. Korean Journal of Adult Nursing 2005;17(1):128-38.
Sohlberg 1987 \{published data only\}

Sohlberg MM, Mateer CA. Effectiveness of an attentiontraining program. Journal of Clinical and Experimental Neuropsychology 1987;9(2):117-30.

Sokhela 1999 \{published data only\}

Sokhela NE. The integration of comprehensive psychiatric / mental health care into the primary health system: diagnosis and treatment. Journal of Advanced Nursing 1999;30(1): 229-37.

Staples 2011 \{published data only\} Staples JK, Abdel AJA, Gordon JS. Mind-body skills groups for posttraumatic stress disorder and depression symptoms in Palestinian children and adolescents in Gaza. International Journal of Stress Management 2011;18(3): 246-62.

Stein 1975 \{published data only\}

Stein L, Test M, Marx AJ. Alternative to the hospital: a controlled study. American Journal of Psychiatry 1975;132: $517-22$.

Stein 2001 \{published data only\} Stein DJ, Wessels C, Zungu-Dirwayi N, Berk M, Wilson $Z$. Value and effectiveness of consumer advocacy groups: a survey of the anxiety disorders support group in South Africa. Depression and Anxiety 2001;13(2):105-7.

Stepakoff 2006 \{published data only\} Stepakoff S, Hubbard J, Katoh M, Falk E, Mikulu J$\mathrm{B}$, Nkhoma $\mathrm{P}$, et al.Trauma healing in refugee camps in Guinea: a psychosocial program for Liberian and Sierra Leonean survivors of torture and war. The American Psychologist 2006;61(8):921-32.

Strain 2001 \{published data only\}

Strain PS, Timm MA. Remediation and prevention of aggression: an evaluation of the regional intervention program over a quarter century. Behavioral Disorders 2001; 26(4):297-313.

\section{Suh 2004 \{published data only\}}

Suh G-H, Ju Y-S, Yeon BK, Shah A. A longitudinal study of Alzheimer's disease: rates of cognitive and functional decline. International Journal of Geriatric Psychiatry 2004; 19:817-24.

Suh 2004a \{published data only\} Suh G-H, Jung HU, Lee CU, Oh BH, Bae JN, Jung H-Y, et al.A prospective, double-blind, community-controlled comparison of three doses of galantamine in the treatment of mild to moderate Alzheimer's disease in a Korean population. Clinical Therapeutics 2004;26(10):1608-18.

Suh 2006 \{published data only\} Suh G-H, Knapp M, Kang C-J. The economic costs of dementia in Korea, 2002. International Journal of Geriatric Psychiatry 2006;21(8):722-8.

Tadaka 2004 \{published data only\} Tadaka E, Kanagawa K. A randomized controlled trial of a group care program for community-dwelling elderly people with dementia. Japan Journal of Nursing Science 2004;1: 19-25.

Non-specialist health worker interventions for the care of mental, neurological and substance-abuse disorders in low- and middle- 
Tang 2010 \{published data only\}

Tang JYM, Wong GHY, Hui CLM, Lam MML, Chiu CPY, Chan SKW, et al.Early intervention for psychosis in Hong Kong - the EASY programme. Early Intervention in Psychiatry 2010;4(3):214-9.

Tareen 2009 \{published data only\}

Tareen A, Mirza I, Minhas A, Minhas F, Rahman A. Developing a child and adolescent mental health service in a low-income country: a global partnership model. Psychiatric Bulletin 2009;33:181-3.

Tezel 2006 \{published data only\}

Tezel A, Gozum S. Comparison of effects of nursing care to problem solving training on levels of depressive symptoms in post partum women. Patient Education and Counseling 2006;63(1-2):64-73.

Tharyan 2005 \{published data only\}

Tharyan P. Traumatic bereavement and the Asian tsunami: perspectives from Tamil Nadu, India. Bereavement Care 2005;24(2):23-6.

Tiwari 2005 \{published data only\}

Tiwari A, Leung WC, Leung TW, Humphreys J, Parker B, Ho PC. A randomised controlled trial of empowerment training for Chinese abused pregnant women in Hong Kong. British Journal of Obstetrics and Gynaecology 2005; 112(9):1249-56.

Tomasevic 1998 \{published data only\}

Tomasevic A. Evaluative study of the psychosocial reinforcement project of the primary health care [Estudio evaluativo del proyecto de reforzamiento psicosocial de la atencion primaria]. Escuela de Salud Publica, Masters thesis, University of Chile 1998:126.

Tran 2008 \{published data only\}

Tran D-S, Zen J, Strobel M, Odermatt P, Preux P-M, Huc P, et al.The challenge of epilepsy control in deprived settings: low compliance and high fatality rates during a communitybased phenobarbital program in rural Laos. Epilepsia 2008; 49(3):539-40.

Tripathy 2010 \{published data only\}

Tripathy P, Nair N, Barnett S, Mahapatra R, Borghi J, Rath $S$, et al.Effect of a participatory intervention with women's groups on birth outcomes and maternal depression in Jharkhand and Orissa, India: a cluster-randomised controlled trial. Lancet 2010;375(9721):1182-92.

Turrisi 2009 \{published data only\} Turrisi R, Larimer ME, Mallett KA, Kilmer JR, Ray AE, Mastroleo NR, et al.A randomized clinical trial evaluating a combined alcohol intervention for high-risk college students. Journal of Studies on Alcohol and Drugs 2009;70 (4):555-67.

Uma 1989 \{published data only\} Uma K, Nagendra HR, Nagarathna R, Vaidehi S, Seethalakshmi R. The integrated approach of yoga: a therapeutic tool for mentally retarded children: a one-year controlled study. Journal of Mental Deficiency Research 1989; 33:415-21.
Uys 1996 \{published data only\}

Uys LR, Zulu RN. An evaluation of the implementation and the effectiveness of case management in the rehabilitation of psychiatric outpatients in South Africa. South African Journal of Psychology 1996;26(4):226-30.

van't Hof 2011 \{published data only\} van't Hof E, Stein DJ, Marks I, Tomlinson M, Cuijpers P. The effectiveness of problem solving therapy in deprived South African communities: results from a pilot study. BMC Psychiatry 2011;11(1):156.

van Emmerik 2002 \{published data only\} van Emmerik AAP, Kamphuis JH, Hulsbosch AM, Emmelkamp PMG. Single session debriefing after psychological trauma: a meta-analysis. Lancet 2002;360 (9335):766-71.

Velleman 2003 \{published data only\} Velleman R, Templeton L. Alcohol, drugs and the family: results from a long-running research programme within the UK. European Addiction Research 2003;9(3):103-12.

Vera 2010 \{published data only\}

Vera M, Perez-Pedrogo C, Huertas SE, Reyes-Rabanillo ML, Juarbe D, Huertas A, et al.Collaborative care for depressed patients with chronic medical conditions: a randomized trial in Puerto Rico. Psychiatric Services 2010;61(2):144-50.

Vermetten 2007 \{published data only\} Vermetten E, Middelkoop CJ, Taal L, Carll EK. Online psychotrauma intervention in the aftermath of the tsunami: acommunity-building effort. In: Carll EK editor(s). Trauma Psychology. Issues in violence, disaster, health, and illness, Vol 1: Violence and disaster. Westport, USA: Praeger Publishers/Greenwood Publishing Group, 2007:255-72.

Vijayakumar 2008 \{published data only\}

Vijayakumar L, Suresh KM. Trained volunteer-delivered mental health support to those bereaved by asian tsunami - an evaluation. International Journal of Social Psychiatry 2008;54(4):293-302.

Vijaykumar 2006 \{published data only\}

Vijaykumar L, Thara R, John S, Chellappa S. Psychosocial interventions after tsunami in Tamil Nadu, India. International Review of Psychiatry 2006;18(3):225-31.

Waitzkin 2011 \{published data only\} Waitzkin H, Getrich C, Heying S, Rodri L, Parmar A, Willging $\mathrm{C}$, et al.Promotoras as mental health practitioners in primary care : a multi-method study of an intervention to address contextual sources of depression. Journal of Community Health 2011;36:316-31.

Walker 2004 \{published data only\} Walker SP, Chang SM, Powell CA, Grantham-McGregor SM. Psychosocial intervention improves the development of term low-birth-weight infants. The Journal of Nutrition 2004;134(6):1417-23.

Wallander 2010 \{published data only\} Wallander JL, McClure E, Biasini F, Goudar SS, Pasha O, Chomba E, et al.Brain research to ameliorate impaired

Non-specialist health worker interventions for the care of mental, neurological and substance-abuse disorders in low- and middle- 
neurodevelopment - home-based intervention trial

(BRAIN-HIT). BMC Pediatrics 2010;10(1):27.

Wang 2006 \{published data only\}

Wang WZ, Wu JZ, Ma GY, Dai XY, Yang B, Wang TP, et al.Efficacy assessment of phenobarbital in epilepsy: a large community-based intervention trial in rural China. Lancet Neurology 2006;5(1):46-52.

Wang 2012 \{published data only\}

Wang L-Q, Chien W-T, Lee IYM. An experimental study on the effectiveness of a mutual support group for family caregivers of a relative with dementia in mainland China. Contemporary Nurse 2012;40(2):210-24.

Wechsberg 2006 \{published data only\}

Wechsberg WM, Luseno WK, Lam WKK, Parry CDH, Morojele NK. Substance use, sexual risk, and violence: HIV prevention intervention with sex workers in Pretoria. AIDS and Behavior 2006;10(2):131-7.

Wechsberg 2008 \{published data only\}

Wechsberg WM, Luseno WK, Karg RS, Young S, Rodman $\mathrm{N}$, Myers B, et al.Alcohol, cannabis, and methamphetamine use and other risk behaviours among Black and Coloured South African women: a small randomized trial in the Western Cape. International Journal of Drug Policy 2008; 19:130-9.

WHO 1996 \{published data only\}

WHO Group Brief Intervention Study. A cross-national trial of brief interventions with heavy drinkers. American Journal of Public Health 1996;86(7):948-55.

Wilson 1981 \{published data only\}

Wilson LG. Utilizing dispersed mental health paraprofessionals for scattered Pacific islands: a Micronesian experience. Community Mental Health Journal 1981;17(2): $161-70$.

Wimo 1997 \{published data only\}

Wimo A. Costs of dementia and dementia care: a review. International Journal of Geriatric Psychiatry 1997;12(8): 841-56.

Wimo 2003 \{published data only\}

Wimo A, Winblad B, Engedal K, Soininen H, Verhey F, Waldemar G, et al.An economic evaluation of donepezil in mild to moderate Alzheimer's disease: results of a 1-year, double-blind, randomized trial. Dementia and Geriatric Cognitive Disorders 2003;15(1):44-54.

Wimo 2007 \{published data only\}

Wimo A, Winblad B, Jonsson L. An estimate of the total worldwide societal costs of dementia in 2005. Alzheimer's and Dementia 2007;3:81-91.

Wolmer 2011 \{published data only\}

Wolmer L, Hamiel D, Barchas JD, Slone M, Laor N. Teacher-delivered resilience-focused intervention in schools with traumatized children following the second Lebanon war. Journal of Traumatic Stress 2011;24(3):309-16.

Wong 2002 \{published data only\}

Wong DFK, Sun SYK, Tse J, Wong F. Evaluating the outcomes of a cognitive-behavioral group intervention model for persons at risk of developing mental health problems in Hong Kong: a pretest-posttest study. Research on Social Work Practice 2002;12(4):534-45.

Wong 2007 \{published data only\}

Wong SYS, Cheung AKY, Lee A, Cheung N, Leung A,

Wong W, et al.Improving general practitioners' interviewing skills in managing patients with depression and anxiety: a randomized controlled clinical trial. Medical Teacher 2007; 29(6):175-83.

Wu 2002 \{published data only\}

Wu Z, Detels R, Zhang J, Li V, Li J. Community-based trial to prevent drug use among youths in Yunnan, China. American Journal of Public Health 2002;92(12):1952-7.

Xiao 2009 \{published data only\}

Xiao L, Wu Z, Wang Y, Chen J. Comparison of quality of life for drug addicts in methadone maintenance treatment clinics, community and compulsory detoxification institutions in Sichuan Province [in Chinese]. Journal of Hygiene Research 2009;38(1):67-70.

Xu 2003 \{published data only\}

Xu F-S, Liu J-X, Zhang S-P, Li J, Su Q. Effects of intervening measures on postpartum depression [in Chinese]. Zhonghua Fu Chan Ke Za Zhi 2003;38(12):724-6.

Yildiz 2003 \{published data only\}

Yildiz M, Tural U, Kurdoglu S, Onder ME. An experience of a clubhouse run by families and volunteers for schizophrenia rehabilitation. Turk Psikiyatri Dergisi 2003;14(4):281-7.

Yoo 2006 \{published data only\}

Yoo Y-S, Kang H-S. Effects of a bereavement intervention program on depression and life satisfaction in middle aged widows in Korea. Journal of Korean Academy of Nursing 2006;36(8):1367-73.

Zakroyeva 2008 \{published data only\} Zakroyeva A, Goldberg D, Gask L, Leese M. Training Russian family physicians in mental health skills. European Journal of General Practice 2008;14(1):19-22.

Zavradashvili 2010 \{published data only\} Zavradashvili N, Donisi V, Grigoletti L, Pertile R, Gelashvili $\mathrm{K}$, Eliashvili $\mathrm{M}$, et al.Is the implementation of assertive community treatment in a low-income country feasible? The experience of Tbilisi, Georgia. Social Psychiatry and Psychiatric Epidemiology 2010;45(8):779-83.

Zencir 2005 \{published data only\} Zencir M, Kuzu N, Beser NG, Ergin A, Catak B, Sahiner T. Cost of Alzheimer's disease in a developing country setting. International Journal of Geriatric Psychiatry 2005;20(7): 616-22.

Zhengyi 1997 \{published data only\} Zhengyi F, Baozhen D, Pei G. General evaluation on community nursing intervention of NIDDM patients [in Chinese]. Shanxi Nursing Journal 1997;11(4):145-6.

References to studies awaiting assessment

Non-specialist health worker interventions for the care of mental, neurological and substance-abuse disorders in low- and middle- 
Abdul 2002 \{published data only\}

Abdul RNH, Radwan SAM, Othman Omayma Abou

BakrAB. Group support and community - based

intervention for caregivers' of Alzheimer's patients.

Alexandria Scientific Nursing Journal 2002;2(1):25-43.

Aljanati 2010 \{published data only\}

Aljanati RE, Martinovic M, Raggio V, Aguiar B, Gonzalez $\mathrm{N}$, Julia OA, et al.Clinical impact of the Specific National Program on patients with Alzheimer's disease. Conference: 14th International Congress of Parkinson's Disease and Movement Disorders Buenos Aires Argentina. 13-17th June.. 2010:S423.

Azizi 2010 \{published data only\}

Azizi M, Lamyian M, Faghihzade S, Nematollahzade $M$. The effect of counseling on anxiety after traumatic childbirth in nulliparous women; a single blind randomized clinical trial. Behbood Journal 2010;14(3):14-5.

Bhadwal 1992 \{published data only\}

Bhadwal SC, Panda PK. The composite effect of a curricular programme on the test anxiety of rural primary school students: a one year study. Educational Review 1992;44(2): 205-20.

Blackmon 1985 \{published data only\}

Blackmon B. Networking community services for elderly clients with alcohol problems. In: Freeman EM editor(s). Social Work Practice for Patients who have Alcohol Problems. Charles C Thomas, Publisher; England, 1985:189-201.

Buttorff 2012 \{published data only\} Buttorff C, Hock RS, Weiss HA, Naik S, Araya R, Kirkwood BR, Chisholm D, Patel V. Economic evaluation of a taskshifting intervention for common mental disorders in India. Bulletin of the World Health Organisation 2012;90:813-821.

Caqueo-Urzar 2010 \{published data only\}

Caqueo-Urzar A, Gutierrez-Maldonado J, Ferrer-

Garca M. No Title. International Journal of Neuropsychopharmacology.Conference: 27th CINP Congress Hong Kong. 6-10 June. 2010:215.

Chang 2010 \{published data only\}

Chang CW. Impact of deinstitutionalisation. Asia-Pacific Psychiatry.Conference: 14th Pacific Rim College of Psychiatrists Scientific Meeting Brisbane, QLD Australia. 28-30 October. 2010:A7.

Cherpitel 2009 \{published data only\}

Cherpitel CJ, Bernstein E, Bernstein J, Moskalewicz J, Swiatkiewicz G. Screening, brief intervention and referral to treatment (SBIRT) in a Polish emergency room: challenges in cultural translation of SBIRT. Journal of Addictions Nursing 2009;20(3):127-31.

Chien 2007a \{published data only\}

Chien W-T. Evaluation of the effects of mutual support and psycho-educational group interventions for family caregivers of people with schizophrenia. http://clinicaltrials.gov/show/ NCT00940394 2007.

Farahat 2010 \{published data only\}

Farahat TM, El-Shafie AM, El-Rasoul Gaafar MMA, ElSherief AM, Farag N. Integrated management of epilepsy among basic school children in Menoufiya governorate. Egypt Journal of Neurology Psychiatry and Neurosurgery 2010; 47(1):49-58.

\section{Hsiao 2009 \{published data only\}}

Hsiao F. The efficacy of the caregiver psychoeducational consultation program in spouse caregivers of resectable colorectal cancer. http://clinicaltrials.gov/show/ NCT01001884 2009.

Kumar 2011 \{published data only\} Kumar CN, Jagadisha T, Kudumallige KS, Basappa KV, Udupi A, Kengeri VK, et al.Four-year follow-up of schizophrenia in a rural community: good outcome and public health implications course of schizophrenia in rural India. Indian Journal of Psychiatry 2011;53(4):4-7.

Lee 2011a \{published data only\}

Lee Y-R, Sung K-T, Kim Y-E. Effects of home-based stress management training on primary caregivers of elderly people with dementia in South Korea. Dementia 2011;11 (2):171-9.

\section{Luna 1984 \{published data only\}}

Luna AJ. Mental health program and pharmacodependence [Programa de salud mental y farmacodependencia in Seminario clinico internacional sobre adicciones a la hoja de coca y sus derivados]. Ministerio de Salud. Colombia. 1984:19-21.

Malakouti 2010 \{published data only\} Malakouti SK. Comparing the outcomes and costeffectiveness of two model of case-manager system for severe mentally ill patients: general practitioner and nurses. Iranian Registry of Clinical Trials. http://www.irct.ir/ searchresult.php?id=1959\&number 2010.

\section{Oh 1997 \{published data only\}}

Oh KS. Effects of a social support group on burden and well-being of mothers of developmentally delayed children. Journal of Korean Academy of Nursing 1997;27(2):389-400.

Sott 1998 \{published data only\}

Sott OW. Evaluation of the patient's cares in the community, after discharge of its first psychiatric internment [Avaliacao dos cuidados do paciente na comunidade, apos alta de sua primeira internacao psiquiatrica]. Masters Thesis. Escola Nacional de Saude Publica, Rio de Janeiro, Brazil 1998:111.

\section{References to ongoing studies}

Ager 2011 \{published data only\}

Ager A, Akesson B, Stark L. The impact of the schoolbased Psychosocial Structured Activities (PSSA) program on conflict-affected children in northern Uganda. Journal of Child Psychology and Psychiatry 2011;52(11):1124-33.

Araya 2010 \{published data only\}

Araya R. Cluster randomised controlled trial of a schoolbased intervention to improve the mental health of lowincome, secondary school students in Santiago, Chile. http: //isrctn.org/ISRCTN194662092010. 
Ayoughi 2012 \{published data only\}

Ayoughi S, Missmahl I, Weierstall R, Elbert T. Provision of mental health services in resource-poor settings: a randomised trial comparing counselling with routine medical treatment in North Afghanistan (Mazar-e-Sharif). BMC Psychiatry 2012;12(1):14.

Chen 2010 \{published data only\}

Chen EYH, Chan G. Community case management for early psychosis: is two year an optimal duration? A randomized controlled study. National Clinical Trials. NCT01202357 2010.

Chen 2011 \{published data only\}

Chen S, Conwell Y, Xu B, Chiu H, Tu X, Ma Y. Depression care management for late-life depression in China primary care: protocol for a randomized controlled trial. Trials 2011;12:121.

Greenfield 2010 \{published data only\} Greenfield SF, Shields A, Connery HS, Livchits V, Yanov SA, Lastimoso CS, et al.Integrated management of physiciandelivered alcohol care for tuberculosis patients: design and implementation. Alcoholism, clinical and experimental research 2010;34(2):317-30.

Kauye (in preparation) \{published data only\}

Kauye F, Rahman A, Jenkins R. Training primary health workers in mental health and its impact on service delivery in a developing country, Malawi: A cluster randomized study. In preparation. 2012

Kobeissi 2011 \{published data only\} Kobeissi L, Araya R, Kak FE, Ghantous Z, Khawaja M, Khoury B, et al.The relaxation exercise and social support trial-rest: study protocol for a randomized community based trial. BMC Psychiatry 2011;11:142.

Logie 2012 \{published data only\}

Logie C. A community-health worker delivered HIV/ STI prevention intervention for internally displaced women in Leogane, Haiti FASY. National Clinical Trials. NCT01492829 2012.

O'Callaghan 2012 \{published data only\} O'Callaghan P, Shannon C. A family-based, resiliencefocused intervention for war-affected communities in northeastern Democratic Republic of Congo. National Clinical Trials. NCT01542398 2012.

\section{Opoka 2008 \{published data only\}}

Opoka RO. Cognitive and psychosocial benefits of caregiver training for Ugandan HIV children. http:// clinicaltrials.gov/show/NCT00889395 2008.

\section{Additional references}

\section{Abas 2003}

Abas M, Baingana F, Broadhead J, Iacoponi E, Vanderpyl J. Common mental disorders and primary health care: current practice in low-income countries. Harvard Review of Psychiatry 2003;11(3):166-73.

\section{Ballini 2010}

Ballini L, Bero L, Eccles MP, Grimshaw J, Gruen RL, Lewin S, et al.Cochrane Effective Practice and Organisation of Care Group. About The Cochrane Collaboration (Cochrane Review Groups (CRGs)). 3. Art. No.: EPOC. http://www.mrw.interscience.wiley.com/cochrane/clabout/ articles/EPOC/frame.html 2010.

Beck 1974

Beck AT, Rial WY, Rickels K. Short form of depression inventory: cross validation. Psychological Reports 1974;34: 1184-6.

Bloom 2011

Bloom DE, Cafiero ET, Jané-Llopis E, Abrahams-Gessel S, Bloom LR, Fathima S, Feigl AB, Gaziano T, Mowafi M, Pandya A, Prettner K, Rosenberg L, Seligman B, Stein AZ, \& Weinstein C. The global economic burden of noncommunicable diseases. Geneva: World Economic Forum 2011.

Boer 2005

Boer PCAM, Wiersma D, Russo S, Bosch RJ.

Paraprofessionals for anxiety and depressive disorders. Cochrane Database of Systematic Reviews 2005, Issue 2. [DOI: 10.1002/14651858.CD004688]

Bolton 2001

Bolton P. Cross-cultural validity and reliability testing of a standard psychiatric assessment instrument without a gold standard. Journal of Nervous and Mental Disease 2001;189: $238-42$.

Bolton 2002

Bolton P, Tang AM. An alternative approach to crosscultural function assessment. Social Psychiatry and Psychiatric Epidemiology 2002;37:537-43.

Bower 2006

Bower P, Gilbody S, Richards D, Fletcher J, Sutton A. Collaborative care for depression in primary care. Making sense of a complex intervention: systematic review and meta-regression. British Journal of Psychiatry 2006;189: 484-93.

\section{CCEMG 2010}

Campbell and Cochrane Economics Methods Group. Training materials. http://www.c-cemg.org/. 2010.

\section{Chisholm 2000a}

Chisholm D, Sekar K, Kishore Kumar KV, Saeed K, James $\mathrm{S}$, Mubbashar $\mathrm{MH}$, et al.Integration of mental health care into primary care. British Journal of Psychiatry 2000;176: 581-8.

Cohen 1988

Cohen J. Statistical power analysis in the behavioral sciences. 2nd Edition. Hillsdale (NJ): Lawrence Erlbaum Associates, Inc., 1988.

Cohen 2003

Cohen A. The effectiveness of mental health services in primary care: the view from the developing world. Geneva: World Health Organization, 2003.

De Vet 2008

De Vet HCW, Eisinga A, Riphagen II, Aertgeerts B, Pewsner D. Chapter 7: Searching for Studies. Cochrane handbook for systematic reviews of diagnostic test accuracy. Version 0.4 [updated September 2008]. The Cochrane Collaboration,

Non-specialist health worker interventions for the care of mental, neurological and substance-abuse disorders in low- and middle- 
28 September 2008:http://srdta.cochrane.org/sites/ srdta.cochrane.org/files/uploads/Chapter07-Searching\%28September-2008\%29.pdf.

\section{Deeks 2009}

Deeks JJ, Higgins JPT, Altman DG. Analysing data and undertaking meta-analysis. In: Higgins JPT, Green $S$ editor (s). Cochrane handbook for systematic reviews of interventions. Version 5.0.2 [updated September 2009]. The Cochrane Collaboration: Available at www.cochrane-handbook.org, 2009:3.4.

Demyttenaere 2004

Demyttenaere K, Bruffaerts R, Posada-Villa J. Prevalence, severity, and unmet need for treatment of mental disorders in the World Health Organization world mental health surveys. Journal of the American Medical Association 2004; 291:2581-2590.

Drummond 2005

Drummond MF, Sculpher MJ, Torrance GW, O’Brien BJ, Stoddart GL. Methods for the economic evaluation of health care programmes. (3rd edition), pp.11. Oxford (UK): Oxford University Press, 2005.

\section{Dybdahl 1996}

Dybdahl R. Group Work Manual. Sarajevo, Bosnia Herzegovina: UNICEF 1996.

\section{Dybdahl 1999}

Dybdahl R. Child development and impact of stress on young children. An intervention programme for mothers. Prepared for the UNICEF/Centre for Crisis Psychology Psychosocial Project 1999.

\section{Foa 2005}

Foa EB, Hembree EA, Cahill SP, Rauch SA, Riggs DS, Feeny NC, et al.Randomised trial of prolonged exposure for post traumatic stress disorder with and without cognitive restructuring: outcome at academic and community clinics. Journal of Consulting and Clinical Psychology 2005;73(5): 953-64.

Fox 2002

Fox S, Tang S. The Sierra Leonean refugee experience: Traumatic events and psychiatric sequelae. Journal of Nervous and Mental Disease 2002;188:490-5.

\section{Goldberg 2009}

Goldberg M, Dill C, Shin JY, Nguyen NV. Reliability and validity of the Vietnamese Vineland adaptive behavior scales with preschool-age children. Research in Developmental Disabilities 2009;30(3):592-602.

GRADE 2007

GRADE Working Group. GRADE Profiler; version 3.6. GRADE Working Group, 2007.

\section{Guyatt 2008}

Guyatt GH, Oxman AD, Vist GE, Kunz R, Falck-Ytter Y, Alonso-Coello P, Schünemann HJ. Rating quality of evidence and strength of recommendations GRADE: an emerging consensus on rating quality of evidence and strength of recommendations. British Medical Journal 2008; 336:924.

\section{Guyatt 2013}

Guyatt GH, Thorlund K, Oxman AD, Walter SD, Patrick D, Furukawa TA, Johnston BC, Karanicolas P, Akl EA, Vist G, Kunzh R, Brozek J, Kupper LL, Martin SL, Meerpohl JJ, Alonso-Coellom P, Christensenn R, Schunemann HJ. GRADE guidelines: 13. preparing summary of findings tables and evidence profiles for continuous outcomes. Journal of Clinical Epidemiology 2013;66:173e183.

\section{Harkness 2009}

Harkness EF, Bower PJ. On-site mental health workers delivering psychological therapy and psychosocial interventions to patients in primary care: effects on the professional practice of primary care providers. Cochrane Database of Systematic Reviews 2009, Issue 1. [DOI: 10.1002/14651858.CD000532.pub2]

\section{Herman 1997}

Herman J. Trauma and Recovery. New York: Basic Books, 1997.

\section{Higgins 2009}

Higgins JPT, Altman DG. Assessing risk of bias in included studies. In: Higgins JPT, Green S editor(s). Cochrane handbook for systematic reviews of interventions. Version 5.0.2 [updated September 2009]. The Cochrane Collaboration, 2009. Available from www.cochrane-handbook.org.

\section{Higgins 2011}

Higgins JPT, Green S. Cochrane Handbook for Systematic Reviews of Interventions. Version 5.1.0. The Cochrane Collaboration, March 2011.

\section{Hu 2006}

Hu TW. An international review of the national cost estimates of mental illness. Journal of Mental Health Policy and Economics 2006;9(1):3-13.

\section{Huntley 2012}

Huntley AL, Araya R, Salisbury C. Group psychological therapies for depression in the community: systematic review and meta-analysis. British Journal of Psychiatry 2012; 200(3):184-90.

\section{Jacob 2007}

Jacob KS, Sharan P, Mirza I, Garrido-Cumbrera M, Seedat S, Mari JJ, et al.Mental health systems in countries: where are we now?. Lancet 2007;370(9592):1061-77.

\section{Jordans 2011}

Jordans MJD, Komproe IH, Tol WA, Susanty D, Vallipuram A, Ntamatumba P, et al.Practice-driven evaluation of a multi-layered psychosocial care package for children in areas of armed conflict. Community Mental Health Journal 2011; 47(3):267-77.

\section{Kakuma 2011}

Kakuma R, Minas H, van Ginneken N, Dal Poz MR, Desiraju K, Morris JE, Saxena S, Scheffler, RM. Human resources for mental health care: current situation and strategies for action. Lancet 2011;378(9803):1654-63.

\section{Khamis 2000}

Khamis V. Child psychological maltreatment in Palestinian families. Child Abuse and Neglect 2000;24:1047-59.

Non-specialist health worker interventions for the care of mental, neurological and substance-abuse disorders in low- and middle- 


\section{Kirsch 2008}

Kirsch I, Deacon BJ, Huedo-Medina TB, Scoboria A, Moore TJ, Johnson BT. Initial severity and antidepressant benefits: a meta-analysis of data submitted to the Food and Drug Administration. PLoS Medicine 2008;5(2):e45. doi: 10.1371/journal.pmed.0050045.

\section{La Trobe 2008}

Cochrane Consumers \& Communication Review Group. Outcomes of interest to the Cochrane consumers and communication review group [La Trobe University]. http:// www.latrobe.edu.au/chcp/assets/downloads/Outcomes.pdf January 2008.

Lancet 2007

Lancet Mental Health Working Group. Scaling-up services for mental disorders: a call for action. Lancet 2007;

Supplement(Global mental health series):S87-98.

\section{Laor 2002}

Laor N, Wolmer L, Kora M, Yucel D, Spirman S, Yazgan Y. Posttraumatic, dissociative and grief symptoms in Turkish children exposed to the 1999 earthquakes. Journal of Nervous and Mental Disease 2002;190:824-32.

Lavis 2009

Lavis JN, Oxman AD, Souza NM, Lewin S, Gruen RL, Fretheim A. SUPPORT Tools for evidence-informed health Policymaking (STP): 9. Assessing the applicability of the findings of a systematic review. Health Research Policy and Systems 2009;7(suppl 1):9.

Lewin 2008

Lewin S, Lavis JN, Oxman AD, Bastias G, Chopra M, Ciapponi A, et al.Supporting the delivery of cost-effective interventions in primary health-care systems in low-income and middle-income countries: an overview of systematic reviews. Lancet 2008;372(9642):928-39.

\section{Lewin 2010}

Lewin S, Munabi-Babigumira S, Glenton C, Daniels K, Bosch-Capblanch X, Van Wyk Brian E, et al.Lay health workers in primary and community health care for maternal and child health and the management of infectious diseases. Cochrane Database of Systematic Reviews 2010, Issue 3. [DOI: 10.1002/14651858.CD004015.pub3]

Lopes Cardozo 2000

Lopes Cardozo B, Vergara A, Agani F, Gotway CA. Mental health, social functioning, and attitudes of Kosovar Albanians following the war in Kosovo. JAMA 2000;284 (5):569-77.

\section{Lucas 2001}

Lucas CP, Zhang H, Fisher PW, Shaffer D, Regier DA, Narrow WE, et al.The DISC Predictive Scales (DPS): efficiently screening for diagnosis. Journal of the American Academy of Child and Adolescent Psychiatry. 2001;40:443-9.

Lukumar 2008

Lukumar P, Wijewardana K, Hermansson J, Lindmark G. Validity and reliability of Tamil version of Strengths and Difficulties Questionnaire self-report. Ceylon Medical Journal 2008;53:48-52.
Mental Health Atlas 2011

WHO. Mental Health Atlas. Geneva: World Health Organisation 2011.

\section{Murray 2012}

Murray CJ, Vos T, Lozano R, et al.Disability-adjusted life years (DALYs) for 291 diseases and injuries in 21 regions, 1990-2010: a systematic analysis for the Global Burden of Disease Study 2010. Lancet 2012;380(9859):2197-223.

\section{Murthy 1987}

Murthy RS. Integration of mental health with primary health care - Indian experience. NIMHANS, Bangalore. Bangalore, 1987.

\section{Murthy 2008}

Murthy RS. Organisation of mental health services universal challenge. In: Varma VK, Kala AK, Gupta $\mathrm{N}$ editor(s). Culture, Personality and Mental Illness - $A$ Perspective of Traditional Societies. New Delhi: Jaypee Brothers, 2008:414-46.

\section{Parker 2008}

Parker AG, Hetrick SE, Purcell R, Gillies D. Consultation liaison in primary practice for mental health problems. Cochrane Database of Systematic Reviews 2008, Issue 2. [DOI: 10.1002/14651858.CD007193]

\section{Patel 2003c}

Patel V. In: Psychiatrists RCo editor(s). Where there is no psychiatrist. A mental health care manual. Glasgow: Bell \& Bain, 2003.

\section{Patel 2007a}

Patel V, Chisholm D, Kirkwood BR, Mabey D. Prioritizing health problems in women in developing countries: comparing the financial burden of reproductive tract infections, anaemia and depressive disorders in a community survey in India. Trop Med Int Health 2007;12(1):130-139.

\section{Patel 2007b}

Patel V, Araya R, Chatterjee S, Chisholm D, Cohen A, De Silva $\mathrm{M}$, et al.Treatment and prevention of mental disorders in low-income and middle-income countries. Lancet 2007; 370(9591):991-1005.

Patel 2007c

Patel V, Flisher AJ, Hetrick S, McGorry P. Mental health of young people: a global public-health challenge. Lancet 2007;369(9569):1302-13.

\section{Patel 2008a}

Patel V, Flisher AJ, Nikapota A, Malhotra S. Promoting child and adolescent mental health in low and middle income countries. Journal of Child Psychology and Psychiatry and Allied Disciplines 2008;49(3):313-34.

Patel 2008b

Patel VH, Kirkwood BR, Pednekar S, Araya R, King M, Chisholm D, et al.Improving the outcomes of primary care attenders with common mental disorders in developing countries: a cluster randomized controlled trial of a collaborative stepped care intervention in Goa, India. Trials 2008;9:4.

Non-specialist health worker interventions for the care of mental, neurological and substance-abuse disorders in low- and middle- 
Prince 2004

Prince M, Graham N, Brodaty H, Rimmer E, Varghese

$\mathrm{M}$, et al.Alzheimer Disease International's 10/66 Dementia Research Group - one model for action research in developing countries. Internation Journal of Geriatric Psychiatry 2004;19(2):178-81.

\section{Prince 2007}

Prince M, Patel V, Saxena S, Maj M, Maselko J, Phillips MR, et al.No health without mental health. Lancet 2007; 370(9590):859-77.

Rahman 2013

Rahman A, Fisher J, Bower P, Luchters S, Tran T, Yasamy MT, et al.Interventions for common perinatal mental disorders in women in low- and middle-income countries: a systematic review and meta-analysis. Bulletin of the World Health Organization 2013;91:593-601.

Reeves 2009

Reeves BC, Deeks JJ, Higgins JPT, Wells GA. Including non-randomized studies. Cochrane handbook for systematic reviews of interventions. Version 5.0.1 (updated September 2009). Available from www.cochranehandbook.org. Chichester, UK: Wiley-Blackwell, 2009.

RevMan 2012

The Nordic Cochrane Centre, The Cochrane Collaboration. Review Manager (RevMan). Version 5.2.. Copenhagen: The Nordic Cochrane Centre, The Cochrane Collaboration, 2012.

Saraceno 2007

Saraceno B, Van Ommeren M, Batniji R, Cohen A, Gureje $\mathrm{O}$, Mahoney J, et al.Barriers to improvement of mental health services in low-income and middle-income countries. Lancet 2007;370(9593):1164-74.

\section{Saxena 2007}

Saxena S, Thornicroft G, Knapp M, Whiteford H. Resources for mental health: scarcity, inequity, and inefficiency. Lancet 2007;370(9590):878-89.

\section{Schünemann 2009}

Schünemann HJ, Oxman AD, Vist GE, Higgins JPT,

Deeks JJ, Glasziou P. Interpreting results and drawing conclusions. In: Higgins JPT, Green S editor(s). Cochrane handbook for systematic reviews of interventions. Version 5.0.1 (updated September 2009). Available from www.cochranehandbook.org. Chichester, UK: Wiley-Blackwell, 2009.

\section{Shemilt 2009}

Shemilt I, Mugford M, Byford S, Drummond M, Eisenstein E, Knapp M, et al.Incorporating economics evidence. In Higgins JPT, Green S (eds), Cochrane handbook for systematic reviews of interventions. Version 5.0.2 [updated September 2009]. Available from www.cochranehandbook.org. The Cochrane Collaboration. Available from www.cochrane-handbook.org.

Shemilt 2010

Shemilt I, Thomas J, Morciano M. A web-based tool for adjusting costs to a specific target currency and price year Evidence \& Policy 2010;6(1):51-9.

\section{Shepperd 2009}

Shepperd S, Doll H, Gowers S, James A, Fazel M, Fitzpatrick R, et al.Alternatives to inpatient mental health care for children and young people. Cochrane Database of Systematic Reviews 2009, Issue 2. [DOI: 10.1002/ 14651858.CD006410.pub2]

\section{Siskind 2010}

Siskind Dan, Araya Ricardo, Kim Jane. Cost-effectiveness of improved primary care treatment of depression in women in Chile. British Journal of Psychiatry 2010;197(4):291-6.

Snyder 1997

Snyder CR, Hoza B, Pelham WE, Rapoff M, Ware L, Danovsky M, et al.The development and validation of the Children's Hope Scale. Journal of Pediatric Psychology 1997; 22:399-421.

\section{Spijker 2002}

Spijker J, de Graaf R, Bijl RV, Beekman ATF, Ormel J, Nolen WA. Duration of major depressive episodes in the general population: results from The Netherlands Mental Health Survey and Incidence Study (NEMESIS). British Journal of Psychiatry 2002;181:208-213.

Spirito 1988

Spirito A, Stark LJ, Williams C. Development of a brief coping checklist for use with paediatric populations. Journal of Pediatric Psychology 1988;13:555-74.

\section{Staub 2005}

Staub E, Pearlman LA, Gubin A, Hagengimana A. Healing, reconciliation, forgiving, and the prevention of violence after genocide or mass killing: An intervention and its experimental evaluation in Rwanda. Journal of Social and Clinical Psychology 2005;24:297-334.

\section{Thornicroft 2004}

Thornicroft G, Tansella M. Components of a modern mental health service: a pragmatic balance of community and hospital care: overview of systematic evidence. British Journal of Psychiatry 2004;185:283-90.

Tol 2011

Tol WA, Barbui C, Galappatti A, Silove D, Betancourt TS, Souza R, et al.Mental health and psychosocial support in humanitarian settings: linking practice and research. Lancet 2011;378(9802):1581-91.

Tol 2011a

Tol WA, Komproe IH, Jordans MJD, Susanty D, de Jong JT. Developing a function impairment measure for children affected by political violence: a mixed methods approach in Indonesia. International Journal of Quality Health Care 2011;23:5-83.

\section{Ukoumunne 1999}

Ukoumunne OC, Gulliford MC, Chinn S, Sterne JA, Burney PG. Methods for evaluating area-wide and organisation-based interventions in health and health care: a systematic review. Health Technology Assessment 1999;3(5): iii-92.

van Steenbergen-Weijenburg 2010 van Steenbergen-Weijenburg KM, van der Feltz-Cornelis CM, Horn EK, van Marwijk HWJ, Beekman ATF, Rutten 
FFH, et al.Cost-effectiveness of collaborative care for the treatment of major depressive disorder in primary care. A systematic review. BMC Health Services Research 2010;10 (1): 19 .

\section{Vos 2012}

Vos T, Flaxman AD, Naghavi M, et al.Years lived with disability (YLDs) for 1160 sequelae of 289 diseases and injuries 1990-2010: a systematic analysis for the Global Burden of Disease Study 2010. Lancet 2012;380(9859): 2163-96.

WHO 1997

World Health Organisation. Composite International

Diagnostic Interview (CIDI). Geneva, Switzerland 1997.

\section{WHO 2001}

WHO. World Health Report - Mental Health. Geneva: World Health Organization, 2001.

\section{WHO 2003a}

WHO. The mental health context. Geneva: World Health Organization, 2003.

\section{WHO 2003b}

WHO. caring for children and adolescents with mental disorders. Setting WHO directions. Geneva: World Health
Organization, 2003.

\section{WHO 2008}

WHO. Scaling up care for mental, neurological, and substance use disorders. mhGAP. Geneva: World Health Organization, 2008.

\section{WHO 2010}

WHO. mhGAP intervention guide for mental, neurological and substance use disorders in non-specialized health settings. http://whqlibdoc.who.int/publications/2010/ 9789241548069' eng.pdf 2010.

\section{Wiley-Exley 2007}

Wiley-Exley E. Evaluations of community mental health care in low- and middle-income countries: a 10-year review of the literature. Social Science and Medicine 2007;64(6): 1231-41.

\section{Woltmann 2012}

Woltmann E, Gorgan-Kaylor A, Perron B, Georges H, Kilbourne AM, Bauer MS. Effectiveness of collaborative chronic care models for mental health conditions across primary, specialty, and behavioral health care settings: systematic review and meta-analysis. American Journal of Psychiatry 2012;169(8):790-804.

* Indicates the major publication for the study 


\section{CHARACTERISTICS OFSTUDIES}

\section{Characteristics of included studies [ordered by study ID]}

\section{Ali 2003 RCT Pakistan}

Methods

Participants

Study design: RCT

Duration of study: Baseline survey January to April 2001 and considering the 8-week intervention must be between May to June-July 2001

Country: Pakistan

Income classification: Low income

Geographical scope: Semi-urban: in Qayoomabad, lower middle class semi-urban community with a population of 80,000 in Karachi

Healthcare setting: Home

Mental health condition: Common mental disorders

Population: Adults

- Age: $18-50$ years

- Gender: Female

- Socioeconomic background: Lower-middle class. Women predominantly aged 26-40 years, half had no formal education, not involved in revenue generation, twothirds had a household income of $>3000$ PKR, nearly $60 \%$ were residing for more than 10 years

- Inclusion criteria: Participant: women 18-50 years old; able to communicate in Urdu, planning to live in the study area for more than 1 year, no bereavement in past 6 weeks

- Exclusion criteria: Participant women: those actively suicidal
Stated purpose: To assess the effect of on levels of anxiety or depression (or both), among women who had attended counselling sessions, provided by briefly trained counsellors of their own community

INTERVENTION:

Name: Counselling

Delivered by:

- Title/name of NSHW/OPHR and number: 21 minimally trained counsellors

- Selection: "Women were informed by word of mouth and by leaflets; out of 73 women who came for interview, 21 selected based on communications skills, motivation, attitude, ability to read and write Urdu and freedom to move in the community“

- Educational background: "Ability to read and write Urdu“ and belonging to local community

- Training: 11 training sessions held over 4 weeks. Each lesson lasted 3 hours and was led by either a family practitioner, a sociologist, a psychiatrist or 3 clinical psychologists. Contents: Basic information regarding anxiety, depression, stress/anger management, and communication/counselling skills. Communication covered active listening, probing and feedback, whereas counselling dealt with supportive problemsolving and cognitive-behavioural techniques. "Manual incorporating the training material is being published and is planned to train master training who could replicate the study in several urban and rural centers"

- Supervision: "Women had ready access to members of the training team 
Ali 2003 RCT Pakistan (Continued)

throughout the study period“"

- Incentives/remuneration: Not specified

Intervention details:

- Duration/frequency: 8 sessions (?possibly weekly). Supportive, cognitive and problem-solving counselling was provided on the day and time convenient of the woman

- Content of intervention: The trained counsellors provided supportive, cognitive and problem solving counselling at the clients residence at convenient time

CONTROL: Usual care, no intervention, just had AKUADS administered at baseline and end of study; however, "as the effectiveness of counselling was proved, for ethical reasons the control group was also counselled" possibly at the end of the study CO-INTERVENTIONS: Nil

Outcomes

Patient: Reduction in Aga Khan University Anxiety and Depression Scale scores

Carer: Not applicable

Process/health worker outcomes: Not specified

Economic outcomes: None

Time points: Baseline and at the end of 8 weeks

Notes

Source of funding: Academic body; Aga Khan University Research Council

Notes on validation of instruments (screening and outcomes): AKUADS (indigenous screening scale, developed from complaints of patients with anxiety/depression, recorded verbatim in Urdu) previously validated against psychiatrist evaluation as gold standard and compared with SRQ

Additional information: None

Handling the data: As per footnotes in data and analysis

Prospective trial registration number: Not specified

Risk of bias

\begin{tabular}{|c|}
\hline Bias \\
\hline
\end{tabular}

Random sequence generation (selection Low risk bias)

Quote: "Every third household was systematically sampled in all of Qayoomabad. [..

.] One woman was randomly chosen from each selected household and screened for anxiety and/or depression. [...] Using computer-generated random numbers, 216 [of 1218 women] cases were randomised to the intervention and 150 to the control group". The initial selection was quasi-random, but then allocation to control or intervention was random

Allocation concealment (selection bias) Unclear risk

Quote: "Computer-generated random numbers"

Comment: Even though sequence generation was centrally done, it was unclear how allocation was concealed

Non-specialist health worker interventions for the care of mental, neurological and substance-abuse disorders in low- and middle- 


\section{Ali 2003 RCT Pakistan (Continued)}

Blinding of participants and personnel Low risk (performance bias)

All outcomes

Blinding of outcome assessment (detection Low risk bias)

objective outcomes

Blinding of outcome assessment (detection Low risk bias)

subjective outcomes

Baseline outcome measurements similar Low risk

Baseline characteristics similar?

Low risk

Incomplete outcome data (attrition bias) High risk

Efficacy data

Safety data (e.g. adverse events)

$\begin{array}{ll}\text { Protection against contamination Low risk } & \text { Lon }\end{array}$

Reliable primary outcome measures Low risk

Selective reporting (reporting bias) Unclear risk
Incomplete outcome data (attrition bias) Unclear risk

Comment: Not able to blind participants or personnel. Unlikely to influence outcomes

Comment: No selective reporting. Not applicable

Comment: Independent data collectors blind to allocation and to the previous scores

Comment: Yes similar, both across intervention and control, and between dropouts and non-dropouts

Comment: Yes, similar. All P values over 0 . 2 comparing dropouts versus non-dropouts and intervention versus control groups

Comment: Intervention: 68\% dropout between baseline and those completing the intervention. Control: 33\% dropout. Though characteristics are similar between dropouts and non-dropouts (including baseline scores), their scores may have been different at follow-up

Comment: No reported safety outcomes

Quote: "The spontaneous decrease in the score [in the control group] could be attributed to the natural history of depression, which waxes and wanes, but a contaminant effect of counselling cannot be ruled out" " the effect of summer holidays occurring during the study period was also considered as possibly "causing contamination"

Comment: AKUADS administered by trained people not delivering intervention. So unlikely to be bias

Comment: No selective reporting. but no protocol to assess if this is the case 
Ali 2003 RCT Pakistan (Continued)

\begin{tabular}{|c|c|}
\hline Other bias & Comment: None detected \\
\hline \multicolumn{2}{|c|}{ Araya 2003 RCT Chile } \\
\hline Methods & $\begin{array}{l}\text { Study design: RCT } \\
\text { Duration of study: March } 2000 \text { to March } 2002\end{array}$ \\
\hline Participants & $\begin{array}{l}\text { Country: Chile } \\
\text { Income classification: Upper middle } \\
\text { Geographical scope: Deprived urban areas in Santiago } \\
\text { Healthcare setting: PC facility that were underfunded and insufficiently resourced } \\
\text { Mental health condition: Women with persistent depression } \\
\text { Population: Women } \\
\text { - Age: } 18-70 \text { years } \\
\text { - Gender: Female } \\
\text { - Socioeconomic background: Majority were housewives from deprived areas } \\
\text { - Inclusion criteria: Age } 18-70 \text { years with current major depression illness ( } 2 \\
\text { screenings of GHQ-12 with a score }>5 \text { at } 2 \text { weeks interval), female PHC patients } \\
\text { - Exclusion criteria: Women who had psychiatric consultation or admission to a } \\
\text { hospital in the } 3 \text { months before the baseline interview, current psychotic symptoms, } \\
\text { serious suicide risks, history of mania, alcohol abuse }\end{array}$ \\
\hline
\end{tabular}

Interventions

Stated purpose: To compare the effectiveness of a stepped-care programme with usual care in primary-care management of depression in low-income women in Santiago, Chile INTERVENTION:

Name: Stepped care

Delivered by:

- Title/name of NSHW/OPHR and number: PC physician and group leaders (non-medical worker)

- Selection:Group leaders and doctors - both were employed in the local PHC units who were selected for the study

- Educational background: Group leaders were a nurse or a social worker. The doctors had a medical degree

- Training:Group leaders - 12 hours training by the principal investigator psychiatrist. Content - not specified. Doctors -4 hours of training by psychiatrist to understand the brief pharmacotherapy protocol (medical algorithm of fluoxetine, amitriptyline, imipramine) and initial and follow-up assessments

- Supervision: Group leader - 8 hours supervision by the principal investigator over the course of the intervention

- Incentives/remuneration: Are none as they are employees Intervention details:

- Duration/frequency: Psychoeducation: 7 weekly sessions and 2 booster sessions (weeks 9 and 12) each lasting 75 minutes, groups of 20 participants

- Content of intervention: Group leaders provides psychoeducation, which consists of information on symptoms, causes of depressions, treatments available, positive activities, problem-solving techniques, basic cognitive and relapse-prevention techniques; patients given a manual on session contents and examples/exercises.

Follow-up by group leaders: monitoring medication adherence, attendance at follow-

Non-specialist health worker interventions for the care of mental, neurological and substance-abuse disorders in low- and middle- 
up visits for patients receiving pharmacotherapy. They also refer to the doctor if HDRS score $>12$ at 6 weeks with psychoeducation. Doctors: detect and diagnose using their brief pharmacotherapy protocol and then prescribe according to the medical algorithm, and then follow-up the patients

CONTROL: Usual care: normally available services in PC clinic: included antidepressant medication, referral to specialist (usually takes 2 months to be seen by psychiatrist); given guidelines on treating depression in PC before initiation of study. No services restricted/ with held

CO-INTERVENTIONS: None

Outcomes

Patient: GHQ-12 screening, MINI, Diagnosis for DSM-IV, HDRS* - Severity of depression, SF-36 - scores for mental health, emotional role, social functioning, vitality

Carer: None

Process/health worker outcomes: None

Economic outcomes: None

(* study's primary outcomes)

Time points: Baseline, 3 months, 6 months

$\begin{array}{ll}\text { Notes } & \text { Source of funding: US National Institute of Mental Health } \\ & \text { Notes on validation of instruments (screening and outcomes): All instruments vali- } \\ \text { dated } & \text { Additional information: Information from authors: no study protocol so unable to } \\ \text { check primary and secondary outcomes } \\ \text { Handling the data: As per footnotes in data and analysis } \\ \text { Prospective trial registration number: None (only National Institute of Mental Health } \\ \text { proposal) }\end{array}$

Risk of bias

\begin{tabular}{|c|c|c|}
\hline Bias & Authors' judgement & Support for judgement \\
\hline $\begin{array}{l}\text { Random sequence generation (selection } \\
\text { bias) }\end{array}$ & Low risk & $\begin{array}{l}\text { Quote: "Randomisation of patients was } \\
\text { stratified by clinic, done in blocks of } 20 \text { us- } \\
\text { ing computer-generated random number" } \\
\text { Comment: Probably done }\end{array}$ \\
\hline
\end{tabular}

Allocation concealment (selection bias) Low risk

Blinding of participants and personnel Low risk (performance bias)

All outcomes
Quote: “Individuals who recruited patients were neither involved in nor aware of the procedure used to generate allocations. Allocations in numbered sealed envelopes in each clinic and opened by an individual who had not recruited patients"

Comment: Probably done

Comment: Patients and personnel not blinded but unlikely to have any effect on outcome 


\section{Araya 2003 RCT Chile (Continued)}

\begin{tabular}{|c|c|c|}
\hline $\begin{array}{l}\text { Blinding of outcome assessment (detection } \\
\text { bias) } \\
\text { objective outcomes }\end{array}$ & Low risk & Comment: None \\
\hline $\begin{array}{l}\text { Blinding of outcome assessment (detection } \\
\text { bias) } \\
\text { subjective outcomes }\end{array}$ & Low risk & $\begin{array}{l}\text { Quotes: } \\
\text { "At baseline, a clinician administered the } \\
\text { three assessments" } \\
\text { "Follow-up interviews were done by an in- } \\
\text { dependent clinician blinded to treatment } \\
\text { assignment" } \\
\text { "Rates of participation in the intervention } \\
\text { programme were high, and participation } \\
\text { in blinded outcome assessments exceeded } \\
85 \% \text { in both groups" }\end{array}$ \\
\hline Baseline outcome measurements similar & Low risk & $\begin{array}{l}\text { Comment: Similar baseline outcome } \\
\text { measurements. No adjustment therefore } \\
\text { needed }\end{array}$ \\
\hline Baseline characteristics similar? & Low risk & Comment: Similar baseline characteristics \\
\hline $\begin{array}{l}\text { Incomplete outcome data (attrition bias) } \\
\text { Efficacy data }\end{array}$ & Low risk & $\begin{array}{l}\text { Comment: } 3 \text { months: } 18 \text { (stepped care) vs } \\
11 \text { (usual care); } 6 \text { months: } 16 \text { vs } 13 \text { out of } \\
120 \text { patients in each group. This represents } \\
\text { more than } 80 \% \text { follow-up rate }\end{array}$ \\
\hline
\end{tabular}

Incomplete outcome data (attrition bias) Unclear risk

Safety data (e.g. adverse events)

Protection against contamination

High risk

Reliable primary outcome measures

Low risk

Low risk

Low risk

Selective reporting (reporting bias) Low ris

Author information: "There was a record kept [of adverse outcomes] but do not know where it is"

Comment: 3 clinics that can have both intervention and control people and the same GP could be delivering both interventions, so theoretical risk of contamination

Comment: There is no mention of their being a reliability test/agreement between physicians in using the HDRS score or the SF-36. However, also low dropout rate

Comment: No protocol. Author suggests all outcomes reported 
- Age: Mother: reproductive age: mean (SD): 26 years (7.1); child: mean age (SD): 18.4 months (4.5)

- Gender: Female; child: both

- Socioeconomic background: $40 \%$ completed high school, moderately crowded environments with few possessions and mean sanitation facilities

- Inclusion criteria: Mothers of undernourished children enrolled in nutrition clinics in government health centres. All 12 nutrition clinics in the urban areas of the included parishes were included. Children: aged 9-13 months, weight-for-age $<-1.5$ ( $\mathrm{z}$ score) and birthweight $>1.8 \mathrm{~kg}$, singleton, absence of chronic disease or obvious disability

- Exclusion criteria: Not specified

Stated purpose: To determine the effect of early childhood stimulation with undernourished children and their mothers on maternal depression

INTERVENTION:

Name: Early home stimulation programme

Delivered by:

- Title/name of NSHW/OPHR and number: $18 \mathrm{CHAs}$

- Selection: Paraprofessionals employed in government health centres. Selected by public health nurses in each clinic to be involved in the home stimulation programme. They were people whom the nurses thought would be interested and reasonably competent at the task

- Educational background: Most CHWs would have either not completed high school or have completed high school with no examinations

- Training: 5-day introductory workshop (over 4 weeks provided by government) conducted with CHAs before start of programme on health and nutrition. The study team provided an additional 2 weeks training covering child development, parenting issues and how to conduct the intervention. All CHAs received a manual and a set of homemade toys and these were used in the training. A further 4-day refresher training was held midway through the study

- Supervision: The supervisor observed each CHA conduct a visit once a month and visited health centre every 2 weeks to discuss the programme and review the records of each visit. Supervised by main author $(\mathrm{HBH})$ - degree in education and Master of Science from Centre of International Child Health and studying for Doctor of Philosophy degree at the time

- Incentives/remuneration: Employed by government

Intervention details:

- Duration/frequency: CHAs conducted house visits weekly for 30 minutes lasted 
Baker-H 2005 CRCT Jamaica (Continued)

for 1 year

- Content of intervention: Home visits (demonstrating age-appropriate activities to improve mothers' knowledge and practices of child rearing/parental self esteem, encouragement, make sure they experience success, empathy)

CONTROL: Usual care: which is standard health and nutrition care (from nutrition clinics). This was also provided to intervention group

CO-INTERVENTIONS: As above (usual care)

Outcomes

Patient: (Child) Griffiths Mental Development Scales*; child anthropometry: z scores (National Center for Health Statistics references) for height for age, weight for height and weight for age

Carer: (mother) CES-D* - for assessing frequency of depressive symptoms; Peabody Picture Vocabulary Test-Revised (for mother's vocabulary)

Process/health worker outcomes: None

Economic outcomes: None

(*: study's primary outcomes)

Time points: For mothers: baseline and 1 year follow-up

Notes

Source of funding: Thrasher Research Fund, USA, with subsidiary grants from the British High Commission-DFID, Jamaica and the University of the West Indies Mona Campus Research and Publication Fund. The Ministry of Health Jamaica supported the CHAs

Notes on validation of instruments: Validated international instruments but not for the Jamaican population

Additional information: No published study protocol

Handling the data: As per footnotes in data and analysis

Prospective trial registration number: None

Risk of bias

$\begin{array}{lll}\text { Bias Authors' judgement Support for judgement } & \text { Autgents }\end{array}$

Random sequence generation (selection Low risk bias)

Quote: "The 12 clinics were stratified into two groups by size and randomly assigned to intervention and control groups. Preliminary investigation of records indicated that there should have been sufficient children attending the clinics in Kingston and St Andrew to fulfil the sample size requirements. However, fewer children were available than had been anticipated, especially in the centres assigned to intervention. Therefore six clinics in urban areas of the adjacent parish, St Catherine, were also enrolled. Four were randomly assigned to intervention and two to control, to ensure equal numbers of children in the intervention and control groups, making a total of 11 intervention clinics and seven control

Non-specialist health worker interventions for the care of mental, neurological and substance-abuse disorders in low- and middle- 
Baker-H 2005 CRCT Jamaica (Continued)

\begin{tabular}{|c|c|c|}
\hline & & $\begin{array}{l}\text { clinics" } \\
\text { Information from author: Using a com- } \\
\text { puter generated, simple randomisation se- } \\
\text { quence } \\
\text { Comment: Despite extra clinics later as- } \\
\text { signed, this was done randomly }\end{array}$ \\
\hline Allocation concealment (selection bias) & Low risk & $\begin{array}{l}\text { Information from author: Allocation } \\
\text { done by an independent statistician }\end{array}$ \\
\hline $\begin{array}{l}\text { Blinding of participants and personnel } \\
\text { (performance bias) } \\
\text { All outcomes }\end{array}$ & Low risk & $\begin{array}{l}\text { Comment: No blinding but this would not } \\
\text { be feasible for such an intervention. Un- } \\
\text { likely to affect outcomes }\end{array}$ \\
\hline
\end{tabular}

Blinding of outcome assessment (detection Low risk

bias)

Comment: $\mathrm{z}$ score anthropometric mea-

objective outcomes

surements standardised and validated

Blinding of outcome assessment (detection Low risk

bias)

subjective outcomes

Comment: CED evaluation done by the interviewers and were unaware of the mothers' intervention status. For children, "assessed by one of two persons who tested equal numbers from each group and were unaware of the children's group"

\begin{tabular}{l|ll}
\hline Baseline outcome measurements similar & Low risk & Comment: All similar \\
\hline Baseline characteristics similar? & High risk & $\begin{array}{l}\text { Comment: In the intervention group } \\
\text { more fathers live at home }(30 \text { vs. } 21 \%) \text { and } \\
\text { more mothers completed high school }(28 \\
\text { vs. 23\%). This may add to the support of } \\
\text { the mothers, but it is unlikely to have a } \\
\text { large effect on the outcomes because base- } \\
\text { line outcomes are similar. However, these } \\
\text { differences are not adjusted for in the sum- } \\
\text { mary statistics }\end{array}$ \\
\end{tabular}

Incomplete outcome data (attrition bias) Low risk Efficacy data

Comment: Intervention 64/70 completed follow up and for control 61/69; This is more than $20 \%$ completion rate

Incomplete outcome data (attrition bias) Unclear risk

Comment: No adverse events reported

Safety data (e.g. adverse events)

Protection against contamination Low risk

Comment: This is a cluster trial so patients unlikely to discuss the intervention

Additional information from author: The CHAs did not have training together in groups at the time so they would not

Non-specialist health worker interventions for the care of mental, neurological and substance-abuse disorders in low- and middle- 
Baker-H 2005 CRCT Jamaica (Continued)

have come into contact with CHAs from control clinics in a formal setting

Reliable primary outcome measures High risk

Quote: "The test-retest (intraclass correlation coefficient) for the depression scale over a two week period was $\mathrm{R}=0.71(\mathrm{n}=20)$. The internal reliability (Chronbach's alpha) of the scale was $\mathrm{a}=0.90(\mathrm{n}=125)$. Two interviewers administered the questionnaires in the study; they were unaware of the mothers' intervention status. Interobserver agreement was $.90 \%$ for each question (n $=22$ )"

Comment: Reliable tools and interobserver agreement and interviewers unaware of allocation status. However, the CES-D instrument has not been validated in the Jamaican population and does not measure clinical depression, rather depressive symptoms. Several of the mothers may not have been depressed. The reliability of these figures for assessing depression levels is low

Selective reporting (reporting bias) Low risk

Comment: Child outcomes are reported in a subsequent paper that author sent to me Powell 2004

\begin{tabular}{|c|c|c|}
\hline Other bias & Low risk & Comment: No other bias detected \\
\hline
\end{tabular}

Bass 2012 CBA Indonesia

Methods

Study design: CBA study, unit of allocation by geographic area and unit of analysis by individual

Duration of study: August 2007 to January 2008

Participants

Country: Indonesia

Income classification: Lower-middle

Geographical scope: Rural, 6 villages around the central town of Bireuen District in Aceh, selected for having historically high rates of torture and where other NGOs were not currently providing services. Villages were paired based on distance from the urban district centre. This district was considered 1 of the strongholds of the Free Aceh Movement (Gerakan Aceh Merdeka (GAM) and these villages were frequently attacked by the Indonesian military throughout the 1980s and 1990s. These populations are situated far from the sea and were not directly affected by the disastrous effects of the 2004 tsunami. They were, however, affected by the unequal distribution of resources provided to the tsunami survivors, which according to the United Nations led to local discontent and perceptions of inequality with regard to international aid distribution

Healthcare setting: Community groups

Mental health condition: Anxiety and depression. As PTSD cardinal symptoms did

Non-specialist health worker interventions for the care of mental, neurological and substance-abuse disorders in low- and middle- 
Bass 2012 CBA Indonesia (Continued)

not come up much, they did not measure PTSD.

Population: Adults

- Age: > 18 years, most aged 30-69 years

- Gender: Both

- Socioeconomic background: The populations in the study villages were exposed to systematic human rights violations, with entire villages experiencing torture through direct experience, torture of family members, witnessing of torture and arbitrary killings, or a combination of these. Majority were married (79\%)

- Inclusion criteria: Total symptom scale > 38 and eligible cases also had to indicate at least some level of functional impairment, as assessed by a score ( 0 on either the local function or the adapted WHODAS II measure)

- Exclusion criteria: Not specified

Stated purpose: 1. to investigate the impact of the group counselling intervention on reducing the severity of mental symptoms and associated dysfunction. 2. to investigate potential moderating effects of gender and age because the literature in general, as well as recent cross-cultural studies, have shown some differential effects of gender and age with certain treatments

\section{INTERVENTION:}

Name: Problem-Solving Counseling (PSC) programme

Delivered by:

- Title/name of NSHW/OPHR and number: RATA (A local torture survivor and treatment organisation) counsellors, number not specified

- Selection: Local lay individuals

- Educational background: No formal degrees or education prior to training

- Training: Initial 5-day training by ICMC (International Catholic Migration Commission) on the programme components. They then provided individual counselling to torture survivors in non-study communities for 3 months to improve their skills with regular supervision to ensure proper implementation. This was followed by a second period of training on implementing the programme in a group format, including skills for group management. The group intervention was provided by pairs of counsellors working together. The manualised training was developed by ICMC Indonesia: topics such as qualities of an effective helper, confidentiality, empathy, listening and responding, questioning and problem management skills, stress and coping, and information specifically on the consequences and needs of torture survivors, featured

- Supervision: Group and individual counsellor supervision was provided throughout the study, not specified by whom

- Incentives/remuneration: Not specified Intervention details:

- Duration/frequency: 8 weekly group sessions. Duration of intervention 4 months: September to December 2007

- Content of intervention: Talking groups/group counselling in community settings. Client-guided problem-solving approach to the problems selected by the group of clients at the time the groups were created (non-specific counselling). Sessions 1 and 2: introduction of intervention, expectations, current problems related to distress identified. Study participants selected the focal problems. Sessions 3-6: discussions, sharing experiences, coping strategies identification and participants through discussions lead to understand how to cope; session 7: self evaluation of positive and 
negative changes; session 8: looking towards the future; conducted by 2 counsellors due to training purposes "the process was designed so that if it became necessary to replace a counsellor, new counsellors would be trained and then matched with more experienced counsellors to continue running the groups"

CONTROL: Wait-list control

CO-INTERVENTIONS: Change in service delivery. Prior to the initiation of the intervention, the counsellors conducted programme introduction sessions in the community (called socialisation sessions) in each study village, to introduce the community to the organisation and the services that would be provided. This was done to improve the acceptance of these services within the community and inform potential participants of the dates on which the interviewers would conduct the eligibility screenings. These community presentations were open to everyone in the village

Outcomes

Patient: Adapted HSCL - severity of depression and anxiety); adapted WHODAS II; newly developed scale on coping mechanisms (coping); local function scale; SCL somatic WHO scale (somatic symptoms)

(for screening: total symptom scale (a combination of all 3 scales mentioned under instruments for assessment)

Carer: $n / a$

Process/health worker outcomes: None

Economic outcomes: None

Time points: Baseline, 1 month pre intervention, 1 month post intervention (5 months following baseline) SCL, and local function scale: qualitative study done applying free listing and key informant qualitative interviewing to identify local signs and symptoms that mapped on to domains of anxiety, depression and somatoform disorders, these were used to draft the assessment of the 44-item questionnaire; it is unspecified if validated in the local setting. Local function scale also only validated during study in a pilot study and Pearson's coefficients done

WHODAS: adaptations using elements of Bolton and Tang (26) and other groups (20, $27,28)$. Items that population identified during prior qualitative study as typical daily carer tasks/community activities

Coping scale: based on qualitative study

Additional information: Contact for protocol

Handling the data: As per footnotes in data and analysis

Prospective trial registration number: None given

Risk of bias

\begin{tabular}{|c|c|c|}
\hline Bias & Authors' judgement & Support for judgement \\
\hline $\begin{array}{l}\text { Random sequence generation (selection } \\
\text { bias) }\end{array}$ & High risk & $\begin{array}{l}\text { Quote: "A local torture survivor and treat- } \\
\text { ment organization (RATA) helped identify } \\
\text { potential study villages, selecting those with } \\
\text { historically high rates of torture and where } \\
\text { other NGOs were not currently providing }\end{array}$ \\
\hline
\end{tabular}

Non-specialist health worker interventions for the care of mental, neurological and substance-abuse disorders in low- and middle- 
Bass 2012 CBA Indonesia (Continued)

services and therefore the need was greatest. Villages were paired based on distance to the urban district center. Table 1 presents the population structures of the study villages. The designation of intervention or control status was made in discussion with ICMC and RATA staff, who had to consider which villages would be more or less accessible during the rainy season in which the first round of services were provided, with more accessible villages given priority as intervention sites"

$\begin{array}{lll}\text { Allocation concealment (selection bias) } & \text { High risk } & \text { Comment: This was a CBA study }\end{array}$

Blinding of participants and personnel Low risk

Comment: Not done but unlikely to affect (performance bias)

outcomes

All outcomes

Blinding of outcome assessment (detection Low risk

bias)

objective outcomes

Blinding of outcome assessment (detection Low risk bias)

subjective outcomes

Baseline outcome measurements similar High risk
Quote: "The designation of intervention or control village was not shared with the interviewers until after data collection was complete, preventing any knowledge of which villages would get services first from biasing the baseline assessments"

Baseline outcome measurements similar

Quote: “The symptom scales are similar across the intervention and control groups, while the functional impairment levels differ, with the controls having higher rates of impairment among both men and women. " Differed for females on local functions scale and for all on WHODAS items (both for all eligible participants and for those actual participants who took part in $\geq 2$ sessions)

\begin{tabular}{lll}
\hline Baseline characteristics similar? & Low risk & Comment: All similar \\
\hline $\begin{array}{l}\text { Incomplete outcome data (attrition bias) } \\
\text { Efficacy data }\end{array}$ & Low risk & $\begin{array}{l}\text { Comment: } 79 \%(333 / 420) \text { of those eligi- } \\
\text { ble completed the full study. This is close } \\
\text { to } 80 \% \text { so is likely to be representative. In } \\
\text { addition, baseline characteristics and out- } \\
\text { comes did not differ significantly between } \\
\text { all eligible participants and these actual par- }\end{array}$
\end{tabular}


Bass 2012 CBA Indonesia (Continued)

ticipants

\begin{tabular}{|c|c|c|}
\hline $\begin{array}{l}\text { Incomplete outcome data (attrition bias) } \\
\text { Safety data (e.g. adverse events) }\end{array}$ & High risk & $\begin{array}{l}\text { Comment: Poorly powered study and } 1 \\
\text { outcome (social functioning in women) } \\
\text { seems to have a detrimental effect in } \\
\text { women. No adverse outcomes specifically } \\
\text { reported }\end{array}$ \\
\hline Protection against contamination & Low risk & $\begin{array}{l}\text { Comment: Clusters of interventions and } \\
\text { controls (controls are also hard to access), } \\
\text { so unlikely contamination }\end{array}$ \\
\hline
\end{tabular}

Reliable primary outcome measures High risk

Quote 1: "With regard to reliability, correlation coefficients were adequate at 0.65 for the anxiety subscale, 0.68 for the somatic subscale, and strong at 0.91 for the depression subscale. Cronbach alpha scores (a measure of internal reliability) ranged from 0.81 to 0.87 for the three scales. Alpha scores of 0.70 generally indicate adequate internal consistency"

Quote 2: "Adjusted for baseline symptom score, sex, age and group clustering"

Quote 3: "In our power calculations, we did not take the group clustering into account, and thus we may have underestimated the sample size required to show significant differences between the program conditions. However, an analysis of the intra-class correlation coefficients indicates that the variance due to clustering was minimal, thereby not affecting the comparisons significantly"

Selective reporting (reporting bias) Unclear risk

Comment: No protocol so unknown if there is selective reporting

Other bias High risk

Comment: In addition, villages selected for intervention group were more accessible to study team. This could have introduced biases due to unknown factors associated with ease of access

Non-specialist health worker interventions for the care of mental, neurological and substance-abuse disorders in low- and middle- 
Methods
Study design: Cluster RCT (unit of allocation: school setting; unit of analysis: individual patient)

Duration of study: February to May 2006
Participants
Country: Sri Lanka

Income classification: Low income

Geographical scope: Southern coast of Sri Lanka, small town of Welligama

Healthcare setting: Schools

Mental health condition: PTSD

Population: Children/adolescents

- Age: 9-14 years school

- Gender: Both male and female

- Socioeconomic background: Almost all the children in this school lost their homes and many lost family members or relatives in the tsunami

- Inclusion criteria: Aged 9-14 years; all exposed to tsunami

- Exclusion criteria: Not specified

\section{INTERVENTION:}

Name: ES-Sl (ERASE Stress Srilanka)

Delivered by:

- Title/name of NSHW/OPHR and number: 12 home-room teachers (12 trained but 6 took part in intervention and 6 in wait-list control)

- Selection: Teachers at the chosen school

- Educational background: Primary and secondary school teachers

- Training: ES-Sl course 3 days of 8-hour training (24 hours in total). Trainers were study researchers too

- Supervision: Throughout the application of the programme, teachers were supervised on a weekly basis by 2 local mental health professionals previously trained by the researchers to insure programme fidelity; monitoring protocol adherence done by trainers). During the first 2 sessions of the intervention, all teachers in the active group participated in two 3-hour supervisory sessions delivered by the trainers and assisted by 2 local mental health professionals to insure reliability of application of the protocol and to overcome potential problems. Adherence to protocol was monitored during these sessions, which included a point-by-point discussion of the training procedure by the trainers. Because the trainers could not remain in Sri Lanka for the entire intervention period, further fidelity was monitored by the local professionals and by periodic phone and Internet supervision with the first author (R.B.)

- Incentives/remuneration: Not specified Intervention details:

- Duration/frequency: The twelve 90-minute sessions (18 hours) delivered on a weekly basis

- Content of intervention: Each teacher in charge of 1 class only (12-16 students). The 12 sessions included homework review, warm-up exercises, experiential group activity, psycho-educational presentations, practical coping skills training, and a closure exercise followed by a new home assignment. Each teacher was given a manual CONTROL: Wait-list religious class control but where teachers had received the training for the intervention at baseline (risk of spillover effect). Due to perform intervention on other 6 classes the following year

CO-INTERVENTIONS: As above (usual care) 


\section{Berger2009 CRCT SriLanka (Continued)}

\begin{tabular}{|c|c|}
\hline Outcomes & $\begin{array}{l}\text { Patient: (Child) } 1.2 \text { objective exposure-related questions analysed as } 2 \text { Guttman scales } \\
\$ ; \text {; } \text { subjective exposure: Pat - Horencyck questionnaire } \$ \text {; } 3 \text {. Significant distress, help- } \\
\text { lessness and horror: } 3 \text { questions querying whether participants experienced any of those } \\
\text { emotions as related to the tsunami, using a 5-point scale from } 1 \text { (did not experience this } \\
\text { emotion at all) to } 5 \text { (experienced this emotion often). So as to avoid overinclusion, } 1 \\
\text { score of at least } 4 \text { was necessary to fulfil criterion A2 of PTSD } \$ \text {; } 4 \text {. major trauma life } \\
\text { questionnaire } \$ ; 5 \text {. UCLA PTSD index; } 6 \text {. subjective functional impairment: } 7 \text { items } \\
\text { derived from the Child DIS. 5-point scale ranging from } 1 \text { (not at all impaired) to } 5 \text { (very } \\
\text { much impaired); } 7 \text {. Somatic complaints related to terrorism: } 5 \text { yes/no categorical items } \\
\text { from the Diagnostic Predictive Scales } \$ ; 8 \text {. Hope: } 6 \text {-item self report questionnaire } \$ ; ~ 9 \text {. } \\
\text { Depression: } 7 \text { - item brief BDI } \\
\text { Carer: None } \\
\text { Process/health worker outcomes: None } \\
\text { Economic outcomes: None } \\
\text { (*: primary outcomes; } \$ \text { : outcomes that we have not reported in this review) } \\
\text { Time points: None }\end{array}$ \\
\hline Notes & $\begin{array}{l}\text { Source of funding: Not specified } \\
\text { Notes on validation of instruments: Instruments } 1,2 \text { and } 4 \text { are not validated. Instru- } \\
\text { ment } 3 \text { only validated in Israeli settings. 5. UCLA PTSD index: validated in Sri Lankan } \\
\text { population). Instruments 6-9: internal reliability only for current setting, validated else- } \\
\text { where in other settings (Beck 1974; Lucas 2001; Snyder 1997) } \\
\text { Additional information: None } \\
\text { Handling the data: As per footnotes in data and analysis } \\
\text { Prospective trial registration number: Not specified }\end{array}$ \\
\hline
\end{tabular}

\section{Risk of bias}

\begin{tabular}{lll}
\hline Bias & Authors' judgement & Support for judgement \\
\hline $\begin{array}{l}\text { Random sequence generation (selection } \\
\text { bias) }\end{array}$ & Low risk & $\begin{array}{l}\text { Quote: "The randomisation procedure was } \\
\text { done by coin tossing and choosing } 1 \text { class } \\
\text { for each age group" }\end{array}$ \\
\hline Allocation concealment (selection bias) & High risk & $\begin{array}{l}\text { Comment: There was no allocation con- } \\
\text { cealment }\end{array}$
\end{tabular}

Blinding of participants and personnel Low risk (performance bias)

All outcomes

Blinding of outcome assessment (detection Low risk bias)

objective outcomes
Blinding of outcome assessment (detection Low risk bias)

subjective outcomes
Comment: There was no blinding of teachers or students but this is unlikely to affect outcomes

Comment: No objective outcomes 


\section{Berger2009 CRCT SriLanka (Continued)}

\begin{tabular}{|c|c|c|}
\hline Baseline outcome measurements similar & Low risk & $\begin{array}{l}\text { Quote: "Further analyses show no differ- } \\
\text { ence in outcome measures at the first assess- } \\
\text { ment between the ES-SL and WL [wait- } \\
\text { list] groups ( table 1)" }\end{array}$ \\
\hline Baseline characteristics similar? & Low risk & $\begin{array}{l}\text { Quote: "No differences between the ES- } \\
\text { SL experimental group and the WL [wish- } \\
\text { list] control group were found for gender, } \\
\text { grade level and personal or important other } \\
\text { exposure to tsunami" }\end{array}$ \\
\hline $\begin{array}{l}\text { Incomplete outcome data (attrition bias) } \\
\text { Efficacy data }\end{array}$ & Low risk & Quote: "There were no missing data" \\
\hline $\begin{array}{l}\text { Incomplete outcome data (attrition bias) } \\
\text { Safety data (e.g. adverse events) }\end{array}$ & Unclear risk & $\begin{array}{l}\text { Comment: No safety outcomes/adverse } \\
\text { events reported in this study }\end{array}$ \\
\hline Protection against contamination & High risk & $\begin{array}{l}\text { Quote: "There may have been a spillover } \\
\text { effect since all the homeroom teachers par- } \\
\text { ticipated in the training" }\end{array}$ \\
\hline Reliable primary outcome measures & High risk & $\begin{array}{l}\text { Comment: UCLA PTSD index validated } \\
\text { in Sri Lankan population; BDI, hope and } \\
\text { DPS (somatic), and CDIS (functional im- } \\
\text { pairment) validated in other settings but } \\
\text { only have internal reliability scores in this } \\
\text { setting/study. No internal reliability data or } \\
\text { validation for other local scales/question- } \\
\text { naires. That is many tools have not been } \\
\text { validated in local context. All apart from } \\
\text { PTSD may not be reliable }\end{array}$ \\
\hline Selective reporting (reporting bias) & Unclear risk & $\begin{array}{l}\text { Comment: Not mentioned and not able to } \\
\text { find protocol }\end{array}$ \\
\hline Other bias & High risk & $\begin{array}{l}\text { Comment: High risk as clustering error not } \\
\text { adjusted for }\end{array}$ \\
\hline
\end{tabular}

Bolton 2003 C-RCT Uganda

Methods

Study design: Cluster randomised, parallel group, gender-stratified, controlled clinical trial (unit of randomisation: village; unit of analysis: individual)

Duration of study: February 2002 to July 2002; 6-month follow-up completed in January 2003

Participants

Country: Uganda

Income classification: Low income

Non-specialist health worker interventions for the care of mental, neurological and substance-abuse disorders in low- and middle- 
Bolton 2003 C-RCT Uganda (Continued)

Geographical scope: 30 villages in Rakkai Province and contiguous half of Masaka province in South West Uganda; rural

Healthcare setting: Community (community centres, churches, open spaces)

Mental health condition: Depression (DSM IV depression and sub-syndromal depression)

Population: Patients

- Age: Adults (> 18 years); mean age ranged from 27 years (SD 13.5) to 66 years (SD 10.5)

- Gender: Both (stratified for gender)

- Socioeconomic background: Not stated except for education (mean 4.7 years (SD 2.8) Intervention; 3.9 years (SD 3.3) control)

- Inclusion criteria: A 3-stage screening: Stage 1: (by trained local World Vision staff) identified 20 people from the selected 15 villages ( 8 for males and 5 for females) with depressive symptoms in local idiom; Stage 2: same interviews visited identified people, and if they admitted to having 1 of 2 locally approximate depressive conditions, informed consent was sought; Stage 3: eligibility expanded to include subsyndromal depression by DSM IV criteria (less 1 DSM criterion); screening for depression was done by 10 trained and experienced local World Vision staff using a composite instrument (Bolton 2004) consisting of the HSCL (to assess depressive symptomatology and diagnose DSM IV Major Depression (excluding criteria related to exclusion of medical causes and drug effects) using a previously validated algorithm), a locally developed culturally appropriate instrument to assess functional impairment (separately for women and for men), and ethnographically validated questions that assessed significant distress and duration of depression

- Exclusion criteria: Absence of symptoms of depression; age $<18$ years, unwillingness to meet weekly (additional criteria revised after screening commenced) people very different in age from the rest included in a village; and those appearing currently suicidal

Interventions

Stated purpose: To test the efficacy of a manual-based, time-limited group psychotherapeutic approach in relieving depressive symptoms and improving functioning; and to demonstrate that psychotherapy trials are feasible in Sub-Saharan Africa

INTERVENTION:

Name: Group Interpersonal Therapy for Uganda (IPT-G-U), 116 people (of 163 in 15 villages originally randomised, and 139 invited to participate; 107 completed intervention and follow-up);

Delivered by: NSHW/LHW

- Title/name of NSHW/OPHR and number: Group leader (9/10 who completed training)

- Selection: Local person of the same sex as the sex-segregated group; nonclinicians fluent in English and Luganda employed by World Vision

- Educational background: Completed high school (college-level)

- Training: Duration: 2 weeks intensive training. Trained by 2 faculty members of the New York State Psychiatric Institute (members of the team led by Myrna Weissman that developed ITP and the Group adaptation of IPT) assisted by a trained psychologist and an experienced group therapist employed by World Vision. Content of training: participating in local adaptations of the IPT manual; explanations of the treatment process and the contract; explanations of the role of group leaders in helping members in identifying problem areas and discussing locally acceptable variations to

Non-specialist health worker interventions for the care of mental, neurological and substance-abuse disorders in low- and middle- 
Bolton 2003 C-RCT Uganda (Continued)

absolute confidentiality; identification of, and agreement about, interpersonal problem areas likely to be encountered in group work according to the 4 domains in IPT; using the principles of IPT to identify personal problems and supporting each other to find options and in implementation. Format: didactic teaching and experiential group processes with role plays and group exercises

- Supervision: By local World Vision mental health professionals involved in training; format and duration not described

- Incentives/remuneration: Weekly payment for 16 weeks (amount not stated) Intervention details:

- Duration/frequency: 16 weekly 90 minute sessions

- Content of intervention: Group work led by group leader who first diagnoses depression; works with group member to identify problem areas associated with current symptoms and identify the 4 areas of interpersonal difficulties that served as triggers for the depression; weekly review of mood and encouragement of participant's description of events that could link to the mood; facilitation of support and solutions from group members.

CONTROL: Treatment as usual (treatment by local traditional healers, no treatment, or in rare cases, hospitalisation), 138 people (of 178 randomised in 15 villages and 145 invited to participate; 117 completed intervention and follow-up)

CO-INTERVENTIONS: No restrictions on additional interventions (utilisation and nature of any not described)

Patient: Screening: HSCL and local functional impairment scale. Outcomes: Prevalence of DSM IV Major Depression (excluding criteria related to exclusion of medical causes and drug effects (using Mollica DSM-IV algorithm for A, C and E criteria)*; HSCL mean scores; Functional Impairment scores (sex-specific 9-item questionnaire); depression in subgroups continuing informal group meetings between 2 weeks and 6 months versus subgroup not meeting after group intervention

Carer: Not applicable

Process/health worker outcomes: No direct outcomes reported: indirect outcomes are the results of the trial

Economic outcomes: Not reported

Time points: Initial assessment 2 weeks after intervention; follow-up at 6 months (*: primary outcomes)

Source of funding: Supported by World Vision, Washington, DC; Psychotherapy Core of the Child Intervention Research Center Columbia University (NIMH grant \#5P30 MH60570); Center for International Emergency Disaster and Refugee Studies, Johns Hopkins Bloomberg School of Public Health; Mellon Foundation

Notes on validation of instruments (screening and outcomes): All screening instruments and outcome measures locally adapted and validated in previous exercises and published; the HSCL scale consisted of 14 items, with 4 responses for each item related to the degree of distress due to a particular symptom (range 0-42 points); higher scores indicate more severe depression; the function scale consisted of 9 items with 5 responses for each item indicating degree of difficulty in completing the activity (range $0-36$ points) ; higher scores indicate more dysfunction

Additional information: IPT attendance was high: 54\% attended at least 14/16 sessions; $4 \%$ attended $\leq 10$ sessions

Handling the data: As per footnotes in data and analysis 
Bolton 2003 C-RCT Uganda (Continued)

Prospective trial registration number: Not prospectively registered

Risk of bias

$\begin{array}{lll}\text { Bias } & \text { Authors' judgement } & \text { Support for judgement }\end{array}$

Random sequence generation (selection Low risk bias)

Quote from report: "Random assignment was performed by enumerating the villages and using a random number table to determine study allocation"

Comment: Cluster randomisation of 30 villages to 15 in each arm was done using a random number table; the 30 villages of 154 eligible villages were chosen for a previous prevalence study (Bolton 2002, unpublished) that used weighted random sampling based on government census data

Allocation concealment (selection bias) Unclear risk

Quote from report: "Each list began with those who met the original diagnostic criteria, followed by those who fell short by a single criterion, in order of decreasing depression score. Interviewers visited each person in the order they appeared on the list. The interviewer re-read the consent form, advised the person about the study group to which their village had been allocated, and asked them to confirm their willingness to continue in the study. Interviewers continued down the list until they had at least 8 participants (at which point they did not contact the remainder of the list) or until they reached the end of the list"

Comment: Allocation of participants was not concealed though cluster randomisation of villages was the unit of randomisation; the eligibility criteria were modified to exclude people whose age varied widely from the rest of those selected in each village to ensure better outcomes with group IPT (based on previous experiences)

Blinding of participants and personnel Low risk (performance bias)

All outcomes

Comment: Participants and personnel were not blinded; however, cluster randomisation would ensure minimal risk of performance bias since villages where intervention was given were separate from villages randomised to usual care

Non-specialist health worker interventions for the care of mental, neurological and substance-abuse disorders in low- and middle- 
Bolton 2003 C-RCT Uganda (Continued)

Blinding of outcome assessment (detection Low risk bias)

objective outcomes

Blinding of outcome assessment (detection Low risk bias)

subjective outcomes
Comment: There were no objective outcomes
Quote from report: "The baseline assessments were conducted in the villages, with the randomisation of village groups to intervention or control or control status done afterwards to ensure that interviewers were not aware of participant trial status at baseline. In an effort to keep interviewers unaware of the participants' intervention status, the post-intervention and 6-month follow-up assessments were conducted at a centrally located community centre. At these assessments, trial participants were transferred from their villages and were asked not to divulge either their village of origin or their treatment assignment status. To reduce measurement error that might have arisen from different interviewing styles, study participants were interviewed by the same interviewer at each stage of the study"

Comment: The period from recruitment to first assessment was 18 weeks and to second assessment was a further 6 months. There is a possibility that the recruiter (who did not administer the intervention) may have guessed allocation for the first assessment in a few instances but this unlikely to have altered results significantly given the magnitude of the differences in results between groups

Baseline outcome measurements similar Low risk
Quote from 6-month follow-up report: "At baseline $86 \%$ of participants in the intervention group met the modified diagnostic criteria for major depressive disorder and $94 \%$ of those in the control group met these criteria (prevalence difference was not significant)"

Quote from primary report: "However, there was a significant difference in the proportions who met the original depression diagnostic criteria, both among those who completed the study and all those on the original lists of eligible participants (TA- 
Bolton 2003 C-RCT Uganda (Continued)

\begin{tabular}{|c|c|c|}
\hline & & $\begin{array}{l}\text { BLE 1). (Tests for differences in baseline } \\
\text { characteristics were performed using stan- } \\
\text { dard significance tests and were not ad- } \\
\text { justed for cluster effects. However, because } \\
\text { we found a positive correlation between } \\
\text { clusters, adjusting for cluster effects would } \\
\text { tend to reduce variance and cause group } \\
\text { differences to be even less significant than } \\
\text { the values reported herein)" } \\
\text { Comment: Discrepancy in interpretation } \\
\text { of baseline differences in the } 2 \text { reports; re- } \\
\text { sults adjusted for clustering and for base- } \\
\text { line outcome differences }\end{array}$ \\
\hline Baseline characteristics similar? & Low risk & $\begin{array}{l}\text { Comment: No differences in age or educa- } \\
\text { tion, or symptoms duration }\end{array}$ \\
\hline $\begin{array}{l}\text { Incomplete outcome data (attrition bias) } \\
\text { Efficacy data }\end{array}$ & Low risk & $\begin{array}{l}\text { Quote from 6-month follow-up report: } \\
\text { "Six months after the post-intervention } \\
103(96 \%) \text { of the } 107 \text { participants in the } \\
\text { intervention group who completed the trial } \\
\text { and } 113(97 \%) \text { of the } 117 \text { completed the } \\
\text { trial and } 113(97 \%) \text { of the } 117 \text { controls } \\
\text { were reassessed" } \\
\text { Comment: Attrition was high in both } \\
\text { groups due to the } 3 \text {-stage screening process; } \\
\text { however, } 116 / 163 \text { eligible and randomised } \\
\text { to IPT consented to participate; } 132 / 178 \\
\text { randomised to control consented to par- } \\
\text { ticipate) results did not differ in completer } \\
\text { analyses and in } 2 \text { sets of ITT analyses }\end{array}$ \\
\hline $\begin{array}{l}\text { Incomplete outcome data (attrition bias) } \\
\text { Safety data (e.g. adverse events) }\end{array}$ & Unclear risk & Comment: No safety data reported \\
\hline Protection against contamination & Low risk & $\begin{array}{l}\text { Comment: Cluster randomisation of inter- } \\
\text { vention arms precluded contamination }\end{array}$ \\
\hline Reliable primary outcome measures & Low risk & $\begin{array}{l}\text { Comment: Culturally adapted and vali- } \\
\text { dated measures used }\end{array}$ \\
\hline Selective reporting (reporting bias) & Low risk & $\begin{array}{l}\text { Comment: Trial not prospectively regis- } \\
\text { tered, but all pre-stated outcomes were re- } \\
\text { ported }\end{array}$ \\
\hline Other bias & Low risk & Comment: No other biases were detected \\
\hline
\end{tabular}

Non-specialist health worker interventions for the care of mental, neurological and substance-abuse disorders in low- and middle- 
Participants

Interventions
Country: Uganda

Income classification: Low income

Geographical scope: 2 camps (Awer and Unyama) for internally displaced people near Gulu town in northern Uganda; semi-rural; > 20,000 inhabitants each; minimal socioeconomic facilities

Healthcare setting: Group meetings

Mental health condition: Anxiety, depression, conduct problems and some PTSD symptoms

Population: Adolescents

- Age: 14-17 years

- Gender: Both

- Socioeconomic background: Acholi youth from socioeconomically deprived backgrounds living in camps for displaced youths

- Inclusion criteria: Age 14-17 years, scored > 32 on depression scale; $>0$ on the function scale; had symptoms $>1$ month; camp resident for previous month

- Exclusion criteria: Inability to be interviewed due to physical or cognitive difficulties, severe suicidal ideation or behaviour

Stated purpose: To assess effect of locally feasible interventions on depression, anxiety and conduct problem symptoms among adolescent survivors of war and displacement in northern Uganda

\section{INTERVENTION 1:}

Name: G-IPT (psychotherapy-based intervention); 105 people randomised (103 enrolled)

Delivered by: LHW

- Title/name of NSHW/OPHR and number: G-IPT facilitator; 12 people

- Selection: Same gender as groups; local Acholi, spoke both English and the local language Luo, and had minimal previous mental health intervention experience

- Educational background: Not stated

- Training: 2 weeks of intensive training by Columbia University faculty using a locally adapted G-IPT treatment manual (unpublished)

- Supervision: Weekly direct supervision by World Vision Uganda staff and weekly phone supervision of written case notes for adherence to study protocol with study personnel in the US; supervisors had previous IPT experience and received weekly telephone supervision with US trainer

- Incentives/remuneration: Not stated Intervention details:

- Duration/frequency: 16 weekly group meetings lasting 90-180 minutes (preceded by 1-2 individual meetings to explain treatment and draw up a treatment plan)

- Content of intervention: 6-8 same sex groups of adolescent Acholi youths per facilitator; manualised G-IPT based on the concept that depressive episodes are related to difficulties in 1 or more of 4 interpersonal areas: grief, interpersonal disputes, role transitions and interpersonal deficits. The focus is on improving depressive symptoms and functioning by identifying the interpersonal problems most relevant to the current depression and assisting the individual in building skills to manage those problems. 
"The flow and organization of the IPT-G sessions was organized in three phases: The initial phase (corresponding roughly to sessions 1-4) focused on building rapport, setting personal treatment goals and learning to identify mood states. The middle or working phase (corresponding roughly to sessions 5-12) involved exploring major issues related to grief, transitions, disputes and building interpersonal skills and connections among group members. The final, closure phase (corresponding roughly to sessions 13-16) was dedicated to preparing for the end of the IPT-G intervention and the close of formal group meetings. During this final phase, participants in the IPT-G intervention groups were encouraged to discuss how they might continue to provide support and connection to one another after the formal ending of the group (if this topic arose naturally)"

\section{INTERVENTION 2:}

Name: Creative play (activity based intervention); 105 people randomised (99 enrolled) Delivered by: NSHW

- Title/name of NSHW/OPHR and number: Creative play facilitator, 2 people

- Selection: War Child Holland staff (selection not described)

- Educational background: Not stated

- Training (contents, duration and by whom): Not stated

- Supervision: Weekly or bi-monthly supervision by War Child Holland psychosocial specialist who reported bimonthly by telephone with US study personnel

- Incentives/remuneration: Not stated Intervention details:

- Duration/frequency: 16 weekly group meetings lasting 90-80 minutes (preceded by 1-2 individual sessions where treatment was explained)

- Content of intervention: 4 groups (2 per camp) of 25-30 adolescents of both genders per group; based on War Child Holland manual adapted for adolescents with depression; for war-affected youth, based on the premise that a youth's resilience is strengthened by verbal and nonverbal expression of thoughts and feelings through ageappropriate creative activities such as, songs, art, role plays, music, sports, games and debates. Each activity served specific psychosocial goals and after the activities, facilitators led discussions on what the participants and facilitators thought about the activity as a means of drawing real-life lessons. "Sessions 1-4 focused on getting to know one another and setting the group rules. Sessions 5-12 were more in-depth and focused on issues in the group, in particular the interrelationships between the adolescents in the group and developing opportunities for self-expression. Sessions 1315 were dedicated to closure and preparing for a closing inter-generational event. The final CP [creative play] session (session 16) was an inter-generational event where caregivers were invited to attend along with the young people. This final session at each camp was hosted by one of the young people serving as Master of Ceremonies and facilitated by the participating young people themselves. The youth facilitated some of their CP activities for the family members who attended"

CONTROL: Wait-list controls, 104 people (102 enrolled); received no specific intervention but were free to access any services or programmes that they would have received in the absence of the study

CO-INTERVENTIONS: Not stated

Outcomes
Patient: (Locally developed) Acholi Psychosocial Assessment Instrument depression symptom scale scores*; improvements in anxiety symptoms, conduct problems and functioning on the APAI (minimum score for clinically significant symptoms on the APAI = 32; maximum score 105; higher scores $=$ more symptoms); functional impairment 
Bolton 2007 RCT Uganda (Continued)

scores: (range 0-36 for girls (9 items) and 0-20 for boys (5 items) with higher scores representing a greater degree of impairment); qualitative interviews $₫$

Carer: Not applicable

Process/health worker outcomes: Not assessed

Economic outcomes: Not reported

${ }^{*}$ : primary outcomes; $\$$ : outcomes that we have not reported in this review)

Time points: Baseline, 2 weeks to 1 month of completing interventions

Notes

Source of funding: World Vision and War Child Holland; the Ruth and David Levine Foundation

Notes on validation of instruments (screening and outcomes): APAI locally developed: Scale reliability and validity were evaluated for a sub-sample (178 people) of the adolescents interviewed for trial eligibility (667 people). Cronbach alpha (a measure of internal reliability) was 0.92 . Concurrent validity established by comparing depression symptoms scale scores between cases and non-cases identified by carer-youth pairs and threshold scale score of 32 identified (1 SD below mean score for cases); Test-re-test reliability for the depression symptom scale was 0.84 (in 30 of convenience sub-sample re-administered the APAI after 5 days)

Additional information (e.g. provide by authors, existence of a published study protocol): None

Handling the data: As per footnotes in data and analysis

Prospective trial registration number: Not prospectively registered

Risk of bias

Bias

Authors' judgement

Support for judgement

Random sequence generation (selection Low risk bias)

Quote: "Eligible youths were then randomly assigned to a study group. Random allocation was done by computerized generation of a random number between 1 and 400 for each eligible participant, ordering them by number and assigning the first third to IPT-G, the second third to $\mathrm{CP}$ and the final third to the wait-control group." "Of the total sample screened $(\mathrm{N}=667), 300$ individuals met original inclusion criteria, were stratified by camp and sex, and randomised to a study group. Of these 300, 290 were enrolled in the study. Of the remaining 10 individuals, 1 was already involved in the $\mathrm{CP}$ program in a neighbouring camp, 4 could not be located, and 5 refused. To meet our original sample size (300), we randomised an additional 38 individuals whose depression symptom scores were between 28 and 31 points. This relaxation of a trial eligibility

Non-specialist health worker interventions for the care of mental, neurological and substance-abuse disorders in low- and middle- 
Bolton 2007 RCT Uganda (Continued)

\begin{tabular}{|c|c|c|}
\hline & & $\begin{array}{l}\text { criterion is acceptable when study design } \\
\text { consequences are minimal. } 19 \text { The first } 14 \\
\text { individuals all consented and therefore, the } \\
\text { remainder were not approached" } \\
\text { Comment: Very few people are in this non- } \\
\text { randomised group. unlikely to make any } \\
\text { difference to the outcomes }\end{array}$ \\
\hline Allocation concealment (selection bias) & Unclear risk & $\begin{array}{l}\text { Quote: "Eligible youths were then ran- } \\
\text { domly assigned to a study group. Random } \\
\text { allocation was done by computerized gen- } \\
\text { eration of a random number between } 1 \text { and } \\
400 \text { for each eligible participant, ordering } \\
\text { them by number and assigning the first } \\
\text { third to IPT-G, the second third to CP and } \\
\text { the final third to the wait-control group" } \\
\text { Comment: Not specified who allocated } \\
\text { them and whether the allocation was con- } \\
\text { cealed to them; however, there were no ma- } \\
\text { jor differences in baseline prognostic vari- } \\
\text { ables or outcome measures }\end{array}$ \\
\hline
\end{tabular}

Blinding of participants and personnel Low risk

Comment: No blinding of participants or (performance bias)

personnel but unlikely to affect outcome

All outcomes

Blinding of outcome assessment (detection Low risk

Comment: No objective outcomes

bias)

objective outcomes

Blinding of outcome assessment (detection Low risk bias)

subjective outcomes

Quote from report: "Interviewers were blinded to interviewees' intervention status"

Comment: Outcome assessor were blinded to allocation

Baseline outcome measurements similar Low risk

Baseline characteristics similar? High risk

Comment: Except for a slightly older age among wait-list controls, the 3 study groups did not vary significantly, but age not adjusted for in statistical analysis

Incomplete outcome data (attrition bias) Low risk Efficacy data

Quote: “The study instrument was re-administered to $282(90 \%)$ of the original 314 participants within 1 month of completing both interventions"

Comment: $304 / 314$ enrolled and $261(82$ $+89+90)$, i.e. $83 \%$ completed analysis

Non-specialist health worker interventions for the care of mental, neurological and substance-abuse disorders in low- and middle- 
Bolton 2007 RCT Uganda (Continued)

\begin{tabular}{|c|c|c|}
\hline $\begin{array}{l}\text { Incomplete outcome data (attrition bias) } \\
\text { Safety data (e.g. adverse events) }\end{array}$ & Unclear risk & $\begin{array}{l}\text { Comment: None reported or mentioned } \\
\text { in the paper }\end{array}$ \\
\hline Protection against contamination & High risk & $\begin{array}{l}\text { Comment: Only } 2 \text { camps chosen with } \\
\text { refugee settings. Likely to be contamina- } \\
\text { tion (no clustering) }\end{array}$ \\
\hline Reliable primary outcome measures & Low risk & $\begin{array}{l}\text { Comment: Acceptable scores for APAI on } \\
\text { criterion and concurrent validity; internal } \\
\text { reliability and test-re-test reliability }\end{array}$ \\
\hline Selective reporting (reporting bias) & Low risk & $\begin{array}{l}\text { Comment: All outcomes found over both } \\
\text { report and paper though conduct not re- } \\
\text { ported in published article. These were } \\
\text { available when asked for from author }\end{array}$ \\
\hline Other bias & Low risk & Comment: None detected \\
\hline
\end{tabular}

\section{Brown 2009 CBA Rwanda}

\begin{tabular}{|c|c|}
\hline Methods & $\begin{array}{l}\text { Study design: CBA study } \\
\text { Duration of study: Late } 2003-2006\end{array}$ \\
\hline Participants & $\begin{array}{l}\text { Country: Rwanda } \\
\text { Income classification: Low income } \\
\text { Geographical scope: Gikongoro province in rural south-western Rwanda, } 1 \text { of the } \\
\text { poorest regions of the country where World Vision Rwanda had begun a basic needs } \\
\text { programme (providing range of goods and services) for youth-headed households in } \\
2001 \\
\text { Healthcare setting: House-hold level } \\
\text { Mental health condition: Grief, depression } \\
\text { Population: Youth who head households (because of the AIDS pandemic and genocide) } \\
\text { - Age: } 12-24 \text { years } \\
\text { - Gender: Both } \\
\text { - Socioeconomic background: Poor, about } 50 \% \text { have }<3 \text { years' education } \\
\text { - Inclusion criteria: Age } 12-24 \text { years, had to be heads of their households } \\
\text { - Exclusion criteria: Not mentioned }\end{array}$ \\
\hline
\end{tabular}

Interventions

Stated purpose: Study tested a model of adult mentorship and support to improve psychosocial outcomes among youth-headed households

\section{INTERVENTION:}

Name: Mentoring programme

Delivered by:

- Title/name of NSHW/OPHR and number: 156 adult mentor volunteers $(60 \%$ male)

- Selection: Through nomination from youth who serve as heads of households and other trusted community members in the project area

- Educational background: Not specified 
Carer: Not relevant

Process/health worker outcomes: None

Economic outcomes: None

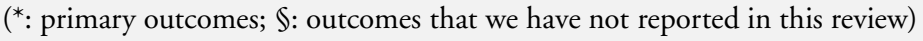

Time points: Baseline and follow-up after 2 years

\begin{tabular}{l|l|l}
\hline Bias & Authors' judgement & Support for judgement \\
\hline $\begin{array}{l}\text { Random sequence generation (selection } \\
\text { bias) }\end{array}$ & High risk & $\begin{array}{l}\text { Comment: Non-random method was } \\
\text { used. It was a CBA study }\end{array}$ \\
\hline Allocation concealment (selection bias) & High risk & Comment: There is no randomisation of \\
& & $\begin{array}{l}\text { allocation of districts or allocation of con- } \\
\text { cealment. These were chosen according to } \\
\text { those delivering basic needs programme } \\
\text { (convenience). and in discussion it men- } \\
\text { tions that } 1 \text { of the districts was closer to } \\
\text { World Vision headquarters so may account } \\
\text { for the difference in baseline outcomes and } \\
\text { characteristics }\end{array}$
\end{tabular}


Brown 2009 CBA Rwanda (Continued)

Blinding of participants and personnel Low risk (performance bias)

All outcomes
Comment: It is not possible to blind participant or personnel for such an intervention and outcome unlikely to be affected by blinding

Comment: No objective outcomes

Quote: "Even though the intervention group was worse off on many key variables relative to the comparison group at baseline, for most of the outcomes the intervention group improved, whereas the comparison group remained unchanged or in some cases worsened"

Comment: There is insufficient information on who the researchers were who assessed the outcomes

Baseline outcome measurements similar Low risk
Quote: "The intervention and control groups were not equivalent at baseline with respect to key background and outcome variables: the intervention group was significantly older (21 years vs. 20 years, $\mathrm{p}<$ 0.001 ) and significantly worse off than the comparison group on many outcomes, including having less adult support, greater marginalisation and higher levels of grief and depression. These differences may be explained partially by the higher levels of parental loss due to genocide also found in these areas. However, the intervention group had significantly higher levels of education and asset ownership and had received a greater number of services from WVR than the comparison group, a difference which may be attributable in part to the fact one of the two districts within the intervention group was in closer proximity to a main town and WVR offices"

Comment: These differences were controlled for in regression analyses
Baseline characteristics similar? Low risk
Quote: "The intervention and control groups were not equivalent at baseline with respect to key background and outcome variables: the intervention group was significantly older $(21$ years vs. 20 years, $\mathrm{p}<$ 
Incomplete outcome data (attrition bias) High risk Efficacy data
0.001 ) and significantly worse off than the comparison group on many outcomes, including having less adult support, greater marginalisation and higher levels of grief and depression. These differences may be explained partially by the higher levels of parental loss due to genocide also found in these areas. However, the intervention group had significantly higher levels of education and asset ownership and had received a greater number of services from WVR than the comparison group, a difference which may be attributable in part to the fact one of the two districts within the intervention group was in closer proximity to a main town and WVR offices"

Comment: These differences were controlled for in regression analyses

Quote: Attendance "was not always possible, due to changes in household formation associated with marriage and migration"

Comment: Of 692 included in first survey, 593 were in the follow up sample. This is more than $20 \%$ dropout rate

Incomplete outcome data (attrition bias) Unclear risk

Comment: No adverse outcomes reported Safety data (e.g. adverse events)

$\begin{array}{ll}\text { Protection against contamination Low risk } & \text { Lon }\end{array}$

Comment: Allocation by districts so unlikely to be any contamination

Reliable primary outcome measures High risk

Comment: The outcome is obtained from key psychosocial outcomes measuring scale: 1 . perceptions of adult support, 2. marginalisation, 3. grief and 4. symptoms of depression. Alpha level over 0.65 considered acceptable. Grief alpha: 0.66; adult support; 0.85; marginalisation: 0.77; depression: 0.86 . There is moderately good inter-rater reliability (except for grief) for these scores though they were not validated

Selective reporting (reporting bias) Low risk
Comment: Every aspect was reported mentioned in methods was reported. There is no access to a study protocol to check 
Brown 2009 CBA Rwanda (Continued)

Other bias

High risk
Comment: The participants received a token incentive after the interview. This could have made much difference to the outcomes

Chen 2000 RCT Taiwan

\begin{tabular}{ll} 
Methods & $\begin{array}{l}\text { Study design: RCT } \\
\text { Duration of study: Not mentioned }\end{array}$ \\
\hline Participants & $\begin{array}{l}\text { Country: Taiwan, China } \\
\text { Income classification: Middle income } \\
\text { Geographical scope: Urban } \\
\text { Healthcare setting: Postnatal wards } \\
\text { Mental health condition: Postnatal depression } \\
\text { Population: Mothers at days } 2 \text { or } 3 \text { post-partum } \\
\text { - Age: Mothers: > 18 years } \\
\text { - Gender: Female } \\
\text { - Socioeconomic background: Half had senior high school qualification, just over } \\
\text { half were housewives, more or less equal numbers of high, middle and low social classes } \\
\text { - Inclusion criteria: } 1 .>18 \text { years of age; } 2 \text { survival of the infant; } 3 \text {. at least a junior } \\
\text { high school education; and } 4 \text {. BDI score above the depression cut-off point of } 9 / 10 \\
\text { - Exclusion criteria: Not specified }\end{array}$ \\
\hline
\end{tabular}

Interventions

Stated purpose: To investigate the psychosocial effects of a support group programme on postnatally distressed mothers in Taiwan

INTERVENTION:

Name: Support group intervention

Delivered by (NSHW or OPHR and title)

- Title/name of NSHW/OPHR and number: Registered nurse was the group leader

- Selection: Not specified

- Educational background: Trained nurse

- Training: Not specified

- Supervision: Not specified

- Incentives/remuneration: Not specified

Intervention details:

- Duration/frequency: Groups met for 4 weekly sessions, each of 1.5-2 hours' duration, held during the day

- Content of intervention (by types of health worker and per patient/carers: The primary goal of the group was to bring women into contact with other women having similar experiences, so they could share problems and conflicts and talk about solutions. Each week a different topic area was given primary emphasis, although if other issues arose, these were also discussed. If distressed mothers became engaged in another topic that had not been planned, the scheduled topic was deferred for 1 week. The 4 sessions comprised discussions that centred around transition to motherhood, postnatal stress management, communication skills and life planning. Session 1: transition to motherhood; Session 2: postnatal stress management; Session 3: 
Chen 2000 RCT Taiwan (Continued)

communication skills; session 4: life planning. A crèche was provided, and drinks and biscuits were offered to help make the sessions as friendly and relaxed as possible. There were no fees for attending these support group meetings

CONTROL: Usual care: the control group did not receive a support group intervention (58 women)

\section{CO-INTERVENTIONS: None}

Outcomes

Patient: Taiwanese BDI*; Taiwanese Perceived Stress Scale \$; Taiwanese Interpersonal Support Evaluation List short form $\$$ (to assess the availability of support along 4 dimensions: tangible aid, appraisal, self esteem and belonging); Coopersmith's SEI $₫$ (to measure evaluative attitudes toward the self in social, academic, family and personal areas of experience)

Carer: none

Process/health worker outcomes: none

Economic outcomes: none

(*: primary outcomes; $\$$ : outcomes that we have not reported in this review)

Time points: baseline; 4 weeks

$\begin{array}{ll}\text { Notes } & \text { Source of funding: National Science Council, Taipei, Taiwan } \\ \text { Notes on validation of instruments (screening and outcomes): Yes all valid in Tai- } \\ \text { wanese settings } \\ \text { Additional information (e.g. provide by authors, existence of a published study } \\ \text { protocol): None } \\ \text { Handling the data: As per footnotes in data and analysis } \\ \text { Prospective trial registration number: None }\end{array}$

\section{Risk of bias}

\begin{tabular}{|c|c|c|}
\hline Bias & Authors' judgement & Support for judgement \\
\hline $\begin{array}{l}\text { Random sequence generation (selection } \\
\text { bias) }\end{array}$ & Unclear risk & $\begin{array}{l}\text { Quote: "The women who met the inclu- } \\
\text { sion criteria were randomly assigned to ei- } \\
\text { ther the support or control groups" } \\
\text { Comment: Not mentioned about se- } \\
\text { quence generation }\end{array}$ \\
\hline
\end{tabular}

$\begin{array}{lll}\text { Allocation concealment (selection bias) Unclear risk } & \text { Comment: Not mentioned }\end{array}$

Blinding of participants and personnel Low risk (performance bias)

Comment: Not blinded but unlikely to af-

All outcomes

Blinding of outcome assessment (detection Low risk bias)

objective outcomes

Blinding of outcome assessment (detection High risk bias)

subjective outcomes

Non-specialist health worker interventions for the care of mental, neurological and substance-abuse disorders in low- and middle- 
Chen 2000 RCT Taiwan (Continued)

\begin{tabular}{|c|c|c|}
\hline Baseline outcome measurements similar & Low risk & Comment: Baseline characteristics similar \\
\hline Baseline characteristics similar? & Low risk & $\begin{array}{l}\text { Quote: "There were no significant differ- } \\
\text { ences in the demographic characteristics of } \\
\text { the experimental and control groups" }\end{array}$ \\
\hline $\begin{array}{l}\text { Incomplete outcome data (attrition bias) } \\
\text { Efficacy data }\end{array}$ & High risk & $\begin{array}{l}\text { Comment: Many people did not consent } \\
\text { to participate (consent was done after ran- } \\
\text { domisation) so high dropout rate after ran- } \\
\text { domisation }\end{array}$ \\
\hline $\begin{array}{l}\text { Incomplete outcome data (attrition bias) } \\
\text { Safety data (e.g. adverse events) }\end{array}$ & Unclear risk & $\begin{array}{l}\text { Comment: No adverse outcomes men- } \\
\text { tioned }\end{array}$ \\
\hline Protection against contamination & Unclear risk & $\begin{array}{l}\text { Comment: Women from } 2 \text { postnatal } \\
\text { wards. It is possible that the control and in- } \\
\text { tervention groups would have had exposure } \\
\text { to each other (e.g. if they had peer meet- } \\
\text { ings, etc.) }\end{array}$ \\
\hline Reliable primary outcome measures & Low risk & Comment: All tools validated \\
\hline Selective reporting (reporting bias) & Unclear risk & Comment: No access to protocol \\
\hline Other bias & Low risk & Comment: None detected \\
\hline
\end{tabular}

\section{Dias 2008 RCT India}

\begin{tabular}{l|l}
\hline Methods & $\begin{array}{l}\text { Study design: RCT } \\
\text { Duration of study: Unknown }\end{array}$ \\
\hline Participants & $\begin{array}{l}\text { Country: India } \\
\text { Income classification: Lower middle } \\
\text { Geographical scope: Taluka semi-urban } \\
\text { Healthcare setting: Home-based care } \\
\text { Mental health condition: Dementia } \\
\text { Population: Patient and carer dyads } \\
\text { - Age: Carers around 53 years; patients with dementia around } 78 \text { years } \\
\text { - Gender: Both } \\
\text { - Socioeconomic background: 40\% of patients with dementia and 20\% of carers } \\
\text { had below primary education. Most (90\%) unable to afford paid help } \\
\text { - Inclusion criteria: Using Clinical Dementia Rating scale: mild to moderate } \\
\text { dementia; carers: identified person by the family } \\
\text { - Exclusion criteria: Clinical Dementia Rating scale: severe dementia or severe co- } \\
\text { morbid physical health condition }\end{array}$ \\
\hline
\end{tabular}

Non-specialist health worker interventions for the care of mental, neurological and substance-abuse disorders in low- and middle- 
Dias 2008 RCT India (Continued)

Interventions
Stated purpose: Testing the effectiveness of the 10/66 intervention in reducing carer burden, promoting carer mental health and reducing behaviour problems in elderly people with dementia

INTERVENTION:

Name: 10/66 Flexible stepped-care brief carer intervention

Delivered by:

- Title/name of NSHW/OPHR and number: 4 HCAs (2 in each taluks) and 1

LHC (shared by both taluks)

- Selection: HCA: knowledge of local language, being literate, motivated to involve in community care of older people. LC: She was part of the intervention team/authors; member of the Dementia Society in Goa

- Educational background: HCA: passed higher secondary school, LC: not specified

- Training: HCA: intensive training module over 1 week developed/adapted to local settings. Trained in key skills including listening and counselling skills, bereavement counselling, stress management and health advice for common health problems. Trained by author (geriatrician/epidemiologist) and LHC. LHC: not specified

- Supervision: for HCA: meetings every 2 weeks with psychiatrist and LC. The HCA would meet the psychiatrist twice a month to give update on person with dementia, especially if they were taking medication. In addition, met with the LC every 2 weeks to share experiences, support one another and problem solve difficult situations. LC: supervised by the psychiatrists

- Incentives/remuneration: LC: Rs 5000/month. HCA: not specified; psychiatrist remunerated Rs 3000/month for monitoring/supervising LCs

Intervention details:

- Duration/frequency: Home visits at least every 2 weeks for 6 months

- Content of intervention (by types of health worker and per patient/carers:

HCAs: Intervention for carers: psychoeducation plus follow-up and some counselling skills. Patients or carers (or both) had follow-up with the psychiatrist and patients may be prescribed medication

CONTROL: Control arm dyads received only education and information regarding dementia and were then placed on a waiting list to receive the intervention after 6 months CO-INTERVENTIONS: Both intervention and control were free to utilise existing health services during this time

Outcomes

Patient: Severity of behavioural problems (NPI-S); functional ability of the subject (Everyday Abilities Scales for India)

Carer: Carer mental health (GHQ score)*; carer perceived burden (ZBS); carer distress due to problem behaviours (NPI-D)

Process/health worker outcomes: Process indicators: mean number of visits by HCA, visits by psychiatrists, use of medication not reported

Economic outcomes: Protocol mentions primary outcome: cost of illness but not reported

${ }^{*}$ : primary outcomes of study)

Time points: 3 and 6 months after baseline 
Dias 2008 RCT India (Continued)

\begin{tabular}{|c|c|c|}
\hline Notes & \multicolumn{2}{|c|}{$\begin{array}{l}\text { Source of funding: WHO } \\
\text { Notes on validation of instruments (screening and outcomes): All were validated } \\
\text { (Dias 2004) } \\
\text { Additional information: Authors provided supplementary information on supervision, } \\
\text { remuneration and other elements. We had access to the study protocol } \\
\text { Handling the data: As per footnotes in data and analysis } \\
\text { Prospective trial registration number: NCT00479271 }\end{array}$} \\
\hline \multicolumn{3}{|l|}{ Risk of bias } \\
\hline Bias & Authors' judgement & Support for judgement \\
\hline $\begin{array}{l}\text { Random sequence generation (selection } \\
\text { bias) }\end{array}$ & Low risk & $\begin{array}{l}\text { Quote: "Randomization of dyads compris- } \\
\text { ing the person with dementia and their } \\
\text { principal caregiver was carried out by an } \\
\text { independent person, based on simple ran- } \\
\text { dom number tables, either to the interven- } \\
\text { tion or waiting list group" } \\
\text { Comment: It was carried out using simple } \\
\text { random number tables }\end{array}$ \\
\hline Allocation concealment (selection bias) & Low risk & $\begin{array}{l}\text { Comment: The allocation was done by an } \\
\text { 'independent person' }\end{array}$ \\
\hline $\begin{array}{l}\text { Blinding of participants and personnel } \\
\text { (performance bias) } \\
\text { All outcomes }\end{array}$ & Low risk & $\begin{array}{l}\text { Patients and carers recommended by the } \\
\text { family and personnel knew who was allo- } \\
\text { cated to the intervention. The personnel } \\
\text { did not take part in the measuring the out- } \\
\text { come so it does not affect the outcome }\end{array}$ \\
\hline
\end{tabular}

Blinding of outcome assessment (detection Low risk bias)

objective outcomes

Blinding of outcome assessment (detection Low risk bias)

subjective outcomes
Comment: Mortality is an objective outcome and was reported completely. Agree with low risk assessment

Quote: "Outcome evaluations were carried out by researchers who were masked to the allocation status until the end of the project. We attempted to blind outcome evaluations by ensuring that allocation status was kept in a separate office from the outcome evaluation teams. We had also instructed the families not to divulge information on the visits by the Home Care Advisor. However, we anticipated that some unmasking would occur because both the intervention and outcome evaluations were home-based. In order to evaluate the masking process, researchers were asked to guess 
Dias 2008 RCT India (Continued)

\begin{tabular}{|c|c|c|}
\hline & & $\begin{array}{l}\text { the intervention status. Another limitation } \\
\text { in trials of this nature is that the researchers } \\
\text { did, during the course of their outcome } \\
\text { evaluation, correctly guess the allocation } \\
\text { status in nearly two-thirds of individuals } \\
\text { because of the information on health care } \\
\text { use which typically led some care-givers to } \\
\text { share contacts with the intervention team" } \\
\text { Comment: Authors have mentioned the } \\
\text { possibility of unmasking and measure they } \\
\text { took to minimise this }\end{array}$ \\
\hline Baseline outcome measurements similar & Low risk & $\begin{array}{l}\text { Comment: There were differences in out- } \\
\text { come measures at baseline: mean GHQ } \\
\text { scores was different - higher in the interven- } \\
\text { tion group (Table } 2 \text { ). This difference was } \\
\text { adjusted for in subsequent analyses }\end{array}$ \\
\hline Baseline characteristics similar? & Low risk & $\begin{array}{l}\text { Comment: There were no baseline differ- } \\
\text { ences in SES and psychiatric co-morbid- } \\
\text { ity. Outcome measures at baseline were also } \\
\text { similar }\end{array}$ \\
\hline $\begin{array}{l}\text { Incomplete outcome data (attrition bias) } \\
\text { Efficacy data }\end{array}$ & Low risk & $\begin{array}{l}\text { Comment: There was }>20 \% \text { dropout rate } \\
\text { (only } 59 \text { remain at follow-up compared } \\
\text { with } 81 \text { randomised) but this was a small } \\
\text { sample size. The most common causes of } \\
\text { death were stroke ( } 4 \text { people), pneumonia } \\
\text { ( } 4 \text { people), myocardial infarction ( } 3 \text { peo- } \\
\text { ple) and septicaemia ( } 2 \text { people). } 2 \text { families } \\
\text { moved out of the study area and } 2 \text { refused } \\
\text { to continue with the trial. However, there } \\
\text { was no significant difference in the baseline } \\
\text { characteristics of those who died or were } \\
\text { alive to the end of the trial (P value = } 0.05 \\
\text { for GHQ, NPI-S, NPI-D, Everyday Abili- } \\
\text { ties Scales for India and ZBS scores) }\end{array}$ \\
\hline $\begin{array}{l}\text { Incomplete outcome data (attrition bias) } \\
\text { Safety data (e.g. adverse events) }\end{array}$ & Unclear risk & $\begin{array}{l}\text { Comment: The deaths were reported but } \\
\text { the intervention adverse effects on the car- } \\
\text { ers not specified }\end{array}$ \\
\hline Protection against contamination & Unclear risk & $\begin{array}{l}\text { Comment: There was insufficient infor- } \\
\text { mation about how close the intervention } \\
\text { group and control group were placed (e. } \\
\text { g. where they in same village or necessarily } \\
\text { mean to share the details of dementia care } \\
\text { in Goa (cultural view) - (contacted author } \\
\text { for this) }\end{array}$ \\
\hline
\end{tabular}

Non-specialist health worker interventions for the care of mental, neurological and substance-abuse disorders in low- and middle- 
Dias 2008 RCT India (Continued)

\begin{tabular}{|c|c|c|}
\hline Reliable primary outcome measures & High risk & $\begin{array}{l}\text { Quote: "Limitation in trials of this nature } \\
\text { is that the researchers did, during the course } \\
\text { of their outcome evaluation, correctly guess } \\
\text { the allocation status in nearly two-thirds of } \\
\text { individuals because of the information on } \\
\text { health care use which typically led some } \\
\text { care-givers to share contacts with the inter- } \\
\text { vention team" } \\
\text { Comment: This may have led to the re- } \\
\text { searchers being biased in the analysis of the } \\
\text { outcomes }\end{array}$ \\
\hline
\end{tabular}

Selective reporting (reporting bias) High risk

Comment: They have not reported the cost of illness or process indicators: mean number of visits by home care advisor, visits by psychiatrists, use of medication. The protocol mentions primary outcomes as being: 1. carer mental health, 2. carer burden, 3 . behaviour problems and activities of daily living in elderly people with dementia, 4 . costs of illness but in the results section the last point is not reported

\begin{tabular}{lll}
\hline Other bias & Low risk & Comment: None detected \\
\hline
\end{tabular}

Dybdahl 2001 RCT Bosnia

Methods

Study design: Randomised, 2-sided, parallel group, open-label, assessor-blinded, controlled, trial (unit of randomisation: mother-child dyads; unit of analysis: individuals)

Duration of study: 1995-1996

Participants

Country: Bosnia

Income classification: Middle income

Geographical scope: Urban (town of Tuzla, multiethnic industrial town in north eastern Bosnia)

Healthcare setting: Home (1 refugee settlement, and private accommodation for refugees)

Mental health condition: Child mental health (PTSD, mental health, behavioural problems, scholastic difficulties)

Population: Mother-child dyads (internally displaced refugees)

- Age: Mothers: mean 30.7 years (SD 4.9), range 20-44 years; children: mean age 5.5 years (SD 0.7)

- Gender: Both (children 48 girls, 39 boys)

- Socioeconomic background: Mothers: $85 \%$ urban origin, education $14 \%$ illiterate (mean 5.3 years, SD 2.8; range $0-14$ years), married 63\%, widowed 36\%, divorced $1 \%$, living in private accommodation $60 \%$, refugee camp $40 \%$

- Inclusion criteria: Internally displaced Bosnian mothers with a child aged 5-6 years

Non-specialist health worker interventions for the care of mental, neurological and substance-abuse disorders in low- and middle- 
Dybdahl 2001 RCT Bosnia (Continued)

- Exclusion criteria: Not participating in any other intervention programme; unlikely to move out of the area before November 1996

Interventions

Stated purpose: To provide early childhood care and education and as well as psychosocial support to traumatised children by working with their mothers to help them resolve grief and improve parenting and providing a well-functioning family environment, utilising non-medical professionals in a post-conflict situation

INTERVENTION:

Name: Psychosocial intervention (+ basic medical care), 42 people

Delivered by: OPHR

- Title/name of NSHW/OPHR and number: Group leaders; 5 preschool teachers trained for the study

- Selection: Not specified in this report

- Educational background: As above

- Training (contents, duration and by whom): In a group of 3-8 group leaders by a mental health professional. Duration: 5-day workshop. Before arrival, the participants received basic information about the programme, its background and aims. Content: participants introduced to one another, receive written material, introductory training in some of the key issues, such as trauma, child development and the importance of interaction and communication (mother-child) two 3-hour seminars. Then 3 days of more detailed description of the programme and reinforcing through group work, demonstrations, role-plays and discussion the above topics (roles of caretaker, trauma and its effects on adults and children, groups and group dynamics, supervision, logbook)

- Supervision: Weekly group meetings (with 6-8 group leaders with a supervisor (a mental health professional) (and later twice a month)

- Incentives/remuneration: As above

Intervention details:

- Duration/frequency: Group leader met weekly with 2 groups of mothers (5 per group) for 5 months; 1 additional visit to each mother at her home at start of programme

- Content of intervention: Group work using a manual-based approach derived from therapeutic discussions with war-traumatised women at the Psychological Centre in Tuzla (1993-1996), and the ICDP; semi-structured group discussions introduced by group leaders dedicated to providing information about trauma and trauma reactions in adults and children, as well as suggestions for how to meet common post-traumatic needs and problems, with an emphasis on strengthening participants' own coping strategies, and reinforcing existing normal basic communication and interaction skills. Direct attention was given to the mothers and their mental health, to their beliefs and knowledge about children, and the reactions and needs of adults and children following traumatic events

CONTROL: Non-intervention group; participated in evaluations and received free basic medical care (45 people)

CO-INTERVENTIONS: Free basic medical care by local physicians provided for both groups; vitamins or iron were given to 52 children (66\% in intervention group; $81 \%$ in control group) 
Dybdahl 2001 RCT Bosnia (Continued)

points); Mother's rating of concentration problems $\$$ (yes/no); Raven’s Coloured Progressive Matrices $\$$; Children's interview (modified Birleson Depressive Inventory; modified by removing 2 of 13 items; scored 0-32; 11 used as cut-off for depression); well-being $\$$; Psychologists' observations $\$$ (video-rated; 14 items; 4-point scale; scored on 2 factorsproblems 0-32; resources 0-16); Anthropometrics: haemoglobin $\$$

Mothers: Perceived Social Support; IES; well-being $\mathbb{\$}$

Process/health worker outcomes: Not reported

Economic outcomes: Not reported

(*: primary outcomes of the study; $§$ : outcomes that we have not reported in this review) Outcomes not used in quantitative synthesis: War Trauma Questionnaire (given at baseline)

Time points: Baseline, 5-6 months after recruitment

Source of funding: UNICEF; University of Tromso

Notes on validation of instruments (screening and outcomes): Mother's rating of child's concentration and concentration problems; perceived social support - not validated separately. IES scores: not diagnostic of PTSD but some literature suggests IES score above 33 suggestive of PTSD

Additional information: Group work described in Dybdahl 1996; Dybdahl 1999

Handling the data: As per footnotes in data and analysis

Prospective trial registration number: Not registered

Risk of bias

Bias

Authors' judgement

Support for judgement

Random sequence generation (selection Low risk bias)

Quote from report: "The assignment was random. All the names of the motherchild dyads were written on pieces of paper, which were folded, mixed together, and then separated into two piles at random so that one pile formed the intervention group and the other pile formed the control group"

$\begin{array}{lll}\text { Allocation concealment (selection bias) Unclear risk } & \text { Comment: Not stated }\end{array}$

Blinding of participants and personnel Unclear risk (performance bias)

Comment: Participants and intervention All outcomes

Blinding of outcome assessment (detection Low risk bias)

objective outcomes
Comment: Physical and psychosocial outcomes were conducted by teams of physicians and experienced health workers assistants not involved in delivering interventions and blind to interventions 


\section{Dybdahl 2001 RCT Bosnia (Continued)}

\begin{tabular}{l|l|l}
\hline $\begin{array}{l}\text { Blinding of outcome assessment (detection } \\
\text { bias) } \\
\text { subjective outcomes }\end{array}$ & Low risk & $\begin{array}{l}\text { Comment: Physical and psychosocial out- } \\
\text { comes were conducted by teams of physi- } \\
\text { cians and experienced health workers assis- } \\
\text { tants not involved in delivering interven- } \\
\text { tions and blind to interventions }\end{array}$ \\
\hline
\end{tabular}

Baseline outcome measurements similar Low risk

Efficacy data

Comment: Baseline imbalances in prognostic variables noted for psychosocial support for mothers and well-being (but not statistically significant); and children's haemoglobin (P value $=0.3$ ); however, analyses were for differences in groups for changes from baseline

Comment: Mothers in refugee camps reported more war trauma and were more likely to be widowed during the conflict

Quote: “Twelve of the families dropped out of the study and did not participate in scheduled interventions: 7 from the intervention group, and 5 from the control group. Several of the mothers and children did not complete all tests at both test periods for a variety of reasons; thus the number of participants varied from test to test" Comment: Denominators not provided by intervention or control for each of the tests

Incomplete outcome data (attrition bias) Unclear risk Safety data (e.g. adverse events)

Comment: No safety data provided; it was assumed that intervention group results would be better than control group a priori

Protection against contamination Unclear risk

Comment: Mothers in refugee camps could have discussed contents of the intervention and supported mothers in control group

\begin{tabular}{|c|c|c|}
\hline Reliable primary outcome measures & High risk & $\begin{array}{l}\text { Comment: Many outcome measures used } \\
\text { were not previously validated; some had } \\
\text { poor psychometric properties (BDI) }\end{array}$ \\
\hline Selective reporting (reporting bias) & Low risk & $\begin{array}{l}\text { Comment: Protocol not available but all } \\
\text { measures stated in methods were reported }\end{array}$ \\
\hline Other bias & High risk & $\begin{array}{l}\text { Comment: Multiple statistical analyses } \\
\text { used without pre-specified primary or sec- } \\
\text { ondary outcomes; analyses corrected for } \\
\text { multiple comparisons yielded non-signif- }\end{array}$ \\
\hline
\end{tabular}

Non-specialist health worker interventions for the care of mental, neurological and substance-abuse disorders in low- and middleincome countries (Review)

Copyright $\odot 2013$ The Cochrane Collaboration. Published by John Wiley \& Sons, Ltd. 
Dybdahl 2001 RCT Bosnia (Continued)

cant results

Ertl 2011 RCT Uganda

Methods

Participants
Study design: RCT

Duration of study: Trial conducted between November 2007 and October 2009 (last follow-up). Preceded/overlapped by an epidemiological survey July 2007 to April 2008

Country: Uganda

Income classification: Low income

Geographical scope: Rural and urban, takes place in IDP camps and new settlement areas in 3 regions of Northern Uganda: Anaka: rural area with the most documented rebel activity, Awer: urban relatively safe area close to large town called Gulu, Padibe: rural (long distance from Gulu and was more affected by the war)

Healthcare setting: Home

Mental health condition: Child mental disorder, PTSD

Population: Patient, children/adolescents (child soldiers)

- Age: $12-25$ years; mean age 18.66 years (SD 3.77)

- Gender: Both

- Socioeconomic background: Former child soldiers, mean economic status in Euros (as measured by household possessions weighted by current local market prices divided by household size): EUR44-55

- Inclusion criteria: Clinical diagnosis of PTSD derived from expert interviews, member of the group of formerly abducted people or former child soldiers. To keep the trial naturalistic we did not exclude patients with suicidal ideation, substance abuse, or depression

- Exclusion criteria: Current substance dependence, mental retardation, psychotic disorder

Interventions

Stated purpose: Aim of this study was to examine whether individual-based, traumafocused NET is feasible and effective in reducing PTSD symptoms in traumatised former child soldiers living in the IDP camps of Northern Uganda when carried out by trained local lay therapists directly in the communities

INTERVENTION 1:

Name: NET

Delivered by:

- Title/name of NSHW/OPHR and number: Local lay counsellors, 14 (7 women and 7 men)

- Selection: Not specified

- Educational background: Not specified

- Training: Training in and performance of NET were as outlined by an adapted field version of the manual, duration and trainers: unspecified

- Supervision: "Treatment fidelity and therapeutic competence were monitored by case discussions in supervision meetings, observation and evaluation of treatment sessions via video recordings, and review of the obligatory treatment process notes for each session. In the case of NET, testimonies were additionally reviewed to check for trauma focus and richness of detail", not specified by whom

- Incentives/remuneration: Not specified 


\section{Intervention details:}

- Duration/frequency: 8 sessions of individual therapy, "Sessions lasted between 90 and 120 minutes and were scheduled 3 times a week"

- Content of intervention: "We chose an individual-based over a group-based treatment, because we expected this approach to better meet the requirements of former child soldiers, who present with high levels of PTSD as well as mistrust." "Narrative exposure therapy is a short-term, trauma-focused treatment developed for use in low-resource countries affected by crises and conflict. Intended for survivors of multiple trauma, this therapy results in the detailed documentation of the patients' lives as part of the therapy process." "Irrespective of treatment condition, the first session included psychoeducation on PTSD, its symptoms and consequences for the individual, and explanation of the rationale for narrative exposure therapy or academic catch-up". Participant constructs chronological account of self biography with therapist, reconstruct fragmented memories of traumatic events and to habituate INTERVENTION 2:

Name: Academic catch-up training

Delivered by

- Title/name of NSHW/OPHR and number: Local lay counsellors, 14 (7 women and 7 men)

- Selection: Not specified by whom

- Educational background: Not specified by whom

- Training: Written guidelines that summarised basic counselling skills and session outlines for the academic catch-up training, duration and trainers unspecified

- Supervision: "Treatment fidelity and therapeutic competence were monitored by case discussions in supervision meetings, observation and evaluation of treatment sessions via video recordings, and review of the obligatory treatment process notes for each session". Not specified by whom

- Incentives/remuneration: Not specified by whom Intervention details:

- Duration/frequency: 8 sessions of individual therapy, "Sessions lasted between 90 and 120 minutes and were scheduled 3 times a week"

- Content of intervention: "Carried out according to written guidelines that summarized basic counselling skills and session outlines for the academic catch-up training". "Irrespective of treatment condition, the first session included psychoeducation on PTSD, its symptoms and consequences for the individual, and explanation of the rationale for narrative exposure therapy or academic catch-up". "An intensive English catch-up course using the official Ugandan schoolbooks for different skill levels was developed. The evaluation of process notes revealed that the counsellors spent $55 \%$ of the total time allocated for academic catch-up doing academic training. The rest of the time was equally dedicated to psychoeducation, conducting discussions on coping with symptoms, and dealing with current problems. None of the counsellors deviated from the restriction that they should not focus on traumatic experiences in this condition. In the last session, the participants received the English textbooks and exercise books they had been working on with their counsellors"

CONTROL: Wait-list control, 10 received suicide intervention due to suicidal ideation. "After the 12-month follow-up, each waiting-list and academic catch-up participant still presenting with PTSD was offered narrative exposure therapy"

CO-INTERVENTIONS: Wait list had suicide intervention for those who exhibited high levels of suicide ideation (10 people)

Non-specialist health worker interventions for the care of mental, neurological and substance-abuse disorders in low- and middle- 
Ertl 2011 RCT Uganda (Continued)

\begin{tabular}{|c|c|}
\hline Outcomes & $\begin{array}{l}\text { Patient: PTSD symptom load* (Clinician-Administered PTSD scale; CAPS), functional } \\
\text { impairment*(CAPS); guilt } ₫ \text { (CAPS), symptoms of depression (MINI Neuropsychiatric } \\
\text { Interview for depression module A; MINI), suicidal ideation (MINI), stigmatisation } \S \\
\text { (Perceived Stigmatization Questionnaire; PSQ) } \\
\text { Carer: n/a } \\
\text { Process/health worker outcomes: "Treatment fidelity and therapeutic competence were } \\
\text { monitored by case discussions in supervision meetings, observation and evaluation of } \\
\text { treatment sessions via video recordings, and review of the obligatory treatment process } \\
\text { notes for each session. In the case of narrative exposure therapy, testimonies were addi- } \\
\text { tionally reviewed to check for trauma focus and richness of detail. No deviations from } \\
\text { the study protocol were noted", none reported in study } \$ \\
\text { Economic outcomes: None } \\
\text { (*: primary outcomes of the study; } \$ \text { : outcomes that we have not reported in this review) } \\
\text { Time points: Baseline, } 3-, 6-\text {, and } 12 \text {-month follow-up }\end{array}$ \\
\hline
\end{tabular}

Notes

Source of funding: This study was supported by the NGO vivo and by funding from the DFG (Deutsche Forschungsgemeinschaft) and the Ein Herz für Kinder foundation Notes on validation of instruments (screening and outcomes): CAPS and MINI validated, PSQ not validated

Additional information: clinicaltrialsgov/show/NCT00552006

Handling the data: As per footnotes in data and analysis

Prospective trial registration number: NCT00552006

Risk of bias

\begin{tabular}{l|l|l}
\hline Bias & Authors judgement & Support for judgement \\
\hline $\begin{array}{l}\text { Random sequence generation (selection } \\
\text { bias) }\end{array}$ & Unclear risk & $\begin{array}{l}\text { Comment: Randomly selected but does } \\
\text { not specify as to how }\end{array}$ \\
\hline $\begin{array}{l}\text { Allocation concealment (selection bias) } \\
\begin{array}{l}\text { Blinding of participants and personnel } \\
\text { (performance bias) } \\
\text { All outcomes }\end{array}\end{array}$ & Unclear risk & Comment: Unspecified \\
\hline
\end{tabular}

Blinding of outcome assessment (detection Low risk

Comment: No objective outcomes

bias)

objective outcomes

Blinding of outcome assessment (detection Low risk bias)

subjective outcomes

Quote: "Pretreatment assessments as well as follow-up assessments at 3 months, 6 months, and 12 months after treatment were conducted by 13 clinical psychologists blinded to treatment conditions"

Baseline outcome measurements similar Low risk

Comment: No statistical differences Quote: “There were no systematic pre-

Non-specialist health worker interventions for the care of mental, neurological and substance-abuse disorders in low- and middleincome countries (Review)

Copyright $(2013$ The Cochrane Collaboration. Published by John Wiley \& Sons, Ltd. 
Ertl 2011 RCT Uganda (Continued)

\begin{tabular}{|c|c|c|}
\hline & & $\begin{array}{l}\text { treatment differences in sociodemographic } \\
\text { data, traumatic load, and psychological im- } \\
\text { pairment between the } 3 \text { groups" }\end{array}$ \\
\hline Baseline characteristics similar? & Low risk & $\begin{array}{l}\text { Comment: No statistical differences } \\
\text { Quote: "There were no systematic pre- } \\
\text { treatment differences in sociodemographic } \\
\text { data, traumatic load, and psychological im- } \\
\text { pairment between the } 3 \text { groups" }\end{array}$ \\
\hline
\end{tabular}

Incomplete outcome data (attrition bias) Low risk

Efficacy data

Comment: 3-month follow-up: 26 included and 2 discontinued in NET, 24 included, 2 discontinued and 1 died in academic catch-up (ACU) at 6 months' follow-up: 26 included in NET, 23 included, 1 not found in ACU at 12 months' followup: 25 included 1 loss to follow-up in NET, 23 in NET. All 28 wait-list participants remained throughout treatment

Quote: “Apart from providing participants with the written documentation of their lives or with the English textbooks and exercise books, no incentives were offered. During follow-up periods, individuals who had relocated far from the former IDP camps were refunded travel expenses"

Comment: This would have reduced attrition. Unlikely to have affected outcomes

Incomplete outcome data (attrition bias) Low risk Safety data (e.g. adverse events)

Comment: No negative effects of NET were observed in this trial. Clinically reliable aggravation of symptoms was not present in the NET group but was present in $4.4 \%$ of the academic catch-up and 10 . $7 \%$ of the waiting-list participants

Protection against contamination Low risk

Comment: Lay counsellors were instructed not to integrate treatment material from NET to ACU

Reliable primary outcome measures Unclear risk

Quote: “Further, the trial might have been underpowered to detect significant treatment effects for most of the secondary outcome variables"

Comment: In addition, have little information on validity of these instruments in the context of the trial

Non-specialist health worker interventions for the care of mental, neurological and substance-abuse disorders in low- and middle- 
Ertl 2011 RCT Uganda (Continued)

\begin{tabular}{l|ll} 
Selective reporting (reporting bias) & Low risk & $\begin{array}{l}\text { Comment: All pre-specified outcomes in } \\
\text { protocol reported }\end{array}$ \\
\hline Other bias & Low risk & Comment: None detected \\
\hline
\end{tabular}

Fritsch 2007 RCT Chile

Methods

Study design: RCT

Duration of study: 6 months

Participants

Country: Chile

Income classification: Upper middle income

Geographical scope: Urban (Santiago)

Healthcare setting: $5 \mathrm{PHC}$ clinics

Mental health condition: Major depression

Population:

- Age: 18-70 years

- Gender: Female

- Socioeconomic background: About 30\% employed, $8 \%$ unemployed, $5 \%$ student

- Inclusion criteria: As above, with depression for 3 months (screening with GHQ-12 ( $\geq 5$ ) twice, 2 weeks apart), and at least 1 child aged 6-16 living with her

- Exclusion criteria: Abuse/dependence on alcohol or drugs, bipolar disorder, psychotic symptoms (present or past), suicidal ideation, pregnancy, physical or mental disabilities that would hamper their participation in the study

Interventions

Stated purpose: To compare a monitored pharmacotherapy intervention with current treatment in PC

INTERVENTION:

Name: Monitored pharmacotherapy

Delivered by

- Title/name of NSHW/OPHR and number: 5 generalist doctors/GP (1 per practice) and non-professional trained staff from the 5 clinics

- Selection: Based on practice selection

- Educational background: Qualified doctors

- Training: For doctors: 6 hours of training by the principal investigators; for nonprofessional trained staff: 2 hours

- Supervision: Doctors had permanent monitoring by the principal investigators. In addition, doctors participated in monthly meetings with a psychiatrist to discuss cases

- Incentives/remuneration: Not specified Intervention details:

- Duration/frequency: Regular visits to GP by patients

- Content of intervention: Regular visits to the GP with pharmacotherapy structured using clinical algorithms (use of available antidepressants: fluoxetine, amitriptyline, imipramine). Regular telephone contact by non-professional but trained personnel who did education, monitoring of drug intake and side effects and to remind/reinforce the need for regular follow-up with the doctor

Non-specialist health worker interventions for the care of mental, neurological and substance-abuse disorders in low- and middle- 
Fritsch 2007 RCT Chile (Continued)

CONTROL: Usual care: based on the Ministry of Health's programme for treatment of depression in PC: consultations with GPs, pharmacotherapy, individual or group psychotherapy with psychologists, and referral to psychiatrists

CO-INTERVENTIONS: None

Outcomes

Patient: Diagnosis of depression (MINI), severity of symptoms (HDRS), QoL (SF-36)

Carer: None

Process/health worker outcomes: None

Economic outcomes: None

Time points: 3 and 6 months

$\begin{array}{ll}\text { Notes } & \text { Source of funding: Fondecyt, Chile } \\ \text { Notes on validation of instruments: All instruments validated internationally and in } \\ \text { Chilean setting } \\ \text { Additional information: No protocol } \\ \text { Handling the data: As per footnotes in data and analysis }\end{array}$

Risk of bias

\begin{tabular}{lll}
\hline Bias & Authors judgement & Support for judgement \\
\hline $\begin{array}{l}\text { Random sequence generation (selection } \\
\text { bias) }\end{array}$ & Low risk & $\begin{array}{l}\text { Comment: Patients were assigned ran- } \\
\text { domly. This took place at the individ- } \\
\text { ual level, using computer systems managed } \\
\text { from a central level }\end{array}$ \\
\hline Allocation concealment (selection bias) & Low risk & $\begin{array}{l}\text { Comment: Patients were assigned ran- } \\
\text { domly. This took place at the individ- } \\
\text { ual level, using computer systems managed } \\
\text { from a central level }\end{array}$ \\
\hline
\end{tabular}

Blinding of participants and personnel Low risk (performance bias)

All outcomes

Blinding of outcome assessment (detection Low risk bias)

objective outcomes

Blinding of outcome assessment (detection Low risk

bias)

subjective outcomes

Baseline outcome measurements similar Low risk
Comment: Due to the nature of the intervention, the participants could not be blinded to the intervention and this is unlikely to create any bias to the results

Comment: There were no objective outcomes

Comment: Assessors were not involved in the design of the study, did not know the study hypotheses, and were blinded to group assignment

Comment: The 2 study groups did not vary significantly 
Fritsch 2007 RCT Chile (Continued)

\begin{tabular}{|c|c|c|}
\hline Baseline characteristics similar? & Low risk & $\begin{array}{l}\text { Comment: The } 2 \text { study groups did not } \\
\text { vary significantly }\end{array}$ \\
\hline $\begin{array}{l}\text { Incomplete outcome data (attrition bias) } \\
\text { Efficacy data }\end{array}$ & Unclear risk & $\begin{array}{l}\text { Comment: The MINI scores are not re- } \\
\text { ported at follow-up. In addition, the au- } \\
\text { thor does not show the comparative tables } \\
\text { of the results at } 3 \text { and } 6 \text { months (only in- } \\
\text { dividual figures per allocated group but no } \\
\text { summary statistics) }\end{array}$ \\
\hline $\begin{array}{l}\text { Incomplete outcome data (attrition bias) } \\
\text { Safety data (e.g. adverse events) }\end{array}$ & High risk & $\begin{array}{l}\text { Comment: We have incomplete informa- } \\
\text { tion }\end{array}$ \\
\hline Protection against contamination & Unclear risk & $\begin{array}{l}\text { Comment: We have incomplete informa- } \\
\text { tion and we are not sure if the GPs in this } \\
\text { setting may be doing both intervention and } \\
\text { control interventions }\end{array}$ \\
\hline Reliable primary outcome measures & Low risk & $\begin{array}{l}\text { Comment: These tools are known to be } \\
\text { validated from previous studies }\end{array}$ \\
\hline Selective reporting (reporting bias) & Low risk & $\begin{array}{l}\text { Comment: No selective reporting, though } \\
\text { some reporting of things that would be use- } \\
\text { ful not done like costs of psychiatric drugs }\end{array}$ \\
\hline Other bias & Low risk & Comment: No other sources of bias \\
\hline
\end{tabular}

\section{Gavrilova 2009 RCT Russia}

Methods

Study design: Randomised, parallel group, single-blind, controlled, clinical trial Duration of study: 2000-2004

\section{Participants}

\section{Country: Russia}

Income classification: Middle

Geographical scope: Urban (Moscow - South administrative district, patients registered in 3 general practices)

Healthcare setting: Group community training

Mental health condition: Dementia

Population: Patient-carer dyad

- Age: Patients: > 65 years; carers' mean age 61.5 years (SD 17.6)

- Gender: Both

- Socioeconomic background: Not specified

- Inclusion criteria: Patients > 65 years; met DSM-IV criteria for dementia

- Exclusion criteria: Serious current physical illness, no family carer, $>1$ person with dementia in same household 


\section{INTERVENTION:}

Name: 10/66 brief carer intervention

Delivered by:

- Title/name of NSHW/OPHR: Newly qualified doctors (number not specified)

- Selection: Not specified

- Educational background: Medical degree

- Training (contents, duration and by whom): 2-day training, using the 10/66 intervention manual (includes vignettes, role plays, live interviews).

- Supervision: Not specified.

- Incentives/remuneration: Not specified.

Intervention details:

- Duration/frequency: 5 weekly 30-minute sessions

- Content of intervention: Intervention for carers. Content (manualised approach): 3 modules: assessment of cognitive and functional impairment, carers' knowledge and understanding, care arrangements ( 1 session), basic education about dementia illness, what to expect in future, local available resources (2 sessions), training regarding dealing with specific problem behaviours (2 sessions)

CONTROL: Usual medical care (on a wait-list for the intervention)

CO-INTERVENTIONS: Medical care for both intervention and control

Random sequence generation (selection Low risk bias)
Quote from report: "Randomisation was carried out in London, with the codes transmitted immediately back to the Moscow centre by e-mail. We used a stratified permuted block method to ensure as fare as possible an even distribution of baseline caregiver strain assessed using the Zarit Burden Interview" 
Gavrilova 2009 RCT Russia (Continued)

Comment: Central randomisation apparently computer generated

\begin{tabular}{|c|c|c|}
\hline Allocation concealment (selection bias) & Unclear risk & $\begin{array}{l}\text { Comment: Not reported } \\
\text { Comment: Even though sequence genera- } \\
\text { tion was centrally done, it was unclear how } \\
\text { allocation was concealed }\end{array}$ \\
\hline
\end{tabular}

Blinding of participants and personnel Low risk (performance bias)

Comment: The control group was a waitAll outcomes list so differential interventions were unlikely

Blinding of outcome assessment (detection Low risk bias)

objective outcomes

Blinding of outcome assessment (detection Low risk

bias)

subjective outcomes

Comment: This was an open-label trial; however, the assessors were blind to treatment allocation

\begin{tabular}{l|ll}
\hline Baseline outcome measurements similar & Low risk & Comment: All similar \\
\hline Baseline characteristics similar? & Low risk & $\begin{array}{l}\text { Comment: All similar. There were baseline } \\
\text { imbalances in the degree of care needed by } \\
\text { the patients in control group. However, this } \\
\text { was adjusted in statistical analysis }\end{array}$ \\
\hline
\end{tabular}

Incomplete outcome data (attrition bias) Low risk Efficacy data

Comment: Attition was low in both groups (only deaths) and adjusted for in statistical analysis

Incomplete outcome data (attrition bias) Unclear risk

Comment: No safety outcomes reported

Safety data (e.g. adverse events)

Protection against contamination $\quad$ Low risk

Comment: Wait-list control so unlikely to be contamination

Reliable primary outcome measures Unclear risk

Comment: All outcome measures were validated. We do not know if tools were translated/methods used

Selective reporting (reporting bias) Low risk

Comment: Trial prospectively registered. All pre-stated outcomes reported

Other bias

Low risk

Comment: None detected 
Healthcare setting: Small group school setting-high school

Mental health condition: PTSD

Population: Patient (adolescents only)

- Age: 14-18 years; mean age 16.3 years

- Gender: Both, significantly more girls than boys

- Socioeconomic background: War-traumatised area with students who had lost both or 1 parent and $90 \%$ of the homes of that area were destroyed

- Inclusion criteria: Students having PTSD as defined according to a scoring algorithm of the HTQ previously described by the Harvard Refugee Trauma group and used in a Kosovar Albanian population. This definition of PTSD requires a score of 3 or 4 , on a Likert scale of $1-4$, on at least 1 of the 4 of the re-experiencing symptoms (Criterion B), at least 3 of the 7 avoidance and numbing symptoms (Criterion C), and at least 2 of the 5 arousal symptoms (Criterion D) in addition to exposure to a traumatic event (Criterion A)

- Exclusion criteria: No specific exclusion criteria. Students having PTSD symptoms as defined above may participate in the study

Stated purpose: To determine whether participation in a mind-body skills group programme based on psychological self care, mind-body techniques and self expression decreases symptoms of PTSD

\section{INTERVENTION 1:}

Name: Mind-body school-based skill group

Delivered by:

- Title/name of NSHW/OPHR and number: 4 high-school teachers

- Selection: Information from author: "The teachers were self-selected"

- Educational background: Information from author: "All graduated from the university but did not have advanced degrees. They would have whatever certification is required to teach high school in Kosovo"

- Training: 2 part, 10-day intensive training undertaken in 1999-2000; Washington DC-based faculty of the centre for Mind-body medicine (CMBM). Info from author: "When we went to Kosovo after the war to train health professionals, the teachers from this village came to our training and brought the mind-body techniques back to their school in the rural village and began using them with their students. We did one pilot study before we did the RCT"

- Supervision: CMBM's Kosovo faculty of psychiatrist and psychologist

- Incentives/remuneration: Information from author: "they were paid a small stipend"

Intervention details:

- Duration/frequency: 12 sessions for 2 hours twice a week for 6 weeks

- Content of intervention: Self expression and personal sharing with instruction in and use of meditative and imaginative mind body techniques; given in small group sessions (about 10 students per group) Format is now manualised. The aim is not to 
Gordon 2008 RCT Kosovo (Continued)

discuss traumatic events but create a supportive environment in which self awareness, sharing and listening are encouraged, teach them self care techniques, and give them skills to deal with traumatic events in their daily life, and to understand the trauma they suffered

CONTROL: Wait-list control group, who received the 12 session mind-body skills after the first intervention group finished their 12 sessions

CO-INTERVENTIONS: None

Patient: HTQ
Carer: n/a
Process/health worker outcomes: None
Economic outcomes: None reported
Time points: Baseline (pre-intervention), immediately post-intervention (i.e. after 6
weeks), 3-month follow-up after the intervention

Source of funding: The Center for Mind-Body Medicine listed as sponsor on protocol Notes on validation of instruments (screening and outcomes): Used previously in Kosovo as described in Lopes Cardozo 2000

Additional information: clinicaltrials.gov/ct2/show/NCT00136357? term=NCT00136357

Handling the data (e.g. imputed values/other calculations we have made): None Prospective trial registration number: NCT00136357

Risk of bias

\begin{tabular}{l|l|l}
\hline Bias & Authors' judgement & Support for judgement \\
\hline $\begin{array}{l}\text { Random sequence generation (selection } \\
\text { bias) }\end{array}$ & Low risk & $\begin{array}{l}\text { Quote: "Students were stratified according } \\
\text { to gender and randomly assigned by the re- } \\
\text { search director using random numbers gen- } \\
\text { erated by Microsoft Excel 2003" }\end{array}$ \\
\hline Allocation concealment (selection bias) & High risk & $\begin{array}{l}\text { Quote: "The list of assigned groups was } \\
\text { given to the teachers, who then notified the } \\
\text { students of their group assignment" } \\
\text { Comment: No allocation concealment }\end{array}$ \\
\hline
\end{tabular}

Blinding of participants and personnel Low risk (performance bias)

All outcomes
Comment: Not blinded but unlikely to affect the outcomes

Comment: No objective outcomes

Blinding of outcome assessment (detection Low risk bias)

objective outcomes

Blinding of outcome assessment (detection Unclear risk bias)

subjective outcomes
Quote: "While it is possible that students wanted to please the teachers by reporting a decrease in symptomatology after the groups, the teachers' experience, and that 
Gordon 2008 RCT Kosovo (Continued)

of the observers was that greater familiarity with the teachers, on the contrary, facilitated more frank discussions and sharing of problems and symptoms after as well as before and during the intervention"

Comment: Teachers both performed intervention and delivered the instruments but given explanation above, may be classified as unclear risk

\begin{tabular}{lll}
\hline Baseline outcome measurements similar & Low risk & Comment: All similar \\
\hline Baseline characteristics similar? & Low risk & $\begin{array}{l}\text { Information from author: Age, sex and } \\
\text { baseline PTSD were all similar }\end{array}$ \\
\hline
\end{tabular}

Incomplete outcome data (attrition bias) Low risk Efficacy data Safety data (e.g. adverse events)
Incomplete outcome data (attrition bias) Low risk

Comment: Low dropout rate

Information from author: "We did not look at any adverse outcomes. The teachers received ongoing supervision. The supervisors and teachers would have notified us if any adverse events occurred as required by the IRB, but there were none. We did not formally record this"

Comment: Low risk

Comment: Just 1 school in the study, so may have been contamination. However, control group received intervention as soon as intervention group had finished. Some of the results (e.g. arousal) suggest improvement in control group before they received intervention which may suggest contamination

Reliable primary outcome measures Low risk
Quote: "The study's main limitation was the lack of inclusion of a trauma exposure scale in the actual interviews with participants. This was done deliberately, so as not to obligate the students to discuss the traumatic events they had experienced". Due to the pervasive violence and universal homes' destruction it was assumed all students had traumatic exposure to events

Comment: However, scale used for PTSD is reliable and validated. Consider this to be low risk 


\section{Gordon 2008 RCT Kosovo (Continued)}

\begin{tabular}{l|ll} 
Selective reporting (reporting bias) & Low risk & $\begin{array}{l}\text { Comment: Only 1 outcome on the proto- } \\
\text { col, HTQ }\end{array}$ \\
\hline Other bias & Low risk & Comment: None detected \\
\hline
\end{tabular}

Hirani 2010 CRCT Pakistan

Methods

Participants

Study design: Cluster RCT (unit of allocation: residents with similar SES, ethnicity, education and income level. Unit of randomisation: individual)

Duration of study: 2000-2004

Income classification: Low income

Geographical scope: Urban (inner city slum area of Karachi (Pakistan) a sprawling metropolis of 18 million residents located in Arabian sea)

Healthcare setting: Adult literacy centres (ACLs)

Mental health condition: Depression

Population: Women. The community was selected for ESB intervention testing due to the availability of nearby factories and employment opportunities for women following the ESB

- Age: 25-35 years

- Gender: Female

- Socioeconomic background: Economically disadvantaged women. Most women reported $<4$ years of formal education and most women were not employed.

Household size was 6-10 people for most women and monthly household income averaged USD55.00 US

- Inclusion criteria: Women in adult literacy programmes in each of the randomly chosen clusters were recruited into the study and 25-35 years

- Exclusion criteria: Not specified

Interventions

Stated purpose: To provide an evidence-based intervention to address the $\mathrm{PHC}$ problems confronting women in Pakistan and worldwide: depression and violence. Specifically, we tested the differential effectiveness of a community-derived intervention of ESB, developed through community-based participatory methods against an evidence-based empirically tested counselling model

INTERVENTION 1:

Name: ESB intervention

Delivered by:

- Title/name of NSHW/OPHR: CHWs

- Selection: Not specified

- Educational background: Not specified

- Training (contents, duration and by whom): 21 hours training, included skillbuilding on components of the intervention as well as research ethics of privacy and confidentiality

- Supervision: Not specified

- Incentives/remuneration: Not specified Intervention details:

- Duration/frequency: 8 weekly at the adult literacy centres (for both Intervention 
1 and 2)

- Content of intervention (by types of health worker and per patient/carers: Skills for employment attainment and retention such as, effective communication, balancing personal and work life and time management, conflict resolution, dealing with abuse and harassment, enhancing self efficacy, effective parenting, and personal hygiene and grooming

\section{INTERVENTION 2:}

Name: Group counselling intervention

Delivered by:

- Title/name of NSHW/OPHR: CHWs

- Selection: Not specified

- Educational background: Not specified

- Training (contents, duration and by whom): 21 hours of training; included skill-building on components of the intervention as well as research ethics of privacy and confidentiality

- Supervision: Not specified

- Incentives/remuneration: Not specified Intervention details:

- Duration/frequency: 8 weekly at the adult literacy centres (for both Intervention 1 and 2)

- Content of intervention(by types of health worker and per patient/carers: Covered effective communication, balancing personal and work life and time management, conflict resolution, dealing with abuse and harassment, enhancing self efficacy, effective parenting and personal hygiene and grooming

CONTROL: Usual care (the control group received no intervention) CO-INTERVENTIONS: None

Patient: Depression (BDI-II, IPV $₫$ (questionnaire - instrument developed by WHO guidelines and modified based on the Pakistani national gender indicators list for violence again women and self efficacy $₫$ (GSE Scale; employment status $\$$

Carer: Not applicable

Process/health worker outcomes: Not assessed

Economic outcomes (and where these can be found, e.g. ref or table number): Not reported

Time points: Baseline, 8 weeks

No mention of primary or secondary outcomes

(*: primary outcomes of the study; $\$$ : outcomes that we have not reported in this review) 
Hirani 2010 CRCT Pakistan (Continued)

Random sequence generation (selection Low risk bias)
Comment: It is cluster sampling

Quote: "A three-arm randomised controlled trial with cluster randomisation sampling was followed, whereby blocks of similar ethnic, language, and cultural affiliated families were randomised to an intervention". "The methodology of cluster randomisation maintained the internal validity of the research by preventing the contamination of interventions among the study groups." "Since our study was conducted in a densely populated urban community, randomisation at the individual level could result in women randomly assigned to different intervention groups living next door. Therefore, intra-class sampling was followed to maximize homogeneity and decrease the variance in the data. For intra-class sampling, the community was divided into eighteen clusters. Each cluster was defined according to residents with a similar socio-economic status, ethnicity, education, and income level. Three sets of two adjacent similar clusters were randomly assigned to the interventions of economic skill-building, counselling and control group. Each cluster had several hundred adult women. The randomisation took place maintaining the community based participatory approach and the internal validity of the research remained strong"

Allocation concealment (selection bias) Unclear risk

Comment: No mention of whether the allocation of clusters was concealed

Comment: No blinding but this is unlikely to affect outcomes

Comment: No objective outcomes

Blinding of outcome assessment (detection Low risk bias)

objective outcomes

Blinding of outcome assessment (detection Unclear risk bias)

subjective outcomes
Comment: All instruments were self reported (not mentioned if there was an interviewer to administer them), so unlikely that there was any blinding

Non-specialist health worker interventions for the care of mental, neurological and substance-abuse disorders in low- and middle- 
Hirani 2010 CRCT Pakistan (Continued)

\begin{tabular}{|c|c|c|}
\hline Baseline outcome measurements similar & Unclear risk & $\begin{array}{l}\text { Comment: Unable to say as no baseline } \\
\text { data reported }\end{array}$ \\
\hline Baseline characteristics similar? & Low risk & $\begin{array}{l}\text { Quote: "No significant differences existed } \\
\text { in demographic characteristics between the } \\
\text { groups. Most of the women were between } \\
25 \text { and } 35 \text { years of age. Most women re- } \\
\text { ported less than } 4 \text { years of formal educa- } \\
\text { tion and most women were not employed. } \\
\text { Household size was between } 6 \text { and } 10 \text { per- } \\
\text { sons for most women and monthly house- } \\
\text { hold income averaged } \$ 55.00 \text { dollars US" } \\
\text { Comment: However, there is no table of } \\
\text { characteristics of participants so unable to } \\
\text { make a truly informed comment on this }\end{array}$ \\
\hline $\begin{array}{l}\text { Incomplete outcome data (attrition bias) } \\
\text { Efficacy data }\end{array}$ & Unclear risk & $\begin{array}{l}\text { Quote: "Twenty four women began and } \\
\text { completed the first } 8 \text {-week intervention } \\
\text { sessions and outcome measures, specifically } \\
7 \text { women received counselling, } 9 \text { women } \\
\text { received economic skill- building, and } 8 \\
\text { women were in the control group" } \\
\text { Comment: However, no information on } \\
\text { dropouts }\end{array}$ \\
\hline
\end{tabular}

Incomplete outcome data (attrition bias) Unclear risk Safety data (e.g. adverse events)

Comment: No safety outcomes reported. Not sure if they were looked for or not. No protocol available

Protection against contamination Low risk

Quote: "The methodology of cluster randomisation maintained the internal validity of the research by preventing the contamination of interventions among the study groups. However, cluster randomised trials can often prevent contamination between intervention and control groups" and .29-31 Since our study was conducted in a densely populated urban community, randomisation at the individual level could result in women "randomly assigned to different intervention groups living next door. Therefore, intra-class sampling was followed to maximize homogeneity and decrease the variance in the data. For intra-class sampling, the community was divided into eighteen clusters. Each cluster was defined according to residents with a similar socio-economic status, ethnic- 
Hirani 2010 CRCT Pakistan (Continued)

ity, education, and income level. Three sets of two adjacent similar clusters were randomly assigned to the interventions of economic skill-building, counselling and control group. Each cluster had several hundred adult women. The randomisation took place maintaining the community based participatory approach and the internal validity of the research remained strong"

Reliable primary outcome measures Unclear risk

Selective reporting (reporting bias)

Unclear risk
Comment: The tools do not seem validated for the setting in which they are used, so difficult to know if they are reliable

\begin{tabular}{l|l|l} 
& & $\begin{array}{l}\text { to check with protocol to check if these are } \\
\text { also the prespecified outcomes }\end{array}$ \\
\hline Other bias & Low risk & Comment: No other bias
\end{tabular}

Jenkins 2012 C-RCT Kenya

Methods

Study design: Cluster RCT, allocated by clinic, analysed at individual level for patient outcome, analysed at clinic level for GHQ cases

Duration of study: Conducted in 2010

Participants

Country: Kenya
Income classification: Low income
Geographical scope: Urban and rural; Nyanza province, Kenya, as
where the national training programme $2005 / 2010$ had hither to train
thus most clinics were eligible for study. The districts of Siaya, Bon
were selected, allocated around Kisumu near Lake Victoria
Healthcare setting: PC facilities (dispensaries and PHC centres)
Mental health condition: All mental disorders
Population: Patients (adults and children), anyone attending PHC
Age: > 16 years

Gender: Both

Socioeconomic background: Livelihoods were based on subsistence farming, an extensive fishing industry along the lake, and some commercial business. The majority tribe is Luo. The area was the site of significant election violence in January 2007

Inclusion criteria: The sample framework was the Ministry of Health list of all publicly funded primary care facilities in Siaya, Bondo and Rachuonya districts in Nyanza province. The criteria for entry for clinics was that they were in the Ministry of Health list of PHCs, and were publicly funded. Criterion for entry for patients was that they were over 16 years

Exclusion criteria: Centres where staff had previously received training from the KMTC mental health training programme were excluded from the study; publicly funded. Cri- 
terion for entry for patients was that they were over 16 years of age; criteria for exclusion were dementia and learning disability of such severity as to be unable to complete the questionnaires; life threatening illness; did not speak the language spoken by the researchers; and refusal to co-operate

Interventions
Stated purpose: To conduct a phase 2 exploratory trial as a cluster RCT, testing the effect of a low-cost training intervention, integrated with the national health sector reforms, 1 . on the competencies of primary care staff to recognise mental disorders, treat and make appropriate referrals to the scarce specialist services and 2. on recovery (improved health and social outcomes and quality of life) of clients

\section{INTERVENTION:}

Name: PC mental health training

Delivered by:

Title/name of NSHW/OPHR and number: PHC staff (all nurses and clinical officers (doctors) eligible for training); 2 in each centre

Selection: Self selection: 2 invited from each centre

Educational background: Nurses and clinical officers at PHC

Training: RJ trained local trainers (3 courses) to deliver the course to frontline workers, in 2005 (By RJ) and gave them a refresher course in 2009 ( 40 hours in total). The trainers had done the KMTC mental health training and had been delivering training since then. These trainers included 20 senior staff from Kenya medical college (KMTC) (i.e. from Nairobi, provincial medical training colleges and the Ministry of Health rural health training centres). They were supplied with good practice guidelines and handouts to those who attended the training course, and the project also provided a training course on mental health for the local district public health nurses. Course structure: comprehensive structured interactive mental health training programme for 5 days. Curriculum and teaching materials developed by the WHO Collaborating Centre in dialogue with Kenya partners, based on the Kenya adaptation of the WHO primary carePC guidelines. Ccontent: 5 modules: 1 . core concepts of $\mathrm{MH}$, MDs, their contribution to physical health economic and social outcomes; 2 . core skills (examination, communication, assessment, managing difficult cases/ violence/bad news); 3. neurological disorders (epilepsy, Parkinson's disease, headache, dementia, toxic confusional states), 4. psychiatric disorders (content based on the WHO primary care PC guidelines for mental health, Kenya adaptation); 5. system issues of policy; legislation; links between mental health and child health, reproductive health, HIV and malaria; roles and responsibilities; health management information systems; working with community health worker CHWs and with traditional healers; and integration of mental health into annual operational plans. Use of role plays ( 25 each), theory, discussion, videos, emphasis on acquisition of practical skills and competencies for assessment, diagnosis and management)

Supervision: No supervision available from district level and poor medication supply Incentives/remuneration: "Each health facility is staffed by one or more nurses and clinical officers on Ministry of Health salaries, and around 15-20 community health workers are not remunerated by the Ministry by the Ministry of Health but are now expected to receive small remuneration from the community"

Intervention details:

Duration/frequency: Varying depending on patient

Content of intervention: Diagnosis and treatment with medicines, and follow-up

CONTROL: Usual care, PHCs that had not received prior KMTC training, neither 
Jenkins 2012 C-RCT Kenya (Continued)

were given training during this intervention

CO-INTERVENTIONS: Patients in both intervention and the control groups were treated as the health worker routinely decided, based on their knowledge, experience and training

Outcomes

Notes
Patient: GHQ change in patients (neurotic symptoms, including morbid rating), EQ5D $\$$ (health outcome for wide range of health conditions and treatments), WHODAS II (disability according to ICF)

Carer: $\mathrm{n} / \mathrm{a}$

Process/health worker outcomes: GHQ identification index of clinics: detection rate of mental disorder (agreement/disagreement of staff diagnosis with patient rated GHQ score cut-off)

Economic outcomes: None reported

Time points: Baseline ( 3 months post training), 3 months ( 6 months post training)

${ }^{*}$ : primary outcomes of the study; $\$$ : outcomes that we have not reported in this review)

Source of funding: Nuffield Foundation and Department for International Development (UK)

Notes on validation of instruments: All instruments available in English and Kiswahili, and all validated in local setting. GHQ: widely validated in Africa; WHODAS II: validated (Ref from WHO, www.who.int/classifications/icf/en/); EUROQOL 5D: "The special validated calculator used in this project is derived from normative data from Zimbabwe for the EQ" Global Forum for Health Research (2002) The 10/9 Report on Health Research, 2001-2002. Geneva, Switzerland

Additional information: www.controlled-trials.com/ISRCTN53515024

Handling the data: As per footnotes in data and analysis

Prospective trial registration number: ISRCTN53515024

Risk of bias

\begin{tabular}{l|l} 
Bias Authors' judgement Support for judgement & Autgents
\end{tabular}

Random sequence generation (selection Low risk bias)
Quote 1: "All public level 2 and 3 health facilities were eligible for randomisation, which was done by DK and the Great Lakes University Knowledge Management and Research Department, using a table of random numbers drawn from JT McLure and F Dietrich 1994, Statistics, Macmillan College Publishing Co. pp 909-911”

Quote 2: "A random sample of 99 centres were selected stratified by health facility level, which were then randomly allocated to intervention and control groups, resulting in 33 dispensaries and 16 health centres in the intervention group and 37 dispensaries and 13 health centres in the control group" 
Jenkins 2012 C-RCT Kenya (Continued)

Allocation concealment (selection bias) Low risk

Blinding of participants and personnel Low risk (performance bias)

All outcomes
Information from the author: Allocation to intervention and controls was concealed from the research assistants

Quote: "The clinic staff were not blind as to whether they had received the training. We did not run a quantitative check on whether recruited clinic clients were aware of the trained status of their health workers" Comment: However, unlikely to affect outcome. This was performed in real conditions in Kenya

Blinding of outcome assessment (detection Low risk bias)

objective outcomes

Blinding of outcome assessment (detection Low risk bias)

subjective outcomes

Comment: No objective outcomes

Quote: "The research assistants were blind to whether the clinic staff had received the mental health training course, and to whether clients were attending clinics with trained or untrained staff. JA, who organised the research assistants in the field, was not blind to the clinic status"

\begin{tabular}{l|ll}
\hline Baseline outcome measurements similar & Low risk & Comment: All similar \\
\hline Baseline characteristics similar? & Low risk & $\begin{array}{l}\text { Quote: "The groups were generally similar } \\
\text { on these parameters except that interven- } \\
\text { tion clinics had more availability of benzo- } \\
\text { diazepines, and more clients who were un- } \\
\text { married" } \\
\text { Comment: These were adjusted for in the } \\
\text { analysis }\end{array}$ \\
\hline
\end{tabular}

Incomplete outcome data (attrition bias) Low risk Efficacy data

Quote: "To reduce the possibility of attrition bias [31], we paid the 12 participants per cluster $£ 2$ per day to complete their initial assessment day ( 3 months after training of the health workers) and follow up day 12 weeks later, as compensation for their transport costs and time"

Comment: In addition, dropout rate very small (> 90\% retention rate)

Incomplete outcome data (attrition bias) Low risk Safety data (e.g. adverse events)

Information from author: No major adverse events were noted, e.g. suicides. NB The trial was of training, not of a specific medicine or specific intervention 
Jenkins 2012 C-RCT Kenya (Continued)

\begin{tabular}{|c|c|c|}
\hline Protection against contamination & Low risk & $\begin{array}{l}\text { Quote: "Randomisation was conducted at } \\
\text { the cluster level, namely PHC level rather } \\
\text { than individual health worker level. If ran- } \\
\text { domisation had taken place at individual } \\
\text { health worker level, the risk of contamina- } \\
\text { tion between the practice of trained and } \\
\text { untrained staff would be high, since they } \\
\text { work closely in small teams" } \\
\text { Comment: In addition, mentioned that } \\
\text { there is other training happening simul- } \\
\text { taneously (HIV, malaria, nutrition, paedi- } \\
\text { atrics) but none of them covered mental } \\
\text { health issues (HIV training was only about } \\
\text { pre-post test counselling for HIV) }\end{array}$ \\
\hline Reliable primary outcome measures & High risk & $\begin{array}{l}\text { Comment: GHQ-12 is a screening instru- } \\
\text { ment and was used here as a diagnostic and } \\
\text { symptom severity scorer }\end{array}$ \\
\hline Selective reporting (reporting bias) & Low risk & $\begin{array}{l}\text { Comment: Outcomes in protocol and in } \\
\text { paper are the same }\end{array}$ \\
\hline Other bias & Low risk & $\begin{array}{l}\text { Comment: The first author confirmed that } \\
\text { all results "adjusted for clustering" }\end{array}$ \\
\hline
\end{tabular}

Jordans 2010 C-RCT Nepal

Methods

Study design: Cluster RCT, unit of allocation: schools, unit of analysis: individual Duration of study: December 2006 to March 2007

Participants

\author{
Country: Nepal \\ Income classification: Low income \\ Geographical scope: 4 districts of rural south-western Nepal (Banke, Dang, Bardia, \\ Kailali) \\ Healthcare setting: School \\ Mental health condition: Psychosocial distress (including PTSD symptoms) \\ Population: Patient (children/adolescents)
}

Age: 11-14 years

Gender: Both, more girls in treatment group

Socioeconomic background: Significant differences in groups despite randomisation: more brahmins in treatment group, Terai caste in wait-list (none in intervention group). Higher education among treatment group. Religion and place of residence were statistically different but of minimal importance: the majority were Hindu in both groups and lived in a village other to their original village

Inclusion criteria: School-aged children, positive Child Psychosocial Distress Screener score (cut-off score unspecified)

Exclusion criteria: Psychiatric problems (mutism, mental retardation, dissociative dis-

Non-specialist health worker interventions for the care of mental, neurological and substance-abuse disorders in low- and middle- 
Jordans 2010 C-RCT Nepal (Continued)

orders, epilepsy without medication, panic or phobic disorders, and child psychosis), schools excluded if they were in Village Development Committees (VDCs) where the intervention was already implemented and schools in adjoining VDCs to avoid contamination

Interventions

Stated purpose: To assess the efficacy of CBIs among school-going children in rural Nepal as a psychosocial intervention to address children affected by armed conflict in LAMIC

INTERVENTION:

Name: CBI

Delivered by:

- Title/name of NSHW/OPHR and number: 16 paraprofessional interventionists / facilitators

- Selection: Gender-balanced group, from targeted communities

- Educational background: Based on previous experience and affinity to work with children

- Training: 15-day skills-oriented course (duration and trainers not specified)

- Supervision: Regular supervision by experienced counsellor

- Incentives/remuneration: Information from author: The facilitators received a monthly remuneration of $4000 \mathrm{NPR}$ for running the CBI sessions Intervention details:

- Duration/frequency: 5 weeks, 15 sessions (about 60-minute sessions)

- Content of intervention: Protocolised group intervention; eclectic intervention based on concepts from creative-expressive and experiential therapy, co-operative play and CBT. Use of the same manual as for Tol 2008 (Center for Trauma Psychology in Boston)

CONTROL: Usual care (wait-list control)

CO-INTERVENTIONS: CBI was offered as part of a multilayered care system that included activities geared towards strengthening community resilience through parental support groups, recreational activities, community sensitisation and psycho-education (tier 1), the CBI to target children with elevated psychosocial distress upon primary screening (tier 2), and individual supportive and problem-solving counselling and referral to psychiatric care (if available) for children, mainly referred on from the group intervention, in need of more individualised or specialised care (tier 3) gression), CPSS (Child PTSD)*, DSRS*, SDQ* $\$$. Secondary outcomes:Concern for other scale $\$$ (prosocial behaviour), Children's Function Impairment (protocol mentioned secondary outcomes would also be daily functioning and self efficacy - these are not reported here)

Carer: $\mathrm{n} / \mathrm{a}$

Process/health worker outcomes: Not assessed

Economic outcomes: None

Time points: Baseline and 3-month follow-up

(*: primary outcomes of the study; $§$ : outcomes that we have not reported in this review) 
CPDS, was developed for Nepali context specifically and described in Bolton 2002

Additional information: www.controlled-trials.com/ISRCTN48004304/

ISRCTN48004304

Handling the data: As per footnotes in data and analysis

Prospective trial registration number: SRCTN48004304

Risk of bias

Bias

Random sequence generation (selection Low risk

bias)

\section{Support for judgement}

Quote: "Allocation to study conditions followed a three-step procedure. First, districts were randomly allocated to either $\mathrm{CBI}$ or control condition (2 CBI districts, 2 waitlist districts). Second, two schools per district were randomly selected from a list of all eligible schools. Exclusion criteria for schools were (a) schools in Village Development Committees (VDC; the smallest administrative unit in Nepal) where CBI had already been implemented and schools in adjoining VDCs to avoid contamination; (b) schools in parts of the district with large geographic or ethnic differences compared to the majority of the district to increase group homogeneity within districts. Third, children were randomly selected from a list of all children aged 1114 years in the school. The randomisation was done, without imposing a randomisation constraint, by use of computer-generated random numbers (in SPSS) by the research team in Amsterdam. Out of 53 eligible schools, 8 were randomly selected with a total of 1367 eligible children of whom 149 were absent and 30 refused"

Allocation concealment (selection bias) High risk
Quote: "Randomisation was done, without imposing a randomisation constraint, by use of computer-generated random numbers (in SPSS) by the research team in Amsterdam"

Comment: Schools, districts and students randomised through computer-generated random numbers by research team in Amsterdam but still not clear whether at the point of allocation whether the allocation concealed 
Blinding of participants and personnel Low risk (performance bias)

All outcomes
Comment: School children and teachers could not be blinded due to nature of intervention. But outcomes unlikely to be affected by blinding

Blinding of outcome assessment (detection Low risk bias)

objective outcomes

Blinding of outcome assessment (detection High risk bias)

subjective outcomes

Comment: No objective outcomes

Comment: Research assistants not blinded to treatment condition; interviewed children's self report (children not blinded to treatment condition)

Baseline outcome measurements similar Unclear risk

Baseline characteristics similar? Low risk

Low risk

Incomplete outcome data (attritio
Safety data (e.g. adverse events)

Incomplete outcome data (attrition bias) Low risk Efficacy data

Comment: Lost to follow-up at T2, 2 in treatment group, 0 in wait-list control group

Comment: Report that no significant baseline differences between boys and girls on outcomes but data not presented between control and intervention groups. Baseline outcome measures seem similar between both groups (table 2) except perhaps SCARED, physical aggression and prosocial behaviour. In addition, noted in limitations that SCARED reliability between assessors was poor, so may not be reliable

Comment: Baseline differences in gender, ethnicity, religion, place of residence and level of education which were adjusted for

Information from author: "There were no adverse outcomes"

$\begin{array}{ll}\text { Protection against contamination Low risk } & \text { Lon }\end{array}$

Low risk
Comment: Cluster design which is unlikely to lead to contamination and waitlist control

Quote: "Internal reliability of some of the instruments was low, especially for the SCARED-5, which hampers pre-post intervention comparisons"

Comment: Inter-rater reliability between assessors was 0.891 for dichotomous items and 0.972 for continuous 
Jordans 2010 C-RCT Nepal (Continued)

\begin{tabular}{l|l} 
Selective reporting (reporting bias) Low risk
\end{tabular}

Comment: 2 secondary outcomes reported in protocol are not reported in results (self efficacy and daily functioning)

Author response: "With regards to the secondary outcomes; (a) 'daily functioning' has been included in the paper but has been renamed as 'functional impairment' (following the paper that was written on the development and validation of that scale); (b) 'self-efficacy' was included in the protocol, but no instrument was found with sufficient cross-cultural validity. As a result we have opted to include a 'coping scale (KIDCOPE)', which was not included in the reporting because of unforeseen problems with the analyses (i.e. we were not able to adequately analyse the combined response format of dichotomous and ordinal scales per respondents of the KID-COPE)"

Comment: Good explanation

Comment: ICC done and adjustment for clustering

\section{Li 1989 RCT China}

\begin{tabular}{ll} 
Methods & $\begin{array}{l}\text { Study design: RCT } \\
\text { Duration of study: April-August } 1986\end{array}$ \\
\hline
\end{tabular}

Participants

Country: China

Income classification: Upper middle

Geographical scope: Rural. The trial conducted in 2 provinces:

1. Beijing: Bei Cangxiang Township, Da Xing County in Beijing. It is $40 \mathrm{~km}$ from the downtown areas of Beijing. The local resident had middle-level living standard

2. Si Chuan Province: 3 townships (Shi Jian, Feng An, and Jian Xin) in Ren Shou County. These townships were remote hilly terrain, which are $30 \mathrm{~km}$ from the county town and transportation not convenient. The local resident's living standard was low and medical condition was poor

Healthcare setting: Community. Epilepsy patients identified through door-to-door visits by village doctors

Mental health condition: Epilepsy patients

Population (mention if patient, carer or dyads): Epilepsy patients

- Age: 4-64 years

- Gender: Male 21, female 19

- Socioeconomic background: 24 patients were from poor remote rural area and 16 patients were from middle-level rural area

- Inclusion criteria: 1 . Athermal (primary or secondary) systemic rigidity clonus type grand mal epilepsy; 2. epileptic seizure more than 3 times within 3 months before 
Li 1989 RCT China (Continued)

enrolment, and at least 1 time that someone witness

- Exclusion criteria: 1 . Seizures during pregnancy; 2. febrile seizure; 3. weight $<10$ $\mathrm{kg} ; 4$. $<2$ years old; 5. progressive disease of the nervous system; 6. serious mental disorder or mental deficiency; 7. attention deficit hyperactivity disorder (ADHD); 8. diseases of heart, liver or kidney, or severe hypertension; 9. history of status epilepticus; 10. undergoing regular western medicine treatment based on psychiatrist judgements; 11. epileptic seizure related to alcohol or drug dependence

Interventions

Stated purpose: To compare the effectiveness of epilepsy treatment regimen provided by trained village doctors with treatment by psychiatrists, as well as patient's reliance on village doctors with psychiatrists

INTERVENTION:

Name: Standard epilepsy treatment regimen provided by village doctors

Delivered by NSHW

- Title/name of NSHW/OPHR and number: Trained village doctors

- Selection: Selected village doctors and trained for 3-5 days

- Educational background: Not mentioned

- Training (contents, duration and by whom): 3-5 days training on the standard treatment regimen and how to deal with common side effects

- Supervision: Not mentioned

- Incentives/remuneration: Not mentioned

Intervention details:

- Duration/frequency: Treatment regimen was 3-4 months, and follow-up the patients once every 2 weeks

- Content of intervention: Village doctors identified patients through door-todoor visits. Their diagnosis was then checked by a psychiatrist. Village doctors then provided standard regimen of phenobarbital for epilepsy

CONTROL: Psychiatrists provided phenobarbital treatment and can adjust the dosage according to the patients disease severity

CO-INTERVENTIONS: None

Outcomes

Patient: Number of epileptic seizure per month*; adverse events; treatment adherence $₫$ (number of patients taking medicine according to prescription, number of patients with return visit on time)

Carer: None

Process/health worker outcomes: None

Economic outcomes: None

(*: primary outcomes of the study; $\$$ : outcomes that we have not reported in this review)

Time points: Baseline, 3 months, 4 months

Notes

Source of funding: WHO

Notes on validation of instruments (screening and outcomes): None

Additional information (e.g. provide by authors, existence of a published study protocol): No study protocol so unable to check primary and second outcomes

Handling the data: As per footnotes in data and analysis

Prospective trial registration number: None (only feasibility trial)

Risk of bias

Non-specialist health worker interventions for the care of mental, neurological and substance-abuse disorders in low- and middle- 


\section{Li 1989 RCT China (Continued)}

\begin{tabular}{|c|c|c|}
\hline Bias & Authors' judgement & Support for judgement \\
\hline $\begin{array}{l}\text { Random sequence generation (selection } \\
\text { bias) }\end{array}$ & Unclear risk & $\begin{array}{l}\text { Comment: Not specified how the random } \\
\text { sequence generated }\end{array}$ \\
\hline Allocation concealment (selection bias) & Unclear risk & Comment: Not specified \\
\hline $\begin{array}{l}\text { Blinding of participants and personnel } \\
\text { (performance bias) } \\
\text { All outcomes }\end{array}$ & Low risk & $\begin{array}{l}\text { Comment: Patients were not blinded as } \\
\text { it compared the phenobarbital treatment } \\
\text { provided by different health providers }\end{array}$ \\
\hline $\begin{array}{l}\text { Blinding of outcome assessment (detection } \\
\text { bias) } \\
\text { objective outcomes }\end{array}$ & Low risk & $\begin{array}{l}\text { Comment: Not specified whether outcome } \\
\text { assessors blinded or not, but the outcomes } \\
\text { were mainly objective outcomes }\end{array}$ \\
\hline $\begin{array}{l}\text { Blinding of outcome assessment (detection } \\
\text { bias) } \\
\text { subjective outcomes }\end{array}$ & Low risk & Comment: None \\
\hline Baseline outcome measurements similar & Unclear risk & $\begin{array}{l}\text { Comment: Not reported if the baseline } \\
\text { outcome measurements were similar }\end{array}$ \\
\hline Baseline characteristics similar? & Unclear risk & $\begin{array}{l}\text { Comment: Not clear about whether base- } \\
\text { line characteristics substantially different } \\
\text { between } 2 \text { groups }\end{array}$ \\
\hline $\begin{array}{l}\text { Incomplete outcome data (attrition bias) } \\
\text { Efficacy data }\end{array}$ & Low risk & Comment: $100 \%$ follow-up \\
\hline $\begin{array}{l}\text { Incomplete outcome data (attrition bias) } \\
\text { Safety data (e.g. adverse events) }\end{array}$ & Low risk & $\begin{array}{l}\text { Quote: " } 2 \text { person-time were not visit doc- } \\
\text { tor on time" } \\
\text { Comment: Safety outcome measures ob- } \\
\text { tained for more than } 80 \% \text { of subjects ran- } \\
\text { domised }\end{array}$ \\
\hline Protection against contamination & Low risk & Comment: Community randomisation \\
\hline Reliable primary outcome measures & Unclear risk & $\begin{array}{l}\text { Comments: Reliability is not reported for } \\
\text { outcome measures }\end{array}$ \\
\hline Selective reporting (reporting bias) & Low risk & $\begin{array}{l}\text { Comment: No protocol. Author suggests } \\
\text { all outcomes reported }\end{array}$ \\
\hline Other bias & Low risk & Comment: None \\
\hline
\end{tabular}


- Age: 6-11 and 12-17 years recruited in equal proportions for each age range in intervention group

- Gender: Both

- Socioeconomic background: Conflict area, previous studies report $93 \%$ of children report not feeling safe, $45 \%$ had personal experience with violence from conflict, tension in territories and subject to military incursions, curfews, and restricted movement of populations

- Inclusion criteria: Children were recruited to the study at the time of registering for programme activities when they commenced in 2003

- Exclusion criteria: Not specified dren exposed to political conflict involving structured activities, supported by provision of equipment and training of facilitators

INTERVENTION:

Name: Structured activities for children

\section{Delivered by:}

- Title/name of NSHW/OPHR and number: Local young adult volunteers (number not specified)

- Selection: Not specified

- Educational background: Not specified

- Training: Training facilitated and funded by 2 NGOs. The content and duration of the training is not specified

- Supervision: Not specified

- Incentives/remuneration: Not specified Intervention details:

- Duration/frequency: Week nights and weekend, during school holidays there were week-long camps, duration unspecified

- Content of intervention: "The interventions sought to support the resilience of children living in this situation, and principally addressed this by enabling (through provision of equipment and training) the delivery of structured activities." "The focus was to provide children and their parents with greater opportunities to participate in recreational, cultural and other non-formal activities in a safe setting. The children's activities included after-school recreation activities in a community setting, 'connectivity' activities (e.g., summer camps, using the internet to put children in touch with other children in different settings, etc.), and for one of the nongovernment organisations, the establishment of 'safe play' areas (Loughry and Ager, 
2004). The activities for the children's parents included information classes as well as opportunities to join with their children in structured recreational activities." But also both organisations had different emphases and time frames: "Both non-government organisations trained local young adult volunteers in how to conduct structured activities for children. Emphasis was given to cultural and recreational activities, such as traditional dancing, art work, sports, drama and puppetry, though after-school educational activities were also covered. Once trained, these volunteers facilitated these activities in local recreation centres on week nights and weekends. In school holidays, these activities formed the basis of week-long holiday camps. The training and material for these activities were facilitated by funding from the two non-government organisations. In addition, one of the non-government organisations focused on activities that were designed to increase the children's sense of 'connectivity' with Palestinian children in other geographical areas as well as with children living abroad. This was done through the provision of computers with internet access and training in the use of the internet, as well as organised outings to other community centres. The other non-government organisation emphasised the development of 'safe' outdoor settings. These settings were playgrounds equipped with recreation equipment and supervised by adults"

CONTROL: Usual care, comparison group sites, families receiving non-psychosocial services (e.g. water and sanitation assistance) from same 2 NGOs as in intervention group

CO-INTERVENTIONS: See above, the interventions provided at the 2 NGO locations differed - in addition to the base cultural/recreational activity intervention, 1 focused on activities designed to increase connectivity with other geographical areas through computers, and 1 emphasised safe outdoors settings with playgrounds/recreational equipment

Outcomes

Patient:CBCL (parent report), Hopefulness (component of the Child Adolescent Measurement Scale; child report), PSS (child report)

Carer: n/a

Process/health worker outcomes: Not reported

Economic outcomes: None

Time points: Baseline and 12 months

Notes

Source of funding: Bill \& Melinda Gates Foundation

Notes on validation of instruments: CBCL taken from University of Vermont where it had been translated and used before in Arabic; PSS was designed for Palestinian population and validated (Khamis 2000), unspecified for Child Adolescent Measurement Scale (Khamis 2000)

Additional information: No protocol found

Handling the data: As per footnotes in data and analysis

Prospective trial registration number: None given

Risk of bias

\begin{tabular}{|c|c|c|}
\hline Bias & Authors' judgement & Support for judgement \\
\hline $\begin{array}{l}\text { Random sequence generation (selection } \\
\text { bias) }\end{array}$ & High risk & Comment: Non-randomised intervention \\
\hline
\end{tabular}

Non-specialist health worker interventions for the care of mental, neurological and substance-abuse disorders in low- and middle- 


\section{Loughry 2006 CBA Palestin (Continued)}

\begin{tabular}{|c|c|c|}
\hline Allocation concealment (selection bias) & High risk & Comment: CBA study \\
\hline $\begin{array}{l}\text { Blinding of participants and personnel } \\
\text { (performance bias) } \\
\text { All outcomes }\end{array}$ & Unclear risk & $\begin{array}{l}\text { Comment: Unspecified if personnel were } \\
\text { blinded }\end{array}$ \\
\hline $\begin{array}{l}\text { Blinding of outcome assessment (detection } \\
\text { bias) } \\
\text { objective outcomes }\end{array}$ & Low risk & Comment: No objective outcomes \\
\hline $\begin{array}{l}\text { Blinding of outcome assessment (detection } \\
\text { bias) } \\
\text { subjective outcomes }\end{array}$ & High risk & $\begin{array}{l}\text { Quote: "The interviewers had purposefully } \\
\text { not been informed of the goals of the in- } \\
\text { tervention" } \\
\text { Comment: Child and parental report of } \\
\text { outcomes (not blind to intervention) as } \\
\text { part of interview done by interviewers un- } \\
\text { informed of intervention goals }\end{array}$ \\
\hline Baseline outcome measurements similar & High risk & $\begin{array}{l}\text { Comment: Intervention group had higher } \\
\text { hopefulness and PSS scores than compari- } \\
\text { son at baseline (P value }<0.01)\end{array}$ \\
\hline Baseline characteristics similar? & High risk & $\begin{array}{l}\text { Quote: "Intervention and comparison } \\
\text { groups were broadly well matched in terms } \\
\text { of the five outcomes measures. Adjusting } \\
\text { for alpha at } .05 / 514.01 \text {, it was found } \\
\text { that there was no difference in the CBCL } \\
\text { Total, Internalising or Externalising prob- } \\
\text { lem scores at baseline between the chil- } \\
\text { dren who subsequently took the interven- } \\
\text { tion and those who did not ( } \mathrm{F}(1,398) 14 \\
.00, \mathrm{p}>.01, \mathrm{~F}(1,398) 14.25, \mathrm{p}>.01 \text {, } \\
\text { and } \mathrm{F}(1,398) 141.08, \mathrm{p}>.01 \text {, respectively) } \\
\text {. However, the children in the intervention } \\
\text { group did begin with higher hopefulness } \\
\text { and PSS scores than those in the compari- } \\
\text { son group (F(1,396) } 1419.55, \mathrm{p}<.01 \text {, and } \\
\mathrm{F}(1,397) 1413.39, \mathrm{p}<.01, \text { respectively)" } \\
\text { Comment: Difference between interven- } \\
\text { tion and comparison groups }\end{array}$ \\
\hline
\end{tabular}

Incomplete outcome data (attrition bias) Unclear risk Efficacy data

Incomplete outcome data (attrition bias) Unclear risk Safety data (e.g. adverse events)
Comment: Not clear in report of data if there was lost to follow-up

Comment: No adverse outcomes reported 


\section{Loughry 2006 CBA Palestin (Continued)}

\begin{tabular}{|c|c|c|}
\hline Protection against contamination & High risk & $\begin{array}{l}\text { Comment: Other humanitarian efforts on- } \\
\text { going, including other CBI programmes } \\
\text { across Palestinian schools during study pe- } \\
\text { riod, risk of outside contamination (al- } \\
\text { though contamination between interven- } \\
\text { tion and control groups minimal) }\end{array}$ \\
\hline Reliable primary outcome measures & Low risk & $\begin{array}{l}\text { Comment: Good internal reliability re- } \\
\text { ported for PSS and Hopefulness scale and } \\
\text { tools validated (see reference under notes) }\end{array}$ \\
\hline Selective reporting (reporting bias) & Low risk & $\begin{array}{l}\text { Comment: Outcomes reported in methods } \\
\text { reported in results }\end{array}$ \\
\hline Other bias & Low risk & None reported \\
\hline \multicolumn{3}{|l|}{ Lyketsos1999CBA Argentina } \\
\hline Methods & \multicolumn{2}{|c|}{$\begin{array}{l}\text { Study design: CBA study (PC officers vs. psychiatric clinic) } \\
\text { Duration of study: Not mentioned but study done in } 1996 \text { published in } 1999\end{array}$} \\
\hline Participants & \multicolumn{2}{|c|}{$\begin{array}{l}\text { Country: Argentina } \\
\text { Income classification: Upper-middle income country } \\
\text { Geographical scope: Urban, in Buenos Aires. } \\
\text { Healthcare setting: PC (intervention) and psychiatric hospital practice (control) } \\
\text { Mental health condition: Major depressive disorder } \\
\text { Population: Adults } \\
\text { - Age: > } 18 \text { years (not specified; also included > } 60 \text { years) } \\
\text { - Gender: Both } \\
\text { - Socioeconomic background: Not specified } \\
\text { - Inclusion criteria: Quote: "Males and females older than } 18 \text { years; females with } \\
\text { childbearing potential with a negative pregnancy test who practiced successful } \\
\text { contraception for at least } 3 \text { months before entering the study; patients met DSM-IV } \\
\text { criteria for major depressive disorder (MDD); a score of at least } 10 \text { points on the 17- } \\
\text { item Hamilton Depression Rating Scale" } \\
\text { - Exclusion criteria: Women who were pregnant, lactating, or of childbearing } \\
\text { potential; not using reliable contraception; or who intended to become pregnant } \\
\text { within } 3 \text { months of study entry. Diagnosis of seizure disorder, organic brain disease, } \\
\text { malignancy, schizophrenia, psychotic disorder, anorexia, nervosa, or bulimia nervosa, } \\
\text { severe allergies or multiple adverse drug reactions by history, hypertensive patients } \\
\text { being treated with reserpine or alpha methyldopa, other clinically significant current } \\
\text { active medical disorder that would interfere with study participation, known } \\
\text { hypersensitivity to sertraline or lactose, history of alcoholism, drug abuse, personality } \\
\text { disorder, poor motivation, or other emotional problems likely to invalidate informed } \\
\text { consent, very high current suicidal risk, in the opinion of the treating physician }\end{array}$} \\
\hline
\end{tabular}

Non-specialist health worker interventions for the care of mental, neurological and substance-abuse disorders in low- and middle- 
Lyketsos1999CBA Argentina (Continued)

$\begin{array}{ll}\text { Interventions } & \text { Stated purpose: To compare the clinical profile of patients with major depression seen in } \\ \text { PC office practice with that of those seen in psychiatric office practice and to investigate } \\ \text { whether comparable treatment outcomes regarding depression remission can be achieved } \\ \text { in both settings, using a structured, open-label pharmacological intervention }\end{array}$

INTERVENTION 1:

Name: Response to sertraline (antidepressant) in PC

Delivered by

- Title/name of NSHW/OPHR and number: 113 PC physicians

- Selection: Volunteers to contribute cases to study

- Educational background: Physicians with a medical degree

- Training: All participating physicians received half a day of training by a boardcertified psychiatrist in the diagnosis of MDD and in the scoring of the HDRS. They were also provided with a checklist of depressive symptoms to assist in the diagnosis of MDD

- Supervision: By support staff "who visited the practices to provide medication supplies, answer design questions, and collect the data"

- Incentives/remuneration: Not specified Intervention details:

- Duration/frequency: Acute intervention; prescribing the drug sertraline and enhancing the dose. Protocol consisted of: "sertraline beginning at $50 \mathrm{mg}$ per day for 4 weeks. After 4 weeks (at follow-up Day 29), the treating physicians had the choice of increasing the dose of sertraline to $100 \mathrm{mg}$." There was no psychological intervention offered

- Content of intervention: Pharmacological intervention as above CONTROL: Specialist (gold standard); psychiatrist office practitioners who also were trained in using the protocol (using DSM-IV and HDRS, and then sertraline $50 \mathrm{mg}$ with an option to increase dose to $100 \mathrm{mg} 4$ weeks later)

CO-INTERVENTIONS: None

Outcomes

Patient: Clinical outcomes after treatment with antidepressants. The primary outcome measure was the HDRS*. Secondary outcome measures included rates and severity of adverse events, reasons for discontinuation $\$$, compliance $\$$ (> $80 \%$ of doses taken), and the number of patients who required antidepressant dose escalation at the day 29 visit $\$$ Carer: Not applicable

Process/health worker outcomes: None

Economic outcomes (and where these can be found e.g. ref or table number): Not done

${ }^{*}$ : primary outcomes of the study; $\$$ : outcomes that we have not reported in this review) Time points: The patients were seen for follow-up at days 8, 15, 29 and 56 after initiation of treatment

Notes

Source of funding: Grants from CEMIC and from the Pfizer Corporation.

Notes on validation of instruments: HDRS with a cut-off score of 10 was used; but not specified whether version used was locally validated or not

Additional information: Nothing of significance, no published protocol

Handling the data: As per footnotes in data and analysis

Prospective trial registration number: Not mentioned

\section{Risk of bias}

Non-specialist health worker interventions for the care of mental, neurological and substance-abuse disorders in low- and middle- 
Lyketsos1999CBA Argentina (Continued)

\begin{tabular}{|c|c|c|}
\hline Bias & Authors' judgement & Support for judgement \\
\hline $\begin{array}{l}\text { Random sequence generation (selection } \\
\text { bias) }\end{array}$ & High risk & $\begin{array}{l}\text { Quote: "This was a consecutive series of } \\
\text { patients who met the ... inclusion and ex- } \\
\text { clusion criteria" }\end{array}$ \\
\hline Allocation concealment (selection bias) & High risk & $\begin{array}{l}\text { Quote: "Comparative, open-label study of } \\
\text { patients" } \\
\text { Comment: No allocation concealment }\end{array}$ \\
\hline $\begin{array}{l}\text { Blinding of participants and personnel } \\
\text { (performance bias) } \\
\text { All outcomes }\end{array}$ & Unclear risk & $\begin{array}{l}\text { Comment: No blinding. No placebo, so } \\
\text { not sure if blinding would have had an im- } \\
\text { pact on outcomes }\end{array}$ \\
\hline
\end{tabular}

Blinding of outcome assessment (detection Low risk

Comment: Adverse events reported

bias)

objective outcomes

Blinding of outcome assessment (detection High risk bias)

subjective outcomes

Quote: "Provided with a checklist of depressive symptoms to assist in the diagnosis of MDD"

Comment: Standard instrument utilised and administered after training. However, these are administered by the clinician (physician or psychiatrist) so not blinded. So high risk

Baseline outcome measurements similar Low risk

Comment: Baseline primary outcome similar

Baseline characteristics similar?

High risk

Quote: "The patients in primary care were older by an average of 8 years, more likely to have active medical illnesses, less likely to be abusing alcohol, and less likely to have received prior treatment for depression during the present episode. The two groups were comparable on other variables" viz. gender and mean days in present episode

Incomplete outcome data (attrition bias) Low risk

Comment: Only about $15 \%$ dropout rate Efficacy data

Incomplete outcome data (attrition bias) Low risk Safety data (e.g. adverse events)

$\begin{array}{ll}\text { Protection against contamination } & \text { Unclear risk }\end{array}$

Comment: Adverse events reported

Comment: Exclusion criteria did not mention excluding participants seeking psychiatric treatment. So perhaps there was a risk

Non-specialist health worker interventions for the care of mental, neurological and substance-abuse disorders in low- and middleincome countries (Review)

Copyright $\odot 2013$ The Cochrane Collaboration. Published by John Wiley \& Sons, Ltd. 
Lyketsos1999CBA Argentina (Continued)

\begin{tabular}{|c|c|c|}
\hline & & $\begin{array}{l}\text { that patients could have been included in } \\
\text { both arms. This risk was low; however, it } \\
\text { is unclear whether patients could still have } \\
\text { seen a psychiatrist }\end{array}$ \\
\hline Reliable primary outcome measures & Low risk & $\begin{array}{l}\text { Quote: "The patients in the study were not } \\
\text { evaluated in a structured diagnostic exam- } \\
\text { nation. This would have been expensive in } \\
\text { this size study. To guard against diagnos- } \\
\text { tic inaccuracies, the physicians making di- } \\
\text { agnoses were trained in diagnostic assess- } \\
\text { ment for the study and in rating the Ham- } \\
\text { D [HDRS], and a minimal rating of } 10 \text { on } \\
\text { the Ham-D was required for study inclu- } \\
\text { sion" }\end{array}$ \\
\hline Selective reporting (reporting bias) & Unclear risk & $\begin{array}{l}\text { Comment: No selective reporting. but no } \\
\text { protocol, so check with authors to check } \\
\text { if pre-intervention specified outcomes have } \\
\text { been reported }\end{array}$ \\
\hline Other bias & Low risk & Comment: None detected \\
\hline
\end{tabular}

\section{Neuner 2008 NRCT Uganda}

Methods

Study design: Quasi-randomised, parallel group, assessor blinded, 3-armed, controlled clinical trial

Duration of study: 2003-2004

\begin{tabular}{l} 
Participants \\
$\begin{array}{l}\text { Country: Uganda } \\
\text { Income classification: Low } \\
\text { Geographical scope: Nakivale refugee settlements in Uganda for Somali and Rwandan } \\
\text { refugees; semi-rural (2 refugee camps close to base hospital) } \\
\text { Healthcare setting: Home-based care } \\
\text { Mental health condition: PTSD } \\
\text { Population: Patients } \\
\text { - Age: }>18 \text { years; mean age } 34-36 \text { years (SD 12-14 years) in the } 3 \text { groups } \\
\text { - Gender: Both } \\
\text { - Socioeconomic background: Refugees from Somalia and Rwanda } \\
\text { - Inclusion criteria: Fulfilling DSM IV criteria for PTSD (assessed using the PDS; } \\
\text { consent to participate } \\
\text { - Exclusion criteria: Drug abuse, obvious mental retardation; psychosis }\end{array}$ \\
\hline
\end{tabular}

Interventions

Stated purpose: To evaluate whether trained counsellors from the local afflicted population can effectively deliver a manual-based approach to counselling victims of civil war trauma, and to compare the structured manual-based approach with a more flexible approach or no specific intervention

\section{INTERVENTION 1:}

Name: NET; 111 people

Non-specialist health worker interventions for the care of mental, neurological and substance-abuse disorders in low- and middle- 
Delivered by: LHW (residents of refugee camps trained in counselling for the study)

- Title/name of NSHW/OPHR and number: Counsellors ( 9 in total; Somali and Rwandan refugees; 5 women, 4 men; mean age 27 years)

- Selection: Literacy in English and their mother tongue; ability to empathise with their clients; strong motivation

- Educational background: Secondary school (7); primary school (1); university (1)

- Training: 6 weeks of general counselling skills; NET and TC given by 5 post-doc and doctoral university personnel from Germany and Uganda; used the NET manual, and case discussions. 5 of the trainees had PTSD ( 3 lifetime, 2 current) and were given individual NET by trainees

- Supervision: Weekly case and personal supervision by trainers; treatment adherence monitored by case discussions during supervision, direct observation of treatment sessions, and review of patient testimonies and treatment protocols

- Incentives/remuneration: Not stated in this report Intervention details:

- Duration/frequency: 6 sessions (2 per week for 3 weeks); 1-2 hours' duration

- Content of intervention: Manualised, structured reconstruction of chronology of biography incorporating traumatic events into a coherent narrative; emphasis on reliving and describing emotional, physiological, cognitive and behavioural reactions to traumatic events; and habituation of reactions. Final narrative report (psychoeducation about PTSD in initial sessions; written rationale about relationship between PTSD and multiple past trauma; written chronological autobiography of traumatic experiences given to participant)

INTERVENTION 2:

Name: TC; 111 people

Delivered by: LHW (residents of refugee camps trained in counselling for the study; same as those who gave NET)

- Title/name of NSHW/OPHR and number: Counsellors ( 9 in total; Somali and Rwandan refugees; 5 women, 4 men; mean age 27 years)

- Selection: As above

- Educational background: As above

- Training: Flexible, less directive approach than NET; developed through discussions with trainees and by experienced senior counsellor from Uganda; training sessions focused on the psychological and social needs, conflicts and current life problems of clients; related current problems to past traumatic experiences; counsellors also trained in non-directive active listening; problems solving; exploring coping skills and grief interventions

- Supervision: Weekly supervision assisted by experienced senior Ugandan counsellor

- Incentives/remuneration: Not stated

Intervention details:

- Duration/frequency: 6 sessions (2 per week for 3 weeks) 1-2 hours' duration

- Content of intervention: Not manualised but used a flexible approach focusing on current psychological and social needs of clients; NET considered a part of this approach but was not mandatory. Psycho-education about PTSD in initial sessions; written rationale about relationship between PTSD and multiple past trauma developed; final report contained in mother tongue of participant the current and past

Non-specialist health worker interventions for the care of mental, neurological and substance-abuse disorders in low- and middle- 
Neuner 2008 NRCT Uganda (Continued)

problems discussed with the counsellor and possible solutions and coping strategies.

CONTROL: Monitoring group (no treatment) who were told they would be eligible for NET or TC if they proved effective; 55 people

CO-INTERVENTIONS: Not stated

Outcomes

Notes
Patient: 1. PDS (Foa 2005; contains 17 items of DSM IV for PTSD; translated and linguistically adapted; standard methods to translate and back-translate from Afsomali and Kinyaruwanda; (methods published separately); used to make DSM IV diagnoses of PTSD at baseline, 3 months and 6 months by 12 trained research assistants blind to allocation; 2. Expert evaluation: using PTSD section of the Composite International Diagnostic Interview (WHO 1997), by PhD level psychologists or graduate students (number not stated) at 9 months, blind to allocation; 3. Physical health checklist; sum of scores of symptoms of common illnesses over last 4 weeks (not validated)

Carer: Not applicable

Process/health worker outcomes: Not reported

Economic outcomes (and where these can be found, e.g. ref or table number): Not reported

Time points: Baseline for all: 3, 6 and 9 months for intervention groups; 3 and 9 months for monitoring group

Source of funding: German funding agencies (DFG; BMZ)

Notes on validation of instruments (screening and outcomes): Psychological outcomes validated; physical symptoms checklist not validated

Additional information (e.g. provide by authors, existence of a published study protocol): Translation of instruments published

Handling the data: As per footnotes in data and analysis

Prospective trial registration number: Not prospectively registered

\section{Risk of bias}

\section{Bias}

Random sequence generation (selection High risk bias)

\begin{tabular}{ll} 
Bias & Authors' \\
\hline $\begin{array}{l}\text { Random sequence generation (selection } \\
\text { bias) }\end{array}$ & High risk \\
\hline
\end{tabular}

\section{Authors' judgement}

\section{Support for judgement}

Quote from report: "The list of participants was ordered randomly; the first 4 were consecutively assigned to NET (narrative exposure therapy), TC (trauma counselling). NET, TC and the fifth was assigned to the MG (monitoring) group. This procedure was repeated until all 277 participants were assigned"

Comment: Alternate assignment; prone to prediction of next allocation and to high risk of bias; baseline imbalances in nationalities due to lack of stratification

Comment: Allocation not concealed; participants were approached at home and allocated treatments after randomisation; baseline imbalances in prognostic variables 
evident

Blinding of participants and personnel High risk (performance bias)

All outcomes

objective outcomes

Blinding of outcome assessment (detection Low risk bias)

subjective outcomes

Baseline outcome measurements similar Unclear risk
High risk
Comment: Open-label trial; group supervision of cases also precludes effective blinding; counsellors used both interventions, risk of contamination present, as well as of differential interventions

Comment: Outcome assessors were not aware of treatment allocation; no objective outcomes used

Comment: Outcome assessors were blind to allocation

\begin{tabular}{|c|c|c|}
\hline & & $\begin{array}{l}\text { tion of Somali and Rwandan refugees in in- } \\
\text { tervention groups with highest } \% \text { of Rwan- } \\
\text { dan nationals in monitoring group ( } 79 \%) \\
\text {, and lowest in NET group ( } 32 \%) \text { (P } \\
\text { value }<0.01 \text { ). Somali participants had more } \\
\text { trauma than Rwandan participants; analy- } \\
\text { ses in report adjusted for this difference but } \\
\text { is unlikely to have eliminated risk of bias }\end{array}$ \\
\hline $\begin{array}{l}\text { Incomplete outcome data (attrition bias) } \\
\text { Efficacy data }\end{array}$ & High risk & $\begin{array}{l}\text { Comment: Dropouts more than } 65 \% \text { in } \\
\text { all groups; significantly high differential } \\
\text { dropout rates }\end{array}$ \\
\hline $\begin{array}{l}\text { Incomplete outcome data (attrition bias) } \\
\text { Safety data (e.g. adverse events) }\end{array}$ & Low risk & Comment: No safety data reported \\
\hline Protection against contamination & High risk & $\begin{array}{l}\text { Comment: Contamination likely as same } \\
\text { therapists used NET and TC; NET was a } \\
\text { manualised treatment and TC is expected } \\
\text { to incorporate NET; but it is also possi- } \\
\text { ble that participants discussed treatments } \\
\text { among themselves in the refugee camps, } \\
\text { and further contaminated the fidelity of the } \\
\text { interventions }\end{array}$ \\
\hline
\end{tabular}

Non-specialist health worker interventions for the care of mental, neurological and substance-abuse disorders in low- and middle- 
Neuner 2008 NRCT Uganda (Continued)

\begin{tabular}{lll}
\hline Reliable primary outcome measures & Low risk & $\begin{array}{l}\text { Comment: Main psychological outcomes } \\
\text { used validated tools }\end{array}$ \\
\hline Selective reporting (reporting bias) & Unclear risk & $\begin{array}{l}\text { Comment: Trial not prospectively regis- } \\
\text { tered; protocol not available; yet we could } \\
\text { detect no evidence of selective reporting }\end{array}$ \\
\hline Other bias & Low risk & Comment: None detected \\
\hline
\end{tabular}

Noknoy 2010 RCT Thailand

\begin{tabular}{l|l}
\hline Methods & $\begin{array}{l}\text { Study design: RCT } \\
\text { Duration of study: } 2003-2004\end{array}$ \\
\hline Participants & $\begin{array}{l}\text { Country: Thailand } \\
\text { Income classification: Upper-middle } \\
\text { Geographical scope: Rural } \\
\text { Healthcare setting: PCUs - } 7 \text { in north-east Thailand and } 1 \text { in central Thailand } \\
\text { Mental health condition: Hazardous drinking } \\
\text { Population: Hazardous drinkers } \\
\text { - Age: } 18-65 \text { years } \\
\text { - Gender: Both, but majority (91\%) male } \\
\text { - Socioeconomic background: Predominantly have primary and secondary } \\
\text { education, married } \\
\text { - Inclusion criteria: } 18-65 \text { years old, AUDIT score } \geq 8 \\
\text { - Exclusion criteria: Alcohol-dependent patients (DSM-IV criteria), history of } \\
\text { liver disease, history or regular early morning drinking, recent extremely high } \\
\text { consumption }>120 \mathrm{~g} \text { for men or }>80 \mathrm{~g} \text { women), neurological and psychiatric } \\
\text { disorders, pregnant women, outside age range }\end{array}$ \\
\hline
\end{tabular}

Interventions

Stated purpose: Determine effectiveness of MET for hazardous drinkers in PCU settings INTERVENTION 1:

Name: MET

Delivered by

- Title/name of NSHW/OPHR and number: 8 nurses

- Selection: Nurses from each of the selected PCUs (only 1 nurse per PCU)

- Educational background: Nursing degree

- Training (contents, duration and by whom): 6 hours' training by a psychiatrist, and consisted of understanding the standard drink measurement, the stage of change and MET

- Supervision: Not specifically planned but nurses could contact main author (GP working in PC) by telephone for any difficulties or clarifications

- Incentives/remuneration: None Intervention details:

- Duration/frequency: 3 scheduled sessions: on day 1, at 2 weeks and at 6 weeks after the baseline evaluation. Each session comprised 15 minutes of counselling

- Content of intervention: Evaluation of the patient's ability to change his drinking habits according to the stage of change. For patients in the pre-contemplation

Non-specialist health worker interventions for the care of mental, neurological and substance-abuse disorders in low- and middleincome countries (Review)

Copyright $\odot 2013$ The Cochrane Collaboration. Published by John Wiley \& Sons, Ltd. 
stage, the main technique was feedback, using reflection and questioning skills to elicit self motivational statements. If change was contemplated, the study nurse would work with the patient's ambivalence using a pros and cons technique. At the same time, an empathic counselling style and encouragement of the patient's self efficacy were used to support change in drinking behaviour. Subsequently, each participant's readiness to change drinking behaviour was assessed. If in the determination stage, options on how to reduce drinking behaviour were provided

CONTROL: Patients without MET intervention, who were told that the trial focused on health behaviours, which included questions on smoking, exercise, eating behaviour, weight and alcohol use (to minimise intervention effect on health behaviour)

CO-INTERVENTIONS: None

Patient: AUDIT tool (for screening); outcome measures: health survey questionnaire that included amount of alcohol consumption in the previous week*, measured in 4 ways (mean drinking/per drinking day/previous week, hazardous drinking/drinking day/ previous week, mean drinking/per week, hazardous drinking/per week) and number of episodes of binge drinking in 7 days, frequency of accidents and traffic accidents and frequency of being drunk in the last month. GGT: blood test for evaluation of current drinking severity $\$$, and honesty/accuracy of patient information through collateral informant interviews $\S$

\section{Carer: None}

Process/health worker outcomes: None

Economic outcomes: None

$\left({ }^{*}\right.$ : primary outcomes of the study; $\$$ : outcomes that we have not reported in this review)

Time points: Baseline, 6 weeks (post-intervention), 3 months, 6 months

\section{Notes}

Source of funding: Thai Health Promotion Foundation

Notes on validation of instruments: AUDIT is validated but not the health survey

Additional information: Provided by authors for characteristics of NSHWs and intervention

Handling the data: As per footnotes in data and analysis

Prospective trial registration number: No protocol

\section{Risk of bias}

\begin{tabular}{|l|l|l}
\hline Bias & Authors' judgement & Support for judgement \\
\hline $\begin{array}{l}\text { Random sequence generation (selection } \\
\text { bias) }\end{array}$ & Low risk & $\begin{array}{l}\text { Quote: "The unit of randomisation was } \\
\text { the individual patient. Randomization of } \\
\text { subjects to the intervention and control } \\
\text { groups was carried out from the Coordi- } \\
\text { nating Centre in Phramong-Kutklao Hos- } \\
\text { pital in Bangkok using a standard randomi- } \\
\text { sation table. Each PCU had both control } \\
\text { and intervention groups. In order to keep } \\
\text { both groups of similar size, random alloca- } \\
\text { tion was done in blocks. On average, the } \\
\text { trial was to have 6-8 participants in each } \\
\text { study condition in each PCU" }\end{array}$ \\
\hline
\end{tabular}


Noknoy 2010 RCT Thailand (Continued)

\begin{tabular}{|c|c|c|}
\hline Allocation concealment (selection bias) & Low risk & $\begin{array}{l}\text { Quote: "Randomization codes were dis- } \\
\text { tributed to each PCU in sealed envelopes. } \\
\text { Eligible study participants were enrolled by } \\
\text { health personnel when subjects" }\end{array}$ \\
\hline $\begin{array}{l}\text { Blinding of participants and personnel } \\
\text { (performance bias) } \\
\text { All outcomes }\end{array}$ & Low risk & $\begin{array}{l}\text { Quote: "In order to minimize the in- } \\
\text { tervention effect of the research proce- } \\
\text { dures, the subjects randomised into the } \\
\text { control condition were told that the trial } \\
\text { focused on health behaviours, which in- } \\
\text { cluded questions on smoking, exercise, eat- } \\
\text { ing behaviour, weight and alcohol use. The } \\
\text { study interviewers at follow-up visits were } \\
\text { not aware of the assignment allocation of } \\
\text { the study participants" }\end{array}$ \\
\hline
\end{tabular}

Blinding of outcome assessment (detection Low risk

Comment: Less than $20 \%$ of those were bias) meant to have GGT test dropped out

objective outcomes

Blinding of outcome assessment (detection High risk

Comment: The tools were not validated bias)

subjective outcomes

\begin{tabular}{|c|c|c|}
\hline Baseline outcome measurements similar & Low risk & Comment: All similar \\
\hline Baseline characteristics similar? & Low risk & Comment: All similar \\
\hline $\begin{array}{l}\text { Incomplete outcome data (attrition bias) } \\
\text { Efficacy data }\end{array}$ & High risk & $\begin{array}{l}\text { Comment: There is }<20 \% \text { dropout rate in } \\
\text { intervention between baseline and last fol- } \\
\text { low-up; however, there is more than } 20 \% \\
\text { dropout rate in the control group. May af- } \\
\text { fect the outcomes. No mention about anal- } \\
\text { ysis of the control dropouts, therefore clas- } \\
\text { sify as high risk }\end{array}$ \\
\hline $\begin{array}{l}\text { Incomplete outcome data (attrition bias) } \\
\text { Safety data (e.g. adverse events) }\end{array}$ & High risk & $\begin{array}{l}\text { Information from author: "We didn't } \\
\text { look for the adverse event. However, I have } \\
\text { found that there was problems there were } \\
\text { some cases of participants that need to be } \\
\text { excluded as it was not originally detected" } \\
\text { Comment: they have not searched for ad- } \\
\text { verse events of the intervention }\end{array}$ \\
\hline Protection against contamination & Low risk & $\begin{array}{l}\text { Quote: "In order to minimize the in- } \\
\text { tervention effect of the research proce- } \\
\text { dures, the subjects randomised into the } \\
\text { control condition were told that the trial } \\
\text { focused on health behaviours, which in- }\end{array}$ \\
\hline
\end{tabular}

Non-specialist health worker interventions for the care of mental, neurological and substance-abuse disorders in low- and middle- 
Noknoy 2010 RCT Thailand (Continued)

\begin{tabular}{|c|c|c|}
\hline & & $\begin{array}{l}\text { cluded questions on smoking, exercise, eat- } \\
\text { ing behaviour, weight and alcohol use" } \\
\text { Comment: Also unlikely contamination } \\
\text { between groups as dispersed communities } \\
\text { (clinics) and not aware the intervention was } \\
\text { regarding alcohol necessarily }\end{array}$ \\
\hline Reliable primary outcome measures & High risk & $\begin{array}{l}\text { Comment: Many subjective outcomes but } \\
\text { GGT objective - but not always specific to } \\
\text { alcohol disease }\end{array}$ \\
\hline Selective reporting (reporting bias) & Low risk & Comment: All outcomes reported \\
\hline Other bias & High risk & $\begin{array}{l}\text { Comment: The trial showed an increase in } \\
\text { GGT at } 6 \text { months in both groups possibly } \\
\text { because baseline data was collected just af- } \\
\text { ter'Kao Pansaa' a 3-month Buddhist retreat } \\
\text { where it is customary for people to avoid } \\
\text { wrongdoing including reducing drinking }\end{array}$ \\
\hline
\end{tabular}

Papas 2011 RCT Kenya

Methods

Study design: Randomised, gender-stratified, parallel group, open-label, controlled clinical trial

Duration of study: February to December 2009

Participants

\section{Country: Kenya}

Income classification: Low income

Geographical scope: Urban, HIV clinic affiliated with Moi Teaching and Referral Hospital, Eldoret, Kenya

Healthcare setting: Outpatient clinic

Mental health condition: Hazardous use of alcohol or binge drinking

Population: Patients

- Age: $\geq 18$ years

- Gender: Both

- Socioeconomic background: Not specified

- Inclusion criteria: $\geq 18$ years, enrolment as an AMPATH HIV outpatient attending the Eldoret clinic affiliated with Moi Teaching and Referral Hospital, hazardous or binge drinking criteria (score $\geq 3$ on the AUDIT-C, or more than 6 drinks per occasion at least monthly), any alcohol use in the past 30 days, being antiretroviral eligible or antiretroviral initiated in the past 12 months, spoken knowledge of Kiswahili, living within 1 hour's travelling distance from the clinic, no plans to move further away during the study period and being available during the weekly group time

- Exclusion criteria: Active psychosis or suicidal, attendance in the past year at an existing AMPATH alcohol peer support group or participation in the study's group CBT pre-pilot development 
Papas 2011 RCT Kenya (Continued)

Interventions

Stated purpose: To use CBT due to empirical evidence of success in reducing risky behaviours in African HIV-infected people, and its structured format that makes it feasible to train paraprofessionals

INTERVENTION 1:

Name: CBT, 42 people

Delivered by: OPHR

- Title/name of NSHW/OPHR and number: 2 CBT counsellor (1 male; 1 female)

- Selection: Knowledge of English and Kswahili; essays and role plays to assess empathy, emotional perceptiveness; good communication skills and analytical abilities; met certification criteria for CBT training (adherence and competence)

- Educational background: High-school

- Training (contents, duration and by whom): Trained by study personnel; 175 hours of training; classes, role plays, videotaped feedback with medical students as simulated patients; assessment of adherence and competency using the YACS

- Supervision: 300 hours of supervision prior to trial; during the trial, all CBT group sessions were videotaped and monitored weekly by 1 experienced CBT supervisor. Supervision was conducted via telephone during the latter stages of trial. $50 \%$ of sessions with men and women, respectively (18 sessions) were selected randomly, translated into English, with random back-translation verification, and rated by 2 highly experienced YACS raters from the Yale Psychotherapy Development Center

- Incentives/remuneration: Not stated Intervention details:

- Duration/frequency: 6 weekly, gender stratified 90-minute group CBT sessions; 7 participants per group delivered by same-sex CBT counsellor

- Content of intervention: Manual-based CBT. Abstinence from alcohol was set as goal and a quit date was decided during the second session; behavioural analysis; risky behaviours and alcohol refusal skills reinforced

CONTROL: Routine medical care provided by the clinic (33 people)

CO-INTERVENTIONS: Not reported

Outcomes

Patient: Percentage of drinking days*; mean drinks per drinking days; abstinence at longest follow-up $\$$; adherence to CBT sessions $\$$

Carer: Not applicable

Process/health worker outcomes: Adherence and competence to CBT

Economic outcomes: Not reported

(*: primary outcomes of the study; $\$$ : outcomes that we have not reported in this review)

Time points: 30 days; 60 days; 90 days post treatment

Notes

Source of funding: National Institute on Alcohol Abuse and Alcoholism-funded grant (R21AA016884) USAID-AMPATH Partnership from the United States Agency for International Development (President's Emergency Plan for AIDS Relief and P50DA09241)

Notes on validation of instruments (screening and outcomes): Validated outcome tools

Additional information (e.g. provide by authors, existence of a published study protocol): Not applicable

Handling the data: As per footnotes in data and analysis

Prospective trial registration number: Clinicaltrials.gov identifier: NCT00792519

Non-specialist health worker interventions for the care of mental, neurological and substance-abuse disorders in low- and middle-

income countries (Review)

Copyright $\odot 2013$ The Cochrane Collaboration. Published by John Wiley \& Sons, Ltd. 
Papas 2011 RCT Kenya (Continued)

\section{Risk of bias}

\begin{tabular}{|c|c|c|}
\hline Bias & Authors' judgement & Support for judgement \\
\hline $\begin{array}{l}\text { Random sequence generation (selection } \\
\text { bias) }\end{array}$ & Low risk & $\begin{array}{l}\text { Quote: "A stratified simple randomization } \\
\text { procedure was used to form gender-strati- } \\
\text { fied cohorts. Within gender-based cohorts, } \\
\text { participants were assigned randomly until a } \\
\text { minimum was achieved of seven CBT and } \\
\text { five usual care participants, thereby creat- } \\
\text { ing some waiting time. A group of seven } \\
\text { was required for CBT to enhance participa- } \\
\text { tion, while fewer were required for the in- } \\
\text { dividual usual care condition to minimize } \\
\text { waiting time before treatment initiation" }\end{array}$ \\
\hline Allocation concealment (selection bias) & Low risk & $\begin{array}{l}\text { Quote: "Each participant was randomized } \\
\text { after she or he drew from a jar a paper with } \\
\text { the name of the condition. The papers were } \\
\text { prepared by study administrators to con- } \\
\text { ceal the name of the condition during the } \\
\text { drawing, which was supervised by staff" } \\
\text { Comment: Allocation was possibly con- } \\
\text { cealed }\end{array}$ \\
\hline
\end{tabular}

Blinding of participants and personnel Low risk

Comment: not blinded but also was not (performance bias) possible. No likely effect on outcomes

All outcomes

Blinding of outcome assessment (detection Low risk bias)

objective outcomes
Comment: Three alcohol saliva tests came back positive during treatment phase. This showed concordance with patient's self reported or scored outcomes

Blinding of outcome assessment (detection Unclear risk bias)

subjective outcomes

\begin{tabular}{ll}
\hline Baseline outcome measurements similar & Low risk \\
\hline Baseline characteristics similar? & Low risk \\
\hline $\begin{array}{l}\text { Incomplete outcome data (attrition bias) } \\
\text { Efficacy data }\end{array}$ & Low risk
\end{tabular}

Quote: "Non-blinded research assistants both recruited and interviewed participants; none delivered study interventions" Comment: Unlikely that if they did not deliver the intervention that there was much bias

Comment: All similar

Comment: All similar

Comment: $36 / 42$ completed intervention; $32 / 33$ stayed in control (completers), i.e. less than $20 \%$ drop outs 
Papas 2011 RCT Kenya (Continued)

\begin{tabular}{l|l|l}
\hline $\begin{array}{l}\text { Incomplete outcome data (attrition bias) } \\
\text { Safety data (e.g. adverse events) }\end{array}$ & Unclear risk & Comment: Not reported \\
\hline Protection against contamination & Low risk & $\begin{array}{l}\text { Comment: RCT occurring just in one } \\
\text { clinic. The lessons from CBT therapy could } \\
\text { therefore have been shared within the pop- } \\
\text { ulation between controls and those in CBT } \\
\text { intervention. However a large number of } \\
\text { people were enrolled at the clinic suggest- } \\
\text { ing its geographical remit is very wide }\end{array}$ \\
\hline
\end{tabular}

Reliable primary outcome measures Low risk

Quote 1: "An initial sample of six tapes was rated by two independent raters and indicated a high level of inter-rater reliability (mean intraclass correlation coefficients) across both adherence (mean $=0$. $98)$ and competence $($ mean $=0.95)$ "

Quote 2: "Overall level of drinking was low in the trial, i.e. at the 90-day followup $69 \%$ of CBT participants reported abstinence and PDD was 5\%. There were six positive saliva tests, three in CBT and three in usual care; five occurred during the treatment phase"

Comment: This latter statement suggests good correlation between physical objective findings (saliva tests) and the scored outcomes

Selective reporting (reporting bias) Low risk

Comment: All outcomes mentioned in methods section were reported. The trial was prospectively registered and primary outcomes were identical: quantity and frequency of alcohol use

\begin{tabular}{lll}
\hline Other bias & Low risk & Comment: None detected \\
\hline
\end{tabular}

\section{Paranthaman2010CBAMalaysi}

Methods

Study design: CBA study

Duration of study: Not specified which years of recruitment and intervention. Intervention length: 6 months

Participants

Country: Malaysia

Income classification: Middle

Geographical scope: Urban/semi-urban

Healthcare setting: Community psychiatric free-standing clinic

Mental health condition: Schizophrenia

Non-specialist health worker interventions for the care of mental, neurological and substance-abuse disorders in low- and middle- 
Population: Patient carer dyads

- Age: Patient mean age (SD): 41.5 years (14.2), carers: mean age (SD) 53.1 years (13.5)

- Gender: Both

- Socioeconomic background: Majority of carers and patients had above secondary education; half have a household income $<$ RM1000

- Inclusion criteria: Patients were well enough to be on follow-up in the community for long-term antipsychotic therapy. Carers also understood either Malay or English language

- Exclusion criteria: Carers who had co-morbidity of substance abuse or having uncontrolled or unstable medical illness requiring admission, and those who had already undergone a structured psychoeducation programme improving knowledge of carers, decreasing the carers' burden and reducing patient readmission rates as well as the rate of default to follow-up

\section{INTERVENTION 1:}

Name: 5 module psychoeducation programme for carers

Delivered by:

- Title/name of NSHW/OPHR and number: Health staff: medical assistants and staff nurses who were involved in the care of patients with schizophrenia

- Selection: Those who spoke English or Malay

- Educational background: Not specified

- Training: Workshop for health assistants on how to do psychoeducation for carers. Done by psychiatrists, and psychoeducation team members. Consists of 5 modules: understanding the illness, treatment, prevention of relapse, handling crisis and health life, diet and exercise

- Supervision: Supervised training done initially under care of specialist, after which the programme was carried out on their own. For the purpose of the study, fidelity testing was done to ensure compliance to the actual module

- Incentives/remuneration: Information from author: "No incentives were given other than training"

Intervention details:

- Duration/frequency: Delivered to carers over a period of 2 weeks

- Content of intervention: Psychoeducation delivered by health staff using audiovisual aids (e.g. PowerPoint presentations), charts or booklets. Carers encouraged to participate actively and ask for clarifications

CONTROL: Carers in the control group received standard treatment that consisted of history taking for symptoms of relapse, noting concomitant complaints, prescribing medication and giving appointment for patients. No additional formal psychoeducation was given for either patient or family in this group

CO-INTERVENTIONS: Standard medical treatment

Patient: DSM-IV: diagnosis*; default rates

Carer: Change in knowledge of carers $\$$ (pre-post test knowledge scores); change in carers burden (FBIS - which contains 5 sections: a. assistance in daily living: severity and burden; b. supervision: severity and burden; c. financial expenditure: severe debt, financial burden; $d$. impact on daily routine for past 1 month; e. worry

Process/health worker outcomes: Re-admission rates (relapse rates) 
Paranthaman2010CBAMalaysi (Continued)

Economic outcomes: None (apart from measuring FBIS financial burden (as above) (*: primary outcomes of the study; $\$$ : outcomes that we have not reported in this review) Time points: Baseline, 3 months, 6 months for all outcomes, and in addition post-test scores for knowledge of carers

Notes

Source of funding: National Institute of Health, Malaysia and the Perak State Health Department

Notes on validation of instruments (screening and outcomes): DSM-IV and FBIS validated (originals used, not translated)

Additional information (e.g. provide by authors, existence of a published study protocol): Not specified

Handling the data: As per footnotes in data and analysis

Prospective trial registration number: None

Risk of bias

\begin{tabular}{|c|c|c|}
\hline Bias & Authors' judgement & Support for judgement \\
\hline $\begin{array}{l}\text { Random sequence generation (selection } \\
\text { bias) }\end{array}$ & High risk & $\begin{array}{l}\text { Quote: "Clinics were assigned to the inter- } \\
\text { vention or control group allocation at the } \\
\text { onset (three clinics each), and subjects were } \\
\text { recruited in each clinic by convenient sam- } \\
\text { pling. No randomisation was done within } \\
\text { each clinic as researchers felt contamination } \\
\text { bias could not be adequately addressed if } \\
\text { both intervention and control subjects were } \\
\text { recruited from the same clinic. Interven- } \\
\text { tion clinic was chosen based on geograph- } \\
\text { ical accessibility to researchers" } \\
\text { Comment: Cluster sampling and conve- } \\
\text { nience sampling, i.e. is not random so it is } \\
\text { high risk }\end{array}$ \\
\hline
\end{tabular}

Allocation concealment (selection bias) High risk

Comment: No information about allocation concealment. there probably is none as it was not randomised

Blinding of participants and personnel Low risk (performance bias)

Comment: Would not be possible to blind participants or personnel. Unlikely to affect

All outcomes outcomes

Blinding of outcome assessment (detection Low risk bias)

Comment: There are objective outcomes objective outcomes for patient (re-admission rate and defaulting from follow-up), which are reported for $>80 \%$ of people

Blinding of outcome assessment (detection High risk bias)

subjective outcomes

Quote: “Assessments were conducted by staff in the clinics, and hence not blinded to the group allocation status"

Non-specialist health worker interventions for the care of mental, neurological and substance-abuse disorders in low- and middleincome countries (Review)

Copyright $\odot 2013$ The Cochrane Collaboration. Published by John Wiley \& Sons, Ltd. 


\section{Paranthaman2010CBAMalaysi (Continued)}

\begin{tabular}{|c|c|c|}
\hline Baseline outcome measurements similar & Low risk & Comment: All similar \\
\hline Baseline characteristics similar? & High risk & $\begin{array}{l}\text { Quote: "As the intervention and con- } \\
\text { trol group differed significantly in gen- } \\
\text { der, household income and duration as a } \\
\text { caregiver, all subsequent analysis was done } \\
\text { within each group and not between groups" } \\
\text { Comment: The baseline characteristics are } \\
\text { dissimilar so cannot compare the groups }\end{array}$ \\
\hline $\begin{array}{l}\text { Incomplete outcome data (attrition bias) } \\
\text { Efficacy data }\end{array}$ & Low risk & $\begin{array}{l}\text { Quote: "There were five dropouts: two } \\
\text { were dropped due to pretest inadvertently } \\
\text { missed in the recruitment period, one pa- } \\
\text { tient passed away due to dengue fever mid- } \\
\text { way through the study, one caregiver de- } \\
\text { veloped stroke and was unable to care for } \\
\text { the patient and one caregiver was unable to } \\
\text { complete the study questionnaire as he was } \\
\text { untraceable." } \\
\text { Comment: Only } 5 / 109 \text { dropouts }(<20 \%) \\
\text { so low risk of affecting outcome data }\end{array}$ \\
\hline $\begin{array}{l}\text { Incomplete outcome data (attrition bias) } \\
\text { Safety data (e.g. adverse events) }\end{array}$ & Low risk & $\begin{array}{l}\text { Comment: There are defaulter rates and } \\
\text { readmission/relapse rates reported }\end{array}$ \\
\hline Protection against contamination & Low risk & $\begin{array}{l}\text { Information from author: "To ensure } \\
\text { no contamination, the intervention/con- } \\
\text { trol was carried out in different clinic pop- } \\
\text { ulations with different staff involved and } \\
\text { these clinics were also geographically physi- } \\
\text { cally separate. The control population were } \\
\text { not exposed to the intervention module to } \\
\text { our best knowledge" } \\
\text { Comment: In addition, control carers were } \\
\text { on a waiting list so there may not have been } \\
\text { that much curiosity to find out what was } \\
\text { going on in intervention group }\end{array}$ \\
\hline Reliable primary outcome measures & Low risk & $\begin{array}{l}\text { Comment: No inter-rater reliability re- } \\
\text { ported but low risk as validated tools }\end{array}$ \\
\hline Selective reporting (reporting bias) & Low risk & $\begin{array}{l}\text { Comment: All outcomes mentioned in } \\
\text { methods are reported in results. Not able } \\
\text { to source protocol }\end{array}$ \\
\hline Other bias & Low risk & Comment: None detected \\
\hline
\end{tabular}

Non-specialist health worker interventions for the care of mental, neurological and substance-abuse disorders in low- and middle- 
Methods

Participants
Study design: RCT (cluster trial - unit allocation - health facility (PHC or GP), analysis - individual)

Duration of study: April 2007 to September 2009

Country: India

Income classification: Lower-middle income country

Geographical scope: Urban and rural

Healthcare setting: PC facilities, i.e. all facilities with space and primary and privacy for LHCs regular outpatient clinics not involved in preliminary phases of the project. There were government PHC facilities and private GP settings

Mental health condition: Common mental disorders

Population: Patients

- Age: > 17

- Gender: Both

- Socioeconomic background: Predominantly female, married and one-third widower, nearly half of them $<1$ year of education or illiterate

- Inclusion criteria: 1 . Adults $>17$ years, speaking Konkani, Marathi, Hindi, English, need not need medical attention, did not have difficulty with hearing, speaking, cognition, not already screened in the previous weeks, not receiving intervention. 2. Those who screened positive for common mental disorders with the GHQ-12; with a previously validated cut-off of > 5) and who expected to be resident of Goa for subsequent 12 months

- Exclusion criteria: Had a cognitive or sensory impairment that made participation in the evaluation difficult. Not speaking Konkani, Marathi, Hindi or English

Interventions
Stated purpose: The MANAS trial aimed to test the effectiveness of an intervention led by LHCs in PC settings to improve outcomes of people with these disorders INTERVENTION:

Name: Collaborative stepped-care intervention - Phase 1 (12 government PHCs) and Phase 2 (12 private GP facilities)

Delivered by (NSHW or OPHR and title)

- Title/name of NSHW/OPHR and number: 1. LHC; 2. GP and PHC physicians

- Selection:LHC: A woman fluent in the local languages, have excellent communication skills and be available for consultations on a regular basis in the clinics; GP/PHC physician: those located at the selected facilities

- Educational background:LHC: Graduates - locally recruited, graduate nonmedical worker; GP/PHC physician: registered medical GP as per the a priori eligibility criteria

- Training:LHC: Training component included how to deliver the various treatments, including counselling skills, psychoeducation, yoga and IPT. Their training was based on a draft manual developed for the intervention. Duration: 2 months' training. Trained by research team. GP/PHC physician: half a day of training and given a manual

- Supervision: of LHCs and GPs/PHC physicians: Clinical specialist (psychiatrist) visited about once a month and was also available for consultation on the telephone to discuss cases

- Incentives/remuneration: Not mentioned Intervention details: 
- Duration/frequency: Both phases carried out consecutively between April 2007 and September 2009

- Content of intervention: LHCs provided psychoeducation: Psychoeducation taught patients strategies to alleviate symptoms, such as breathing exercises for anxiety symptoms and scheduling activities for symptoms of depression. Encouraging adherence to treatments for these disorders and providing information about social and welfare organisations when needed were other key components of psychoeducation. Individual (not group) IPT was also provided by the LHC as the psychological treatment of choice. Focus on interpersonal problems such as grief, disputes and role transitions. A minimum of 6 sessions, with an optimum of 8 and a maximum of 12 sessions, was offered to each eligible participant. Interpersonal psychotherapy was reserved only for patients who had moderate or severe common mental disorders, and was offered as an alternative to, or in addition to, antidepressant drugs for those who did not respond to antidepressant treatment. Physician/GP roles: prescribe antidepressants according to a protocol for moderate to severe depression (private GPs could prescribe their drug of choice, PHC doctors had to use available drug). The other key roles of the physicians were to encourage patients to meet the LHC, to avoid the use of unnecessary drugs, and to provide usual care for any co-existing physical health problems. Referral: Referral to the clinical specialist was reserved for patients who were assessed as having a high suicide risk at any stage, were unresponsive to the earlier treatments, posed diagnostic dilemmas, had substantial co-morbidity with alcohol dependence, had other associated substantial medical problems, or for whom the PC physician requested a consultation

CONTROL: Enhanced usual care: physicians and patients in usual care practices received screening results and were given the treatment manual prepared for PC physicians. Physicians were allowed to start treatments of their choice

CO-INTERVENTIONS: None

Outcomes

Patient: Screening: GHQ-12. Primary outcome: CIS-R*: generates 2 outputs: an ICD10 diagnosis derived from a computer algorithm and a total score indicating the overall severity of symptoms

Carer: None

Process/health worker outcomes: None

Economic outcomes: None

(*: primary outcomes of the study)

Time points: Baseline 0 and follow-up 6 months, 12 months

Notes

Source of funding: The MANAS project was funded by a Wellcome Trust Fellowship in clinical sciences

Notes on validation of instruments (screening and outcomes): Validated GHQ in Goan setting but not specified for the CIS-R

Additional information (e.g. provide by authors, existence of a published study protocol): Yes

Handling the data: As per footnotes in data and analysis

Prospective trial registration number: NCT00446407 
Patel 2010 C-RCT India (Continued)

Random sequence generation (selection Low risk bias)
Quote 1: "Facilities were stratified into three strata; urban with a visiting psychiatrist (VP), rural with a VP, rural without a VP. Two intervention and two control PHCs were selected at random from each stratum, using on-line software by the MANAS trial statistician (HW). A given seed number was used to enable the randomisation procedure to be reproduced. This guards against mis-allocation or changes in allocation at a later stage"

Quote 2: "For phase 1, 17 facilities in Goa met these inclusion criteria, of which 12 were randomly selected for inclusion in the trial. PHC facilities were first stratified by the presence or absence of a visiting psychiatrist and then randomised within four strata defined by size"

Quote 3: "12 of the 22 eligible GP facilities were randomly selected for phase 2 of the trial. The 12 GP facilities were randomised within two strata defined by size. For both phases, facilities were randomly allocated within each stratum to either the intervention or control arm using a 1:1 allocation ratio using a computer-generated randomisation sequence"

Allocation concealment (selection bias) Low risk

Blinding of participants and personnel Low risk (performance bias)

All outcomes

Quote 1: "Randomly allocating unique patient IDs [identification number] so that there is no association between the ID number and the facility identity"

Quote 2: "Assessing the efficacy of blinding (through asking assessors to guess which arm the participant is allocated to) at the end of the trial"

Comment: It was a cluster randomised trial but the non-blinding of participants was unlikely to affect the outcome

Quote: "Health assistant completes baseline CIS-R schedule"

bias)

objective outcomes

Blinding of outcome assessment (detection Low risk

bias)

subjective outcomes

Quote 1: "Masking of the research assessor maximised by; undertaking assessment at home; randomly allocating clinic identification numbers to patients so that there was

Non-specialist health worker interventions for the care of mental, neurological and substance-abuse disorders in low- and middle- 
Patel 2010 C-RCT India (Continued)

\begin{tabular}{|c|c|c|}
\hline & & $\begin{array}{l}\text { no association between their number and } \\
\text { identity of the facility; outcome assessment } \\
\text { by an independent association and under- } \\
\text { taking primary outcome assessment before } \\
\text { all assessment" } \\
\text { Quote 2: "Emphasizing to assessors that all } \\
\text { patients are receiving an intervention (not } \\
\text { specifying whether this is enhanced care or } \\
\text { Collaborative Stepped Care) and that there } \\
\text { is genuine equipoise about which is better. } \\
\text { but also: health assistant completes baseline } \\
\text { CIS-R schedule" }\end{array}$ \\
\hline Baseline outcome measurements similar & Low risk & $\begin{array}{l}\text { Quote 1: "We recorded little intra-cluster } \\
\text { correlation ( } 0.03 \text { ), and the coefficient of } \\
\text { variation (k) for prevalence of these disor- } \\
\text { ders at baseline in all patients who screened } \\
\text { positive was } 0.08 \text { " } \\
\text { Quote 2: "Although participants in the en- } \\
\text { hanced usual care group were more likely to } \\
\text { have depression, the proportion of patients } \\
\text { with these disorders according to ICD-10 } \\
\text { and mean CIS-R scores were similar" }\end{array}$ \\
\hline Baseline characteristics similar? & Low risk & $\begin{array}{l}\text { Quote 1: "Characteristics of patients dif- } \\
\text { fered by clinic type" } \\
\text { Quote 2: "Distribution of these disorders } \\
\text { between groups was similar; although par- } \\
\text { ticipants in the enhanced usual care group } \\
\text { were more likely to have depression, the } \\
\text { proportion of patients with these disor- } \\
\text { ders according to ICD-10 and mean CISR } \\
\text { scores were similar" } \\
\text { Comment: baseline characteristics were } \\
\text { dissimilar but adjusted for in the analysis }\end{array}$ \\
\hline $\begin{array}{l}\text { Incomplete outcome data (attrition bias) } \\
\text { Efficacy data }\end{array}$ & Unclear risk & $\begin{array}{l}\text { Quote: "1160 participants ( } 85 \%) \text { in the } \\
\text { collaborative stepped-care group and } 1269 \\
(88 \%) \text { in the control group completed the } \\
\text { 6-month outcome assessment" } \\
\text { Comment: Low risk at } 6 \text { months, but high } \\
\text { risk at } 12 \text { months: significant difference } \\
\text { in attrition between collaborative care and } \\
\text { control groups ( } 81 \% \text { vs. } 77 \% \text {; Palue = } 0 \text {. } \\
01) \text {, which may not be clinically significant } \\
\text { but nevertheless no stated reasons for this } \\
\text { variation in dropout }\end{array}$ \\
\hline
\end{tabular}

Non-specialist health worker interventions for the care of mental, neurological and substance-abuse disorders in low- and middle- 
Patel 2010 C-RCT India (Continued)

\begin{tabular}{|c|c|c|}
\hline $\begin{array}{l}\text { Incomplete outcome data (attrition bias) } \\
\text { Safety data (e.g. adverse events) }\end{array}$ & Low risk & $\begin{array}{l}\text { Quote 1: "No stopping rules are proposed } \\
\text { because serious adverse events are not ex- } \\
\text { pected in the trial since none of the treat- } \\
\text { ments being offered are experimental or as- } \\
\text { sociated with serious outcomes" } \\
\text { Quote 2: "There were seven serious adverse } \\
\text { events (three deaths and four suicide at- } \\
\text { tempts) in the collaborative stepped- care } \\
\text { group and } 12 \text { in the enhanced usual care } \\
\text { group (six deaths and six suicide attempts) } \\
\text {. None of the deaths were from suicide" }\end{array}$ \\
\hline Protection against contamination & Low risk & $\begin{array}{l}\text { Quote: "We do not anticipate a significant } \\
\text { risk of contamination, i.e. patients moving } \\
\text { from an Enhanced usual care control facil- } \\
\text { ity to an intervention facility, due to the ge- } \\
\text { ographical spread of facilities, and because } \\
\text { no publicity will be produced regarding the } \\
\text { availability of the intervention in other fa- } \\
\text { cilities" }\end{array}$ \\
\hline
\end{tabular}

Reliable primary outcome measures Low risk

Quote 1: "Process indicators assessing the fidelity and quality of the intervention were obtained from four sources: the separate clinical records maintained by the lay health counsellor and the clinical specialist, antidepressant use from the clinic records, and quality assessments done for every component of the intervention. Quality assessments for intervention components were made by direct observation or through transcripts of sessions, and were rated by senior clinicians. The only possible process indicator in the enhanced usual care group was antidepressant use"

Quote 2: "From results it seems like those who needed antidepressants got them, but those who were on IPT - most completed first session (95-98\%) but v [very] few completed the required minimal standard of 6 sessions (PHC: 33\%; GP: 0\%). As the intervention was not effectively delivered for psychotherapy it may be difficult to assess the outcomes in those receiving it (i.e. those with mild to moderate symptoms)"

Selective reporting (reporting bias) Low risk

Comment: No selective reporting

Non-specialist health worker interventions for the care of mental, neurological and substance-abuse disorders in low- and middleincome countries (Review)

Copyright $\odot 2013$ The Cochrane Collaboration. Published by John Wiley \& Sons, Ltd. 
Patel 2010 C-RCT India (Continued)

$\begin{array}{lll}\text { Other bias } & \text { Low risk } & \text { Comment: No other bias detected }\end{array}$

Rahman 2008 CRCT Pakistan

Methods

Participants
Study design: Cluster RCT single-blind study with 2 parallel groups (Unit of allocation - union council clusters, unit of analysis - individual)

Duration of study: Enrollment between April 2005 and March 2006

Country: Pakistan

Income classification: Low-income country

Geographical scope: Rural area of Pakistan where there was subsistence farming

Healthcare setting: Home

Mental health condition: Antenatal depression in 3rd trimester

Population:

- Age: $16-45$ years

- Gender: Female

- Socioeconomic background: $68 \%$ of the cases and controls were poor; nearly $40 \%$ of them relying on well without pump; $55 \%$ relied on the field for toilets and "subsistence farming, supplemented by one or more of the men serving in the armed forces or working as government employees, or as semi-skilled or unskilled labourers in the cities". "Male and female literacy rates are $79.6 \%$ and $48.6 \%$ respectively". "Infant mortality rates are 84 per 1000 live births"

- Inclusion criteria: Participants were women in the 40 Union Councils who were aged 16-45 years, married, and in their third trimester of pregnancy. They were enrolled from lists of participants compiled from official registers kept with the Lady Health Workers

- Exclusion criteria: Women with a diagnosed serious medical condition requiring inpatient or outpatient treatment, pregnancy-related illness (except for common conditions, such as anaemia), substantial physical or learning disability, and postpartum or other form of psychosis

Interventions

Stated purpose: To develop and deliver a psychological intervention to depressed mothers and their infants through non-specialist village-based health workers

INTERVENTION:

Name: Thinking healthy programme

Delivered by

- Title/name of NSHW/OPHR and number: 40 Lady Health Workers

- Selection: Existing staff in the union councils were trained to deliver the intervention

- Educational background: Completed secondary schools

- Training: 2-day workshop and 1-day refresher 3 months after the first training was all given by the study team psychiatrist. Here and now problem-solving CBT was used with a manual that used culturally appropriate illustrations. Included in the training were the 3 steps that helped in avoiding direct confrontation with the mothers and manage illiterate mothers

- Supervision: Research team meetings in which "health workers brainstorm for solutions and discuss their successes and failures in a supportive environment"

- Incentives/remuneration: Not mentioned 
Rahman 2008 CRCT Pakistan (Continued)

\section{Intervention details:}

- Duration/frequency: Session every week for 4 weeks in the last month of pregnancy, 3 sessions in the first postnatal month and nine 1 monthly sessions thereafter

- Content of intervention: 3-step approach: 1. identify unhealthy unhelpful thinking styles and behaviours, 2. replacing these with helpful or healthy thinking; 3 . activities and 'homework' to help mothers practice healthy thinking CONTROL: Enhanced usual care: "control clusters received an equal number of visits in exactly the same way as those in the intervention group, but by routinely trained Lady Health Workers (two for each Union Council)"

CO-INTERVENTIONS: None

Patient: Infant weight and height at 6 and 12 months $* \$$, maternal depression, exclusive breastfeeding $\$$, number of diarrhoeal episodes in the infants in the 2 weeks before interview $\$$, records of immunisation $₫$ (with or without up-to-date immunisation status) , use of contraception $₫$ and if both parents set aside time every day to play with their infant $\$$

Carer (mother): Structured clinical interview for DSM IV diagnosis (screening); HDRS (for outcomes); brief disability questionnaire $\$$; global assessment of functioning questionnaire; multidimensional scale for perceived social support $\$$

Process/health worker outcomes: None

Economic outcomes: None

(*: primary outcomes of the study; $\$$ : outcomes that we have not reported in this review) Time points: Baseline, 6 and 12 months

Source of funding: This research was funded by a career development fellowship awarded to Atif Rahman by the Wellcome Trust, UK

Notes on validation of instruments (screening and outcomes): Structured clinical interview for DSM IV diagnosis and HDRS are internationally validated, but not specified if validated for the Pakistani settings. Other mother outcome scales not validated. Child outcome tools validated

Additional information (e.g. provide by authors, existence of a published study protocol): Study protocol is not present

Handling the data: As per footnotes in data and analysis

Prospective trial registration number: ISRCTN65316374

\section{Risk of bias}

\section{Bias}

Random sequence generation (selection Low risk bias)

\section{Support for judgement}

Quote: "40 Union Councils in the two subdistricts of the study area. These subdistricts were geographically contiguous and ethnically, culturally, and socio-economically homogeneous. All the units were eligible for randomisation, which was done by an independent trial centre in Islamabad, before recruitment of participants. These administrative units were assigned by 
Rahman 2008 CRCT Pakistan (Continued)

random allocation with a table of random numbers by a researcher who was not involved in the study and who was unaware of the identity of the Union Councils. Lady Health Workers from each Union Council were enrolled to participate in the study before randomisation"

Comment: Adequate

Allocation concealment (selection bias) Low risk
Quote: "The interviewers were unaware of the allocation status of the Union Councils (because they had no contact with the team that did the randomisation), and we took care to ensure they remained so; none of the interviewers resided in the study area, and throughout the duration of the study they had no contact with the Lady Health Workers or any other health personnel in the study area. Mothers were asked not to tell the interviewers anything about their sessions with Lady Health Workers"

Comment: Adequate

Quote: "Mothers in the control clusters received an equal number of visits in exactly the same way as those in the intervention group, but by routinely trained Lady Health Workers (two for each Union Council). These health workers in both groups received monthly supervision, and were monitored by the research team to ensure that they were attending the scheduled visits. In practice, the Lady Health Workers seldom provide such structured and monitored care in the community. The control group thus received what would be regarded as ideal care, which we called enhanced routine care"

Blinding of outcome assessment (detection Low risk bias)

objective outcomes

Quote: “All infant outcomes were assessed by researchers unaware of the psychiatric status of the mother"

Comment: > 80\% subjects and deemed low risk

Blinding of outcome assessment (detection Unclear risk bias)

subjective outcomes
Quote: “The interviewers were unaware of the allocation status of the Union Councils (because they had no contact with the team that did the randomisation), and we took

Non-specialist health worker interventions for the care of mental, neurological and substance-abuse disorders in low- and middle- 
Rahman 2008 CRCT Pakistan (Continued)

\begin{tabular}{|c|c|c|}
\hline & & $\begin{array}{l}\text { care to ensure they remained so; none of } \\
\text { the interviewers resided in the study area, } \\
\text { and throughout the duration of the study } \\
\text { they had no contact with the Lady Health } \\
\text { Workers or any other health personnel in } \\
\text { the study area. Mothers were asked not to } \\
\text { tell the interviewers anything about their } \\
\text { sessions with Lady Health Workers" } \\
\text { Comment: Likely low risk, though a small } \\
\text { risk that mothers may have told aspects of } \\
\text { their interactions with LHWs to interview- } \\
\text { ers }\end{array}$ \\
\hline Baseline outcome measurements similar & Low risk & Comment: All similar \\
\hline Baseline characteristics similar? & Low risk & Comment: All similar \\
\hline $\begin{array}{l}\text { Incomplete outcome data (attrition bias) } \\
\text { Efficacy data }\end{array}$ & Low risk & $\begin{array}{l}\text { Comment: All outcomes stated they would } \\
\text { collect, are reported }\end{array}$ \\
\hline $\begin{array}{l}\text { Incomplete outcome data (attrition bias) } \\
\text { Safety data (e.g. adverse events) }\end{array}$ & Unclear risk & Comment: None mentioned \\
\hline Protection against contamination & Low risk & $\begin{array}{l}\text { Quote: "Normally one Basic Health Unit } \\
\text { provides primary health care for one Union } \\
\text { Council and all affiliated Lady Health } \\
\text { Workers work in villages within that Union } \\
\text { Council only. Supervision of health work- } \\
\text { ers takes place in the Union Council. Thus } \\
\text { the risk of contamination of the control } \\
\text { group with the intervention is negligible" }\end{array}$ \\
\hline Reliable primary outcome measures & Low risk & $\begin{array}{l}\text { Quote: "Growth data were converted into } \\
\text { SDs (Z scores) for weight and length with } \\
\text { Epi Info } 2002 \text { (version 3.4.1)" }\end{array}$ \\
\hline Selective reporting (reporting bias) & Low risk & Comment: No selective reporting \\
\hline Other bias & Low risk & $\begin{array}{l}\text { Comment: No other obvious sources of } \\
\text { bias }\end{array}$ \\
\hline
\end{tabular}

Rojas 2007 RCT Chile

Methods Study design: Single-blind parallel RCT

Duration of study: June 2004 to 2006

Participants Country: Chile

Income classification: Upper middle income

Non-specialist health worker interventions for the care of mental, neurological and substance-abuse disorders in low- and middleincome countries (Review)

Copyright $\odot 2013$ The Cochrane Collaboration. Published by John Wiley \& Sons, Ltd. 
Rojas 2007 RCT Chile (Continued)

Geographical scope: Urban-deprived urban area of Santiago

Healthcare setting: 3 PHC clinics

Mental health condition: Postnatal depression

Population: Women

- Age: mean (SD) 26.7 (SD 6.4)

- Gender: Female

- Socioeconomic background: Low-income women and majority were housewives

- Inclusion criteria: Scoring $\geq 10$ on EPDS at 2-week intervals and $\geq 18$ years women with children younger than 1 -year of age and who meet DSM-IV criteria for major depression

- Exclusion criteria: Women who received treatment for depression during their current postnatal period if they were pregnant, psychotic symptoms, serious suicide risks, history of mania, alcohol or drug abuse

Interventions

Stated purpose: Compared the effectiveness of a multicomponent intervention with usual care to treat postnatal depression in low-income mothers in primary care clinics in Santiago, Chile (protocol mentioned they would look at infant outcomes. There are no infant outcomes mentioned in the protocol or have they been reported in the results paper)

INTERVENTION:

Name: Multicomponent intervention

Delivered by

- Title/name of NSHW/OPHR and number: Physician doctor, group leaders (midwives) and nurse, and a designated trained non-professional person

- Selection: Doctor - from the PHC selected, group leaders and non-professionals not specified

- Educational background: Doctor - medical degree, group leaders and nonprofessionals - not specified

- Training: Group leaders - 8 hours of training. Non-professional - not specified

- Supervision: Doctor - 1 hour of supervision every week by research psychiatrist, group leaders - supervision every week by the doctor and the non-professionals - not specified

- Incentives/remuneration: Not specified Intervention details:

- Duration/frequency: The group sessions consisted of 1 session per week for 8 weeks (maximum 20 attendants with every session lasting 50 minutes). Women received medical appointments at 2 and 4 weeks and subsequently every month for the first 6 months

- Content of intervention (by types of health worker and per patient/ carers;doctor: Protocol: First choice of drug was fluoxetine $(20-40 \mathrm{mg} /$ day) but sertraline (50-100 mg/day) was also available for those who did not respond to fluoxetine or were breastfeeding. All medication was supplied free in both groups. Group leaders: Psychoeducation intervention, which consisted of information about symptoms and treatments, problem solving and simple behavioural activation, and cognitive techniques. All topics were presented with examples relevant to the postnatal period. Groups consisted of 1 session per week for 8 weeks (maximum 20 attendants), with every session lasting 50 minutes. The groups followed a structured format with every session covering something different but with plenty of time for sharing experiences. There was poor attendance of these psychoeducational sessions: "mean

Non-specialist health worker interventions for the care of mental, neurological and substance-abuse disorders in low- and middle- 
Rojas 2007 RCT Chile (Continued)

number of multicomponent intervention group sessions attended was 2.7 of eight (SD $3 \cdot 1$ ), and attendance was not associated with the EPDS score", "women taking medication attended slightly more sessions". Group leaders: they delivered the sessions but had no further contact with patients. Doctor was ultimately responsible for the group, non-professional: designated trained, non-professional person monitored attendance at consultations and group sessions and provided support and advice about antidepressant use following a structured format. If any problems were detected, patients were advised to see their doctors and some assistance was provided to obtain medical appointments sooner if deemed essential

CONTROL: Usual care: "Usual care included all services normally available in the clinics, including antidepressant drugs, brief psychotherapeutic interventions, medical consultations, or external referral for specialty treatment. Although all these options are potentially part of usual care, in reality medication and consultation remain the main treatment methods; psychotherapy and specialty referrals are rarely offered. Doctors in the usual care group were informed of the baseline assessment but no further information was provided"

CO-INTERVENTIONS: None

Outcomes

Patient: EPDS* - used twice for screening (2 weeks apart), MINI (used to establish inclusion and exclusion criteria in people screened twice, SF-36 - secondary outcome (has 4 dimensions - mental health, social functioning, emotional role and vitality)

Carer: n/a

Process/health worker outcomes: No process

Economic outcomes: None

(*: primary outcomes of the study)

Time points: Baseline, 3 and 6 months

Notes

Source of funding: Fondo Nacional de Desarrollo Científico y Tecnológico, Chile Notes on validation of instruments (screening and outcomes): All are validated but not specified if the EPDS and MINI were translated/validated in that setting

Additional information (e.g. provide by authors, existence of a published study protocol): None

Handling the data: As per footnotes in data and analysis

Prospective trial registration number: NCT00518830

Risk of bias

Bias

Random sequence generation (selection Low risk bias)
Authors' judgement

income countries (Review)

Copyright $\Subset 2013$ The Cochrane Collaboration. Published by John Wiley \& Sons, Ltd.

\section{Support for judgement}

Quote from the paper: "The clinics participating in the trial were chosen for practical reasons rather than randomly selected, which could affect the generalisability of our findings"

Comment: 1 . But these are deemed fairly representative of PHCs in deprived urban areas in Santiago;

2. The number were computer generated random numbers, 
Rojas 2007 RCT Chile (Continued)

therefore, low risk of bias

Allocation concealment (selection bias) Low risk

Blinding of participants and personnel Low risk (performance bias)

All outcomes
Quote: "Allocations were kept in numbered sealed envelopes in every clinic, opened by a person"

Comment: Neither control nor intervention groups were blinded for the intervention. The usual care group could receive medical consultation and so differential intervention were unlikely

Blinding of outcome assessment (detection Low risk

Comment: No objective outcomes used

bias)

objective outcomes

Blinding of outcome assessment (detection Low risk bias)

subjective outcomes

\begin{tabular}{l|ll}
\hline $\begin{array}{l}\text { Baseline outcome measurements similar } \\
\text { Baseline characteristics similar? }\end{array}$ & Low risk & $\begin{array}{l}\text { Comment: Yes, baseline outcome measure- } \\
\text { ment similar }\end{array}$ \\
\hline $\begin{array}{l}\text { Incomplete outcome data (attrition bias) } \\
\text { Efficacy data }\end{array}$ & Low risk & Comment: Yes: all were similar \\
\hline
\end{tabular}

Incomplete outcome data (attrition bias) Unclear risk

Safety data (e.g. adverse events)

Protection against contamination

Low risk
Comment: No information on safety data

Quote: "Staff recruiting patients were neither involved in nor aware of the procedure used to generate allocations"

Comment: Yes, baseline outcome measureComment: Yes: all were similar

Comment: $90 \%$ of women randomly asment in both groups but not adjusted for

Quote: "Since women in both groups attended the same centres, some degree of contamination could possibly have occurred, but we tried to reduce this possibility by allocating patients in each group to different doctors. Our previous experience with clinical trials in these settings shows that after a few weeks, the pressure of work is so intense that participating clinicians only remember things when constantly reminded. If there were contamination it would have been more likely in early stages of the study, which is when we found the largest differences, than in late stages" Comment: Though there is no wait-list control, they have tried to minimise con- 
Rojas 2007 RCT Chile (Continued)

tamination by allocating patients to different doctors. Unlikely to be that much contamination

Reliable primary outcome measures Low risk

Comment: Validated tools were used and were shown to be reliable in other studies including previous RCT (Araya 2003)

Selective reporting (reporting bias) Low risk

Comment: The trial prospectively registered. All pre-stated outcomes reported

Other bias

Low risk

Comment: No other bias

Scholte 2011 CBA Rwanda

Methods

Participants
Study design: CBA study

Duration of study: This study took place from October 2007 to September 2008, preceded by a pilot study over 2005-2006

Country: Rwanda

Income classification: Low-income

Geographical scope: Rural, Gicumbi district in northern Rwanda, post-genocide population

Healthcare setting: Community groups: sociotherapy groups were set up in the community after individuals enrolled in the programme

Mental health condition: Mental health outcomes post-war; high mean SRQ-20 scores in entire group at baseline; study also separately examines the $63 \%$ females and $37 \%$ males scoring above cut-off at baseline for common mental disorders (include depression and anxiety)

Population: Patient

- Age: $\geq 16$ years ; mean age 34.9 (range 16-76 years) intervention: mean age 38.5 years (range 16-73 years) control These values were for entire sample, rather than subset meeting cut-off at baseline

- Gender: Both

- Socioeconomic background: SES determined differently for control and intervention groups, interviewers scored SES by judging the state of the houses in person, intervention group: interviewed at the spot of their meetings and asked to describe the state of their houses themselves; placed into groups of marginal $(6 \%$ intervention vs. $13 \%$ control), poor ( $83 \%$ intervention vs. $66 \%$ control), and sufficient ( $11 \%$ intervention vs. $21 \%$ control), similar education characteristics $(50 \%$ had no schooling) This was in entire sample, rather than the subset meeting cut-off at baseline

- Inclusion criteria: From protocol: 1 . Within a 6-year period all areas of Byumba province were covered by the sociotherapy programme. The sequence of areas was dictated by matters of actual convenience, and determined by the programme's local counterpart; 2 . Group participants were aged $\geq 16$ years; 3 . The composition of groups was mixed (both sexes, various ethnic backgrounds, wide age distribution); no strict criteria for participation in a sociotherapy group existed

- Exclusion criteria: No exclusion criteria. Purposeful decision as people are paranoid there and would not open up before being within a group therapy session 
Scholte 2011 CBA Rwanda (Continued)

Interventions
Stated purpose: Aims to establish the effects of a therapeutic group intervention called sociotherapy, which is specifically tailored to traumatised survivors of systematic violence displaying a broad spectrum of affective and cognitive disturbances

INTERVENTION: Sociotherapy programme

Delivered by:

- Title/name of NSHW/OPHR and number: Trained Rwandan community

leaders, number not specified

- Selection: Local people

- Educational background: Familiar with region's history and current living situation

- Training: 3 months of training from Equator (Dutch agency) staff

- Supervision: "Regularly supervised" by Equator staff

- Incentives/remuneration: No fees, travel expenses reimbursed

Intervention details: Group therapy

- Duration/frequency: $10-15$ people in each group, 45 groups total, weekly meetings over 15 weeks, lasting 3 hours each

- Content of intervention: "The technique therapeutically uses interaction between individuals and their social environment to help subjects to reassess and redefine values, norms, relations and possible collaborations. The principal premise is that reaching a certain level of mutual respect, trust and care in group interaction helps to increase the problem solving capacity and subjective mental health in individual group participants....In non-clinical, international settings it is essential to continuously tailor it to the actual context and group (so the intervention is not strictly protocolised). Group leaders are allowed to attune their routines to the characteristics of their groups (e.g., degree of trust, nature of problems) and to their own affinity and experience, putting different emphases on elements like rules, role plays, and spirituality. For example, group leaders who are pastors may stimulate praying and singing, while teachers may encourage role plays and debate about social rules; others again may take a less active role, supporting the group to share experiences. There were some core principles, however, that all group leaders complied to: two-way communication, shared leadership, consensus in decision-making, and social learning through actual social interaction. Additionally, each subsequent phase of a group had a different focus, notably safety, trust, care, respect, rules and memories"

CONTROL: Selection of controls: to ensure equivalence at baseline with regard to SRQ-20 score, the following was done. "We identified five regions within Gicumbi district where the programme was not or had not been running so far, or for practical reasons would not start over the upcoming year. It could be assumed the inhabitants of these regions had experienced similar trauma exposure. Here, we randomly selected respondents through convenience sampling. Interviewers started at the top of a hill or in the centre of a village and each walked down a different footpath towards scattered houses or huts. An equal number of men and women, at home or in the fields, were randomly chosen and asked to participate. Finally 251 respondents were interviewed. After analysis of the data collected, we selected a group of 100 out of these for which the distribution of SRQ-20 scores matched that of the intervention group. For this purpose we used 8 clusters of scores $(0-1,2-3,4-5,6-7,8-9,10-12,13-15,16-20)$ and from each cluster randomly selected a number of respondents equal to the corresponding cluster in the experimental group. This final selection of 100 constituted our definite control 
Scholte 2011 CBA Rwanda (Continued)

group"

CO-INTERVENTIONS: None

Outcomes

Patient: SRQ-20 for common mental disorders

Carer: $\mathrm{n} / \mathrm{a}$

Process/health worker outcomes: Not assessed

Economic outcomes: None

Time points: Measurements were taken pre and post intervention and at 8 months'

follow-up

Notes

Source of funding: Partly by grant from Health Research Development Counsel, Department Prevention Programme (ZonMW), OOG- Geestkracht (ZonMW: 60-6010598-117), partly by Cordaid and partly by a Prins Bernhard Cultuurfonds grant

Notes on validation of instruments: WHO developed questionnaire, translated and back translated for the study, "Reliability was considered to be good (Cronbach's $a=0$. 83). The optimal cut-off point was $7 / 8$ for men and $9 / 10$ for women (manuscript under review). We also validated the SRQ-20 for its capacity to assess change in symptom severity over time. The instruments factor structure proved to be time invariant; the number of factors, factor loadings and covariances of factors remained equal over time" Additional information: www.trialregister.nl/trialreg/admin/rctview.asp? TC $=1120$ Handling the data (e.g. imputed values/other calculations we have made): None Prospective trial registration number: Nederlands Trial Register 1120

Risk of bias

\begin{tabular}{|c|c|c|}
\hline Bias & Authors' judgement & Support for judgement \\
\hline $\begin{array}{l}\text { Random sequence generation (selection } \\
\text { bias) }\end{array}$ & High risk & $\begin{array}{l}\text { Comment: CBA study; after enrolling par- } \\
\text { ticipants with interest or referral to the in- } \\
\text { tervention, } 45 \text { groups created (number not } \\
\text { specified), } 10 \text { groups selected out of conve- } \\
\text { nience balanced by gender and ratio, } 100 \\
\text { were randomly selected from } 133 \text { total par- } \\
\text { ticipants in the } 10 \text { groups, controls were } \\
\text { matched with the } 100 \text { based on SRQ-20 } \\
\text { score, gender and age }\end{array}$ \\
\hline Allocation concealment (selection bias) & High risk & Comment: CBA study \\
\hline
\end{tabular}

Blinding of participants and personnel Low risk (performance bias)

Comment: Personnel conducting intervention were not blinded, neither were participants. This is unlikely to affect outcomes

Blinding of outcome assessment (detection Low risk

Comment: No objective outcomes

bias)

objective outcomes

Non-specialist health worker interventions for the care of mental, neurological and substance-abuse disorders in low- and middle- 


\section{Scholte 2011 CBA Rwanda (Continued)}

\begin{tabular}{l|l|l}
$\begin{array}{l}\text { Blinding of outcome assessment (detection } \\
\text { bias) }\end{array}$ & Low risk & $\begin{array}{l}\text { Comment: Personnel conducting inter- } \\
\text { subjective outcomes }\end{array}$ \\
\hline
\end{tabular}

Baseline outcome measurements similar Low risk

Baseline characteristics similar?

Unclear risk
Comment: Participants were matched for outcome measure so no group differences observed $(\mathrm{P}$ value $=0.83)$

Comment: SES was significantly different between groups. This applied to entire sample only (not just those whom we report, i.e. those who are probable cases). Unclear for this group whether any group differences

Incomplete outcome data (attrition bias) Unclear risk Efficacy data

Incomplete outcome data (attrition bias) Low risk Safety data (e.g. adverse events)

\begin{tabular}{ll}
\hline Protection against contamination $\quad$ Low risk
\end{tabular}

Unclear risk

Unclear risk

Uncher risk
Comment: Loss to follow-up similar in intervention and control (19 of 100 in intervention vs 27 of 100 in control); however, control group follow-up was below $80 \%$, therefore, high risk

This applies to entire sample only. As above

Comment: No adverse outcomes observed

Comment: Control group were selected from 5 regions where programme was not running and would not start for the upcoming year

\begin{tabular}{l|l|l}
\hline Reliable primary outcome measures & Unclear risk & Comment: No kappas given \\
\hline Selective reporting (reporting bias) & Unclear risk & $\begin{array}{l}\text { Comment: In the protocol, it mentioned } \\
\text { several secondary outcomes that were not } \\
\text { reported (social functioning, as measured } \\
\text { by the Byumba Social Functioning Ques- } \\
\text { tionnaire. Social functioning will also be } \\
\text { measured with the MOS Social Function- } \\
\text { ing Scale 36 items (SF-36); Social capital, } \\
\text { as measured by use of the SA-SCAT. Alco- } \\
\text { hol use, as measured by use of the AUDIT- } \\
\text { C. IPV, as measured by using elements of } \\
\text { the CTS2S: the 'negotiation', 'psycholog- } \\
\text { ical aggression', 'physical assault' and 'in- } \\
\text { jury' scales }\end{array}$ \\
& &
\end{tabular}

$\begin{array}{lll}\text { Other bias } & \text { Low risk } \quad \text { Comment: None detected }\end{array}$

Non-specialist health worker interventions for the care of mental, neurological and substance-abuse disorders in low- and middle- 
- Gender: Both

- Socioeconomic background: Majority of participants rated low average and average economic status (as observed by interviewer based on housing conditions), majority had high-school education or more, control more high-school educated and intervention group more junior college participants

- Inclusion criteria: 3-6 year olds with IDs identified by teachers in kindergarten programmes or by records of community health clinics

- Exclusion criteria: Severe physical disability (such as microcephaly or severe physical disability too severe to receive intervention services)

Stated purpose: This study was conducted to examine the impact of a 1-year intervention for children with ID in Vietnam

\section{INTERVENTION 1:}

Name: Portage curriculum for preschool children

Delivered by:

- Title/name of NSHW/OPHR and number: 11 teachers

- Selection: Recruited from primary special education schools

- Educational background: 4 years of experience with children with intellectual/ developmental disabilities

- Training (contents, duration and by whom): 3 months of weekly training on Portage Program curriculum before intervention, monthly review sessions after intervention

- Supervision: Random visits by experienced supervisors; also monitoring done by parent who signed weekly sheets to testify of the teacher's visit

- Incentives/remuneration: Weekly payment upon receiving signed teaching objective from intervention session

Intervention details:

- Duration/frequency: Weekly 1-hour sessions over 1 year

- Content of intervention: "Typically teachers hold a 1-h session each week, which can be broken down into three small components. First, they review the homework assignment by having the parents demonstrate the previously assigned homework with their children. Second, teachers review one or two new teaching objectives they wrote with the parents and demonstrate the steps to achieve a desired behaviour by demonstrating the objectives. These new objectives become the newly assigned homework for the parents, who try them with their children and receive coaching and feedback on their work. Assurance of parent compliance in carrying out the programme was made by teachers, who reviewed the daily homework checklist parents completed and who observed parents demonstrating their work with their children during their next visit" 
CONTROL: Usual care (wait-list control). Peers who attended the kindergarten with no added parental training

CO-INTERVENTIONS: None

Outcomes

\section{Patient: 1984 VABS}

Carer: Not assessed

Process/health worker outcomes: Not assessed

Economic outcomes: None

Time points: Baseline, 6 months, 12 months

\begin{tabular}{|c|c|c|}
\hline Notes & \multicolumn{2}{|c|}{$\begin{array}{l}\text { Source of funding: Partially supported by funding from KFR-2005-J01702 in Korea and } \\
\text { a travel grant by the Center for International Rehabilitation and Research Information } \\
\text { Exchange in the US } \\
\text { Notes on validation of instruments: Screening and outcome instruments the same, } \\
\text { VABS evaluated for content and semantic equivalence by } 3 \text { bilingual Vietnamese, "The } \\
\text { Cronbach alpha values of the scale over three assessments are } 0.94 \text { to } 0.96 \text { for the com- } \\
\text { munication, } 0.95 \text { to } 0.97 \text { for the daily living skills, } 0.91 \text { to } 0.95 \text { for socialisation and } 0 . \\
95 \text { to } 0.97 \text { for motor skills. The validity of the Vietnamese Vineland version was assessed } \\
\text { in another study with children with typical development" (Goldberg 2009) } \\
\text { Additional information: Protocol not available online } \\
\text { Handling the data: As per footnotes in data and analysis } \\
\text { Prospective trial registration number: KFR-2005-J01702 }\end{array}$} \\
\hline \multicolumn{3}{|l|}{ Risk of bias } \\
\hline Bias & Authors' judgement & Support for judgement \\
\hline $\begin{array}{l}\text { Random sequence generation (selection } \\
\text { bias) }\end{array}$ & Low risk & $\begin{array}{l}\text { Quote: "After matching on gender, they } \\
\text { (participants) were randomly assigned" } \\
\text { Author response: Done by coin toss ran- } \\
\text { domisation } \\
\text { Comment: Minimal risk as there are so } \\
\text { few people that are not randomised that it } \\
\text { would not make significant difference }\end{array}$ \\
\hline Allocation concealment (selection bias) & Unclear risk & $\begin{array}{l}\text { Comment: Authors swapped } 1 \text { mother } \\
\text { that was randomised to the wait-list con- } \\
\text { trol group for } 1 \text { mother randomised to the } \\
\text { intervention group. This would reveal allo- } \\
\text { cation for that } 1 \text { person and the sample size } \\
\text { is small ( } 37 \text { participants). This may affect } \\
\text { the outcome }\end{array}$ \\
\hline
\end{tabular}

Blinding of participants and personnel Low risk (performance bias)

All outcomes
Comment: Not possible to blind participants and personnel to the intervention. Unlikely to affect the outcome 


\section{Shin 2009 RCT Vietnam (Continued)}

\begin{tabular}{|c|c|c|}
\hline $\begin{array}{l}\text { Blinding of outcome assessment (detection } \\
\text { bias) } \\
\text { objective outcomes }\end{array}$ & Low risk & Comment: No objective outcomes \\
\hline $\begin{array}{l}\text { Blinding of outcome assessment (detection } \\
\text { bias) } \\
\text { subjective outcomes }\end{array}$ & High risk & $\begin{array}{l}\text { Comment: Assessment of outcomes made } \\
\text { through interviews with mothers con- } \\
\text { ducted by teachers who already knew chil- } \\
\text { dren }\end{array}$ \\
\hline Baseline outcome measurements similar & Low risk & $\begin{array}{l}\text { Quote: "No significant differences between } \\
\text { the intervention and control groups in any } \\
\text { of the domains of adaptive behaviour mea- } \\
\text { sured by the Vineland" }\end{array}$ \\
\hline Baseline characteristics similar? & Low risk & $\begin{array}{l}\text { Comment: Intervention and control sim- } \\
\text { ilar in child disability categories, age, gen- } \\
\text { der, mother education and SES; children } \\
\text { who stayed home compared with those in } \\
\text { kindergarten had lower adaptive function- } \\
\text { ing but } 1 \text {. not statistically significant and } 2 \text {. } \\
\text { taken into account during post hoc analysis }\end{array}$ \\
\hline $\begin{array}{l}\text { Incomplete outcome data (attrition bias) } \\
\text { Efficacy data }\end{array}$ & Low risk & $\begin{array}{l}\text { Comment: Number at each follow-up for } \\
\text { both groups the same throughout the study } \\
\text { follow-up periods, } 16 \text { in intervention and } \\
14 \text { in control }\end{array}$ \\
\hline
\end{tabular}

Incomplete outcome data (attrition bias) Unclear risk Comment: No adverse outcomes reported Safety data (e.g. adverse events)

Protection against contamination Low risk

Comment: Individual home visits to families by teachers, unlikely that contamination occurred, control families were to receive intervention eventually (wait-list controls)

Reliable primary outcome measures Low risk

Comment: Author does not specify kappa agreement values, JP emailed to request clarification and kappa values were not done. However, the tool has been validated (see notes above)

Selective reporting (reporting bias) Low risk

Comment: All outcome scores on VABS reported

$\begin{array}{lll}\text { Other bias } & \text { Low risk } & \text { Comment: No other bias detected }\end{array}$


Mental health condition: Methamphetamine use

Population: Adults, initial index participants recruited who then also brought in 'network' participants

- Age: 18-25 years old (median 19 years (interquartile range: 18-20))

- Gender: Both male and females (75\% male)

- Socioeconomic background: About a one-third worked, one-third students, one-third unemployed; primarily Buddhist (97.1\%), and ethnically Thai (99.2\%). A majority $(63.8 \%)$ reported living with their parents. Participants' education level was low, with only $39 \%$ reporting being currently in school and a median of 9 (interquartile range: 9-11) years of schooling)

- Inclusion criteria: Index participants: between the ages of 18 and 25 years at screening, used methamphetamine at least 3 times and had sex at least 3 times in the past 3 months, and were able to enrol at least 1 of their sex or drug network members in the study within 45 days of screening). Network participants: between the ages of 18 and 25 years at screening and had used methamphetamine at least 3 times or had sex with the index participant at least 3 times in the last 3 months

- Exclusion criteria: Refused to have blood drawn or provide urine, if they were enrolled in another prevention study, or if they refused to provide locator information vention ("peer education" condition) with a best practice standard life skills curriculum ("life-skills" condition) on methamphetamine use, sexual risks and incident STIs

\section{INTERVENTION 1:}

Name: Peer education condition

Delivered by:

- Title/name of NSHW/OPHR and number: 6 peer educators

- Selection: 2 facilitators with 1 back up (totalling 6 facilitators) who were in their early 20 s and had been a part of the ethnography team in the study's first phase

- Educational background: Not specified

- Training: Facilitators were trained by the study's first and third authors in an intensive one-week long training session. The curriculum was being implemented using a manual. Copies of the manuals for the peer education and life skills conditions are available in Thai and English from the study authors

- Supervision: Not specified

- Incentives/remuneration: Not specified

Intervention details: (according to NSHWs/OPHRs and whether aimed at carers or patients (or both))

- Duration/frequency: Seven 2-hour session for each group undertaken by the facilitators over 1 month with twice-weekly sessions. Participants in the peer education condition also attended 2 booster sessions that occurred 3 and 6 months after study entry 
- Content of intervention: "Peer education condition was based on theory, informed by an extensive 18-month formative research phase, and built upon our previous intervention experience in Thailand and USA." "The peer education condition aimed to teach participants to think critically about and reduce their methamphetamine use and sexual risk behaviours. Participants were taught communication skills that they practiced in role plays during the sessions and used to convey methamphetamine and risk reduction messages to specific social network members that were identified through a social network inventory administered at baseline. The first session aimed to build group cohesion and identity, through having the group establish its own "group rules" to follow during the ensuing sessions. During this session, participants delineated how methamphetamine affected themselves, their social network, and their family. The second session focused on social influences in initiating methamphetamine use and taught participants a set of communication tools that were reinforced and used throughout the subsequent sessions in designated role plays and videos. The third and fourth sessions focused on sexual risk reduction, sexually transmitted infections (STIs), and communication skills in sexual situations. The fifth session focused on stigma and examined methamphetamine's effects on participants' families and the broader community. Because of the intervention's focus on creating a positive and constructive role for participants, the sixth session was dedicated to participants being involved in a community service project, which was chosen by each group. These projects lasted two to four hours and included painting or cleaning temples, garbage clean-up in villages, renovating a village play ground, and weeding a community garden. During the seventh and final session, participants reviewed the content from the previous sessions and graduated from the project. Sessions were comprised of interactive teaching modules, instructive games, and problem-solving activities. Sessions ended with assigning peer education homework in which participants would discuss a specific issue with specific peers (MA-using and/or sexual partners), which were reviewed at the beginning of the next session"

CONTROL: A best practice intervention: a life skills building approach based on a skills building approach. "It was largely derived from cognitive behavioral psychology, which is widely used with youth in drug treatment and juvenile justice settings in Thailand. Juvenile justice staff were consulted throughout the development of the life skills condition. The sessions focused on the causes and consequences of methamphetamine use at the individual level, with specific attention to stress in the role of drug use. 1 session focused on STIs and sex risk behaviours. The sessions placed no emphasis on communicating the session content to social network members. The first session focused on examining the role of methamphetamine in participants' life. The second session reviewed problem-solving tools and friendships. The third session focused on the physiological effects of methamphetamine use. The fourth session addressed STIs and safer sex practices. The fifth session considered stress and coping. The sixth session focused on managing emotions and self worth. The last session reviewed the intervention and participants graduated"

CO-INTERVENTIONS: Prevention of STIs as part of what the peer educator sessions comprised during the 3 months prior to the interview*; 2. Use of condom for either vaginal or anal sex $*$; 3 . Presence of a laboratory-confirmed STI* $\$$. In German (2012) the main outcome is depression scores (using CES-D scale) 
Sutcliffe2009RCT Thailand (Continued)

\section{Carer: None}

Process/health worker outcomes: Not mentioned

Economic outcomes: Not mentioned

(*: primary outcomes of the study; $\$$ : outcomes that we have not reported in this review)

Time points: Baseline, 3 months, 6 months, 9 months and 12 months

Source of funding: National Institutes of Health (1 R01 DA14702)

Notes on validation of instruments (screening and outcomes): No screening instruments used. "Methods to enhance the reliability of self-reported behaviours included: 1) using unique study ID's to maintain confidentiality during data collection; and 2) using a brief recall period (three months and 30 days). In addition, STI testing at the 12 month visit provided a biological outcome measure". CES-D validated in Thai setting (Trangkasombat and Nukhew 1998) with a cut-off score of 22 (range 0-60)

Additional information (e.g. provide by authors, existence of a published study protocol): None

Handling the data: As per footnotes in data and analysis

Prospective trial registration number: Registration not mentioned

\section{Risk of bias}

Bias Authors' judgement

Random sequence generation (selection High risk bias)

\section{Support for judgement}

Quote: "Nonrandom sampling recruitment methods". "Some risk of sampling bias due to recruitment time periods and locations"'

Quote: "Randomization of index members occurred at the end of the baseline visit. Indexes were randomised to either the peer education or the life skills condition within 45 days of their baseline visit. Randomization occurred in blocks (cohorts) once a minimum of 16 and a maximum of 24 index participants had been enrolled, and randomisation sequences for each cohort were generated by a computer program. Scheduling for the first session occurred within two weeks of randomisation. In total, 21 cohorts were randomised over a period of 15 months. As this was a peer network intervention and we were interested in examining the effects of index participants on their network members' risk behaviours, network members were not randomised to attend the peer education or life skills sessions. Their involvement was limited to the baseline and four follow-up visit assessments" 
Sutcliffe2009RCT Thailand (Continued)

Allocation concealment (selection bias) Unclear risk
Quote: Randomisation of index members occurred at the end of the baseline visit. Indexes were randomised to either the peer education or the life skills condition within 45 days of their baseline visit. Randomisation occurred in blocks (cohorts) once a minimum of 16 and a maximum of 24 index participants had been enrolled, and randomisation sequences for each cohort were generated by a computer programme Comment: Allocation of randomisation in blocks. Not mentioned if this was in sealed envelopes, etc.; among those excluded (as found in CONSORT diagram 6 were randomised but attended the wrong arm of the trial

Comment: Participants and personnel not blinded to intervention. This is unlikely to affect outcomes

(performance bias)

All outcomes

Comment: Only biological tests for STIs not for methamphetamines

bias)

objective outcomes

Blinding of outcome assessment (detection High risk bias)

subjective outcomes

Baseline outcome measurements similar High risk
Quote: "Behavioral data were collected through self-report and it is possible that social desirability influenced participants' responses, particularly in light of the recent 'war on drugs"'

Quote 2: "Interviewers were blind to the participant's group allocation"

Comment: Behavioural data self reporting is likely to bias the outcome assessment

Quote: "There were few significant differences in demographic or reported drug use patterns between participants randomised to the peer education compared to the life skills condition. A significantly higher percentage of participants in the peer education condition compared to those in the life skills condition reported drinking problems ( $77 \%$ vs. $71 \%, \mathrm{p}<0.05)$, condom use at last vaginal sex act $(38 \%$ vs. $31 \%, \mathrm{p}<0$. $05)$ and "always " using condoms in the past 30 days $(22 \%$ vs. $16 \%, \mathrm{p}<0.05)$ " 
Sutcliffe2009RCT Thailand (Continued)

Baseline characteristics similar? High risk
Quote: “There were few significant differences in demographic or reported drug use patterns between participants randomised to the peer education compared to the life skills condition. A significantly higher percentage of participants in the peer education condition compared to those in the life skills condition reported drinking problems $(77 \%$ vs. $71 \%, \mathrm{p}<0.05)$ "

Comment:

Sociodemographic details similar but differences in drinking problems

Incomplete outcome data (attrition bias) Low risk Efficacy data

Quote: "At each of the four follow-up visits, follow-up was greater or equal to $90 \%$ (range: $89 \%$ - 95\%) for index participants and $86 \%$ (range: $85 \%-91 \%$ ) for network participants in both arms. Among index and network members in both arms, there was at least an $89 \%$ retention rate at the 12-month follow-up"

Comment: Mean $10 \%$ dropout (11\% in intervention group, $9 \%$ in control group) reported but reasons not specified; however, low dropout rate so unlikely to affect outcomes

Incomplete outcome data (attrition bias) Unclear risk

Comment: Not mentioned

Safety data (e.g. adverse events)

Protection against contamination

High risk

Quote: "There is the possibility that tight social networks were randomised to both control and intervention arms, leading to a high degree of contamination that resulted in a bias towards the null"

Quote 2: "It is highly probable that contamination occurred between the two study arms. Based on our observation at the study house, many participants enrolled in the study with or were referred to the study by their friends who could have been randomized to different study arms"

Reliable primary outcome measures High risk
Quote: "Behavioral data were collected through self-report and it is possible that social desirability influenced participants' responses, particularly in light of the recent 'war on drugs' "

Comment: Behavioural data self reporting 
Sutcliffe2009RCT Thailand (Continued)

is likely to bias the reliability of outcomes

\begin{tabular}{|c|c|c|}
\hline Selective reporting (reporting bias) & Unclear risk & $\begin{array}{l}\text { Comment: All stated outcomes reported. } \\
\text { No protocol to check if pre-specified out- } \\
\text { comes are reported }\end{array}$ \\
\hline
\end{tabular}

Other bias $\quad$ High risk

Quote: "Session attendance and follow-up rates were consistently high in both arms indicating a high level of interest; 'perhaps the comparison arm was too similar to the intervention with its parallel, albeit not as intense'; resulted in the arrest and forced treatment of thousands of drug users, as well as the extrajudicial killings of over 2500 people. In this context, it was difficult not to provide a comparison condition that was meaningful to the study participants and that provided them with important risk reduction information delivered in a humane and respectful manner"

Comment: The intervention and comparisons were too similar

Thabet 2005 CBA Palestine

\begin{tabular}{ll} 
Methods & $\begin{array}{l}\text { Study design: CBA study } \\
\text { Duration of study: Not specified, but conducted over } 6 \text { months during ongoing war }\end{array}$ \\
\hline
\end{tabular}

Participants Country: Palestinian territories (Gaza Strip)

Income classification: Lower-middle

Geographical scope: Urban and rural in North Gaza and mid-zone, study population from 6 refugee camps ( 1 camp with teacher education could not be assessed because of road closure)

Healthcare setting: Schools

Mental health condition: PTSD

Population: children

- Age: 9-15 years

- Gender: Both

- Socioeconomic background: Large family size, low SES

- Inclusion criteria: Selected from an earlier epidemiological study, attending

UNRWA schools for refugees, and they had moderate to severe PTSD reactions at time of survey (even if PTSD scores had reduced to 'mild' range by the time of intervention

- Exclusion criteria: Not mentioned

Interventions

Stated purpose: Evaluate the short-term impact of a group crisis intervention for children living in a zone of ongoing war conflict

INTERVENTION 1:

Name: Crisis intervention

Delivered by

Non-specialist health worker interventions for the care of mental, neurological and substance-abuse disorders in low- and middle- 
Thabet 2005 CBA Palestine (Continued)

- Title/name of NSHW/OPHR and number: Social worker (and psychologist as a specialist) both acting as facilitators

- Selection: Not specified

- Educational background: Not specified

- Training: No training, but moderation by lead psychiatrist

- Supervision: By lead child psychiatrist

- Incentives/remuneration: Not specified Intervention details:

- Duration/frequency: 7 weekly sessions

- Content of intervention: Debriefing and cognitive techniques for children. Adjusted to the nature of trauma (ongoing political conflict), sociocultural circumstances, and children's developmental ability, by using free drawing, talking about their traumatic experiences and feelings, writing about traumatic events, storytelling, games, and role-play related to the conflict. Children were encouraged to use these communication techniques to describe their direct experience of trauma, losses suffered during the conflict, and the impact of trauma on their family, peers and their community. Children could, thus, talk about events that led to trauma, their perceived impact (feelings), and resulting symptoms (such as anxiety and nightmares). There was guidance and facilitation by the group moderators, as well as some traumaspecific exercises, but there was no specific structure or order of group themes. Facilitators also referred patients who had more serious symptoms (prolonged bereavement reaction or suicidal ideation)

\section{INTERVENTION 2:}

Name: Teacher education

Delivered by:

- Title/name of NSHW/OPHR and number: Teachers (number not specified)

- Selection: Not specified

- Educational background: Not specified

- Training (contents, duration and by whom): 4 training sessions by main author (consultant child psychiatrist). Contents: meaning of trauma, consequences, and how to deal with such problems

- Supervision: Not specified

- Incentives/remuneration: Not specified

Intervention details:

- Duration/frequency: Over 4 sessions

- Content of intervention: Teachers provided information to children on the impact of trauma on different areas of the child's life, and aimed, through education, to normalise the child's response. They also referred patients who had more serious symptoms (prolonged bereavement reaction or suicidal ideation)

CONTROL: Usual care (no intervention), but were on wait-list for crisis intervention after the follow-up

CO-INTERVENTIONS: None

Time points: baseline and 3 months (post intervention) 
Thabet 2005 CBA Palestine (Continued)

\begin{tabular}{|c|c|c|}
\hline Notes & \multicolumn{2}{|c|}{$\begin{array}{l}\text { Source of funding: Not mentioned } \\
\text { Notes on validation of instruments: Both tools validated in settings } \\
\text { Additional information (e.g. provide by authors, existence of a published study } \\
\text { protocol): None } \\
\text { Handling the data: As per footnotes in data and analysis } \\
\text { Prospective trial registration number: None }\end{array}$} \\
\hline \multicolumn{3}{|l|}{ Risk of bias } \\
\hline Bias & Authors' judgement & Support for judgement \\
\hline $\begin{array}{l}\text { Random sequence generation (selection } \\
\text { bias) }\end{array}$ & High risk & $\begin{array}{l}\text { Comment: This was a non-random } \\
\text { method }\end{array}$ \\
\hline Allocation concealment (selection bias) & High risk & $\begin{array}{l}\text { Comment: This was a CBA study so is la- } \\
\text { belled as high risk }\end{array}$ \\
\hline $\begin{array}{l}\text { Blinding of participants and personnel } \\
\text { (performance bias) } \\
\text { All outcomes }\end{array}$ & Low risk & $\begin{array}{l}\text { Comment: No blinding which would not } \\
\text { be possible, but unlikely to have an effect } \\
\text { on outcomes }\end{array}$ \\
\hline $\begin{array}{l}\text { Blinding of outcome assessment (detection } \\
\text { bias) } \\
\text { objective outcomes }\end{array}$ & Low risk & Comment: No objective outcomes \\
\hline $\begin{array}{l}\text { Blinding of outcome assessment (detection } \\
\text { bias) } \\
\text { subjective outcomes }\end{array}$ & Unclear risk & $\begin{array}{l}\text { Comment: It is not clear if the team mea- } \\
\text { suring outcomes are the same or not as } \\
\text { those moderating the intervention }\end{array}$ \\
\hline Baseline outcome measurements similar & High risk & $\begin{array}{l}\text { Comment: In education group, there are } \\
\text { lower rates of 'likely depression' and higher } \\
\text { rates of 'likely PTSD' even though the } \\
\text { means are roughly similar. They are not ad- } \\
\text { justed for }\end{array}$ \\
\hline Baseline characteristics similar? & Low risk & $\begin{array}{l}\text { Quote: "The large family size and low so- } \\
\text { cioeconomic status were striking across the } \\
\text { sample. The three groups did not differ sig- } \\
\text { nificantly on parental employment status, } \\
\text { family size, or family income. As stated ear- } \\
\text { lier, there were only female pupils in the ed- } \\
\text { ucation group. The mean age significantly } \\
\text { differed between the three group but it does } \\
\text { not have the impact on the outcome" } \\
\text { Comment: These were adjusted for }\end{array}$ \\
\hline
\end{tabular}

Non-specialist health worker interventions for the care of mental, neurological and substance-abuse disorders in low- and middle- 
Thabet 2005 CBA Palestine (Continued)

\begin{tabular}{l|l}
$\begin{array}{l}\text { Incomplete outcome data (attrition bias) } \\
\text { Efficacy data }\end{array}$ & Low risk
\end{tabular}$\quad \begin{aligned} & \text { Comment: There seems to be } 100 \% \text { fol- } \\
& \text { low-up and all outcomes seem to have been } \\
& \text { reported }\end{aligned}$

Incomplete outcome data (attrition bias) Unclear risk $\quad$ Comment: Not mentioned

Safety data (e.g. adverse events)

Protection against contamination Low risk

Comment: The groups are in different geographical locations so low risk of contamination

Reliable primary outcome measures Low risk

Comment: Validated scores used though no inter-rater reliability reported

Selective reporting (reporting bias) Low risk

Comment: Not able to find protocol, but compared with methods section, all outcomes reported

Other bias

Low risk

Comment: None detected

Tiwari 2010 RCT China

Methods

Study design: RCT

Duration of study: February 2007 to June 2009

Participants

Country: Hong Kong, China

Income classification: Middle income

Geographical scope: Urban and rural parts covers 3 districts of Hong Kong

Healthcare setting: Community setting

Mental health condition: Depression

Population

- Age: $\geq 18$ years

- Gender: Female

- Socioeconomic background: $72 \%$ of women had financial hardship, more women in intervention group received comprehensive social security assistance $(33 \%$ vs. $9 \%$ ) and said they were in need of financial support (65\%). Most had a minimum of 13 years' education, over half born in mainland China but most $70 \%$ resident in Hong Kong for $<7$ years, majority were married, $50 \%$ had $\leq 1$ child, $15 \%$ had chronic illness, $30 \%$ employed ( $80 \%$ of partners employed)

- Inclusion criteria: Women aged $\geq 18$ years who resided or worked in 1 of the districts covered by the community centre, screened positive for IPV (using the Chinese Abuse Assessment Screen)

- Exclusion criteria: Women were excluded from the study if they could not communicate in Cantonese or Putonghua, the 2 main Hong Kong dialects used in this study for administering the intervention and collecting data 
Tiwari 2010 RCT China (Continued)

Interventions
Stated purpose: To determine whether an advocacy intervention would improve the depressive symptoms of Chinese women survivors of IPV

INTERVENTION:

Name: Less intensive advocacy intervention

Delivered by:

- Title/name of NSHW/OPHR and number: Research assistants (social workers)

the number of research assistants not specified

- Selection: Not mentioned

- Educational background: Masters in social work

- Training (contents, duration and by whom): 5 days' training: how to institute the intervention in culturally appropriate, empathetic manner base on empowerment and social support protocols. 2 investigators (PhDs from school of nursing) trained, materials used were protocols, etc. as per under training for intervention

- Supervision: Checking by 2 investigators on telephone logs

- Incentives/remuneration: Not specified Intervention details:

- Duration/frequency: First component: delivered for 30 minutes as 1-to-1 interview by a research assistant at beginning of 12-week intervention. Second component: telephone social support: 12 weekly telephone calls (by research assistant) and 24-hour access to hotline for additional social support

- Content of intervention: 2 components: 1 . empowerment protection, enhance choice making and problem solving - Dutton's empowerment model (modified from Parker model of Abuse Prevention Protocol) - given an empowerment pamphlet (reinforce info provided). 2. telephone social support: (based on Cohen's social support theory); and 24-hour access to hotline for additional social support. In addition, free to choose other care/services

CONTROL: Enhanced usual care, i.e. usual community services provided by community centre or its outreach sites - supportive services but not designed for abused women CO-INTERVENTIONS: None

Patient: Change in depressive symptoms* (Chinese version of the BDI II)* between baseline and 9 months. Changes in IPV $₫$ (Chinese Revised Conflict Tactics Scales), health-related QoL (12-Item Short Form Health Survey), and perceived social support $\$$ (Interpersonal Support Evaluation List) between baseline and 9 months

Carer: Not applicable

Process/health worker outcomes: None

Economic outcomes: None

(*: primary outcomes of the study; $\$$ : outcomes that we have not reported in this review)

Time points: baseline, 3 months and 9 months

Notes

Source of funding: This study was supported by the Health and Health Services Research Fund awarded by the Food and Health Bureau of the Hong Kong SAR Government (project 04060741)

Notes on validation of instruments (screening and outcomes): Validated tools

Additional information: Study protocol

Handling the data: As per footnotes in data and analysis

Prospective trial registration number: NCT01054898

\section{Risk of bias}

Non-specialist health worker interventions for the care of mental, neurological and substance-abuse disorders in low- and middle- 
Tiwari 2010 RCT China (Continued)

\begin{tabular}{|c|c|c|}
\hline Bias & Authors' judgement & Support for judgement \\
\hline $\begin{array}{l}\text { Random sequence generation (selection } \\
\text { bias) }\end{array}$ & Low risk & $\begin{array}{l}\text { Quote: "Participants were randomised (1: } \\
\text { 1) to the intervention or control group ac- } \\
\text { cording to a list of random permutations } \\
\text { prepared by computer-generated blocked } \\
\text { randomisation performed by a research } \\
\text { staff member who had not been involved } \\
\text { in participant recruitment" }\end{array}$ \\
\hline Allocation concealment (selection bias) & Low risk & $\begin{array}{l}\text { Quote: "The block size was kept secure by } \\
\text { the randomiser, and the order of allocation } \\
\text { was centrally controlled to avoid any bias } \\
\text { in selection." } \\
\text { "The allocation sequence was concealed in } \\
\text { opaque envelopes. At the time of randomi- } \\
\text { sation, the research assistant who had suc- } \\
\text { cessfully recruited a participant called the } \\
\text { site investigator, who then opened the enve- } \\
\text { lope containing the group assignment. To } \\
\text { ensure random assignment, no detail was } \\
\text { provided to the site investigator about the } \\
\text { identity of the participant" }\end{array}$ \\
\hline
\end{tabular}

Blinding of participants and personnel Low risk Comment: Not blinded but unlikely to af(performance bias) fect outcomes

All outcomes

Blinding of outcome assessment (detection Low risk bias)

objective outcomes

Blinding of outcome assessment (detection Low risk bias)

subjective outcomes

Comment: No objective outcomes

Quote: "Blinding appeared to be sustained, because none of the assessors knew the group assignment of the participants until they came to the last question, which solicited the participants' evaluation of the intervention or usual community services"

Comment: All instruments involve scales/ judgements of assessor. But assessors were not involved in the design of the study, did not know the study hypotheses, and were blinded to group assignment

\begin{tabular}{l|ll}
\hline Baseline outcome measurements similar & Low risk & Comment: All similar \\
\hline Baseline characteristics similar? & Low risk & $\begin{array}{l}\text { Comment: All parameters similar except } \\
\text { intervention group had significantly more } \\
\text { access to social security insurance than did }\end{array}$
\end{tabular}

Non-specialist health worker interventions for the care of mental, neurological and substance-abuse disorders in low- and middle- 
Tiwari 2010 RCT China (Continued)

\begin{tabular}{|c|c|c|}
\hline & & $\begin{array}{l}\text { control group. This was unlikely to make a } \\
\text { big difference to the outcome of the inter- } \\
\text { vention, however, particularly as the out- } \\
\text { come was that there was not much dif- } \\
\text { ference between intervention and control } \\
\text { groups }\end{array}$ \\
\hline $\begin{array}{l}\text { Incomplete outcome data (attrition bias) } \\
\text { Efficacy data }\end{array}$ & Low risk & $\begin{array}{l}\text { Comment: No dropouts after randomisa- } \\
\text { tion. } 2 \text { eligible refused to participate before } \\
\text { randomisation. Low dropout because of in- } \\
\text { tensive tracking system they had in place. } \\
88 \% \text { of women received all } 12 \text { weeks of } \\
\text { telephone support. No participant received } \\
<10 \text { weeks }\end{array}$ \\
\hline $\begin{array}{l}\text { Incomplete outcome data (attrition bias) } \\
\text { Safety data (e.g. adverse events) }\end{array}$ & Unclear risk & Comment: No adverse outcomes reported \\
\hline Protection against contamination & Low risk & $\begin{array}{l}\text { Comment: } 1 \text {-to- } 1 \text { interview done so inter- } \\
\text { vention unlikely to have contaminated con- } \\
\text { trol. IPV women often isolated and did not } \\
\text { discuss their situation (as also discussed by } \\
\text { authors in discussion) }\end{array}$ \\
\hline Reliable primary outcome measures & Low risk & $\begin{array}{l}\text { Quote: "In addition, across the length of } \\
\text { the study, } 15 \% \text { of the telephone logs in- } \\
\text { cluding the needs expressed and the re- } \\
\text { sponses provided were randomly checked } \\
\text { for adherence to the protocol. If adher- } \\
\text { ence dropped below } 90 \% \text {, retraining and } \\
\text { observation were conducted until a return } \\
\text { to } 90 \% \text { or greater adherence was achieved. } \\
\text { The random checks revealed that adher- } \\
\text { ence did not drop below } 90 \% \text {. But also } \\
\text { many scales with self-reported outcomes } \\
\text { which are less reliable" }\end{array}$ \\
\hline Selective reporting (reporting bias) & Low risk & Comment: No selective reporting \\
\hline Other bias & Low risk & Comment: None detected \\
\hline \multicolumn{3}{|l|}{ Tol 2008 C-RCT Indonesia } \\
\hline Methods & \multicolumn{2}{|c|}{$\begin{array}{l}\text { Study design: Cluster RCT, schools as unit of allocation, individuals as unit of analysis } \\
\text { Duration of study: March to December } 2006\end{array}$} \\
\hline Participants & \multicolumn{2}{|c|}{$\begin{array}{l}\text { Country: Indonesia } \\
\text { Income classification: Lower-middle } \\
\text { Geographical scope: Rural, in Poso district of Central Sulawesi }\end{array}$} \\
\hline
\end{tabular}

Non-specialist health worker interventions for the care of mental, neurological and substance-abuse disorders in low- and middle- 
Tol 2008 C-RCT Indonesia (Continued)

Healthcare setting: School

Mental health condition: PTSDsymptoms

Population: Patient

- Age: 8-13 years (80\% between 9 and 11 years)

- Gender: Both (50/50 boys and girls in sample)

- Socioeconomic background: $25 \%$ of population in province below poverty line and living off agriculture, $20 \%$ intervention group and 30\% control group displaced, most houses had 4.5 household members, most suffered about 4 violent event types on average. $31 \%$ Muslim; 47\% Protestant

- Inclusion criteria: Children screened for exposure to traumatic events, PTSD symptoms or depressive anxiety symptoms, with the use of symptom checklists

- Exclusion criteria: Serious psychopathology and psychiatric disorders (mutism, retardation, psychotic symptoms) or incapability to function in a group (conduct disorders, harming others), as judged by local psychosocial counsellors

Interventions

Stated purpose: To assess the efficacy of a school-based intervention designed for conflict-exposed children, implemented in a low-income setting

INTERVENTION:

Name: CBI

Delivered by:

- Title/name of NSHW/OPHR and number: Paraprofessional interventionists (number not specified)

- Selection: Selected from local target communities, based on selection procedure assessing social skills through role-plays

- Educational background: At least a high school education, without former mental health background but some experience as volunteers in humanitarian programmes

- Training: 2-week training programme, trained by national staff working for partnering humanitarian organisation Church World Services, based on a manual developed by the Centre for Trauma Psychology in Boston which conforms to current expert-based consensus and similar school-based interventions

- Supervision: Unspecified

- Incentives/remuneration: Unspecified

Intervention details:

- Duration/frequency: 15 sessions with groups of 15 children over 5 weeks manualised CBI

- Content of intervention: manualised CBI, CBT and creative-expressive techniques in a structured format: week 1: psychoeducation; week 2: stabilisation awareness self esteem; weeks 3 and 4 trauma narrative; week 5: reconnecting child and group to social context/ resiliency, etc. and sharing trauma stories

CONTROL: Usual care (wait-list control)

CO-INTERVENTIONS: None

Outcomes

Patient: PTSD (CPSS) and depressive symptoms (DSRS)*

Secondary outcomes:

1. Anxiety (SCARED)

2. Aggression (Children's Aggression Scale for Parents) $\$$

3. Daily functioning (Children's Function Impairment)

4. Social support (Social Support Inventory Scheme; SSIS) $\$$

Non-specialist health worker interventions for the care of mental, neurological and substance-abuse disorders in low- and middle- 


\section{Coping (Kidcope) $\$$}

6. Functioning (Impairment in functioning)

7. Hope (Children’s Hope Scale) $\$$

Carer: $\mathrm{n} / \mathrm{a}$

Process/health worker outcomes: None reported

Economic outcomes: Treatment outcome, treatment satisfaction, therapist burden, level of selection to care, care package cost (see: Jordans 2011)

(*: primary outcomes of the study; $\$$ : outcomes that we have not reported in this review)

Time points: Baseline, 1 week, 6 months

$\begin{array}{ll}\text { Notes } & \text { Source of funding: PLAN Netherlands } \\ \text { Notes on validation of instruments: Validated in local context, "to measure internal } \\ \text { reliability, we used a Cronbach Alpha and for 2-week test-re-test reliability, the Spearman- } \\ \text { Brown coefficient"; screening measure was a self developed symptom checklist which } \\ \text { was not validated against clinical interview } \\ \text { Additional information: www.controlled-trials.com/ISRCTN25172408 } \\ \text { Handling the data: As per footnotes in data and analysis } \\ \text { Prospective trial registration number: ISRCTN25172408 }\end{array}$

Risk of bias

$\begin{array}{lll}\text { Bias } & \text { Authors' judgement } & \text { Support for judgement }\end{array}$

Random sequence generation (selection Low risk bias)
Allocation concealment (selection bias) Low risk

Blinding of participants and personnel Low risk (performance bias)

All outcomes

Blinding of outcome assessment (detection Low risk bias)

objective outcomes

Blinding of outcome assessment (detection High risk bias)

subjective outcomes
Comment: Randomisation using government-provided list of schools, excluded single religious and private schools, random selection using SPSS function

Comment: SPSS allocation function Quote: "Select exact amount of cases randomisation"

Comment: Not possible to blind participants or personnel, but unlikely to affect outcome

Comment: No objective outcomes

Quote: "Assessors were not blinded to treatment status, and this could have biased results"

Comment: Child self ratings with help of assessors who were not blinded to treatment condition 
Tol 2008 C-RCT Indonesia (Continued)

\begin{tabular}{|c|c|c|}
\hline Baseline outcome measurements similar & Low risk & $\begin{array}{l}\text { Comment: No differences except for par- } \\
\text { ent-rated aggression was higher in wait-list } \\
\text { control group }(\mathrm{P} \text { value }=0.03)\end{array}$ \\
\hline Baseline characteristics similar? & Low risk & $\begin{array}{l}\text { Comment: Differences in gender, age and } \\
\% \text { displaced, controlled for in analyses }\end{array}$ \\
\hline $\begin{array}{l}\text { Incomplete outcome data (attrition bias) } \\
\text { Efficacy data }\end{array}$ & Low risk & $\begin{array}{l}\text { Comment: Good follow-up data (more } \\
\text { than } 90 \% \text { ) for } 1 \text { week and } 6 \text { months for } \\
\text { both intervention and control }\end{array}$ \\
\hline $\begin{array}{l}\text { Incomplete outcome data (attrition bias) } \\
\text { Safety data (e.g. adverse events) }\end{array}$ & Unclear risk & Comment: No adverse outcomes reported \\
\hline Protection against contamination & Low risk & $\begin{array}{l}\text { Comment: Randomisation } \\
\text { done by school. In addition, there is a wait- } \\
\text { list control so unlikely for groups to share } \\
\text { information }\end{array}$ \\
\hline Reliable primary outcome measures & Low risk & $\begin{array}{l}\text { Comment: Inter-rater reliability high }(\mathrm{k}= \\
0.901) \text { for dichotomous items and contin- } \\
\text { uous items }(\mathrm{k}=0.988)\end{array}$ \\
\hline Selective reporting (reporting bias) & Low risk & $\begin{array}{l}\text { Comment: Outcomes reported in methods } \\
\text { and in online trial protocol are reported in } \\
\text { results }\end{array}$ \\
\hline Other bias & Low risk & $\begin{array}{l}\text { Comment: ICC done and adjustment for } \\
\text { clustering; intervention fidelity assessed } \\
\text { (89.76\% adherence) }\end{array}$ \\
\hline
\end{tabular}

Tol 2012 C-RCT SriLanka

Methods

Study design: Cluster RCT, unit of allocation by schools, unit of analysis: individuals Duration of study: September 2007 to March 2008

Participants

Country: Sri Lanka

Income classification: Lower-middle

Geographical scope: Urban and rural, Tellippallai and Uduvil divisions of the Jaffna district of northern Sri Lanka

Healthcare setting: School-based group intervention

Mental health condition: PTSD

Population: children/adolescents

- Age: 9-12 years

- Gender: Both

- Socioeconomic background: War-traumatised area with rationed food, and other essential supplies, curfews, road blocks, disappearances, extra judicial killings; "In August 2006, a peace agreement that had been observed since 2002 was abandoned, 

period was characterized by rationed food and other essential supplies, curfews, road blocks, disappearances, extra judicial killings, and skirmishes between the army and Liberation Tigers"

- Inclusion criteria: Those who scored positive using the Child Psychosocial Distress Screener (CPDS); aged 9-12 years; also included children reporting severe mental problems and the latter were provided individual supportive counselling in addition to being enrolled in the study (19 children, 4.8\%)

- Exclusion criteria: Not specified

INTERVENTION:

Name: School-based group intervention

Delivered by:

- Title/name of NSHW/OPHR and number: Non-specialised personnel, number not specified

- Selection: Locally identified

- Educational background: At least a high-school diploma and were selected for their affinity and capacity to work with children as demonstrated in role plays and interview

- Training (contents, duration and by whom): trained 1 year before intervention, manualised intervention, not specified by who

- Supervision: There is some supervision but no details mentioned

- Incentives/remuneration: Not specified Intervention details:

- Duration/frequency: 5-weeks, 15 sessions (about 60-minute sessions). The intervention followed a specific structure within and between sessions, with the following foci: information, safety and control in week 1 (sessions 1-3); stabilisation, awareness and self esteem in week 2 (sessions 4-6); the trauma narrative in week 3 (sessions 7-9); resource identification and coping skills in week 4 (sessions 10-12); and reconnection with the social context and future planning in week 5 (sessions 13-15). Each session is divided into 4 parts, starting and ending with structured movement, songs and dance with the use of a 'parachute' (i.e. large circular coloured fabric). The second part is based on a 'central activity' focused on the main theme of that week (e.g. a drama exercise to identify social supports in the environment, or drawing of traumatic events), and the third part was a co-operative game (i.e. a game in which all children had to participate in order to promote group cohesion

- Content of intervention: The manualised intervention consisted of cognitive behavioural techniques (psychoeducation, strengthening coping and guided exposure to past traumatic events through drawing) and creative expressive elements (cooperative games, structured movement, music, drama and dance) with groups of around 15 children, aimed at decreasing symptoms of common mental disorders and strengthening protective factors

CONTROL: Wait list control

CO-INTERVENTIONS: The intervention was part of a larger public mental health programme for children affected by war, including primary and tertiary prevention approaches 
Tol 2012 C-RCT SriLanka (Continued)

Outcomes
Patient: CPSS*, DSRS (depression scale)*, SCARED-5 (anxiety)*, SDQ \$, psychological complaints, functional impairment scale, exposure to violence and daily stressors local scale $\$$, KIDCOPE (daily stressors) $\$$

Carer: $\mathrm{n} / \mathrm{a}$

Process/health worker outcomes: None

Economic outcomes: Treatment outcome, treatment satisfaction, therapist burden, level of selection to care, care package cost; see: Jordans 2011

(*: primary outcomes of the study; $\$$ : outcomes that we have not reported in this review)

Time points: Baseline, 1 week, 3 months

\section{Source of funding: PLAN Netherlands}

Notes on validation of instruments (screening and outcomes):

Primary outcome measures: primary outcome measures for PTSD, depression and anxiety have unknown local criterion validity

Secondary outcome measures:

SDQ: validation in tamil (Lukumar 2008)

Psychological complaints: Not validated

Functional impairment scale: Validated in Tol 2011a

Exposure to violence and daily stressors local scale: not mentioned if validated

KIDCOPE (daily stressors): validated in Spirito 1988

Additional information: none

Handling the data: As per footnotes in data and analysis

Prospective trial registration number: None given

Risk of bias

\begin{tabular}{|c|c|c|}
\hline Bias & Authors' judgement & Support for judgement \\
\hline
\end{tabular}

Random sequence generation (selection Unclear risk bias)
Quote: "We used a two-step randomisation procedure. First, within district divisions, we randomly allocated each division to either the intervention or waitlist control condition (see Figure 1). Second, we randomly selected schools for inclusion in the study. All schools on the governmentprovided list were eligible"

Comment: The random sequence generation is not specified

Comment: Not specified

Comment: Not blinded but unlikely to affect outcomes

(performance bias)

All outcomes

Blinding of outcome assessment (detection Low risk

Comment: No objective outcomes bias)

objective outcomes 
Tol 2012 C-RCT SriLanka (Continued)

Blinding of outcome assessment (detection Unclear risk bias)

subjective outcomes
Quote 1: "Group of assessors not involved in service delivery"

Quote 2: "Assessors were not informed about which schools received intervention.

Quote 3: "Although we did not disclose study condition to assessors and we selected research assessors external to intervention activities, we were not able to control possible disclosure of study condition by children participating in the study"

Comment: May have impacted on outcome assessment

\begin{tabular}{|c|c|c|}
\hline Baseline outcome measurements similar & Low risk & Comment: Similar \\
\hline Baseline characteristics similar? & Low risk & $\begin{array}{l}\text { Quote: "We compared demographic char- } \\
\text { acteristics (gender, religion, type of house, } \\
\text { occupation caregiver, household size), ex- } \\
\text { posure to violence, ongoing war-related } \\
\text { stressors, and scores on outcome measures, } \\
\text { and found no statistically significant differ- } \\
\text { ences between study conditions. The sam- } \\
\text { ple consisted of more boys ( } 61.4 \% \text { ) than } \\
\text { girls, was dominantly of Hindu religion } \\
(81.0 \%) \text {, and children were between } 9 \text { and } \\
12 \text { years old (mean } 11.03 \pm 1.05) \text { " } \\
\text { Comment: Similar baseline characteristics } \\
\text { from what text says (though socio-demo- } \\
\text { graphics not present in a table) }\end{array}$ \\
\hline $\begin{array}{l}\text { Incomplete outcome data (attrition bias) } \\
\text { Efficacy data }\end{array}$ & Low risk & $\begin{array}{l}\text { Comment: Very small dropout rate (only } \\
1 / 200 \text { in each of control and intervention } \\
\text { group) }\end{array}$ \\
\hline
\end{tabular}

Incomplete outcome data (attrition bias) Low risk Safety data (e.g. adverse events)

Protection against contamination

Low risk

Quote: "For girls, we found an unintended harmful effect, such that girls in the waitlist condition showed larger improvements in PTSD symptoms than girls in the intervention condition"

Comment: Adverse effects looked for via the intervention

Comment: Cluster trial so low risk of contamination

Reliable primary outcome measures

High risk

Quote: "Our primary outcome measures for PTSD, depression, and anxiety have unknown local criterion validity" "internal re- 
Tol 2012 C-RCT SriLanka (Continued)

liability of some of the measures was slightly less than acceptable (no table for anxiety symptoms)"

Selective reporting (reporting bias) Unclear risk
Comment: Do not have protocol to check against prespecified outcomes. However, outcomes are similar to other studies by the same authors

$\begin{array}{lll}\text { Other bias } & \text { Low risk } & \text { Comment: None detected }\end{array}$

Wolmer 2005 CBA Turkey

Methods

up

Participants
Study design: CBA study

Duration of study: Some months post 1999 earthquake in Turkey, then 3.5 year followup

Country: Turkey

Income classification: Upper-middle

Geographical scope: Urban, East Marmara region heavily affected by earthquake (18, 000 people dead, 150,000 homes destroyed, thousands homeless), village adjacent to Adapazari (Note: the term 'village' was used to describe established displacement areas rather than a rural setting)

Healthcare setting: 3 schools: 1 in the temporary 'Israeli village' established post earthquake by Israeli humanitarian aid where the original intervention took place, and 2 schools equally affected by the earthquake in Adapazari where several of the children who initially received the intervention in the 'Israeli village' had moved to

Mental health condition: PTSD symptoms due to earthquake

Population: Displaced school-aged children

- Age: 9-17 years

- Gender: Both

- Socioeconomic background: Post earthquake area, families displaced in prefabricated houses in temporary villages

- Inclusion criteria: Experienced 1999 earthquake, displaced school-aged children, grades $1-5$

- Exclusion criteria: Not specified
Stated purpose: "Child survivors of a catastrophic earthquake in Turkey were evaluated three and a half years after the event, and three years after a sub-group participated in a teacher-mediated intervention developed by the authors. The goal of this follow-up study was to determine the long-term effectiveness of the original intervention"

INTERVENTION:

Name: School reactivation programme

Delivered by:

- Title/name of NSHW/OPHR and number: 8 teachers (number provided by author)

- Selection: The principal and teachers in a school in the prefabricated 'Israeli village' 
Wolmer 2005 CBA Turkey (Continued)

- Educational background: Trained as teachers

- Training: First, 1 group session (modified debriefing protocol), empowerment activity delivered by study authors; second, taught sessions about issues related to children's responses to trauma and how to implement disaster-related school reactivation programme, introductory training provided by study authors, intervention skills trained by local professional team

- Supervision: Ongoing weekly training, supervision and support from local professional team who conducted intervention training

- Incentives/remuneration: Not described Intervention details:

- Duration/frequency: 1 introductory meeting with parents, then eight 2-hour meetings (over 4 weeks - 2 meetings per week) focused on aspects of recovery process

- Content of intervention: The teachers took charge of class activation in which all children in the class participated. The eight 2-hour meetings focused on various aspects of the recovery process: "restructuring traumatic experiences, dealing with intrusive thoughts, establishing a safe place, learning about the earthquake and preparing for future earthquakes, mourning the ruined city, controlling body sensations, confronting posttraumatic dreams, understanding reactions in the family, coping with loss, guilt, and death, dealing with anger, extracting life lessons, and planning for the future. The programme combined psychoeducational modules, cognitive-behavioral techniques, play activities, and ongoing documentation in personal diaries"

CONTROL: In Wolmer 2003: the control was a group of 101 displaced children from another area not affected by the earthquake. For 2005: they were from a similar background and exposed to the earthquake (from same schools) but had not received the intervention

CO-INTERVENTIONS: None

Outcomes

Notes
Patient: Child Report: CPTSD-RI, Traumatic Dissociation and Grief Scale (TDGS) , mother report (of child): Traumatic Dissociation and Grief Scale (TDGS). Teacher report (of child): daily functioning assessment (academic, social, general conduct)

Carer: $n / a$

Process/health worker outcomes: None reported

Economic outcomes: None

Time points: 3.5 years post earthquake

Source of funding: The Association for Children at Risk, Israel; The American Jewish Joint Distribution Committee; and The American Jewish World Service

Notes on validation of instruments: Validation of CPTSD-RI not specified for local context, no validation report for teacher scale, TDGS developed and used in local context by authors (Laor 2002)

Additional information: Protocol not found

Prospective trial registration number: Not given

\begin{tabular}{l|ll}
\hline Bias & Authors' judgement & Support for judgement \\
\hline $\begin{array}{l}\text { Random sequence generation (selection } \\
\text { bias) }\end{array}$ & High risk & Comment: Non-randomised CBA study
\end{tabular}


Wolmer 2005 CBA Turkey (Continued)

\begin{tabular}{|c|c|c|}
\hline Allocation concealment (selection bias) & High risk & Comment: CBA study \\
\hline $\begin{array}{l}\text { Blinding of participants and personnel } \\
\text { (performance bias) } \\
\text { All outcomes }\end{array}$ & Low risk & $\begin{array}{l}\text { Quote: "It is important to emphasize that } \\
\text { the teachers were unaware of the children's } \\
\text { participation in the School Reactivation } \\
\text { Program" } \\
\text { Comment: Participants could not be } \\
\text { blinded but teachers who filled out ratings } \\
\text { were blinded }\end{array}$ \\
\hline $\begin{array}{l}\text { Blinding of outcome assessment (detection } \\
\text { bias) } \\
\text { objective outcomes }\end{array}$ & Low risk & Comment: No objective outcomes \\
\hline $\begin{array}{l}\text { Blinding of outcome assessment (detection } \\
\text { bias) } \\
\text { subjective outcomes }\end{array}$ & Low risk & $\begin{array}{l}\text { Comment: Self report of children and par- } \\
\text { ents not blinded to treatment group but } \\
\text { teachers were blinded }\end{array}$ \\
\hline Baseline outcome measurements similar & Unclear risk & $\begin{array}{l}\text { Comment: No outcome measurements for } \\
\text { control group so difficult to assess }\end{array}$ \\
\hline Baseline characteristics similar? & Low risk & Comment: Yes they are similar \\
\hline $\begin{array}{l}\text { Incomplete outcome data (attrition bias) } \\
\text { Efficacy data }\end{array}$ & High risk & $\begin{array}{l}\text { Comment: Only proportion of original } \\
\text { participants (in 2003) were included for } \\
\text { follow-up (33\%) }\end{array}$ \\
\hline $\begin{array}{l}\text { Incomplete outcome data (attrition bias) } \\
\text { Safety data (e.g. adverse events) }\end{array}$ & Low risk & $\begin{array}{l}\text { Information from author: "We are not } \\
\text { aware of any adverse outcome" }\end{array}$ \\
\hline Protection against contamination & Low risk & $\begin{array}{l}\text { Comment: Intervention happened in } 1 \\
\text { school only initially, low risk for contami- } \\
\text { nation }\end{array}$ \\
\hline Reliable primary outcome measures & High risk & $\begin{array}{l}\text { Comment: Only the grief scale was done } \\
\text { by both parents and children to correlate. } \\
\text { There are, however, within each measure } \\
\text { no correlation coefficients or measures of } \\
\text { agreement }\end{array}$ \\
\hline Selective reporting (reporting bias) & Low risk & $\begin{array}{l}\text { Comment: Outcomes reported in methods } \\
\text { reported in results }\end{array}$ \\
\hline Other bias & Low risk & Comment: None detected \\
\hline
\end{tabular}

Non-specialist health worker interventions for the care of mental, neurological and substance-abuse disorders in low- and middle- 
- Gender: Both, $44.4 \%$ female

- Socioeconomic background: $48.3 \%$ lived in camps, only $5 \%$ of sample completed $>6$ years of education, ethnic composition was $52 \%$ Hutu and $47.6 \%$ Tutsi, almost all were directly victimised by violence during or since conflict onset in 1993; most not fully literate

- Inclusion criteria: Among future participants of 2 trauma workshops offered by internally displaced people camps

- Exclusion criteria: Not specified

Stated purpose: The current study aimed to evaluate the effects of PTSD psychoeducation within a larger trauma healing and reconciliation intervention in a rural region of Burundi

\section{INTERVENTION 1:}

Name: Workshop with psychoeducation

Delivered by:

- Title/name of NSHW/OPHR: Burundian facilitators, number not specified

- Selection: "Chosen by the nonprofit organisation for their extensive experience with trauma workshop facilitation and for having demographics comparable to participants"

- Educational background: "Rural, poor, many without substantial formal education, and balanced in gender and ethnicity"

- Training: "All facilitators had a full day of training dedicated to the modification of the standard workshop to accommodate planned differences in condition"; not specified by whom

- Supervision: Not specified

- Incentives/remuneration: Not specified Intervention details:

- Duration/frequency: The standard intervention included 2 phases. 6 groups of approximately 20 participants gathered for 3 days, and 1 month later each workshop group reconvened for a full-day follow-up session during which major workshop components were reinforced

- Content of intervention: The 3-day workshop used discussion, experiential exercises aimed at fostering interpersonal exchange, and games to explore themes of trauma, loss, anger, trust and the roots of violence; The Healing and Reconciling Our Communities workshop manual (African Great Lakes Initiative of the Friends Peace Teams, 2006) emphasised that recovery from trauma lies in the restoration of the relations between community members, and in understanding how trauma can affect these relationships and individuals. The Healing and Reconciling Our Communities 
program integrates theoretical frames as described by Herman 1997 and Staub 2005. Each of Herman's 3 stages of recovery from trauma were incorporated within the Healing and Reconciling Our Communities workshop design. There was emphasis on the need for personal recovery and interpersonal reconciliation by means of " $\mathrm{a}$ neighbour-to-neighbour healing process, which must include cognitive and affective engagement with experience in the context of interpersonal support".

Psychoeducational content on the first day of the workshop included a 90-minute presentation and discussion of the 17 specific symptoms of PTSD. An orientation to and solicitation of potential Criterion A (according to the DSM) events was also included. These ideas were reviewed again in the afternoon, and participants shared how they had been affected by the traumatic events they had experienced ( 1 hour additional). Coping with trauma was addressed in terms of teaching relaxation skills with a substantial emphasis on repairing relationships with community members

INTERVENTION 2:

Name: Workshop without psychoeducation

Delivered by:

- Title/name of NSHW/OPHR: Burundian facilitators, number not specified

- Selection: "Chosen by the nonprofit organisation for their extensive experience with trauma workshop facilitation and for having demographics comparable to participants"

- Educational background: "Rural, poor, many without substantial formal education, and balanced in gender and ethnicity"

- Training (contents, duration and by whom): "All facilitators had a full day of training dedicated to the modification of the standard workshop to accommodate planned differences in condition"; not specified by whom

- Supervision: Not specified

- Incentives/remuneration: Not specified Intervention details: (according to NSHWs/OPHRs and whether aimed at carers or patients, or both)

- Duration/frequency: The standard intervention included 2 phases. 6 groups of approximately 20 participants gathered for 3 days, and 1 month later each workshop group reconvened for a full-day follow-up session during which major workshop components were reinforced

- Content of intervention: The active workshop condition with no psychoeducation was identical to that described in intervention 1, with 2 exceptions. First, this condition did not include the introduction of PTSD psychoeducational content. Second, to ensure that both workshop conditions were of equal length, additional time was devoted to an exercise in which participants formed pairs and answered questions provided to them. The assigned topics facilitated communication around perspectives on trust, safety, sense of security, and interethnic relations in the community (e.g. "someone I trust and why", "a time I overcame fear”). Importantly, participants were encouraged to discuss how they have been affected by events during the war, but unlike in the workshop with the psychoeducation condition, facilitators did not augment this discussion with any PTSD psychoeducational content

CONTROL: Wait-list control; received workshops after the second assessment period CO-INTERVENTIONS: None 


\begin{tabular}{|c|c|c|}
\hline & \multicolumn{2}{|c|}{$\begin{array}{l}\text { Carer: } \mathrm{n} / \mathrm{a} \\
\text { Process/health worker outcomes: Facilitators completed a report after each workshop in } \\
\text { reference to the integrity of the condition. Reports indicated that workshop components } \\
\text { were consistent as planned and true to treatment condition. Facilitators did report } 3 \\
\text { instances (in the course of over } 2500 \text { participant-hours) in which a participant proposed } \\
\text { the concept of 'trauma' during a brainstorm about the consequences of the war. As } \\
\text { previously instructed, the facilitators acknowledged the statement, but did not foster } \\
\text { discussion on it } ₫ \\
\text { Economic outcomes: None } \\
\text { (*: primary outcomes of the study; } \S: \text { outcomes that we have not reported in this review) } \\
\text { Time points: } 6 \text { weeks pre intervention and } 2 \text { weeks post intervention }\end{array}$} \\
\hline Notes & \multicolumn{2}{|c|}{$\begin{array}{l}\text { Source of funding: Not specified } \\
\text { Notes on validation of instruments (screening and outcomes): HSCL: for depression } \\
\text { scale a sensitivity of } 0.88 \text { and specificity of } 0.73 \text {; proven to be culturally sensitive with } \\
\text { samples around the world and has demonstrated sufficient validity and reliability (Fox } \\
2002 \text { ); HTQ Part } I V \text { and HTQ- } b \text { : Not validated in local setting; Trauma Discours } \\
\text { exposure interview: Not validated in local setting } \\
\text { Handling the data: As per footnotes in data and analysis } \\
\text { Prospective trial registration number: None }\end{array}$} \\
\hline \multicolumn{3}{|l|}{ Risk of bias } \\
\hline Bias & Authors' judgement & Support for judgement \\
\hline $\begin{array}{l}\text { Random sequence generation (selection } \\
\text { bias) }\end{array}$ & Low risk & $\begin{array}{l}\text { Quote: "Participants were blocked accord- } \\
\text { ing to ethnicity and gender and randomly } \\
\text { assigned to condition" }\end{array}$ \\
\hline Allocation concealment (selection bias) & Low risk & $\begin{array}{l}\text { Quote: "In each community, using a com- } \\
\text { puterized random-number generator, par- } \\
\text { ticipants were assigned to condition ac- } \\
\text { cording to stratified randomisation (by } \\
\text { gender and ethnicity) to either workshop } \\
\text { with psychoeducation, workshop without } \\
\text { psychoeducation, or waitlist control" }\end{array}$ \\
\hline
\end{tabular}

Blinding of participants and personnel Low risk (performance bias)

All outcomes
Quote: "Participants and interviewers (at pre and posttest) were blind to condition assignment"

Comment: No objective outcomes

Blinding of outcome assessment (detection Low risk bias)

objective outcomes

Blinding of outcome assessment (detection Low risk bias)

subjective outcomes
Quote: "Facilitators were not blind to condition as they required awareness of differences in content between conditions"

Comment: Participants were blinded to 
Yeomans 2010 RCT Burundi (Continued)

treatment condition (with psychoeducation or not)

Baseline outcome measurements similar Low risk

Baseline characteristics similar? Low risk
Quote: "There were no significant baseline differences between the three treatment groups across age, gender, ethnicity, symptoms, education level, traumatic events experienced, or on prior exposure to trauma discourse"

Quote: "There were no significant baseline differences between the three treatment groups across age, gender, ethnicity, symptoms, education level, traumatic events experienced, or on prior exposure to trauma discourse"

Quote: "Participants received a small reimbursement for transportation expense only"

Comment: Only a few participants not available at follow-up points

Incomplete outcome data (attrition bias) Unclear risk

Comment: No adverse outcomes reported

Safety data (e.g. adverse events)

Protection against contamination Low risk

Comment: Study done before workshops were delivered later in communities, assessed for prior discourse on trauma

Reliable primary outcome measures Unclear risk

Comment: No kappa values given; also not all tools are validated in local context

Comment: No protocol

$\begin{array}{lll}\text { Selective reporting (reporting bias) } & \text { Unclear risk } & \text { Comment: No protocol }\end{array}$

Other bias $\quad$ Low risk

Quote: "Participants received a small reimbursement for transportation expenses only"

Comment: Unlikely to affect outcomes

\section{Zambori 2002 CBA Hungary}

Methods
Study design: CBA study

Duration of study: Enrollment of GP practices: 1 September 1998 to 1 March 1999. 12 months retrospective and 12 months prospective to the intervention. Finished March 2000 


Country: Hungary
Income classification: Upper-middle income
Geographical scope: Urban (Budapest)
Healthcare setting: PC setting
Mental health condition: Common mental disorders (include anxiety and depression)
Population: Adults attending general practices (intervention) or psychiatrist (control)
- Age: $18-64$ years
- Gender: Both
- Socioeconomic background: No break up
- Inclusion criteria: Anxiety, mood disorders or uncomplicated bereavement
- Exclusion criteria: Mild agoraphobia excluded
and affective disorders (mainly depression) in PC patients after initiation of mental health treatment

\section{INTERVENTION:}

Name: PC vs. psychiatric care for common mental disorders

Delivered by (NSHW or OPHR and title)

- Title/name of NSHW/OPHR and number: GP

- Selection: 12 accepted to participate out of 25 GPs in the 12 practices

- Educational background: Highly qualified

- Training: Already qualified doctors no further training given, interviewers for screening were given 1 week' training

- Supervision: No supervision. GPs in Hungary are able to refer patients to the psychiatrists (information from author)

- Incentives/remuneration: Information from author: no specific incentives/ remuneration

Intervention details:

- Duration/frequency: As per usual consultation

- Content of intervention: Information from author: In Hungary, usual GP care consists of prescribing medications and referring patients to specialist or hospital care if needed. They also provide non-specific psychotherapy in some cases (mostly supportive therapy), but this is not very frequent. Therapy is most often limited to pharmacotherapy

CONTROL: Psychiatric care. Psychiatric diagnosis, care and follow-up CO-INTERVENTIONS: None

Patient: BDI (diagnosis of depression); QLDS; DIS (Diagnostic Interview Schedule Hungarian version)

Carer: Not applicable

Process/health worker outcomes: Number of healthcare visits excluding psychiatric care; number of psychiatric visits, number of days spent in hospital, number of days spent on sick leave

Economic outcomes*: Table 5 and 6: consultation cost; psychiatric drug costs; general prescription drugs cost; laboratory and diagnostic costs; hospitalisation cost $\left(^{*}=\right.$ primary outcomes of the study)

Time points: Baseline and 1 year 
Zambori 2002 CBA Hungary (Continued)

\begin{tabular}{|c|c|c|}
\hline Notes & \multicolumn{2}{|c|}{$\begin{array}{l}\text { Source of funding: Servier Educational Fund } \\
\text { Notes on validation of instruments (screening and outcomes): BDI and QLDS and } \\
\text { DIS have all been validated in the Hungarian version } \\
\text { Additional information: Information from authors acquired to complete above infor- } \\
\text { mation } \\
\text { Handling the data: As per footnotes in data and analysis }\end{array}$} \\
\hline \multicolumn{3}{|l|}{ Risk of bias } \\
\hline Bias & Authors' judgement & Support for judgement \\
\hline $\begin{array}{l}\text { Random sequence generation (selection } \\
\text { bias) }\end{array}$ & High risk & $\begin{array}{l}\text { Comment: No random sequence genera- } \\
\text { tion done }\end{array}$ \\
\hline Allocation concealment (selection bias) & High risk & $\begin{array}{l}\text { Comment: No allocation concealment } \\
\text { done }\end{array}$ \\
\hline $\begin{array}{l}\text { Blinding of participants and personnel } \\
\text { (performance bias) } \\
\text { All outcomes }\end{array}$ & Low risk & Comment: No but unlikely to affect \\
\hline $\begin{array}{l}\text { Blinding of outcome assessment (detection } \\
\text { bias) } \\
\text { objective outcomes }\end{array}$ & Low risk & $\begin{array}{l}\text { Information from author: "The sick } \\
\text { leave/hospitalisation data was collected } \\
\text { from patient's charts at the GP office. As } \\
\text { per Hungarian regulations GP's are respon- } \\
\text { sible for documenting sick-leave for outpa- } \\
\text { tients and also obliged to collect this data } \\
\text { on hospital stays" } \\
\text { Comment: Number of visits and days } \\
\text { spent in hospital/sick at home from records } \\
\text { so objective }\end{array}$ \\
\hline $\begin{array}{l}\text { Blinding of outcome assessment (detection } \\
\text { bias) } \\
\text { subjective outcomes }\end{array}$ & High risk & $\begin{array}{l}\text { Comment: All are from self administered } \\
\text { instruments. Therefore, likely to be some } \\
\text { detection bias }\end{array}$ \\
\hline Baseline outcome measurements similar & High risk & $\begin{array}{l}\text { Quote: "Potential group differences in } \\
\text { severity of psychiatric illness might have } \\
\text { resulted from the fact that the treatment } \\
\text { group was recruited from the first } 1,000 \\
\text { attenders, with an over sampling of pa- } \\
\text { tients with greater disease burden and } \\
\text { health service utilization. Thus, differences } \\
\text { in the severity of illness and reasons not at- } \\
\text { tributable to treatment effects may play a } \\
\text { role in the change in the rate of service use" }\end{array}$ \\
\hline
\end{tabular}

Non-specialist health worker interventions for the care of mental, neurological and substance-abuse disorders in low- and middle- 
Zambori 2002 CBA Hungary (Continued)

Baseline characteristics similar? High risk
Quote 1: "The groups differed in terms of mean age and sex ratios. The mean ages for the treatment group, control group, and treatment-refusal group were 46.3 years, 36.1 years, and 39.5 years respectively. The respective sex ratios (female:male) were 1 : $0.7,1: 0.78$ and 1:0.67. These differences were corrected in the statistical analysis"

Quote 2: "Due to the assignment process, there were significant differences in the baseline characteristics of the groups"
Incomplete outcome data (attrition bias) Unclear risk Efficacy data

Incomplete outcome data (attrition bias) Low risk Safety data (e.g. adverse events)

Protection against contamination

Low risk

Reliable primary outcome measures Low risk
Comment: We have incomplete information on dropouts between year 1 and 2 and authors said that there was not reliable data for this

Information from author: They did not measure adverse outcomes apart from hospitalisation rates

Comment: The GP group was unlikely to have access to the same psychiatrists, though they could go and see other psychiatrists. The psychiatrist group (intervention group) would have access to visiting their GP too. However, low risk of contamination

Selective reporting (reporting bias) Low risk
Comment: Validated tools or objective outcomes used. No information on interrater reliability for DIS scores. BDI and QLDS were self administered and there was no other checking of these facts with other measures which would increase the risk of bias

Information from author: "We had an accepted protocol/research plan that was approved by local ethics committee in place prior to starting the study. We were planning to assess the effect of different intervention on quality of life, but the QoL data obtained from the study was not sufficient for statistical analysis"

In addition, regarding our request for BDI scores at baseline and follow-up and followup numbers for psychiatric visits, the author responded: "We did not publish these 
data because it was unreliable and methodologically biased"

Comment: No selective reporting, though some reporting of things that would be useful not done like costs of psychiatric drugs

AIDS: acquired immunodeficiency syndrome; AMPATH: Academic Model for Providing Access to Healthcare; AUDIT: Alcohol

Use Disorders Identifi cation Test; BDI: Beck Depression Inventory; CBA: controlled before-and-after; CBI: classroom-based intervention; CBCL: Child Behavior Checklist; CBT: cognitive behavioural therapy; CDI: Children's Depression Inventory; CESD: Center for Epidemiological Studies Depression scale; CHA: community health aide; CHW: community health worker; CIS-R: revised Clinical Interview Schedule; CPSS: Child Posttraumatic Stress Scale; CPTSD-RI: Child Post Traumatic Stress Reaction Index; CTS2S: Revised Conflict Tactics Scales, short form; DEMQOL: Dementia Qulaity of Life; DIS: Diagnostic Interview Schedule; DSM: Diagnostic and Statistical Manual of Mental Disorders; DSRS; Depression Self-Rating Scale; EPDS: Edinburgh Postnatal Depression Scale; ESB: economic skill building; FBIS: Family Burden Interview Schedule; GGT: gamma-glutamyl transferase; GHQ-12: General Health Questionnaire; GP: general practitioner; GSE: General Self-Efficacy; HCA: home care advisor; HDRS: Hamilton Depression Rating Scale; HSCL: Hopkins Symptom Checklist; HTQ: Harvard Trauma Questionnaire; ICC: intracluster correlation; ICD: International Classification of Diseases; ICDP: International Child Development Programme; ID: intellectual disability; IES: Impact of Events Scale; IPT: interpersonal therapy; IPT-G: ; IPV: intimate partner violence; ITT: intention to treat; LHC: lay health counsellor; LHW: lay health worker; MDD: major depressive disorder; MET: motivational enhancement therapy; MINI: Mini International Neuropsychiatry Interview; MOS: Medical Outcomes Study; NET: narrative exposure therapy; NGO: non-government organisation; NPI-S: Neuropsychiatric Inventory - severity; NSHW: non-specialist health worker; OPHR: other professionals with health roles; PC: primary care; PCU: primary care unit; PDS: Post-traumatic Stress Diagnostic Scale; PHC: primary health care; PhD: doctor of philosophy; PSS: Parental Support Scale; PTSD: post-traumatic stress disorder; QLDS: Quality of Life in Depression Scale; QoL: quality of life; RCT: randomised controlled trial; SA-SCAT: Social Capital Assessment Tool, short adapted version; SCARED: Screen for Child Anxiety Related Disorders; SCL: Symptom Checklist; SD: standard deviation; SDQ: Strengths and Difficulties Questionnaire; SEI: Self-Esteem Inventory; SES: socioeconomic status; SF: Short Form; SRQ: Self Reporting Questionnaire; STI: sexually transmitted infection; TC: trauma counselling; UCLA: University of California, Los Angeles; UNICEF; United Nations Children's Fund; UNRWA: United Nations Relief and Works Agency; USAID: United States Agency for International Development; VABS: Vineland Adaptive Behavior Scales; WHO: World Health Organization; WHODAS: World Health Organization Disability Assessment Scale; WHOQOL-BREF: World Health Organization Quality of Life-BREF; YACS: Yale Adherence and Competence Scale; ZBI: Zarit Burden Interview; ZBS: Zarit Burden Score.

\section{Characteristics of excluded studies [ordered by study ID]}

\begin{tabular}{l|l}
\hline Study & Reason for exclusion \\
\hline Abiodun 1991 & Study trial had no control \\
\hline Acha 2007 & No control. Related to treatment adherence
\end{tabular}

Non-specialist health worker interventions for the care of mental, neurological and substance-abuse disorders in low- and middleincome countries (Review)

Copyright $(2013$ The Cochrane Collaboration. Published by John Wiley \& Sons, Ltd. 
(Continued)

\begin{tabular}{|c|c|}
\hline Acuda 1992 & $\begin{array}{l}\text { Intervention included both specialists and non-specialists but not able to separate out the } \\
\text { two. Also participants did not have a definite diagnosis of alcohol problem. Intervention } \\
\text { classifies as secondary prevention }\end{array}$ \\
\hline Adamolekun 2000 & The study trial had no control. It was an evaluation \\
\hline Adams 2012 & No control and did not meet ITS criteria \\
\hline Ahn 2004 & The trial intervention and control was mainly led by the non-specialists \\
\hline Ali 2010 & Uncontrolled study (2 groups both given counselling by NSHWs) \\
\hline Alvarado 2011 & No control and did not meet ITS criteria \\
\hline Anand 2005 & $\begin{array}{l}\text { It was related to diagnostic accuracy but not an intervention where the health workers } \\
\text { were involved }\end{array}$ \\
\hline Apil 2011 & High income country (Netherlands) \\
\hline Aravena 2011 & $\begin{array}{l}\text { An evaluation of the clinic after PHC doctors received some specialist training in mental } \\
\text { health. There was no control group in the intervention }\end{array}$ \\
\hline Arcel 1995 & Not a trial \\
\hline Armstrong 2011 & The EPOC study design criteria not met \\
\hline Babor 1992 & $\begin{array}{l}\text { A compilation of RCTs. Though NSHWs were involved, it was not possible to separate } \\
\text { out specialist from non-specialist in the study. Also patients were not defined yet as a } \\
\text { having an alcohol problem, they were heavy drinkers. Classifies as secondary prevention } \\
\text { intervention }\end{array}$ \\
\hline Babor 1994 & As for Babor 1992 \\
\hline Bae 2009 & There was no control group in the intervention \\
\hline Baker-Henningham 2009 & The participants targeted were normal children \\
\hline Bakran 2001 & Intervention was general medical rehabilitation and not mental health rehabilitation \\
\hline Balaji 2011 & $\begin{array}{l}\text { The participants targeted for the intervention were only } 23 \% \text { of youths had probable } \\
\text { baseline depression and we have decided that over } 80 \% \text { of patients at baseline should have } \\
\text { a mental illness }\end{array}$ \\
\hline Ball 2000 & There was no control group \\
\hline Bangirana 2006 & Not a trial \\
\hline
\end{tabular}

Non-specialist health worker interventions for the care of mental, neurological and substance-abuse disorders in low- and middle- 
(Continued)

\begin{tabular}{|c|c|}
\hline Barcala 2009 & $\begin{array}{l}\text { Not a trial. It was permanent training of human resources and the setup of intra- and } \\
\text { intersectional networks }\end{array}$ \\
\hline Becker 2006 & No control group \\
\hline Becker 2007 & No control group and baseline data \\
\hline Becker 2009 & Not all CBA EPOC criteria met \\
\hline Beckerleg 1996 & No control group and three is no baseline data \\
\hline Bedregal 2010 & $\begin{array}{l}\text { A pilot study to investigate the level of children's developments in areas where the Chile } \\
\text { government programme is not taking effect. There is no control to this }\end{array}$ \\
\hline Bellali 2006 & A programme description \\
\hline Berman 1993 & No control group and also ITS criteria not met \\
\hline Bichescu 2007 & A specialist-led intervention (psychology student) \\
\hline Blair 2006 & Does not meet EPOC ITS study \\
\hline Boavida 2000 & No control group and was not an ITS \\
\hline Bochen 2006 & A case study. No control group \\
\hline Bondy 1993 & No control group and was not an ITS \\
\hline Booth 2011 & $\begin{array}{l}\text { Peer educator and network groups could not be considered separately, since the former } \\
\text { were trained to recruit and influence the latter. No NSHWs in comparator. No actual } \\
\text { mental health outcomes }\end{array}$ \\
\hline Boothby 2011 & Not appropriate study design \\
\hline Borucka 2003 & No control group \\
\hline Bower 2001 & Not a trial \\
\hline Boyadjieva 1992 & $\begin{array}{l}\text { Mixed group of specialists and non-specialists intervention and no subgroup differences } \\
\text { data provided }\end{array}$ \\
\hline Bragin 2007 & No control group \\
\hline Brown 2005 & A high-income country \\
\hline Cabildo 1973 & It is the description of mental health service in Mexico and is not an intervention \\
\hline
\end{tabular}

Non-specialist health worker interventions for the care of mental, neurological and substance-abuse disorders in low- and middleincome countries (Review)

Copyright $(2013$ The Cochrane Collaboration. Published by John Wiley \& Sons, Ltd. 
(Continued)

\begin{tabular}{|c|c|}
\hline Caciula 2010 & No control group \\
\hline Caciula 2010a & No control and did not meet ITS criteria \\
\hline Calderon 2008 & No control group and specialist-led intervention \\
\hline Campillo 1992 & $\begin{array}{l}\text { As for all Babor-related studies, secondary prevention and not able to separate out NSHW } \\
\text { from specialist intervention }\end{array}$ \\
\hline Carli 2010 & Secondary prevention initiative. \\
\hline Castellarin 1985 & $\begin{array}{l}\text { An evaluation of a longitudinal study. Not got } 3 \text { points before or after intervention of } \\
\text { study so cannot meet the criteria of ITS design as per the EPOC criteria }\end{array}$ \\
\hline Catani 2009 & $\begin{array}{l}\text { This trial compares } 1 \text { NSHW intervention vs. another NSHW intervention, and no } \\
\text { control }\end{array}$ \\
\hline Cavlek 2006 & No control. No baseline data \\
\hline Cereceda 2011 & Not correct study design \\
\hline Cerny 1975 & A programme description \\
\hline Chang 2000 & CBA but did not meet EPOC criteria. Also researcher-led intervention \\
\hline Chankrachang 2009 & Specialist intervention \\
\hline Chapman 1988 & High-income country \\
\hline
\end{tabular}

Chatterjee 2003 A CBA study but with only 1 control 'site' (because 1 geographic area and 1 outpatient department clinic). Did not meet EPOC inclusion criteria for CBAs

\begin{tabular}{l|l}
\hline Chatterjee 2005 & A carrative account not a trial \\
\hline Chatterjee 2009 & A cohort study. It had only baseline and endpoint measures so cannot classify as ITS \\
\hline Chen 2000a & No role of the non-specialist but was a community support group intervention \\
\hline Chhabra 2010 & A prevention programme \\
\hline Chibanda 2011 & No control and did not meet ITS criteria \\
\hline Chien 2008 & $\begin{array}{l}\text { An intervention by non-specialist health workers but in secondary care unit (hospital } \\
\text { setting) }\end{array}$
\end{tabular}

Chisholm 2000

The study was about economic costs described. It was not linked to an RCT

Non-specialist health worker interventions for the care of mental, neurological and substance-abuse disorders in low- and middle- 
(Continued)

\begin{tabular}{|c|c|}
\hline Cho 2011 & The study design was not appropriate with EPOC criteria \\
\hline Chou 2002 & The intervention was based on family member carer \\
\hline Chow 2010 & The study was performed in a high-income country \\
\hline Chowdhury 2004 & $\begin{array}{l}\text { There was only } 1 \text { PHC in } 1 \text { district so, despite being a CBA, did not meet EPOC criteria. } \\
\text { No control group }\end{array}$ \\
\hline Chowdhury 2005 & $\begin{array}{l}\text { There was only } 1 \text { PHC in } 1 \text { district so, despite being a CBA, did not meet EPOC criteria. } \\
\text { No control group }\end{array}$ \\
\hline Climent 1981 & $\begin{array}{l}\text { There was only } 1 \text { intervention site and } 1 \text { control site so, despite being a CBA, did not } \\
\text { meet EPOC criteria }\end{array}$ \\
\hline Climent 1983 & $\begin{array}{l}\text { Patients were not randomized. There was only } 1 \text { intervention site and } 1 \text { control site so, } \\
\text { despite being a CBA, did not meet EPOC criteria }\end{array}$ \\
\hline Colon de Marti 1993 & A programme description and not a trial \\
\hline Cooper 2002 & A prevention programme and CBA trial without multiple sites \\
\hline Cooper 2009 & $\begin{array}{l}\text { Based on depression as secondary outcome - focus is on attachment style between mother } \\
\text { and infant }\end{array}$ \\
\hline Coyle 1998 & $\begin{array}{l}\text { Paper described a trial but did not actually present data. No subsequent results paper } \\
\text { found }\end{array}$ \\
\hline Crawford 2004 & The setting was a high-income country \\
\hline Cummings 2008 & A high-income country \\
\hline Dabrowski 1998 & A programme description and not a trial \\
\hline Das 2006 & A programme description \\
\hline Davis 1988 & A programme description and not a trial \\
\hline De Arellano 2005 & Study performed in a high-income country \\
\hline De Clercq 2001 & Study design was not appropriate with EPOC criteria \\
\hline De Jong 1996 & $\begin{array}{l}\text { Did not have multiple before and after points and no control group in the intervention } \\
\text { so could not be classified as ITS }\end{array}$ \\
\hline Dernovsek 2010 & A prevention study \\
\hline
\end{tabular}

Non-specialist health worker interventions for the care of mental, neurological and substance-abuse disorders in low- and middleincome countries (Review)

Copyright $\odot 2013$ The Cochrane Collaboration. Published by John Wiley \& Sons, Ltd. 
(Continued)

\begin{tabular}{|c|c|}
\hline Devaramane 2011 & No control group in the intervention \\
\hline Devine 2007 & No control group in the intervention \\
\hline Dias 2004 & Intervention design was not linked to an RCT. This was descriptive \\
\hline Diken 2010 & $\begin{array}{l}\text { A specialist-led intervention where the school counsellors were the specialist in this inter- } \\
\text { vention }\end{array}$ \\
\hline Dorji 2006 & No control group in the intervention \\
\hline Dvorin 1989 & Study design did not fulfil EPOC criteria \\
\hline Eaton 2008 & No control group in the intervention \\
\hline Ehntholt 2005 & Study performed in a high-income country. \\
\hline Eickmann 2003 & A prevention study. A CBA study with only 1 intervention site and so was not appropriate \\
\hline El Gaili 2002 & An evaluation \\
\hline El Sayed 2002 & $\begin{array}{l}\text { ITS but only } 1 \text { baseline time point ( } 3 \text { follow-up time points) and no control. Did not } \\
\text { meet EPOC study design criteria }\end{array}$ \\
\hline Ensink 2007 & $\begin{array}{l}\text { The intervention providers were the specialist such as occupational therapists. The study } \\
\text { design was not appropriate with EPOC criteria }\end{array}$ \\
\hline Erbas 2004 & No control group in the intervention \\
\hline Ezard 2010 & A pilot study and no control group \\
\hline Farooq 2011 & Intervention was based on family member carer \\
\hline Fawzy 2012 & The participants for the trial were recruited in a high-income country (USA) \\
\hline Fayyad 2010 & $\begin{array}{l}\text { CBA study but only } 1 \text { intervention group and no control group in the trial. So not meeting } \\
\text { the EPOC criteria of study design }\end{array}$ \\
\hline Feksi 1991 & No control group \\
\hline
\end{tabular}

Feksi 1991a

The comparison group had the same NSHW intervention. The point of comparison in the trial was not the NSHW

Fernandes 2007

Not a trial with patients. An evaluation of a training course

Fernandes 2011

An evaluation of a training programme for primary care workers (no patient outcomes or implementation in practice)

Non-specialist health worker interventions for the care of mental, neurological and substance-abuse disorders in low- and middleincome countries (Review)

Copyright $\odot 2013$ The Cochrane Collaboration. Published by John Wiley \& Sons, Ltd. 
(Continued)

\begin{tabular}{|c|c|}
\hline Ferrinho 1993 & A narrative description of an experience in South Africa \\
\hline Fischman 1990 & A case study \\
\hline Fleischmann 2008 & $\begin{array}{l}\text { There was a mix group of specialists (psychologists) with non-specialists (general doctors, } \\
\text { nurses) who were delivering the care at primary level in this trial }\end{array}$ \\
\hline Fleming 1999 & Study was in a high-income country \\
\hline Friedlander 1985 & This trial did not have a control group \\
\hline Futterman 2010 & $\begin{array}{l}\text { CBA study but only } 1 \text { intervention site and } 1 \text { control site in the trial. So not meeting the } \\
\text { EPOC criteria of study design }\end{array}$ \\
\hline Gardner 2003 & This is a prevention strategy \\
\hline Gentilello 1999 & A high-income country \\
\hline Ghasseimi 2005 & The intervention providers were the specialists (psychiatrist and mental health team) \\
\hline Ghosh 2004 & Study design did not meet EPOC criteria for any study designs \\
\hline Giannopoulou 2006 & The intervention providers were specialists (psychiatrists and psychiatric nurses) \\
\hline Goenjian 1997 & A pre post intervention and trial had no control group \\
\hline Goldin 2008 & $\begin{array}{l}\text { The intervention appears to compares ratings, no training/intervention to train on 'correct' } \\
\text { diagnosis }\end{array}$ \\
\hline Gondim 2001 & No control group in the intervention \\
\hline Goodfriend 2004 & No control group in the intervention \\
\hline Gordon 2004 & $\begin{array}{l}\text { CBA study but } 3 \text { intervention groups and no control group in the trial. So did not meet } \\
\text { the EPOC criteria of study design }\end{array}$ \\
\hline Gruber 2005 & $\begin{array}{l}\text { CBA study but not got several control sites in the trial. So not meeting the EPOC criteria } \\
\text { of study design }\end{array}$ \\
\hline Guerra 2011 & A specialist delivered intervention (junior psychologist and social workers) \\
\hline Guinhouya 2010 & $\begin{array}{l}\text { CBA study but did not have control sites and did not have } 3 \text { time points in the trial.So } \\
\text { did not meet the EPOC criteria of study design }\end{array}$ \\
\hline Guzman 1985 & A secondary prevention study \\
\hline Hamadani 2006 & A prevention study \\
\hline
\end{tabular}


(Continued)

\begin{tabular}{|c|c|}
\hline Han 2010 & Study design did not meet EPOC criteria and patients not depressed at baseline \\
\hline Harder 2012 & A prevention study \\
\hline Harding 1983 & $\begin{array}{l}\text { ITS trial but less number of before and after follow so cannot be considered as ITS as } \\
\text { per EPOC criteria }\end{array}$ \\
\hline Harris 1985 & Intervention in high-income country \\
\hline Hasanovic 2009 & Cohort study. Did not meet the EPOC criteria of study design \\
\hline Heather 2006 & The study design was not correct and appropriate with EPOC criteria \\
\hline Hegerl 2009 & $\begin{array}{l}\text { CBA study but only } 1 \text { site intervention and } 1 \text { site control so did not meet the EPOC } \\
\text { criteria of study design }\end{array}$ \\
\hline Heh 2003 & $\begin{array}{l}\text { CBA study but only one intervention and control group so did not meet the EPOC } \\
\text { criteria of study design }\end{array}$ \\
\hline Hensel-Dittmann 2011 & High-income country (Germany) \\
\hline Hernandez 2003 & $\begin{array}{l}\text { CBA study but only } 1 \text { intervention site and } 1 \text { control site so did not meet the EPOC } \\
\text { criteria of study design }\end{array}$ \\
\hline Hu 2006а & Mixed community and hospital intervention but predominantly hospital based \\
\hline Idris 2006 & $\begin{array}{l}\text { Participants did not have a mental disorder, they had anxiety relating to mathematics. Not } \\
\text { relevant }\end{array}$ \\
\hline Igreja 2004 & $\begin{array}{l}\text { CBA study but only } 1 \text { site of intervention and control so did not meet the EPOC criteria } \\
\text { of study design }\end{array}$ \\
\hline Ivanets 1992 & $\begin{array}{l}\text { Mixed group of specialists (psychiatrists, } 1 \text { psychologist) and non-specialist ( } 1 \text { health } \\
\text { worker)-led intervention, and was secondary prevention }\end{array}$ \\
\hline Jacob 2007a & The study design did not meet EPOC criteria \\
\hline Jacob $2007 b$ & The study design did not meet EPOC criteria \\
\hline Jain 2010 & The study discussed role of paraprofessionals but was not a trial \\
\hline James 2006 & $\begin{array}{l}\text { The participants targeted did not have mental health problems but were HIV positive. So } \\
\text { HIV was the outset }\end{array}$ \\
\hline Jenkins 2007 & No control (a pre-post intervention) \\
\hline Johnson 2011 & The intervention location was in a high-income country \\
\hline
\end{tabular}

Non-specialist health worker interventions for the care of mental, neurological and substance-abuse disorders in low- and middle- 
(Continued)

\begin{tabular}{l|l}
\hline Jordan 2006 & A case study and not a trial \\
\hline Kaaya 1992 & The intervention location was in a high-income country \\
\hline Kabura 2005 & It had no control (a pre-post intervention) and does not meet EPOC CBA criteria \\
\hline Kaiser 1998 & Programme description and focus on antiepileptics not health workers \\
\hline Kalichman 2007 & $\begin{array}{l}\text { The outcomes were for sexual risk behaviour, which included alcohol consumption but it } \\
\text { was not measuring a mental disorder as such, just sex behaviour following alcohol. This } \\
\text { study classified as prevention related }\end{array}$ \\
\hline Kalichman 2008 & $\begin{array}{l}\text { The outcomes were for sexual risk behaviour, which included alcohol consumption but it } \\
\text { was not measuring a mental disorder as such, just sex behaviour following alcohol. This } \\
\text { study classified as prevention related }\end{array}$ \\
\hline Kalichman 2009 & $\begin{array}{l}\text { The outcomes were for sexual risk behaviour, which included alcohol consumption but it } \\
\text { was not measuring a mental disorder as such, just sex behaviour following alcohol. This } \\
\text { study classified as prevention related }\end{array}$ \\
\hline
\end{tabular}

Karnell 2006

CBA study but 3 intervention sites; 2 control sites (all schools) so did not meet the EPOC criteria of study design

\begin{tabular}{|c|c|}
\hline Kermode 2008 & No comparators in the trial \\
\hline Khamis 2004 & A specialist-delivered intervention (psychology and social work students) \\
\hline Khan 2009 & A case study \\
\hline Kim 2001 & $\begin{array}{l}\text { CBA study but only } 1 \text { intervention and } 1 \text { control site so did not meet the EPOC criteria } \\
\text { of study design }\end{array}$ \\
\hline Kitsumban 2009 & $\begin{array}{l}\text { A specialist-delivered intervention. Also mindfulness may count in the same category as } \\
\text { yoga, which is not considered as mental health intervention }\end{array}$ \\
\hline Klein 2012 & The study design was appropriate with EPOC criteria \\
\hline Kozinzky 2012 & A prevention intervention \\
\hline Kozulin 2010 & A specialist-delivered intervention (the mediator and not the health worker) \\
\hline Kunz 2004 & High-income country (Miami, USA) \\
\hline Lafalaise 2003 & A programme description and not a trial \\
\hline Lara 2003 & $\begin{array}{l}\text { CBA study but no control group and } 2 \text { intervention groups so did not meet the EPOC } \\
\text { criteria of study design }\end{array}$ \\
\hline
\end{tabular}

Non-specialist health worker interventions for the care of mental, neurological and substance-abuse disorders in low- and middle-

income countries (Review)

Copyright $\odot 2013$ The Cochrane Collaboration. Published by John Wiley \& Sons, Ltd. 
(Continued)

\begin{tabular}{|c|c|}
\hline Leitch 2009 & No control group in the intervention \\
\hline Leteka 2003 & This was a study to design an intervention but not to evaluate it. No control group \\
\hline Li 2005 & $\begin{array}{l}\text { CBA study but only } 2 \text { points in time measured after intervention. } 1 \text { baseline point so did } \\
\text { not meet the EPOC criteria of study design. A specialist-delivered intervention. It was } \\
\text { secondary care intervention }\end{array}$ \\
\hline Li 2009 & Did not meet EPOC study design criteria \\
\hline Liu 2010 & No control group. Did not meet EPOC study design criteria \\
\hline Luengo-Fernandez 2011 & Not an RCT \\
\hline Lund 2009 & The economic data were not presented within the context of a trial \\
\hline Machona 1992 & A secondary prevention programme \\
\hline Macic 2010 & A programme description and not a trial \\
\hline Madianos 1999 & It had no control group and was not a trial \\
\hline Maheswaran 1992 & Intervention in a high-income country \\
\hline Mavrommati 2002 & Did not meet the CBA study EPOC criteria \\
\hline McAuliffe 1985 & Did not meet the CBA study EPOC criteria \\
\hline McCorkle 2000 & Intervention in a high-income country \\
\hline Merritt 2007 & Intervention in a high-income country \\
\hline Miller 1981 & Intervention in a high-income country \\
\hline Mishara 2006 & A prevention intervention \\
\hline Mohammad-Alizadeh-Charandabi 2011 & A prevention intervention \\
\hline Montazeri 2001 & The study design was not appropriate with EPOC criteria \\
\hline Montero 1992 & $\begin{array}{l}\text { The intervention led by mixed group of specialists and non-specialists health workers and } \\
\text { is a secondary prevention intervention }\end{array}$ \\
\hline Mooren 2003 & Did not meet the ITS study design EPOC criteria \\
\hline Moretti-Pires 2011 & Did not meet the ITS study design EPOC criteria \\
\hline
\end{tabular}

Non-specialist health worker interventions for the care of mental, neurological and substance-abuse disorders in low- and middle- 
(Continued)

\begin{tabular}{|c|c|}
\hline Morrell 2009 & High-income country (UK) \\
\hline Mueller 2011 & $\begin{array}{l}\text { Does not meet the ITS study design EPOC criteria. A cross-sectional post-intervention } \\
\text { design }\end{array}$ \\
\hline Mufti 1986 & A narrative summary and not a trial \\
\hline Murphy 1997 & A prevention intervention \\
\hline Murthy 2005 & An evaluation and no control group \\
\hline Naeem 2003 & Did not meet the ITS study design EPOC criteria \\
\hline Neuner 2004 & A specialist-led intervention \\
\hline Ng 2008 & $\begin{array}{l}\text { Specialist social worker-ledintervention. Also no control and only } 2 \text { time points measured. } \\
\text { Did not meet EPOC ITS criteria }\end{array}$ \\
\hline Ng 2009 & Did not meet ITS study design within EPOC criteria \\
\hline Nizamie 2009 & $\begin{array}{l}\text { Does not meet ITS study design within EPOC criteria. The intervention is complex with } \\
\text { no indication of what role the community health workers have had in improving the } \\
\text { outcomes. No control group }\end{array}$ \\
\hline Ockene 1999 & intervention in a high-income country \\
\hline Okuyemi 2006 & Intervention in a high-income country \\
\hline Omerov 1999 & Intervention in a high-income country \\
\hline Onbun-Uea 2008 & No control group \\
\hline Ooi 2008 & A specialist-led intervention ( 2 therapists who held postgraduate degrees in psychology) \\
\hline Ould 2009 & No control group \\
\hline Paek 2009 & Specialist intervention and non-CBA study \\
\hline
\end{tabular}

Pai 1985

Did not meet the CBA study design EPOC criteria as it has matched cases and control but not enough sites in the intervention

Pal 2007

The intervention and control delivered by only 1 health worker (social service officer)

Palyska 1987

Does not meet the ITS study design EPOC criteria

Park 2010

The SCL-90-R depression scores could not be interpreted as it was not clear if the patients were depressed

Non-specialist health worker interventions for the care of mental, neurological and substance-abuse disorders in low- and middle-

income countries (Review)

Copyright $\odot 2013$ The Cochrane Collaboration. Published by John Wiley \& Sons, Ltd. 
(Continued)

\begin{tabular}{|c|c|}
\hline Patel 2003a & A specialist-led intervention (therapist was specialist) \\
\hline Patel 2003b & $\begin{array}{l}\text { The intervention was regarding the comparison of psychology vs. fluoxetine treatments, } \\
\text { but not comparisons of health workers. A specialist-led intervention (psychological ther- } \\
\text { apies administered by professional therapist. Decision on medication done by research } \\
\text { team (specialists)) }\end{array}$ \\
\hline Peltzer 2006 & A specialist-led intervention (psychologist trainer) \\
\hline Perrin 2010 & Intervention in a high-income country \\
\hline Perry 1989a & $\begin{array}{l}\text { Did not meet the CBA study design EPOC criteria, which had } 4 \text { countries. A prevention } \\
\text { study as it did not start with heavy drinkers }\end{array}$ \\
\hline Petersen 2012 & The study design was not appropriate with EPOC criteria \\
\hline Placencia 1993 & $\begin{array}{l}\text { The intervention is drug treatment (carbamazepine vs. phenobarbitone) but the compar- } \\
\text { ison was the drugs, not the mode of NSHW delivery. A specialist-led intervention (neu- } \\
\text { rologist makes diagnosis at baseline and initiated treatment. Rural doctor administered, } \\
\text { following doses and changes) }\end{array}$ \\
\hline Powell 2004 & $\begin{array}{l}\text { A prevention study. Participants targeted undernourished children with no baseline mental } \\
\text { health }\end{array}$ \\
\hline Prasetiyawan, 2006 & Not a trial, and it described their work implementing programme \\
\hline Qi 2007 & Case study. Wrong study design \\
\hline Qureshi 2001 & $\begin{array}{l}\text { ITS trial design with no control group for training/intervention of primary care workers } \\
\text { and did not have enough before/after time points for ITS so the study design was not } \\
\text { appropriate with EPOC criteria }\end{array}$ \\
\hline Rahman 1998 & A prevention study \\
\hline Ramos-Cerqueira 2005 & $\begin{array}{l}\text { The intervention was about screening training for non-specialist health workers, but } \\
\text { looked at comparison with specialist diagnosis (accuracy not outcome). The intervention } \\
\text { was the training but there was no control group who did not receive training or pre/post } \\
\text { test of diagnosis }\end{array}$ \\
\hline Ran 2003 & A specialist-led intervention (mainly conducted by the trained psychiatrists) \\
\hline Reay 2012 & High-income country \\
\hline Reay $2012 \mathrm{a}$ & High-income country \\
\hline
\end{tabular}

Rhyne 2002

A descriptive paper, and not a trial

Non-specialist health worker interventions for the care of mental, neurological and substance-abuse disorders in low- and middleincome countries (Review)

Copyright $\odot 2013$ The Cochrane Collaboration. Published by John Wiley \& Sons, Ltd. 
(Continued)

\begin{tabular}{|c|c|}
\hline Rigotti 2009 & It had no control: comparison data of training programmes of 5 countries \\
\hline Rotheram-Borus 2011 & Prevention study for depression and alcohol (as part of a general pregnancy RCT) \\
\hline Rowe 2007 & Intervention in high-income country \\
\hline Sadik 2011 & Pre- post-test of a training programme. No control \\
\hline Saltzman 2001 & Intervention in high-income country \\
\hline Schoenmakers 2010 & A high-income country \\
\hline Schultz 1995 & Not a trial \\
\hline Serrano-Garcia 1991 & It described interventions but none of them were trials \\
\hline Skounti 2009 & Not an intervention and had no control. A diagnostic study \\
\hline Slupczynska-Kossobudzka 1999 & $\begin{array}{l}\text { It had not met the CBA study design criteria which was pre post design with no control } \\
\text { and so the study design was not appropriate with EPOC criteria }\end{array}$ \\
\hline Smith 2008 & A prevention intervention \\
\hline So 2005 & Inappropriate study design (CBA but only 1 site each in control and intervention) \\
\hline Sohlberg 1987 & Intervention in high-income country \\
\hline Sokhela 1999 & Non-controlled ITS but does not have 3 baseline and 3 follow-up time points \\
\hline Staples 2011 & No control group and did not meet ITS criteria \\
\hline Stein 1975 & Study conducted in high-income countries \\
\hline Stein 2001 & Not a trial \\
\hline Stepakoff 2006 & A programme description \\
\hline Strain 2001 & An evaluation but no control group \\
\hline Suh 2004 & Economic study not linked to an included RCT \\
\hline Suh $2004 a$ & Economic study not linked to an included RCT \\
\hline Suh 2006 & $\begin{array}{l}\text { Economic study that was part of an RCT, which did not fit our inclusion criteria (drug } \\
\text { trial) }\end{array}$ \\
\hline Tadaka 2004 & It was a high-income country \\
\hline
\end{tabular}


(Continued)

\begin{tabular}{|c|c|}
\hline Tang 2010 & A programme description and not a trial \\
\hline Tareen 2009 & No control group \\
\hline Tezel 2006 & It was none of these comparators: nurse vs. same nurse in the intervention \\
\hline Tharyan 2005 & It was diagnostic accuracy study \\
\hline Tiwari 2005 & A specialist-led intervention (midwife with a masters degree in counselling) \\
\hline Tomasevic 1998 & Not correct study design as there was no baseline data. An evaluation \\
\hline Tran 2008 & No control group \\
\hline Tripathy 2010 & $\begin{array}{l}\text { No mental health intervention. Having confirmed with author (VP) mental health out- } \\
\text { comes were included after the trial had started, as an add-on }\end{array}$ \\
\hline Turrisi 2009 & It was a high-income country \\
\hline Uma 1989 & Yoga was the intervention and so do not meet our inclusion criteria \\
\hline Uys 1996 & $\begin{array}{l}\text { It did not meet the CBA study design criteria, which has only } 2 \text { clinics, and patients } \\
\text { randomised within each so the study design was not appropriate with EPOC criteria }\end{array}$ \\
\hline van Emmerik 2002 & It was high-income country \\
\hline van't Hof 2011 & No control and did not meet ITS criteria \\
\hline Velleman 2003 & An evaluation and did not have a control group \\
\hline Vera 2010 & $\begin{array}{l}\text { A specialist-led intervention (trained counsellors or psychologists and psychiatrists did all } \\
\text { the interventions) }\end{array}$ \\
\hline Vermetten 2007 & Not a trial and it had no control group \\
\hline Vijayakumar 2008 & Did not meet the EPOC CBA study design criteria \\
\hline Vijaykumar 2006 & Not a trial \\
\hline Waitzkin 2011 & It was high-income country \\
\hline Walker 2004 & A prevention study looking at an intervention on how IQ develops \\
\hline Wallander 2010 & The results were not published and author not replied for further enquiry \\
\hline
\end{tabular}

Non-specialist health worker interventions for the care of mental, neurological and substance-abuse disorders in low- and middle- 
(Continued)

\begin{tabular}{l|l}
\hline Wang 2006 & $\begin{array}{l}\text { An ITS trial design with no control, and not enough time points so the study design was } \\
\text { not appropriate with EPOC criteria. The intervention was a drug (phenobarbital) not a } \\
\text { health worker intervention }\end{array}$ \\
\hline Wang 2012 & A specialist-led intervention (peer support group lead by psychiatric nurse) \\
\hline Wechsberg 2006 & $\begin{array}{l}\text { The outcomes for substance abuse were not related to whether they are disorder-related. } \\
\text { The assessment of substance abuse intake was not done through any validated scales and } \\
\text { there was no indication of who had a substance-use disorder or not (just measured whether } \\
\text { consumption was daily or not, and what type) }\end{array}$ \\
\hline
\end{tabular}

Wechsberg 2008

The outcomes for substance abuse were not related to whether they are disorder-related. The assessment of substance abuse intake was not done through any validated scales and there was no indication of who had a substance-use disorder or not (just measured whether consumption was daily or not, and what type). Also not adequate comparator

WHO 1996

The intervention was led by the mixed group of specialists and non-specialists from the high- and low-income countries

\begin{tabular}{|c|c|}
\hline Wilson 1981 & A case study and not a trial \\
\hline Wimo 1997 & Cost of dementia, not related to an included RCT \\
\hline Wimo 2003 & It was high-income country \\
\hline Wimo 2007 & Cost of dementia, not related to an included RCT \\
\hline Wolmer 2011 & A high-income country \\
\hline Wong 2002 & $\begin{array}{l}\text { A specialist-led intervention (experienced mental health social workers with bachelor's } \\
\text { degrees in social work and post-graduate training in mental health) }\end{array}$ \\
\hline Wong 2007 & Excluded as these were standardised patients (actors), not real setting \\
\hline Wu 2002 & Prevention intervention, not treatment \\
\hline Xiao 2009 & $\begin{array}{l}\text { Drug intervention (methadone and detox drug intervention), and not related to a non- } \\
\text { specialist worker }\end{array}$ \\
\hline Xu 2003 & Prevention study \\
\hline Yildiz 2003 & Uncontrolled before and after study so did not meet EPOC criteria \\
\hline Yoo 2006 & CBA study with only 1 intervention site and 1 control site. Did not meet EPOC criteria \\
\hline Zakroyeva 2008 & $\begin{array}{l}\text { An ITS with only } 1 \text { time point baseline and } 1 \text { time point for effect. The study design did } \\
\text { not meet EPOC criteria }\end{array}$ \\
\hline
\end{tabular}

Non-specialist health worker interventions for the care of mental, neurological and substance-abuse disorders in low- and middle- 
(Continued)

Zavradashvili 2010

Uncontrolled before and after study design. Did not meet EPOC criteria

Zencir 2005

This cost study is not related to any RCT or other trial. It had no intervention and was just a descriptive cost study of carers of people with Alzheimer's disease

Zhengyi 1997

No control group. Did not meet EPOC study design criteria

CBA: controlled before-and-after; EPOC: Effective Practice and Organisation of Care; HIV: human immunodeficiency virus; IPT: interpersonal therapy; IQ: intelligence quotient; ITS: interrupted time series; LMIC: low- and middle-income country; MNS: mental, neurological and substance-abuse; NSHW: non-specialist health worker; PHC: primary healthcare; RCT: randomised controlled trial.

\section{Characteristics of studies awaiting assessment [ordered by study ID]}

\section{Abdul 2002}

\begin{tabular}{ll}
\hline Methods & Unknown \\
\hline Participants & Alzheimer's disease patients \\
\hline Interventions & Group support and community based intervention. ? who are the health workers \\
\hline Outcomes & Unknown \\
\hline Notes & Unable to find anywhere online or in libraries \\
\hline
\end{tabular}

Aljanati 2010

\begin{tabular}{ll}
\hline Methods & Unknown \\
\hline Participants & Parkinson's disease patients in Uruguay \\
\hline Interventions & $\begin{array}{l}\text { Patients benefit from knowledge, information of their resources, group activities where they do not feel alone with } \\
\text { their chronic disease. Family members, often primary carers, receive adequate support. Not known who delivers the } \\
\text { intervention }\end{array}$ \\
\hline Outcomes & Unknown \\
\hline Notes & Author not replied - email returned undelivered \\
\hline
\end{tabular}


Azizi 2010

\begin{tabular}{ll}
\hline Methods & Random allocation of patients \\
\hline Participants & Mothers with traumatic birthing \\
\hline Interventions & Midwifery counselling intervention on anxiety levels of women \\
\hline Outcomes & Levels of anxiety, stress, depression \\
\hline Notes & $\begin{array}{l}\text { Could not find full text. Author not answered. Not sure if this is a preventive study or if majority of included patients } \\
\text { had a mental disorder at baseline }\end{array}$ \\
\hline
\end{tabular}

Bhadwal 1992

\begin{tabular}{ll}
\hline Methods & 3 groups, unsure if randomised \\
\hline Participants & Rural primary school students in India \\
\hline Interventions & Package of certain curricular strategies on cognitive and non-cognitive characteristics (test anxiety) \\
\hline Outcomes & Anxiety levels \\
\hline Notes & Unsure whether NSHW-delivered and of study design. No reply from authors \\
\hline
\end{tabular}

\section{Blackmon 1985}

\begin{tabular}{ll}
\hline Methods & Unknown \\
\hline Participants & Explore problems in drug use by elderly people with particular emphasis on their abuse or addiction to alcohol \\
\hline Interventions & $\begin{array}{l}\text { A comprehensive plan for networking (community) alcoholism treatment and educational services to this underserved } \\
\text { population a comprehensive network of services }\end{array}$ \\
\hline Outcomes & Unknown \\
\hline Notes & Only got abstract of book chapter. Unknown if NSHW-led and which country it is in \\
\hline
\end{tabular}

Buttorff 2012

\begin{tabular}{ll}
\hline Methods & Economic evaluation linked to Patel 2010 C-RCT India \\
\hline Participants & Adults with common mental disorders in primary care settings \\
\hline Interventions & Collaborative stepped care intervention \\
\hline Outcomes & Cost-utility and cost-effectiveness
\end{tabular}




\section{Buttorff 2012 (Continued)}

Notes Was published after the last search was performed

\section{Caqueo-Urzar 2010}

\begin{tabular}{ll}
\hline Methods & Randomised trial \\
\hline Participants & Determine the level of satisfaction of relatives of patients with schizophrenia in Chile \\
\hline Interventions & $\begin{array}{l}1 \text { group comprised } 18 \text { carers who participated in a multifamily intervention programme at a mental health centre. } \\
\text { The second group (waiting list) comprised } 23 \text { carers who would not receive any type of family intervention until the } \\
\text { first group finished the programme }\end{array}$ \\
\hline Outcomes & Carer satisfaction \\
\hline Notes & Abstract. Not sure if care in secondary care settings. Unable to contact author \\
\hline
\end{tabular}

Chang 2010

\begin{tabular}{ll}
\hline Methods & Unknown \\
\hline Participants & Patients with severe mental disorders in Taiwan \\
\hline Interventions & Change from hospital-based model to community-based model \\
\hline Outcomes & Unknown \\
\hline Notes & Abstract. Unsure if meets criteria (?NSHW-led and what study design is). Author not responded \\
\hline
\end{tabular}

\section{Cherpitel 2009}

\begin{tabular}{ll}
\hline Methods & Protocol of an RCT \\
\hline Participants & At risk drinkers and dependent drinkers in Poland \\
\hline Interventions & $\begin{array}{l}\text { Screening, brief intervention, referral and treatment in emergency department by physicians, nurses and assistant } \\
\text { physicians }\end{array}$ \\
\hline Outcomes & Unsure \\
\hline Notes & $\begin{array}{l}\text { Not sure if this classifies as secondary care setting and cannot find the results of this trial. Attempted to contact author } \\
\text { but no response received }\end{array}$ \\
\hline
\end{tabular}


Chien 2007a

\begin{tabular}{ll}
\hline Methods & RCT protocol \\
\hline Participants & Family carers of people with schizophrenia \\
\hline Interventions & Mutual support and psycho-educational group interventions \\
\hline Outcomes & Length of re-hospitalisations; families' perceived social support; patients' symptom severity \\
\hline Notes & $\begin{array}{l}\text { Not sure if this is the same trial as published in 2007 (Chien WT, Wong KF, 2007. A family psychoeducation group } \\
\text { programme for Chinese) } \\
\text { If not, was this done in community or hospital settings. By an NSHW? No reply from author }\end{array}$ \\
\hline
\end{tabular}

Farahat 2010

\begin{tabular}{ll}
\hline Methods & Unknown whether there was a control group \\
\hline Participants & Children with epilepsy \\
\hline Interventions & Integrated programme of epilepsy management was performed on patients, carers and school teachers \\
\hline Outcomes & Prevalence of epilepsy, scholastic achievements, knowledge and practice of epileptic children and their carers \\
\hline Notes & Unable to contact author to find out if NSHW-led intervention and if there was a control group \\
\hline
\end{tabular}

\section{Hsiao 2009}

\begin{tabular}{ll}
\hline Methods & RCT protocol \\
\hline Participants & Spouse carers of resectable colorectal cancer \\
\hline Interventions & Carer psychoeducational consultation programme \\
\hline Outcomes & Short-form 12 health-related quality of life questionnaire, Beck Depression Inventory-II depression scale \\
\hline Notes & $\begin{array}{l}\text { Was this primary or secondary care? Was the psychoeducation programme led by NSHWs? } \\
\text { How many carers at baseline had a diagnosable or borderline mental disorder? }\end{array}$ \\
\hline
\end{tabular}

Kumar 2011

\begin{tabular}{ll}
\hline Methods & Unknown \\
\hline Participants & Patients with schizophrenia in rural India \\
\hline Interventions & Unknown \\
\hline Outcomes & Unknown
\end{tabular}

Non-specialist health worker interventions for the care of mental, neurological and substance-abuse disorders in low- and middle- 


\section{Kumar 2011 (Continued)}

Notes Abstract. Unable to find paper or author

\section{Lee 2011a}

Methods RCT

Participants Carers of people with dementia in Korea

Interventions Stress management training for carers of people with dementia

Outcomes Beck Depression Inventory, Caregiver Stress Burden Interview, Life Satisfaction

Notes Unknown if NSHW-led. Author not replied

\section{Luna 1984}

\begin{tabular}{ll}
\hline Methods & Unknown \\
\hline Participants & Cocaine drug addicts \\
\hline Interventions & Mental health programme and pharmaco-dependence \\
\hline Outcomes & Unknown \\
\hline Notes & $\begin{array}{l}\text { Insufficient information as no abstract. Unable to contact author. Email returned undelivered and no reply to } \\
\text { telephone calls }\end{array}$ \\
\hline
\end{tabular}

\section{Malakouti 2010}

\begin{tabular}{ll}
\hline Methods & RCT protocol \\
\hline Participants & Patients with schizophrenia, schizoaffective disorder and bipolar disorder \\
\hline Interventions & $\begin{array}{l}\text { Intervention 1: GP supervision of drug treatment, etc.; Intervnetion 2: Nurse supervision. Control: usual care (just } \\
\text { psychiatric - no community follow-up) }\end{array}$ \\
\hline Outcomes & $\begin{array}{l}\text { Carer's burden and knowledge. Cost of treatment, health of carer, life-skills of patients, relapse/rehospitalisation rates, } \\
\text { severity of psychopathology }\end{array}$ \\
\hline Notes & Not able to find results. Author not replied \\
\hline
\end{tabular}


Oh 1997

\begin{tabular}{ll}
\hline Methods & group pre-post experimental design \\
\hline Participants & Mothers of developmentally delayed children \\
\hline Interventions & $\begin{array}{l}2 \text { series of 4-weekly meetings for group social support were conducted by the researcher with the intention of } \\
\text { developing a self help group }\end{array}$ \\
\hline Outcomes & Burden, well-being of mothers \\
\hline Notes & Could not find full text (only abstract) and not sure if NSHW-led \\
\hline
\end{tabular}

\begin{tabular}{ll}
\hline Sott $\mathbf{1 9 9 8}$ & \\
\hline Methods & Evaluation (not sure of study design e.g. if ITS) \\
\hline Participants & Patients discharged to community \\
\hline Interventions & $\begin{array}{l}\text { Patients receive 'community assistance'. 1 group is followed up by hospital staff, the other by private (? generalist) } \\
\text { clinics }\end{array}$ \\
\hline Outcomes & Patient outcomes \\
\hline Notes & Cannot contact author to check if correct study design and what 'community assistance' is and if NSHW-led \\
\hline
\end{tabular}

GP: general practitioner; ITS: interrupted time series; NSHW: non-specialist health worker; RCT: randomised controlled trial.

\section{Characteristics of ongoing studies [ordered by study ID]}

\section{Ager 2011}

\begin{tabular}{ll} 
Trial name or title & $\begin{array}{l}\text { The impact of the school-based Psychosocial Structured Activities (PSSA) program on conflict-affected chil- } \\
\text { dren in northern Uganda }\end{array}$ \\
\hline Methods & CBA study \\
\hline Participants & Children who have suffered trauma \\
\hline Interventions & School based PSSA programme \\
\hline Outcomes & Measures of well-being felt by parent, child and teacher \\
\hline Starting date & $2007 / 2008$ \\
\hline
\end{tabular}

Contact information 


\section{Ager 2011 (Continued)}

Notes

Published article that was only detected recently. Appears to be a prevention study. To leave for review update

\section{Araya 2010}

Trial name or title Cluster randomised controlled trial of a school-based intervention to improve the mental health of lowincome, secondary school students in Santiago, Chile

\begin{tabular}{ll} 
Methods & RCT (protocol) \\
\hline Participants & Depressed children aged 13-15 years \\
\hline
\end{tabular}

\begin{tabular}{ll}
\hline Interventions & $\begin{array}{l}\text { Cognitive behavioural therapy-like intervention delivered in the class by trained research workers (psycholo- } \\
\text { gists, teachers, social workers, others) }\end{array}$ \\
\hline Outcomes & $\begin{array}{l}\text { Beck Depression Inventory; Revised Child Anxiety and Depression Scale; school records of academic perfor- } \\
\text { mance }\end{array}$ \\
\hline Starting date & 2009 \\
\hline Contact information & r.araya@bris.ac.uk; www.controlled-trials.com/ISRCTN19466209 \\
\hline Notes &
\end{tabular}

\section{Ayoughi 2012}

Trial name or title Provision of mental health services in resource-poor settings: a randomised trial comparing counselling with routine medical treatment in North Afghanistan (Mazar-e-Sharif)

\begin{tabular}{ll}
\hline Methods & CBA study \\
\hline Participants & $\begin{array}{l}\text { Mentally ill patients from primary health care (excluded were those with neurological disorders, mental } \\
\text { retardation, dementia or schizophrenia) }\end{array}$ \\
\hline Interventions & Lay counsellors delivering counselling to patients \\
\hline Outcomes & Severity of symptoms (HSCL-25 and MINI, stressors and coping mechanism scales \\
\hline Starting date & 2009 \\
\hline Contact information & sarah.ayoughi@uni-konstanz.de \\
\hline Notes & This is already published. Detected recently so decision to include in review update \\
\hline
\end{tabular}


Chen 2010

\begin{tabular}{ll} 
Trial name or title & $\begin{array}{l}\text { Community case management for early psychosis: is two year an optimal duration? A randomized controlled } \\
\text { study }\end{array}$ \\
\hline Methods & RCT (protocol) \\
\hline Participants & Patients with early diagnosis of psychosis in China \\
\hline Interventions & Community case management which includes NSHWs \\
\hline Outcomes & Functioning (social and occupational); symptoms, quality of life and health economics \\
\hline Starting date & July 2010 \\
\hline Contact information & clinicaltrials.gov/show/NCT01202357 \\
\hline Notes &
\end{tabular}

\section{Chen 2011}

Trial name or title Depression care management for late-life depression in China primary care: protocol for a randomised controlled trial

\begin{tabular}{|c|c|}
\hline Methods & RCT (protocol) \\
\hline Participants & Patients with depression in China \\
\hline Interventions & Primary care-based intervention with physicians and care managers (nurses) \\
\hline Outcomes & $\begin{array}{l}\text { Patient outcomes: suicidal ideation, psychopathology, medical health, cognitive function, quality of life and } \\
\text { stigma and satisfaction for the treatment. Provider outcomes: attitudes/knowledge regarding depression and } \\
\text { clinical practices with the treatment guidelines }\end{array}$ \\
\hline Starting date & Unsure \\
\hline Contact information & www.clinicaltrials.gov/ct2/show/NCT01287494 \\
\hline Notes & \\
\hline
\end{tabular}

\section{Greenfield 2010}

\begin{tabular}{ll} 
Trial name or title & $\begin{array}{l}\text { Integrated management of physician-delivered alcohol care for tuberculosis patients: design and implemen- } \\
\text { tation }\end{array}$ \\
\hline Methods & RCT (protocol) \\
\hline
\end{tabular}

Participants People with tuberculosis with alcohol problems in tuberculosis centres in Russia 


\section{Greenfield 2010 (Continued)}

\begin{tabular}{l|l}
\hline Interventions & Brief counselling intervention with or without naltrexone delivered by the tuberculosis physicians \\
\hline Outcomes & Patient and physician outcomes \\
\hline Starting date & 2010 \\
\hline Contact information & sgreenfield@mclean.harvard.edu \\
\hline Notes & Study results not available yet \\
\hline
\end{tabular}

\section{Kauye (in preparation)}

\begin{tabular}{l|l}
\hline Trial name or title & $\begin{array}{l}\text { Training primary health workers in mental health and its impact on service delivery in a developing country, } \\
\text { Malawi: a cluster randomised study }\end{array}$ \\
\hline Methods & Cluster RCT (protocol) \\
\hline Participants & Mentally ill patient in primary care in Malawi \\
\hline Interventions & Collaborative care \\
\hline Outcomes & Patient outcomes \\
\hline Starting date & 2010 \\
\hline Contact information & rachel@olan.org \\
\hline Notes & \\
\hline
\end{tabular}

Kobeissi 2011

\begin{tabular}{ll}
\hline Trial name or title & $\begin{array}{l}\text { The Relaxation Exercise and Social Support Trial - RESST: study protocol for a randomised community based } \\
\text { trial }\end{array}$ \\
\hline Methods & RCT (protocol) \\
\hline Participants & Women with common mental disorders and vaginal discharge in Lebanon \\
\hline Interventions & Relaxation exercises and discussion groups delivered by social workers and psychologists \\
\hline Outcomes & HSCL25; The Scale for Assessment of Somatic Symptoms (SASS) \\
\hline Starting date & Not specified but results not out yet \\
\hline Contact information & Wellcome Trust Registry, www.controlled-trials.com/ISRCTN98441241
\end{tabular}




\section{Kobeissi 2011 (Continued)}

Notes

Logie 2012

\begin{tabular}{ll} 
Trial name or title & $\begin{array}{l}\text { Development and evaluation of a community health worker delivered HIV/STI prevention intervention for } \\
\text { women living in internally displaced persons camps in Leogane, Haiti }\end{array}$ \\
\hline Methods & RCT (protocol) \\
\hline Participants & Internally displaced female adults in Haiti \\
\hline Interventions & Individual and group-based, community health worker delivered \\
\hline Outcomes & $\begin{array}{l}\text { Primary outcome: HIV knowledge; secondary outcomes: depression, substance abuse, resilient coping, rela- } \\
\text { tionship control, social support, condom use, STI knowledge }\end{array}$ \\
\hline Starting date & January 2012 \\
\hline Contact information & clinicaltrials.gov/show/NCT01492829 \\
\hline Notes & \\
\hline
\end{tabular}

\section{O’Callaghan 2012}

\begin{tabular}{ll} 
Trial name or title & $\begin{array}{l}\text { Is a family-based, life skills focused intervention effective in reducing psychological distress and stigma and } \\
\text { improving inter-personal relations and functioning among former LRA abductees and other war-affected } \\
\text { children in their community in Dungu, the Democratic Republic of Congo? }\end{array}$ \\
\hline Methods & RCT (protocol) \\
\hline Participants & Vulnerable children with psychological distress in war-affected Democratic Republic of Congo \\
\hline Interventions & $\begin{array}{l}\text { Family-focused, community-based, resilience-targeting psychosocial intervention delivered by a team of lay } \\
\text { Congolese facilitators }\end{array}$ \\
\hline Outcomes & Reduction in psychological distress; improvement in community, daily and family functioning \\
\hline Starting date & March 2012 \\
\hline Contact information & clinicaltrials.gov/show/NCT01542398 \\
\hline Notes &
\end{tabular}


Opoka 2008

\begin{tabular}{ll}
\hline Trial name or title & Cognitive and psychosocial benefits of caregiver training for Ugandan HIV children \\
\hline Methods & RCT (protocol) \\
\hline Participants & HIV-positive children \\
\hline Interventions & $\begin{array}{l}\text { Mediational intervention for sensitising primary carers delivered by home visitors and social scientists with } \\
\text { minimal training in mental health }\end{array}$ \\
\hline Outcomes & Primary: children's cognitive and psychosocial assessment; secondary: improved caring \\
\hline Starting date & 2012: first year of enrolment \\
\hline Contact information & clinicaltrials.gov/show/NCT00889395 \\
\hline Notes & Need to check if children have baseline mental disorder \\
\hline
\end{tabular}

CBA: controlled before-and-after; HIV: human immunodeficiency virus; HSCL: Hopkins Symptom Checklist; MINI: Mini International Neuropsychiatry Interview; NSHW: non-specialist health worker; RCT: randomised controlled trial; STI: sexually transmitted infection. 
DATA AND ANALYSES

\section{Comparison 1. NSHW-led psychological interventions versus usual care in treating common mental disorders in adults (RCTs)}

\begin{tabular}{|c|c|c|c|c|}
\hline Outcome or subgroup title & $\begin{array}{l}\text { No. of } \\
\text { studies }\end{array}$ & $\begin{array}{c}\text { No. of } \\
\text { participants }\end{array}$ & Statistical method & Effect size \\
\hline $\begin{array}{l}1 \text { Prevalence of depression } \\
\text { (completers) }\end{array}$ & 3 & 1082 & Risk Ratio (Random, 95\% CI) & $0.30[0.14,0.64]$ \\
\hline $\begin{array}{l}1.1 \text { Short term (within } 6 \\
\text { months post intervention) }\end{array}$ & 3 & 1082 & Risk Ratio (Random, 95\% CI) & $0.30[0.14,0.64]$ \\
\hline $\begin{array}{l}2 \text { Prevalence of depression (ITT } \\
\text { sensitivity analysis - assumption } \\
\text { non-completers depressed) }\end{array}$ & 3 & & Risk Ratio (Random, 95\% CI) & Subtotals only \\
\hline $\begin{array}{l}2.1 \text { Short term (within } 6 \\
\text { months post intervention) }\end{array}$ & 3 & 1359 & Risk Ratio (Random, 95\% CI) & $0.61[0.43,0.84]$ \\
\hline $\begin{array}{c}3 \text { Prevalence of depression (ITT } \\
\text { sensitivity analysis - assumption } \\
\text { non-completers not depressed) }\end{array}$ & 3 & & Risk Ratio (Random, 95\% CI) & Subtotals only \\
\hline $\begin{array}{l}\text { 3.1 Short term (within } 6 \\
\text { months post intervention) }\end{array}$ & 3 & 1359 & Risk Ratio (Random, 95\% CI) & $0.39[0.20,0.78]$ \\
\hline $\begin{array}{l}4 \text { Prevalence of depression (ITT } \\
\text { sensitivity analysis - worse-case } \\
\text { scenario intervention group } \\
\text { depressed; control group not } \\
\text { depressed) }\end{array}$ & 3 & & Risk Ratio (Random, 95\% CI) & Subtotals only \\
\hline $\begin{array}{l}\text { 4.1 Short term (within } 6 \\
\text { months post intervention) }\end{array}$ & 3 & 1359 & Risk Ratio (Random, 95\% CI) & $1.11[0.56,2.21]$ \\
\hline $\begin{array}{l}5 \text { Prevalence of depression (ITT } \\
\text { sensitivity analysis - best-case } \\
\text { scenario: intervention group } \\
\text { not depressed; control group all } \\
\text { depressed) }\end{array}$ & 3 & & Risk Ratio (Random, 95\% CI) & Subtotals only \\
\hline $\begin{array}{l}5.1 \text { Short term (within } 6 \\
\text { months post intervention) }\end{array}$ & 3 & 1359 & Risk Ratio (Random, 95\% CI) & $0.20[0.09,0.45]$ \\
\hline $\begin{array}{l}6 \text { Severity of common mental } \\
\text { disorder symptoms (includes } \\
\text { anxiety and depression) }\end{array}$ & 7 & & Std. Mean Difference (Random, 95\% CI) & Subtotals only \\
\hline $\begin{array}{l}\text { 6.1 Short term (within } 6 \\
\text { months post intervention) }\end{array}$ & 6 & 1470 & Std. Mean Difference (Random, 95\% CI) & $-0.75[-1.29,-0.21]$ \\
\hline 6.2 Medium term (1 year) & 2 & 923 & Std. Mean Difference (Random, 95\% CI) & $-0.47[-0.60,-0.34]$ \\
\hline $\begin{array}{l}7 \text { Functional impairment/disability } \\
\text { in common mental disorders }\end{array}$ & 4 & & Std. Mean Difference (Random, 95\% CI) & Subtotals only \\
\hline $\begin{array}{l}\text { 7.1 Short term (within } 6 \\
\text { months post intervention) }\end{array}$ & 4 & 1243 & Std. Mean Difference (Random, 95\% CI) & $-0.33[-0.80,0.13]$ \\
\hline
\end{tabular}

Non-specialist health worker interventions for the care of mental, neurological and substance-abuse disorders in low- and middle- 
7.2 Short term (advocacy

1

Comparison 2. Collaborative care model (NSHWs plus specialist) versus usual care in treating common mental disorders (RCTs)

\begin{tabular}{|c|c|c|c|c|}
\hline Outcome or subgroup title & $\begin{array}{l}\text { No. of } \\
\text { studies }\end{array}$ & $\begin{array}{c}\text { No. of } \\
\text { participants }\end{array}$ & Statistical method & Effect size \\
\hline $\begin{array}{l}1 \text { Prevalence of common mental } \\
\text { disorders (CMDs - includes } \\
\text { anxiety and depression) } \\
\text { (completers-combined) all } \\
\text { facilities and in public and } \\
\text { private facilities }\end{array}$ & 3 & & Risk Ratio (Random, 95\% CI) & Subtotals only \\
\hline $\begin{array}{l}1.1 \text { All facilities short } \\
\text { term (within } 6 \text { months post } \\
\text { intervention) }\end{array}$ & 3 & 2380 & Risk Ratio (Random, 95\% CI) & $0.63[0.44,0.90]$ \\
\hline $\begin{array}{l}\text { 1.2 Public facilities short } \\
\text { term (within } 6 \text { months post } \\
\text { intervention) (subgroup) }\end{array}$ & 3 & 1528 & Risk Ratio (Random, 95\% CI) & $0.57[0.42,0.78]$ \\
\hline $\begin{array}{l}1.3 \text { Private facilities short } \\
\text { term (within } 6 \text { months post } \\
\text { intervention) (subgroup) }\end{array}$ & 1 & 823 & Risk Ratio (Random, 95\% CI) & $1.12[0.68,1.84]$ \\
\hline $\begin{array}{l}1.4 \text { All facilities medium term } \\
\text { (at } 1 \text { year post intervention) }\end{array}$ & 1 & 2009 & Risk Ratio (Random, 95\% CI) & $0.95[0.68,1.33]$ \\
\hline $\begin{array}{l}1.5 \text { Public facilities } \\
\text { medium term (at } 1 \text { year post } \\
\text { intervention) (subgroup) }\end{array}$ & 1 & 1104 & Risk Ratio (Random, 95\% CI) & $0.72[0.39,1.34]$ \\
\hline $\begin{array}{l}1.6 \text { Private facilities at } 1 \text { year } \\
\text { post intervention }\end{array}$ & 1 & 801 & Risk Ratio (Random, 95\% CI) & $1.25[0.76,2.06]$ \\
\hline $\begin{array}{l}2 \text { Severity of symptoms of CMDs } \\
\text { (completers-combined) in all } \\
\text { facilities and in public and } \\
\text { private facilities }\end{array}$ & 5 & & Std. Mean Difference (Random, 95\% CI) & Subtotals only \\
\hline $\begin{array}{l}2.1 \text { All facilities short } \\
\text { term (within } 6 \text { months post } \\
\text { intervention) }\end{array}$ & 5 & 3604 & Std. Mean Difference (Random, 95\% CI) & $-0.31[-0.56,-0.06]$ \\
\hline $\begin{array}{l}2.2 \text { Public facilities short } \\
\text { term (within } 6 \text { months post } \\
\text { intervention) (subgroup) }\end{array}$ & 5 & 2781 & Std. Mean Difference (Random, 95\% CI) & $-0.32[-0.58,-0.07]$ \\
\hline $\begin{array}{l}2.3 \text { Private facilities short } \\
\text { term (within } 6 \text { months post } \\
\text { intervention) (subgroup) }\end{array}$ & 1 & 823 & Std. Mean Difference (Random, 95\% CI) & $0.03[-0.11,0.16]$ \\
\hline
\end{tabular}


2.4 All facilities medium term

(at 1 year post intervention)

2.5 Public facilities

medium term (at 1 year post

intervention) (subgroup)

2.6 Private facilities

medium term (at 1 year post

intervention) (subgroup)

3 Functional impairment/disability in CMD (completers-

combined) all facilities and in

public and private facilities

(SMD)

3.1 All facilities short

term (within 6 months post intervention)

3.2 Public facilities short term (within 6 months post intervention) (subgroup)

3.3 Private facilities short term (within 6 months post intervention) (subgroup)

3.4 All facilities medium term

(at 1 year post intervention)

3.5 Public facilities

medium term (at 1 year post

intervention) (subgroup)

3.6 Private facilities

medium term (at 1 year post

intervention) (subgroup)

4 Suicide attempt for those with CMDs all facilities and in public/private facilities (completers)

4.1 All facilities short term (6 months post intervention)

4.2 Public facilities short term

(6 months post intervention)

4.3 Private facilities short term

(6 months post intervention)

4.4 All facilities medium term

(1 year post intervention)

4.5 Public facilities medium term (1 year post intervention)

4.6 Private facilities medium

term (1 year post intervention)

5 Prevalence of CMDs (only Patel

- sensitivity analysis (SA))

(completers) all facilities and in

public and private facilities
Subtotals only

Subtotals only 
5.1 All facilities short term (within 6 months post intervention)

5.2 Public facilities short term (within 6 months post intervention) (subgroup)

5.3 Private facilities at 6 months post intervention

5.4 All facilities medium term (at 1 year post intervention)

5.5 Public facilities medium term (at 1 year post intervention) (subgroup)

5.6 Private facilities at 1 year post intervention

6 Severity of symptoms in CMD

(only Patel and Jenkins (SA)) in all facilities and in public and private facilities

6.1 All facilities short term (within 6 months post intervention)

6.2 Public facilities short term (within 6 months post intervention) (subgroup)

6.3 Private facilities short term (within 6 months post intervention) (subgroup)

6.4 All facilities medium term (at 1 year post intervention) 6.5 Public facilities medium term (at 1 year post intervention) (subgroup)

6.6 Private facilities medium term (at 1 year post intervention) (subgroup)

7 Prevalence of depression (completers) (SA) all facilities and in public and private facilities

7.1 All facilities short term (within 6 months post intervention)

7.2 Public facilities short term (within 6 months post intervention) (subgroup)

7.3 Private facilities short term (within 6 months post intervention) (subgroup)

7.4 All facilities medium term

(1 year post intervention)
$0.59[0.41,0.85]$

$1.12[0.68,1.84]$

$0.95[0.68,1.33]$

$0.72[0.39,1.34]$

$1.25[0.76,2.06]$

Std. Mean Difference (Random, 95\% CI) Subtotals only

Std. Mean Difference (Random, 95\% CI)

$-0.07[-0.15,-0.00]$

Std. Mean Difference (Random, 95\% CI)

$-0.11[-0.21,-0.00]$

823 Std. Mean Difference (Random, 95\% CI)

$0.03[-0.11,0.16]$

Std. Mean Difference (Random, 95\% CI)

$-0.03[-0.12,0.06]$

1

1104

Std. Mean Difference (Random, 95\% CI)

$-0.07[-0.19,0.05]$

1 801

Std. Mean Difference (Random, 95\% CI)

$0.02[-0.11,0.16]$

Risk Ratio (Random, 95\% CI)

Subtotals only

Risk Ratio (Random, 95\% CI)

$0.61[0.40,0.94]$ 
7.5 Public facilities medium term (1 year post intervention) (subgroup)

7.6 Private facilities medium term (at 1 year post intervention) (subgroup)

8 Severity of symptoms of depression (SA) in all facilities and in public and private facilities

8.1 All facilities short term (within 6 months post intervention)

8.2 Public facilities short term (within 6 months post intervention) (subgroup)

8.3 Private facilities short term (within 6 months post intervention) (subgroup)

8.4 All facilities medium term (at 1 year post intervention)

8.5 Public facilities medium term (at 1 year post intervention) (subgroup)

8.6 Private facilities medium term (at 1 year post intervention) (subgroup)

9 Functional impairment/disability in CMD (SA) all facilities and in public and private facilities (SMD)

9.1 All facilities short term (within 6 months post intervention)

9.2 Public facilities short term (within 6 months post intervention) (subgroup)

9.3 Private facilities short term (within 6 months post intervention) (subgroup)

9.4 All facilities medium term (at 1 year post intervention)

9.5 Public facilities medium term (at 1 year post intervention) (subgroup)

9.6 Private facilities medium term (at 1 year post intervention) (subgroup)
Subtotals only

Std. Mean Difference (Random, 95\% CI)

$-0.39[-0.78,0.01]$

Std. Mean Difference (Random, 95\% CI)

$-0.41[-0.79,-0.04]$

1

Std. Mean Difference (Random, 95\% CI)

$0.16[-0.09,0.41]$

652 Std. Mean Difference (Random, 95\% CI)

$0.11[-0.05,0.26]$

1 398

Std. Mean Difference (Random, 95\% CI)

$-0.09[-0.29,0.12]$

Std. Mean Difference (Random, 95\% CI)

$-0.03[-0.28,0.22]$

Subtotals only

Std. Mean Difference (Random, 95\% CI)

$-0.03[-0.10,0.04]$
Std. Mean Difference (Random, 95\% CI) 
10 Functional

impairment/disability in CMD

(SA) all facilities and in public and private facilities (MD)

10.1 All facilities short term (within 6 months post intervention)

10.2 Public facilities short term (within 6 months post intervention) (subgroup)

10.3 Private facilities short term (within 6 months post intervention) (subgroup)

10.4 All facilities medium term (at 1 year post intervention)

10.5 Public facilities medium term (at 1 year post intervention) (subgroup)

10.6 Private facilities medium term (at 1 year post intervention) (subgroup)

11 Functional impairment/disability in depression (SA) all facilities and in public and private facilities 11.1 All facilities short term (within 6 months post intervention)

11.2 Public facilities short term (within 6 months post intervention) (subgroup)

11.3 Private facilities short term (at 6 months post intervention) (subgroup)

11.4 All facilities medium term (at 1 year post intervention)

11.5 Public facilities medium term (at 1 year post intervention) (subgroup)

11.6 Private facilities medium term (at 1 year post intervention) (subgroup) 


\begin{tabular}{lcccc} 
Outcome or subgroup title & $\begin{array}{c}\text { No. of } \\
\text { studies }\end{array}$ & $\begin{array}{c}\text { No. of } \\
\text { participants }\end{array}$ & Statistical method & Effect size \\
\hline $\begin{array}{l}\text { 1 Severity of symptoms in treating } \\
\text { maternal depression }\end{array}$ & 4 & 1213 & Std. Mean Difference (Random, 95\% CI) & $-0.42[-0.58,-0.26]$ \\
$\quad \begin{array}{l}1.1 \text { NSHW-led interventions } \\
\text { short term (within 3 months }\end{array}$ & 2 & 858 & Std. Mean Difference (Random, 95\% CI) & $-0.50[-0.63,-0.36]$ \\
$\quad \begin{array}{l}\text { post intervention) } \\
\quad 1.2 \text { Collaborative care short }\end{array}$ & 1 & 230 & Std. Mean Difference (Random, 95\% CI) & $-0.22[-0.48,0.04]$ \\
$\quad \begin{array}{l}\text { term (at } 3 \text { months post } \\
\text { intervention) } \\
\quad 1.3 \text { NSHW-led intervention }\end{array}$ & 1 & 125 & Std. Mean Difference (Random, 95\% CI) & $-0.41[-0.76,-0.06]$ \\
$\quad \begin{array}{l}\text { medium term (at 1 year post } \\
\text { intervention) }\end{array}$ & & & & \\
\hline
\end{tabular}

Comparison 4. NSHWs versus specialists in treating depression in adults (controlled before-and-after studies)

\begin{tabular}{|c|c|c|c|c|}
\hline Outcome or subgroup title & $\begin{array}{l}\text { No. of } \\
\text { studies }\end{array}$ & $\begin{array}{c}\text { No. of } \\
\text { participants }\end{array}$ & Statistical method & Effect size \\
\hline $\begin{array}{l}1 \text { Severity of depression short term } \\
\text { (2 months post intervention) }\end{array}$ & 1 & 768 & Mean Difference (IV, Random, 95\% CI) & $-0.90[-1.20,-0.60]$ \\
\hline 2 Frequency of adverse events & 1 & 768 & Risk Ratio (M-H, Random, 95\% CI) & $0.85[0.67,1.07]$ \\
\hline 3 Number of days spent in hospital & 1 & & Mean Difference (IV, Random, 95\% CI) & Subtotals only \\
\hline 3.1 Outcomes at 1 year & 1 & 124 & Mean Difference (IV, Random, 95\% CI) & $-1.79[-3.59,0.01]$ \\
\hline 3.2 Outcomes at 2 years & 1 & 124 & Mean Difference (IV, Random, 95\% CI) & $-0.02[-2.59,2.55]$ \\
\hline $\begin{array}{l}4 \text { Number of days spent on sick } \\
\text { leave }\end{array}$ & 1 & & Mean Difference (IV, Random, 95\% CI) & Subtotals only \\
\hline 4.1 Outcome at 1 year & 1 & 108 & Mean Difference (IV, Random, 95\% CI) & $-3.96[-15.58,7.66]$ \\
\hline 4.2 Outcome at 2 years & 1 & 123 & Mean Difference (IV, Random, 95\% CI) & $14.63[-0.76,30.02]$ \\
\hline
\end{tabular}

Comparison 5. NSHW-led psychological interventions versus usual care in treating adults with post-traumatic stress disorder (RCT and NRCT)

\begin{tabular}{lcccc} 
Outcome or subgroup title & $\begin{array}{c}\text { No. of } \\
\text { studies }\end{array}$ & $\begin{array}{c}\text { No. of } \\
\text { participants }\end{array}$ & Statistical method & Effect size \\
\hline $\begin{array}{l}1 \text { Prevalence of post-traumatic } \\
\text { stress disorder (PTSD) }\end{array}$ & 1 & & Risk Ratio (M-H, Fixed, 95\% CI) & Subtotals only \\
$\quad \begin{array}{l}1.1 \text { LHW-led narrative } \\
\text { exposure therapy short term (6 }\end{array}$ & 1 & 62 & Risk Ratio (M-H, Fixed, 95\% CI) & $0.48[0.27,0.85]$ \\
$\quad$ months post intervention) & & & & \\
\hline
\end{tabular}

Non-specialist health worker interventions for the care of mental, neurological and substance-abuse disorders in low- and middle- 
1.2 LHW-led trauma counselling short term (6 months post intervention) 2 Severity of PTSD symptoms 2.1 Short term LHW-led counselling with PTSD psychoeducation (6 months post intervention)

2.2 Short term (Yeomans second arm) (2 weeks post intervention)

2.3 Short term (Neuner first arm - narrative exposure therapy) (6 months post intervention)

3 Severity of depression

3.1 LHW-led workshop with psychoeducation short term (2 weeks post intervention) 3.2 LHW-led workshop without psychoeducation short term (2 weeks post intervention)

\section{Comparison 6. NSHWs versus usual care in improving dementia patients' and carers' outcomes (RCTs)}

\begin{tabular}{lcclc} 
Outcome or subgroup title & $\begin{array}{c}\text { No. of } \\
\text { studies }\end{array}$ & $\begin{array}{c}\text { No. of } \\
\text { participants }\end{array}$ & \multicolumn{1}{c}{ Statistical method } & Effect size \\
\hline $\begin{array}{l}\text { 1 Severity of behavioural problem } \\
\quad \text { patient) }\end{array}$ & 2 & 134 & Std. Mean Difference (Random, 95\% CI) & $-0.26[-0.60,0.08]$ \\
2 Patient functional ability & 1 & 81 & Mean Difference (Random, 95\% CI) & $-0.24[-0.67,0.20]$ \\
3 Patient quality of life & 1 & 53 & Mean Difference (Random, 95\% CI) & $-0.43[-0.98,0.12]$ \\
4 Carer mental health status & 2 & 134 & Std. Mean Difference (Random, 95\% CI) & $-0.42[-0.76,-0.08]$ \\
5 Carer burden & 2 & 134 & Std. Mean Difference (Random, 95\% CI) & $-0.50[-0.84,-0.15]$ \\
6 Carer distress & 2 & 134 & Std. Mean Difference (Random, 95\% CI) & $-0.47[-0.82,-0.13]$ \\
7 Carer quality of life & 1 & 53 & Mean Difference (Random, 95\% CI) & $-0.37[-0.92,0.17]$ \\
\hline
\end{tabular}


Comparison 7. NSHW-led brief alcohol interventions versus usual care for adults with alcohol-use disorders (RCTs)

\begin{tabular}{|c|c|c|c|c|}
\hline Outcome or subgroup title & $\begin{array}{l}\text { No. of } \\
\text { studies }\end{array}$ & $\begin{array}{c}\text { No. of } \\
\text { participants }\end{array}$ & Statistical method & Effect size \\
\hline $\begin{array}{l}1 \text { Amount of alcohol consumed } \\
\text { (MD) }\end{array}$ & 2 & 167 & Mean Difference (Random, 95\% CI) & $-1.68[-2.79,-0.57]$ \\
\hline 2 Frequency of binge drinking & 1 & 92 & Mean Difference (IV, Random, 95\% CI) & $-0.50[-1.14,0.14]$ \\
\hline 3 Adverse consequences & 2 & 160 & Risk Ratio (M-H, Random, 95\% CI) & $0.77[0.11,5.29]$ \\
\hline 3.1 Road traffic accidents & 1 & 92 & Risk Ratio (M-H, Random, 95\% CI) & $0.36[0.12,1.08]$ \\
\hline 3.2 Withdrawal symptoms & 1 & 68 & Risk Ratio (M-H, Random, 95\% CI) & $2.67[0.29,24.37]$ \\
\hline
\end{tabular}

Comparison 8. NSHWs/OPHRs versus usual care in conducting interventions for children with post-traumatic stress and depression (RCTs)

\begin{tabular}{|c|c|c|c|c|}
\hline Outcome or subgroup title & $\begin{array}{l}\text { No. of } \\
\text { studies }\end{array}$ & $\begin{array}{c}\text { No. of } \\
\text { participants }\end{array}$ & Statistical method & Effect size \\
\hline $\begin{array}{l}1 \text { Severity of PTSD symptoms - } \\
\text { teacher/LHW-led interventions } \\
\text { (SMDs) }\end{array}$ & 3 & & Std. Mean Difference (Random, 95\% CI) & Subtotals only \\
\hline $\begin{array}{l}1.1 \text { Short term (within } 6 \\
\text { months post intervention) }\end{array}$ & 3 & 298 & Std. Mean Difference (Random, 95\% CI) & $-0.89[-1.49,-0.30]$ \\
\hline $\begin{array}{l}\text { 1.2 Short term (Ertl } \\
\text { second arm) ( } 5 \text { months post } \\
\text { intervention) }\end{array}$ & 1 & 51 & Std. Mean Difference (Random, 95\% CI) & $-0.12[-0.67,0.44]$ \\
\hline $\begin{array}{l}\text { 1.3 Medium term (Ertl } \\
\text { first arm) ( } 11 \text { months post } \\
\text { intervention) }\end{array}$ & 1 & 53 & Std. Mean Difference (Random, 95\% CI) & $-0.45[-0.99,0.10]$ \\
\hline $\begin{array}{l}\text { 1.4 Medium term (Ertl } \\
\text { second arm) (11 months post } \\
\text { intervention) }\end{array}$ & 1 & 51 & Std. Mean Difference (Random, 95\% CI) & $-0.04[-0.59,0.52]$ \\
\hline $\begin{array}{l}2 \text { Severity of PTSD symptoms } \\
\text { - classroom-based LHW } \\
\text { interventions (MCDs) }\end{array}$ & 3 & & Mean Change Difference (Random, 95\% CI) & Subtotals only \\
\hline $\begin{array}{l}2.1 \text { Short term (within } 6 \\
\text { months post intervention) }\end{array}$ & 3 & 1090 & Mean Change Difference (Random, 95\% CI) & $-0.56[-2.82,1.70]$ \\
\hline $\begin{array}{l}3 \text { Severity of PTSD symptoms } \\
\text { - classroom-based LHW } \\
\text { interventions - boys/girls }\end{array}$ & 1 & 399 & Mean Change Difference (Random, 95\% CI) & $1.40[-1.58,4.37]$ \\
\hline $\begin{array}{l}\text { 3.1 Short term (boys) (within } \\
6 \text { months post intervention) }\end{array}$ & 1 & 245 & Mean Change Difference (Random, 95\% CI) & $0.0[-2.02,2.02]$ \\
\hline $\begin{array}{l}\text { 3.2 Short term (girls) (within } \\
6 \text { months post intervention) }\end{array}$ & 1 & 154 & Mean Change Difference (Random, 95\% CI) & $3.05[0.39,5.71]$ \\
\hline $\begin{array}{l}4 \text { Severity of depressive symptoms - } \\
\text { teacher/LHW-led interventions } \\
\text { (SMDs) }\end{array}$ & 4 & & Std. Mean Difference (Random, 95\% CI) & Subtotals only \\
\hline
\end{tabular}

Non-specialist health worker interventions for the care of mental, neurological and substance-abuse disorders in low- and middle- 
4.1 Short term (within 6 months post intervention)

4.2 Short term (Bolton second arm) (6 months post intervention)

4.3 Short term (Ertl second arm) (5 months post intervention)

4.4 Medium term (Ertl first arm) (11 months post intervention)

4.5 Medium term (Ertl second arm) (11 months post intervention)

5 Severity of depressive symptoms - classroom-based LHW interventions (MCDs)

5.1 Short term (within 6 months post intervention)

6 Severity of depressive symptoms (MCDs) Tol 2012 boys/girls

6.1 Short term (boys) (within 6 months post intervention)

6.2 Short term (girls) (within 6 months post intervention)

7 Severity of anxiety symptoms classroom-based intervention (within 6 months post intervention)

7.1 Short term (within 6 months post intervention)

8 Severity of anxiety symptoms classroom-based intervention boys/girls

8.1 Short term (boys) (within 6 months post intervention) 8.2 Short term (girls) (within 6 months post intervention)

9 Functional impairment teacher/LHW-led interventions

9.1 Short term (within 6 months post intervention) 9.2 Medium term (11 months post intervention)

10 Functional impairment LHW-led - classroom-based intervention

10.1 Short term (within 6 months post intervention)
Mean Change Difference (Random, 95\% CI)

1092

Mean Change Difference (Random, 95\% CI)

Std. Mean Difference (Random, 95\% CI)

Mean Change Difference (Random, 95\% CI)

Mean Change Difference (Random, 95\% CI)
Std. Mean Difference (Random, 95\% CI)
Subtotals only

$-0.18[-0.33,-0.03]$

$0.27[-0.58,1.12]$

$-0.02[-1.18,1.14]$

$0.61[-0.63,1.85]$

$-0.34[-0.75,0.07]$

$-0.34[-0.75,0.07]$

$-0.22[-1.09,0.65]$

$-0.63[-1.23,-0.03]$

$0.26[-0.53,1.05]$

Subtotals only

$-0.61[-1.13,-0.08]$

$-0.69[-1.25,-0.14]$

Subtotals only

$-0.81[-1.48,-0.13]$

Non-specialist health worker interventions for the care of mental, neurological and substance-abuse disorders in low- and middle- 


\begin{tabular}{|c|c|c|c|c|}
\hline $\begin{array}{l}11 \text { Functional impairment } \\
\text { - classroom-based LHW } \\
\text { intervention - boys/girls }\end{array}$ & 1 & 399 & Mean Difference (Random, 95\% CI) & $-0.94[-1.80,-0.08]$ \\
\hline $\begin{array}{l}11.1 \text { Short term (boys) (within } \\
6 \text { months post intervention) }\end{array}$ & 1 & 245 & Mean Difference (Random, 95\% CI) & $-1.19[-2.23,-0.15]$ \\
\hline $\begin{array}{l}11.2 \text { Short term (girls) (within } \\
6 \text { months post intervention) }\end{array}$ & 1 & 154 & Mean Difference (Random, 95\% CI) & $-0.40[-1.93,1.13]$ \\
\hline
\end{tabular}

\section{Analysis I.I. Comparison I NSHW-led psychological interventions versus usual care in treating common mental disorders in adults (RCTs), Outcome I Prevalence of depression (completers).}

Review: Non-specialist health worker interventions for the care of mental, neurological and substance-abuse disorders in low- and middle-income countries

Comparison: I NSHW-led psychological interventions versus usual care in treating common mental disorders in adults (RCTs)

Outcome: I Prevalence of depression (completers)

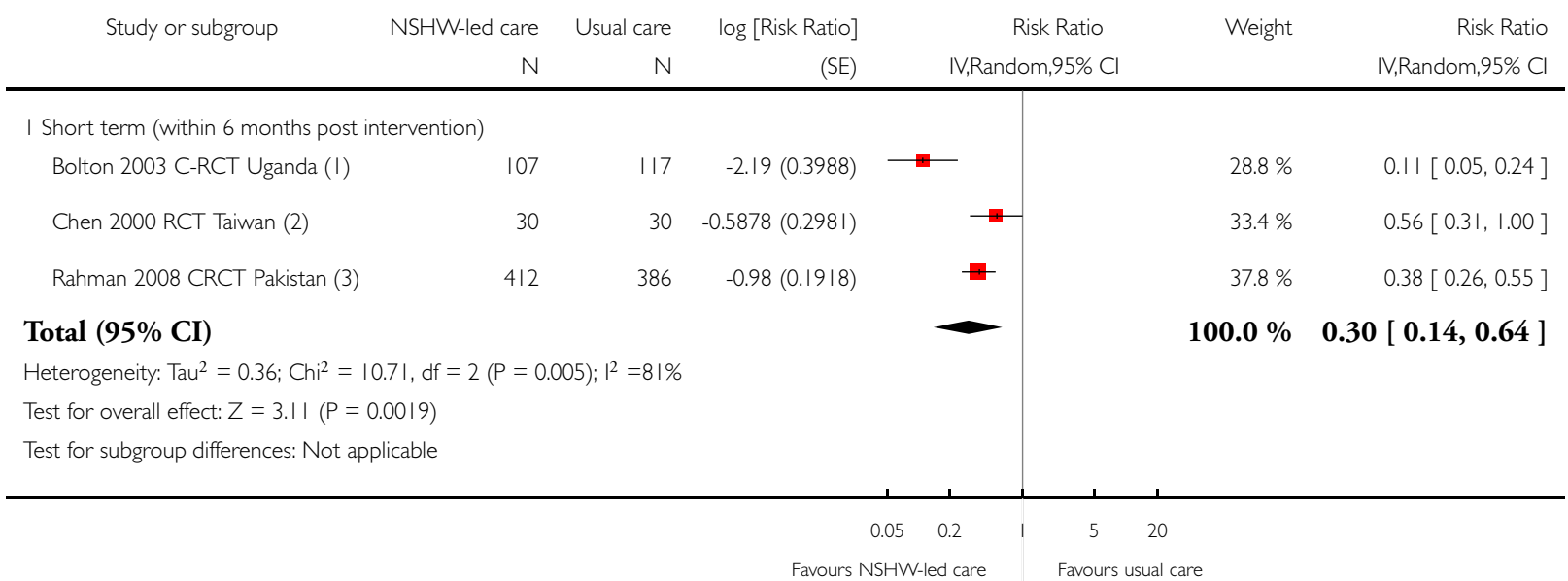

(I) Lay health worker (LHW) led group interpersonal therapy (G-IPT); DSM-IV Mollica criteria; 2 wks post-interv transformed adj ORs to adj log RR and calculated SE of $\log R$.

(2) Nurse-led psychosocial intervention; Taiwanese BDI; BDI score > I0; RR entered (immediately post intervention).

(3) LHW-led CBT-like intervention; Hamilton Depression Rating Scale; 2 months post intervention; transformed adjusted ORs into log risk ratios (95\% Cl); ICC = 0.047. 
Analysis I.2. Comparison I NSHW-led psychological interventions versus usual care in treating common mental disorders in adults (RCTs), Outcome 2 Prevalence of depression (ITT sensitivity analysis - assumption non-completers depressed).

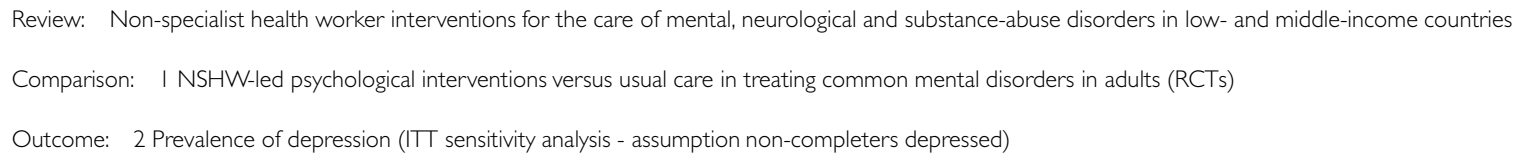

I Short term (within 6 months post intervention)

Bolton 2003 C-RCT Uganda (I)

Chen 2000 RCT Taiwan (2)

Rahman 2008 CRCT Pakistan (3)

Subtotal (95\% CI)

Heterogeneity: $\mathrm{Tau}^{2}=0.07 ; \mathrm{Chi}^{2}=10.45, \mathrm{df}=2(\mathrm{P}=0.0 \mathrm{I}) ; \mathrm{I}^{2}=81 \%$

Test for overall effect: $Z=2.97(P=0.0030)$

Test for subgroup differences: Not applicable

$\begin{array}{lll}163 & 178 & -0.5971(0.1567) \\ 57 & 58 & -0.2003(0.1182) \\ 463 & 440 & -0.7159(0.1132)\end{array}$

$\begin{array}{ll}30.6 \% & 0.55[0.40,0.75] \\ 34.5 \% & 0.82[0.65,1.03] \\ 35.0 \% & 0.49[0.39,0.61]\end{array}$

$100.0 \% \quad 0.61[0.43,0.84]$ per Bolton 2007))

(2) Nurse-led psychosocial intervention; Taiwanese BDI (Beck's depression inventory); BDI score >I0; immediately post-interv; events (BDI>I0)/ITT denominator

(3) LHW-led CBT-like interv; Hamilton Depr Rating scale (> I 7); 2 mths post-interv; prev of depr (completers+( $\mathrm{N}$ of ITT-N of completers), cluster-adjusted using ICC=0.047 
Analysis I.3. Comparison I NSHW-led psychological interventions versus usual care in treating common mental disorders in adults (RCTs), Outcome 3 Prevalence of depression (ITT sensitivity analysis - assumption non-completers not depressed).

Review: Non-specialist health worker interventions for the care of mental, neurological and substance-abuse disorders in low- and middle-income countries

Comparison: I NSHW-led psychological interventions versus usual care in treating common mental disorders in adults (RCTs)

Outcome: 3 Prevalence of depression (ITT sensitivity analysis - assumption non-completers not depressed)

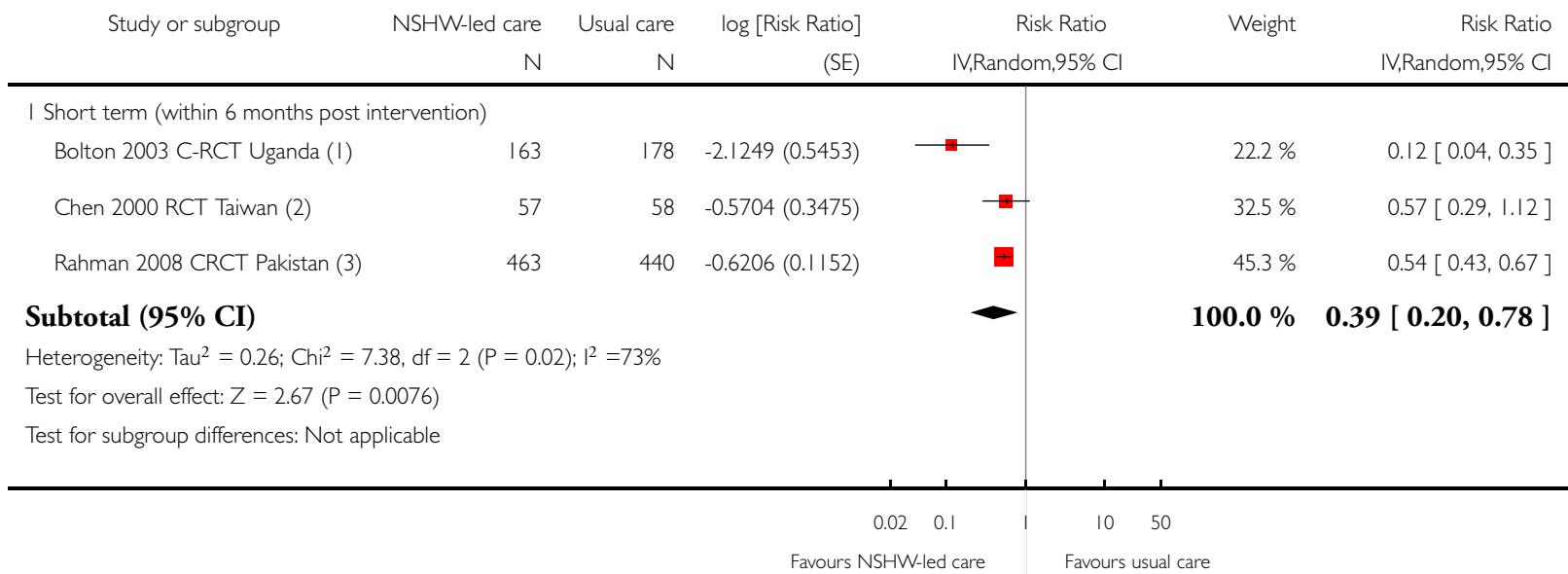

(I) LHW led G-IPT; DSM-IV Mollica criteria; 2 weeks post interv; events (prevalence at follow up)/ITT denominator, cluster-adjusted using ICC=0.099

(2) Nurse-led psychosocial intervention; Taiwanese BDI (Beck's depression inventory); proportions with BDI score > I0; prevalence of depression (completers)/ N of ITI

(3) LHW-led CBT-like intervention; Hamilton Depression Rating scale (>17); 2 months post-interv; events (prevalence at follow up)/ITT denominator; cluster-adjusted using ICC $=0.047$ 
Analysis I.4. Comparison I NSHW-led psychological interventions versus usual care in treating common mental disorders in adults (RCTs), Outcome 4 Prevalence of depression (ITT sensitivity analysis - worse-case scenario intervention group depressed; control group not depressed).

Review: Non-specialist health worker interventions for the care of mental, neurological and substance-abuse disorders in low- and middle-income countries

Comparison: I NSHW-led psychological interventions versus usual care in treating common mental disorders in adults (RCTs)

Outcome: 4 Prevalence of depression (ITT sensitivity analysis - worse-case scenario intervention group depressed; control group not depressed)

\begin{tabular}{|c|c|c|c|c|c|c|c|}
\hline \multirow[t]{2}{*}{ Study or subgroup } & NSHW-led care & Usual care & log [Risk Ratio] & \multicolumn{2}{|c|}{ Risk Ratio } & Weight & Risk Ratio \\
\hline & $N$ & $N$ & (SE) & \multicolumn{2}{|c|}{ IV,Random,95\% Cl } & & IV,Random,95\% Cl \\
\hline \multicolumn{8}{|c|}{ I Short term (within 6 months post intervention) } \\
\hline Bolton 2003 C-RCT Uganda (I) & 163 & 178 & $0.0723(0.2)$ & & ? & $32.8 \%$ & $1.07[0.73,1.59]$ \\
\hline Chen 2000 RCT Taiwan (2) & 57 & 58 & $0.7379(0.2186)$ & & 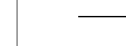 & $32.1 \%$ & $2.09[1.36,3.21]$ \\
\hline Rahman 2008 CRCT Pakistan (3) & 463 & 440 & $-0.447(0.1216)$ & + & & $35.1 \%$ & $0.64[0.50,0.81]$ \\
\hline \multicolumn{6}{|l|}{ Subtotal (95\% CI) } & $100.0 \%$ & $1.11[0.56,2.21]$ \\
\hline \multicolumn{8}{|c|}{ Heterogeneity: $\operatorname{Tau}^{2}=0.34 ; \mathrm{Chi}^{2}=23.55, \mathrm{df}=2(\mathrm{P}<0.0000 \mathrm{I}) ; \mathrm{I}^{2}=92 \%$} \\
\hline \multicolumn{8}{|c|}{ Test for overall effect: $Z=0.29(P=0.77)$} \\
\hline \multicolumn{8}{|c|}{ Test for subgroup differences: Not applicable } \\
\hline & & & & 0.7 & 1.5 & 2 & \\
\hline
\end{tabular}

(I) LHW led G-IPT; DSM-IV Mollica criteria; 2 weeks post intervention, Prevalence of depression events, cluster-adjusted with ICC=0.099

(2) Nurse-led psychosocial intervention; Taiwanese BDI (Beck's depression inventory); proportions with BDI score > I0; immediately post-intervention events of depression prevalence

(3) LHW-led CBT-like intervention; Hamilton Depression Rating scale (> 17); 2 months post-interv; events of prevalence of depression, cluster-adjusted with ICC=0.047 
Analysis I.5. Comparison I NSHW-led psychological interventions versus usual care in treating common mental disorders in adults (RCTs), Outcome 5 Prevalence of depression (ITT sensitivity analysis - best-case scenario: intervention group not depressed; control group all depressed).

Review: Non-specialist health worker interventions for the care of mental, neurological and substance-abuse disorders in low- and middle-income countries

Comparison: I NSHW-led psychological interventions versus usual care in treating common mental disorders in adults (RCTs)

Outcome: 5 Prevalence of depression (ITT sensitivity analysis - best-case scenario: intervention group not depressed; control group all depressed)

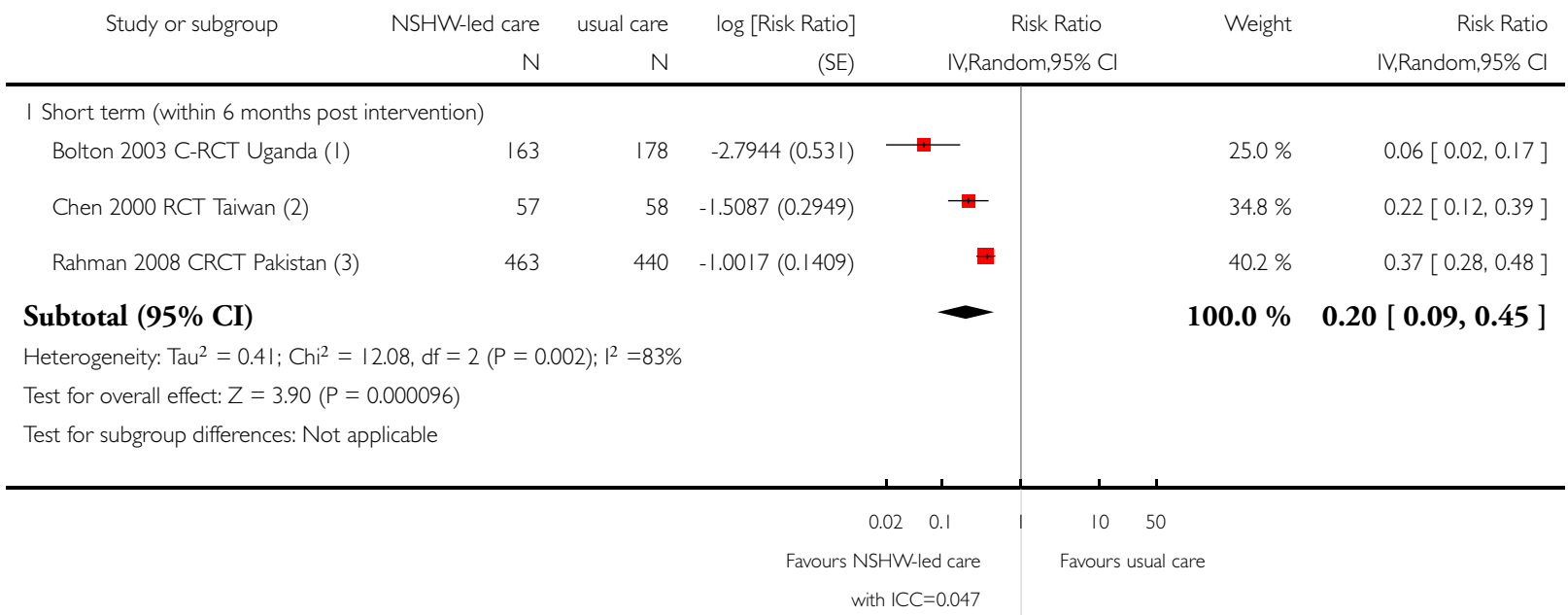

(I) LHW led G-IPT; DSM-IV Mollica criteria; 2 weeks post interv; prev of depression events; events of prevalence of depression, cluster-adjusted with ICC=0.099

(2) Nurse-led psychosocial intervention; Taiwanese BDI (Beck's depression inventory); proportions with BDI score >I 0; immediately post-interv prev of depression events

(3) LHW-led CBT-like intervention; Hamilton Depression Rating scale (> 17); 2 months post-interv;prev of depression events;events of prevalence of depression, clusteradjusted 
Analysis I.6. Comparison I NSHW-led psychological interventions versus usual care in treating common mental disorders in adults (RCTs), Outcome 6 Severity of common mental disorder symptoms (includes anxiety and depression).

Review: Non-specialist health worker interventions for the care of mental, neurological and substance-abuse disorders in low- and middle-income countries

Comparison: I NSHW-led psychological interventions versus usual care in treating common mental disorders in adults (RCTs)

Outcome: 6 Severity of common mental disorder symptoms (includes anxiety and depression)

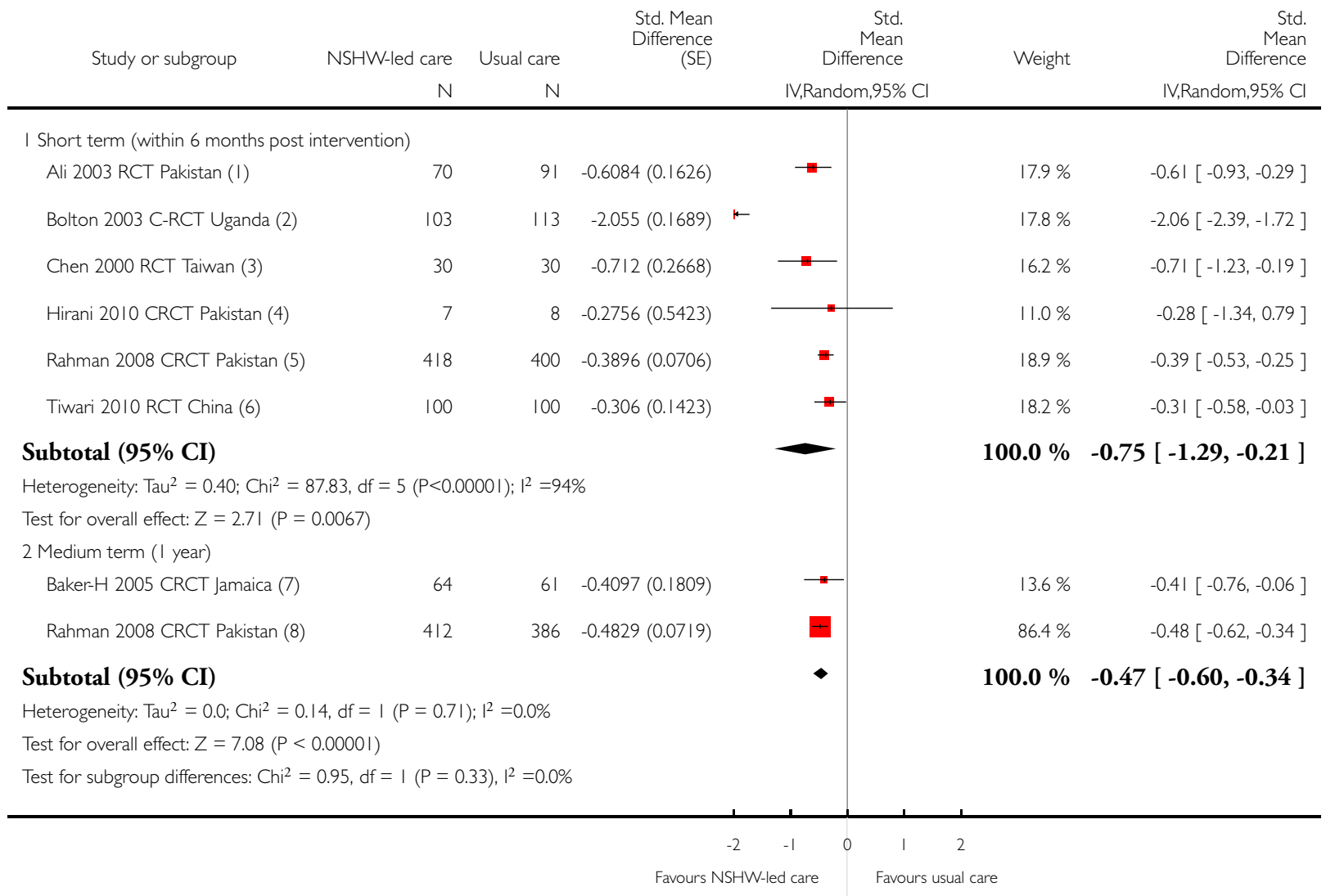

Non-specialist health worker interventions for the care of mental, neurological and substance-abuse disorders in low- and middle- 
(I) Lay health worker (LHW)-led adapted cognitive behavioural therapy (CBT); AKUADS score (anx and depr); mean and standard deviation(SD) (2 mths post-interv)).

(2) LHW-led group interpersonal therapy (G-IPT); Hopkins Symptom Checklist (HSCL); adj mean difference (adj for clustering, baseline depression) at 6 mths post-interv.

(3) Nurse-led psychosocial intervention;Taiwanese Beck's Depression Inventory (BDI); t-tests (immediately post intervention)

(4) LHW-led counselling; BDI; means and SDs at 2 months post intervention; cluster-adjusted (intracluster correlation coefficient (ICC) = 0.056 to calculate design effect)

(5) LHW-led CBT depressed mothers; Hamilton Depression Rating Scale; mean difference (MD) scores adjusted for clustering and baseline effects (2 months post intervention).

(6) Social worker-led advocacy intervention; Chinese BDI; adjusted MD (baseline to combined immediately post intervantion to 6 months post intervention).

(7) LHW-led home visits; Center for Epidemiological Studies - Depression scale (CES-D); means SDs adjusted for clustering (information from author) (I year post intervention).

(8) LHW-led CBT depressed mothers; Hamilton Depression Rating Scale; MD scores adjusted for clustering and baseline effects (8 months post intervention).

\section{Analysis I.7. Comparison I NSHW-led psychological interventions versus usual care in treating common} mental disorders in adults (RCTs), Outcome 7 Functional impairment/disability in common mental disorders.

Review: Non-specialist health worker interventions for the care of mental, neurological and substance-abuse disorders in low- and middle-income countries

Comparison: I NSHW-led psychological interventions versus usual care in treating common mental disorders in adults (RCTs)

Outcome: 7 Functional impairment/disability in common mental disorders

\begin{tabular}{|c|c|c|c|c|c|c|c|}
\hline \multirow[t]{2}{*}{ Study or subgroup } & NSHW-led care & usual care & $\begin{array}{r}\text { Std. Mean } \\
\text { Difference } \\
(\mathrm{SE})\end{array}$ & & $\begin{array}{r}\text { Std. } \\
\text { Mean } \\
\text { ifference }\end{array}$ & \multirow[t]{2}{*}{ Weight } & \multirow{2}{*}{$\begin{array}{r}\text { Std. } \\
\text { Mean } \\
\text { Difference } \\
\text { IV,Random,95\% Cl }\end{array}$} \\
\hline & $\mathrm{N}$ & N & \multicolumn{3}{|c|}{ IV,Random,95\% Cl } & & \\
\hline \multicolumn{8}{|c|}{ I Short term (within 6 months post intervention) } \\
\hline Bolton 2003 C-RCT Uganda (I) & 103 & 107 & $-0.9502(0.1458)$ & 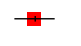 & & $28.5 \%$ & $-0.95[-1.24,-0.66]$ \\
\hline Hirani 2010 CRCT Pakistan (2) & 7 & 8 & $0.3584(0.5446)$ & & & $11.9 \%$ & $0.36[-0.71,1.43]$ \\
\hline Rahman 2008 CRCT Pakistan (3) & 418 & 400 & $-0.4446(0.0708)$ & + & & $31.0 \%$ & $-0.44[-0.58,-0.31]$ \\
\hline Tiwari 2010 RCT China (4) & 100 & 100 & $0.1127(0.1415)$ & & 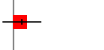 & $28.6 \%$ & $0.11[-0.16,0.39]$ \\
\hline Subtotal $(95 \% \mathrm{CI})$ & & & & & & $100.0 \%$ & $-0.33[-0.80,0.13]$ \\
\hline \multicolumn{8}{|c|}{ Heterogeneity: $\operatorname{Tau}^{2}=0.18 ; \mathrm{Chi}^{2}=29.56, \mathrm{df}=3(\mathrm{P}<0.0000 \mathrm{I}) ; \mathrm{I}^{2}=90 \%$} \\
\hline \multicolumn{8}{|c|}{ Test for overall effect: $Z=1.41(P=0.16)$} \\
\hline \multicolumn{8}{|c|}{2 Short term (advocacy empowerment physical functioning) short term (6 months post intervention) } \\
\hline Tiwari 2010 RCT China (5) & 100 & 100 & $0.0798(0.1415)$ & & & $100.0 \%$ & $0.08[-0.20,0.36]$ \\
\hline Subtotal (95\% CI) & & & & & & $100.0 \%$ & $0.08[-0.20,0.36]$ \\
\hline \multicolumn{8}{|c|}{ Heterogeneity: not applicable } \\
\hline \multicolumn{8}{|c|}{ Test for overall effect: $Z=0.56(P=0.57)$} \\
\hline \multicolumn{8}{|c|}{3 Medium term ( 8 months post intervention) } \\
\hline & & & -2 & -1 & 0 & 2 & \\
\hline \multicolumn{8}{|c|}{ Favours NSHW-led care } \\
\hline
\end{tabular}

Non-specialist health worker interventions for the care of mental, neurological and substance-abuse disorders in low- and middle- 


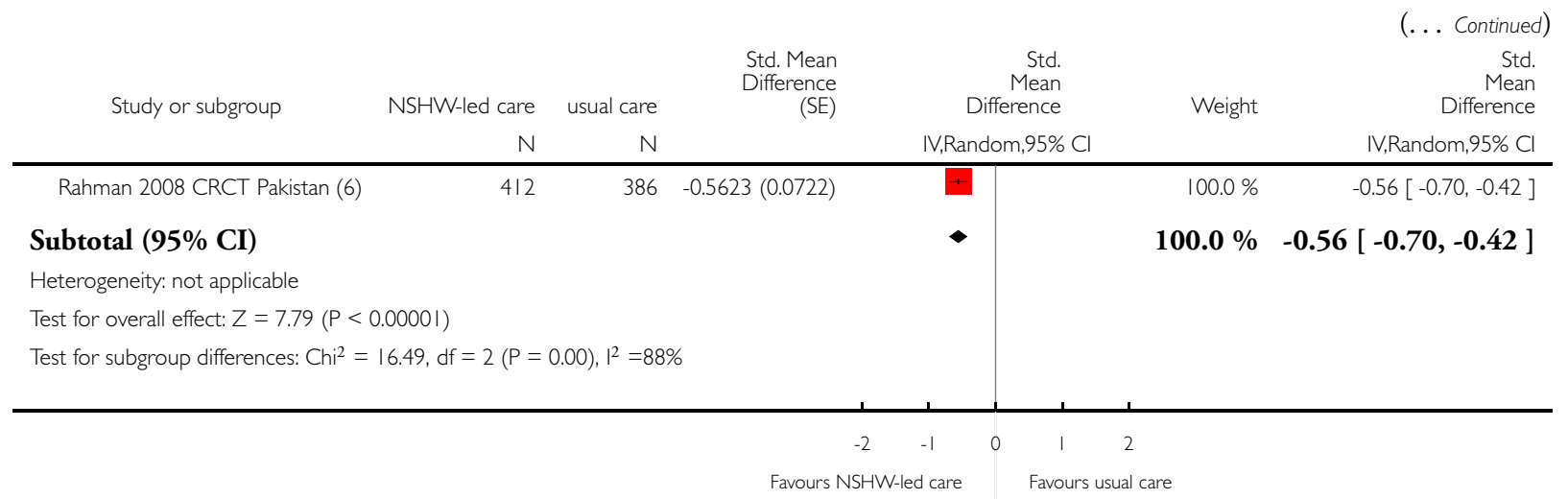

(I) LHW-led G-IPT; sex-specific functional impairment questionnaire;high score=high impairment; MD adj clustering/baseline scores (6 m post interv)

(2) LHW-led (counselling gr); General self-efficacy scale (GSE). higher score=greater efficacy -SMD reversed. 2 m post-interv, cluster-adjusted using ICC=0.056 (Rahman)

(3) LHW-led CBT-like interv; Global assessmt of functioning (GAF) scale (high score=better functioning -direction of effect reversed). Adj MDs+Cls 2 mths post-interv

(4) Social worker-led advocacy; SF-12 mental component; adjusted MD (baseline to combined immediately post-int to 6 mths post-interv)

(5) Social worker-led advocacy interv; SF- 12 physical component; adjusted MD (baseline to combined immediately post-int to 6 mths post-interv)

(6) LHW-led CBT-like interv; GAF scale (high score=better functioning -direction of effect reversed). Adj MDs+Cls 8 mths post-interv 
Analysis 2.I. Comparison 2 Collaborative care model (NSHWs plus specialist) versus usual care in treating common mental disorders (RCTs), Outcome I Prevalence of common mental disorders (CMDs - includes anxiety and depression) (completers-combined) all facilities and in public and private facilities.

Review: Non-specialist health worker interventions for the care of mental, neurological and substance-abuse disorders in low- and middle-income countries

Comparison: 2 Collaborative care model (NSHWs plus specialist) versus usual care in treating common mental disorders (RCTs)

Outcome: I Prevalence of common mental disorders (CMDs - includes anxiety and depression) (completers-combined) all facilities and in public and private facilities

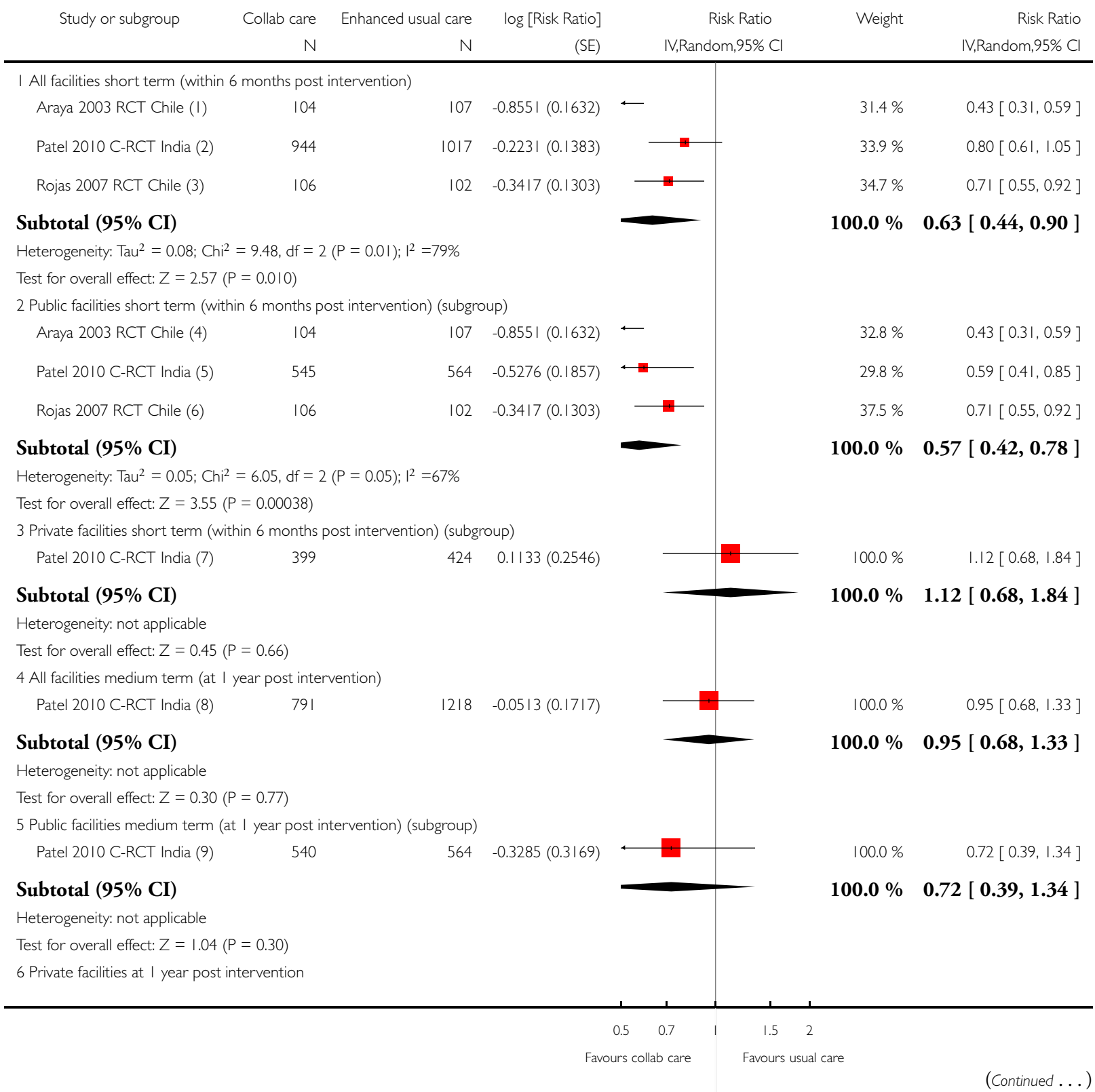

Non-specialist health worker interventions for the care of mental, neurological and substance-abuse disorders in low- and middle- 


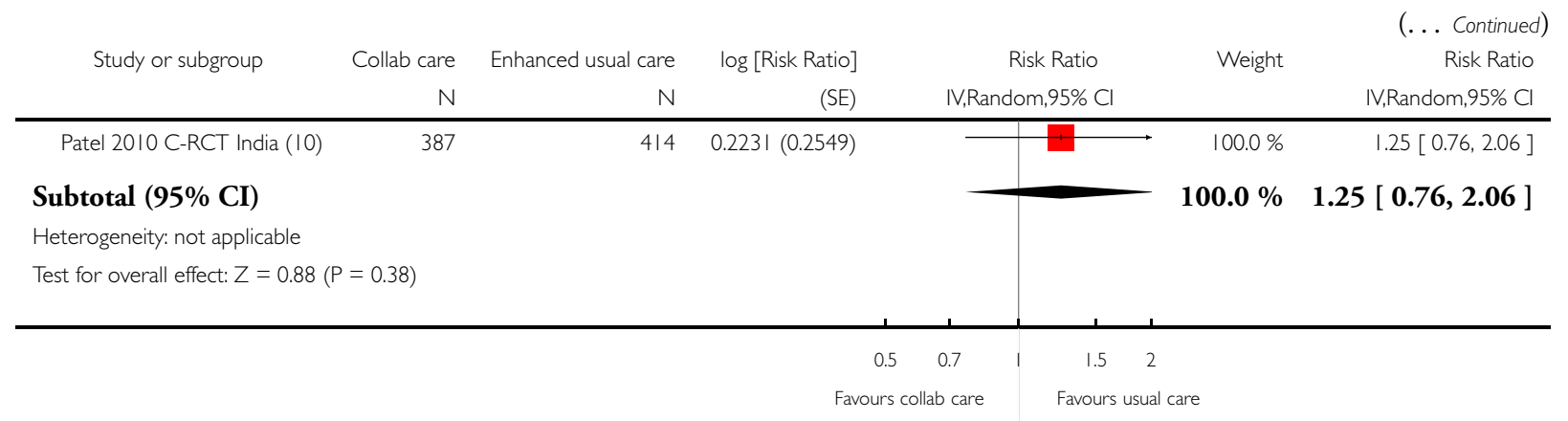

( I) Collab model for persistent recurrent depression;HRDS scores<8 (ie recovered) at 6 months; transformed to fit with Patel prevalence=total - nb recovered

(2) stepped care for CMDs; CIS-R; 6 month prevalence of CMDs adjusted RRs

(3) collab care for post natal depression; nb of patients with EPDS 6 point reduction 3 mths post-int (=6 mths post baseline).prev of depression (total - nb recovered)

(4) Collab model for persistent recurrent depression;HRDS scores $<8$ (ie recovered) at 6 months; transformed to fit with Patel prevalence by doing total - nb recovered

(5) stepped care for CMDs; CIS-R; 6 month prevalence of CMDs adjusted RRs

(6) collab care post natal depr; nb of patients with EPDS 6 point reduction at 3 mths post-int (=6 months post baseline).prevalence of depression (total - nb recovered)

(7) stepped care for CMDs; CIS-R; 6 month prevalence of CMDs adjusted RRs

(8) stepped care for CMDs; CIS-R; adjusted RR for non-recovered completers

(9) stepped care for CMDs; CIS-R; numbers and totals for CMDs; adjusted RR for non-recovered (completers)

( I0) stepped care for CMDs; CIS-R;adjusted RR for non-recovered (completers)

Non-specialist health worker interventions for the care of mental, neurological and substance-abuse disorders in low- and middle- 
Analysis 2.2. Comparison 2 Collaborative care model (NSHWs plus specialist) versus usual care in treating common mental disorders (RCTs), Outcome 2 Severity of symptoms of CMDs (completers-combined) in all facilities and in public and private facilities.

Review: Non-specialist health worker interventions for the care of mental, neurological and substance-abuse disorders in low- and middle-income countries

Comparison: 2 Collaborative care model (NSHWs plus specialist) versus usual care in treating common mental disorders (RCTs)

Outcome: 2 Severity of symptoms of CMDs (completers-combined) in all facilities and in public and private facilities

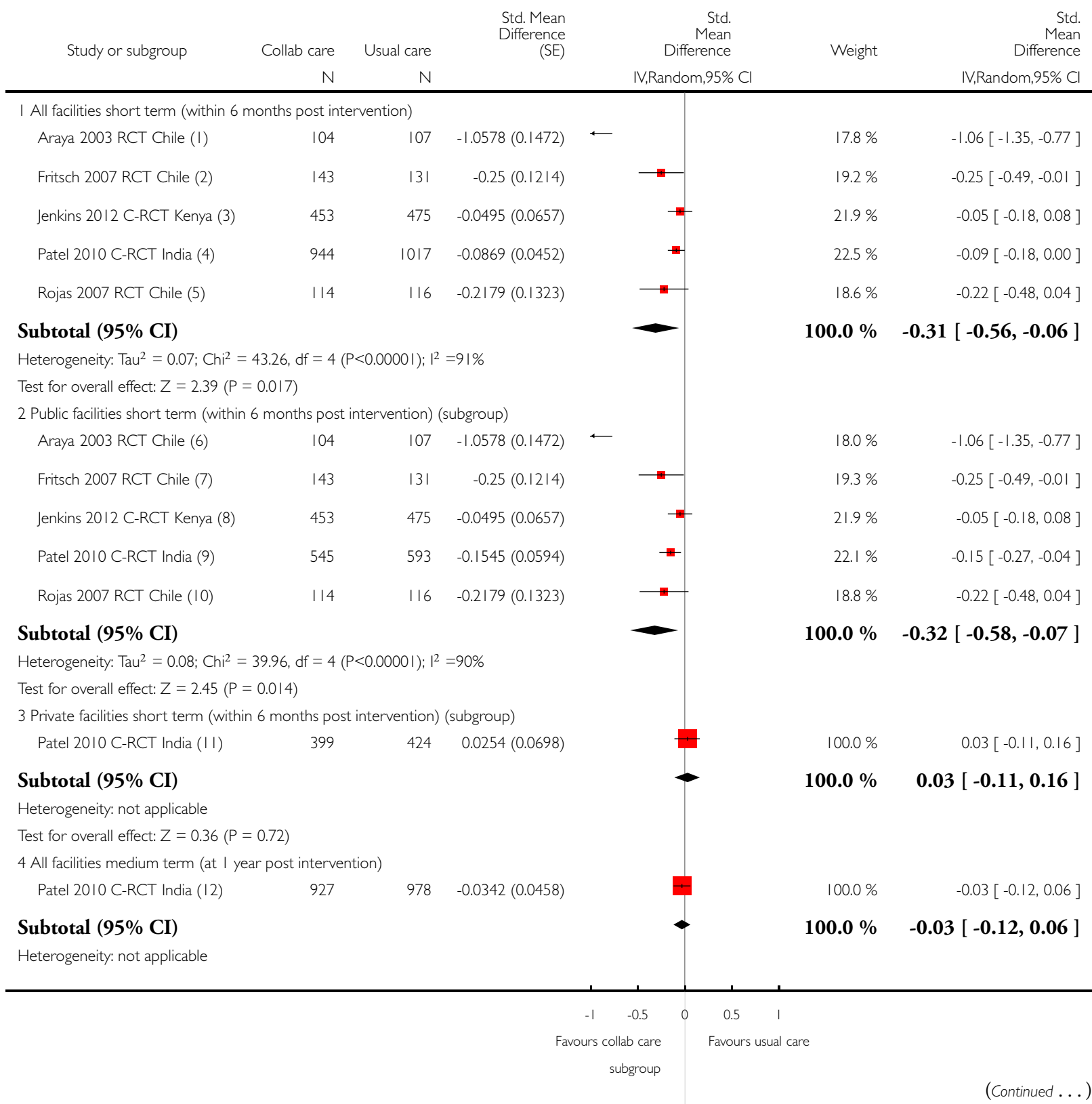

Non-specialist health worker interventions for the care of mental, neurological and substance-abuse disorders in low- and middle- 


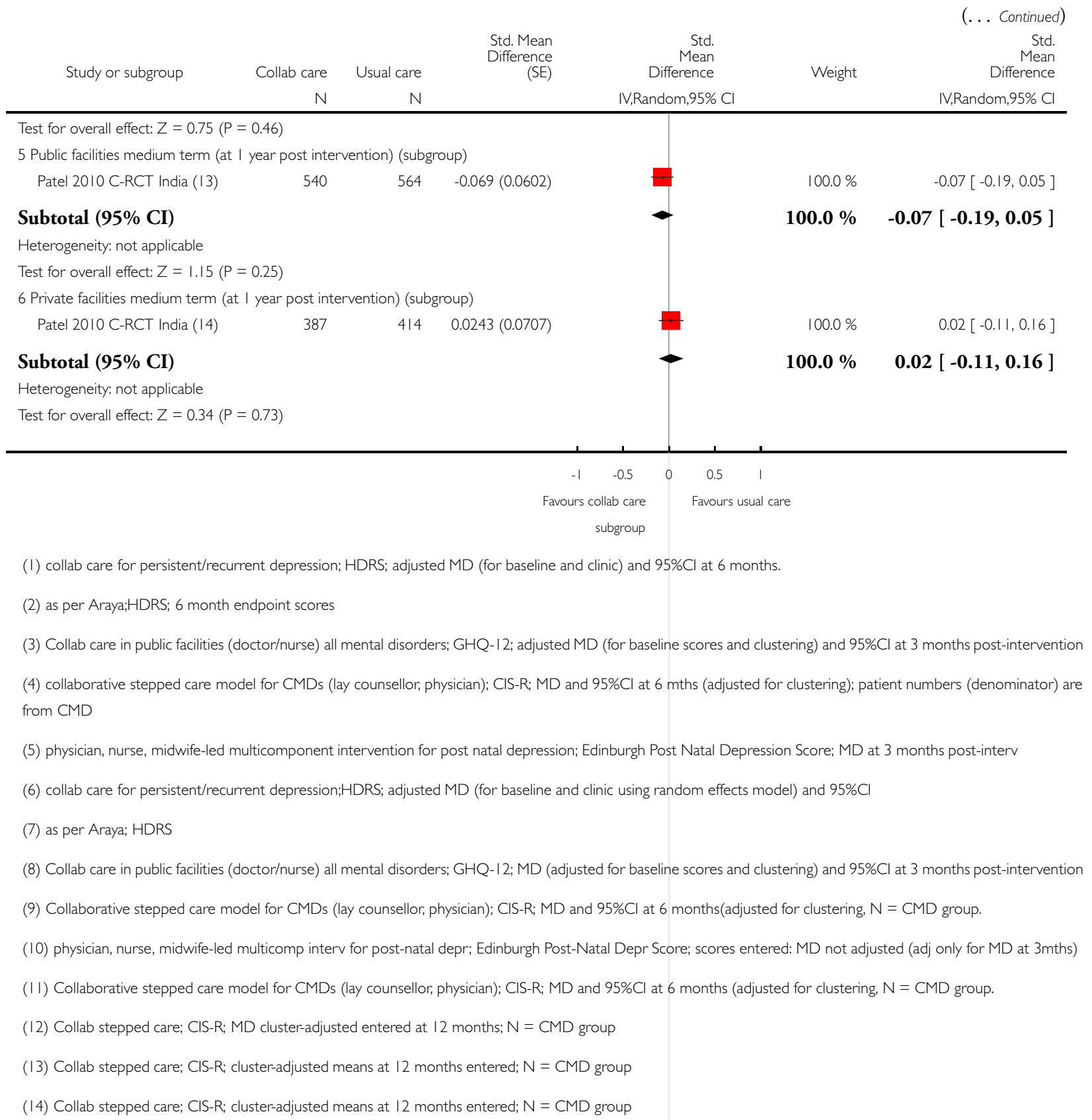

Non-specialist health worker interventions for the care of mental, neurological and substance-abuse disorders in low- and middle- 
Analysis 2.3. Comparison 2 Collaborative care model (NSHWs plus specialist) versus usual care in treating common mental disorders (RCTs), Outcome 3 Functional impairment/disability in CMD (completerscombined) all facilities and in public and private facilities (SMD).

Review: Non-specialist health worker interventions for the care of mental, neurological and substance-abuse disorders in low- and middle-income countries

Comparison: 2 Collaborative care model (NSHWs plus specialist) versus usual care in treating common mental disorders (RCTs)

Outcome: 3 Functional impairment/disability in CMD (completers- combined) all facilities and in public and private facilities (SMD)

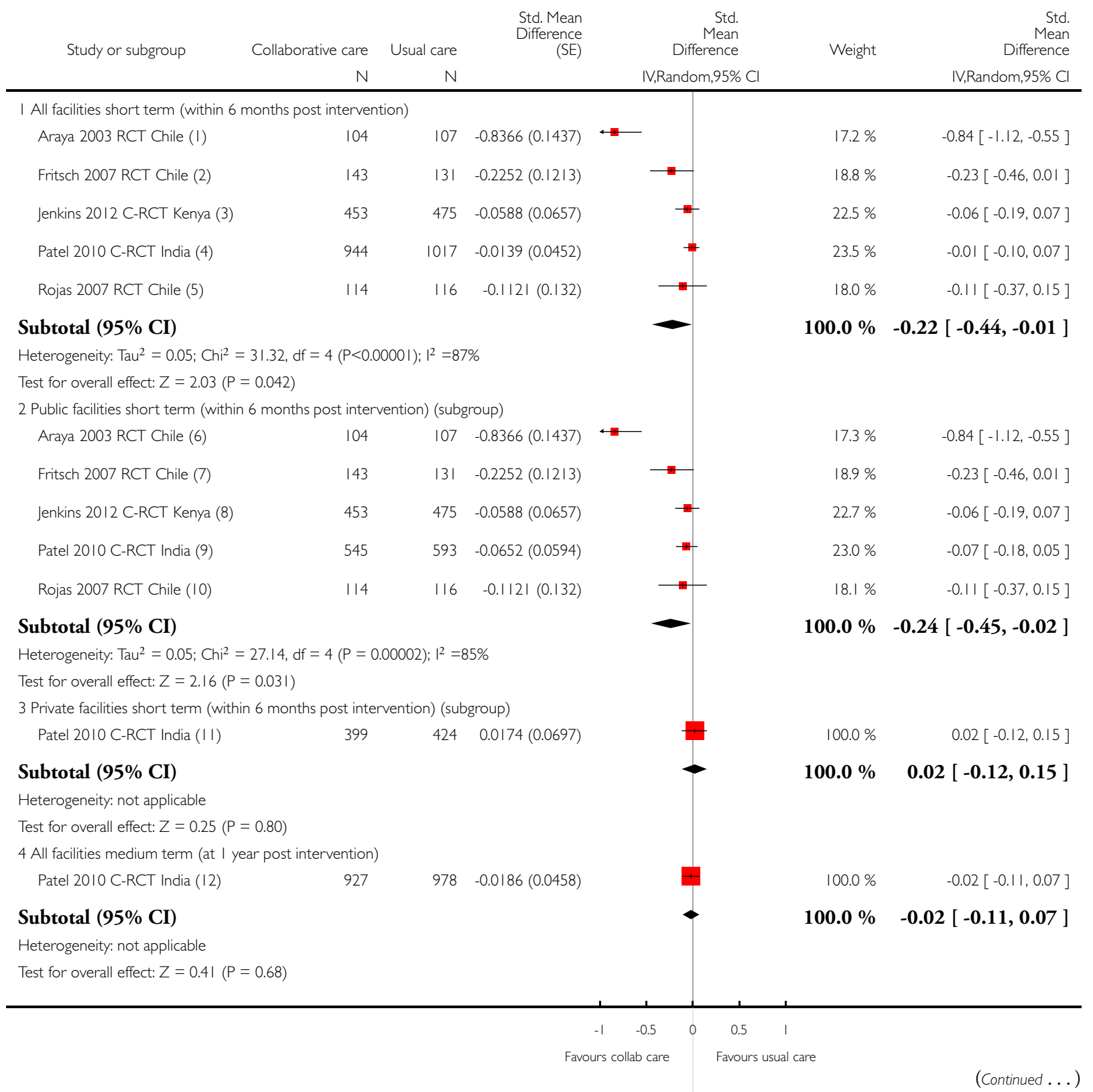

Non-specialist health worker interventions for the care of mental, neurological and substance-abuse disorders in low- and middle- 


\begin{tabular}{|c|c|c|c|c|c|c|}
\hline \multirow[t]{2}{*}{ Study or subgroup } & Collaborative care & Usual care & $\begin{array}{r}\text { Std. Mean } \\
\text { Difference } \\
\text { (SE) }\end{array}$ & $\begin{array}{r}\text { Std. } \\
\text { Mean } \\
\text { Difference }\end{array}$ & \multirow[t]{2}{*}{ Weight } & $\begin{array}{r}\text { (... Continued) } \\
\text { Std. } \\
\text { Mean } \\
\text { Difference }\end{array}$ \\
\hline & $\mathrm{N}$ & $\mathrm{N}$ & \multicolumn{2}{|r|}{ IV,Random,95\% Cl } & & IV,Random,95\% Cl \\
\hline \multicolumn{7}{|c|}{5 Public facilities medium term (at I year post intervention) (subgroup) } \\
\hline Patel 20 I0 C-RCT India (I3) & 540 & 564 & $-0.0503(0.0602)$ & & $100.0 \%$ & $-0.05[-0.17,0.07]$ \\
\hline Subtotal $(95 \% \mathrm{CI})$ & & & & & $100.0 \%$ & $-0.05[-0.17,0.07]$ \\
\hline \multicolumn{7}{|l|}{ Heterogeneity: not applicable } \\
\hline \multicolumn{7}{|c|}{ Test for overall effect: $Z=0.84(P=0.40)$} \\
\hline \multicolumn{7}{|c|}{6 Private facilities medium term (at I year post intervention) (subgroup) } \\
\hline Patel 2010 C-RCT India (14) & 387 & 414 & $0.0307(0.0707)$ & & $100.0 \%$ & $0.03[-0.11,0.17]$ \\
\hline Subtotal $(95 \% \mathrm{CI})$ & & & & 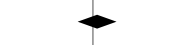 & $100.0 \%$ & $0.03[-0.11,0.17]$ \\
\hline \multicolumn{7}{|l|}{ Heterogeneity: not applicable } \\
\hline \multicolumn{7}{|c|}{ Test for overall effect: $Z=0.43(P=0.66)$} \\
\hline \multicolumn{7}{|c|}{ Test for subgroup differences: $\mathrm{Chi}^{2}=7.93, \mathrm{df}=5(\mathrm{P}=0.16), \mathrm{I}^{2}=37 \%$} \\
\hline & & & & -0.5 & | & \\
\hline
\end{tabular}

(I) collab care for persistent/recurrent depression; SF 36 social function (High score=better functioning); endpoint scores 6 mths adj for baseline/clinic

(2) Collab care for persistent/recurrent depression;SF 36 social functioning; endpoint scores at 6 months

(3) Collab care in public facilities by doctor or nurse, all mental disorders; WHODAS II (36 item); MD (adjusted for baseline scores+clustering) and 95\%Cls 3m post-interv;

(4) collab stepped care model (lay counsellor, physician); WHODAS II ( 12 item) (high score=higher disability); endpoint scores: cluster-adjusted means and Cls at 6 months

(5) physician, nurse, midwife-led multicomponent intervention for post natal depression; SF-36 soc functioning; MD (CI) at 6 months

(6) collab care for persistent/recurrent depression; SF 36 social function (High score=better functioning); endpoint scores 6 mths adj for baseline/clinic

(7) collab care for persistent/recurrent depression; SF 36 social functioning; endpoint scores at 6 months reversed as need impairment scores not recovered)

(8) Collab care public facilities by doctor or nurse, all mental disorders; WHODAS II ; MD (adjusted for baseline scores and clustering) and 95\%Cls 3m post-interv

(9) collab stepped care model (lay counsellor, physician); WHODAS II; endpoint scores: cluster-adjustedmeans and Cls at 6 months

(10) physician, nurse, midwife-led multicomponent intervention for post natal depression; SF-36 soc functioning; MD (Cl) at 6 months

( I I) collaborative stepped care model (lay counsellor, physician); WHODAS II; endpoint scores:adjusted means and Cls at 6 months

(12) collab stepped care model (lay counsellor, physician); WHODAS II; endpoint scores:Cluster-adjusted means and Cls at 12 months

(13) collaborative stepped care model (lay counsellor, physician); WHODAS II; cluster-adjusted endpoint scores:means and Cls at I2 months

(14) collaborative stepped care model (lay counsellor, physician); WHODAS II; endpoint scores:means and Cls at 12 months

Non-specialist health worker interventions for the care of mental, neurological and substance-abuse disorders in low- and middle- 
Analysis 2.4. Comparison 2 Collaborative care model (NSHWs plus specialist) versus usual care in treating common mental disorders (RCTs), Outcome 4 Suicide attempt for those with CMDs all facilities and in public/private facilities (completers).

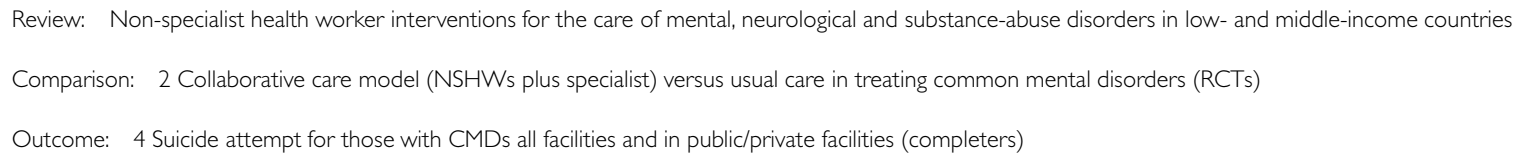

Subtotal (95\% CI)

Total events: 7 (Collab care), 10 (Usual care)

Heterogeneity: not applicable

Test for overall effect: $Z=0.58(P=0.57)$

2 Public facilities short term (6 months post intervention)

Patel 2010 C-RCT India (2) I I I/545 I8/593

Subtotal (95\% CI)

545

Total events: I I (Collab care), 18 (Usual care)

Heterogeneity: not applicable

Test for overall effect: $Z=1.08(P=0.28)$

3 Private facilities short term (6 months post intervention)

Patel 2010 C-RCT India (3) 2/399

399

Subtotal (95\% CI)

Total events: 2 (Collab care), 3 (Usual care)

Heterogeneity: not applicable

Test for overall effect: $Z=0.38(P=0.70)$

4 All facilities medium term (I year post intervention)

Patel 20I0 C-RCT India (4) 8/927

Subtotal (95\% CI)

927

Total events: 8 (Collab care), 15 (Usual care)

Heterogeneity: not applicable

Test for overall effect: $Z=1.32(P=0.19)$

5 Public facilities medium term ( $\mid$ year post intervention)

Patel 2010 C-RCT India (5) 3/540

Subtotal (95\% CI)

540

Total events: 3 (Collab care), 4 (Usual care)

Heterogeneity: not applicable

Test for overall effect: $Z=0.32(P=0.75)$

6 Private facilities medium term (I year post intervention)

1017

$3 / 424$

593

424

15/978

978

$\underset{+}{\longrightarrow}$

$\mathrm{Cl}$

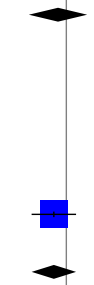

$100.0 \%$

$0.66[0.32,1.40]$

$100.0 \%$

0.66 [ $0.32,1.40$ ]

$100.0 \%$

$0.75[0.29,1.97]$

$100.0 \%$

$0.71[0.12,4.22]$

$100.0 \%$

$0.71[0.12,4.22$ ]

$100.0 \%$

$0.56[0.24,1.32]$

$100.0 \%$

$0.56[0.24,1.32$ ]

$100.0 \%$

$0.78[0.18,3.48]$

$4 / 564$

564

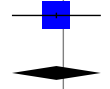

$100.0 \%$

$0.78[0.18,3.48]$

Non-specialist health worker interventions for the care of mental, neurological and substance-abuse disorders in low- and middle- 


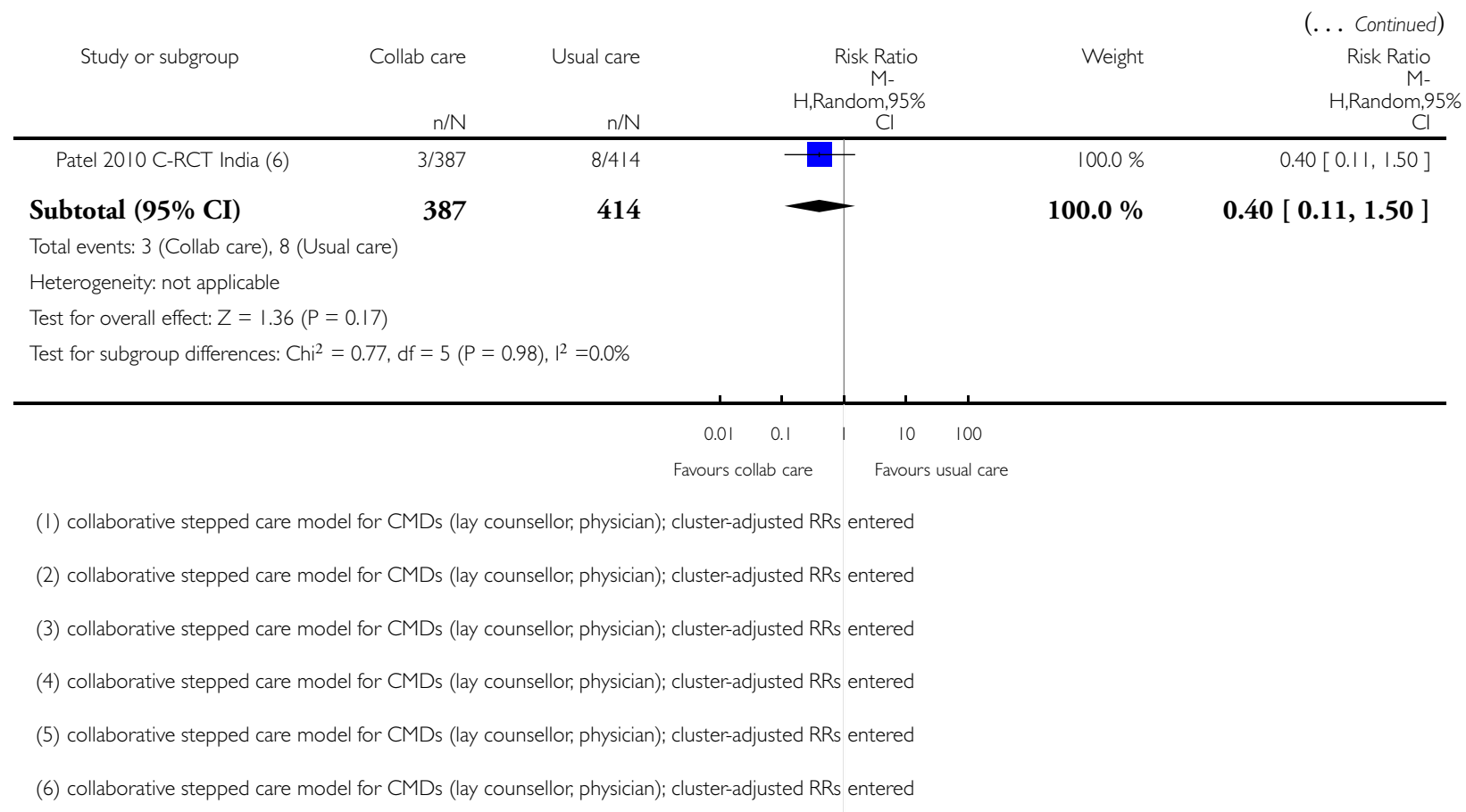


Analysis 2.5. Comparison 2 Collaborative care model (NSHWs plus specialist) versus usual care in treating common mental disorders (RCTs), Outcome 5 Prevalence of CMDs (only Patel - sensitivity analysis (SA)) (completers) all facilities and in public and private facilities.

Review: Non-specialist health worker interventions for the care of mental, neurological and substance-abuse disorders in low- and middle-income countries

Comparison: 2 Collaborative care model (NSHWs plus specialist) versus usual care in treating common mental disorders (RCTs)

Outcome: 5 Prevalence of CMDs (only Patel - sensitivity analysis (SA)) (completers) all facilities and in public and private facilities

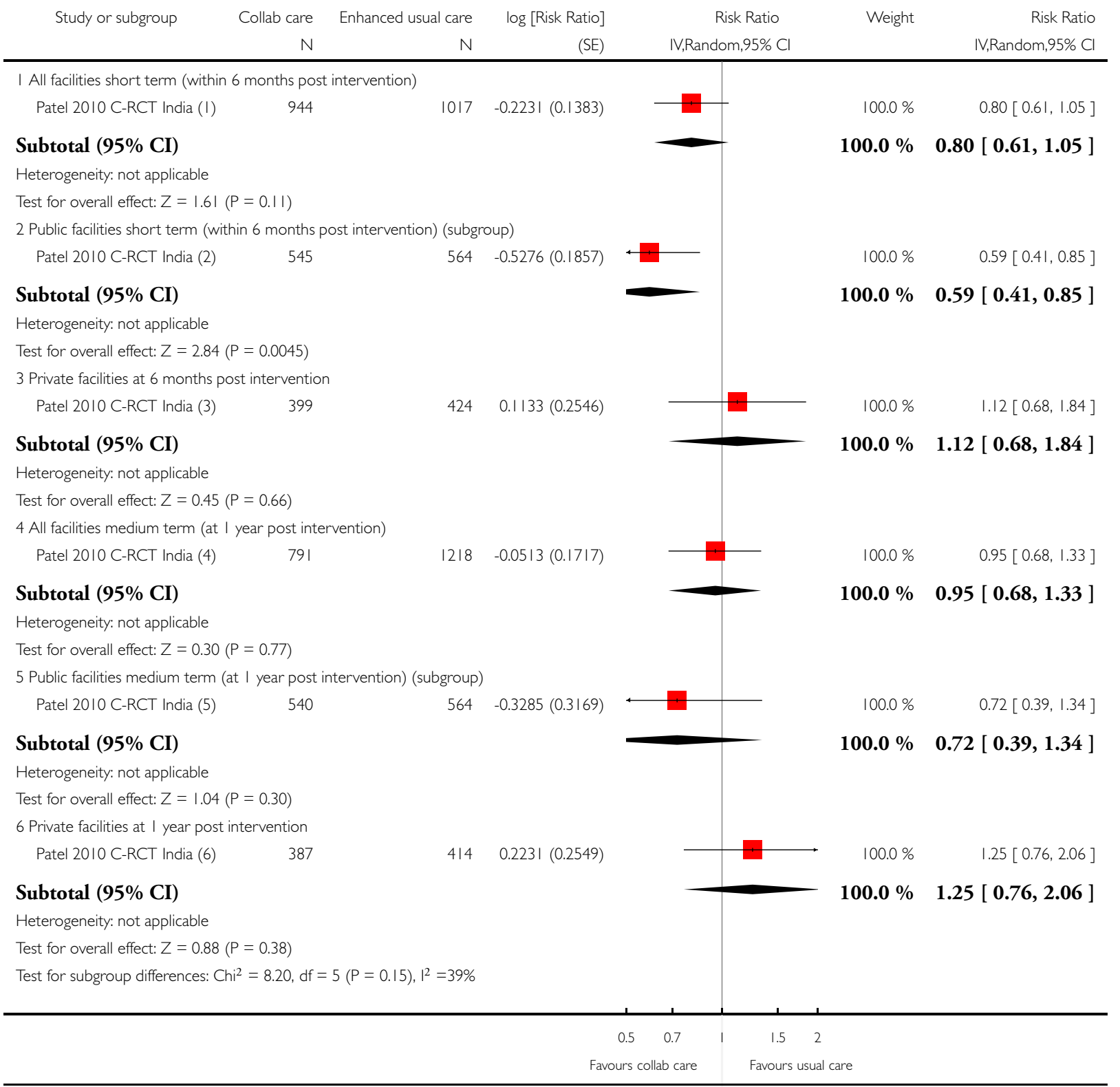

Non-specialist health worker interventions for the care of mental, neurological and substance-abuse disorders in low- and middle- 
(I) stepped care for CMDs; CIS-R; 6 month prevalence of CMDs adjusted RRs

(2) stepped care for CMDs; CIS-R; 6 month prevalence of CMDs adjusted RRs

(3) stepped care for CMDs; CIS-R; 6 month prevalence of CMDs adjusted RRs

(4) stepped care for CMDs; CIS-R; adjusted RR for non-recovered completers

(5) stepped care for CMDs; CIS-R; numbers and totals for CMDs; adjusted RR for non-recovered (completers)

(6) stepped care for CMDs; CIS-R;adjusted RR for non-recovered (completers)

Analysis 2.6. Comparison 2 Collaborative care model (NSHWs plus specialist) versus usual care in treating common mental disorders (RCTs), Outcome 6 Severity of symptoms in CMD (only Patel and Jenkins (SA)) in all facilities and in public and private facilities.

Review: Non-specialist health worker interventions for the care of mental, neurological and substance-abuse disorders in low- and middle-income countries

Comparison: 2 Collaborative care model (NSHWs plus specialist) versus usual care in treating common mental disorders (RCTs)

Outcome: 6 Severity of symptoms in CMD (only Patel and Jenkins (SA)) in all facilities and in public and private facilities

\begin{tabular}{|c|c|c|c|c|c|c|c|}
\hline \multirow[t]{2}{*}{ Study or subgroup } & Collab care & Enhanced usual care & $\begin{array}{r}\text { Std. Mean } \\
\text { Difference } \\
(\mathrm{SE})\end{array}$ & & $\begin{array}{r}\text { Std. } \\
\text { Mean } \\
\text { ifference }\end{array}$ & Weight & $\begin{array}{r}\text { Std. } \\
\text { Mean } \\
\text { Difference }\end{array}$ \\
\hline & N & $\mathrm{N}$ & \multicolumn{4}{|c|}{ IV,Random,95\% Cl } & IV,Random,95\% Cl \\
\hline \multicolumn{8}{|c|}{ I All facilities short term (within 6 months post intervention) } \\
\hline Jenkins 2012 C-RCT Kenya (I) & 453 & 475 & $-0.0495(0.0657)$ & + & & $32.1 \%$ & $-0.05[-0.18,0.08]$ \\
\hline Patel 2010 C-RCT India (2) & 944 & 1017 & $-0.0869(0.0452)$ & & & $67.9 \%$ & $-0.09[-0.18,0.00]$ \\
\hline \multicolumn{6}{|c|}{ Subtotal (95\% CI) } & $100.0 \%$ & $-0.07[-0.15,0.00]$ \\
\hline \multicolumn{8}{|c|}{ Heterogeneity: $\mathrm{Tau}^{2}=0.0 ; \mathrm{Chi}^{2}=0.22, \mathrm{df}=\mathrm{I}(\mathrm{P}=0.64) ; \mathrm{I}^{2}=0.0 \%$} \\
\hline \multicolumn{8}{|c|}{ Test for overall effect: $Z=2.01(P=0.044)$} \\
\hline \multicolumn{8}{|c|}{2 Public facilities short term (within 6 months post intervention) (subgroup) } \\
\hline Jenkins 2012 C-RCT Kenya (3) & 453 & 475 & $-0.0495(0.0657)$ & & & $46.4 \%$ & $-0.05[-0.18,0.08]$ \\
\hline Patel 2010 C-RCT India (4) & 545 & 593 & $-0.1545(0.0594)$ & $\hookrightarrow$ & & $53.6 \%$ & $-0.15[-0.27,-0.04]$ \\
\hline \multicolumn{5}{|l|}{ Subtotal (95\% CI) } & & $100.0 \%$ & $-0.11[-0.21,0.00]$ \\
\hline \multicolumn{8}{|c|}{ Heterogeneity: $\mathrm{Tau}^{2}=0.00 ; \mathrm{Chi}^{2}=1.4 \mathrm{I}, \mathrm{df}=\mathrm{I}(\mathrm{P}=0.24) ; \mathrm{I}^{2}=29 \%$} \\
\hline \multicolumn{8}{|c|}{ Test for overall effect: $Z=2.02(P=0.043)$} \\
\hline \multicolumn{8}{|c|}{3 Private facilities short term (within 6 months post intervention) (subgroup) } \\
\hline Patel 2010 C-RCT India (5) & 399 & 424 & $0.0254(0.0698)$ & & & $100.0 \%$ & $0.03[-0.11,0.16]$ \\
\hline \multicolumn{8}{|l|}{ Subtotal (95\% CI) } \\
\hline & & & & -0.1 & 0.1 & 0.2 & \\
\hline \multicolumn{8}{|c|}{ Favours collab care } \\
\hline
\end{tabular}

Non-specialist health worker interventions for the care of mental, neurological and substance-abuse disorders in low- and middle- 


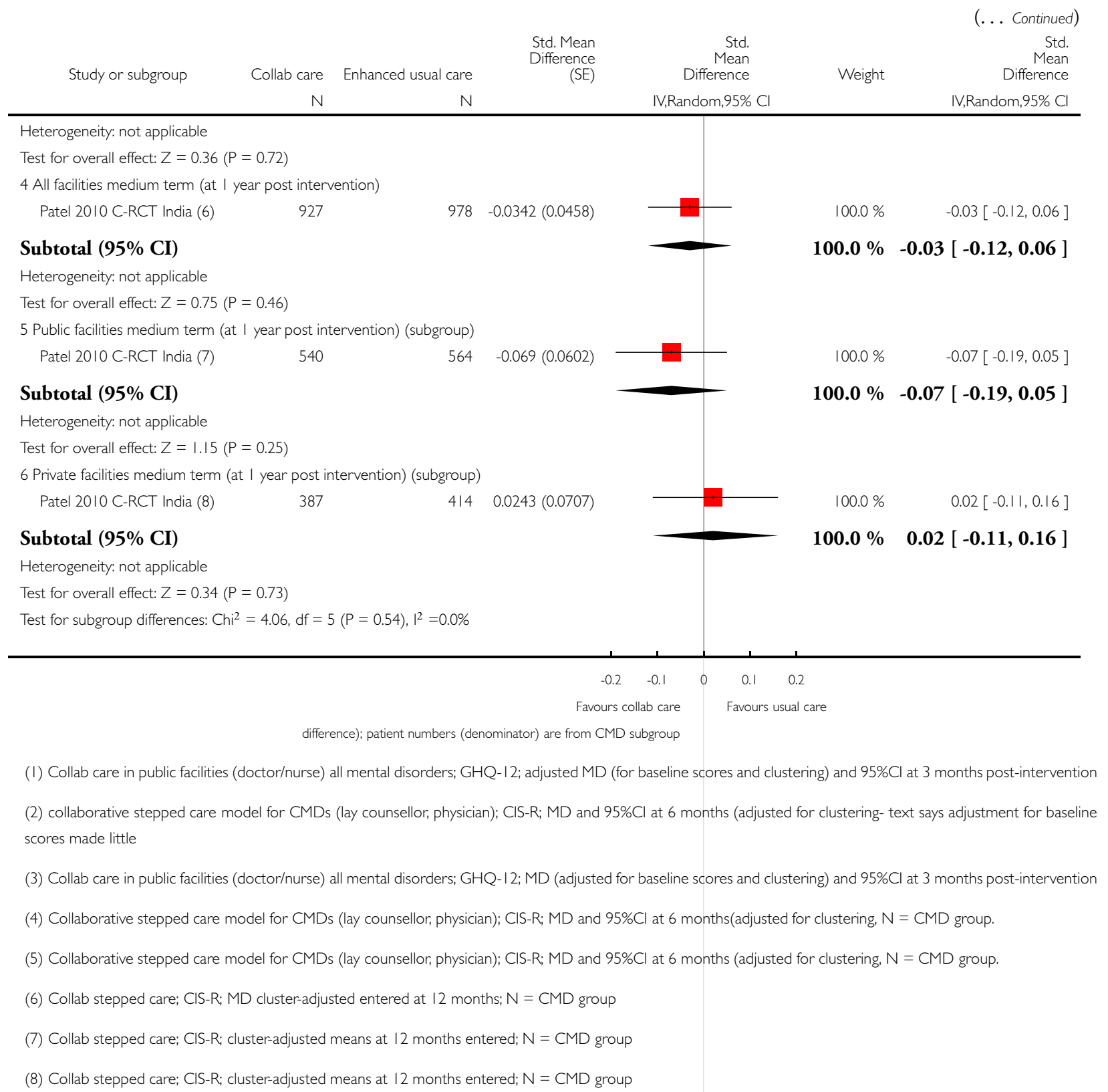

Non-specialist health worker interventions for the care of mental, neurological and substance-abuse disorders in low- and middle- 
Analysis 2.7. Comparison 2 Collaborative care model (NSHWs plus specialist) versus usual care in treating common mental disorders (RCTs), Outcome 7 Prevalence of depression (completers) (SA) all facilities and in public and private facilities.

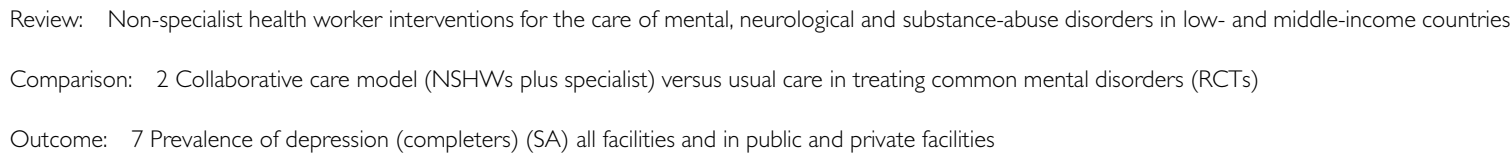

Patel 20I0 C-RCT India (2) $\quad 257 \quad 416 \quad-0.0726(0.4056)$

$\begin{array}{llll}\text { Rojas } 2007 \text { RCT Chile (3) } \quad 106 & 102 & -0.3417(0.1303)\end{array}$

Subtotal $(\mathbf{9 5 \%} \mathrm{CI})$

Heterogeneity: $\operatorname{Tau}^{2}=0.10 ; \mathrm{Chi}^{2}=7.30, \mathrm{df}=2(\mathrm{P}=0.03) ; \mathrm{I}^{2}=73 \%$

Test for overall effect: $Z=2.24(P=0.025)$

2 Public facilities short term (within 6 months post intervention) (subgroup)

$\begin{array}{lccc}\text { Araya } 2003 \text { RCT Chile (4) } & 104 & 107 & -0.8551 \text { (0.1632) } \\ \text { Patel 2010 C-RCT India (5) } & 144 & 265 & -0.5798(0.5004) \\ \text { Rojas 2007 RCT Chile (6) } & 106 & 102 & -0.3417(0.1303)\end{array}$

Subtotal $(95 \%$ CI $)$

Heterogeneity: Tau $^{2}=0.08 ; \mathrm{Chi}^{2}=6.05, \mathrm{df}=2(\mathrm{P}=0.05) ; \mathrm{I}^{2}=67 \%$

Test for overall effect: $Z=2.81(P=0.0049)$

3 Private facilities short term (within 6 months post intervention) (subgroup)

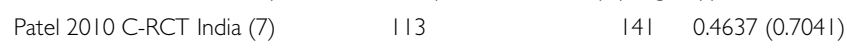

Subtotal (95\% CI)

Heterogeneity: not applicable

Test for overall effect: $Z=0.66(P=0.5 \mathrm{I})$

4 All facilities medium term (I year post intervention)

Patel 20I0 C-RCT India (8) $\quad 250 \quad 402-0.0513(0.1717)$

Subtotal (95\% CI)

Heterogeneity: not applicable

Test for overall effect: $Z=0.30(P=0.77)$

5 Public facilities medium term ( 1 year post intervention) (subgroup)

Patel 20I0 C-RCT India (9) $\quad 144 \quad 254 \quad-0.3285$ (0.3169)

Subtotal (95\% CI)

Heterogeneity: not applicable

Test for overall effect: $Z=1.04(P=0.30)$

6 Private facilities medium term (at I year post intervention) (subgroup)

\begin{tabular}{rrr}
$39.1 \%$ & $0.43[0.31,0.59]$ \\
$18.5 \%$ & $0.93[0.42,2.06]$ \\
$42.4 \%$ & $0.71[0.55,0.92]$ \\
\hline $\mathbf{1 0 0 . 0} \%$ & $\mathbf{0 . 6 1}[\mathbf{0 . 4 0 , 0 . 9 4}]$
\end{tabular}

$41.3 \% \quad 0.43[0.31,0.59]$

$13.2 \% \quad 0.56[0.21,1.49]$

$45.5 \% \quad 0.71[0.55,0.92]$

$100.0 \% \quad 0.56[0.37,0.84$ ]

$100.0 \% \quad 1.59[0.40,6.32]$

$100.0 \% 1.59[0.40,6.32$ ]

$100.0 \% \quad 0.95[0.68,1.33]$

$100.0 \% \quad 0.95[0.68,1.33$ ]

$100.0 \% \quad 0.72[0.39,1.34]$

$100.0 \% \quad 0.72[0.39,1.34]$

Non-specialist health worker interventions for the care of mental, neurological and substance-abuse disorders in low- and middle- 


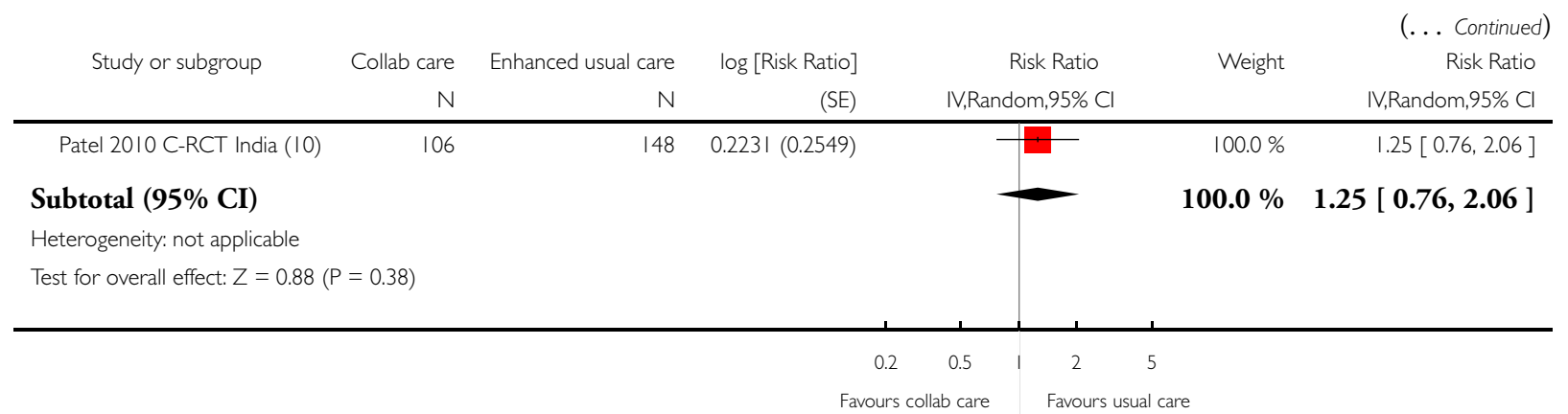

(I) Collab model for persistent recurrent depression;HRDS scores $<8$ (ie recovered) at 6 months; transformed to fit with Patel prevalence=total - nb recovered

(2) stepped care for CMDs; CIS-R; 6 month prevalence of depression, cluster-adjusted RRs.

(3) collab care for post natal depression; nb of patients with EPDS 6 point reduction 3 mths post-int (=6 mths post baseline).prev of depression (total - nb recovered)

(4) Collab model for persistent recurrent depression;HRDS scores $<8$ (ie recovered) at 6 months; transformed to fit with Patel prevalence by doing total - nb recovered

(5) stepped care for CMDs; CIS-R; 6 mth prev depression cluster-adjusted RRs

(6) collab care post natal depr; $\mathrm{nb}$ of patients with EPDS 6 point reduction at 3 mths post-int (=6 months post baseline).prevalence of depression (total - nb recovered)

(7) stepped care for CMDs; CIS-R; cluster-adjusted RRs

(8) stepped care for CMDs. CIS-R; 12 month prevalence of depression adjusted RRs (info from author)

(9) stepped care for CMDs; CIS-R; adjusted RR for non-recovered

(I0) stepped care for CMDs; CIS-R; adjusted RR for non-recovered 
Analysis 2.8. Comparison 2 Collaborative care model (NSHWs plus specialist) versus usual care in treating common mental disorders (RCTs), Outcome 8 Severity of symptoms of depression (SA) in all facilities and in public and private facilities.

Review: Non-specialist health worker interventions for the care of mental, neurological and substance-abuse disorders in low- and middle-income countries

Comparison: 2 Collaborative care model (NSHWs plus specialist) versus usual care in treating common mental disorders (RCTs)

Outcome: 8 Severity of symptoms of depression (SA) in all facilities and in public and private facilities

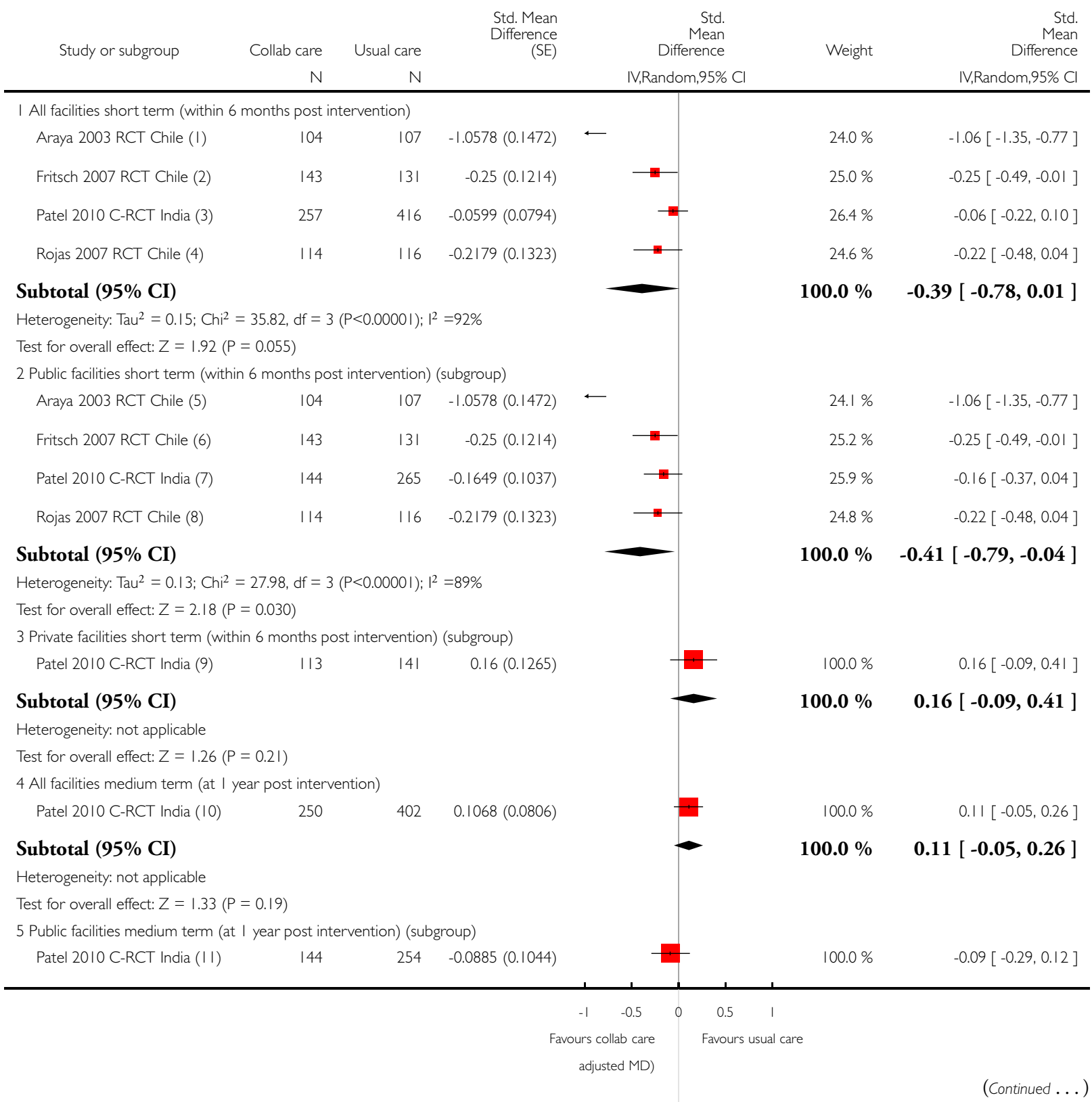

Non-specialist health worker interventions for the care of mental, neurological and substance-abuse disorders in low- and middle- 


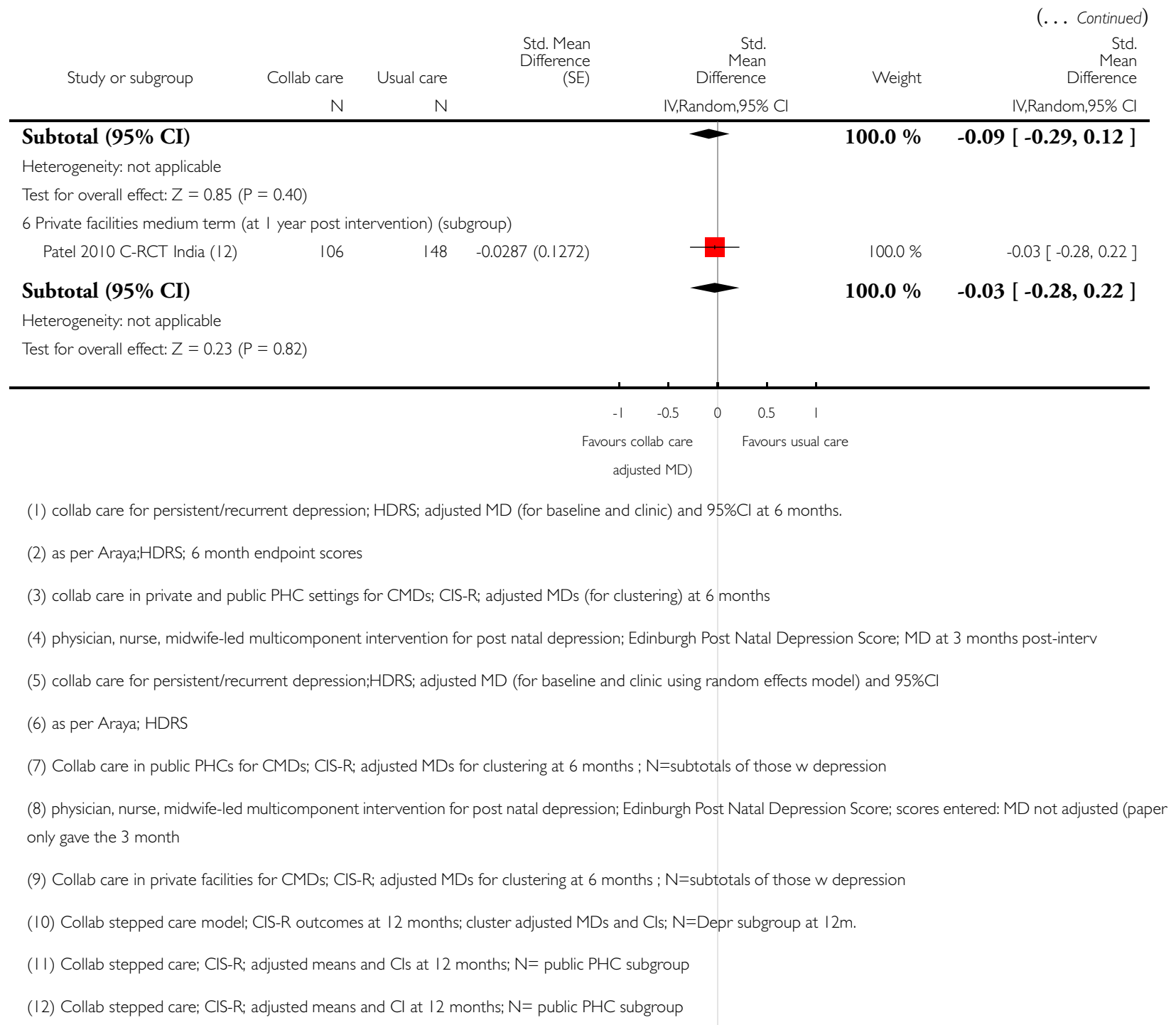

Non-specialist health worker interventions for the care of mental, neurological and substance-abuse disorders in low- and middle- 
Analysis 2.9. Comparison 2 Collaborative care model (NSHWs plus specialist) versus usual care in treating common mental disorders (RCTs), Outcome 9 Functional impairment/disability in CMD (SA) all facilities and in public and private facilities (SMD).

Review: Non-specialist health worker interventions for the care of mental, neurological and substance-abuse disorders in low- and middle-income countries

Comparison: 2 Collaborative care model (NSHWs plus specialist) versus usual care in treating common mental disorders (RCTs)

Outcome: 9 Functional impairment/disability in CMD (SA) all facilities and in public and private facilities (SMD)

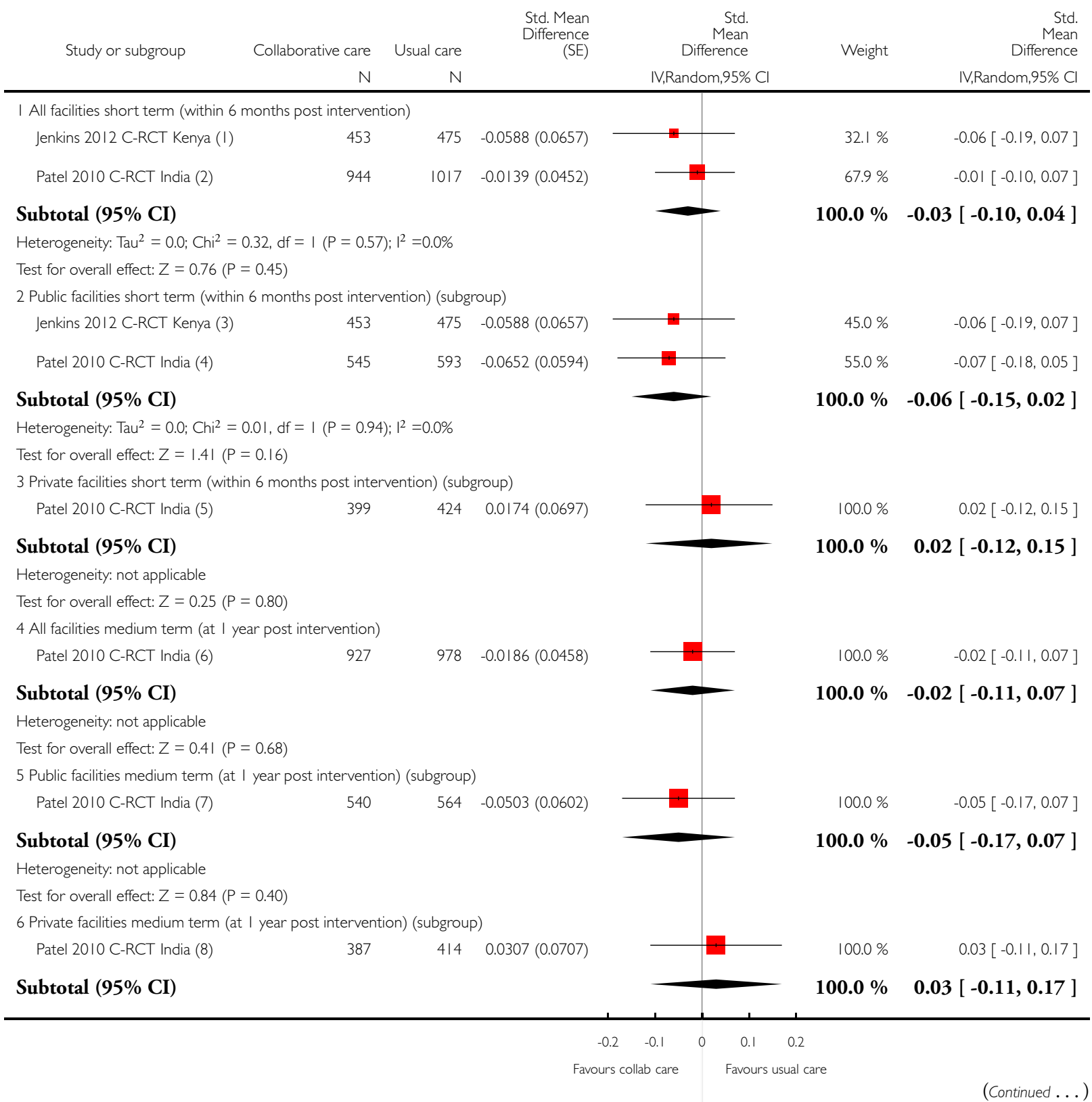

Non-specialist health worker interventions for the care of mental, neurological and substance-abuse disorders in low- and middle- 


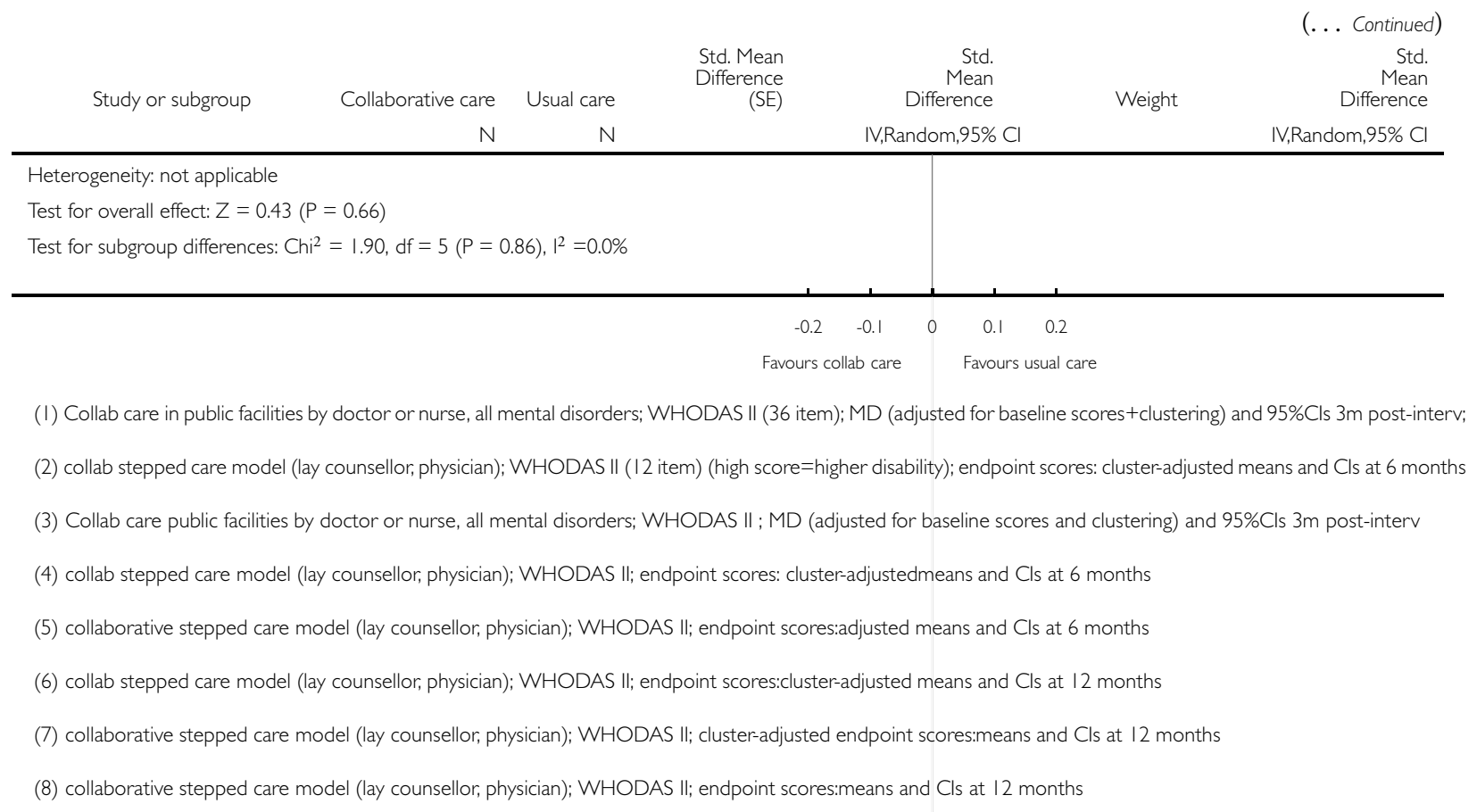

Non-specialist health worker interventions for the care of mental, neurological and substance-abuse disorders in low- and middle- 
Analysis 2.10. Comparison 2 Collaborative care model (NSHWs plus specialist) versus usual care in treating common mental disorders (RCTs), Outcome 10 Functional impairment/disability in CMD (SA) all facilities and in public and private facilities (MD).

Review: Non-specialist health worker interventions for the care of mental, neurological and substance-abuse disorders in low- and middle-income countries

Comparison: 2 Collaborative care model (NSHWs plus specialist) versus usual care in treating common mental disorders (RCTs)

Outcome: 10 Functional impairment/disability in CMD (SA) all facilities and in public and private facilities (MD)

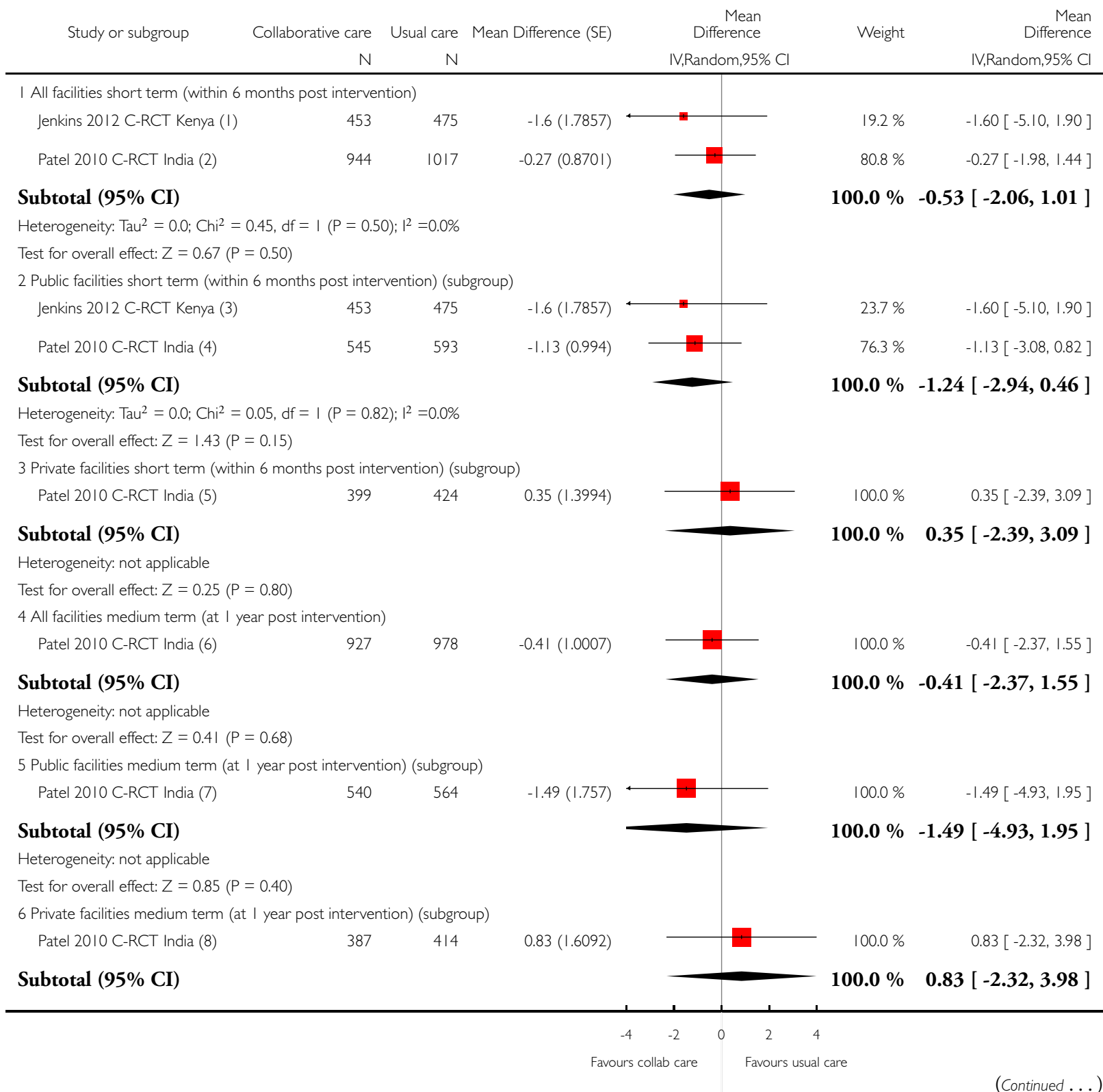

Non-specialist health worker interventions for the care of mental, neurological and substance-abuse disorders in low- and middle- 


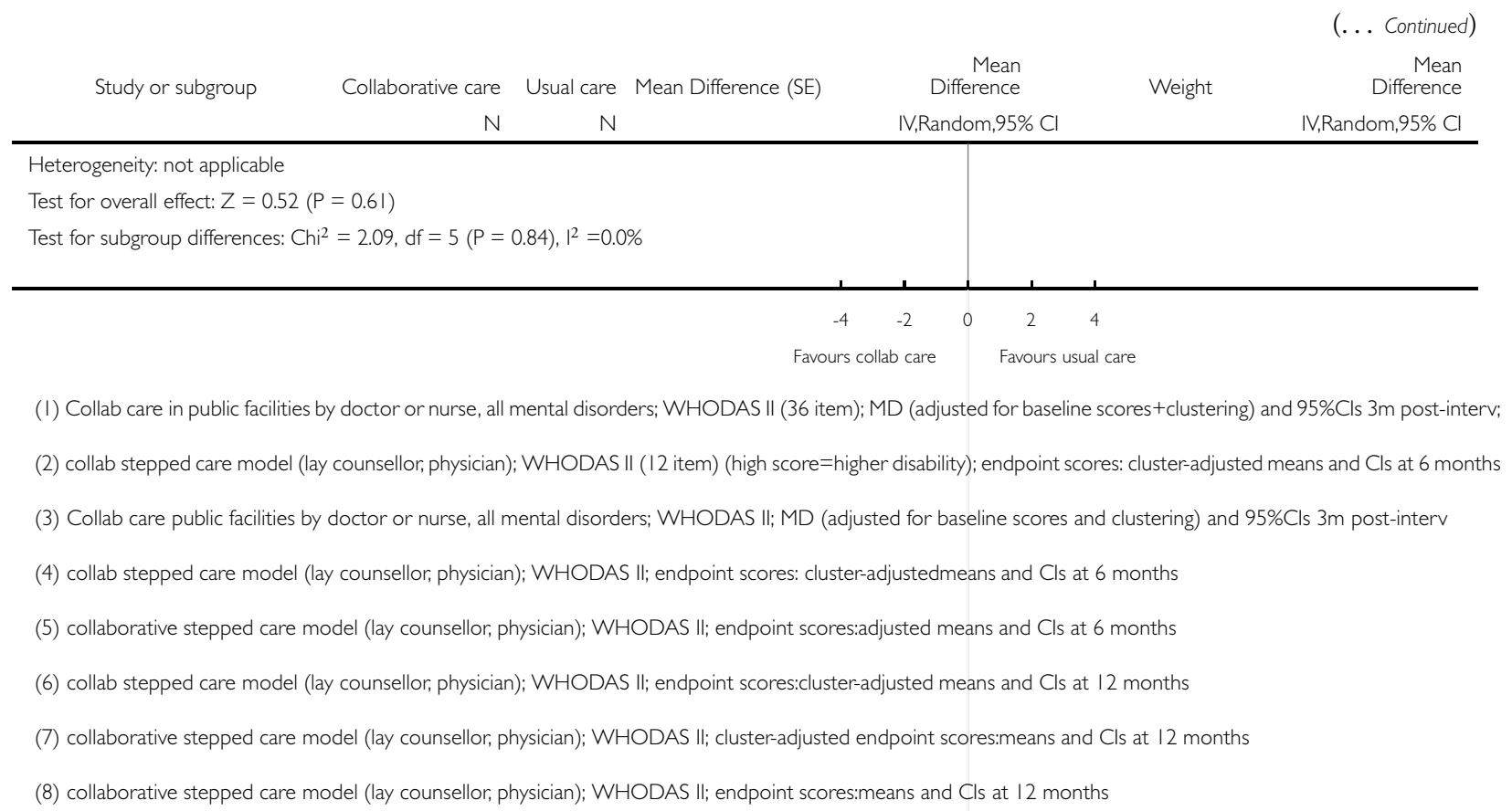

Non-specialist health worker interventions for the care of mental, neurological and substance-abuse disorders in low- and middle- 
Analysis 2.1 I. Comparison 2 Collaborative care model (NSHWs plus specialist) versus usual care in treating common mental disorders (RCTs), Outcome I I Functional impairment/disability in depression (SA) all facilities and in public and private facilities.

Review: Non-specialist health worker interventions for the care of mental, neurological and substance-abuse disorders in low- and middle-income countries

Comparison: 2 Collaborative care model (NSHWs plus specialist) versus usual care in treating common mental disorders (RCTs)

Outcome: II Functional impairment/disability in depression (SA) all facilities and in public and private facilities

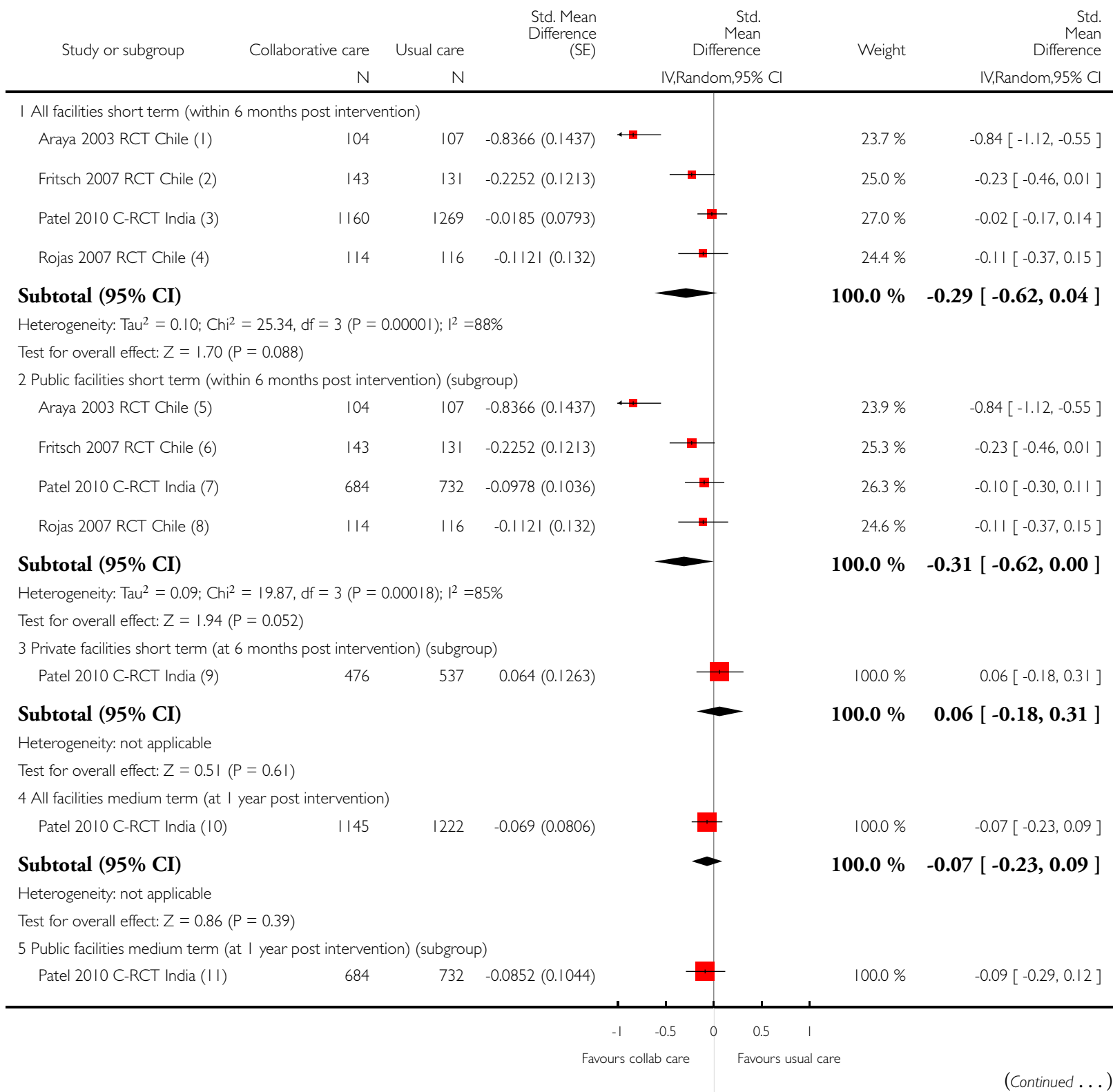




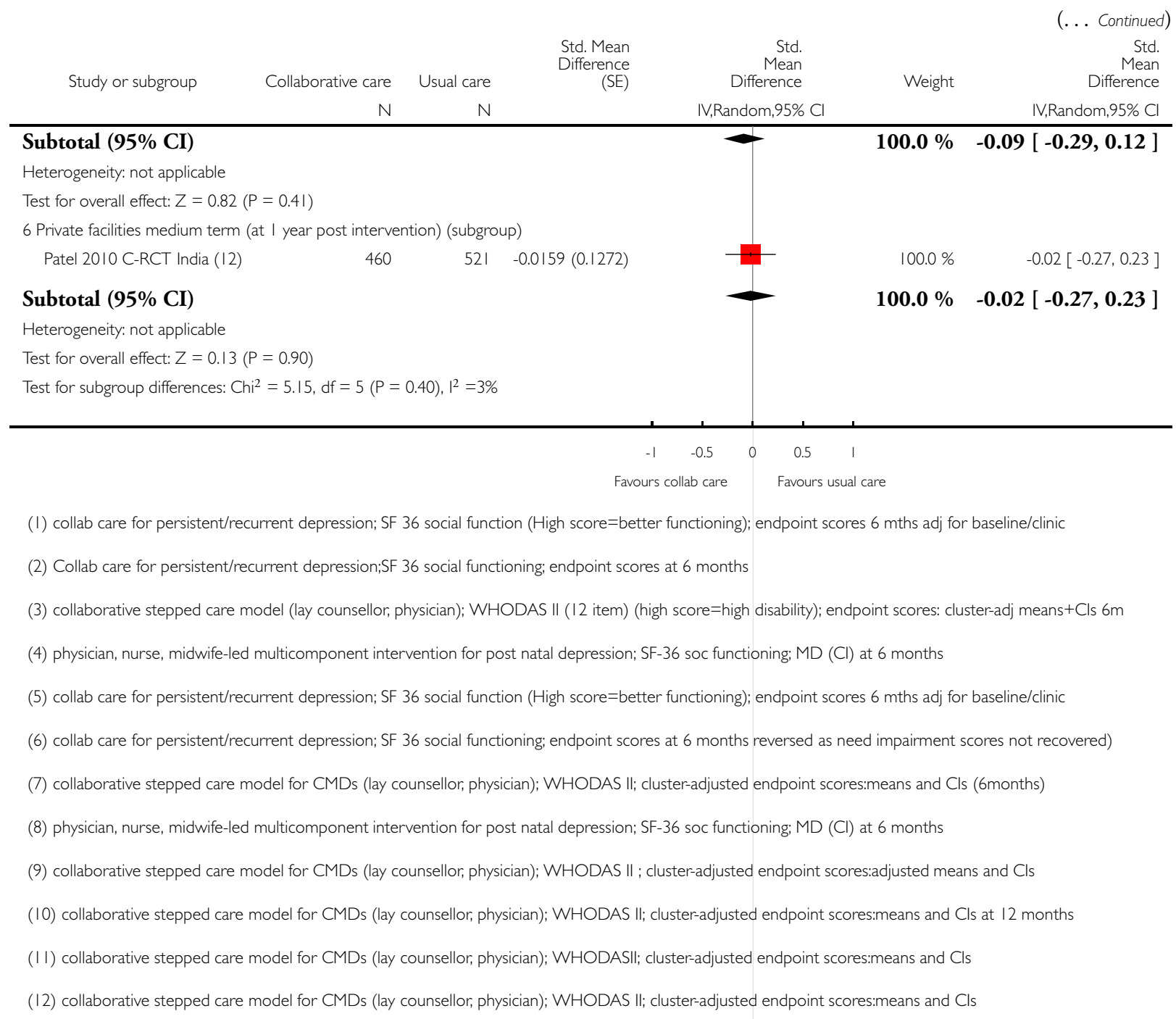

Non-specialist health worker interventions for the care of mental, neurological and substance-abuse disorders in low- and middle- 


\section{Analysis 3.I. Comparison 3 NSHWs versus usual care in treating maternal depression (RCTs), Outcome I Severity of symptoms in treating maternal depression.}

Review: Non-specialist health worker interventions for the care of mental, neurological and substance-abuse disorders in low- and middle-income countries

Comparison: 3 NSHWs versus usual care in treating maternal depression (RCTs)

Outcome: I Severity of symptoms in treating maternal depression

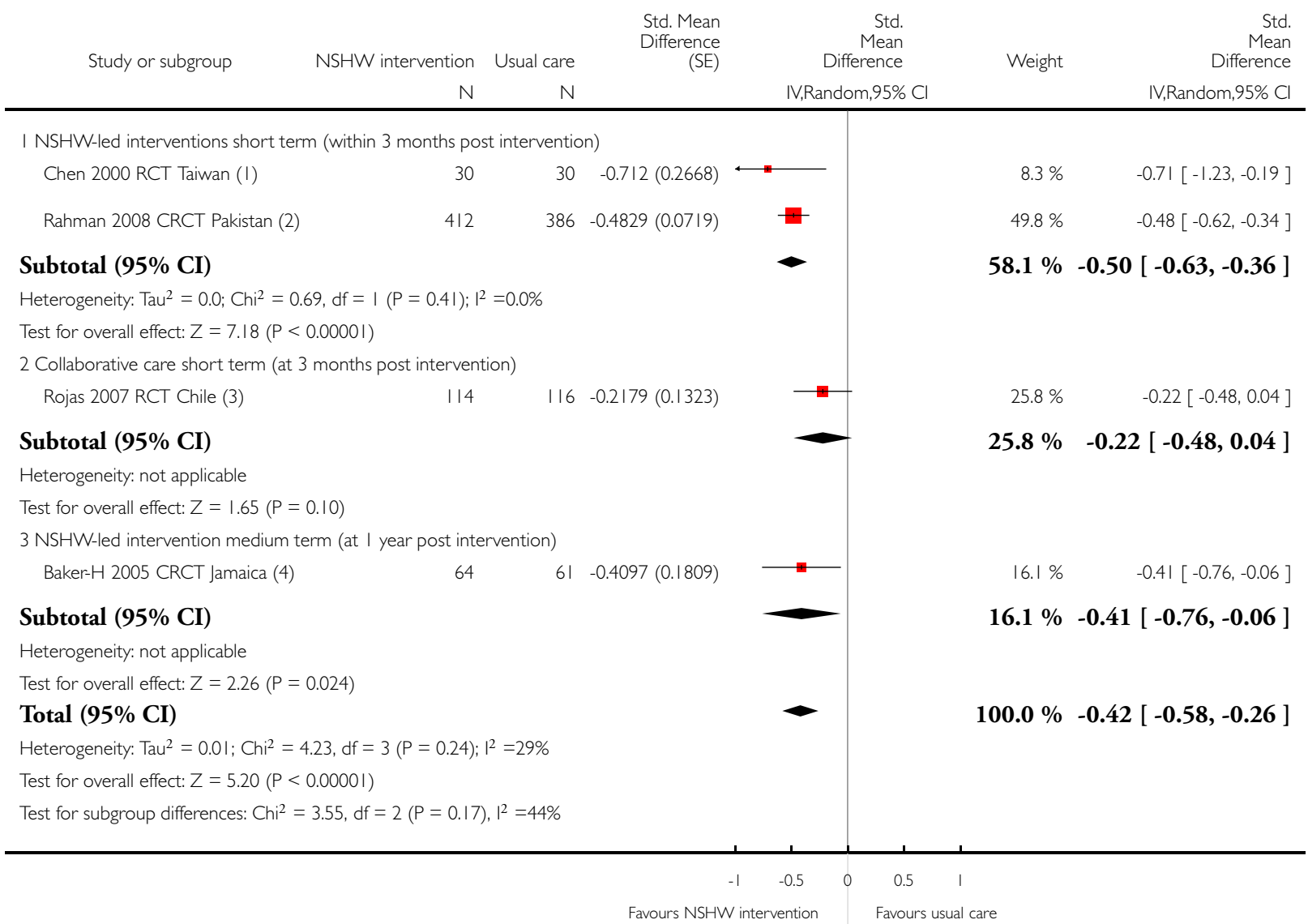

( I) Nurse-led psychosocial intervention;Taiwanese BDI (Beck's depression inventory); t-tests (unadjusted) immediately post intervention

(2) LHW-led CBT; Hamilton depression rating scale; endpoint scores at 2 months post-interv adjusted for clustering, and baseline characteristics

(3) Physician, nurse, midwife-led multicomponent intervention. Edinburgh Post Natal Depression Score; MD 3 months post-intervention

(4) LHW-led home visits (psychosocial intervention); Centre for epidemiological studies depression scale (CES-D); Means `SDs adjusted for clustering (info from author) 
Analysis 4.I. Comparison 4 NSHWs versus specialists in treating depression in adults (controlled beforeand-after studies), Outcome I Severity of depression short term ( 2 months post intervention).

Review: Non-specialist health worker interventions for the care of mental, neurological and substance-abuse disorders in low- and middle-income countries

Comparison: 4 NSHWs versus specialists in treating depression in adults (controlled before-and-after studies)

Outcome: I Severity of depression short term (2 months post intervention)

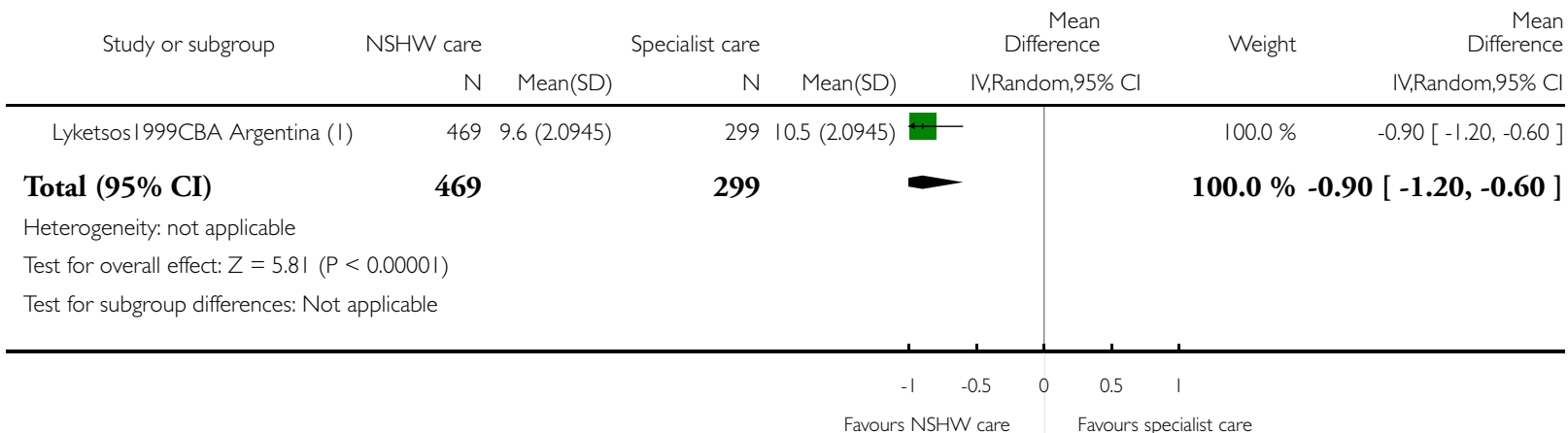

(I) GP vs psychiatrist in applying a protocol using sertraline; Hamilton-D ; scores at 56 days: mean and t-test.

Analysis 4.2. Comparison 4 NSHWs versus specialists in treating depression in adults (controlled beforeand-after studies), Outcome 2 Frequency of adverse events.

Review: Non-specialist health worker interventions for the care of mental, neurological and substance-abuse disorders in low- and middle-income countries

Comparison: 4 NSHWs versus specialists in treating depression in adults (controlled before-and-after studies)

Outcome: 2 Frequency of adverse events

\begin{tabular}{crr} 
Study or subgroup & NSHW care & Specialist car \\
& $n / N$ & $n / N$ \\
\hline Lyketsos 1999CBA Argentina (I) & $1 / 4 / 469$ & $86 / 299$ \\
Total (95\% CI) & $\mathbf{4 6 9}$ & $\mathbf{2 9 9}$
\end{tabular}

Total events: II 4 (NSHW care), 86 (Specialist care)

Heterogeneity: not applicable

Test for overall effect: $Z=1.38(P=0.17)$

Test for subgroup differences: Not applicable

\begin{tabular}{|c|c|c|}
\hline $\begin{array}{r}\text { Risk Ratio } \\
\text { M- } \\
\text { H,Random,95\% } \\
\text { Cl }\end{array}$ & Weight & $\begin{array}{c}\text { Risk Ratio } \\
\text { M- } \\
\text { H,Random,95\% } \\
\text { Cl }\end{array}$ \\
\hline 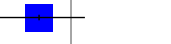 & $100.0 \%$ & $0.85[0.67,1.07]$ \\
\hline & $100.0 \%$ & $0.85[0.67,1.07]$ \\
\hline
\end{tabular}


(I) GP vs psychiatrist in applying a protocol using sertraline; nb of mild, moderate and severe outcome events at 56 days.

Analysis 4.3. Comparison 4 NSHWs versus specialists in treating depression in adults (controlled beforeand-after studies), Outcome 3 Number of days spent in hospital.

Review: Non-specialist health worker interventions for the care of mental, neurological and substance-abuse disorders in low- and middle-income countries

Comparison: 4 NSHWs versus specialists in treating depression in adults (controlled before-and-after studies)

Outcome: 3 Number of days spent in hospital

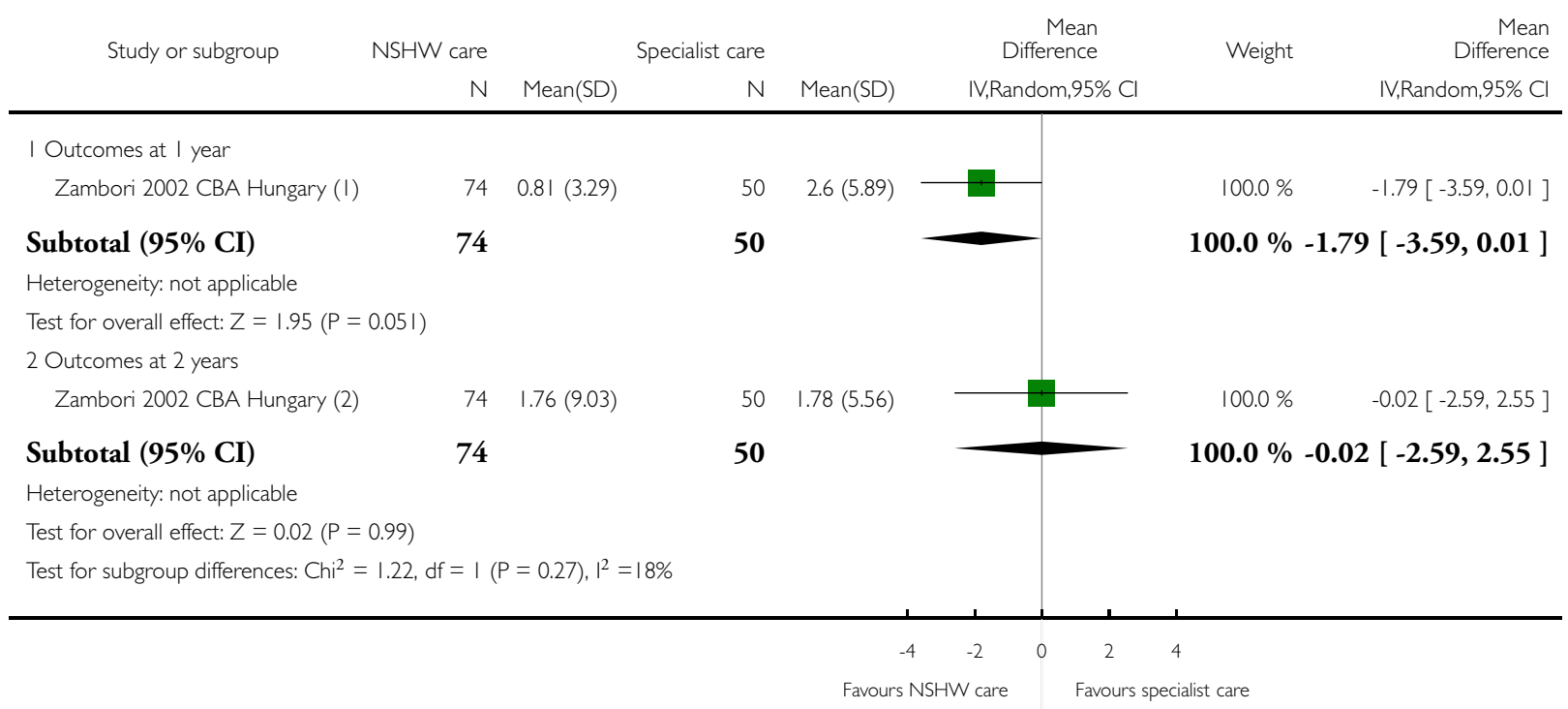

( I) normal GP care vs specialist care; outcomes at I year.

(2) normal GP care vs specialist care; outcomes at 2 years. 
Analysis 4.4. Comparison 4 NSHWs versus specialists in treating depression in adults (controlled beforeand-after studies), Outcome 4 Number of days spent on sick leave.

Review: Non-specialist health worker interventions for the care of mental, neurological and substance-abuse disorders in low- and middle-income countries

Comparison: 4 NSHWs versus specialists in treating depression in adults (controlled before-and-after studies)

Outcome: 4 Number of days spent on sick leave

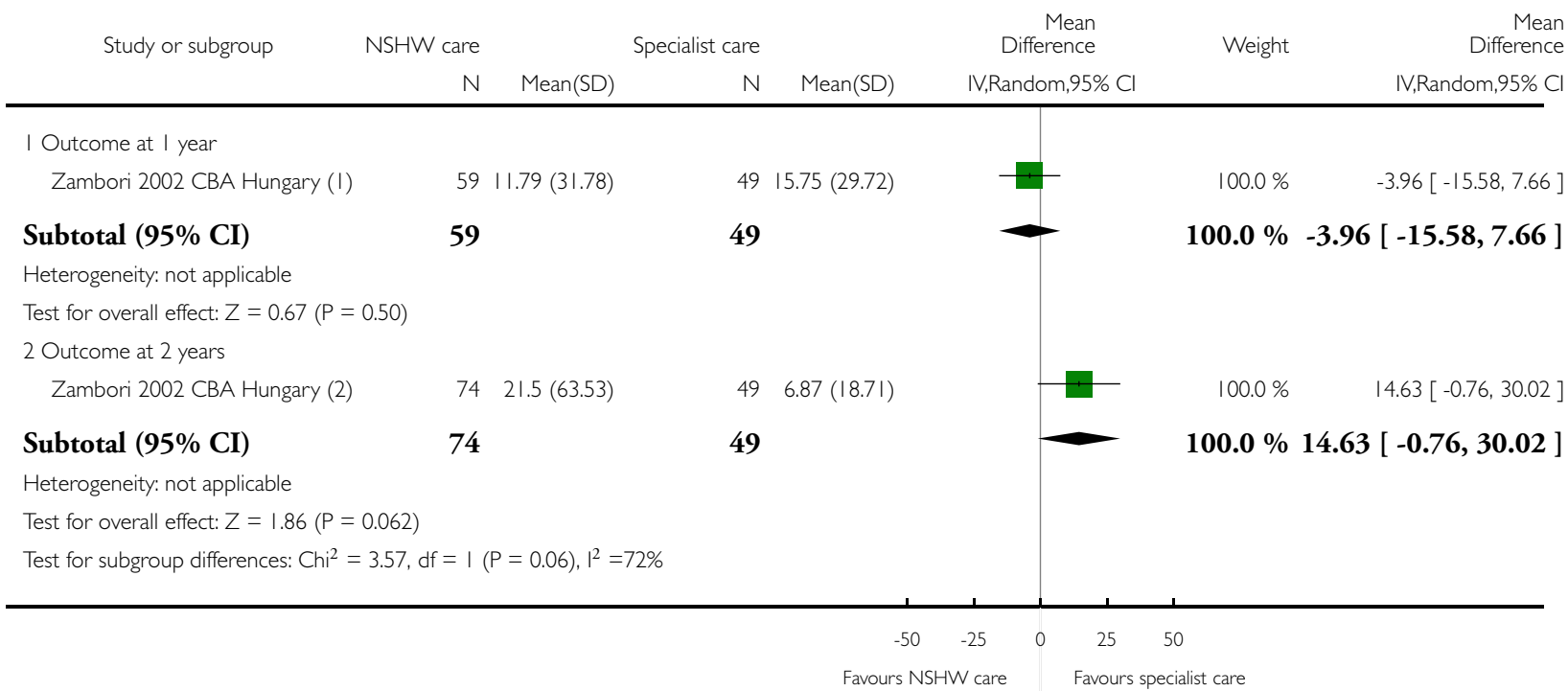

( I) normal GP care vs specialist care; numbers of days spent on sickleave.

(2) normal GP care vs specialist care; numbers of days spent on sickleave. 
Analysis 5.I. Comparison 5 NSHW-led psychological interventions versus usual care in treating adults with post-traumatic stress disorder (RCT and NRCT), Outcome I Prevalence of post-traumatic stress disorder (PTSD).

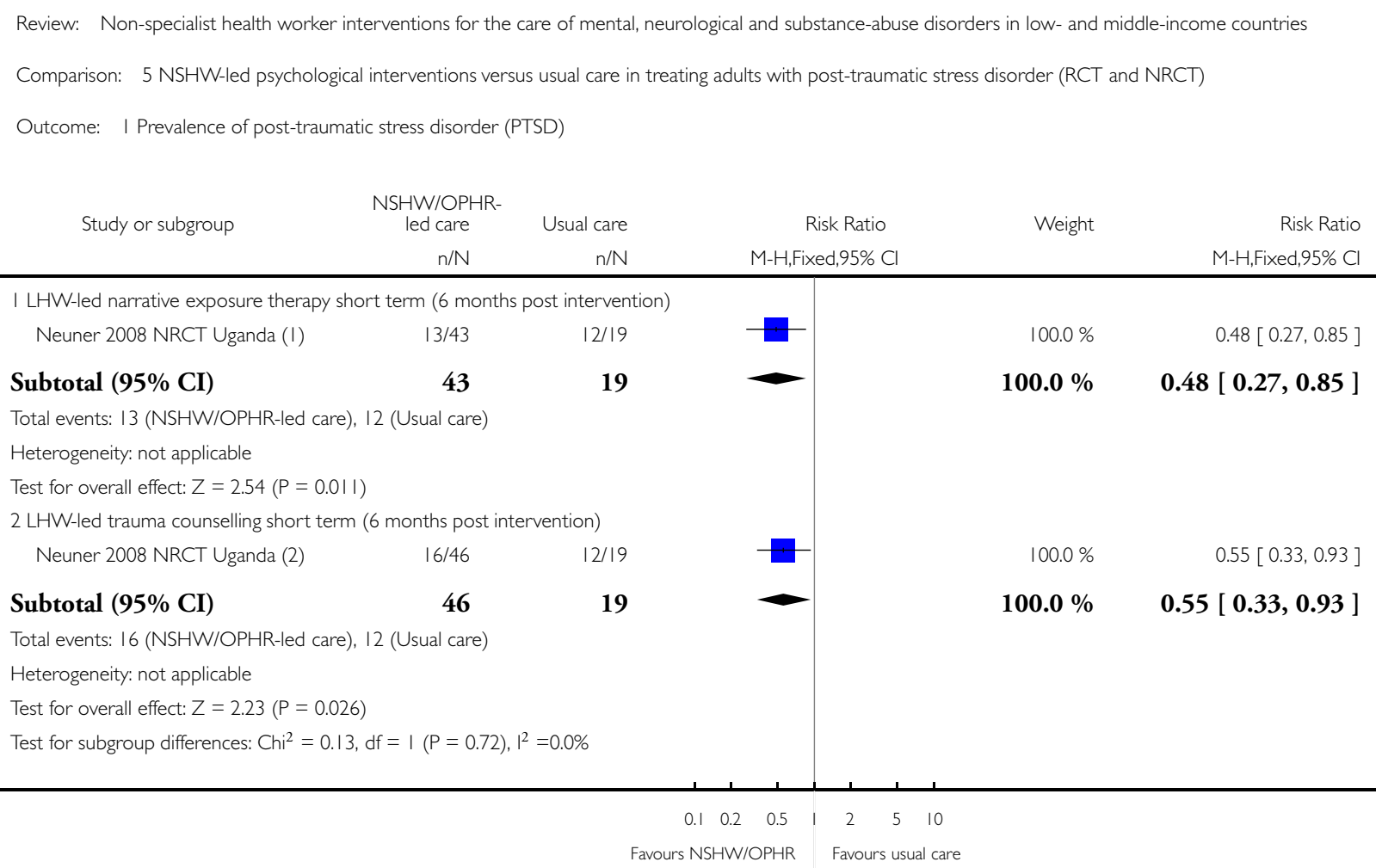

(I) LHW-led narrative exposure therapy; DSM-IV from CIDI; Nb with PTSD at 9 months post-interv (not adjusted); N=completers

(2) LHW-led trauma counselling with non-specific counselling); DSM-IV from CIDI; Nb with PTSD at 9 months post-interv (not adjusted); N=completers. 


\section{Analysis 5.2. Comparison 5 NSHW-led psychological interventions versus usual care in treating adults with post-traumatic stress disorder (RCT and NRCT), Outcome 2 Severity of PTSD symptoms.}

Review: Non-specialist health worker interventions for the care of mental, neurological and substance-abuse disorders in low- and middle-income countries

Comparison: 5 NSHW-led psychological interventions versus usual care in treating adults with post-traumatic stress disorder (RCT and NRCT)

Outcome: 2 Severity of PTSD symptoms

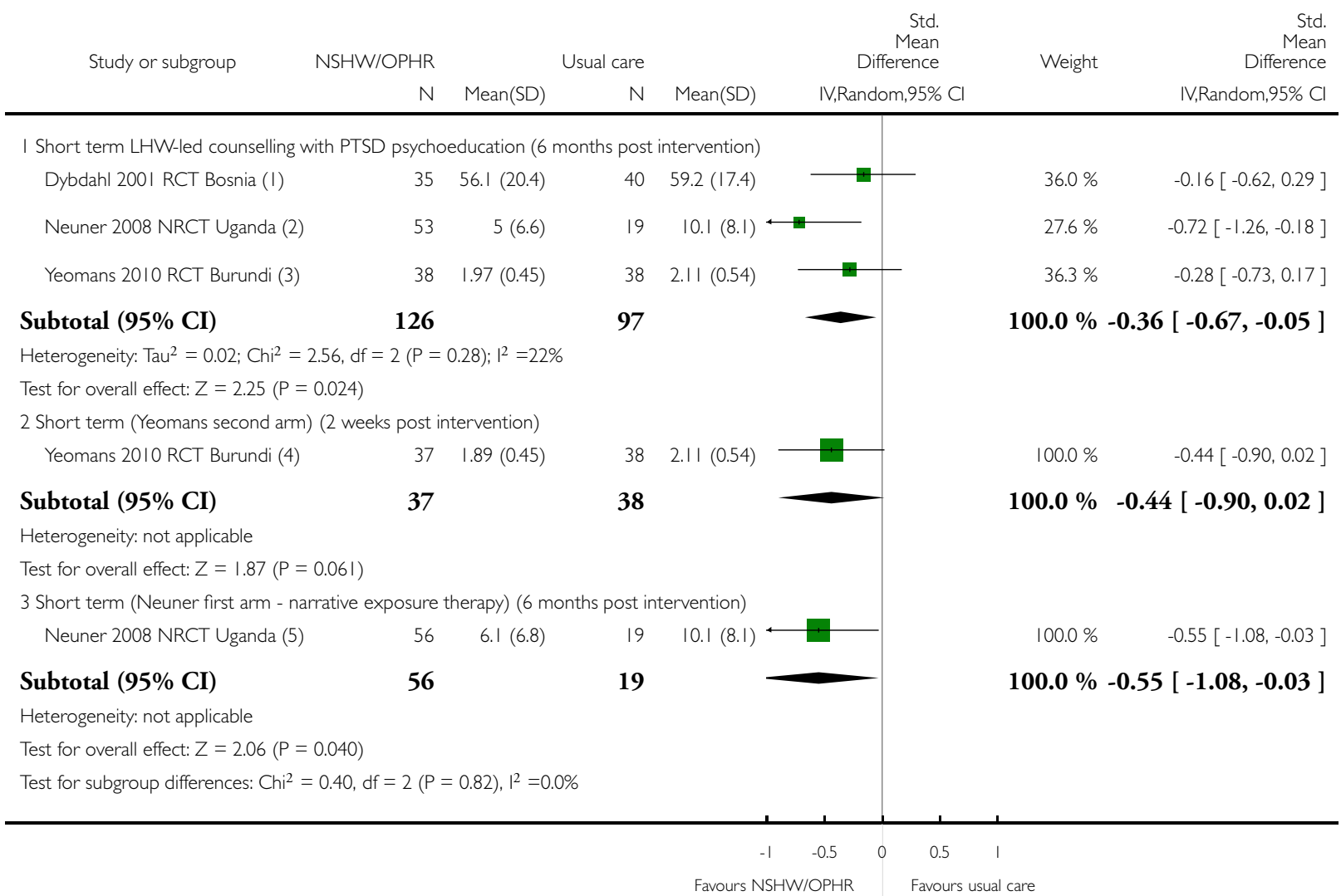

(I) Teacher-led psychosocial intervention for children+mothers; IES (Impact of events scale):mothers symptoms; mean and SD 5-6 months post intervention.

(2) LHW-led trauma non-specific trauma counselling; Post traumatic Stress Diagnostic Scale; mean (SD) at 6 months; $N=$ completers

(3) LHW-led workshop with psychoeducation; HTQ scores (Harvard Trauma Questionnaire); means, SDs at 2 weeks post interv

(4) LHW-led workshop without psychoeducation; HTQ scores (Harvard Trauma Questionnaire); means, SDs at 2 weeks post-interv; N=completers

(5) LHW-led narrative exposure therapy; Post traumatic Stress Diagnostic Scale; mean (SD)s at 6 months

Non-specialist health worker interventions for the care of mental, neurological and substance-abuse disorders in low- and middle- 
Analysis 5.3. Comparison 5 NSHW-led psychological interventions versus usual care in treating adults with post-traumatic stress disorder (RCT and NRCT), Outcome 3 Severity of depression.

Review: Non-specialist health worker interventions for the care of mental, neurological and substance-abuse disorders in low- and middle-income countries

Comparison: 5 NSHW-led psychological interventions versus usual care in treating adults with post-traumatic stress disorder (RCT and NRCT)

Outcome: 3 Severity of depression

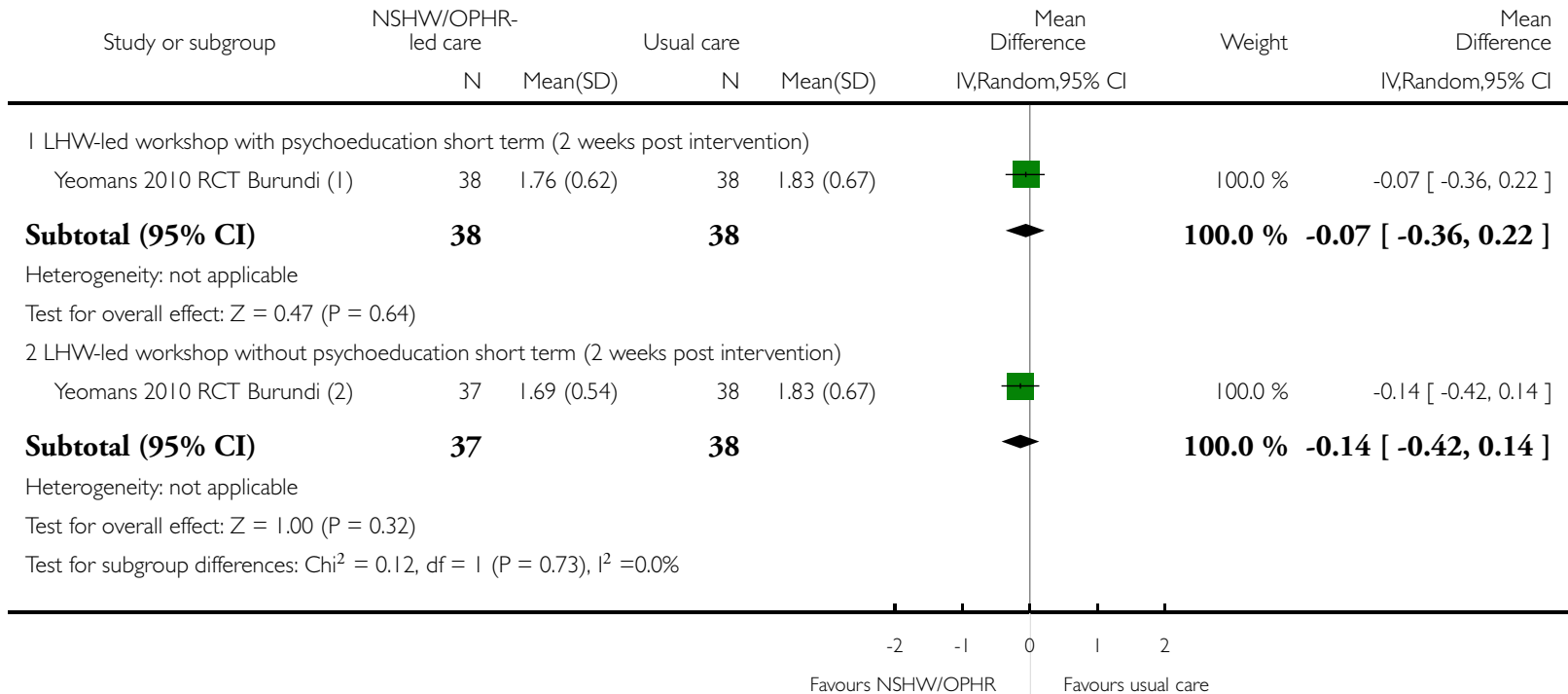

(I) LHW-led workshop with psychoeducation; HSCL-25 (Hopkins Symptom checklist); 2 weeks post-interv, means, SDs (not adjusted)

(2) LHW-led workshop with psychoeducation; HSCL-25 (Hopkins Symptom checklist); 2 weeks post-interv, means, SDs (not adjusted)

Non-specialist health worker interventions for the care of mental, neurological and substance-abuse disorders in low- and middle- 
Analysis 6.I. Comparison 6 NSHWs versus usual care in improving dementia patients' and carers' outcomes (RCTs), Outcome I Severity of behavioural problem (patient).

Review: Non-specialist health worker interventions for the care of mental, neurological and substance-abuse disorders in low- and middle-income countries

Comparison: 6 NSHWs versus usual care in improving dementia patients' and carers' outcomes (RCTs)

Outcome: I Severity of behavioural problem (patient)

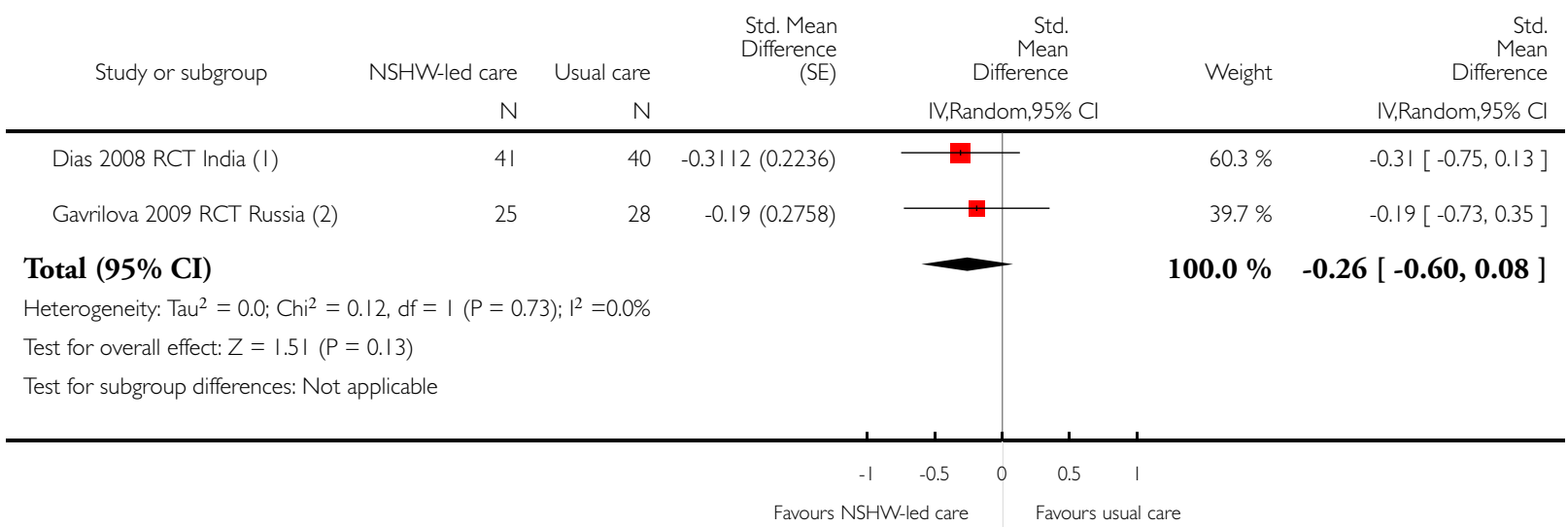

(I) LHW-led brief carer intervention. NPI-S (neuropsychiatric inventory - severity); adjusted effect sizes at 6 months post-interv

(2) Doctor-led brief carer intervention. NPI-S (neuropsychiatric inventory - severity); adjusted effect sizes (standardised MD) and Cls at 6 months post-interv.

\section{Analysis 6.2. Comparison 6 NSHWs versus usual care in improving dementia patients' and carers' outcomes (RCTs), Outcome 2 Patient functional ability.}

Review: Non-specialist health worker interventions for the care of mental, neurological and substance-abuse disorders in low- and middle-income countries

Comparison: 6 NSHWs versus usual care in improving dementia patients' and carers' outcomes (RCTs)

Outcome: 2 Patient functional ability

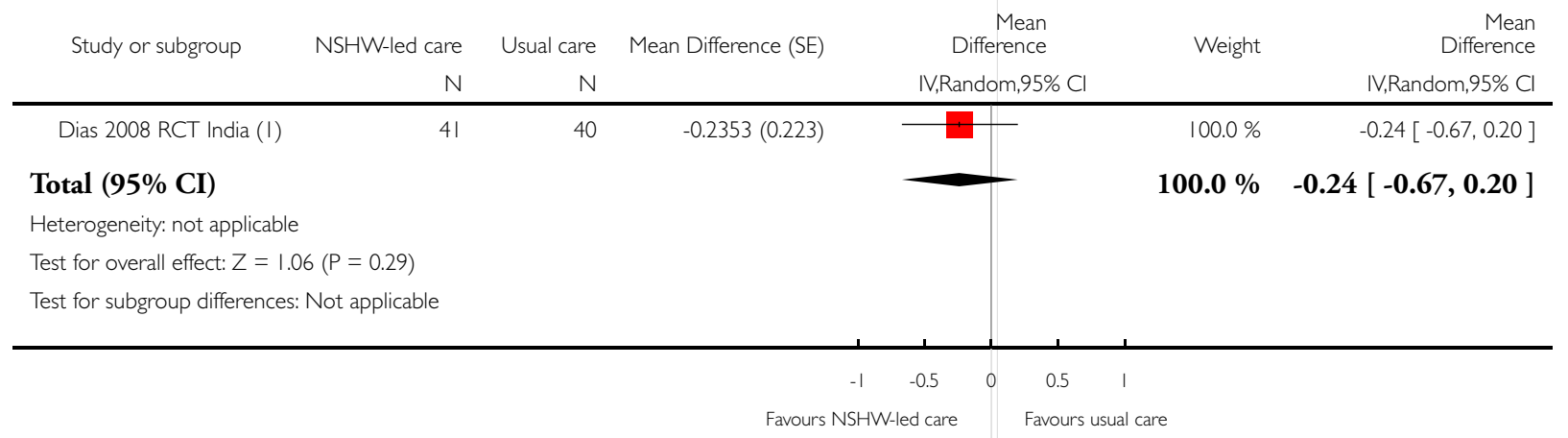

Non-specialist health worker interventions for the care of mental, neurological and substance-abuse disorders in low- and middle- 
(I) LHW-led brief carer intervention. EASI (everyday abilities scales for India); outcome at 6 months

\section{Analysis 6.3. Comparison 6 NSHWs versus usual care in improving dementia patients' and carers' outcomes (RCTs), Outcome 3 Patient quality of life.}

Review: Non-specialist health worker interventions for the care of mental, neurological and substance-abuse disorders in low- and middle-income countries Comparison: 6 NSHWs versus usual care in improving dementia patients' and carers' outcomes (RCTs)

Outcome: 3 Patient quality of life

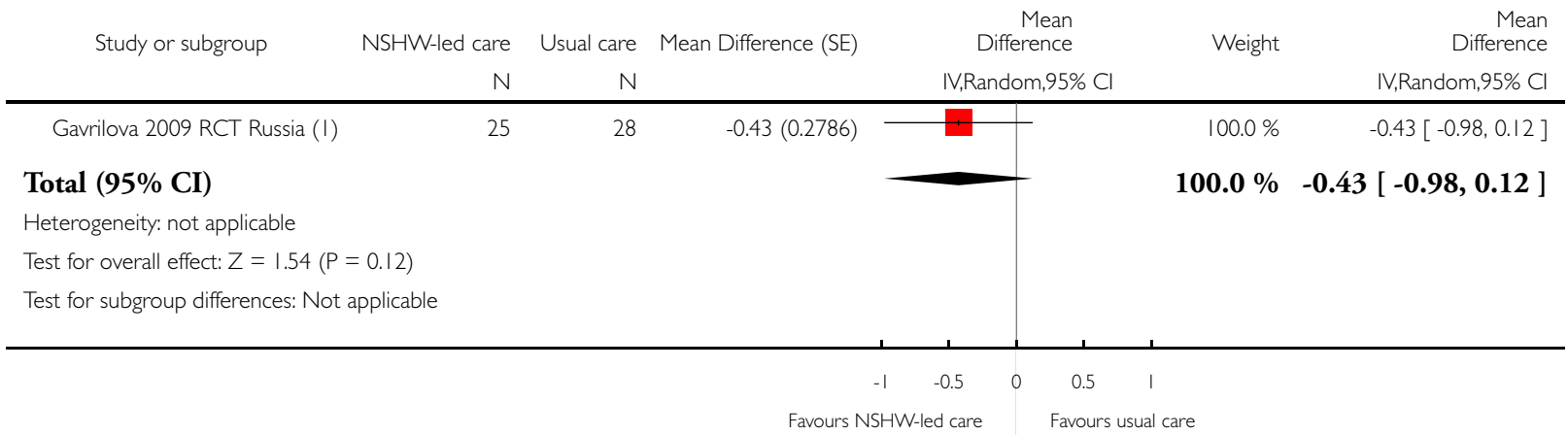

( I) doctor-led brief carer intervention. DEMQOL; adjusted effect sizes (standardised MD) and Cls at 6 months post-interv 


\section{Analysis 6.4. Comparison 6 NSHWs versus usual care in improving dementia patients' and carers' outcomes (RCTs), Outcome 4 Carer mental health status.}

Review: Non-specialist health worker interventions for the care of mental, neurological and substance-abuse disorders in low- and middle-income countries

Comparison: 6 NSHWs versus usual care in improving dementia patients' and carers' outcomes (RCTs)

Outcome: 4 Carer mental health status

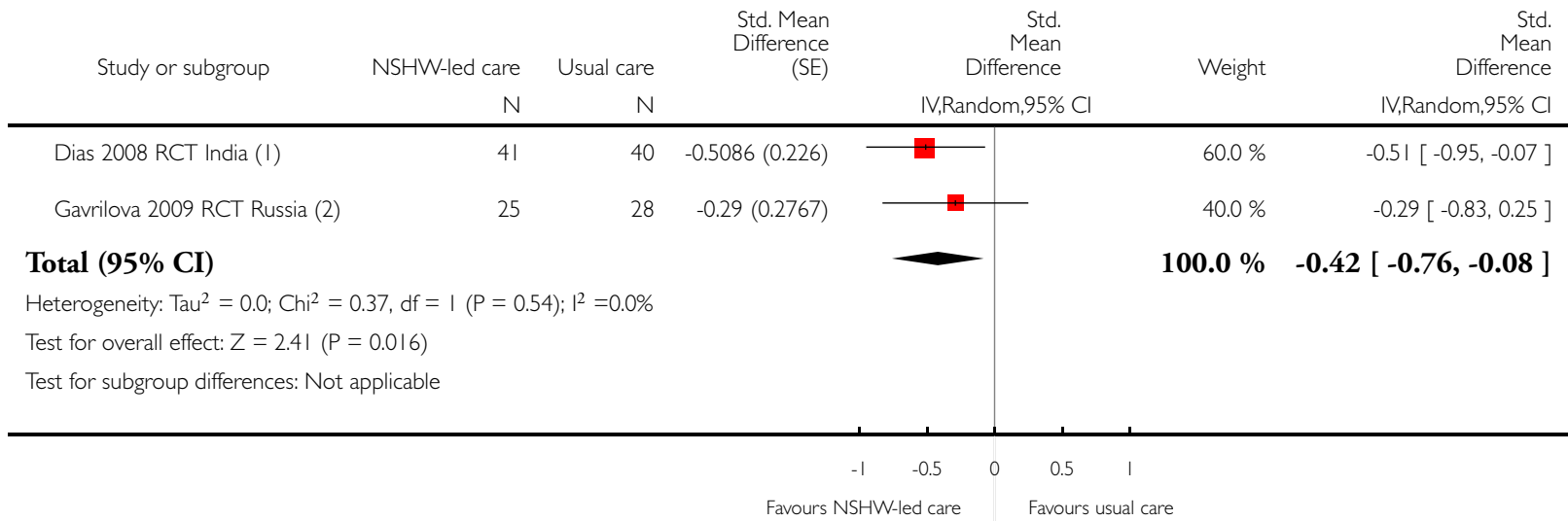

(I) LHW-led brief carer intervention. GHQ-12 score; outcome at 6 months

(2) doctor-led brief carer intervention. SRQ-20 score; adjusted effect sizes (standardised MD) and Cls at 6 months post-interv

\section{Analysis 6.5. Comparison 6 NSHWs versus usual care in improving dementia patients' and carers' outcomes (RCTs), Outcome 5 Carer burden.}

Review: Non-specialist health worker interventions for the care of mental, neurological and substance-abuse disorders in low- and middle-income countries

Comparison: 6 NSHWs versus usual care in improving dementia patients' and carers' outcomes (RCTs)

Outcome: 5 Carer burden

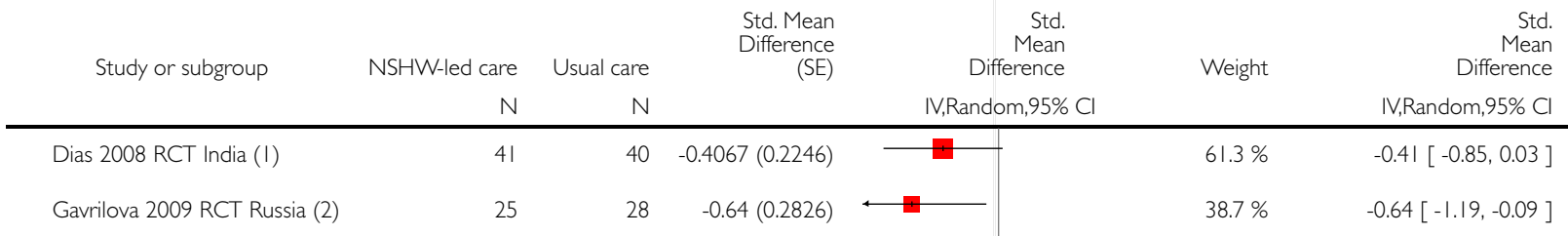

Total $(95 \% \mathrm{CI})$

$\mathbf{1 0 0 . 0} \%-\mathbf{0 . 5 0}[-\mathbf{- 0 . 8 4},-\mathbf{0 . 1 5}]$

Heterogeneity: $\mathrm{Tau}^{2}=0.0 ; \mathrm{Chi}^{2}=0.42, \mathrm{df}=\mathrm{I}(\mathrm{P}=0.52) ; \mathrm{I}^{2}=0.0 \%$

Test for overall effect: $Z=2.83(P=0.0047)$

Test for subgroup differences: Not applicable

Favours NSHW-led care Favours usual care

Non-specialist health worker interventions for the care of mental, neurological and substance-abuse disorders in low- and middle- 
(I) LHW-led brief carer intervention. Zarit burden interview (ZBI); adjusted coefficient size at 6 months.

(2) doctor-led brief carer intervention. Zarit burden interview (ZBI); adjusted effect sizes (standardised MD) and Cls at 6 months post-interv

\section{Analysis 6.6. Comparison 6 NSHWs versus usual care in improving dementia patients' and carers' outcomes (RCTs), Outcome 6 Carer distress.}

Review: Non-specialist health worker interventions for the care of mental, neurological and substance-abuse disorders in low- and middle-income countries

Comparison: 6 NSHWs versus usual care in improving dementia patients' and carers' outcomes (RCTs)

Outcome: 6 Carer distress

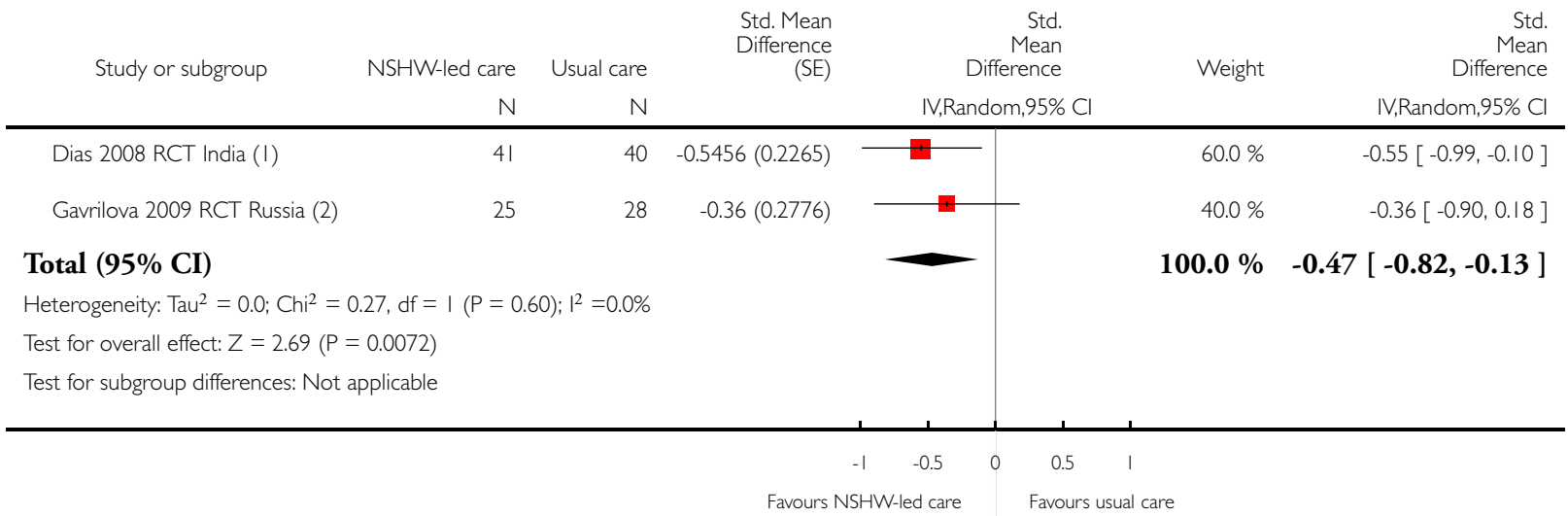

(I) LHW-led brief carer intervention; NPI-D (Neuropsychiatric Interview - distress of carers); outcome at 6 months

(2) doctor-led brief carer intervention. NPI-D (Neuropsychiatric Interview - distress of carers); adjusted effect sizes (standardised MD) and Cls at 6 months post-interv 
Analysis 6.7. Comparison 6 NSHWs versus usual care in improving dementia patients' and carers' outcomes (RCTs), Outcome 7 Carer quality of life.

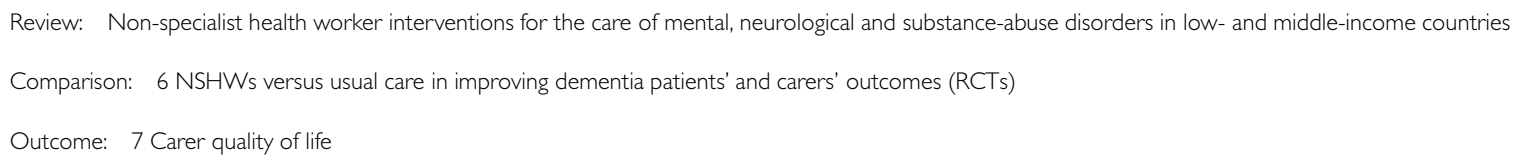

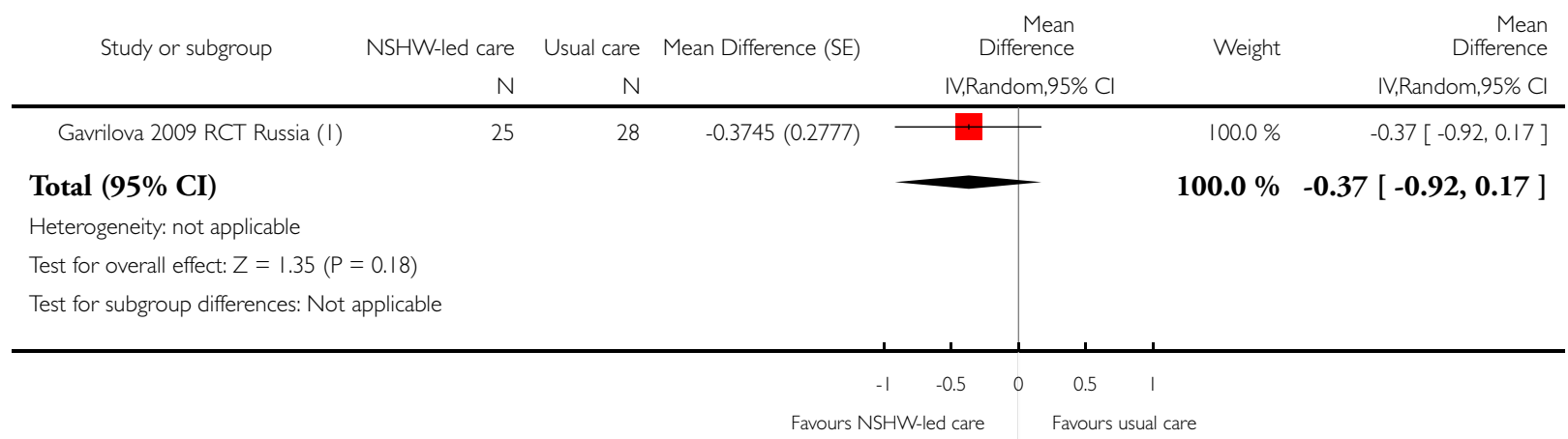

( I) doctor-led brief carer intervention. WHOQOL-BREF (psychological reported) (assumption adjusted effect size is adj MD); outcome at 6 months

Analysis 7.I. Comparison 7 NSHW-led brief alcohol interventions versus usual care for adults with alcoholuse disorders (RCTs), Outcome I Amount of alcohol consumed (MD).

Review: Non-specialist health worker interventions for the care of mental, neurological and substance-abuse disorders in low- and middle-income countries

Comparison: $7 \mathrm{NSHW}$-led brief alcohol interventions versus usual care for adults with alcohol-use disorders (RCTs)

Outcome: I Amount of alcohol consumed (MD)

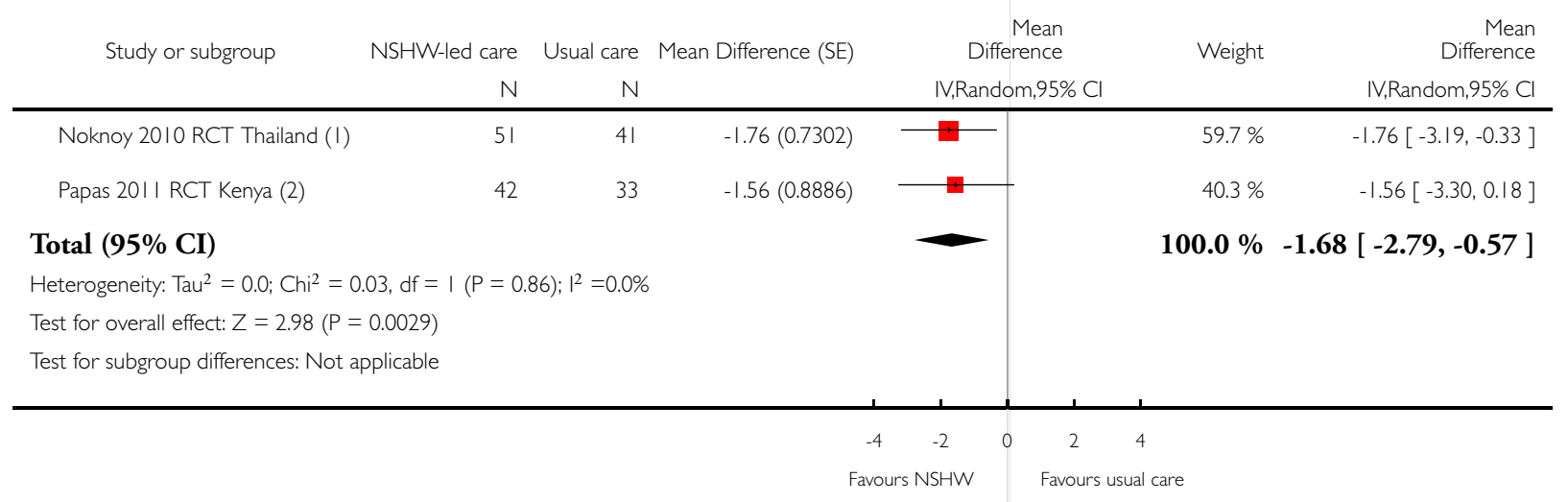

Non-specialist health worker interventions for the care of mental, neurological and substance-abuse disorders in low- and middle- 
(I) Nurse-led motivational enhancement therapy (MET); number of drinks/ drinking day in previous week; 6 month outcome scores mean, SD (non adjusted)

(2) LHW-led adapted CBT and education; average drinks/drinking day (past 30 days); 3 month post-interv means and SD (unadjusted)

Analysis 7.2. Comparison 7 NSHW-led brief alcohol interventions versus usual care for adults with alcoholuse disorders (RCTs), Outcome 2 Frequency of binge drinking.

Review: Non-specialist health worker interventions for the care of mental, neurological and substance-abuse disorders in low- and middle-income countries

Comparison: $7 \mathrm{NSHW}$-led brief alcohol interventions versus usual care for adults with alcohol-use disorders (RCTs)

Outcome: 2 Frequency of binge drinking

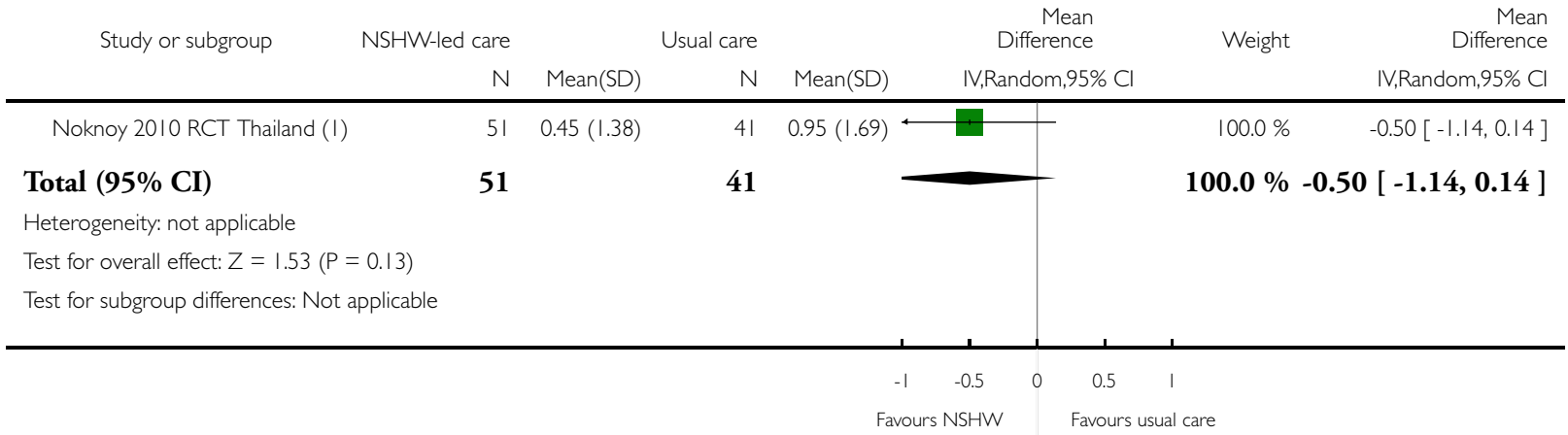

(I) Nurse-led motivational enhancement therapy (MET); frequency of binge drinking in past week; 6 month outcome scores mean, SD (non adjusted) 
Analysis 7.3. Comparison 7 NSHW-led brief alcohol interventions versus usual care for adults with alcoholuse disorders (RCTs), Outcome 3 Adverse consequences.

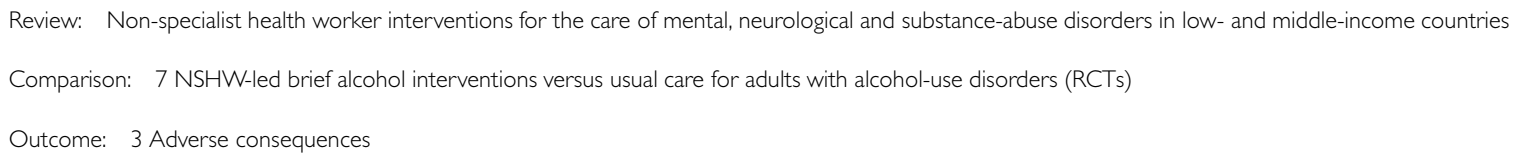

Total events: 4 (NSHW), 9 (Usual care)

Heterogeneity: not applicable

Test for overall effect: $Z=1.83(P=0.068)$

2 Withdrawal symptoms

Papas 201 I RCT Kenya (2)

Subtotal (95\% CI)

Total events: 3 (NSHW), I (Usual care)

Heterogeneity: not applicable

Test for overall effect: $Z=0.87(P=0.38)$

Total (95\% CI)

$\rightarrow$

Total events: 7 (NSHW), 10 (Usual care)

Heterogeneity: $\operatorname{Tau}^{2}=1.25 ; \mathrm{Chi}^{2}=2.57, \mathrm{df}=1(\mathrm{P}=0.1 \mathrm{I}) ; \mathrm{I}^{2}=61 \%$

Test for overall effect: $Z=0.26(P=0.79)$

Test for subgroup differences: $\mathrm{Chi}^{2}=2.54, \mathrm{df}=\mathrm{I}(\mathrm{P}=0.1 \mathrm{I}), \mathrm{I}^{2}=61 \%$

Weight

Risk Ratio

$H$,Random $95 \%$

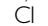

$61.7 \%$

$0.36[0.12,1.08]$

$61.7 \%$

$0.36[0.12,1.08]$

$38.3 \%$

$2.67[0.29,24.37]$

$38.3 \%$

$2.67[0.29,24.37]$

$100.0 \%$

$0.77[0.11,5.29]$

(I) Nurse-led motivational enhancement therapy (MET); nb of accidents/road-traffic accidents over 6 months

(2) LHW-led adapted CBT and education; nb of withdrawal symptoms at 3 month post-intervention. 


\section{Analysis 8.1. Comparison 8 NSHWs/OPHRs versus usual care in conducting interventions for children with post-traumatic stress and depression (RCTs), Outcome I Severity of PTSD symptoms - teacher/LHW-led interventions (SMDs).}

Review: Non-specialist health worker interventions for the care of mental, neurological and substance-abuse disorders in low- and middle-income countries

Comparison: $8 \mathrm{NSHWs} / \mathrm{OPHRs}$ versus usual care in conducting interventions for children with post-traumatic stress and depression (RCTs)

Outcome: I Severity of PTSD symptoms - teacher/LHW-led interventions (SMDs)

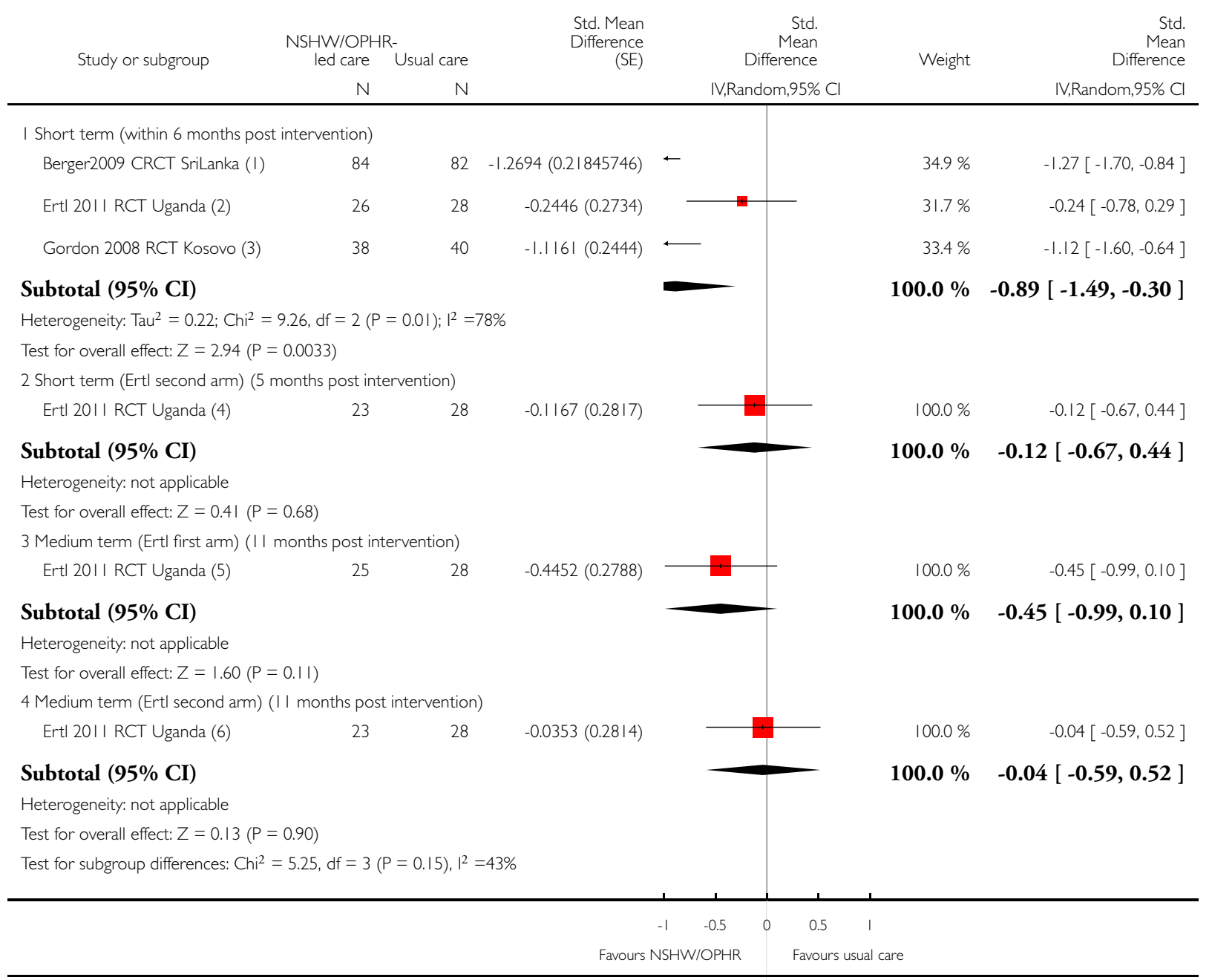

Non-specialist health worker interventions for the care of mental, neurological and substance-abuse disorders in low- and middle- 
(I) Teacher-led stress programme; basic version of the UCLA PTSD index (DSM-IV); mean and SD at 2 months, cluster-adjusted with ICC=0.05 to calculate design effect

(2) LHW-led narrative exposure therapy; Clinician administered PTSD scale (CAPS); unadjusted mean and SDs; 5 mths post-intervention

(3) Teacher-led mind body skills programme; HTQ (Harvard Trauma questionnaire); immediate post intervention unadjusted means and Cls.

(4) LHW-led Academic catch-up; Clinician administered PTSD scale (CAPS); Unadjusted mean and SEs; 5 mths post-intervention

(5) LHW-led narrative exposure therapy; Clinician administered PTSD scale (CAPS); unadjusted mean and SEs; II mths post-intervention

(6) LHW-led academic catch-up; Clinician administered PTSD scale (CAPS); unadjusted mean and SEs; I I mths post-intervention

\section{Analysis 8.2. Comparison 8 NSHWs/OPHRs versus usual care in conducting interventions for children with post-traumatic stress and depression (RCTs), Outcome 2 Severity of PTSD symptoms - classroom-based LHW interventions (MCDs).}

Review: Non-specialist health worker interventions for the care of mental, neurological and substance-abuse disorders in low- and middle-income countries

Comparison: $8 \mathrm{NSHWs/OPHRs} \mathrm{versus} \mathrm{usual} \mathrm{care} \mathrm{in} \mathrm{conducting} \mathrm{interventions} \mathrm{for} \mathrm{children} \mathrm{with} \mathrm{post-traumatic} \mathrm{stress} \mathrm{and} \mathrm{depression} \mathrm{(RCTs)}$

Outcome: 2 Severity of PTSD symptoms - classroom-based LHW interventions (MCDs)

\begin{tabular}{|c|c|c|c|c|c|c|}
\hline \multirow[t]{2}{*}{ Study or subgroup } & $\begin{array}{l}\text { NSHW/OPHR- } \\
\text { led care }\end{array}$ & Usual care & $\begin{array}{r}\text { Mean Change } \\
\text { Difference } \\
(\mathrm{SE})\end{array}$ & $\begin{array}{r}\text { Mean } \\
\text { Change } \\
\text { Difference }\end{array}$ & \multirow[t]{2}{*}{ Weight } & $\begin{array}{r}\text { Mean } \\
\text { Change } \\
\text { Difference }\end{array}$ \\
\hline & $\mathrm{N}$ & $\mathrm{N}$ & \multicolumn{2}{|r|}{ IV,Random,95\% Cl } & & IV,Random,95\% Cl \\
\hline \multicolumn{7}{|c|}{ term (within 6 months post intervention) } \\
\hline ins 2010 C-RCT Nepal (I) & 164 & 161 & $-0.13(0.85)$ & & $33.4 \%$ & $-0.13[-1.80,1.54]$ \\
\hline 008 C-RCT Indonesia (2) & 177 & 191 & $-2.78(0.89)$ & $\longleftarrow$ & $32.9 \%$ & $-2.78[-4.52,-1.04]$ \\
\hline 012 C-RCT SriLanka (3) & 198 & 199 & $1.18(0.8318)$ & ए & $33.7 \%$ & $1.18[-0.45,2.81]$ \\
\hline
\end{tabular}

Subtotal $(95 \% \mathrm{CI})$

Heterogeneity: $\mathrm{Tau}^{2}=3.24 ; \mathrm{Chi}^{2}=10.83, \mathrm{df}=2(\mathrm{P}=0.004) ; \mathrm{I}^{2}=82 \%$

Test for overall effect: $Z=0.49(P=0.63)$

Test for subgroup differences: Not applicable

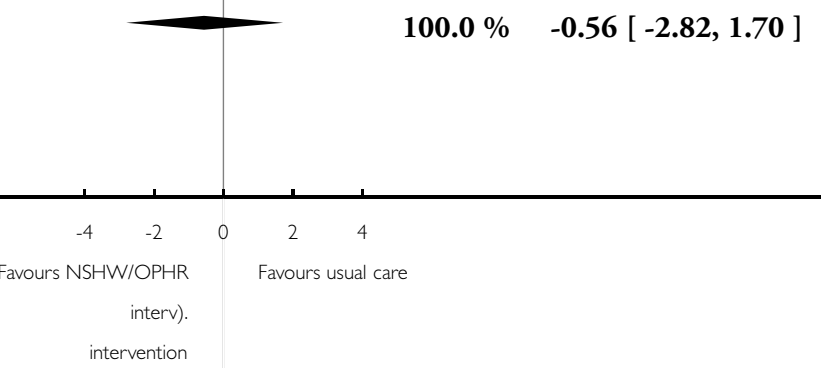

(I) LHW-led classroom based intervention (CBI); CPSS (Child posttraumatic stress scale); I month post-interv: MCD adj for clustering/SE. reversed direction of effect (+ve result favours

(2) LHW-led classroom based intervention (CBI); CPSS; 6 months post-interv: Mean Change Difference adjusted for clustering and SE.

(3) LHW-led CBI; CPSS; 3 months post interv; Cluster-adjusted mean change differences boys and girls. ICC $=0.002$., reversed direction of effect as a positive result favours 
Analysis 8.3. Comparison 8 NSHWs/OPHRs versus usual care in conducting interventions for children with post-traumatic stress and depression (RCTs), Outcome 3 Severity of PTSD symptoms - classroom-based LHW interventions - boys/girls.

Review: Non-specialist health worker interventions for the care of mental, neurological and substance-abuse disorders in low- and middle-income countries

Comparison: $8 \mathrm{NSHWs} / \mathrm{OPHRs}$ versus usual care in conducting interventions for children with post-traumatic stress and depression (RCTs)

Outcome: 3 Severity of PTSD symptoms - classroom-based LHW interventions - boys/girls

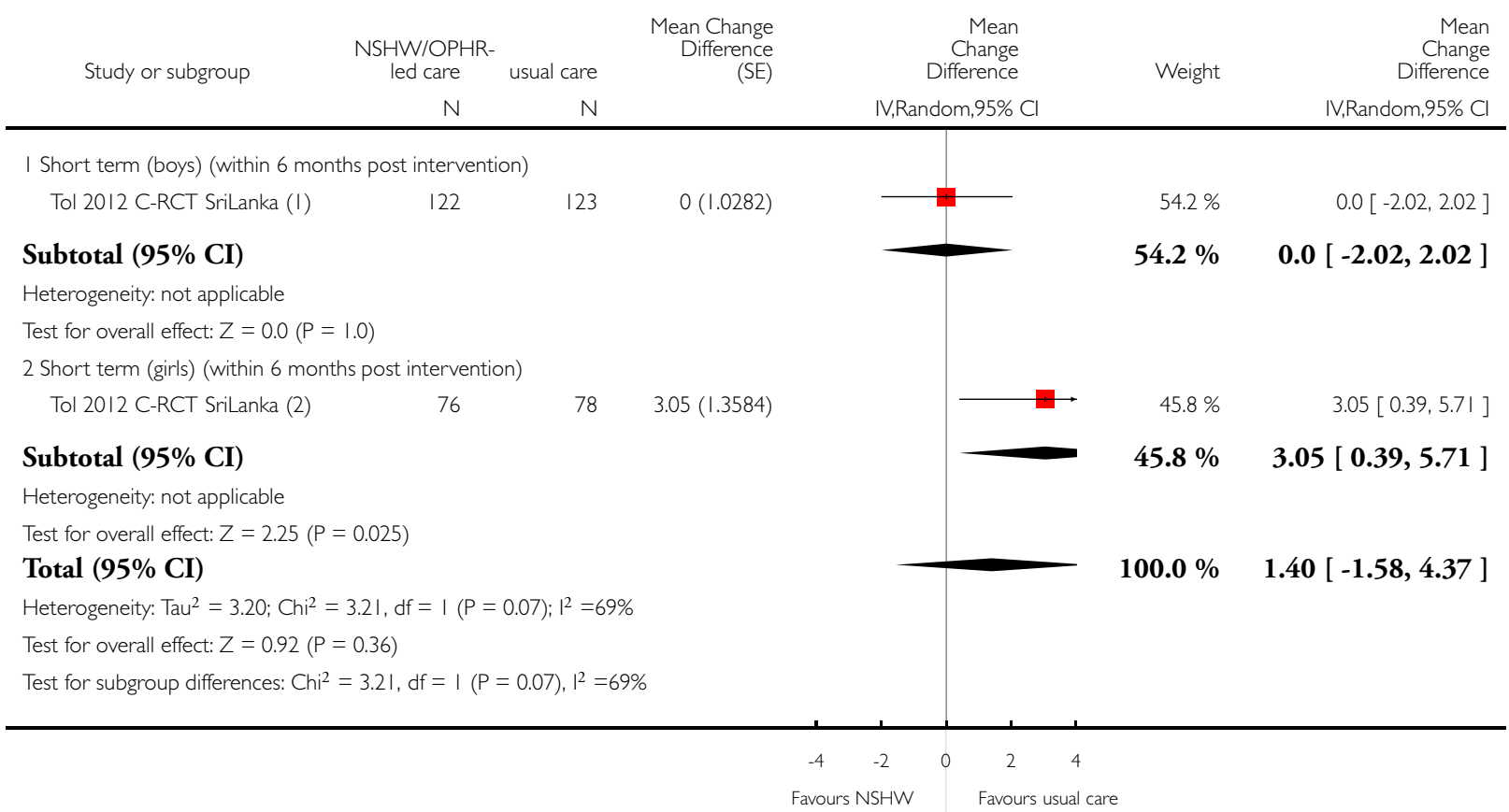

(I) LHW-led CBI; CPSS; 3 m post interv; cluster-adj mean change scores and SDs calculated with ICC $=0.002$, reversed direction of effect (+ve result favours interv)

(2) LHW-led CBI; CPSS; 3 m post interv; cluster-adj mean change scores and SDs calculated with ICC $=0.002$, reversed direction of effect (+ve result favours interv) 
Analysis 8.4. Comparison 8 NSHWs/OPHRs versus usual care in conducting interventions for children with post-traumatic stress and depression (RCTs), Outcome 4 Severity of depressive symptoms - teacher/LHW-led interventions (SMDs).

Review: Non-specialist health worker interventions for the care of mental, neurological and substance-abuse disorders in low- and middle-income countries

Comparison: $8 \mathrm{NSHWs} / \mathrm{OPHRs}$ versus usual care in conducting interventions for children with post-traumatic stress and depression (RCTs)

Outcome: 4 Severity of depressive symptoms - teacher/LHW-led interventions (SMDs)

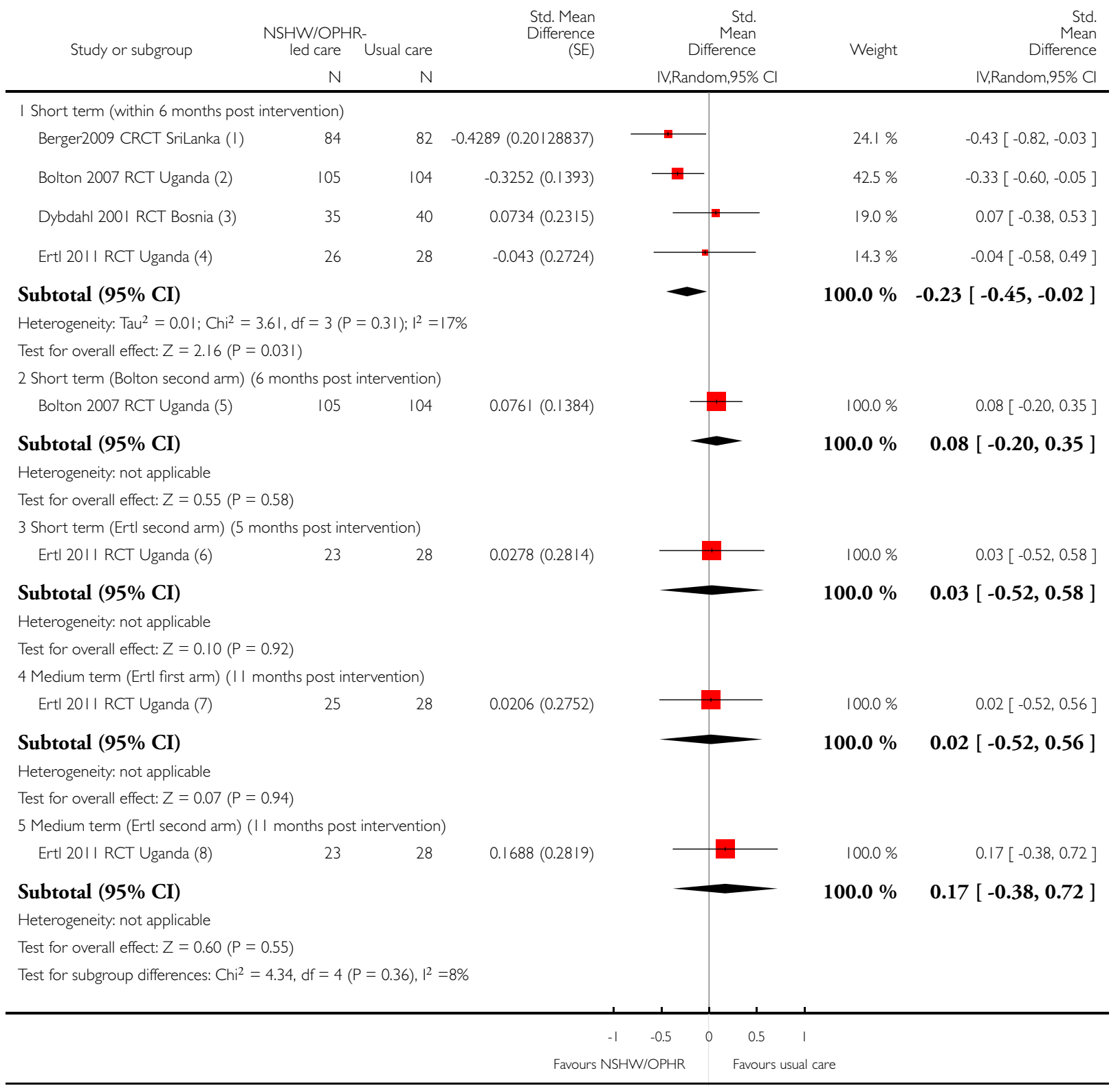

Non-specialist health worker interventions for the care of mental, neurological and substance-abuse disorders in low- and middle- 
(I) Teacher-led stress programme; BDI (Beck Depression Inventory); mean and SD at 2 months, cluster-adjusted with ICC=0.05 to calculate design effect

(2) LHW-led IPT-G; Acholi Psychosocial Assessment Instrument; adjusted mean score change and SE (ITT) (I month post-interv)

(3) Teacher-led psychosocial intervention for children and mothers; Birleson's Depression inventory; mean and SD 5-6 months post intervention.

(4) LHW-led narrative exposure therapy; Mini International and Neuropsychiatric Interview (MINI); unadjusted mean and SEs; 5 mths post-intervention

(5) LHW-led Creative play; Acholi Psychosocial Assessmt Instrument; adjusted mean score change and SE (completers)(I mth post-interv)

(6) LHW-led academic catch-up; MINI; Unadjusted mean and SDs; 5 mths post-intervention

(7) LHW-led narrative exposure therapy; MINI; unadjusted mean and SDs; I I mths post-intervention

(8) LHW-led academic catch up; MINI; unadjusted mean and SDs; II mths post-intervention

Analysis 8.5. Comparison 8 NSHWs/OPHRs versus usual care in conducting interventions for children with post-traumatic stress and depression (RCTs), Outcome 5 Severity of depressive symptoms - classroom-based LHW interventions (MCDs).

Review: Non-specialist health worker interventions for the care of mental, neurological and substance-abuse disorders in low- and middle-income countries

Comparison: $8 \mathrm{NSHWs/OPHRs} \mathrm{versus} \mathrm{usual} \mathrm{care} \mathrm{in} \mathrm{conducting} \mathrm{interventions} \mathrm{for} \mathrm{children} \mathrm{with} \mathrm{post-traumatic} \mathrm{stress} \mathrm{and} \mathrm{depression} \mathrm{(RCTs)}$

Outcome: 5 Severity of depressive symptoms - classroom-based LHW interventions (MCDs)

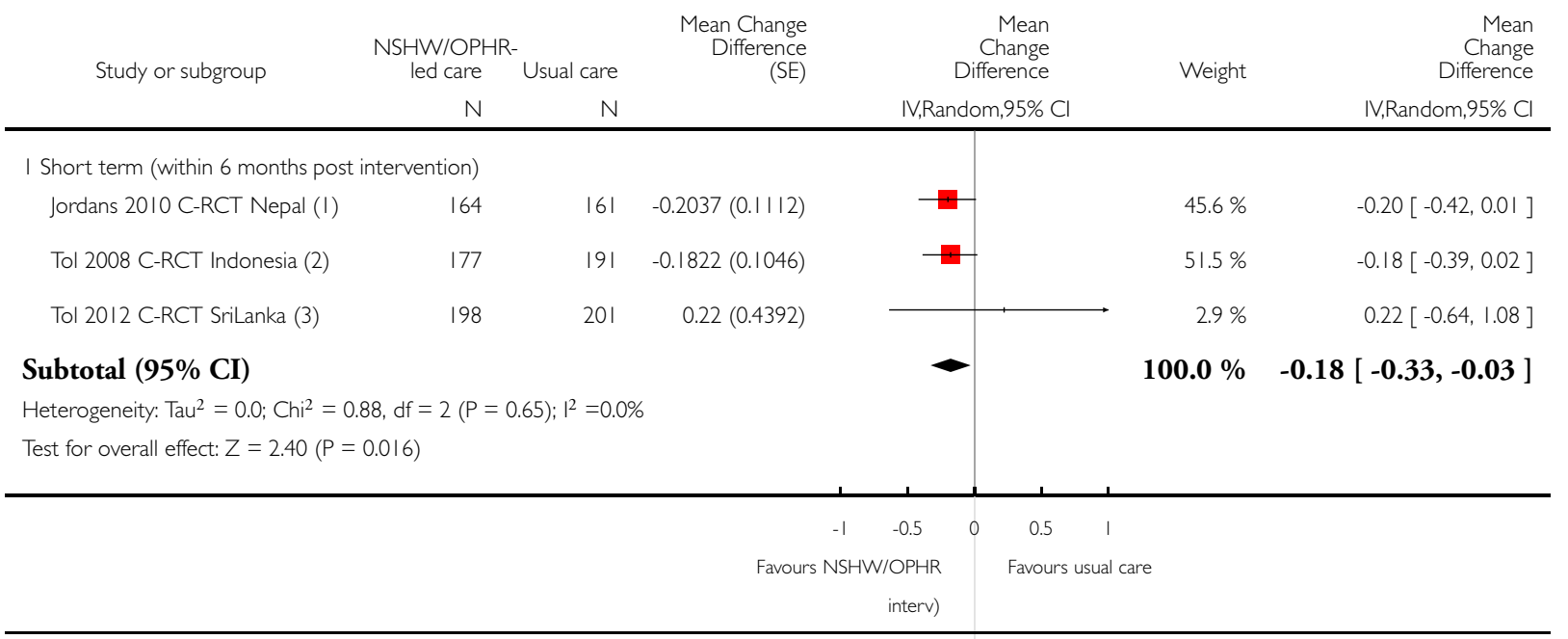

Non-specialist health worker interventions for the care of mental, neurological and substance-abuse disorders in low- and middleincome countries (Review)

Copyright $\odot 2013$ The Cochrane Collaboration. Published by John Wiley \& Sons, Ltd. 
(I) LHW-led classroom based intervention (CBI); DSRS (depression self-rating scale); cluster-adjusted MCD (SE) I mth post-interv. reversed direction of effect (+ve result favours

(2) LHW-led classroom based intervention (CBI);DSRS; 6 months post interv: M Change diff adjusted for clustering and SE.

(3) LHW-led CBI; DSRS; 3 months post interv; cluster-adjusted mean change differences and SDs. ICC $=0.000$, reversed direction of effect as a positive result favours intervention

Analysis 8.6. Comparison 8 NSHWs/OPHRs versus usual care in conducting interventions for children with post-traumatic stress and depression (RCTs), Outcome 6 Severity of depressive symptoms (MCDs) Tol 2012 boys/girls.

Review: Non-specialist health worker interventions for the care of mental, neurological and substance-abuse disorders in low- and middle-income countries

Comparison: $8 \mathrm{NSHWs} / \mathrm{OPHRs}$ versus usual care in conducting interventions for children with post-traumatic stress and depression (RCTs)

Outcome: 6 Severity of depressive symptoms (MCDs) Tol 2012 boys/girls

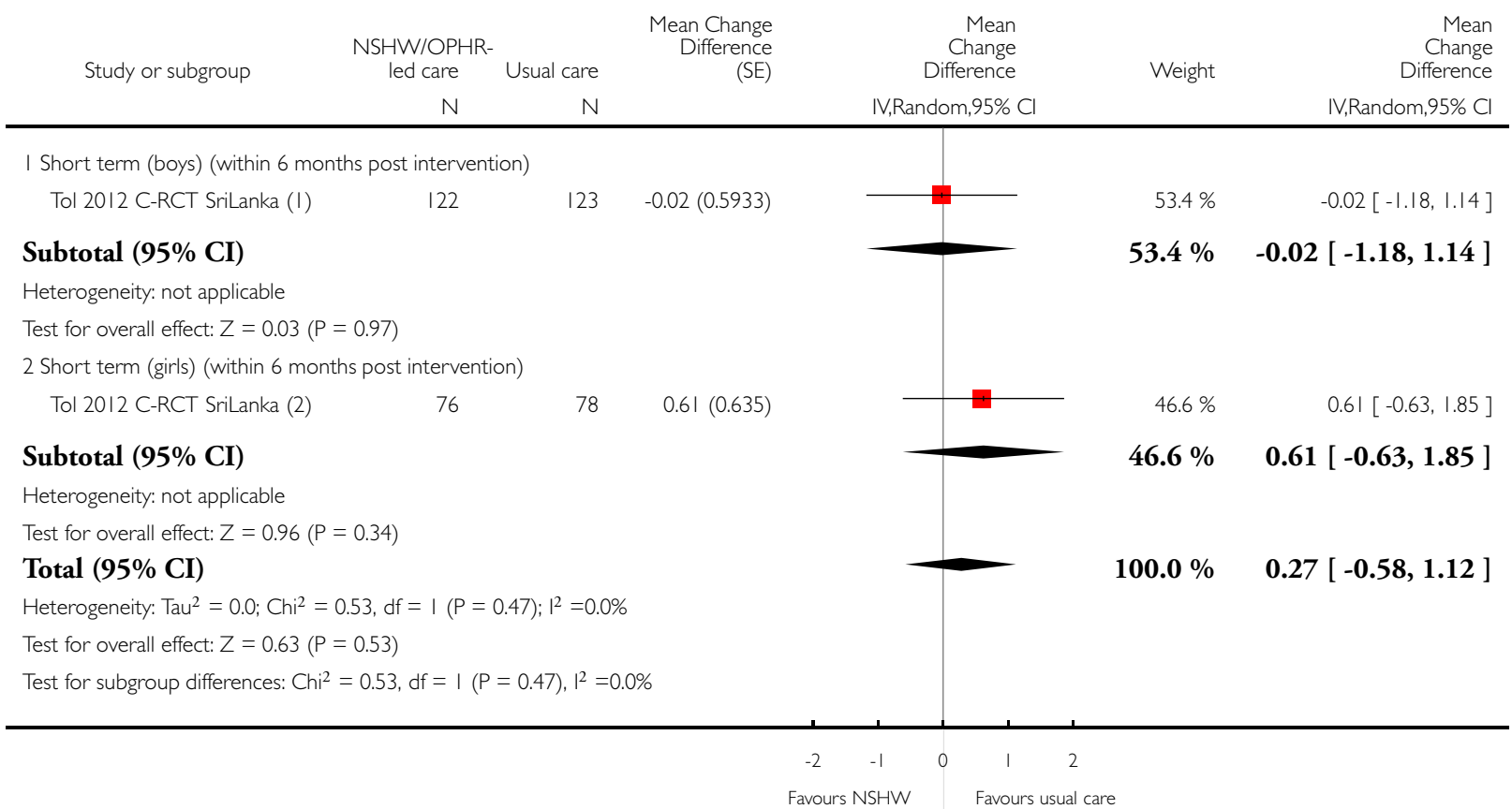

(I) LHW-led CBI; DSRS; 3 months post interv; cluster-adjusted mean change diff and SDs calculated with ICC=0, reversed direction of effect

(2) LHW-led CBI; DSRS; 3 months post interv; cluster-adjusted mean change diff and SDs calculated with ICC=0, reversed direction of effect as a positive result favours intervention 
Analysis 8.7. Comparison 8 NSHWs/OPHRs versus usual care in conducting interventions for children with post-traumatic stress and depression (RCTs), Outcome 7 Severity of anxiety symptoms - classroom-based intervention (within 6 months post intervention).

Review: Non-specialist health worker interventions for the care of mental, neurological and substance-abuse disorders in low- and middle-income countries

Comparison: $8 \mathrm{NSHWs} / \mathrm{OPHRs}$ versus usual care in conducting interventions for children with post-traumatic stress and depression (RCTs)

Outcome: 7 Severity of anxiety symptoms - classroom-based intervention (within 6 months post intervention)

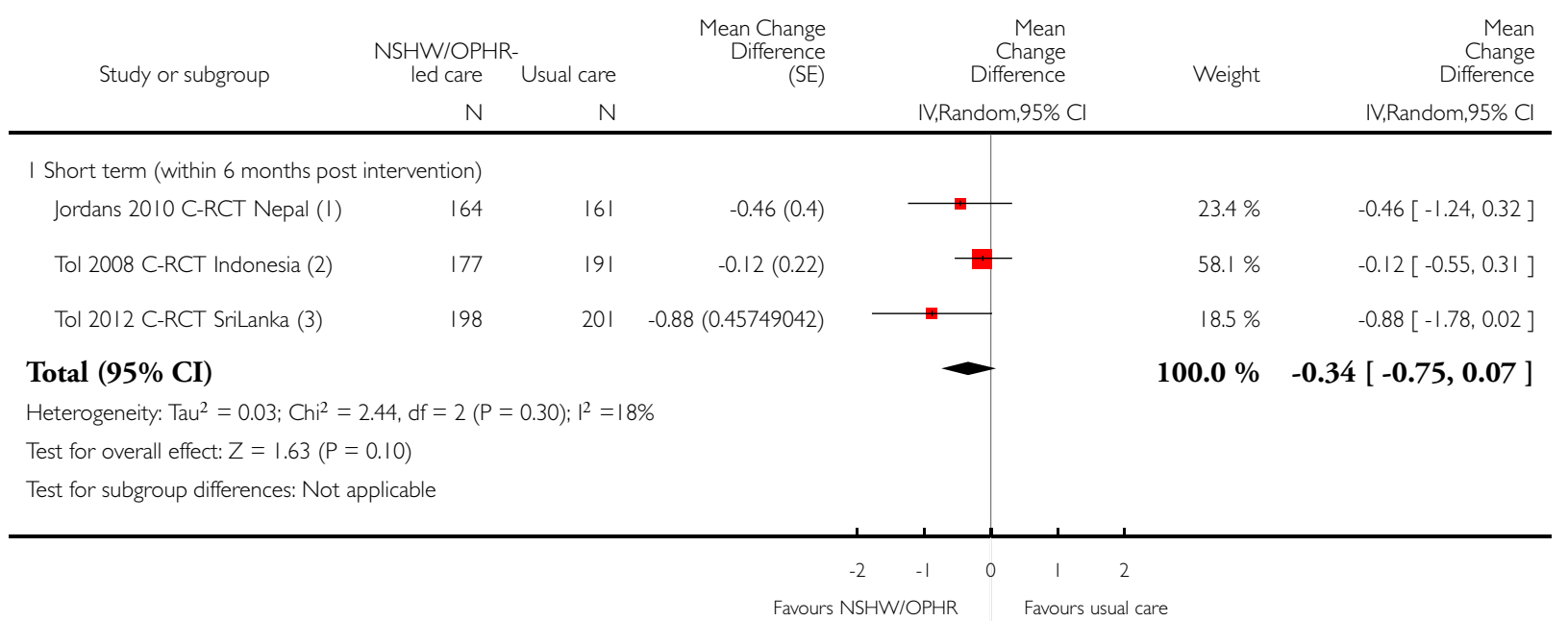

( I) LHW-led classroom based intervention (CBI); SCARED-5; cluster adj MchangeDiff I m post-interv, reversed direction of effect as a +ve result favours intervention

(2) LHW-led CBI; SCARED-5; 6 m post-interv Mean Change Diff adjusted for clustering and SE.

(3) LHW-led CBI; SCARED-5; 3 m post interv; cluster-adj mean change diff+SDs calculated with ICC $=0.005$, reversed direction of effect as a +ve result favours intervention 
Analysis 8.8. Comparison 8 NSHWs/OPHRs versus usual care in conducting interventions for children with post-traumatic stress and depression (RCTs), Outcome 8 Severity of anxiety symptoms - classroom-based intervention - boys/girls.

Review: Non-specialist health worker interventions for the care of mental, neurological and substance-abuse disorders in low- and middle-income countries

Comparison: $8 \mathrm{NSHWs} / \mathrm{OPHRs}$ versus usual care in conducting interventions for children with post-traumatic stress and depression (RCTs)

Outcome: 8 Severity of anxiety symptoms - classroom-based intervention - boys/girls

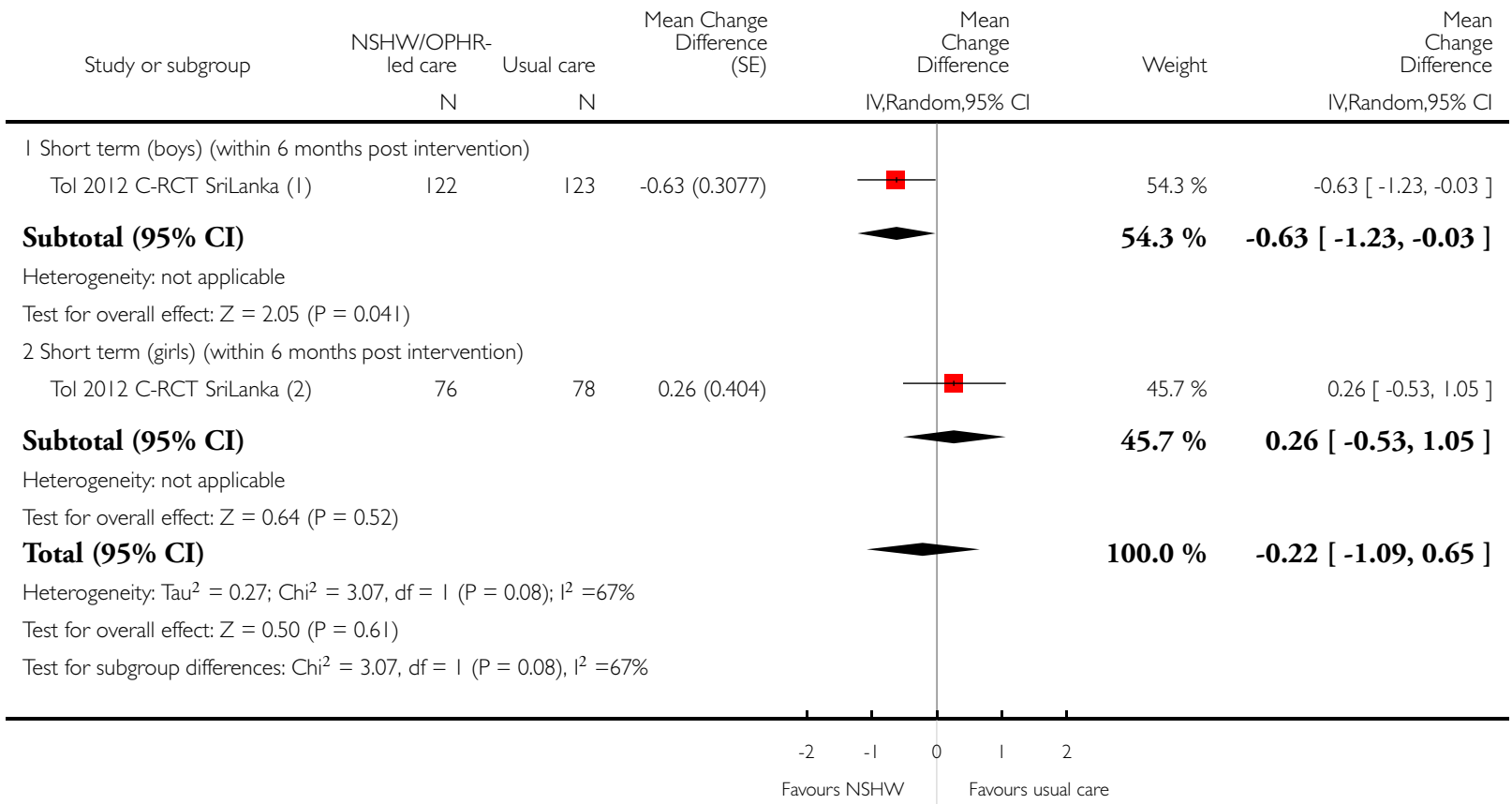

(I) LHW-led CBI; SCARED-5; 3 m post interv; cluster-adj mean change diff+SDs calculated with ICC $=0.005$. reversed direction of effect as a +ve result favours interv

(2) LHW-led CBI; SCARED-5; 3 m post interv; cluster-adj mean change diff+SDs calculated with ICC $=0.005$. reversed direction of effect as a +ve result favours interv 
Analysis 8.9. Comparison 8 NSHWs/OPHRs versus usual care in conducting interventions for children with post-traumatic stress and depression (RCTs), Outcome 9 Functional impairment teacher/LHW-led interventions.

Review: Non-specialist health worker interventions for the care of mental, neurological and substance-abuse disorders in low- and middle-income countries

Comparison: $8 \mathrm{NSHWs/OPHRs} \mathrm{versus} \mathrm{usual} \mathrm{care} \mathrm{in} \mathrm{conducting} \mathrm{interventions} \mathrm{for} \mathrm{children} \mathrm{with} \mathrm{post-traumatic} \mathrm{stress} \mathrm{and} \mathrm{depression} \mathrm{(RCTs)}$

Outcome: 9 Functional impairment teacher/LHW-led interventions

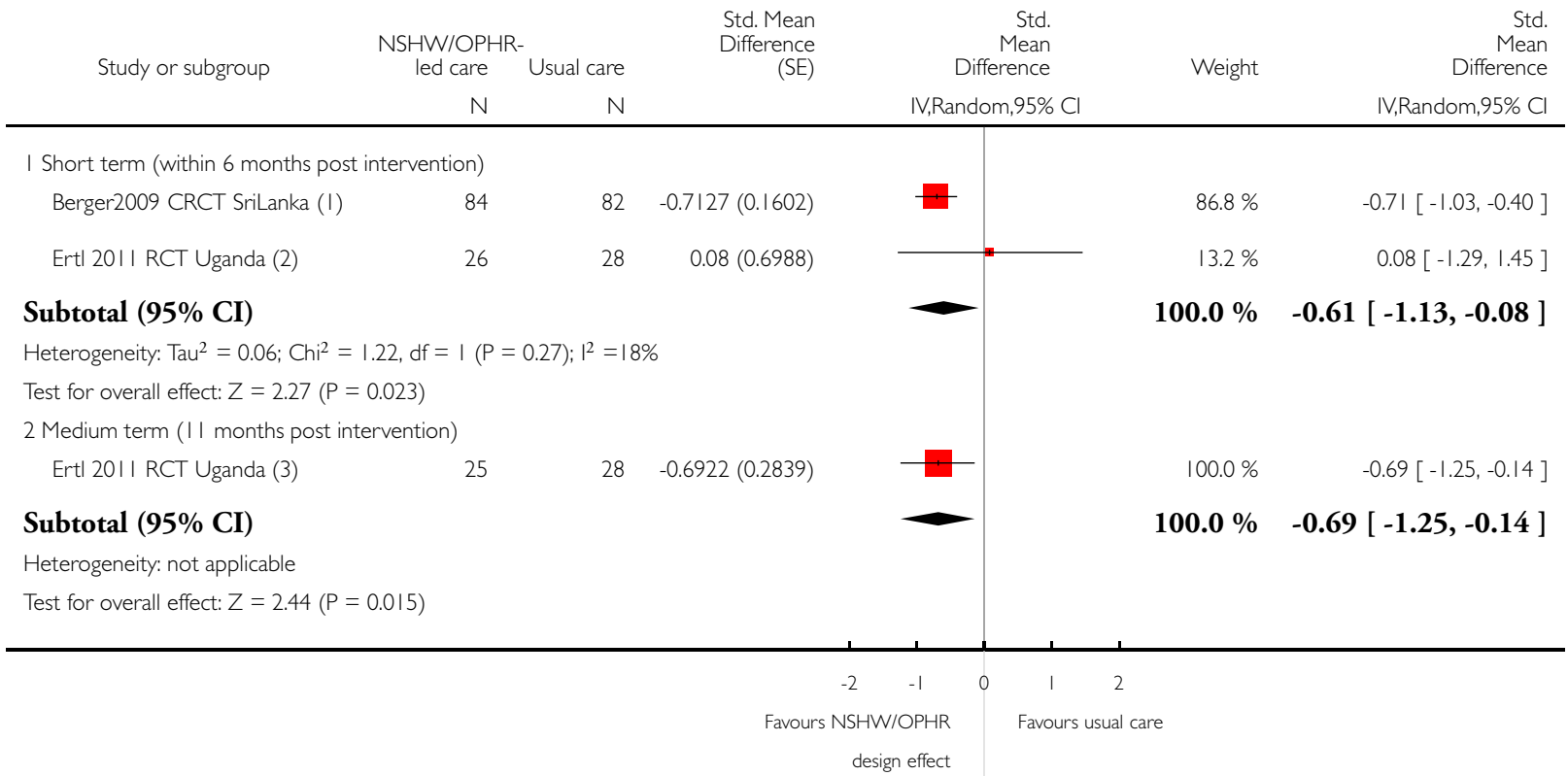

(I) Teacher-led stress programme;CDIS (child diagnostic intervew schedule) not validated in local context; mean and SD at 2 months, cluster-adjusted with ICC=0.05 to calculate

(2) LHW-led narrative exposure therapy; Clinician administered PTSD scale (CAPS)-functional impairment score; unadjusted means and SDs; 5 months post-interv

(3) LHW-led narrative exposure therapy; Clinician administered PTSD scale (CAPS)-functional impairment score; unadjusted means and SDs 
Analysis 8.10. Comparison 8 NSHWs/OPHRs versus usual care in conducting interventions for children with post-traumatic stress and depression (RCTs), Outcome 10 Functional impairment LHW-led - classroombased intervention.

Review: Non-specialist health worker interventions for the care of mental, neurological and substance-abuse disorders in low- and middle-income countries

Comparison: $8 \mathrm{NSHWs} / \mathrm{OPHRs}$ versus usual care in conducting interventions for children with post-traumatic stress and depression (RCTs)

Outcome: 10 Functional impairment LHW-led - classroom-based intervention

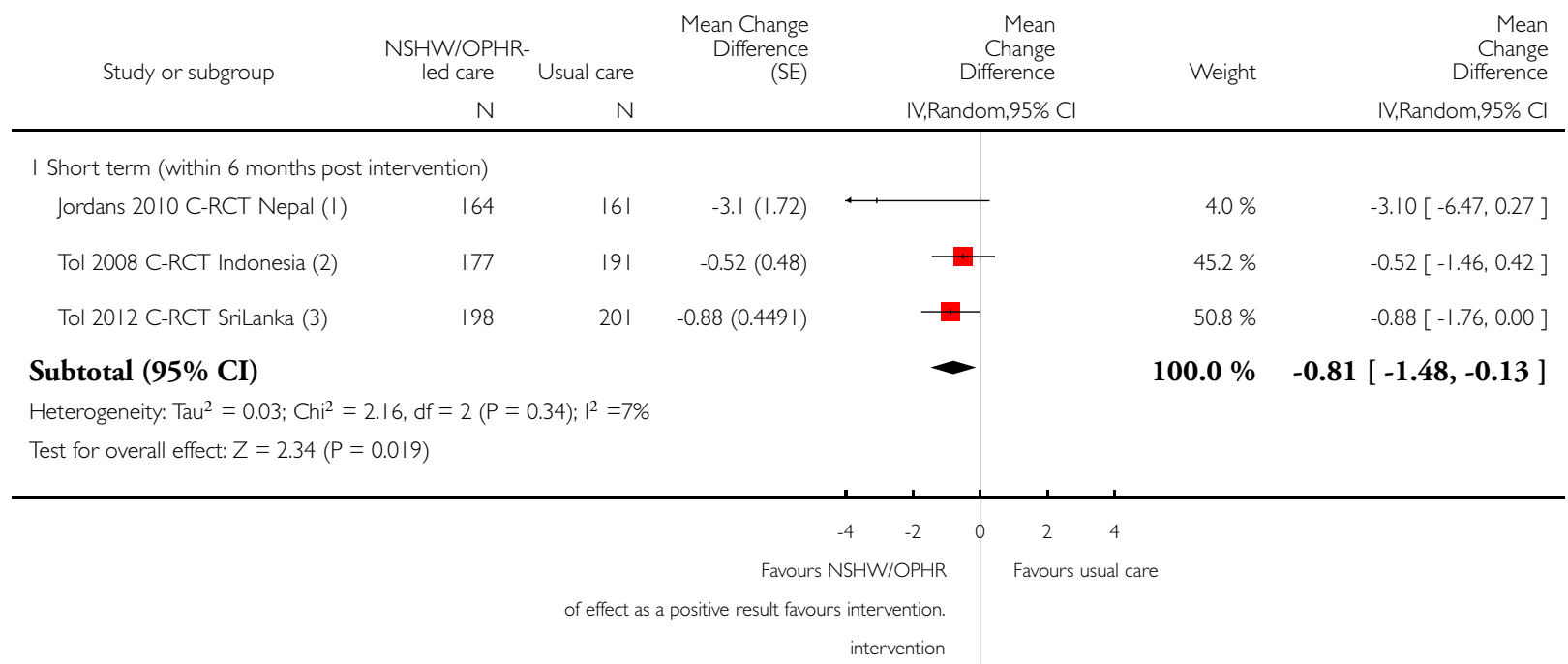

(I) LHW-led classroom based intervention (CBI); CFI (children's function impairment); I month post intervention: MchangeDiff (change scores) adjusted for clustering and SE, reversed direction

(2) LHW-led CBI;Functional Impairment scale (FIS); 6 months post interv: Mchange diff adjusted for clustering and SE.

(3) LHW-led CBI; Functional Impairment Scale (FIS); 3 months post interv; cluster-adjusted mean change diff and SDs. ICC $=0.003$, reversed direction of effect as a positive result favours 
Analysis 8.II. Comparison 8 NSHWs/OPHRs versus usual care in conducting interventions for children with post-traumatic stress and depression (RCTs), Outcome I I Functional impairment - classroom-based LHW intervention - boys/girls.

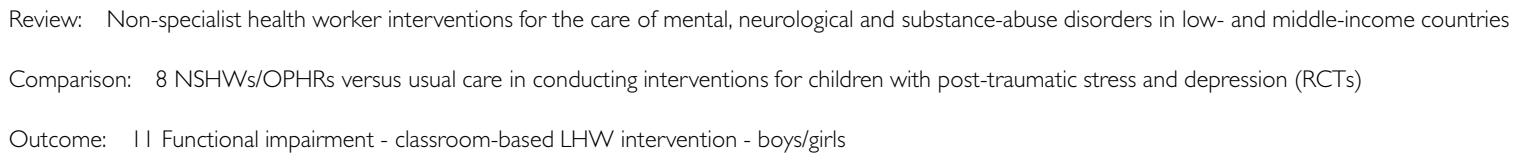

I Short term (boys) (within 6 months post intervention) Tol 2012 C-RCT SriLanka (I)

122 123 $-1.19(0.5312)$
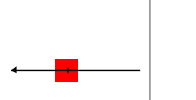

Subtotal (95\% CI)

Heterogeneity: not applicable

Test for overall effect: $Z=2.24(P=0.025)$

2 Short term (girls) (within 6 months post intervention)

$\begin{array}{llll}\text { Tol } 2012 \text { C-RCT SriLanka (2) } & 76 & 78 & -0.4(0.7823)\end{array}$

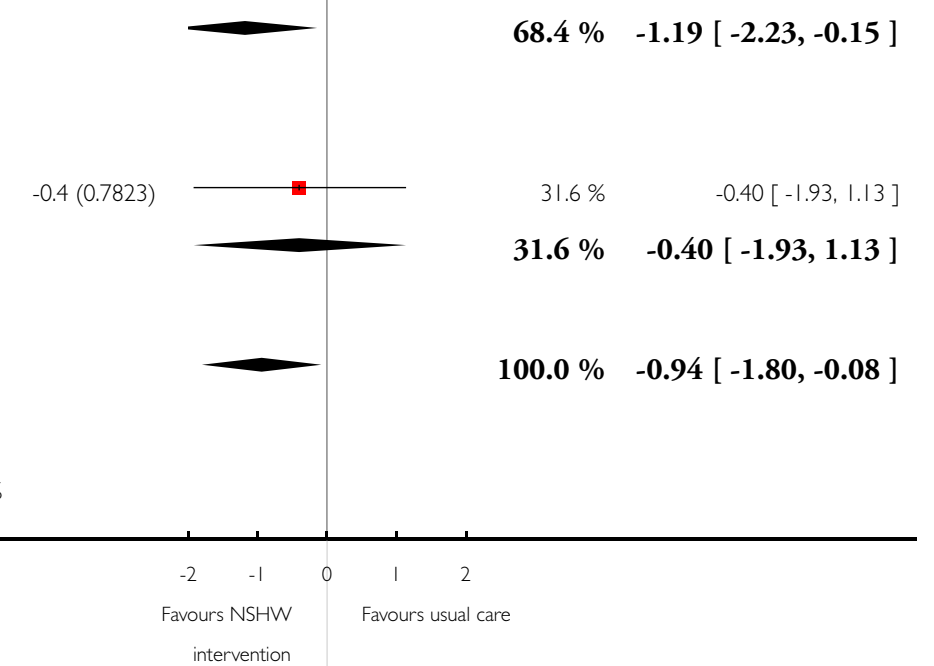

$68.4 \%$

$-1.19[-2.23,-0.15]$

ubtotal $(95 \%$ CI $)$

Heterogeneity: not applicable

Test for overall effect: $Z=0.5 \mathrm{I}(P=0.6 \mathrm{I})$

Total (95\% CI)

Heterogeneity: $\mathrm{Tau}^{2}=0.0 ; \mathrm{Chi}^{2}=0.70, \mathrm{df}=\mathrm{I}(\mathrm{P}=0.40) ; \mathrm{I}^{2}=0.0 \%$

Test for overall effect: $Z=2.14(P=0.032)$

Test for subgroup differences: $\mathrm{Chi}^{2}=0.70, \mathrm{df}=\mathrm{I}(\mathrm{P}=0.40), \mathrm{I}^{2}=0.0 \%$

(I) LHW-led CBI; Functional impairment scale (FIS); 3 months post interv; cluster-adjusted mean change diff and SDs. ICC=0.003, reversed direction of effect as a positive result favours

(2) LHW-led CBI; FIS; 3 months post interv; cluster-adjusted mean change diffs and SDs. ICC $=0.003$, reversed direction of effect as a positive result favours intervention

\section{ADDITIONAL TABLES}

Table 1. Definitions

Adult

Patients who were $\geq 18$ years old. However, if some studies had an age range from, for example, 16 years upwards and the majority of participants are over 18 years, we included these study participants as adults

Children and adolescents

Children (from birth to 18 years) were considered as a separate group of participants as they have 1 . different patterns of psychopathology/mental disorders; 2 . different help-seeking be- 
Table 1. Definitions (Continued)

haviours that would, therefore, require different interventions, in different settings (e.g. schools) and a different approach to careworker interventions (such as teacher-led interventions)

Mental, neurological and substance-abuse (MNS) disorders

This review included MNS disorders as defined by any criteria within included papers. For the purpose of subgroup analysis, we subcategorised these disorders using the International Classification of Diseases (ICD)-10 criteria for mental and behavioural disorders and epilepsy in adults (the related ICD-10 code is listed in brackets). These categories are most likely to be used in LMIC mental health service delivery, and are based on Patel's classification (Patel 2003c), and the World Health Organization (WHO)

MNS disorder categorisation (WHO 2008)

1. Common mental disorders

Mild to moderate mood (affective) disorders (F32-38)

Neurotic, stress-related and somatoform disorders (F40-49)

Behavioural syndromes associated with physiological disturbances and physical factors (F50-59)

2. Severe mental disorders

Schizophrenia, schizotypal and delusional disorders (F20-F29)

Bipolar affective disorder (F31)

Severe depressive episode with/without psychosis (F32.2, F32.3)

3. Neuropsychiatric disorders

Organic, including symptomatic, mental disorders (includes dementia) (F1-9)

Mental retardation (F70-79)

Epilepsy (G40)

4. Disorders caused by substance abuse

Mental and behavioural disorders due to psychoactive substance use (F10-19)

5. Mental disorders specifically related to childhood/development Conduct disorders

Developmental disorders

Eating disorders

Pervasive developmental disorders

The diagnosis could be made in clinical practice or in the context of the trial

First level care, primary care and community

First level of contact with formal health services were community-based interventions or primary care interventions (or both) , on their own or attached to hospital settings, provided they had no specialist input apart from supervision (modified from Wiley-Exley 2007). This would include individuals with mental illness living in the community and programmes in outpatient clinics or primary care practices. This would not include programmes in hospitals unless the programmes in the hospitals were providing care to outpatients (i.e. generalists in outpatient departments) Community: as mentioned above detection of mental disorders in all age groups were often done outside the health facility, for

Non-specialist health worker interventions for the care of mental, neurological and substance-abuse disorders in low- and middle- 
Table 1. Definitions (Continued)

example through school, training and other community settings. Therefore, we considered interventions outside the health sector

Low- and middle-income country (LMIC)

Non-specialist health workers (NSHWs)
Any country that has ever been an LMIC, as defined by the World Bank lists of LMICs

Health workers who were not specialised in MNS disorders or have not received in-depth professional specialist training in this clinical area. These included doctors, nurses, auxiliary nurses, lay health workers, as well as allied health personnel such as social workers, occupational therapists. This category did not include professional specialist health workers such as psychiatrists, neurologists, psychiatric nurses or mental health social workers. For inclusion, NSHWs received some training in MNS disorders (in either the control or the intervention group), but this would not constitute a professional category. The authors made a judgement of what constitutes 'some training'. Examples of 'some training' may be an undergraduate module or a short course in mental health

Other professionals with health roles (OPHRs)

People who were involved as community-level workers but were not within the health sector, as many people, particularly adolescents and young adults, have low contact with health workers. This category included teachers/trainers/support workers from schools and colleges, and other volunteers or workers within community-based networks or non-governmental organisations. These OPHRs have an important role particularly in the promotion of mental health and detection of mental disorders (Patel 2007c; Patel 2008a; WHO 2003a)

We excluded studies that looked at informal care provided by family members or extended members only to members of his or her own family (i.e. who were unavailable to other members of the community) from this review. As previously highlighted in Lewin;s Cochrane review, "these interventions are qualitatively different from other LHW [lay health worker] interventions included in this review given that parents or spouses have an established close relationship with those receiving care which could affect the process and effects of the intervention" (Lewin 2010).

\section{Clinical interventions}

1. Detection (recognition and diagnosis) of illness, including screening

2. Acute interventions: drug treatment, non-drug treatment/care (such as specific psychological therapies, or interventions with psychosocial components like counselling, psychoeducation, coping skills, etc.), referral

3. Follow-up, rehabilitation

\section{Service interventions}

These include change in staffing, or change in mechanism of mental health service delivery (e.g. extension of mental health services through camps and such other outreach services, mobile vans, etc. 
Table 1. Definitions (Continued)

Table 2. Risk of bias economic studies - CHEC list criteria

\begin{tabular}{|c|c|}
\hline Study & Risk of bias issues \\
\hline Araya 2003 RCT Chile & $\begin{array}{l}\text { - time horizon }<1 \text { year } \\
\text { - a societal perspective would have been more appropriate } \\
\text { - not all relevant costs reported } \\
\text { - not all relevant outcomes included (only ambulatory, not hospital) } \\
\text { - no discounting }\end{array}$ \\
\hline Jordans 2010 C-RCT Nepal & $\begin{array}{l}\text { - no discounting } \\
\text { - no sensitivity analysis } \\
\text { - not all important variables listed } \\
\text { - no discussion of ethical/distributional issues }\end{array}$ \\
\hline Zambori 2002 CBA Hungary & $\begin{array}{l}\text { - the competing alternatives were not described } \\
\text { - time horizon at } 1 \text { year was not appropriate (needs to be longer) } \\
\text { - not all relevant outcomes assessed (e.g. effect of treatment on severity, number of healthcare visits } \\
\text { to psychiatrist) } \\
\text { - outcomes not measured appropriately (self reporting meant low response; standard prices used may } \\
\text { not reflect actual prices) } \\
\text { - outcomes not valued (only the short-term outcome) } \\
\text { - no sensitivity analysis } \\
\text { - conclusions do not all follow from results }\end{array}$ \\
\hline
\end{tabular}

Table 3. Outcomes of studies not assigned to meta-analyses

\begin{tabular}{|c|c|c|c|c|c|}
\hline $\begin{array}{l}\text { Study, and } \\
\text { outcomes measured } \\
\text { and tools }\end{array}$ & $\begin{array}{l}\text { Intervention data } \\
\text { [no. of participants] }\end{array}$ & Control data & $\begin{array}{l}\text { Measure of effect } \\
(95 \% \mathrm{CI})\end{array}$ & P value & Authors' conclusions \\
\hline $\begin{array}{l}\text { Brown } 2009 \text { CBA } \\
\text { Rwanda(depres- } \\
\text { sion in youth) }\end{array}$ & $\begin{array}{l}\text { Mentoring pro- } \\
\text { gramme by LHW }\end{array}$ & Usual care & - & - & - \\
\hline $\begin{array}{l}\text { Sever- } \\
\text { ity of depression at } \\
2 \text { years (mean) mea- } \\
\text { sured using CID-S }\end{array}$ & $\begin{array}{l}\text { Mean } \\
\text { [no. of participants] } \\
23.27 \\
{[347]}\end{array}$ & $\begin{array}{l}\text { Mean } \\
\text { [no. of participants] } \\
23.28 \\
{[345]}\end{array}$ & - & 0.99 & $\begin{array}{l}\text { Reduction in intervention } \\
\text { group but not in control group } \\
\text { (at baseline higher score in in- } \\
\text { tervention group). However, the } \\
\text { score indicates continuing levels } \\
\text { of depression in both groups }\end{array}$ \\
\hline $\begin{array}{l}\text { Levels of marginali- } \\
\text { sation at } \\
2 \text { years (mean) mea- }\end{array}$ & 3.35 & 3.13 & - & - & $\begin{array}{l}\text { Improved scores in intervention } \\
\text { group, which are no different to } \\
\text { control group }\end{array}$ \\
\hline
\end{tabular}

Non-specialist health worker interventions for the care of mental, neurological and substance-abuse disorders in low- and middle- 
Table 3. Outcomes of studies not assigned to meta-analyses (Continued)

sured using a nonvalidated marginali-

sation scale

Levels of grief at $2 \quad 3.42$ years (mean) measured using a nonvalidated 7 point grief scale
3.38

Baseline lower levels of grief in the control group. No change at the end of the intervention though grief increased in control group and remained stable in the intervention group

\begin{tabular}{l|l|l|}
\hline $\begin{array}{l}\text { Li } 1989 \text { RCT China } \\
\text { (epilepsy - adults }\end{array}$ & Village doctors & Psychiatrists \\
and children) &
\end{tabular}

Ef-

fective epilepsy control with phenobarbital after 3 months

No. seizures/month [no. of participants] 12 [20]

Total number of ad- No. events verse events after 3 months [no. of participants] 19 [20]
No. seizures/month -

[no. of participants] 11

[20]

No. events

[no. of participants]

39

[20]

\begin{tabular}{|c|c|c|c|c|c|}
\hline $\begin{array}{l}\text { Paran- } \\
\text { thaman } 2010 \mathrm{CBAMa} \\
\text { (people } \\
\text { with schizophrenia } \\
\text { and their carers) }\end{array}$ & $\begin{array}{l}\text { Medical assistants/ } \\
\text { nurses }\end{array}$ & Usual care & MD (95\% CI) & Pvalue & Authors' conclusions \\
\hline $\begin{array}{l}\text { Carer burden (activ- } \\
\text { ities in daily living) } \\
\text { (mean) at } 6 \text { months. } \\
\text { Measured using the } \\
\text { Family Burden In- } \\
\text { terview schedule }\end{array}$ & $\begin{array}{l}\text { Mean (SD) } \\
\text { [no. of participants] } \\
9.41(3.99) \\
{[54]}\end{array}$ & $\begin{array}{l}\text { Mean }(\mathrm{SD}) \\
\text { [no. of participants] } \\
8.93(4.47)\end{array}$ & $0.48(-1.11$ to 2.07$)$ & 0.55 & $\begin{array}{l}\text { Mostly there are similar scores } \\
\text { between control and interven- } \\
\text { tion groups }\end{array}$ \\
\hline
\end{tabular}

Carer assistance in

$0.83(-0.94$ to 2.60$)$

daily living severity -

ADL at 6 months measured using the Family Burden Interview Schedule

\begin{tabular}{|l|l|l|l}
\hline Re-admission rates & $\begin{array}{l}\text { No. (events) } \\
\text { [no. of participants] }\end{array}$ & $\begin{array}{l}\text { No. (events) } \\
\text { [no. of participants] }\end{array}$ \\
3 & 5 \\
{$[54]$} & {$[55]$} & \\
\hline
\end{tabular}


Table 3. Outcomes of studies not assigned to meta-analyses (Continued)

\begin{tabular}{|c|c|c|c|c|c|}
\hline $\begin{array}{l}\text { Defaulting from fol- } \\
\text { low-up }\end{array}$ & $\begin{array}{l}\text { No. (events) } \\
\text { [no. of participants] } \\
6 \\
{[54]}\end{array}$ & $\begin{array}{l}\text { No. (events) } \\
\text { [no. of participants] } \\
14 \\
{[55]}\end{array}$ & - & 0.03 & $\begin{array}{l}\text { important improvement in fol- } \\
\text { low-up rate for intervention } \\
\text { group }\end{array}$ \\
\hline $\begin{array}{l}\text { Shin } 2009 \\
\text { RCT Vietnam(chil- } \\
\text { dren with intellec- } \\
\text { tual disabilities) }\end{array}$ & $\begin{array}{l}\text { Teacher- } \\
\text { led portage pro- } \\
\text { gramme (OPHRs) }\end{array}$ & Usual care & MD (95\% CI) & P value & Authors' conclusions \\
\hline
\end{tabular}

\begin{tabular}{|c|c|c|c|c|c|}
\hline $\begin{array}{l}\text { Functional impair- } \\
\text { ment (motor skills) } \\
\text { at } 6 \text { months (sim- } \\
\text { ilar at } 12 \text { months) } \\
\text { measured using the } \\
\text { Vineland Adaptive } \\
\text { Behaviour Scales }\end{array}$ & $\begin{array}{l}\text { Mean }(\mathrm{SD}) \\
\text { [no. of participants] } \\
47.6(16.8) \\
{[16]}\end{array}$ & $\begin{array}{l}\text { Mean }(\mathrm{SD}) \\
\text { [no. of participants] } \\
49(15.4) \\
{[14]}\end{array}$ & $\begin{array}{l}-1.40(-12.93 \text { to } 10 . \\
13)\end{array}$ & 0.81 & $\begin{array}{l}\text { No significant difference for any } \\
\text { mental outcomes but some im- } \\
\text { provement for motor and per- } \\
\text { sonal care outcomes if looked at } \\
\text { time x effect interaction) }\end{array}$ \\
\hline $\begin{array}{l}\text { Functional impair- } \\
\text { ment (social skills) } \\
\text { at } 6 \text { months (sim- } \\
\text { ilar at } 12 \text { months) } \\
\text { measured using the } \\
\text { Vineland Adaptive } \\
\text { Behaviour Scales }\end{array}$ & $\begin{array}{l}47.1(15.5) \\
{[16]}\end{array}$ & $\begin{array}{l}46.3(18.3) \\
{[14]}\end{array}$ & $\begin{array}{l}0.80(-11.51 \text { to } 13 . \\
11)\end{array}$ & 0.93 & - \\
\hline
\end{tabular}

\begin{tabular}{|c|c|c|c|c|c|}
\hline $\begin{array}{l}\text { Be- } \\
\text { havioural changes at } \\
6 \text { months (similar } \\
\text { at } 12 \text { months) mea- } \\
\text { sured } \\
\text { using the Vineland } \\
\text { Adaptive Behaviour } \\
\text { Scales }\end{array}$ & $\begin{array}{l}55.6(10.5) \\
{[16]}\end{array}$ & $\begin{array}{l}55.7(10) \\
{[14]}\end{array}$ & $\begin{array}{l}-0.10 \quad(-7.44 \text { to } 7 . \\
24)\end{array}$ & 0.98 & - \\
\hline $\begin{array}{l}\text { Sutcliffe } 2009 \mathrm{RCT} \\
\text { Thailand } \\
\text { (people with drug } \\
\text { abuse disorder) }\end{array}$ & $\begin{array}{l}\text { Peer educa- } \\
\text { tor-led psychoedu- } \\
\text { cation (LHWs) }\end{array}$ & $\begin{array}{l}\text { Usual care (life } \\
\text { skills training) }\end{array}$ & RR/MD (95\% CI) & P value & Authors' conclusions \\
\hline $\begin{array}{l}\text { Metham- } \\
\text { phetamine use at } 6 \\
\text { months (similar re- } \\
\text { sults at } 3,9 \text { and } 12 \\
\text { months) }\end{array}$ & $\begin{array}{l}\text { No. } \\
\text { [no. of participants] } \\
272 \\
{[442]}\end{array}$ & $\begin{array}{l}\text { No. } \\
\text { [no. of participants] } \\
267 \\
{[440]}\end{array}$ & $\begin{array}{l}\text { RR } 1.01(0.91 \text { to } 1 . \\
13)\end{array}$ & 0.79 & $\begin{array}{l}\text { Randomised peer education, so- } \\
\text { cial network intervention and } \\
\text { control (social skills training) are } \\
\text { both associated with reductions } \\
\text { in methamphetamine use and } \\
\text { increases in condom use over } \\
12 \text { months among a sample of } \\
\text { young Thai people }\end{array}$ \\
\hline
\end{tabular}




\begin{tabular}{|c|c|c|c|c|c|}
\hline $\begin{array}{l}\text { Recovery of depres- } \\
\text { sive symptoms at } 12 \\
\text { months (index pa- } \\
\text { tient) measured us- } \\
\text { ing CES-D score }\end{array}$ & $\begin{array}{l}\text { Mean }(\mathrm{SD}) \\
\text { [no. of participants] } \\
15.7(9.7) \\
{[209]}\end{array}$ & $\begin{array}{l}\text { Mean }(\mathrm{SD}) \\
\text { [no. of participants] } \\
17.9(9.3) \\
{[206]}\end{array}$ & $\begin{array}{l}\text { MD }-2.20(-4.03 \text { to } \\
-0.37)\end{array}$ & - & $\begin{array}{l}\text { The effect was strongly observed } \\
\text { amount intervention index par- } \\
\text { ticipants compared with both } \\
\text { control and network partici- } \\
\text { pants }\end{array}$ \\
\hline $\begin{array}{l}\text { Recovery of depres- } \\
\text { sive symptoms at } 12 \\
\text { months (index and } \\
\text { net- } \\
\text { work patient com- } \\
\text { bined) measured us- } \\
\text { ing CES-D score }\end{array}$ & $\begin{array}{l}\text { [no. of participants] } \\
\text { [495] }\end{array}$ & $\begin{array}{l}\text { [no. of participants] } \\
\text { [488] }\end{array}$ & $\begin{array}{l}\mathrm{MD}-1.05[-3.20 \text { to } \\
1.11]\end{array}$ & - & $\begin{array}{l}\text { Contrary to expectation, mea } \\
\text { and in CES-D score change did } \\
\text { not substantially differ between } \\
\text { intervention network partici- } \\
\text { pants and control network par- } \\
\text { ticipants. Thus, there is no evi- } \\
\text { dence that the differential inter- } \\
\text { vention effect on depression dif- } \\
\text { fuses to network members }\end{array}$ \\
\hline $\begin{array}{l}\text { Prevalence } \\
\text { of depression at } 12 \\
\text { months (index pa- } \\
\text { tient) measured us- } \\
\text { ing CES-D score }\end{array}$ & $\begin{array}{l}\text { Events }(\text { No.) } \\
\text { [no. of participants] } \\
57 \\
{[209]}\end{array}$ & $\begin{array}{l}\text { Events (No.) } \\
\text { [no. of participants] } \\
70 \\
{[206]}\end{array}$ & $\begin{array}{l}\text { RR } 0.80(0.60 \text { to } 1 . \\
07)\end{array}$ & - & - \\
\hline $\begin{array}{l}\text { Preva- } \\
\text { lence of depression } \\
\text { at } 12 \text { months (index } \\
\text { and network patient } \\
\text { combined) mea- } \\
\text { sured using CES-D } \\
\text { score }\end{array}$ & $\begin{array}{l}\text { [no. of participants] } \\
\text { [495] }\end{array}$ & $\begin{array}{l}\text { [no. of participants] } \\
\text { [488] }\end{array}$ & $\begin{array}{l}\text { RR } 0.88 \text { ( } 0.73 \text { to } 1 . \\
06)\end{array}$ & - & - \\
\hline $\begin{array}{l}\text { Hirani } 2010 \text { CRCT } \\
\text { Pakistan(adults } \\
\text { with depression, } \\
\text { economic } \\
\text { skills building in- } \\
\text { tervention arm) }\end{array}$ & $\begin{array}{l}\text { NSHW- } \\
\text { led economics skill } \\
\text { building } \\
\mathrm{n}=9\end{array}$ & $\begin{array}{l}\text { Usual care } \\
\mathrm{n}=\mathbf{8}\end{array}$ & SMD $(95 \%$ CI $)$ & - & $\begin{array}{l}\text { Comment: these are presented } \\
\text { as SMDs (calculated in } \\
\text { RevMan, to compare with other } \\
\text { SMDs in comparison } 1.6 \text { and } 1 . \\
7 \text { ) }\end{array}$ \\
\hline $\begin{array}{l}\text { Severity of depres- } \\
\text { sive symptoms mea- } \\
\text { sured using Becks } \\
\text { Depression Inven- } \\
\text { tory II }\end{array}$ & $\begin{array}{l}\text { Mean (SD) } \\
20.1(11.3)\end{array}$ & $\begin{array}{l}\text { Mean (SD) } \\
27.63(9.1)\end{array}$ & $\begin{array}{l}\text { SMD }-0.69(-1.73 \\
\text { to } 0.35)\end{array}$ & - & $\begin{array}{l}\text { This study documents im- } \\
\text { proved self efficacy and em- } \\
\text { ployment for women enrolled } \\
\text { in economic skill-building com- } \\
\text { pared with general counselling } \\
\text { and to control }\end{array}$ \\
\hline $\begin{array}{l}\text { Functional impair- } \\
\text { ment measured us- } \\
\text { ing the General Self- } \\
\text { Efficacy scale }\end{array}$ & $28.7(6.2)$ & $21.63(3.8)$ & $\begin{array}{l}\text { SMD }-1.29(-2.41 \\
\text { to }-0.16)\end{array}$ & - & - \\
\hline
\end{tabular}

Non-specialist health worker interventions for the care of mental, neurological and substance-abuse disorders in low- and middle- 
CES-D: Center for Epidemiological Studies Depression scale; CID-S: Composite International Diagnostic-Screener; CI: confidence interval; LHW: lay health workers; MD: mean difference; No.: number; OPHR: other professionals with health roles; RR: risk ratio; SD: standard deviation; SMD: standardised mean difference.

Table 4. SoF 1: NSHW-led psychological interventions compared with usual care in treating common mental disorders in adults in low- and middle-income countries (RCTs)

What are the effects of NSHW-led psychological interventions for treating common mental disorders in adults in low- and middle- income countries? (additional outcomes to comparison 1)

Patient or population: Adults with common mental disorders (depression or anxiety, or both)

Settings: Low- and middle-income countries (China, Jamaica, Pakistan, Taiwan, Uganda)

Intervention: NSHWs conducting psychological interventions

Comparison: Usual care

\begin{tabular}{|c|c|c|c|c|c|c|}
\hline \multirow[t]{3}{*}{ Outcomes } & \multicolumn{2}{|c|}{$\begin{array}{l}\text { Illustrative comparative risks* } \\
(95 \% \mathrm{CI})\end{array}$} & \multirow[t]{3}{*}{$\begin{array}{l}\text { Effect estimate } \\
(95 \% \mathrm{CI})\end{array}$} & \multirow{3}{*}{$\begin{array}{l}\text { No of Partici- } \\
\text { pants } \\
\text { (studies) }\end{array}$} & \multirow{3}{*}{$\begin{array}{l}\text { Quality of the } \\
\text { evidence } \\
\text { (GRADE) }\end{array}$} & \multirow[t]{3}{*}{ Comments } \\
\hline & Assumed risk & $\begin{array}{l}\text { Corresponding } \\
\text { risk }\end{array}$ & & & & \\
\hline & Usual care & NSHWs & & & & \\
\hline $\begin{array}{l}\text { Severity } \\
\text { of CMD symp- } \\
\text { toms - all inter- } \\
\text { ventions } \\
\text { short term (0-6 } \\
\text { months) } \\
\text { measured using } \\
\text { various depres- } \\
\text { sion rating scales } \\
1\end{array}$ & - & $\begin{array}{l}\text { The mean sever- } \\
\text { ity } \\
\text { of CMD symp- } \\
\text { toms - NSHW } \\
\text { interventions } \\
\text { short term was } \\
\text { 0. } \\
75 \text { standard de- } \\
\text { viations lower } \\
(1.29 \text { to } 0.21 \\
\text { lower) }\end{array}$ & $\begin{array}{l}\text { SMD }-0.75(-1 \\
29 \text { to }-0.21)\end{array}$ & $\begin{array}{l}1470 \\
\text { (6 studies) }\end{array}$ & $\begin{array}{l}\oplus \bigcirc \bigcirc \bigcirc \\
\text { very low }\end{array}$ & - \\
\hline $\begin{array}{l}\text { Severity } \\
\text { of CMD symp- } \\
\text { toms - all inter- } \\
\text { ventions } \\
\text { medium term } \\
\text { (12 months) } \\
\text { mea- } \\
\text { sured using vari- } \\
\text { ous CMD rating } \\
\text { scales } 5\end{array}$ & - & $\begin{array}{l}\text { The mean sever- } \\
\text { ity } \\
\text { of CMD symp- } \\
\text { toms - NSHW } \\
\text { interventions } \\
\text { medium term } \\
\text { was } \\
\mathbf{0 .} \\
\mathbf{4 7} \text { standard de- } \\
\text { viations lower } \\
(0.60 \text { to } 0.34 \\
\text { lower) }\end{array}$ & $\begin{array}{l}\text { SMD }-0.47(-0 . \\
60 \text { to }-0.34)\end{array}$ & $\begin{array}{l}923 \\
\text { (2 studies) }\end{array}$ & $\begin{array}{l}\oplus \oplus \oplus \bigcirc \\
\text { moderate }\end{array}$ & - \\
\hline
\end{tabular}


Table 4. SoF 1: NSHW-led psychological interventions compared with usual care in treating common mental disorders in adults in low- and middle-income countries (RCTs) (Continued)

\begin{tabular}{|c|c|c|c|c|c|}
\hline $\begin{array}{l}\text { Functional im- } \\
\text { pairment/dis- } \\
\text { ability in adults } \\
\text { with } \\
\text { CMD - NSHW } \\
\text { interventions } \\
\text { short term (2-6 } \\
\text { months) } \\
\text { measured us- } \\
\text { ing various func- } \\
\text { tional impair- } \\
\text { ment scales }\end{array}$ & $\begin{array}{l}\text { The mean func- } \\
\text { tional impair- } \\
\text { ment of adults } \\
\text { with } \\
\text { CMD - NSHW } \\
\text { interventions } \\
\text { short term was } \\
\text { 0. } \\
\mathbf{3 3} \text { standard de- } \\
\text { viations lower } \\
(0.80 \text { lower to } 0 . \\
13 \text { higher })\end{array}$ & $\begin{array}{l}\text { SMD }-0.33(-0 . \\
80 \text { to } 0.13)\end{array}$ & $\begin{array}{l}1243 \\
\text { (4 studies) }\end{array}$ & $\begin{array}{l}\oplus \bigcirc \bigcirc \bigcirc \\
\text { very low } 9,10,11\end{array}$ & . \\
\hline $\begin{array}{l}\text { Functional im- } \\
\text { pairment/ } \\
\text { disability in de- } \\
\text { pression/ } \\
\text { CMD (adults) } \\
\text { - NSHW inter- } \\
\text { ventions } \\
\text { medium term } \\
\text { (2-6 months) } \\
\text { measured using } \\
\text { the Global As- } \\
\text { sess- } \\
\text { ment of Func- } \\
\text { tioning scale }\end{array}$ & $\begin{array}{l}\text { The mean func- } \\
\text { tional impair- } \\
\text { ment of adults } \\
\text { with } \\
\text { CMD - NSHW } \\
\text { interventions } \\
\text { medium term } \\
\text { was } \\
\mathbf{0 .} \\
\mathbf{5 6} \text { standard de- } \\
\text { viations lower } \\
(0.70 \text { to } 0.42 \\
\text { lower) }\end{array}$ & $\begin{array}{l}\text { SMD }-0.56(-0 . \\
70 \text { to }-0.42)\end{array}$ & $\begin{array}{l}798 \\
\text { (1 study) }\end{array}$ & $\begin{array}{l}\oplus \oplus \oplus \bigcirc \\
\text { moderate }^{12}\end{array}$ & . \\
\hline
\end{tabular}

*The basis for the assumed risk is the mean control group risk across studies for pooled results and the control group risk for single studies. The corresponding risk (and its $95 \%$ confidence interval) is based on the assumed risk in the comparison group and the relative effect of the intervention (and its 95\% CI).

CMD: common mental disorders; CI: confidence interval; NSHW: non-specialist health worker; SMD: standardised mean difference.

GRADE Working Group grades of evidence

High quality: Further research is very unlikely to change our confidence in the estimate of effect.

Moderate quality: Further research is likely to have an important impact on our confidence in the estimate of effect and may change the estimate.

Low quality: Further research is very likely to have an important impact on our confidence in the estimate of effect and is likely to change the estimate.

Very low quality: We are very uncertain about the estimate.

${ }^{1}$ Chen 2000 RCT Taiwan, Hirani 2010 CRCT Pakistan and Tiwari 2010 RCT China used the Beck's Depression Inventory; Rahman 2008 CRCT Pakistan used the Hamilton Depression Rating Scale; Ali 2003 RCT Pakistan used the AKUADS; Bolton 2003 C-RCT Uganda used the Hopkins Symptom Checklist (HSCL).

${ }^{2}$ Serious study limitations: Two of the six trials in this analysis were judged at high risk of bias and one was unclear about possible risk of bias. Chen 2000 RCT Taiwan had unclear sequence generation and allocation concealment, all were self reported outcomes, there was possible contamination and there was a high dropout rate after randomisation, with no analysis of dropout versus non-dropout differences; Hirani 2010 CRCT Pakistan was unclear regarding allocation concealment, there was no blinding of outcome assessment (self reported outcomes), it was unclear if baseline measures and characteristics were similar in both groups; and the report provided

Non-specialist health worker interventions for the care of mental, neurological and substance-abuse disorders in low- and middle- 
no information on dropouts. Bolton 2003 C-RCT Uganda was not clear about allocation concealment and quasi randomisation of individuals within clusters (though randomisation of clusters) may have introduced bias. The three trials contributed $45 \%$ of the weight in the pooled analysis. Downgraded by 1.

${ }^{3}$ Serious inconsistency: $\mathrm{I}^{2}$ statistic $=94 \%$. However, the inconsistency related to the magnitude of benefit favouring NSHW interventions rather than in the direction of effect. Downgraded by 1 .

${ }^{4}$ Serious imprecision: The $95 \%$ CI for the pooled estimates indicates appreciable benefit and non-appreciable benefit for collaborative care (appreciable SMD $=\geq 0.5$; non-appreciable benefit $\leq 0.2$ ). Downgraded by 1 .

${ }^{5}$ Baker-H 2005 CRCT Jamaica used the CED-S; Rahman 2008 CRCT Pakistan used the Hamilton Depression Rating Scale.

${ }^{6}$ No serious study limitations: The CES-D used in Baker-H 2005 CRCT Jamaica is not validated in the Jamaican population and is not a measure of clinical depression but just identifies depressive symptoms. Most women were not likely to have been depressed. Also in this study, there were unadjusted differences in baseline characteristics. However, this study contributed only $14 \%$ weight to the pooled results and removal of this study did not alter the direction or precision of the effect estimate. Not downgraded.

${ }^{7}$ Serious indirectness: The two trials included were the only two of the six trials that compared this intervention that had data over the medium term, and only one used a validated outcome measure. Downgraded by 1 .

${ }^{8}$ Bolton 2003 C-RCT Uganda used a sex-specific Functional Impairment Questionnaire; Rahman 2008 CRCT Pakistan used the Global Assessment of Functioning (GAF) scale, Hirani 2010 CRCT Pakistan used the General Self-efficacy Scale; Tiwari 2010 RCT China used the Short Form- 12 (SF-12) (mental and physical components).

${ }^{9}$ Serious study limitations: Two of the four studies were at risk of bias. Bolton 2003 C-RCT Uganda was not clear about allocation concealment and quasi-randomisation of individuals within clusters (though randomisation of clusters) may have introduced bias. Hirani 2010 CRCT Pakistan was unclear regarding allocation concealment, there was no blinding of outcome assessment (self reported outcomes), it was unclear if baseline measures and characteristics were similar in both groups; and the report provided no information on dropouts. Downgraded by 1 .

${ }^{10}$ Very serious inconsistency: $\mathrm{I}^{2}$ statistic $=90 \%$. The inconsistency related to the direction of effect between interventions and was unexplained. Downgraded by 2 .

${ }^{11}$ Serious imprecision: the $95 \%$ CI of the pooled estimate showed appreciable benefit for interventions (appreciable SMD $=0.5$ ) and non-appreciable benefit for control. Downgraded by 1 .

12 Serious imprecision: the $95 \%$ CI of the pooled estimate shows non-appreciable benefit for psychological interventions and usual care (appreciable SMD = 0.5). However, the data for this outcome were from only one trial. Downgraded by 1.

Table 5. SoF: NSHW-led interventions compared with usual care in treating common mental disorders in adults in low- and middle-income countries (CBAs)

What are the effects of NSHWs conducting single interventions compared with usual care in treating common mental disorders for mental health care in low- and middle-income countries? (additional CBA outcomes to comparison 1)

Patient or population: Adults with CMDs (such as depression and anxiety)

Settings: Low- and middle-income countries (Indonesia, Rwanda)

Intervention: NSHWs conducting single interventions

Comparison: Usual care

\begin{tabular}{|c|c|c|c|c|c|c|}
\hline \multirow[t]{3}{*}{ Outcomes } & \multicolumn{2}{|c|}{$\begin{array}{l}\text { Illustrative comparative risks* } \\
(95 \% \mathrm{CI})\end{array}$} & \multirow[t]{3}{*}{$\begin{array}{l}\text { Relative effect } \\
(95 \% \text { CI })\end{array}$} & \multirow{3}{*}{$\begin{array}{l}\text { No of Partici- } \\
\text { pants } \\
\text { (studies) }\end{array}$} & \multirow{3}{*}{$\begin{array}{l}\text { Quality of the } \\
\text { evidence } \\
\text { (GRADE) }\end{array}$} & \multirow[t]{3}{*}{ Comments } \\
\hline & Assumed risk & $\begin{array}{l}\text { Corresponding } \\
\text { risk }\end{array}$ & & & & \\
\hline & Usual care & NSHWs & & & & \\
\hline
\end{tabular}

Non-specialist health worker interventions for the care of mental, neurological and substance-abuse disorders in low- and middle- 
Table 5. SoF: NSHW-led interventions compared with usual care in treating common mental disorders in adults in low- and middle-income countries (CBAs) (Continued)

\begin{tabular}{|c|c|c|c|c|c|c|}
\hline $\begin{array}{l}\text { Severity of com- } \\
\text { mon men- } \\
\text { tal disorders - } \\
\text { short } \\
\text { term (within } 6 \\
\text { months) } \\
\text { measured us- } \\
\text { ing CMD rating } \\
\text { scales }{ }^{1}\end{array}$ & - & $\begin{array}{l}\text { The mean sever- } \\
\text { ity } \\
\text { of CMDs - short } \\
\text { term (within } 6 \\
\text { months post in- } \\
\text { tervention) } \\
\text { in the interven- } \\
\text { tion groups was } \\
\mathbf{0 .} \\
\mathbf{0 8} \text { standard de- } \\
\text { viations lower } \\
(0.25 \text { lower to } 0 \text {. } \\
09 \text { higher) }\end{array}$ & $\begin{array}{l}\text { SMD }-0.08(-0 . \\
25 \text { to } 0.09)\end{array}$ & $\begin{array}{l}533 \\
\text { (2 studies) }\end{array}$ & $\begin{array}{l}\oplus \bigcirc \bigcirc \bigcirc \\
\text { very low } \text { low }^{2,3}\end{array}$ & - \\
\hline $\begin{array}{l}\text { Sever- } \\
\text { ity of CMDs - } \\
\text { medium term (8 } \\
\text { months) } \\
\text { measured using } \\
\text { SRQ-20 }\end{array}$ & - & $\begin{array}{l}\text { The mean sever- } \\
\text { ity of CMDs - } \\
\text { medium term (6 } \\
\text { months to } 1 \text { year } \\
\text { post } \\
\text { intervention) in } \\
\text { the intervention } \\
\text { groups was } \\
\text { 0. } \\
\mathbf{3 2} \text { standard de- } \\
\text { viations lower } \\
(0.6 \text { to } 0.04 \\
\text { lower) }\end{array}$ & $\begin{array}{l}\text { SMD }-0.32 \\
(-0.6 \text { to }-0.04)\end{array}$ & $\begin{array}{l}200 \\
\text { (1 study) }\end{array}$ & $\begin{array}{l}\oplus \bigcirc \bigcirc \bigcirc \\
\text { very low }\end{array}$ & - \\
\hline $\begin{array}{l}\text { Functional im- } \\
\text { pairment - male } \\
\text { short term (1 } \\
\text { month) } \\
\text { measured using } \\
\text { WHO- } \\
\text { DAS (adapted) } \\
11 \text { items }\end{array}$ & - & $\begin{array}{l}\text { The mean func- } \\
\text { tional im- } \\
\text { pairment - male } \\
\text { short } \\
\text { term (within } 6 \\
\text { months of inter- } \\
\text { vention) } \\
\text { in the interven- } \\
\text { tion groups was } \\
\text { 0. } \\
\mathbf{3 2} \text { standard de- } \\
\text { viations lower } \\
(0.65 \text { lower to } 0 \text {. } \\
02 \text { higher) }\end{array}$ & $\begin{array}{l}\text { SMD }-0.32(-0 . \\
65 \text { to } 0.02)\end{array}$ & $\begin{array}{l}141 \\
\text { (1 study) }\end{array}$ & $\begin{array}{l}\oplus \bigcirc \bigcirc \bigcirc \\
\text { very low }\end{array}$ & - \\
\hline $\begin{array}{l}\text { Functional im- } \\
\text { pairment - fe- } \\
\text { male short term } \\
\text { (1 month) } \\
\text { measured using } \\
\text { WHO- }\end{array}$ & - & $\begin{array}{l}\text { The mean func- } \\
\text { tional } \\
\text { impairment - fe- } \\
\text { male short term } \\
\text { (within } 6\end{array}$ & $\begin{array}{l}\text { SMD }-0.34(-0 . \\
63 \text { to }-0.06)\end{array}$ & $\begin{array}{l}192 \\
\text { (1 study) }\end{array}$ & $\begin{array}{l}\oplus \bigcirc \bigcirc \bigcirc \\
\text { very low }\end{array}$ & - \\
\hline
\end{tabular}

Non-specialist health worker interventions for the care of mental, neurological and substance-abuse disorders in low- and middle- 
Table 5. SoF: NSHW-led interventions compared with usual care in treating common mental disorders in adults in low- and middle-income countries (CBAs) (Continued)

\begin{tabular}{l|l}
\hline DAS (adapted) & months of inter- \\
11 items & vention) \\
& in the interven- \\
tion groups was & $\mathbf{0 .}$ \\
& $\mathbf{3 4}$ standard de- \\
viations lower \\
$(0.63$ to 0.06 \\
lower)
\end{tabular}

*The basis for the assumed risk (e.g. the median control group risk across studies) is provided in footnotes. The corresponding risk (and its $95 \%$ confidence interval) is based on the assumed risk in the comparison group and the relative effect of the intervention (and its $95 \% \mathrm{CI}$ ).

CI: confidence interval; CMD: common mental disorders; NSHW: non-specialist health worker; SMD: standardised mean difference; SRQ: Self Reporting Questionnaire; WHODAS: World Health Organization Disability Assessment Scale.

GRADE Working Group grades of evidence

High quality: Further research is very unlikely to change our confidence in the estimate of effect.

Moderate quality: Further research is likely to have an important impact on our confidence in the estimate of effect and may change the estimate.

Low quality: Further research is very likely to have an important impact on our confidence in the estimate of effect and is likely to change the estimate.

Very low quality: We are very uncertain about the estimate.

${ }^{1}$ Bass 2012 CBA Indonesia: Hopkins Symptom Checklist 25; Scholte 2011 CBA Rwanda: SRQ-20.

${ }^{2}$ Very serious risk of bias: Bass 2012 CBA Indonesia: controlled before-and-after study so non-random and not concealed. Also differences in baseline outcomes for girls, and doubt about reliability of primary outcomes as tool not properly validated. Scholte 2011 CBA Rwanda: controlled before-and-after study so non-randomised and no concealment. Also unclear risk for incomplete outcome data, there are baseline differences in outcomes and in characteristics not all adjusted for, and high rate of loss to follow-up with no analysis of group lost to follow-up. Downgraded by 2 .

${ }^{3}$ No imprecision: Non-appreciable benefit for either intervention or control group.

${ }^{4}$ No explanation was provided.

${ }^{5}$ No imprecision: Appreciable and non-appreciable benefit for intervention.

${ }^{6}$ Very serious risk of bias: Bass 2012 CBA Indonesia: controlled before-and-after study so non-random and not concealed. Also differences in baseline outcomes for girls, and doubt about reliability of primary outcomes as tool not properly validated. Downgraded by 2 .

${ }^{7}$ Serious imprecision: Appreciable benefit for intervention and non-appreciable benefit for usual care. Downgrade by 1.

Table 6. SoF 2: Collaborative care model (NSHWs plus specialist) compared with usual care in treating common mental disorders in adults in low- and middle-income countries (RCTs)

What are the effects of a collaborative care model (NSHW plus specialist supervision) for mental health care in adults with common mental disorders low- and middle-income countries? (additional outcomes to comparison 2)

Patient or population: Adults ( $\geq 18$ years) with common mental disorders (includes anxiety or depression, or both)

Settings: Middle-income countries (Chile, India)

Intervention: Collaborative care model (NSHW plus specialist supervision)

Comparison: Enhanced usual care

Non-specialist health worker interventions for the care of mental, neurological and substance-abuse disorders in low- and middle- 
Table 6. SoF 2: Collaborative care model (NSHWs plus specialist) compared with usual care in treating common mental disorders in adults in low- and middle-income countries (RCTs) (Continued)

\begin{tabular}{|c|c|c|c|c|c|c|}
\hline \multirow[t]{3}{*}{ Outcomes } & \multicolumn{2}{|c|}{$\begin{array}{l}\text { Illustrative comparative risks* } \\
(95 \% \mathrm{CI})\end{array}$} & \multirow[t]{3}{*}{$\begin{array}{l}\text { Effect estimate } \\
(95 \% \mathrm{CI})\end{array}$} & \multirow{3}{*}{$\begin{array}{l}\text { No of Partici- } \\
\text { pants } \\
\text { (studies) }\end{array}$} & \multirow{3}{*}{$\begin{array}{l}\text { Quality of the } \\
\text { evidence } \\
\text { (GRADE) }\end{array}$} & \multirow[t]{3}{*}{ Comments } \\
\hline & Assumed risk & $\begin{array}{l}\text { Corresponding } \\
\text { risk }\end{array}$ & & & & \\
\hline & usual care & $\begin{array}{l}\text { Collaborative } \\
\text { care model }\end{array}$ & & & & \\
\hline $\begin{array}{l}\text { Prevalence of } \\
\text { CMDs medium } \\
\text { term } \\
\text { months) } \\
\text { measured using } \\
\text { CIS-R generated } \\
\text { ICD-10 diagno- } \\
\text { sis for CMD }\end{array}$ & See comment & See comment & $\begin{array}{l}\text { RR } 0.95 \\
(0.68 \text { to } 1.33)\end{array}$ & $\begin{array}{l}2009 \\
\text { (1 study) }\end{array}$ & $\begin{array}{l}\oplus \oplus \bigcirc \bigcirc \\
\text { low }^{1}\end{array}$ & $\begin{array}{l}\text { Patel } 2010 \text { C- } \\
\text { RCT India did } \\
\text { not reveal signif- } \\
\text { icant differences } \\
\text { in the prevalence } \\
\text { of depression } \\
\text { with both inter- } \\
\text { ventions in pub- } \\
\text { lic or private care } \\
\text { facilities }\end{array}$ \\
\hline $\begin{array}{l}\text { Severity } \\
\text { of symptoms in } \\
\text { CMD } \\
\text { short term (2-6 } \\
\text { months) } \\
\text { mea- } \\
\text { sured using vari- } \\
\text { ous rating scales }{ }^{2}\end{array}$ & - & $\begin{array}{l}\text { The mean sever- } \\
\text { ity of symptoms } \\
\text { in CMD with } \\
\text { collaborative } \\
\text { care was } \\
\text { 0. } \\
\mathbf{3 1} \text { standard de- } \\
\text { viations lower } \\
(0.56 \text { to } 0.06 \\
\text { lower })\end{array}$ & $\begin{array}{l}\text { SMD }-\mathbf{0 . 3 1}(-0 . \\
56 \text { to }-0.06)\end{array}$ & $\begin{array}{l}3604 \\
\text { (5 studies) }\end{array}$ & $\begin{array}{l}\oplus \bigcirc \bigcirc \bigcirc \\
\text { very low } \text { low }^{3,4,5}\end{array}$ & - \\
\hline $\begin{array}{l}\text { Severity } \\
\text { of symptoms in } \\
\text { CMD } \\
\text { medium term } \\
\text { (12 months) } \\
\text { measured us- } \\
\text { ing CIS-R rating } \\
\text { scale }\end{array}$ & - & $\begin{array}{l}\text { The mean sever- } \\
\text { ity of symptoms } \\
\text { in CMD with } \\
\text { collaborative } \\
\text { care was } \\
\text { 0. } \\
\mathbf{0 3} \text { standard de- } \\
\text { viations lower } \\
(0.12 \text { lower to } 0 \text {. } \\
06 \text { higher) }\end{array}$ & $\begin{array}{l}\text { SMD }-\mathbf{0 . 0 3}(-0 . \\
12 \text { to } 0.06)\end{array}$ & $\begin{array}{l}1905 \\
\text { (1 study) }\end{array}$ & $\begin{array}{l}\oplus \oplus \oplus \bigcirc \\
\text { moderate }^{6}\end{array}$ & - \\
\hline $\begin{array}{l}\text { Functional im- } \\
\text { pairment/dis- } \\
\text { ability in CMD } \\
\text { short term (2-6 } \\
\text { months) }\end{array}$ & - & $\begin{array}{l}\text { The mean func- } \\
\text { tional } \\
\text { impairment/dis- } \\
\text { ability in CMD } \\
\text { with collabora- }\end{array}$ & $\begin{array}{l}\text { SMD }-\mathbf{0 . 2 2}(-0 . \\
44 \text { to }-0.01)\end{array}$ & $\begin{array}{l}3604 \\
\text { (5 studies) }\end{array}$ & $\begin{array}{l}\oplus \bigcirc \bigcirc \bigcirc \\
\text { very low } \text { low }^{5,9}\end{array}$ & - \\
\hline
\end{tabular}

Non-specialist health worker interventions for the care of mental, neurological and substance-abuse disorders in low- and middle- 
Table 6. SoF 2: Collaborative care model (NSHWs plus specialist) compared with usual care in treating common mental disorders in adults in low- and middle-income countries (RCTs) (Continued)

\begin{tabular}{|c|c|c|c|c|c|}
\hline $\begin{array}{l}\text { measured us- } \\
\text { ing various func- } \\
\text { tional disability } \\
\text { scores }^{7}\end{array}$ & $\begin{array}{l}\text { tive care was } \\
\mathbf{0 .} \\
\mathbf{2 2} \text { standard de- } \\
\text { viations lower } \\
(0.44 \text { to } 0.01 \\
\text { lower })\end{array}$ & & & & \\
\hline $\begin{array}{l}\text { Functional im- } \\
\text { pairment/dis- } \\
\text { ability in CMD } \\
\text { medium term } \\
\text { (12 months) } \\
\text { measured using } \\
\text { WHODAS II } \\
\text { scores }\end{array}$ & $\begin{array}{l}\text { The mean func- } \\
\text { tional } \\
\text { impairment/dis- } \\
\text { ability in CMD } \\
\text { with collabora- } \\
\text { tive care was } \\
\mathbf{0 .} \\
\mathbf{0 2} \text { standard de- } \\
\text { viations lower } \\
(0.11 \text { lower to } 0 . \\
07 \text { higher) }\end{array}$ & $\begin{array}{l}\text { SMD }-\mathbf{0 . 0 2}(-0 . \\
11 \text { to } 0.07)\end{array}$ & $\begin{array}{l}1905 \\
\text { (1 study) }\end{array}$ & $\begin{array}{l}\oplus \oplus \oplus \bigcirc \\
\text { moderate }^{6}\end{array}$ & \\
\hline
\end{tabular}

*The basis for the assumed risk is the mean control group risk across studies for pooled results and the control group risk for single studies. The corresponding risk (and its $95 \%$ confidence interval) is based on the assumed risk in the comparison group and the relative effect of the intervention (and its $95 \% \mathrm{CI}$ ).

CI: confidence interval; CIS-D: Composite International Diagnostic-Screener; CMD: common mental disorders; ICD: International Classification of Diseases; NSHW: non-specialist health worker; RR: risk ratio; SMD: standardised mean difference; WHODAS: World Health Organization Disability Assessment Scale.

GRADE Working Group grades of evidence

High quality: Further research is very unlikely to change our confidence in the estimate of effect.

Moderate quality: Further research is likely to have an important impact on our confidence in the estimate of effect and may change the estimate.

Low quality: Further research is very likely to have an important impact on our confidence in the estimate of effect and is likely to change the estimate.

Very low quality: We are very uncertain about the estimate.

${ }^{1}$ Very serious imprecision: The 95\% CI for the pooled estimates indicates appreciable benefit for collaborative care and appreciable benefit for usual care. Downgraded by 2 .

2 Jenkins 2012 C-RCT Kenya used the General Health Questionnaire (GHQ)-12. Patel 2010 C-RCT India used CIS-R to generate ICD-10 depression diagnoses; Fritsch 2007 RCT Chile and Araya 2003 RCT Chile used the Hamilton Depression Rating Scale (HDRS); Rojas 2007 RCT Chile used the: Edinburgh Postnatal Depression Scale (EPDS).

${ }^{3}$ Serious study limitations: In Araya 2003 RCT Chile and possibly in Fritsch 2007 RCT Chile general practitioners (GPs) did both interventions, so there was a high risk of contamination; this would have reduced the potential benefits with collaborative care in two of the four trials in the meta-analysis. Downgraded by 1.

${ }^{4}$ Serious inconsistency. The $\mathrm{I}^{2}$ statistic $=91 \%$ with Araya 2003 RCT Chile clearly an outlier, contributing to this unexplained inconsistency. However, the inconsistency related to the magnitude of benefit favouring collaborative care rather than in the direction of effect. Downgraded by 1 .

${ }^{5}$ Serious indirectness: Jenkins 2012 C-RCT Kenya used the GHQ-12 to grade severity of symptoms; the GHQ is a screening instrument that is validated to screen for CMDs; its use to rate the severity of depression is less reliable). Downgraded by 1. 
${ }^{6}$ Serious imprecision: The $95 \%$ CI for the pooled estimates indicates no appreciable benefit for collaborative care $(<0.2)$ and nonappreciable benefit for usual care. The data come from one study (Patel 2010 C-RCT India), and therefore imprecise. Downgraded by 1.

7 Jenkins 2012 C-RCT Kenya used WHODAS II long version (36 items); Patel 2010 C-RCT India used the WHODAS II short version (12 items); Araya 2003 RCT Chile; Fritsch 2007 RCT Chile; and Rojas 2007 RCT Chile used SF-36 social functioning component. ${ }^{8}$ Serious study limitations: In Araya 2003 RCT Chile and probably Fritsch 2007 RCT Chile, GPs did both intervention and control interventions so there was a high risk of contamination. Downgraded by 1 .

9 Serious inconsistency. The $\mathrm{I}^{2}$ statistic $=87 \%$ with Araya 2003 RCT Chile clearly an outlier, contributing to this unexplained inconsistency. However, the inconsistency related to the magnitude of benefit favouring collaborative care rather than in the direction of effect. Downgraded by 1 .

Table 7. SoF 3: NSHWs compared with usual care for treating maternal depression (RCTs)

What are the effects of NSHW-led interventions for treating maternal depression in low- and middle-income countries? (additional outcomes for comparison 3)

Patient or population: Adult women with maternal depression

Settings: Low- and middle-income countries (Chile, Jamaica, Pakistan, Taiwan)

Intervention: NSHW-led interventions

Comparison: Usual care

\begin{tabular}{|c|c|c|c|c|c|c|}
\hline \multirow[t]{3}{*}{ Outcomes } & \multicolumn{2}{|c|}{$\begin{array}{l}\text { Illustrative comparative risks* } \\
(95 \% \mathrm{CI})\end{array}$} & \multirow[t]{3}{*}{$\begin{array}{l}\text { Estimate effect } \\
(95 \% \mathrm{CI})\end{array}$} & \multirow{3}{*}{$\begin{array}{l}\text { No of Partici- } \\
\text { pants } \\
\text { (studies) }\end{array}$} & \multirow{3}{*}{$\begin{array}{l}\text { Quality of the } \\
\text { evidence } \\
\text { (GRADE) }\end{array}$} & \multirow[t]{3}{*}{ Comments } \\
\hline & Assumed risk & $\begin{array}{l}\text { Corresponding } \\
\text { risk }\end{array}$ & & & & \\
\hline & Usual care & NSHWs & & & & \\
\hline $\begin{array}{l}\text { Severity } \\
\text { of symptoms of } \\
\text { perina- } \\
\text { tal depression - } \\
\text { NSHW led-psy- } \\
\text { chological } \\
\text { interventions } \\
\text { short term (0-2 } \\
\text { months) } \\
\text { measured using } \\
\text { depression rating } \\
\text { scales }{ }^{1}\end{array}$ & - & $\begin{array}{l}\text { The mean sever- } \\
\text { ity of symptoms } \\
\text { of peri- } \\
\text { natal depression } \\
\text { - short term with } \\
\text { NSHW-led in- } \\
\text { terventions was } \\
\mathbf{0 . 5} \text { standard de- } \\
\text { viations lower } \\
(0.63 \text { to } 0.36 \\
\text { lower) }\end{array}$ & $\begin{array}{l}\text { SMD }-0.5(-0.63 \\
\text { to }-0.36)\end{array}$ & $\begin{array}{l}858 \\
\text { (2 studies) }\end{array}$ & $\begin{array}{l}\oplus \oplus \oplus \oplus \\
\text { high }^{2,3}\end{array}$ & $\begin{array}{l}\text { Note } \\
\text { that a small clin- } \\
\text { ically appreciable } \\
\text { benefit was set at } \\
\text { SMD < } 0.2 \text {, and } \\
\text { a moderate ben- } \\
\text { efit at SMD of } 0 \text {. } \\
5 \text { to } 0.8 \text { (Cohen } \\
1988 \text { ) }\end{array}$ \\
\hline $\begin{array}{l}\text { Severity } \\
\text { of symptoms of } \\
\text { perina- } \\
\text { tal depression - } \\
\text { NSHW led-psy- } \\
\text { chological } \\
\text { interventions } \\
\text { medium term }\end{array}$ & - & $\begin{array}{l}\text { The mean sever- } \\
\text { ity of symptoms } \\
\text { of peri- } \\
\text { natal depression } \\
\text { - medium term } \\
\text { - with NSHW- } \\
\text { led interventions }\end{array}$ & $\begin{array}{l}\text { SMD }-0.41(-0 . \\
76 \text { to }-0.06)\end{array}$ & $\begin{array}{l}125 \\
\text { (1 study) }\end{array}$ & $\begin{array}{l}\oplus \bigcirc \bigcirc \bigcirc \\
\text { low }^{5,6}\end{array}$ & - \\
\hline
\end{tabular}

Non-specialist health worker interventions for the care of mental, neurological and substance-abuse disorders in low- and middle- 
Table 7. SoF 3: NSHWs compared with usual care for treating maternal depression (RCTs) (Continued)

\begin{tabular}{|c|c|c|c|c|c|}
\hline $\begin{array}{l}\text { (12 months) } \\
\text { measured using a } \\
\text { depression scale }\end{array}$ & $\begin{array}{l}\text { was } \\
\mathbf{0 .} \\
\mathbf{4 1} \text { standard de- } \\
\text { viations lower } \\
(0.76 \text { to } 0.06 \\
\text { lower })\end{array}$ & & & & \\
\hline $\begin{array}{l}\text { Sever- } \\
\text { ity of symptoms } \\
\text { of perinatal de- } \\
\text { pression - col- } \\
\text { laborative care- } \\
\text { short term (3 } \\
\text { months) } \\
\text { depression rating } \\
\text { scale - EPDS }\end{array}$ & $\begin{array}{l}\text { The mean sever- } \\
\text { ity of symptoms } \\
\text { of } \\
\text { perinatal depres- } \\
\text { sion - short term } \\
\text { - with collabora- } \\
\text { tive care was } \\
\mathbf{0 .} \\
\mathbf{2 2} \text { standard de- } \\
\text { viations lower } \\
(0.48 \text { lower to } 0 \text {. } \\
04 \text { higher) }\end{array}$ & $\begin{array}{l}\text { SMD }-0.22(-0 . \\
48 \text { to } 0.04)\end{array}$ & $\begin{array}{l}230 \\
\text { (1 study) }\end{array}$ & $\begin{array}{l}\oplus \oplus \oplus \bigcirc \\
\text { moderate }^{7}\end{array}$ & - \\
\hline
\end{tabular}

*The basis for the assumed risk is the mean control group risk across studies for pooled results and the control group risk for single studies. The corresponding risk (and its $95 \%$ confidence interval) is based on the assumed risk in the comparison group and the relative effect of the intervention (and its 95\% CI).

CI: confidence interval; EPDS: Edinburgh Postnatal Depression Score; NSHW: non-specialist health worker; SMD: standardised mean difference.

GRADE Working Group grades of evidence

High quality: Further research is very unlikely to change our confidence in the estimate of effect.

Moderate quality: Further research is likely to have an important impact on our confidence in the estimate of effect and may change the estimate.

Low quality: Further research is very likely to have an important impact on our confidence in the estimate of effect and is likely to change the estimate.

Very low quality: We are very uncertain about the estimate.

${ }^{1}$ Chen 2000 RCT Taiwan: Taiwanese Beck Depression Inventory; Rahman 2008 CRCT Pakistan: Hamilton Depression Rating Scale (HDRS).

${ }^{2}$ No serious study limitations: Chen 2000 RCT Taiwan was unclear for sequence generation and allocation concealment, all were self reported outcomes, there was possible contamination and there was a high dropout rate after randomisation, with no analysis of differences between dropout versus non-dropouts differences. However, Rahman 2008 CRCT Pakistan had no serious study limitations and contributed most of the weight to the pooled analysis. Removal of data from Chen 2000 RCT Taiwan did not appreciably change effect estimates. Not downgraded.

${ }^{3}$ No serious imprecision: appreciable benefit seen at SMD $=0.2$ for Rahman 2008 CRCT Pakistan with HDRS and 0.5 for Chen 2000 RCT Taiwan with the BDI. The 95\% CI includes appreciable benefit for NSHW-led interventions. Not downgraded.

${ }^{4}$ Baker-H 2005 CRCT Jamaica: CES-D.

${ }^{5}$ Serious study limitations: Baker-H 2005 CRCT Jamaica had unadjusted differences in baseline characteristics. Downgraded by 1.

${ }^{6}$ Serious imprecision: The $95 \%$ CI of the effect estimate indicated appreciable and non-appreciable benefit with NSHW-led interventions, and the sample size was small. Downgraded by 1.

${ }^{7}$ Serious imprecision: The 95\% CI of the effect estimate indicated appreciable and non-appreciable benefit with collaborative care, and the sample size was small. Downgraded by 1. 
Table 8. SoF 4: NSHWs compared with specialists in treating depression in adults in low- and middle-income countries (CBAs)

What are the effects of NSHWs compared with specialists in treating depression for mental health care in low- and middleincome countries? (additional outcomes for comparison 4)

Patient or population: Adults with depression

Settings: Middle-income countries (Hungary and Argentina)

Intervention: NSHWs providing pharmacological intervention

Comparison: Specialists providing pharmacological intervention

\begin{tabular}{|c|c|c|c|c|c|c|}
\hline \multirow[t]{3}{*}{ Outcomes } & \multicolumn{2}{|c|}{$\begin{array}{l}\text { Illustrative comparative risks* } \\
(95 \% \mathrm{CI})\end{array}$} & \multirow[t]{3}{*}{$\begin{array}{l}\text { Relative effect } \\
(95 \% \text { CI })\end{array}$} & \multirow{3}{*}{$\begin{array}{l}\text { No of Partici- } \\
\text { pants } \\
\text { (studies) }\end{array}$} & \multirow{3}{*}{$\begin{array}{l}\text { Quality of the } \\
\text { evidence } \\
\text { (GRADE) }\end{array}$} & \multirow[t]{3}{*}{ Comments } \\
\hline & Assumed risk & $\begin{array}{l}\text { Corresponding } \\
\text { risk }\end{array}$ & & & & \\
\hline & Specialists & NSHWs & & & & \\
\hline $\begin{array}{l}\text { Fre- } \\
\text { quency of ad- } \\
\text { verse events (at } \\
\mathbf{5 6} \text { days) } \\
\text { measured using } \\
\text { the number of } \\
\text { mild, moderate } \\
\text { and severe ad- } \\
\text { verse events }\end{array}$ & 288 per 1000 & $\begin{array}{l}\mathbf{2 4 5} \text { per } \mathbf{1 0 0 0} \\
(193 \text { to } 308)\end{array}$ & $\begin{array}{l}\text { RR } 0.85 \\
(0.67 \text { to } 1.07)\end{array}$ & $\begin{array}{l}768 \\
\text { (1 study) }\end{array}$ & $\begin{array}{l}\oplus \bigcirc \bigcirc \bigcirc \\
\text { very low } \mathbf{l o w}^{1,2}\end{array}$ & $\begin{array}{l}\text { Note } \\
\text { that a small clin- } \\
\text { ically appreciable } \\
\text { benefit was set at } \\
\text { SMD }<0.2 \text {, and } \\
\text { a moderate ben- } \\
\text { efit at SMD of } 0 \text {. } \\
5 \text { to } 0.8 \text { (Cohen } \\
1988 \text { ) }\end{array}$ \\
\hline $\begin{array}{l}\text { Number of days } \\
\text { spent in hospi- } \\
\text { tal (at } 1 \text { year) }\end{array}$ & - & $\begin{array}{l}\text { The mean num- } \\
\text { ber of days spent } \\
\text { in } \\
\text { hospital at } 1 \text { year } \\
\text { in the NSHW } \\
\text { group was } \\
1.79 \text { lower } \\
\text { ( } 3.59 \text { lower to } 0 . \\
01 \text { higher) }\end{array}$ & $\begin{array}{l}\text { MD }-\mathbf{1 . 7 9}(-3 \\
59 \text { to } 0.01)\end{array}$ & $\begin{array}{l}124 \\
\text { (1 study) }\end{array}$ & $\begin{array}{l}\oplus \bigcirc \bigcirc \bigcirc \\
\text { very low }\end{array}$ & - \\
\hline $\begin{array}{l}\text { Number of days } \\
\text { spent in hospi- } \\
\text { tal (at } 2 \text { years) }\end{array}$ & - & $\begin{array}{l}\text { The mean num- } \\
\text { ber of days spent } \\
\text { in hospital at } 2 \\
\text { years } \\
\text { in the NSHW } \\
\text { group was } \\
\mathbf{0 . 0 2} \text { lower } \\
\text { ( } 2.59 \text { lower to } 2 \text {. } \\
55 \text { higher) }\end{array}$ & $\begin{array}{l}\text { MD }-\mathbf{0 . 0 2}(-2 . \\
59 \text { to } 2.55)\end{array}$ & $\begin{array}{l}124 \\
\text { (1 study) }\end{array}$ & $\begin{array}{l}\oplus \bigcirc \bigcirc \bigcirc \\
\text { very low } \\
\text { lo, }\end{array}$ & - \\
\hline
\end{tabular}

Non-specialist health worker interventions for the care of mental, neurological and substance-abuse disorders in low- and middle- 
Table 8. SoF 4: NSHWs compared with specialists in treating depression in adults in low- and middle-income countries (CBAs) (Continued)

\begin{tabular}{|c|c|c|c|c|c|c|}
\hline $\begin{array}{l}\text { Number of days } \\
\text { spent on sick } \\
\text { leave (at } 1 \text { year) }\end{array}$ & - & $\begin{array}{l}\text { The mean num- } \\
\text { ber of days spent } \\
\text { on sick leave at } 1 \\
\text { year } \\
\text { in the NSHW } \\
\text { group was } \\
3.96 \text { lower } \\
\text { (15.58 lower to } \\
7.66 \text { higher) }\end{array}$ & $\begin{array}{l}\text { MD }-3.96(-15 . \\
58 \text { to } 7.66)\end{array}$ & $\begin{array}{l}108 \\
\text { (1 study) }\end{array}$ & $\begin{array}{l}\oplus \bigcirc \bigcirc \bigcirc \\
\text { very low } \text { low }^{3,5}\end{array}$ & - \\
\hline $\begin{array}{l}\text { Number of days } \\
\text { spent on sick } \\
\text { leave (at } 2 \text { years) }\end{array}$ & - & $\begin{array}{l}\text { The mean num- } \\
\text { ber of days spent } \\
\text { on sick leave at } \\
2 \text { years in the } \\
\text { NSHW group } \\
\text { was } \\
\mathbf{1 4 . 6 3} \text { higher } \\
(0.76 \text { lower to } \\
30.02 \text { higher })\end{array}$ & $\begin{array}{l}\text { MD } 14.63(-0 . \\
76 \text { to } 30.02)\end{array}$ & $\begin{array}{l}123 \\
\text { (1 study) }\end{array}$ & $\begin{array}{l}\oplus \bigcirc \bigcirc \bigcirc \\
\text { very low }\end{array}$ & - \\
\hline
\end{tabular}

*The basis for the assumed risk is the risk in the control group. The corresponding risk (and its $95 \%$ confidence interval) is based on the assumed risk in the comparison group and the relative effect of the intervention (and its 95\% CI).

CBA: controlled before-and-after; CI: confidence interval; MD: mean difference; NSHW: non-specialist health worker; RR: risk ratio; SMD: standardised mean difference.

GRADE Working Group grades of evidence

High quality: Further research is very unlikely to change our confidence in the estimate of effect.

Moderate quality: Further research is likely to have an important impact on our confidence in the estimate of effect and may change the estimate.

Low quality: Further research is very likely to have an important impact on our confidence in the estimate of effect and is likely to change the estimate.

Very low quality: We are very uncertain about the estimate.

${ }^{1}$ Very serious study limitations: Lyketsos1999CBA Argentina was a CBA study so selection bias was likely. There was a risk of contamination and outcome assessments were done by same physicians doing the intervention. Downgraded by 2 .

${ }^{2}$ Serious imprecision: The $95 \%$ CI for the pooled estimates indicates appreciable and non-appreciable harms with the interventions. In addition, results are only from one study. Downgraded by 1 .

${ }^{3}$ Very serious study limitations: Zambori 2002 CBA Hungary was a CBA study so selection is likely; and there were significant difference in baseline outcomes and baseline characteristics. Downgraded by 2 .

${ }^{4}$ Serious imprecision: The $95 \%$ CI for the pooled estimates indicates appreciable benefit for the interventions and non-appreciable benefit for the control. In addition, results were only from one study. Downgraded by 1 .

${ }^{5}$ Very serious imprecision: Shows appreciable benefit for both interventions. Also results were only from one study. Downgraded by 2.

${ }^{6}$ Serious imprecision: Shows appreciable benefit for specialists and non-appreciable benefit for NSHWs. In addition, results were only from one study. Downgraded by 1. 
Table 9. SoF 5: NSHW and OPHR-led psychological interventions compared with usual care in treating adults with PTSD (NRCT and RCTs)

What are the effects of NSHWs/OPHRs compared with usual mental health care in low- and middle-income countries for data from an NRCT in adults with PTSD?

Patient or population: Adults with PTSD

Settings: Low- and middle-income countries (Burundi, Uganda)

Intervention: NSHWs and OPHRs delivering psychological interventions (narrative exposure therapy, trauma counselling and workshops with psychoeducation)

Comparison: Usual care

\begin{tabular}{|c|c|c|c|c|c|c|}
\hline \multirow[t]{3}{*}{ Outcomes } & \multicolumn{2}{|c|}{$\begin{array}{l}\text { Illustrative comparative risks* } \\
(95 \% \mathrm{CI})\end{array}$} & \multirow[t]{3}{*}{$\begin{array}{l}\text { Relative effect } \\
(95 \% \mathrm{CI})\end{array}$} & \multirow{3}{*}{$\begin{array}{l}\text { No of Partici- } \\
\text { pants } \\
\text { (studies) }\end{array}$} & \multirow{3}{*}{$\begin{array}{l}\text { Quality of the } \\
\text { evidence } \\
\text { (GRADE) }\end{array}$} & \multirow[t]{3}{*}{ Comments } \\
\hline & Assumed risk & $\begin{array}{l}\text { Corresponding } \\
\text { risk }\end{array}$ & & & & \\
\hline & Usual care & $\begin{array}{l}\text { NSHWs/ } \\
\text { OPHRs }\end{array}$ & & & & \\
\hline $\begin{array}{l}\text { Prevalence of } \\
\text { PTSD in LHW- } \\
\text { led narrative ex- } \\
\text { posure therapy, } \\
\text { medium term (9 } \\
\text { months) } \\
\text { measured using } \\
\text { the PTSD diag- } \\
\text { nostic tool } \\
\text { - DSM-IV from } \\
\text { CIDI }\end{array}$ & 632 per 1000 & $\begin{array}{l}\mathbf{3 0 3} \text { per } \mathbf{1 0 0 0} \\
(171 \text { to } 537)\end{array}$ & $\begin{array}{l}\text { RR } 0.48 \\
(0.27 \text { to } 0.85)\end{array}$ & $\begin{array}{l}62 \\
\text { (1 study) }\end{array}$ & $\begin{array}{l}\oplus \oplus \bigcirc \bigcirc \\
\text { low }^{1,2}\end{array}$ & - \\
\hline $\begin{array}{l}\text { Preva- } \\
\text { lence of PTSD } \\
\text { in a LHW-led } \\
\text { trauma coun- } \\
\text { selling, medium } \\
\text { term (mean } 9 \\
\text { months) } \\
\text { measured using } \\
\text { the PTSD diag- } \\
\text { nostic tool } \\
\text { - DSM-IV from } \\
\text { CIDI }\end{array}$ & 632 per 1000 & $\begin{array}{l}348 \text { per } 1000 \\
(209 \text { to } 588)\end{array}$ & $\begin{array}{l}\text { RR } 0.55 \\
(0.33 \text { to } 0.93)\end{array}$ & $\begin{array}{l}65 \\
\text { (1 study) }\end{array}$ & $\begin{array}{l}\oplus \oplus \bigcirc \bigcirc \\
\text { low }^{1,2}\end{array}$ & - \\
\hline $\begin{array}{l}\text { Severity } \\
\text { of PTSD symp- } \\
\text { toms in LHW- } \\
\text { led psychologi- }\end{array}$ & - & $\begin{array}{l}\text { The mean sever- } \\
\text { ity of PTSD with } \\
\text { narrative } \\
\text { exposure therapy }\end{array}$ & $\begin{array}{l}\text { SMD }-0.55(-1 . \\
08 \text { to }-0.03)\end{array}$ & $\begin{array}{l}75 \\
\text { (1 study) }\end{array}$ & $\begin{array}{l}\oplus \oplus \bigcirc \bigcirc \\
\mathbf{l o w}^{1,3}\end{array}$ & - \\
\hline
\end{tabular}

Non-specialist health worker interventions for the care of mental, neurological and substance-abuse disorders in low- and middle- 
Table 9. SoF 5: NSHW and OPHR-led psychological interventions compared with usual care in treating adults with PTSD (NRCT and RCTs) (Continued)

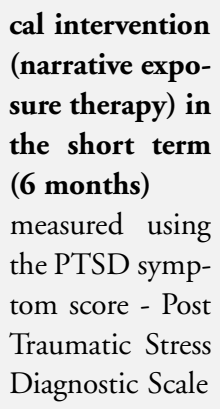

in the short term

was

0.

55 standard deviations lower

(1.08 to 0.03

lower)

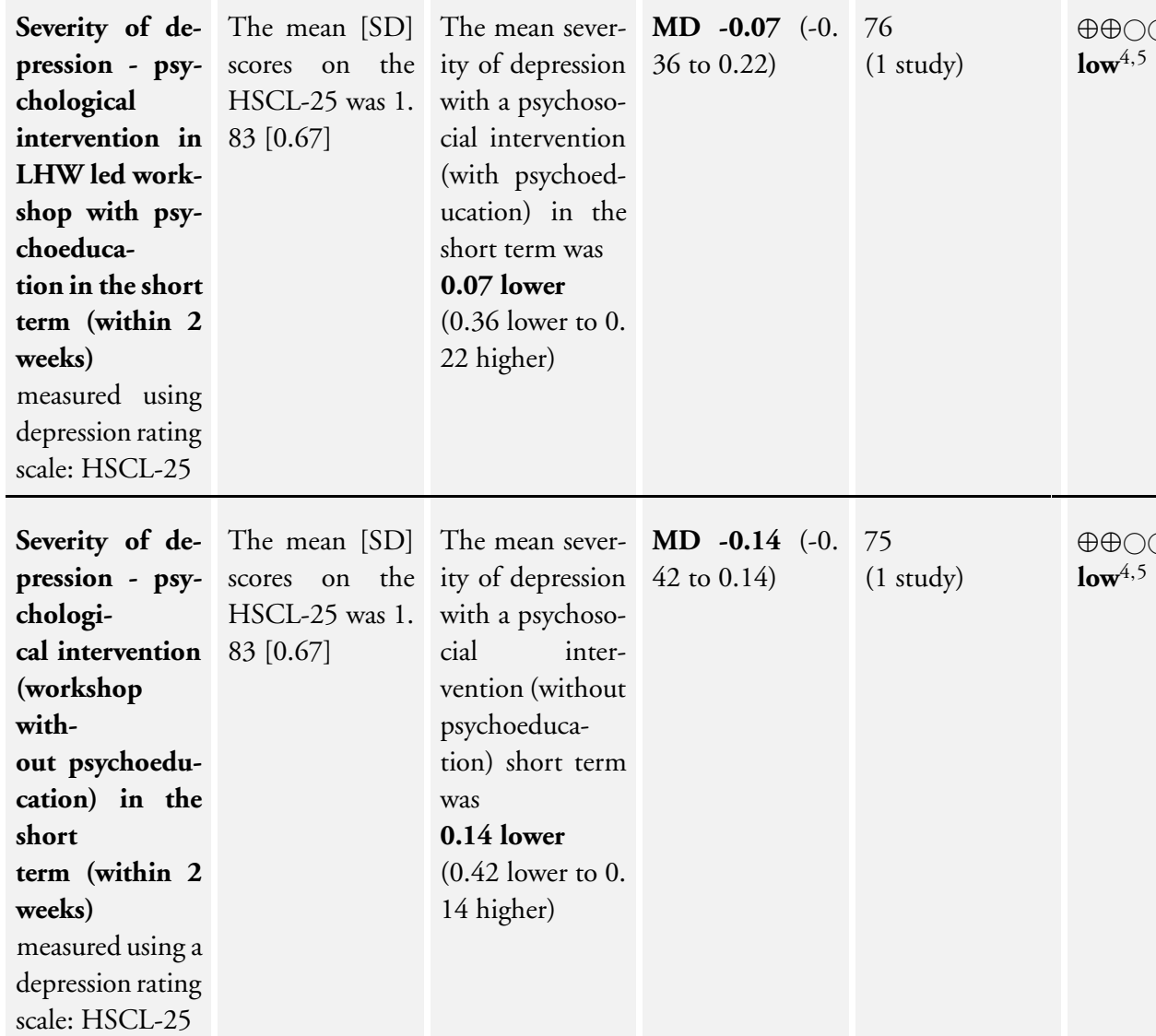

*The basis for the assumed risk is the median control group risk or mean control group risk across studies for pooled estimates and the control group risk for single studies. The corresponding risk (and its $95 \%$ confidence interval) is based on the assumed risk in the comparison group and the relative effect of the intervention (and its $95 \% \mathrm{CI}$ ).

CI: confidence interval; CIDI: Composite International Diagnostic Interview; DSM: Diagnostic and Statistical Manual of Mental Disorders; HSCL: Hopkins Symptom Checklist; LHW: lay health worker; MD: mean difference; NRCT: non-randomised controlled trial; NSHW: non-specialist health worker; OPHR: other professionals with health roles; PTSD: post-traumatic stress disorder; RCT: randomised controlled trial; RR: risk ratio; SD: standard deviation; SMD: standardised mean difference. 
Table 9. SoF 5: NSHW and OPHR-led psychological interventions compared with usual care in treating adults with PTSD (NRCT and RCTs) (Continued)

GRADE Working Group grades of evidence

High quality: Further research is very unlikely to change our confidence in the estimate of effect.

Moderate quality: Further research is likely to have an important impact on our confidence in the estimate of effect and may change the estimate.

Low quality: Further research is very likely to have an important impact on our confidence in the estimate of effect and is likely to change the estimate.

Very low quality: We are very uncertain about the estimate.

${ }^{1}$ Serious study limitations: Neuner 2008 NRCT Uganda no allocation concealment, randomisation has no sequence generation. High dropout rate and different between groups, different baseline characteristics and likely contamination. Downgraded by 1.

${ }^{2}$ Serious imprecision: The $95 \%$ CI of the effect estimate indicated appreciable and non-appreciable benefit with the intervention, and the sample size was small. Downgraded by 1 .

${ }^{3}$ Serious imprecision: The 95\% CI of the effect estimates demonstrated appreciable and non-appreciable benefit with intervention and the sample size (75 participants) was small. Downgraded by 1 .

${ }^{4}$ Serious study limitations: Yeomans 2010 RCT Burundi: unvalidated Harvard Trauma Questionnaire in the local context (only validated in Burundi) so may affect reliability of outcomes. Downgraded by 1 .

${ }^{5}$ Serious imprecision: The $95 \%$ CI of the effect estimates demonstrated non-appreciable benefit for both intervention and usual care and the sample size was small. Downgraded by 1 .

Table 10. SoF 6: NSHWs compared with usual care in improving dementia patients' and carers' outcomes in low- and middleincome countries (RCTs)

What are the effects of NSHW-led care in improving dementia patients' and carers' outcomes for mental health care in lowand middle-income countries? (additional outcomes for comparison 6)

Patient or population: Patients with dementia and their carers

Settings: Middle-income countries (India, Russia)

Intervention: NSHWs delivering brief intervention to carers

Comparison: Usual care

\begin{tabular}{|c|c|c|c|c|c|c|}
\hline \multirow[t]{3}{*}{ Outcomes } & \multicolumn{2}{|c|}{$\begin{array}{l}\text { Illustrative comparative risks* } \\
(95 \% \mathrm{CI})\end{array}$} & \multirow[t]{3}{*}{$\begin{array}{l}\text { Estimate effect } \\
(95 \% \mathrm{CI})\end{array}$} & \multirow{3}{*}{$\begin{array}{l}\text { No of Partici- } \\
\text { pants } \\
\text { (studies) }\end{array}$} & \multirow{3}{*}{$\begin{array}{l}\text { Quality of the } \\
\text { evidence } \\
\text { (GRADE) }\end{array}$} & \multirow[t]{3}{*}{ Comments } \\
\hline & Assumed risk & $\begin{array}{l}\text { Corresponding } \\
\text { risk }\end{array}$ & & & & \\
\hline & Usual care & NSHWs & & & & \\
\hline $\begin{array}{l}\text { Patient func- } \\
\text { tional ability (at } \\
\mathbf{6} \text { months) } \\
\text { mea- } \\
\text { sured using the } \\
\text { functional abil- } \\
\text { ity scale (EASI) }\end{array}$ & - & $\begin{array}{l}\text { The mean pa- } \\
\text { tient functional } \\
\text { ability with this } \\
\text { brief carer inter- } \\
\text { vention was } \\
\text { 0. } \\
\mathbf{2 4} \text { standard de- }\end{array}$ & $\begin{array}{l}\text { MD }-\mathbf{0 . 2 4}(-0 . \\
67 \text { to } 0.20)\end{array}$ & $\begin{array}{l}81 \\
\text { (1 study) }\end{array}$ & $\begin{array}{l}\oplus \oplus \oplus \bigcirc \\
\text { moderate }\end{array}$ & $\begin{array}{l}\text { Note } \\
\text { that a small clin- } \\
\text { ically appreciable } \\
\text { benefit was set at } \\
\text { SMD }<0.2 \text {, and } \\
\text { a moderate ben- } \\
\text { efit at SMD of } 0 \text {. }\end{array}$ \\
\hline
\end{tabular}


Table 10. SoF 6: NSHWs compared with usual care in improving dementia patients' and carers' outcomes in low- and middleincome countries (RCTs) (Continued)

viations lower

(0.67 lower to 0 .

5 to 0.8 (Cohen

20 higher)

\begin{tabular}{|c|c|c|c|c|c|c|}
\hline $\begin{array}{l}\text { Patient QoL (at } \\
\mathbf{6} \text { months) } \\
\text { measured } \\
\text { using the qual- } \\
\text { ity of life score } \\
\text { (DEMQOL) }\end{array}$ & - & $\begin{array}{l}\text { The mean pa- } \\
\text { tient QoL with } \\
\text { this brief carer } \\
\text { intervention was } \\
\text { 0. } \\
43 \text { standard de- } \\
\text { viations lower } \\
\text { ( } 0.98 \text { lower to } 0 . \\
12 \text { higher) }\end{array}$ & $\begin{array}{l}\text { MD } \mathbf{- 0 . 4 3}(-0 . \\
98 \text { to } 0.12)\end{array}$ & $\begin{array}{l}53 \\
\text { (1 study) }\end{array}$ & $\begin{array}{l}\oplus \oplus \oplus \bigcirc \\
\text { moderate }\end{array}$ & - \\
\hline $\begin{array}{l}\text { Carer mental } \\
\text { health status (at } \\
\mathbf{6} \text { months) } \\
\text { measured using } \\
\text { general men- } \\
\text { tal health status } \\
\text { scores }^{4}\end{array}$ & - & $\begin{array}{l}\text { The mean carer } \\
\text { mental health } \\
\text { status with this } \\
\text { brief carer inter- } \\
\text { vention was } \\
\mathbf{0 .} \\
\mathbf{4 2} \text { standard de- } \\
\text { viations lower } \\
(0.76 \text { to } 0.08 \\
\text { lower })\end{array}$ & $\begin{array}{l}\text { SMD }-\mathbf{0 . 4 2}(-0 . \\
76 \text { to }-0.08)\end{array}$ & $\begin{array}{l}134 \\
\text { (2 studies) }\end{array}$ & $\begin{array}{l}\oplus \oplus \oplus \bigcirc \\
\text { moderate }^{5}\end{array}$ & - \\
\hline $\begin{array}{l}\text { Carer burden } \\
\text { (at } 6 \text { months) } \\
\text { measured using a } \\
\text { burden } \\
\text { scale (Zarit Bur- } \\
\text { den Interview) }\end{array}$ & - & $\begin{array}{l}\text { The mean carer } \\
\text { burden in the } \\
\text { brief carer inter- } \\
\text { vention was } \\
\text { 0. } \\
50 \text { standard de- } \\
\text { viations lower } \\
(0.84 \text { to } 0.15 \\
\text { lower) }\end{array}$ & $\begin{array}{l}\text { SMD }-\mathbf{0 . 5 0}(-0 . \\
84 \text { to }-0.15)\end{array}$ & $\begin{array}{l}134 \\
\text { (2 studies) }\end{array}$ & $\begin{array}{l}\oplus \oplus \oplus \bigcirc \\
\text { moderate }^{5}\end{array}$ & - \\
\hline $\begin{array}{l}\text { Carer distress } \\
\text { (at } \mathbf{6} \text { months) } \\
\text { measured using } \\
\text { the carer distress } \\
\text { scale: (NPI-D) }\end{array}$ & - & $\begin{array}{l}\text { The mean carer } \\
\text { distress with this } \\
\text { brief carer inter- } \\
\text { vention was } \\
\mathbf{0 .} \\
47 \text { standard de- } \\
\text { viations lower } \\
(0.82 \text { to } 0.13 \\
\text { lower })\end{array}$ & $\begin{array}{l}\text { SMD }-\mathbf{0 . 4 7}(-0 . \\
82 \text { to }-0.13)\end{array}$ & $\begin{array}{l}134 \\
\text { (2 studies) }\end{array}$ & $\begin{array}{l}\oplus \oplus \oplus \bigcirc \\
\text { moderate }^{5}\end{array}$ & - \\
\hline $\begin{array}{l}\text { Carer QoL (at } 6 \\
\text { months) } \\
\text { measured using } \\
\text { the }\end{array}$ & - & $\begin{array}{l}\text { The mean carer } \\
\text { quality of life in } \\
\text { this brief carer } \\
\text { intervention was }\end{array}$ & $\begin{array}{l}\text { MD }-\mathbf{0 . 3 7} \quad(-0 . \\
92 \text { to } 0.17)\end{array}$ & $\begin{array}{l}53 \\
\text { (1 study) }\end{array}$ & $\begin{array}{l}\oplus \oplus \oplus \bigcirc \\
\text { moderate }\end{array}$ & - \\
\hline
\end{tabular}

Non-specialist health worker interventions for the care of mental, neurological and substance-abuse disorders in low- and middle- 
Table 10. SoF 6: NSHWs compared with usual care in improving dementia patients' and carers' outcomes in low- and middleincome countries (RCTs) (Continued)

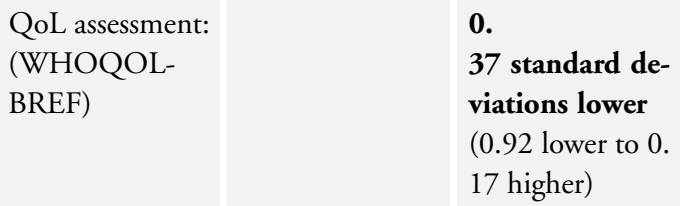

*The basis for the assumed risk is the mean control group risk across studies for pooled results and the control group risk for single studies. The corresponding risk (and its $95 \%$ confidence interval) is based on the assumed risk in the comparison group and the relative effect of the intervention (and its $95 \% \mathrm{CI}$ ).

CI: confidence interval; DEMQOL: Dementia Quality of Life; EASI: Everyday Abilities Scales for India; MD: mean difference; NPID: Neuropsychiatric Inventory - Dementia; NSHW: non-specialist health worker; QoL: quality of life; RCT: randomised controlled trial; SMD: standardised mean difference; WHOQOL-BREF: World Health Organization Quality of Life-BREF.

GRADE Working Group grades of evidence

High quality: Further research is very unlikely to change our confidence in the estimate of effect.

Moderate quality: Further research is likely to have an important impact on our confidence in the estimate of effect and may change the estimate.

Low quality: Further research is very likely to have an important impact on our confidence in the estimate of effect and is likely to change the estimate.

Very low quality: We are very uncertain about the estimate.

${ }^{1}$ No indirectness: there is only one study therefore the generalisability of this results to other settings is compromised. However, this also resulted in imprecision in the effect estimate, and hence the quality of evidence was not further downgraded.

${ }^{2}$ Serious imprecision. The $95 \%$ CI of the MD indicated appreciable benefits for NSHWs/intervention and non-appreciable benefits for usual care. The sample size is also small. Downgraded by 1 .

${ }^{3}$ No study limitations. Gavrilova 2009 RCT Russia was unclear whether allocation was concealed. However, no serious baseline differences in characteristics or outcomes were seen. Not downgraded.

${ }^{4}$ Dias 2008 RCT India: General Health Questionnaire (GHQ)-12; Gavrilova 2009 RCT Russia Self Reporting Questionnaire (SRQ)20.

${ }^{5}$ Serious imprecision. The $95 \%$ CI for the mean difference indicated appreciable and non-appreciable benefits with the interventions. Downgraded by 1 .

Table 11. SoF 7: NSHW-led brief alcohol interventions compared with usual care for adults with alcohol-use disorders (RCTs)

What are the effects of NSHWs in delivering brief alcohol interventions in RCTs for alcohol-use disorders? (additional outcomes for comparison 7)

Patient or population: Patients with alcohol-use disorders

Settings: Low- and middle-income countries (Thailand, Kenya)

Intervention: NSHWs in delivering brief alcohol interventions

Comparison: Usual care

\begin{tabular}{|l|lll|l|l|l}
\hline Outcomes & $\begin{array}{l}\text { Illustrative } \\
(95 \% \mathrm{CI})\end{array}$ & comparative & risks* & $\begin{array}{l}\text { Relative effect } \\
(95 \% \mathrm{CI})\end{array}$ & $\begin{array}{l}\text { No of Partici- } \\
\text { pants } \\
\text { (studies) }\end{array}$ & $\begin{array}{l}\text { Quality of the Comments } \\
\text { evidence } \\
\text { (GRADE) }\end{array}$
\end{tabular}


Table 11. SoF 7: NSHW-led brief alcohol interventions compared with usual care for adults with alcohol-use disorders (RCTs) (Continued)

\begin{tabular}{|c|c|c|c|c|c|c|}
\hline & Assumed risk & $\begin{array}{l}\text { Corresponding } \\
\text { risk }\end{array}$ & & & & \\
\hline & Usual care & NSHWs & & & & \\
\hline $\begin{array}{l}\text { Frequency of } \\
\text { binge drinking } \\
\text { (3-6 months) } \\
\text { measured using } \\
\text { the frequency of } \\
\text { binge drinking } \\
\text { in the past week }\end{array}$ & - & $\begin{array}{l}\text { The mean fre- } \\
\text { quency of binge } \\
\text { drinking } \\
\text { in the interven- } \\
\text { tion groups was } \\
\text { 0.50 lower } \\
\text { (1.14 lower to } 0 . \\
14 \text { higher) }\end{array}$ & $\begin{array}{l}\text { MD }-\mathbf{0 . 5 0} \\
(-1.14 \text { to } 0.14)\end{array}$ & $\begin{array}{l}92 \\
\text { (1 study) }\end{array}$ & $\begin{array}{l}\oplus \oplus \bigcirc \bigcirc \\
\text { low }^{1,2}\end{array}$ & - \\
\hline $\begin{array}{l}\text { Adverse conse- } \\
\text { quences - RTAs } \\
\text { (at } 6 \text { months) } \\
\text { measured using } \\
\text { the number of } \\
\text { RTAs }\end{array}$ & 220 per 1000 & $\begin{array}{l}\mathbf{7 9} \text { per } \mathbf{1 0 0 0} \\
(26 \text { to } 238)\end{array}$ & $\begin{array}{l}\text { RR } 0.36 \\
(0.12 \text { to } 1.08)\end{array}$ & $\begin{array}{l}92 \\
\text { (1 study) }\end{array}$ & $\begin{array}{l}\oplus \oplus \bigcirc \bigcirc \\
\mathbf{l o w}^{1,2}\end{array}$ & - \\
\hline $\begin{array}{l}\text { Adverse conse- } \\
\text { quences - with- } \\
\text { drawal } \\
\text { symptoms (at } 3 \\
\text { months) } \\
\text { measured using } \\
\text { the number of } \\
\text { withdrawal } \\
\text { symptoms }\end{array}$ & 31 per 1000 & $\begin{array}{l}\mathbf{8 3} \text { per } \mathbf{1 0 0 0} \\
(9 \text { to } 755)\end{array}$ & $\begin{array}{l}\text { RR 2.67 } \\
(0.29 \text { to } 24.37)\end{array}$ & $\begin{array}{l}68 \\
\text { (1 study) }\end{array}$ & $\begin{array}{l}\oplus \bigcirc \bigcirc \bigcirc \\
\text { very low } \text { low }^{1,3}\end{array}$ & - \\
\hline
\end{tabular}

*The basis for the assumed risk is the mean control group risk across studies for pooled data or the control group risk for individual studies. The corresponding risk (and its $95 \%$ confidence interval) is based on the assumed risk in the comparison group and the relative effect of the intervention (and its $95 \% \mathrm{CI}$ ).

CI: confidence interval; MD: mean difference; NSHW: non-specialist health worker; RCT: randomised controlled trial; RR: risk ratio; RTA: road traffic accident.

GRADE Working Group grades of evidence

High quality: Further research is very unlikely to change our confidence in the estimate of effect.

Moderate quality: Further research is likely to have an important impact on our confidence in the estimate of effect and may change the estimate.

Low quality: Further research is very likely to have an important impact on our confidence in the estimate of effect and is likely to change the estimate.

Very low quality: We are very uncertain about the estimate.

${ }^{1}$ Serious study limitations: Noknoy 2010 RCT Thailand: high dropout rate with no information on whether they are different to completers, no validated tools in the setting, so unreliable primary outcomes. Papas 2011 RCT Kenya: unclear about whether the non-blinding of outcome assessors would have impacted on study. Downgraded by 1 . 
${ }^{2}$ Serious imprecision: The $95 \%$ CI of the effect estimates indicates an appreciable benefit for NSHW care and non-appreciable benefit for usual care. The sample size was also small. Downgraded by 1 .

${ }^{3}$ Very serious imprecision: The $95 \%$ CI of the effect estimate indicates appreciable benefit for NSHW care and for usual care and also the study had a very small sample size. Downgraded by 2 .

Table 12. SoF 8: NSHWs/OPHRs compared with usual care in conducting interventions for children with post-traumatic stress disorder and depression (RCTs)

What are the effects of NSHWs/OPHRs conducting interventions for children with PTSD from RCTs in low- and middleincome countries? (additional outcomes for comparison 8)

Patient or population: Children/adolescents with PTSD and related depressive/anxiety symptoms Settings: Low- and middle-income countries (Bosnia, Indonesia, Kosovo, Nepal, Sri Lanka, Uganda)

Intervention: NSHWs/OPHRs delivering psychological and psychosocial interventions

Comparison: Usual care

\begin{tabular}{|c|c|c|c|c|c|c|}
\hline \multirow[t]{3}{*}{ Outcomes } & \multicolumn{2}{|c|}{$\begin{array}{l}\text { Illustrative comparative risks* } \\
(95 \% \mathrm{CI})\end{array}$} & \multirow[t]{3}{*}{$\begin{array}{l}\text { Estimate effect } \\
(95 \% \mathrm{CI})\end{array}$} & \multirow{3}{*}{$\begin{array}{l}\text { No of Partici- } \\
\text { pants } \\
\text { (studies) }\end{array}$} & \multirow{3}{*}{$\begin{array}{l}\text { Quality of the } \\
\text { evidence } \\
\text { (GRADE) }\end{array}$} & \multirow[t]{3}{*}{ Comments } \\
\hline & Assumed risk & $\begin{array}{l}\text { Corresponding } \\
\text { risk }\end{array}$ & & & & \\
\hline & Usual care & $\begin{array}{l}\text { NSHWs/ } \\
\text { OPHRs }\end{array}$ & & & & \\
\hline $\begin{array}{l}\text { Severity } \\
\text { of PTSD symp- } \\
\text { toms in LHW- } \\
\text { led classroom- } \\
\text { based interven- } \\
\text { tion, short term } \\
\text { (1-6 months) } \\
\text { measured using } \\
\text { the CPSS }\end{array}$ & - & $\begin{array}{l}\text { The MCD in } \\
\text { severity of PTSD } \\
\text { symptoms in } \\
\text { classroom-based } \\
\text { LHW-led inter- } \\
\text { vention groups } \\
\text { was } \\
\mathbf{0 . 5 6} \text { lower } \\
\text { ( } 2.82 \text { lower to } 1 . \\
7 \text { higher) }\end{array}$ & $\begin{array}{l}\text { MCD }-\mathbf{0 . 5 6}(-2 . \\
82 \text { to } 1.70)\end{array}$ & $\begin{array}{l}1090 \\
\text { (3 studies) }\end{array}$ & $\begin{array}{l}\oplus \bigcirc \bigcirc \bigcirc \\
\text { very low }\end{array}$ & - \\
\hline $\begin{array}{l}\text { Severity } \\
\text { of PTSD symp- } \\
\text { toms } \\
\text { in narrative ex- } \\
\text { posure therapy, } \\
\text { medium term } \\
\text { (11 months) } \\
\text { measured using } \\
\text { the CAPS }\end{array}$ & - & $\begin{array}{l}\text { The } \\
\text { mean severity of } \\
\text { depressive symp- } \\
\text { toms in teacher/ } \\
\text { LHW-led inter- } \\
\text { vention groups } \\
\text { was } \\
\mathbf{0 .} \\
\mathbf{4 5} \text { standard de- } \\
\text { viations lower } \\
\text { (0.99 lower to } 0 \text {. } \\
10 \text { higher) }\end{array}$ & $\begin{array}{l}\text { SMD }-\mathbf{0 . 4 5} \\
(-0.99 \text { to } 0.10)\end{array}$ & $\begin{array}{l}53 \\
\text { (1 study) }\end{array}$ & $\begin{array}{l}\oplus \oplus \oplus \bigcirc \\
\text { moderate }^{4,5}\end{array}$ & $\begin{array}{l}\text { Note that a small } \\
\text { clinically appre- } \\
\text { cia- } \\
\text { ble benefit was } \\
\text { set at } S M D<0.2 \text {, } \\
\text { a moderate ben- } \\
\text { efit at SMD of } 0 \text {. } \\
5 \text { to } 0.8 \text {, and a } \\
\text { large benefit }>0 . \\
8 \text { (Cohen } 1988) \text {. }\end{array}$ \\
\hline
\end{tabular}


Table 12. SoF 8: NSHWs/OPHRs compared with usual care in conducting interventions for children with post-traumatic stress disorder and depression (RCTs) (Continued)

\begin{tabular}{|c|c|c|c|c|c|c|}
\hline $\begin{array}{l}\text { Severity of de- } \\
\text { pressive symp- } \\
\text { toms in teacher/ } \\
\text { LHW-led inter- } \\
\text { ventions, } \\
\text { short term (2-6 } \\
\text { months) } \\
\text { measured using } \\
\text { various depres- } \\
\text { sion rating scales } \\
6\end{array}$ & - & $\begin{array}{l}\text { The } \\
\text { mean severity of } \\
\text { depressive symp- } \\
\text { toms in teacher/ } \\
\text { LHW-led inter- } \\
\text { vention groups } \\
\text { was } \\
\mathbf{0 .} \\
\mathbf{2 3} \text { standard de- } \\
\text { viations lower } \\
(0.45 \text { to } 0.22 \\
\text { lower) }\end{array}$ & $\begin{array}{l}\text { SMD }-\mathbf{0 . 2 3}(-0 . \\
45 \text { to }-0.22)\end{array}$ & $\begin{array}{l}504 \\
\text { (4 studies) }\end{array}$ & $\begin{array}{l}\oplus \oplus \bigcirc \bigcirc \\
\mathbf{l o w}^{7,8}\end{array}$ & - \\
\hline $\begin{array}{l}\text { Severity of de- } \\
\text { pressive symp- } \\
\text { toms in } \\
\text { LHW-led class- } \\
\text { room based in- } \\
\text { terventions, } \\
\text { short term (1-6 } \\
\text { months) } \\
\text { measured using } \\
\text { the DSRS }\end{array}$ & - & $\begin{array}{l}\text { The mean sever- } \\
\text { ity of depres- } \\
\text { sive symptoms in } \\
\text { classroom based) } \\
\text { LHW-led inter- } \\
\text { vention groups } \\
\text { was } \\
\mathbf{0 . 1 8} \text { lower } \\
(0.33 \text { to } 0.03 \\
\text { lower })\end{array}$ & $\begin{array}{l}\text { MCD }-\mathbf{0 . 1 8} \\
(-0.33 \text { to }-0.03)\end{array}$ & $\begin{array}{l}1092 \\
\text { (3 studies) }\end{array}$ & $\begin{array}{l}\oplus \oplus \bigcirc \bigcirc \\
\text { low }^{1,8}\end{array}$ & - \\
\hline $\begin{array}{l}\text { Severity of de- } \\
\text { pres- } \\
\text { sive symptoms } \\
\text { in narrative ex- } \\
\text { posure therapy, } \\
\text { medium term } \\
\text { (11 months) } \\
\text { measured } \\
\text { using the MINI } \\
\text { depression rating } \\
\text { scale }\end{array}$ & - & $\begin{array}{l}\text { The } \\
\text { mean severity of } \\
\text { depressive symp- } \\
\text { toms in teacher/ } \\
\text { LHW-led inter- } \\
\text { vention groups } \\
\text { was } \\
\mathbf{0 .} \\
\mathbf{0 2} \text { standard de- } \\
\text { viations lower } \\
\text { (0.52 lower to } 0 . \\
56 \text { higher) }\end{array}$ & $\begin{array}{l}\text { SMD }-\mathbf{0 . 0 2} \\
(-0.52 \text { to } 0.56)\end{array}$ & $\begin{array}{l}53 \text { participants } \\
\text { (1study) }\end{array}$ & $\begin{array}{l}\oplus \oplus \bigcirc \bigcirc \\
\text { low }^{5,9}\end{array}$ & - \\
\hline $\begin{array}{l}\text { Severity of anx- } \\
\text { iety symp- } \\
\text { toms in LHW- } \\
\text { led classroom } \\
\text { based interven- } \\
\text { tion, short term } \\
\text { (1-6 months) } \\
\text { measured by } \\
\text { SCARED }\end{array}$ & - & $\begin{array}{l}\text { The mean sever- } \\
\text { ity of anxi- } \\
\text { ety symptoms in } \\
\text { the intervention } \\
\text { groups was } \\
\mathbf{0 . 3 4} \text { lower } \\
(0.75 \text { lower to } 0 . \\
07 \text { higher) }\end{array}$ & $\begin{array}{l}\text { MD }-\mathbf{0 . 3 4} \\
(-0.75 \text { to } 0.07)\end{array}$ & $\begin{array}{l}1092 \\
\text { (3 studies) }\end{array}$ & $\begin{array}{l}\oplus \bigcirc \bigcirc \bigcirc \\
\text { very low } \mathbf{l o w}^{1,3}\end{array}$ & - \\
\hline
\end{tabular}


Table 12. SoF 8: NSHWs/OPHRs compared with usual care in conducting interventions for children with post-traumatic stress disorder and depression (RCTs) (Continued)

\begin{tabular}{|c|c|c|c|c|c|c|}
\hline $\begin{array}{l}\text { Functional im- } \\
\text { pair- } \\
\text { ment in LHW/ } \\
\text { teacher-led in- } \\
\text { terventions, } \\
\text { short term (1-6 } \\
\text { months) } \\
\text { measured by var- } \\
\text { ious functional } \\
\text { impairment } \\
\text { scales }{ }^{10}\end{array}$ & - & $\begin{array}{l}\text { The mean func- } \\
\text { tional impair- } \\
\text { ment in teacher- } \\
\text { led interventions } \\
\text { was } \\
\mathbf{0 .} \\
\mathbf{6 1} \text { standard de- } \\
\text { viations lower } \\
\text { (1.13 to } 0.08 \\
\text { lower) }\end{array}$ & $\begin{array}{l}\text { SMD }-\mathbf{0 . 6 1} \\
(-1.13 \text { to }-0.08)\end{array}$ & $\begin{array}{l}220 \\
\text { (2 studies) }\end{array}$ & $\begin{array}{l}\oplus \oplus \oplus \bigcirc \\
\text { moderate }^{11,12}\end{array}$ & - \\
\hline $\begin{array}{l}\text { Functional im- } \\
\text { pairment in } \\
\text { LHW-led class- } \\
\text { room-based in- } \\
\text { terventions, } \\
\text { short term (1-6 } \\
\text { months) } \\
\text { measured by var- } \\
\text { ious functional } \\
\text { impairment } \\
\text { scales }{ }^{13}\end{array}$ & - & $\begin{array}{l}\text { The mean func- } \\
\text { tional im- } \\
\text { pairment in CBI } \\
\text { (classroom } \\
\text { based) LHW-led } \\
\text { intervention } \\
\text { groups was } \\
\mathbf{0 . 8 1} \text { lower } \\
\text { (1.48 to } 0.13 \\
\text { lower) }\end{array}$ & $\begin{array}{l}\text { MCD }-\mathbf{0 . 8 1} \\
(-1.48 \text { to }-0.13)\end{array}$ & $\begin{array}{l}1092 \\
\text { (3 studies) }\end{array}$ & $\begin{array}{l}\oplus \oplus \bigcirc \bigcirc \\
\text { low }^{1,8}\end{array}$ & - \\
\hline $\begin{array}{l}\text { Functional im- } \\
\text { pairment, } \\
\text { in narrative ex- } \\
\text { posure therapy, } \\
\text { medium term } \\
\text { (11 months) } \\
\text { measured using } \\
\text { CAPS functional } \\
\text { impairment scale }\end{array}$ & - & $\begin{array}{l}\text { The mean func- } \\
\text { tional impair- } \\
\text { ment in LHW- } \\
\text { led narrative } \\
\text { exposure therapy } \\
\text { was } \\
\mathbf{0 . 6 9} \text { lower } \\
(1.25 \text { to } 0.14 \\
\text { lower) }\end{array}$ & $\begin{array}{l}\text { SMD }-\mathbf{0 . 6 9} \\
(-1.25 \text { to }-0.14)\end{array}$ & $\begin{array}{l}53 \\
\text { (1 study) }\end{array}$ & $\begin{array}{l}\oplus \oplus \oplus \bigcirc \\
\text { moderate }^{14}\end{array}$ & - \\
\hline
\end{tabular}

*The basis for the assumed risk the mean control group risk across studies for pooled results and the control group risk for single studies. The corresponding risk (and its $95 \%$ confidence interval) is based on the assumed risk in the comparison group and the relative effect of the intervention (and its $95 \% \mathrm{CI}$ ).

CAPS: Clinical Administered PTSD Scale; CBI: classroom-room-based intervention; CI: confidence interval; CPSS: Child Posttraumatic Stress Scale; LHW: lay health worker; MCD: mean change difference; MINI: Mini International Neuropsychiatry Interview; NSHWs: non-specialist health worker; OPHRs: other professionals with health roles; PTSD: post-traumatic stress disorder; SMD: standardised mean difference; SCARED: Screen for Child Anxiety Related Disorders.

GRADE Working Group grades of evidence

High quality: Further research is very unlikely to change our confidence in the estimate of effect.

Moderate quality: Further research is likely to have an important impact on our confidence in the estimate of effect and may change the estimate.

Low quality: Further research is very likely to have an important impact on our confidence in the estimate of effect and is likely to change the estimate.

Very low quality: We are very uncertain about the estimate.

Non-specialist health worker interventions for the care of mental, neurological and substance-abuse disorders in low- and middle- 
${ }^{1}$ Very serious study limitations: Tol 2012 C-RCT SriLanka: uncertainty about random sequence generation and allocation concealment. Tol 2008 C-RCT Indonesia: outcome assessment not blind: child self ratings with help of assessors who were not blinded to treatment condition. Jordans 2010 C-RCT Nepal: unclear allocation concealment and non-blinded assessment of outcomes. Downgraded by 2.

${ }^{2}$ Very serious inconsistency: $\mathrm{I}^{2}$ statistic $=82 \%$. The inconsistency is related to the direction of effect. Downgraded by 2 .

${ }^{3}$ Serious imprecision: The $95 \%$ CI for the pooled estimates indicates appreciable benefit for intervention group and non-appreciable benefit for usual care. Downgraded by 1 .

${ }^{4}$ Serious imprecision: The $95 \%$ CI for the pooled estimates indicates appreciable benefit for intervention group and non-appreciable benefit for usual care. The sample size was also small. Downgraded by 1 .

${ }^{5}$ No indirectness: There was only one study, therefore, the generalisability of this results to other settings is compromised. However, this also resulted in imprecision in the effect estimate, and hence the quality of evidence was not further downgraded.

${ }^{6}$ Berger2009 CRCT SriLanka: Becks Depression Inventory; Bolton 2007 RCT Uganda: Acholi Psychosocial Assessment Instrument; Dybdahl 2001 RCT Bosnia: Birleson's depression inventory; Ertl 2011 RCT Uganda: MINI.

7 Very serious study limitations: Berger2009 CRCT SriLanka: no allocation concealment, likely contamination and outcomes not adjusted for clustering; Dybdahl 2001 RCT Bosnia: not clear if allocation concealed; differences in baseline characteristics incomplete outcome data (denominators not provided by intervention or control for each of the tests), and likelihood of contamination as both intervention and control in same camps; Bolton 2007 RCT Uganda had unclear allocation concealment, baseline characteristics (age) were different and not adjusted for in analysis, likely risk of contamination between children in both camps. Downgraded by 2 .

${ }^{8}$ No imprecision: appreciable benefit for LHW/teacher-led care. Not downgraded.

${ }^{9}$ Very serious imprecision: The $95 \%$ CI for the pooled estimates indicates appreciable benefit for intervention group and appreciable benefit for usual care. The sample size is also small. Downgraded by 2 .

${ }^{10}$ Berger2009 CRCT SriLanka:: Child Diagnostic Interview Schedule (CDIS); Ertl 2011 RCT Uganda: CAPS - Functional Impairment Section.

${ }^{11}$ No imprecision: appreciable benefit for LHW/teacher-led care. Not downgraded.

12 Serious study limitations: Berger2009 CRCT SriLanka: no allocation concealment, likely contamination and outcomes not adjusted for clustering. Downgraded by 1 .

13 Jordans 2010 C-RCT Nepal: Children’s Functional Impairment; Tol 2008 C-RCT Indonesia; Tol 2012 C-RCT SriLanka: Functional impairment Score (FIS). Data were change scores and hence SMD could not be used. MD is likely to be misleading but since two of the three trials used the same scale this may not be important. Removal of data from Jordans 2010 C-RCT Nepal did not appreciably alter effect estimates.

${ }^{14}$ Serious imprecision: appreciable benefit for LHW/teacher-led care. However, the sample size is small. Downgraded by 1.

Table 13. SoF: NSHWs/OPHRs compared with usual care in conducting interventions for children with PTSD in low- and middle-income countries (CBAs)

What are the effects of NSHWs/OPHRs compared with usual care in conducting interventions for children with PTSD for mental health care in low- and middle-income countries? (additional CBA outcomes for comparison 8)

Patient or population: Children with PTSD

Settings: Low- and middle-income countries (Palestine, Turkey)

Intervention: Teachers delivering psychoeducational and other interventions

Comparison: Usual care

\begin{tabular}{|l|l|l|l|l|l|}
\hline Outcomes & $\begin{array}{l}\text { Illustrative } \\
(95 \% \mathrm{CI})\end{array}$ & comparative risks* & $\begin{array}{l}\text { Relative effect } \\
(95 \% \mathrm{CI})\end{array}$ & $\begin{array}{l}\text { No of Partici- } \\
\text { pants } \\
\text { (studies) }\end{array}$ & $\begin{array}{l}\text { Quality of the Comments } \\
\text { evidence } \\
\text { (GRADE) }\end{array}$ \\
\cline { 1 - 4 } Assumed risk & $\begin{array}{l}\text { Corresponding } \\
\text { risk }\end{array}$ & &
\end{tabular}

Non-specialist health worker interventions for the care of mental, neurological and substance-abuse disorders in low- and middle- 


\begin{tabular}{|c|c|c|c|c|c|c|}
\hline & Usual care & $\begin{array}{l}\text { NSHWs/ } \\
\text { OPHRs }\end{array}$ & & & & \\
\hline $\begin{array}{l}\text { Severity } \\
\text { of PTSD symp- } \\
\text { toms, medium } \\
\text { and short term } \\
\text { (2-36 months) } \\
\text { measured } \\
\text { using the PTSD } \\
\text { symptom scale - } \\
\text { CPTSD-RI }\end{array}$ & - & $\begin{array}{l}\text { The mean } \\
\text { severity of PTSD } \\
\text { symptoms in the } \\
\text { medium and } \\
\text { short term (com- } \\
\text { bined) in the in- } \\
\text { tervention } \\
\text { groups was } \\
\text { 0.1 standard de- } \\
\text { viations lower } \\
(0.34 \text { lower to } 0 . \\
14 \text { higher) }\end{array}$ & $\begin{array}{l}\text { SMD }-0.10(-0 . \\
34 \text { to } 0.14)\end{array}$ & $\begin{array}{l}351 \\
\text { (2 studies) }\end{array}$ & $\begin{array}{l}\oplus \bigcirc \bigcirc \bigcirc \\
\text { very low }\end{array}$ & - \\
\hline $\begin{array}{l}\text { Severity } \\
\text { of PTSD symp- } \\
\text { toms } \\
\text { - short term (2 } \\
\text { months) } \\
\text { measured } \\
\text { using the PTSD } \\
\text { symptom scale - } \\
\text { CPTSD-RI }\end{array}$ & - & $\begin{array}{l}\text { The mean sever- } \\
\text { ity } \\
\text { of PTSD symp- } \\
\text { toms - short term } \\
\text { (within } \\
2 \text { months) in the } \\
\text { intervention } \\
\text { groups was } \\
\text { 0.1 standard de- } \\
\text { viations higher } \\
\text { (0.41 lower to } 0 \text {. } \\
62 \text { higher) }\end{array}$ & $\begin{array}{l}\text { SMD } 0.1 \quad(-0.41 \\
\text { to } 0.62)\end{array}$ & $\begin{array}{l}64 \\
\text { (1 study) }\end{array}$ & $\begin{array}{l}\oplus \bigcirc \bigcirc \bigcirc \\
\text { very low } \mathbf{l o w}^{1,2}\end{array}$ & $\begin{array}{l}\text { Thabet } 2005 \\
\text { CBA Palestine }\end{array}$ \\
\hline $\begin{array}{l}\text { Severity } \\
\text { of PTSD symp- } \\
\text { toms - long } \\
\text { term (3 years) } \\
\text { measured } \\
\text { using the PTSD } \\
\text { symptom scale - } \\
\text { CPTSD-RI }\end{array}$ & - & $\begin{array}{l}\text { The mean sever- } \\
\text { ity } \\
\text { of PTSD symp- } \\
\text { toms - long term } \\
\text { - in the interven- } \\
\text { tion groups was } \\
\mathbf{0 .} \\
\mathbf{1 6} \text { standard de- } \\
\text { viations lower } \\
(0.43 \text { lower to } 0 \text {. } \\
12 \text { higher) }\end{array}$ & $\begin{array}{l}\text { SMD }-0.16(-0 . \\
43 \text { to } 0.12)\end{array}$ & $\begin{array}{l}287 \\
\text { (1 study) }\end{array}$ & $\begin{array}{l}\oplus \bigcirc \bigcirc \bigcirc \\
\text { very low } \mathbf{l o w}^{1,2}\end{array}$ & $\begin{array}{l}\text { Wolmer } 2005 \\
\text { CBA Turkey }\end{array}$ \\
\hline $\begin{array}{l}\text { Severity } \\
\text { of CMDs (2-12 } \\
\text { months) } \\
\text { measured us- } \\
\text { ing CMD sever- } \\
\text { ity scores }\end{array}$ & - & $\begin{array}{l}\text { The mean sever- } \\
\text { ity of CMDs in } \\
\text { the intervention } \\
\text { groups was } \\
\mathbf{0 .} \\
\mathbf{2 5} \text { standard de- } \\
\text { viations lower } \\
(0.46 \text { to } 0.04\end{array}$ & $\begin{array}{l}\text { SMD }-0.25(-0 \\
46 \text { to }-0.04)\end{array}$ & $\begin{array}{l}459 \\
\text { ( } 2 \text { studies) }\end{array}$ & $\begin{array}{l}\oplus \bigcirc \bigcirc \bigcirc \\
\text { very low } \\
4,5\end{array}$ & $\begin{array}{l}\text { Both Thabet } \\
2005 \text { CBA } \\
\text { Palestine (st) and } \\
\text { Loughry } 2006 \\
\text { CBA Palestin } \\
\text { (LT) }\end{array}$ \\
\hline
\end{tabular}

Non-specialist health worker interventions for the care of mental, neurological and substance-abuse disorders in low- and middle- 
Table 13. SoF: NSHWs/OPHRs compared with usual care in conducting interventions for children with PTSD in low- and middle-income countries (CBAs) (Continued)

\begin{tabular}{|c|c|c|c|c|c|}
\hline & lower) & & & & \\
\hline $\begin{array}{l}\text { Severity } \\
\text { of CMDs psy- } \\
\text { chosocial inter- } \\
\text { ven- } \\
\text { tion, short term } \\
\text { ( } 2 \text { months) } \\
\text { measured using } \\
\text { the Child De- } \\
\text { pression Inven- } \\
\text { tory }\end{array}$ & $\begin{array}{l}\text { The mean sever- } \\
\text { ity of CMDs - } \\
\text { short term - in } \\
\text { psychosocial in- } \\
\text { terventions was } \\
\mathbf{0 .} \\
\mathbf{1 2} \text { standard de- } \\
\text { viations lower } \\
\text { (0.63 lower to } 0 . \\
4 \text { higher) }\end{array}$ & $\begin{array}{l}\text { SMD }-0.12(-0 . \\
63 \text { to } 0.4)\end{array}$ & $\begin{array}{l}64 \\
\text { (1 study) }\end{array}$ & $\begin{array}{l}\oplus \bigcirc \bigcirc \bigcirc \\
\text { very } \text { low }^{4,6}\end{array}$ & $\begin{array}{l}\text { Thabet } 2005 \\
\text { CBA Palestine } \\
\text { only }\end{array}$ \\
\hline $\begin{array}{l}\text { Severity } \\
\text { of CMDs social } \\
\text { intervention } \\
\text { - medium term } \\
\text { (12 months) } \\
\text { measured using } \\
\text { the CBCL in- } \\
\text { ternalising score } \\
\text { (depression, anx- } \\
\text { iety, somatic } \\
\text { symptoms, with- } \\
\text { drawn) }\end{array}$ & $\begin{array}{l}\text { The mean sever- } \\
\text { ity of CMDs - } \\
\text { medium term - } \\
\text { in social inter- } \\
\text { ventions was } \\
\text { 0. } \\
\mathbf{2 7} \text { standard de- } \\
\text { viations lower } \\
(0.5 \text { to } 0.04 \\
\text { lower) }\end{array}$ & $\begin{array}{l}\text { SMD }-0.27(-0.5 \\
\text { to }-0.04)\end{array}$ & $\begin{array}{l}395 \\
\text { (1 study) }\end{array}$ & $\begin{array}{l}\oplus \bigcirc \bigcirc \bigcirc \\
\text { very } \text { low }^{4,5}\end{array}$ & $\begin{array}{l}\text { Loughry } 2006 \\
\text { CBA Palestin } \\
\text { only }\end{array}$ \\
\hline
\end{tabular}

*The basis for the assumed risk (e.g. the median control group risk across studies) is provided in footnotes. The corresponding risk (and its $95 \% \mathrm{CI}$ ) is based on the assumed risk in the comparison group and the relative effect of the intervention (and its $95 \% \mathrm{CI}$ ). CBA: controlled before-and-after; CBCL: Child Behaviour Checklist; CI: confidence interval; CMD: common mental disorders; NSHWs: non-specialist health worker; OPHRs: other professionals with health roles; PTSD: post-traumatic stress disorder; SMD: standardised mean difference.

GRADE Working Group grades of evidence

High quality: Further research is very unlikely to change our confidence in the estimate of effect.

Moderate quality: Further research is likely to have an important impact on our confidence in the estimate of effect and may change the estimate.

Low quality: Further research is very likely to have an important impact on our confidence in the estimate of effect and is likely to change the estimate.

Very low quality: We are very uncertain about the estimate.

${ }^{1}$ No imprecision: Unappreciable benefit for both NSHW care and usual care.

${ }^{2}$ Very serious risk of bias: both CBA studies so non-randomised and no allocation concealment. Also Thabet 2005 CBA Palestine: differences in baseline outcomes; Wolmer 2005 CBA Turkey: unvalidated tools in this setting so uncertain reliability of outcomes.

3 Thabet 2005 CBA Palestine: Child Depression Inventory; Loughry 2006 CBA Palestin: CBCL internalising score (depression, anxiety, somatic symptoms, withdrawn).

${ }^{4}$ Very serious risk of bias: both CBAs so non-randomised and no allocation concealment. Also Thabet 2005 CBA Palestine: differences in baseline outcomes; Loughry 2006 CBA Palestin: differences in baseline outcomes and characteristics and risk of contamination and the intervention between sites differed slightly making comparisons difficult.

${ }^{5}$ No imprecision: Non-appreciable, and possibly appreciable benefit for intervention group.

${ }^{6}$ Very serious imprecision: Appreciable benefit for intervention and non-appreciable benefit for control group.

Non-specialist health worker interventions for the care of mental, neurological and substance-abuse disorders in low- and middle- 
Table 14. Summary of costs and resource use from included studies

\begin{tabular}{|c|c|c|c|c|}
\hline Author/year & $\begin{array}{l}\text { Type of economic eval- } \\
\text { uation }\end{array}$ & Study population & Intervention & Economic results \\
\hline Araya 2003 RCT Chile & $\begin{array}{l}\text { Cost-effectiveness analy- } \\
\text { sis }\end{array}$ & Women with depression & $\begin{array}{l}\text { Collaborative inter- } \\
\text { vention (doctors, non- } \\
\text { medical professionals su- } \\
\text { pervised by psychiatrist) } \\
\text { with stepped care, multi- } \\
\text { component programme } \\
\text { compared with usual } \\
\text { care in depressed women } \\
\text { in Chile }\end{array}$ & $\begin{array}{l}\text { Incremental cost per per- } \\
\text { son for improved care } \\
\text { was USD } 37.6 \text { more than } \\
\text { usual care. Unit cost } \\
\text { to obtain } 1 \text { additional } \\
\text { depression-free day was } \\
\text { USD } 0.75\end{array}$ \\
\hline Jordans 2011 & Cost analysis & $\begin{array}{l}\text { Children with PTSD (7- } \\
15 \text { years) }\end{array}$ & $\begin{array}{l}\text { LHW-led multilayered } \\
\text { package (including class- } \\
\text { room-based inter- } \\
\text { vention, non-therapeu- } \\
\text { tic resilience groups, psy- } \\
\text { choeducation and coun- } \\
\text { selling) (data extracted } \\
\text { from Sri Lanka and In- } \\
\text { donesia as related to Tol } \\
2008 \text { C-RCT Indonesia } \\
\text { and Tol } 2012 \text { C-RCT } \\
\text { SriLanka) }\end{array}$ & $\begin{array}{l}\text { Mean cost per user of to- } \\
\text { tal package: } \\
\text { Indonesia: USD } 21 \text {. } \\
77 \text { ( } 59 \% \text { of which is hu- } \\
\text { man resources cost). Sri } \\
\text { Lanka: USD } 8.85 \text { ( } 56 \% \\
\text { of which is human re- } \\
\text { sources cost) }\end{array}$ \\
\hline $\begin{array}{l}\text { Zambori } 2002 \text { CBA } \\
\text { Hungary }\end{array}$ & Cost analysis & $\begin{array}{l}\text { Patients with anxiety and } \\
\text { mood disorders }\end{array}$ & $\begin{array}{l}\text { Primary } \\
\text { physicians versus psychi- } \\
\text { atrists in prescribing ser- } \\
\text { traline in Hungary }\end{array}$ & $\begin{array}{l}\text { Absenteeism } \\
\text { reduced from } 15.7 \text { to } 6 . \\
8 \text { days and costs of non- } \\
\text { psychiatric prescriptions } \\
\text { decreased from USD138 } \\
\text { to USD91.8 per year. } \\
\text { Laboratory costs } \\
\text { ranged from USD6.4 to } \\
\text { USD11.5 }\end{array}$ \\
\hline
\end{tabular}

LHW: lay health worker; PTSD: post-traumatic stress disorder.

Table 15. Agreements and disagreements with related reviews

\begin{tabular}{|c|c|c|c|}
\hline Author/year & Summary of review & Agreements & Disagreements/differences \\
\hline Parker 2008 & $\begin{array}{l}\text { Reviewed consultation liaison in pri- } \\
\text { mary care - HICs }\end{array}$ & - & $\begin{array}{l}\text { Our review process did not find any } \\
\text { consultation liaison in primary care } \\
\text { in LMICs so results cannot be com- } \\
\text { pared }\end{array}$ \\
\hline
\end{tabular}

Non-specialist health worker interventions for the care of mental, neurological and substance-abuse disorders in low- and middle- 
Table 15. Agreements and disagreements with related reviews (Continued)

\begin{tabular}{|lll} 
Boer 2005 & $\begin{array}{l}\text { Reviewed paraprofessionals in deliv- } \\
\text { ering psychological interventions for } \\
\text { anxiety and depression (HIC only) }\end{array}$ & $\begin{array}{l}\text { Included studies were from HICs Some of their paraprofessionals } \\
\text { only, but support our findings that } \\
\text { non-professional care is generally } \\
\text { equivalent to professional care (this } \\
\text { weviews equivalent of specialist care) }\end{array}$ \\
& $\begin{array}{l}\text { ist health workers in our review } \\
\text { and that non-professional care is }\end{array}$ \\
\hline
\end{tabular}

Bower 2006 Reviewed the effect of collaborative Bower found improvement of ancare models on antidepressant use All included studies were from HICs except for

Araya 2003 RCT Chile tidepressant use, particularly in studies where the case manager had a mental health background, where there was adequate supervision and where there was systematic identification of patients (rather than waiting for a referral)
Woltmann 2012 Review on collaborative care/ They also found a statistically sigchronic care management nificant effect on reduction in de-
We were not able to assess, as did Bower, whether lengths of training, supervision or other intervention characteristics modified these outcomes because only 5 studies were included in this comparison pression severity among the $14 \mathrm{HIC}$ studies that were included in the meta-analysis (SMD 0.31, 95\% CI 0.16 to 0.47 ) (Araya and Patel's studies were included in the narrative review but did not qualify for their meta-analysis). The authors suggested that collaborative care is of moderate benefit; however, Woltmann has estimated a more conservative value of SMD > 0.5 to show moderate benefit (from the analysis of scales and how to interpret their SMDs). Our meta-analyses of collaborative care models suggested similar improvements in symptoms and recovery from depression or CMDs (same direction of effect, and similar magnitude)
Reviewed the effect of CBT and group CBT

Systematic review on mental health interventions in humanitarian settings
Woltman's chronic care management had a stricter definition to our collaborative care definition
Tol 2011
Huntley also found that LHW-led psychological interventions are effective in the short and medium term in reducing symptoms of depression
Huntley described the effect of CBT and group CBT (rather than the effect of NSHWs)

Tol found similar results to our review for school-based interventions for children with PTSD (i.e. no significant benefit) (an extra study
This review differed from ours in that it included studies of both NSHWs/OPHRs and specialists, according to our definitions 
Table 15. Agreements and disagreements with related reviews (Continued)

\begin{tabular}{|c|c|c|c|}
\hline & & $\begin{array}{l}\text { was included in this comparison, } \\
\text { which we had excluded as it did } \\
\text { not meet our NSHW/OPHR defini- } \\
\text { tions). This review went further and } \\
\text { found a statistically significant bene- } \\
\text { fit for improving internalising symp- } \\
\text { toms (SMD }-0.34,95 \% \text { CI }-0.40 \text { to } \\
-0.09 \text { ). For adults, a potential bene- } \\
\text { fit of interventions was also seen }\end{array}$ & \\
\hline Rahman 2013 & $\begin{array}{l}\text { Systematic review on interventions } \\
\text { for common perinatal mental disor- } \\
\text { ders in women in LMICs }\end{array}$ & $\begin{array}{l}\text { This was similar but a more in- } \\
\text { depth review of our perinatal depres- } \\
\text { sion pooled comparison, which also } \\
\text { looked at LHW-led interventions } \\
\text { for mothers with perinatal depres- } \\
\text { sion. Their final pooled outcome } \\
\text { was similar in magnitude and direc- } \\
\text { tion to ours for our perinatal depres- } \\
\text { sion category (SMD - } 0.38,95 \% \mathrm{CI}- \\
0.56 \text { to }-0.21 \text { ) vs, our findings (SMD } \\
-0.42,95 \% \text { CI }-0.58 \text { to }-0.26 \text { ) }\end{array}$ & $\begin{array}{l}\text { This review differed from ours in } \\
\text { that its study's inclusion criteria were } \\
\text { broader as it included studies that } \\
\text { measured maternal (all perinatal dis- } \\
\text { orders) or child (or both) outcomes } \\
\text { even if the intervention was not pri- } \\
\text { marily targeted at these groups. It } \\
\text { also reported child outcomes, which } \\
\text { ours did not }\end{array}$ \\
\hline
\end{tabular}

CBT: cognitive behavioural therapy; CI: confidence interval; CMD: common mental disorders; HIC: high-income country; LHW: lay health worker; LMIC: low- and medium-income countries; NSHW: non-specialist health worker; OPHR: other professionals with health roles; PTSD: post-traumatic stress disorder; SMD: standardised mean difference.

\section{AP PEN DICES}

\section{Appendix I. Search strategies \\ CENTRAL}

\#1 MeSH descriptor Allied Health Personnel, this term only

\#2 MeSH descriptor Community Health Workers, this term only

\#3 MeSH descriptor Nurses' Aides, this term only

\#4 MeSH descriptor Psychiatric Aides, this term only

\#5 MeSH descriptor Caregivers, this term only 
(Continued)

\#6 MeSH descriptor Voluntary Workers, this term only

\#7 MeSH descriptor Community Networks, this term only

\#8 MeSH descriptor Self-Help Groups explode all trees

\#9 MeSH descriptor Social Support, this term only

\#10 MeSH descriptor Health Manpower, this term only

\#11 MeSH descriptor Personnel Staffing and Scheduling, this term only

\#12 (lay NEAR/3 (worker* or visitor* or attendant* or aide or aides or support* or person* or helper* or carer* or caregiver* or "care giver" or "care givers" or consultant* or advisor* or counselor* or counsellor* or assistant* or staff)):ti,ab

\#13 ((voluntary or volunteer*) NEAR/3 (worker* or visitor* or attendant* or aide or aides or support* or person* or helper* or carer* or caregiver* or "care giver" or "care givers" or consultant* or advisor* or counselor* or counsellor* or assistant* or staff) ):ti,ab

\#14 (untrained NEAR/3 (worker* or visitor* or attendant* or aide or aides or support* or person* ${ }^{*}$ or helper* ${ }^{*}$ or carer* ${ }^{*}$ or caregiver* or "care giver" or "care givers" or consultant* or advisor* or counselor* or counsellor* or assistant* or staff or nurse* or doctor* or physician* or therapist*)):ti,ab

\#15 (trained NEAR/3 (worker* or visitor* or attendant* or aide or aides or support* or person* or helper* or carer* or caregiver* or "care giver" or "care givers" or consultant* or advisor* or counselor* or counsellor* or assistant* or staff or nurse* or doctor* or physician* or therapist*)):ti,ab

\#16 (unlicensed NEAR/3 (worker* or visitor* or attendant* or aide or aides or support* or person* or helper* or carer* ${ }^{*}$ or caregiver* or "care giver" or "care givers" or consultant* or advisor* or counselor* or counsellor* or assistant* or staff or nurse* or doctor* or physician* or therapist*)):ti,ab

\#17 ((nonprofessional* or "non professional" or "non professionals) NEAR/3 (worker* or visitor* or attendant* or aide or aides or support* or person* or helper* or carer* or caregiver* or "care giver" or "care givers" or consultant* or advisor* or counselor* or counsellor* or assistant* or staff)):ti,ab

\#18 (("non medical" or "non health" or "non healthcare " or "non health care") NEAR/3 (worker* or visitor* or attendant* or aide or aides or support* or person* or helper* or carer* or caregiver* or "care giver" or "care givers" or consultant* or advisor* or counselor* ${ }^{*}$ or counsellor* or assistant* ${ }^{*}$ staff)):ti,ab

\#19 (community NEAR/3 (worker* or visitor* or attendant* or aide or aides or support* or person* or helper* or carer* or caregiver* or "care giver" or "care givers" or consultant* or advisor* or counselor* or counsellor* or assistant* or staff)):ti,ab

\#20 (paraprofessional* or paramedic or paramedics or "paramedical worker" or "paramedical workers" or "paramedical personnel" or "allied health personnel" or "allied health worker" or "allied health workers" or support NEXT worker* or "non NEXT specialist* or "specially trained" or barefoot NEXT doctor* or nurse* NEXT aide* or psychiatric NEXT aide* or psychiatric NEXT attendant* or social NEXT worker* or teacher* or "school staff” or trainer*):ti,ab

\#21 ((health* or medical*) NEAR/3 (auxiliary or auxiliaries)):ti,ab

Non-specialist health worker interventions for the care of mental, neurological and substance-abuse disorders in low- and middle- 


\section{(Continued)}

\#22 (nurse* NEAR/1 (auxiliary or auxiliaries)):ti,ab

\#23 (informal NEXT (caregiver* or “care giver” or “care givers” or carer*)):ti,ab

\#24 (“self help group” or "self help groups” or “support group” or “support groups”):ti,ab

\#25 ((social or psychosocial) NEXT (care or support)):ti,ab

\#26 (village NEAR/3 worker*):ti,ab

\#27 “community based”:ti,ab

\#28 (community NEAR/3 intervention*):ti,ab

\#29 (“community network” or “community networks”):ti,ab

\#30 ((health or "health care" or healthcare) NEXT manpower):ti,ab

\#31 “human resources”:ti,ab

\#32 (task NEAR/3 shift* or taskshift*):ti,ab

\#33 (staff* NEAR/3 chang*):ti,ab

\#34 \#1 OR \#2 OR \#3 OR \#4 OR \#5 OR \#6 OR \#7 OR \#8 OR \#9 OR \#10 OR \#11 OR \#12 OR \#13 OR \#14 OR \#15 OR \# 16 OR \#17 OR \#18 OR \#19 OR \#20 OR \#21 OR \#22 OR \#23 OR \#24 OR \#25 OR \#26 OR \#27 OR \#28 OR \#29 OR \# 30 OR \#31 OR \#32 OR \#33)

\#35 MeSH descriptor Mentally Ill Persons, this term only

\#36 MeSH descriptor Mentally Disabled Persons, this term only

\#37 MeSH descriptor Mental Disorders explode all trees

\#38 MeSH descriptor Drug Users, this term only

\#39 MeSH descriptor Nervous System Diseases, this term only

\#40 MeSH descriptor Epilepsy, this term only

\#41 MeSH descriptor Mental Health Services, this term only

\#42 MeSH descriptor Community Mental Health Services, this term only

\#43 MeSH descriptor Emergency Services, Psychiatric, this term only

\#44 MeSH descriptor Social Work, Psychiatric, this term only

Non-specialist health worker interventions for the care of mental, neurological and substance-abuse disorders in low- and middleincome countries (Review)

Copyright $\odot 2013$ The Cochrane Collaboration. Published by John Wiley \& Sons, Ltd. 
\#45 ((mentally or psycholog*) NEXT (ill or disabled or handicapped or retarded or disturb* or traumati* or deficient)):ti,ab

\#46 (intellectually NEXT (disabled or handicapped or retarded or deficient)):ti,ab

\#47 (mental NEXT (retardation or deficienc*)):ti,ab

\#48 ((mental or behavioural or behavioral or anxiety or obsessive or compulsive or panic or phobic or schizotypal or delusional or stress or cognitive or cognition or dissociative or personality or "impulse control" or mood or affective or bipolar or depressive or neurotic or paranoid or psychotic or somatoform or neurologic* or nervous or "nervous system" or eating) NEXT (disorder* or illness* or disease*)):ti,ab

\#49 ((“substance related” or alcohol or opioid or morphine or marijuana or heroin or cocaine) NEXT (disorder* or illness* or dependence or abuse or misuse)):ti,ab

\#50 (depression or anxiety or schizophrenia or psychoses or stress NEXT syndrome* or distress NEXT syndrome* or combat NEXT disorder* or war NEXT disorder* or pain NEXT disorder* or dementia or Alzheimer* or epilepsy or down* NEXT syndrome or alcoholism or "substance abuse" or drug NEXT addict* or drug NEXT abus* or "drug misuse" or drug NEXT user*):ti,ab

\#51 (psychiatric NEXT (patient* or service* or care or assistance or help or work)):ti,ab

\#52 ("mental health service" or "mental health services" or "mental health care" or "mental healthcare" or "mental care"):ti,ab

\#53 ((psychiatric or psychosocial) NEXT (service* or care or assistance or help or work)):ti,ab

\#54 \#35 OR \#36 OR \#37 OR \#38 OR \#39 OR \#40 OR \#41 OR \#42 OR \#43 OR \#44 OR \#45 OR \#46 OR \#47 OR \#48 OR \#49 OR \#50 OR \#51 OR\#52 OR\#53)

\#55 MeSH descriptor Developing Countries, this term only

\#56 (Africa or Asia or Caribbean or "West indies" or "South America” or "Latin America” or "Central America"):ti,ab,kw

\#57 Afghanistan or Albania or Algeria or Angola or Antigua or Barbuda or Argentina or Armenia or Armenian or Aruba or Azerbaijan or Bahrain or Bangladesh or Barbados or Benin or Byelarus or Byelorussian or Belarus or Belorussian or Belorussia or Belize or Bhutan or Bolivia or Bosnia or Herzegovina or Hercegovina or Botswana or Brasil or Brazil or Bulgaria or "Burkina Faso" or "Burkina Fasso" or "Upper Volta" or Burundi or Urundi or Cambodia or "Khmer Republic" or Kampuchea or Cameroon or Cameroons or Cameron or Camerons or "Cape Verde" or "Central African Republic" or Chad or Chile or China or Colombia or Comoros or "Comoro Islands" or Comores or Mayotte or Congo or Zaire or "Costa Rica" or "Cote d'Ivoire" or "Ivory Coast" or Croatia or Cuba or Cyprus or Czechoslovakia or "Czech Republic" or Slovakia or "Slovak Republic"):ti, $\mathrm{ab}, \mathrm{kw}$

\#58 (Djibouti or "French Somaliland" or Dominica or "Dominican Republic" or "East Timor" or "East Timur" or "Timor Leste” or Ecuador or Egypt or "United Arab Republic" or "El Salvador" or Eritrea or Estonia or Ethiopia or Fiji or Gabon or "Gabonese Republic" or Gambia or Gaza or Georgia or Georgian or Ghana or "Gold Coast" or Greece or Grenada or Guatemala or Guinea or Guam or Guiana or Guyana or Haiti or Honduras or Hungary or India or Maldives or Indonesia or Iran or Iraq or "Isle of Man" or Jamaica or Jordan or Kazakhstan or Kazakh or Kenya or Kiribati or Korea or Kosovo or Kyrgyzstan or Kirghizia or "Kyrgyz Republic" or Kirghiz or Kirgizstan or "Lao PDR" or Laos or Latvia or Lebanon or Lesotho or Basutoland or Liberia or Libya or Lithuania):ti,ab,kw 
(Continued)

\#59 (Macedonia or Madagascar or "Malagasy Republic" or Malaysia or Malaya or Malay or Sabah or Sarawak or Malawi or Nyasaland or Mali or Malta or "Marshall Islands" or Mauritania or Mauritius or "Agalega Islands" or Mexico or Micronesia or "Middle East" or Moldova or Moldovia or Moldovian or Mongolia or Montenegro or Morocco or Ifni or Mozambique or Myanmar or Myanma or Burma or Namibia or Nepal or "Netherlands Antilles" or "New Caledonia" or Nicaragua or Niger or Nigeria or "Northern Mariana Islands" or Oman or Muscat or Pakistan or Palau or Palestine or Panama or Paraguay or Peru or Philippines or Philipines or Phillipines or Phillippines or Poland or Portugal or "Puerto Rico"):ti,ab,kw

\#60 (Romania or Rumania or Roumania or Russia or Russian or Rwanda or Ruanda or "Saint Kitts" or "St Kitts" or Nevis or "Saint Lucia" or "St Lucia" or "Saint Vincent" or "St Vincent" or Grenadines or Samoa or "Samoan Islands" or "Navigator Island" or "Navigator Islands" or "Sao Tome" or "Saudi Arabia" or Senegal or Serbia or Montenegro or Seychelles or "Sierra Leone" or Slovenia or "Sri Lanka" or Ceylon or "Solomon Islands" or Somalia or "South Africa" or Sudan or Suriname or Surinam or Swaziland or Syria or Tajikistan or Tadzhikistan or Tadjikistan or Tadzhik or Tanzania or Thailand or Togo or "Togolese Republic" or Tonga or Trinidad or Tobago or Tunisia or Turkey or Turkmenistan or Turkmen or Uganda or Ukraine or Uruguay or USSR or "Soviet Union" or "Union of Soviet Socialist Republics" or Uzbekistan or Uzbek or Vanuatu or "New Hebrides" or Venezuela or Vietnam or "Viet Nam" or "West Bank" or Yemen or Yugoslavia or Zambia or Zimbabwe or Rhodesia):ti,ab, $\mathrm{kw}$

\#61 (developing or less* NEXT developed or "under developed" or underdeveloped or "middle income" or low* NEXT income or underserved or "under served" or deprived or poor*) NEXT (countr* or nation* or population* or world):ti,ab,kw

\#62 (developing or less* NEXT developed or "under developed" or underdeveloped or "middle income" or low* NEXT income) NEXT (economy or economies):ti,ab,kw

\#63 low* NEXT (gdp or gnp or "gross domestic" or "gross national"):ti,ab,kw

\#64 (low NEAR/3 middle NEAR/3 countr*):ti,ab,kw

\#65 (lmic or lmics or "third world" or "lami country" or "lami countries"):ti,ab,kw

\#66 ("transitional country" or "transitional countries"):ti,ab,kw

\#67 (\#55 OR \#56 OR \#57 OR \#58 OR \#59 OR \#60 OR \#61 OR \#62 OR \#63 OR \#64 OR \#65 OR \#66)

\#68 (\#34 AND \#54 AND \#67)

\#69 (\#68) [Trials]

MEDLINE In-Process and other non-indexed citations and MEDLINE (OvidSP)

\begin{tabular}{lll}
\hline 1 & Allied Health Personnel/ & 9631 \\
\hline 2 & Community Health Workers/ & 2765 \\
\hline 3 & Nurses' Aides/ & 3368
\end{tabular}

Non-specialist health worker interventions for the care of mental, neurological and substance-abuse disorders in low- and middle- 
(Continued)

\begin{tabular}{lll}
\hline 4 & Psychiatric Aides/ & 367 \\
\hline 5 & Caregivers/ & 18,003 \\
\hline 6 & Voluntary Workers/ & 6700 \\
\hline 7 & Community Networks/ & 4699 \\
\hline 8 & exp Self-Help Groups/ & 8279 \\
\hline 9 & Social Support/ & 45,043 \\
\hline 10 & Health Manpower/ & 10,846 \\
\hline 11 & $\begin{array}{l}\text { "Personnel Staffing and Scheduling"/ } \\
12\end{array}$ & $\begin{array}{l}\text { (lay adj3 (worker? or visitor? or attendant? or aide or aides or } \\
\text { support* or person* or helper? or carer? or caregiver? or care } \\
\text { giver? or consultant? or advisor? or counselor? or counsellor? or } \\
\text { assistant? or staff)).ti,ab }\end{array}$ \\
\hline
\end{tabular}

13 ((voluntary or volunteer?) adj3 (worker? or visitor? or atten2035 dant? or aide or aides or support* or person* or helper? or carer? or caregiver? or care giver? or consultant? or advisor? or counselor? or counsellor? or assistant? or staff)).ti,ab

14 (untrained adj3 (worker? or visitor? or attendant? or aide or 493 aides or support* or person* or helper? or carer? or caregiver? or care giver? or consultant? or advisor? or counselor? or counsellor? or assistant? or staff or nurse? or doctor? or physician? or therapist?)).ti,ab

15 (trained adj3 (worker? or visitor? or attendant? or aide or aides or support* or person* or helper? or carer? or caregiver? or care giver? or consultant? or advisor? or counselor? or counsellor? or assistant? or staff or nurse? or doctor? or physician? or therapist? ) ).ti,ab

16 (unlicensed adj3 (worker? or visitor? or attendant? or aide or aides or support* or person* or helper? or carer? or caregiver? or care giver? or consultant? or advisor? or counselor? or counsellor? or assistant? or staff or nurse? or doctor? or physician? or therapist?)).ti,ab

17 ((nonprofessional? or non professional?) adj3 (worker? or vis- 319 itor? or attendant? or aide or aides or support* or person* or helper? or carer? or caregiver? or care giver? or consultant? or advisor? or counselor? or counsellor? or assistant? or staff)).ti, 
(Continued)

$\mathrm{ab}$

18 ((non medical or non health or non healthcare or non health

care) adj3 (worker? or visitor? or attendant? or aide or aides or support* or person* or helper? or carer? or caregiver? or care giver? or consultant? or advisor? or counselor? or counsellor? or assistant? or staff)).ti,ab

19 (community adj3 (worker? or visitor? or attendant? or aide or 9714 aides or support* or person* or helper? or carer? or caregiver? or care giver? or consultant? or advisor? or counselor? or counsellor? or assistant? or staff)).ti,ab

20 (paraprofessional? or paramedic or paramedics or paramedical 44,576 worker? or paramedical personnel or allied health personnel or allied health worker? or support worker? or non specialist? or specially trained or barefoot doctor? or nurs* aid* or psychiatric aide? or psychiatric attendant? or social worker? or teacher? or school staff or trainer?).ti,ab

21 ((health* or medical*) adj3 (auxiliary or auxiliaries)).ti,ab

22 (nurs* adj1 (auxiliary or auxiliaries)).ti,ab.

23 (informal adj (caregiver? or care giver? or carer?)).ti,ab. 1340

24 (self help group? or support group?).ti,ab. 5301

25 ((social or psychosocial) adj (care or support)).ti,ab.

26 (village adj3 worker?).ti,ab.

27 community based.ti,ab.

28 (community adj3 intervention?).ti,ab.

29 community network?.ti,ab.

30 ((health or health care or healthcare) adj manpower).ti,ab. 768

31 human resources.ti,ab. 3604

32 (task? adj3 shift*).ti,ab.

33 (staff* adj3 chang*).ti,ab.

34 or $1-33$

218,880

Non-specialist health worker interventions for the care of mental, neurological and substance-abuse disorders in low- and middleincome countries (Review)

Copyright $\odot 2013$ The Cochrane Collaboration. Published by John Wiley \& Sons, Ltd. 
(Continued)

\begin{tabular}{lll}
\hline 35 & Mentally Ill Persons/ & 3934 \\
\hline 36 & Mentally Disabled Persons/ & 2063 \\
\hline 37 & exp Mental Disorders/ & 860,728 \\
\hline 38 & Drug Users/ & 744 \\
\hline 39 & Nervous System Diseases/ & 32,711 \\
\hline 40 & Epilepsy/ & 56,267 \\
\hline 41 & Mental Health Services/ & 23,168 \\
\hline 42 & Community Mental Health Services/ & 15,935 \\
\hline 43 & Emergency Services, Psychiatric/ & 2007 \\
\hline 44 & Social Work, Psychiatric/ & 2537 \\
\hline
\end{tabular}

45 ((mentally or psycholog*) adj (ill or disabled or handicapped $\quad 17,550$ or retarded or disturb* or traumati* or deficient)).ti,ab

46 (intellectually adj (disabled or handicapped or retarded or de- 350

ficient)).ti,ab

47 (mental adj (retardation or deficienc*)).ti,ab.

48 ((mental or behavioural or behavioral or anxiety or obsessive or 167,813 compulsive or panic or phobic or schizotypal or delusional or stress or cognitive or cognition or dissociative or personality or impulse control or mood or affective or bipolar or depressive or neurotic or paranoid or psychotic or somatoform or neurologic* or nervous or nervous system or eating) adj (disorder? or illness* or disease?)).ti,ab

49 ((substance related or alcohol or opioid or morphine or marijuana or heroin or cocaine) adj (disorder? or illness* or dependence or abuse or misuse)).ti,ab

50 (depression or anxiety or schizophrenia or psychoses or stress 514,850 syndrome? or distress syndrome? or combat disorder? or war disorder? or pain disorder? or dementia or alzheimer or epilepsy or down syndrome or alcoholism or substance abuse or drug addict* or drug abus* or drug misuse or drug user?).ti,ab

51 (psychiatric adj (patient? or service? or care or assistance or help 17,026 or work)).ti,ab 
52 (mental health service? or mental health care or mental health- 14,476 care or mental care).ti,ab

53 ((psychiatric or psychosocial) adj (service? or care or assistance 8657 or help or work)).ti,ab

$\begin{array}{ll}54 \text { or } / 35-53 & 1,236,906\end{array}$

55 Developing Countries.sh,kf.

56 (Africa or Asia or Caribbean or West Indies or South America or Latin America or Central America).hw,kf,ti,ab,cp

57 (Afghanistan or Albania or Algeria or Angola or Antigua or Barbuda or Argentina or Armenia or Armenian or Aruba or Azerbaijan or Bahrain or Bangladesh or Barbados or Benin or Byelarus or Byelorussian or Belarus or Belorussian or Belorussia or Belize or Bhutan or Bolivia or Bosnia or Herzegovina or Hercegovina or Botswana or Brazil or Brasil or Bulgaria or Burkina Faso or Burkina Fasso or Upper Volta or Burundi or Urundi or Cambodia or Khmer Republic or Kampuchea or Cameroon or Cameroons or Cameron or Camerons or Cape Verde or Central African Republic or Chad or Chile or China or Colombia or Comoros or Comoro Islands or Comores or Mayotte or Congo or Zaire or Costa Rica or Cote d'Ivoire or Ivory Coast or Croatia or Cuba or Cyprus or Czechoslovakia or Czech Republic or Slovakia or Slovak Republic or Djibouti or French Somaliland or Dominica or Dominican Republic or East Timor or East Timur or Timor Leste or Ecuador or Egypt or United Arab Republic or El Salvador or Eritrea or Estonia or Ethiopia or Fiji or Gabon or Gabonese Republic or Gambia or Gaza or Georgia Republic or Georgian Republic or Ghana or Gold Coast or Greece or Grenada or Guatemala or Guinea or Guam or Guiana or Guyana or Haiti or Honduras or Hungary or India or Maldives or Indonesia or Iran or Iraq or Isle of Man or Jamaica or Jordan or Kazakhstan or Kazakh or Kenya or Kiribati or Korea or Kosovo or Kyrgyzstan or Kirghizia or Kyrgyz Republic or Kirghiz or Kirgizstan or Lao PDR or Laos or Latvia or Lebanon or Lesotho or Basutoland or Liberia or Libya or Lithuania or Macedonia or Madagascar or Malagasy Republic or Malaysia or Malaya or Malay or Sabah or Sarawak or Malawi or Nyasaland or Mali or Malta or Marshall Islands or Mauritania or Mauritius or Agalega Islands or Mexico or Micronesia or Middle East or Moldova or Moldovia or Moldovian or Mongolia or Montenegro or Morocco or Ifni or Mozambique or Myanmar or Myanma or Burma or Namibia or Nepal or Netherlands Antilles or New Caledonia or Nicaragua or Niger or Nigeria or Northern Mariana Islands or Oman or Muscat or 
Pakistan or Palau or Palestine or Panama or Paraguay or Peru or Philippines or Philipines or Phillipines or Phillippines or Poland or Portugal or Puerto Rico or Romania or Rumania or Roumania or Russia or Russian or Rwanda or Ruanda or Saint Kitts or St Kitts or Nevis or Saint Lucia or St Lucia or Saint Vincent or St Vincent or Grenadines or Samoa or Samoan Islands or Navigator Island or Navigator Islands or Sao Tome or Saudi Arabia or Senegal or Serbia or Montenegro or Seychelles or Sierra Leone or Slovenia or Sri Lanka or Ceylon or Solomon Islands or Somalia or South Africa or Sudan or Suriname or Surinam or Swaziland or Syria or Tajikistan or Tadzhikistan or Tadjikistan or Tadzhik or Tanzania or Thailand or Togo or Togolese Republic or Tonga or Trinidad or Tobago or Tunisia or Turkey or Turkmenistan or Turkmen or Uganda or Ukraine or Uruguay or USSR or Soviet Union or Union of Soviet Socialist Republics or Uzbekistan or Uzbek or Vanuatu or New Hebrides or Venezuela or Vietnam or Viet Nam or West Bank or Yemen or Yugoslavia or Zambia or Zimbabwe or Rhodesia) .hw,kf,ti,ab,cp

58 ((developing or less* developed or under developed or underdeveloped or middle income or low* income or underserved or under served or deprived or poor*) adj (countr* or nation? or population? or world)).ti,ab

59 ((developing or less* developed or under developed or under- 216 developed or middle income or low* income) adj (economy or economies)).ti,ab

60 (low* adj (gdp or gnp or gross domestic or gross national)).ti, 115 $\mathrm{ab}$

61 (low adj3 middle adj3 countr*).ti,ab.

1901

62 (lmic or lmics or third world or lami countr*).ti,ab. 2881

63 transitional countr*.ti,ab.

64 or/55-63

65 randomized controlled trial.pt.

66 controlled clinical trial.pt.

67 multicenter study.pt.

68 (randomised or randomized or randomly).ti,ab. 462,610

69 placebo.ti,ab. 140,808

Non-specialist health worker interventions for the care of mental, neurological and substance-abuse disorders in low- and middleincome countries (Review)

Copyright $\odot 2013$ The Cochrane Collaboration. Published by John Wiley \& Sons, Ltd. 
(Continued)

\begin{tabular}{|c|c|c|}
\hline 70 & trial.ti,ab. & 304,308 \\
\hline 71 & groups.ti,ab. & $1,194,565$ \\
\hline 72 & intervention*.ti,ab. & 450,065 \\
\hline 73 & evaluat*.ti,ab. & $1,875,064$ \\
\hline 74 & 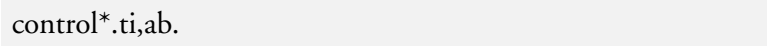 & $2,273,078$ \\
\hline 75 & effect?.ti,ab. & $3,399,512$ \\
\hline 76 & impact.ti,ab. & 394,702 \\
\hline 77 & (time series or time points).ti,ab. & 50,864 \\
\hline 78 & $(($ pretest or pre test) and (posttest or post test)).ti,ab. & 5274 \\
\hline 79 & (quasi experiment* or quasiexperiment*).ti,ab. & 4655 \\
\hline 80 & $\begin{array}{l}\text { ((multicenter or multicentre or multi center or multi centre) } \\
\text { adj study).ti,ab }\end{array}$ & 19,004 \\
\hline 81 & repeated measure*.ti,ab. & 22,128 \\
\hline 82 & or/65-81 & $7,097,338$ \\
\hline 83 & Animals/ & $4,963,387$ \\
\hline 84 & Humans/ & $12,343,636$ \\
\hline 85 & 83 not $(83$ and 84$)$ & $3,640,602$ \\
\hline 86 & 82 not 85 & $5,374,334$ \\
\hline 87 & 34 and 54 and 64 and 86 & 3313 \\
\hline 88 & (diagnos* or detect* or case finding?).ti,ab. & $2,673,184$ \\
\hline 89 & 34 and 54 and 64 and 88 & 1011 \\
\hline 90 & 87 or 89 & 3662 \\
\hline 91 & “comment on".cm. & 507,804 \\
\hline 92 & (systematic review or literature review).ti. & 34,294 \\
\hline 93 & (editorial or comment or meta-analysis or news or review).pt & $2,561,432$ \\
\hline
\end{tabular}

Non-specialist health worker interventions for the care of mental, neurological and substance-abuse disorders in low- and middle- 
(Continued)

94 “cochrane database of systematic reviews".jn. $\quad 8573$

\begin{tabular}{l|l}
95 or/91-94 2,567,662 \\
\hline
\end{tabular}

\begin{tabular}{l|l}
9690 not 95 & 3422
\end{tabular}

EMBASE (OvidSP)

\begin{tabular}{|c|c|c|}
\hline 1 & Paramedical Personnel/ & 10,488 \\
\hline 2 & Health Auxiliary/ & 2282 \\
\hline 3 & Nursing Assistant/ & 3274 \\
\hline 4 & Caregiver/ & 30,543 \\
\hline 5 & Voluntary Worker/ & 5187 \\
\hline 6 & Self Help/ & 10,343 \\
\hline 7 & Social Support/ & 48,504 \\
\hline 8 & Health Care Manpower/ & 9483 \\
\hline 9 & $\begin{array}{l}\text { (lay adj3 (worker? or visitor? or attendant? or aide or aides or } \\
\text { support* or person* or helper? or carer? or caregiver? or care } \\
\text { giver? or consultant? or advisor? or counselor? or counsellor? or } \\
\text { assistant? or staff)).ti,ab }\end{array}$ & 1425 \\
\hline 10 & $\begin{array}{l}\text { ((voluntary or volunteer?) adj3 (worker? or visitor? or atten- } \\
\text { dant? or aide or aides or support* or person* or helper? or carer? } \\
\text { or caregiver? or care giver? or consultant? or advisor? or coun- } \\
\text { selor? or counsellor? or assistant? or staff)).ti,ab }\end{array}$ & 2320 \\
\hline
\end{tabular}

11 (untrained adj3 (worker? or visitor? or attendant? or aide or 517 aides or support* or person* or helper? or carer? or caregiver? or care giver? or consultant? or advisor? or counselor? or counsellor? or assistant? or staff or nurse? or doctor? or physician? or therapist?)).ti,ab

12 (trained adj3 (worker? or visitor? or attendant? or aide or aides 13,341 or support* or person* or helper? or carer? or caregiver? or care giver? or consultant? or advisor? or counselor? or counsellor? or assistant? or staff or nurse? or doctor? or physician? or therapist? )$\cdot \mathrm{ti}, \mathrm{ab}$ 
13 (unlicensed adj3 (worker? or visitor? or attendant? or aide or aides or support* or person* or helper? or carer? or caregiver? or care giver? or consultant? or advisor? or counselor? or counsellor? or assistant? or staff or nurse? or doctor? or physician? or therapist?)).ti,ab

14 ((nonprofessional? or non professional?) adj3 (worker? or visitor? or attendant? or aide or aides or support* or person* or helper? or carer? or caregiver? or care giver? or consultant? or advisor? or counselor? or counsellor? or assistant? or staff)).ti, $\mathrm{ab}$

15 ((non medical or non health or non healthcare or non health care) adj3 (worker? or visitor? or attendant? or aide or aides or support* or person* or helper? or carer? or caregiver? or care giver? or consultant? or advisor? or counselor? or counsellor? or assistant? or staff)).ti,ab

16 (community adj3 (worker? or visitor? or attendant? or aide or aides or support* or person* or helper? or carer? or caregiver? or care giver? or consultant? or advisor? or counselor? or counsellor? or assistant? or staff)).ti,ab

17 (paraprofessional? or paramedic or paramedics or paramedical 51,499 worker? or paramedical personnel or allied health personnel or allied health worker? or support worker? or non specialist? or specially trained or barefoot doctor? or nurs* aid* or psychiatric aide? or psychiatric attendant? or social worker? or teacher? or school staff or trainer?).ti,ab

18 ((health* or medical*) adj3 (auxiliary or auxiliaries)).ti,ab 348

19 (nurs* adj1 (auxiliary or auxiliaries)).ti,ab. 430

20 (informal adj (caregiver? or care giver? or carer?)).ti,ab. 1592

21 (self help group? or support group?).ti,ab. 6863

22 ((social or psychosocial) adj (care or support)).ti,ab. 27,333

23 (village adj3 worker?).ti,ab.

24 community based.ti,ab.

25 (community adj3 intervention?).ti,ab. 5061

26 community network?.ti,ab. 
(Continued)

\begin{tabular}{|c|c|c|}
\hline 27 & ((health or health care or healthcare) adj manpower).ti,ab. & 732 \\
\hline 28 & human resources.ti,ab. & 4047 \\
\hline 29 & (task? adj3 shift*).ti,ab. & 938 \\
\hline 30 & (staff* adj3 chang*).ti,ab. & 1109 \\
\hline 31 & or/ $1-30$ & 236,211 \\
\hline 32 & Mental Patient/ & 15,718 \\
\hline 33 & exp Mental Disease/ & $1,400,373$ \\
\hline 34 & Mental Health Care/ & 16,342 \\
\hline 35 & Home Mental Health Care/ & 115 \\
\hline 36 & Mental Health Service/ & 39,847 \\
\hline 37 & Psychosocial Care/ & 9196 \\
\hline 38 & Neurologic Disease/ & 83,038 \\
\hline 39 & Epilepsy/ & 84,332 \\
\hline
\end{tabular}

40 ((mentally or psycholog*) adj (ill or disabled or handicapped 19,493 or retarded or disturb* or traumati* or deficient)).ti,ab

41 (intellectually adj (disabled or handicapped or retarded or de- 447 ficient)).ti,ab

42 (mental adj (retardation or deficienc $\left.\left.{ }^{*}\right)\right) . t i, a b . \quad 27,388$

43 ((mental or behavioural or behavioral or anxiety or obsessive or 215,937 compulsive or panic or phobic or schizotypal or delusional or stress or cognitive or cognition or dissociative or personality or impulse control or mood or affective or bipolar or depressive or neurotic or paranoid or psychotic or somatoform or neurologic* or nervous or nervous system or eating) adj (disorder? or illness* or disease?)).ti,ab

44 ((substance related or alcohol or opioid or morphine or mari- 28,684 juana or heroin or cocaine) adj (disorder? or illness* or dependence or abuse or misuse)).ti,ab 
45 (depression or anxiety or schizophrenia or psychoses or stress

642,797 syndrome? or distress syndrome? or combat disorder? or war disorder? or pain disorder? or dementia or alzheimer or epilepsy or down syndrome or alcoholism or substance abuse or drug addict* or drug abus* or drug misuse or drug user?).ti,ab

46 (psychiatric adj (patient? or service? or care or assistance or help 20,401 or work)).ti,ab

47 (mental health service? or mental health care or mental health- 17,975 care or mental care).ti,ab

48 ((psychiatric or psychosocial) adj (service? or care or assistance 11,075 or help or work)).ti,ab

50 Developing Country.sh.

51 (Africa or Asia or Caribbean or West Indies or South America or Latin America or Central America).hw,ti,ab,cp

52 (Afghanistan or Albania or Algeria or Angola or Antigua or Barbuda or Argentina or Armenia or Armenian or Aruba or Azerbaijan or Bahrain or Bangladesh or Barbados or Benin or Byelarus or Byelorussian or Belarus or Belorussian or Belorussia or Belize or Bhutan or Bolivia or Bosnia or Herzegovina or Hercegovina or Botswana or Brazil or Brasil or Bulgaria or Burkina Faso or Burkina Fasso or Upper Volta or Burundi or Urundi or Cambodia or Khmer Republic or Kampuchea or Cameroon or Cameroons or Cameron or Camerons or Cape Verde or Central African Republic or Chad or Chile or China or Colombia or Comoros or Comoro Islands or Comores or Mayotte or Congo or Zaire or Costa Rica or Cote d'Ivoire or Ivory Coast or Croatia or Cuba or Cyprus or Czechoslovakia or Czech Republic or Slovakia or Slovak Republic or Djibouti or French Somaliland or Dominica or Dominican Republic or East Timor or East Timur or Timor Leste or Ecuador or Egypt or United Arab Republic or El Salvador or Eritrea or Estonia or Ethiopia or Fiji or Gabon or Gabonese Republic or Gambia or Gaza or Georgia Republic or Georgian Republic or Ghana or Gold Coast or Greece or Grenada or Guatemala or Guinea or Guam or Guiana or Guyana or Haiti or Honduras or Hungary or India or Maldives or Indonesia or Iran or Iraq or Isle of Man or Jamaica or Jordan or Kazakhstan or Kazakh or Kenya or Kiribati or Korea or Kosovo or Kyrgyzstan or Kirghizia or Kyrgyz Republic or Kirghiz or Kirgizstan or Lao PDR or Laos or Latvia or Lebanon or Lesotho or Basutoland or Liberia or Libya or 
Lithuania or Macedonia or Madagascar or Malagasy Republic or Malaysia or Malaya or Malay or Sabah or Sarawak or Malawi or Nyasaland or Mali or Malta or Marshall Islands or Mauritania or Mauritius or Agalega Islands or Mexico or Micronesia or Middle East or Moldova or Moldovia or Moldovian or Mongolia or Montenegro or Morocco or Ifni or Mozambique or Myanmar or Myanma or Burma or Namibia or Nepal or Netherlands Antilles or New Caledonia or Nicaragua or Niger or Nigeria or Northern Mariana Islands or Oman or Muscat or Pakistan or Palau or Palestine or Panama or Paraguay or Peru or Philippines or Philipines or Phillipines or Phillippines or Poland or Portugal or Puerto Rico or Romania or Rumania or Roumania or Russia or Russian or Rwanda or Ruanda or Saint Kitts or St Kitts or Nevis or Saint Lucia or St Lucia or Saint Vincent or St Vincent or Grenadines or Samoa or Samoan Islands or Navigator Island or Navigator Islands or Sao Tome or Saudi Arabia or Senegal or Serbia or Montenegro or Seychelles or Sierra Leone or Slovenia or Sri Lanka or Ceylon or Solomon Islands or Somalia or South Africa or Sudan or Suriname or Surinam or Swaziland or Syria or Tajikistan or Tadzhikistan or Tadjikistan or Tadzhik or Tanzania or Thailand or Togo or Togolese Republic or Tonga or Trinidad or Tobago or Tunisia or Turkey or Turkmenistan or Turkmen or Uganda or Ukraine or Uruguay or USSR or Soviet Union or Union of Soviet Socialist Republics or Uzbekistan or Uzbek or Vanuatu or New Hebrides or Venezuela or Vietnam or Viet Nam or West Bank or Yemen or Yugoslavia or Zambia or Zimbabwe or Rhodesia) .hw,ti,ab,cp

53 ((developing or less* developed or under developed or under- 54,293 developed or middle income or low* income or underserved or under served or deprived or poor*) adj (countr* or nation? or population? or world)).ti,ab

54 ((developing or less* developed or under developed or under- 257 developed or middle income or low* income) adj (economy or economies)).ti,ab

55 (low* adj (gdp or gnp or gross domestic or gross national)).ti, 140 $\mathrm{ab}$

56 (low adj3 middle adj3 countr*).ti,ab. 2153

57 (lmic or lmics or third world or lami countr*).ti,ab.

58 transitional countr*.ti,ab. 99

59 or $/ 50-58$

$2,692,822$

Non-specialist health worker interventions for the care of mental, neurological and substance-abuse disorders in low- and middle- 
(Continued)

\begin{tabular}{|c|c|c|}
\hline 60 & Randomized Controlled Trial/ & 323,003 \\
\hline 61 & Controlled Clinical Trial/ & 389,305 \\
\hline 62 & (randomised or randomized or randomly).ti,ab. & 579,937 \\
\hline 63 & Time Series Analysis/ & 11,636 \\
\hline 64 & (time series or time points).ti,ab. & 63,741 \\
\hline 65 & intervention*.ti,ab. & 562,252 \\
\hline 66 & evaluat*.ti,ab. & $2,316,859$ \\
\hline 67 & 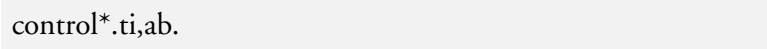 & $2,647,026$ \\
\hline 68 & effect?.ti,ab. & $3,857,543$ \\
\hline 69 & impact.ti,ab. & 504,137 \\
\hline 70 & $(($ pretest or pre test) and (posttest or post test)).ti,ab. & 6116 \\
\hline 71 & (quasi experiment* or quasiexperiment*).ti,ab. & 5334 \\
\hline 72 & $\begin{array}{l}\text { ((multicenter or multicentre or multi center or multi centre) } \\
\text { adj study).ti,ab }\end{array}$ & 25,517 \\
\hline 73 & repeated measure*.ti,ab. & 27,338 \\
\hline 74 & or $/ 60-73$ & $7,718,259$ \\
\hline 75 & Nonhuman/ & $3,853,444$ \\
\hline 76 & 74 not 75 & $5,951,391$ \\
\hline 77 & 31 and 49 and 59 and 76 & 4463 \\
\hline 78 & (diagnos* or detect* or case finding?).ti,ab. & $3,181,233$ \\
\hline 79 & 31 and 49 and 59 and 78 & 1668 \\
\hline 80 & 77 or 79 & 5101 \\
\hline 81 & (systematic review or literature review).ti. & 41,493 \\
\hline 82 & "cochrane database of systematic reviews".jn. & 3773 \\
\hline 83 & 81 or 82 & 45,261 \\
\hline
\end{tabular}

Non-specialist health worker interventions for the care of mental, neurological and substance-abuse disorders in low- and middleincome countries (Review)

Copyright $\odot 2013$ The Cochrane Collaboration. Published by John Wiley \& Sons, Ltd. 
(Continued)

\begin{tabular}{|c|c|c|}
\hline 84 & 80 not 83 & 5078 \\
\hline 85 & limit 84 to embase & 3662 \\
\hline
\end{tabular}

CINAHL (EBSCOhost)

S90 S34 and S56 and S74 and S88 [Exclude MEDLINE records] 781

S89 S34 and S56 and S74 and S88 2326

S88 S75 or S76 or S77 or S78 or S79 or S80 or S81 or S82 or S83 or $S 84$ or $S 85$ or $S 86$ or $S 87$

S87 TI (intervention* or controlled or control W0 group* or compare or compared or before N5 after or pre N5 post or pretest or "pre test" or posttest or "post test" or quasiexperiment* or quasi W0 experiment* or evaluat* or effect or impact or "time series" or time W0 point* or repeated W0 measur* ) OR AB ( intervention* or controlled or control W0 group* or compare or compared or before N5 after or pre N5 post or pretest or "pre test" or posttest or "post test" or quasiexperiment* or quasi W0 experiment* or evaluat* or effect or impact or "time series" or time W0 point* or repeated W0 measur*)

S86 TI ( randomis* or randomiz* or random* W0 allocat*) OR 63,905

$\mathrm{AB}$ ( randomis* or randomiz* or random* W0 allocat*)

S85 MH "Health Services Research"

5825

\begin{tabular}{lll}
\hline S84 & MH “Multicenter Studies” & 5806 \\
\hline S83 & MH “Quasi-Experimental Studies+” & 6116 \\
\hline S82 & MH “Pretest-Posttest Design+” & 18,858 \\
\hline S81 & MH “Experimental Studies” & 11,576 \\
\hline S80 & MH “Nonrandomized Trials” & 126 \\
\hline S79 & MH “Intervention Trials" & 4177 \\
\hline S78 & MH “Clinical Trials” & 74,670 \\
\hline S77 & MH “Randomized Controlled Trials” & 9725 \\
\hline S76 & PT research & 732,410
\end{tabular}

Non-specialist health worker interventions for the care of mental, neurological and substance-abuse disorders in low- and middle- 


\begin{tabular}{ll} 
S75 PT clinical trial & 51,042 \\
\hline
\end{tabular}

S74 S57 or S58 or S59 or S60 or S61 or S62 or S63 or S64 or S65 204,905 or $\mathrm{S} 66$ or $\mathrm{S} 67$ or $\mathrm{S} 68$ or $\mathrm{S} 69$ or $\mathrm{S} 70$ or $\mathrm{S} 71$ or $\mathrm{S} 72$ or $\mathrm{S} 73$

S73 TI transitional W0 countr* OR AB transitional W0 countr*

25

S72 TI ( lmic or lmics or third W0 world or lami W0 countr*) OR AB ( lmic or lmics or third W0 world or lami W0 countr* )

S71 TI low N3 middle N3 countr* OR AB low N3 middle N3 518 countr*

S70 TI ( low* W0 (gdp or gnp or gross W0 domestic or gross 6 W0 national) ) OR AB ( low* W0 (gdp or gnp or gross W0 domestic or gross W0 national) )

S69 TI ( (developing or less* W0 developed or under W0 developed or underdeveloped or middle W0 income or low* W0 income) W0 (economy or economies) ) OR AB ( (developing or less* W0 developed or under W0 developed or underdeveloped or middle W0 income or low* W0 income) W0 (economy or economies) )

S68 TI ( (developing or less* W0 developed or under W0 developed or underdeveloped or middle W0 income or low* W0 income or underserved or under W0 served or deprived or poor*) W0 (countr* or nation or nations or population* or world or area or areas) ) OR AB ( (developing or less* W0 developed or under $\mathrm{W} 0$ developed or underdeveloped or middle W0 income or low* W0 income or underserved or under W0 served or deprived or poor*) W0 (countr* or nation or nations or population* or world or area or areas) )

S67 TI Afghanistan or Bangladesh or Benin or "Burkina Faso" or Burundi or Cambodia or "Central African Republic" or Chad or Comoros or Congo or "Cote d'Ivoire" or Eritrea or Ethiopia or Gambia or Ghana or Guinea or Haiti or India or Kenya or Korea or Kyrgyz or Kyrgyzstan or Lao or Laos or Liberia or Madagascar or Malawi or Mali or Mauritania or Melanesia or Mongolia or Mozambique or Burma or Myanmar or Nepal or Niger or Nigeria or Pakistan or Rwanda or "Salomon Islands" or "Sao Tome" or Senegal or "Sierra Leone" or Somalia or Sudan or Tajikistan or Tanzania or Timor or Togo or Uganda or Uzbekistan or Vietnam or "Viet Nam" or Yemen or Zambia or Zimbabwe 
S66 TX Albania or Algeria or Angola or Armenia or Azerbaijan or Belarus or Bhutan or Bolivia or Bosnia or Herzegovina or "Cape Verde" or Cameroon or China or Colombia or Congo or Cuba or Djibouti or "Dominican Republic" or Ecuador or Egypt or "El Salvador" or Fiji or Gaza or Georgia or Guam or Guatemala or Guyana or Honduras or "Indian Ocean Islands" or Indonesia or Iran or Iraq or Jamaica or Jordan or Kiribati or Lesotho or Macedonia or Maldives or "Marshall Islands" or Micronesia or "Middle East" or Moldova or Morocco or Namibia or Nicaragua or Palestin* or Paraguay or Peru or Philippines or Samoa or "Sri Lanka" or Suriname or Swaziland or Syria or "Syrian Arab Republic" or Thailand or Tonga or Tunisia or Turkmenistan or Ukraine or Vanuatu or "West Bank"

S65 TX "American Samoa” or Argentina or Belize or Botswana or Brazil or Brasil or Bulgaria or Chile or Comoros or "Costa Rica" or Croatia or Dominica or Guinea or Gabon or Grenada or Grenadines or Hungary or Kazakhstan or Latvia or Lebanon or Libia or libyan or Libya or Lithuania or Malaysia or Mauritius or Mayotte or Mexico or Micronesia or Montenegro or Nevis or "Northern Mariana Islands" or Oman or Palau or Panama or Poland or Romania or Russia or "Russian Federation" or Samoa or "Saint Lucia" or "St Lucia" or "Saint Kitts" or "St Kitts" or "Saint Vincent" or "St Vincent" or Serbia or Seychelles or Slovakia or "Slovak Republic" or "South Africa" or Turkey or Uruguay or Venezuela or Yugoslavia
68,169

76,875

S64 TI ( Africa or Asia or "South America" or "Latin America" or 10,039 "Central America") OR AB (Africa or Asia or "South America" or "Latin America" or "Central America" )

\begin{tabular}{|c|c|c|}
\hline S63 & (MH “Asia+”) & 70,391 \\
\hline S62 & (MH “West Indies+”) & 4121 \\
\hline S61 & (MH “South America+") & 18,325 \\
\hline S60 & (MH “Latin America”) & 986 \\
\hline S59 & (MH “Central America+”) & 1715 \\
\hline S58 & (MH “Africa+”) & 23,802 \\
\hline S57 & (MH “Developing Countries”) & 7212 \\
\hline S56 & $\begin{array}{l}S 35 \text { or } S 36 \text { or } S 37 \text { or } S 38 \text { or } S 39 \text { or } S 40 \text { or } S 41 \text { or } S 42 \text { or } S 43 \\
\text { or } S 44 \text { or } S 45 \text { or } S 46 \text { or } S 47 \text { or } S 48 \text { or } S 49 \text { or } S 50 \text { or } S 51 \text { or } \\
S 52 \text { or } S 53 \text { or } S 54 \text { or } S 55\end{array}$ & 268,600 \\
\hline
\end{tabular}

Non-specialist health worker interventions for the care of mental, neurological and substance-abuse disorders in low- and middle- 
S55 TI ( (psychiatric or psychosocial) W0 (service* or care or assis- 2777 tance or help or work) ) OR AB ( (psychiatric or psychosocial) W0 (service* or care or assistance or help or work) )

S54 TI ( (mental W0 health W0 service* or "mental health care" or "mental healthcare" or "mental care") ) OR AB ( (mental W0 health W0 service* or "mental health care" or "mental healthcare" or "mental care") )

S53 TI ( psychiatric W0 (patient* or service* or care or assistance or help or work) ) OR AB ( psychiatric W0 (patient* or service* or care or assistance or help or work) )

S52 TI ( (depression or anxiety or schizophrenia or psychoses or 88,617 stress W0 syndrome* or distress W0 syndrome* or combat W0 disorder* or war W0 disorder* or pain W0 disorder* or dementia or alzheimer or epilepsy or down* W0 syndrome or alcoholism or substance W0 abus* or drug W0 addict* or drug W0 abus* or drug W0 misuse or drug W0 user*) ) OR $\mathrm{AB}$ ( (depression or anxiety or schizophrenia or psychoses or stress W0 syndrome* or distress W0 syndrome* or combat W0 disorder* or war W0 disorder* or pain W0 disorder* or dementia or alzheimer or epilepsy or down* W0 syndrome or alcoholism or substance W0 abus* or drug W0 addict* or drug W0 abus* or drug W0 misuse or drug W0 user*) )

S51 TI ( ("substance related" or alcohol or opioid or morphine or marijuana or heroin or cocaine) W0 (disorder* or illness* or dependence or abuse or misuse) ) OR AB ( (“substance related” or alcohol or opioid or morphine or marijuana or heroin or cocaine) W0 (disorder* or illness* or dependence or abuse or misuse) )

S50 TI ( (mental or behavioural or behavioral or anxiety or obsessive or compulsive or panic or phobic or schizotypal or delusional or stress or cognitive or cognition or dissociative or personality or "impulse control" or mood or affective or bipolar or depressive or neurotic or paranoid or psychotic or somatoform or neurologic* or nervous or eating) W0 (disorder* or illness* or disease*) ) OR AB ( (mental or behavioural or behavioral or anxiety or obsessive or compulsive or panic or phobic or schizotypal or delusional or stress or cognitive or cognition or dissociative or personality or "impulse control" or mood or affective or bipolar or depressive or neurotic or paranoid or psychotic or somatoform or neurologic* or nervous or eating) W0 (disorder* or illness* or disease*) ) 
(Continued)

S49 TI ( mental W0 (retardation or deficienc*) ) OR AB ( mental 1450 W0 (retardation or deficienc*) $\left.{ }^{*}\right)$

S48 TI ( intellectually W0 (disabled or handicapped or retarded or deficient) ) OR AB ( intellectually W0 (disabled or handicapped or retarded or deficient) )

S47 TI ( (mentally or psycholog*) W0 (ill or disabled or handicapped or retarded or disturb* or traumati* or deficient) ) OR AB ( (mentally or psycholog*) W0 (ill or disabled or handicapped or retarded or disturb* or traumati* or deficient) )

\begin{tabular}{|c|c|c|}
\hline S46 & (MH “Social Work, Psychiatric”) & 519 \\
\hline S45 & (MH “Psychiatric Emergencies") & 595 \\
\hline S44 & (MH “Emergency Services, Psychiatric”) & 77 \\
\hline S43 & (MH "Community Mental Health Nursing") & 1628 \\
\hline S42 & (MH “Community Mental Health Services”) & 5226 \\
\hline S41 & (MH “Mental Health Services”) & 14,691 \\
\hline S40 & (MH “Epilepsy”) & 4719 \\
\hline S39 & (MH “Nervous System Diseases") & 2663 \\
\hline S38 & (MH “Substance Abusers+”) & 3348 \\
\hline S37 & (MH “Mentally Disabled Persons") & 1275 \\
\hline S36 & (MH “Psychiatric Patients+") & 7664 \\
\hline S35 & (MH “Mental Disorders+”) & 208,797 \\
\hline S34 & $\begin{array}{l}S 1 \text { or } S 2 \text { or } S 3 \text { or } S 4 \text { or } S 5 \text { or } S 6 \text { or } S 7 \text { or } S 8 \text { or } S 9 \text { or } S 10 \text { or } \\
S 11 \text { or } S 12 \text { or } S 13 \text { or } S 14 \text { or } S 15 \text { or } S 16 \text { or } S 17 \text { or } S 18 \text { or } S 19 \\
\text { or } S 20 \text { or } S 21 \text { or } S 22 \text { or } S 23 \text { or } S 24 \text { or } S 25 \text { or } S 26 \text { or } S 27 \text { or } \\
S 28 \text { or } S 29 \text { or } S 30 \text { or } S 31 \text { or } S 32 \text { or } S 33\end{array}$ & 103,048 \\
\hline S33 & TI staff* N3 chang* OR AB staff* N3 chang* & 886 \\
\hline S32 & $\begin{array}{l}\text { TI ( (task or tasks) N3 shift* ) OR AB ( (task or tasks) N3 } \\
\text { shift }^{*} \text { ) }\end{array}$ & 135 \\
\hline S31 & TI "human resources" OR AB "human resources" & 1490 \\
\hline
\end{tabular}


(Continued)

S30 TI ( (health or healthcare) W0 manpower ) OR AB ( (health 51 or healthcare) W0 manpower )

S29 TI community W0 network* OR AB community W0 net- 105 work*

S28 TI community N3 intervention* OR AB community N3 in- 2298 tervention*

S27 TI "community based" OR AB "community based" 11,426

S26 TI village N3 worker* OR AB village N3 worker* 46

S25 TI ( (social or psychosocial) W0 (care or support) ) OR AB ( 13,431 (social or psychosocial) W0 (care or support) )

S24 TI ( "self help group" or "self help groups" or "support group"

or "support groups" ) OR AB ( "self help group" or "self help groups" or "support group" or "support groups" )

S23 TI ( informal W0 (caregiver* or "care giver" or "care givers" or carer*) ) OR AB ( informal W0 (caregiver* or "care giver" or "care givers" or carer*) )

S22 TI ( nurs* N1 (auxiliary or auxiliaries) ) OR AB ( nurs* N1 271 (auxiliary or auxiliaries) )

S21 TI ( (health* or medical*) N3 (auxiliary or auxiliaries)) OR $\mathrm{AB}\left(\right.$ (health* ${ }^{*}$ or medical $\left.{ }^{*}\right) \mathrm{N} 3$ (auxiliary or auxiliaries) )

S20 TI ( paraprofessional* ${ }^{*}$ or paramedic or paramedics or paramed20,386 ical W0 worker* or paramedical W0 personnel or "allied health personnel" or "allied health worker" or "allied health workers" or support W0 worker* or non W0 specialist* or "specially trained" or barefoot W0 doctor* or nurs* W0 aide* or psychiatric W0 aide* or psychiatric W0 attendant* or social W0 worker* or teacher* or "school staff" or trainer*) OR AB ( paraprofessional* or paramedic or paramedics or paramedical W0 worker* or paramedical W0 personnel or "allied health personnel" or "allied health worker" or "allied health workers" or support W0 worker* or non W0 specialist* or "specially trained" or barefoot W0 doctor* or nurs* W0 aide* or psychiatric W0 aide* or psychiatric W0 attendant* or social W0 worker* or teacher* or "school staff" or trainer*)

S19 TI ( community N3 (worker* or visitor* or attendant* or aide 6052 or aides or support ${ }^{*}$ or person* ${ }^{*}$ or helper* ${ }^{*}$ or carer* ${ }^{*}$ or caregiver* or "care giver" or "care givers" or consultant* or advisor* or 
counselor* or counsellor* or assistant* or staff) ) OR AB ( community $\mathrm{N} 3$ (worker* ${ }^{*}$ or visitor* ${ }^{*}$ or attendant* or aide or aides or support* or person* or helper* or carer* or caregiver* or "care giver" or "care givers" or consultant* or advisor* or counselor* or counsellor* or assistant* or staff) )

S18 TI ( ("non medical" or "non health" or "non healthcare") N3 (worker* or visitor* or attendant* or aide or aides or support* or person* or helper* or carer* or caregiver* or "care giver" or "care givers" or consultant* or advisor* or counselor* or counsellor* or assistant* or staff) ) OR AB ( ("non medical" or "non health" or "non healthcare") N3 (worker* or visitor* or attendant* or aide or aides or support* or person* or helper* or carer* or caregiver* or "care giver" or "care givers" or consultant* or advisor* or counselor* or counsellor* or assistant* or staff) )

S17 TI ( (nonprofessional* or "non professional" or "non professionals") N3 (worker* or visitor* or attendant* or aide or aides or support* or person* or helper* or carer* or caregiver* ${ }^{*}$ or "care giver" or "care givers" or consultant* or advisor* or counselor* or counsellor* or assistant* or staff) ) OR AB ( (nonprofessional* or "non professional" or "non professionals") N3 (worker* or visitor* or attendant* or aide or aides or support* or person* or helper* or carer* or caregiver* or "care giver" or "care givers" or consultant* or advisor* or counselor* or counsellor* or assistant* or staff) )

S16 TI ( unlicensed N3 (worker* or visitor* or attendant* or aide or aides or support* or person* or helper* or carer* or caregiver* or "care giver" or "care givers" or consultant* or advisor* or counselor* or counsellor* or assistant* or staff or nurse* or doctor* or physician* or therapist*) ) OR AB ( unlicensed N3 (worker* or visitor* or attendant* ${ }^{*}$ or aide or aides or support* or person* or helper* or carer* or caregiver* or "care giver" or "care givers" or consultant* or advisor* or counselor* or counsellor* or assistant* or staff or nurse* or doctor* or physician* or therapist*) ) $^{*}$

S15 TI ( trained N3 (worker* or visitor* or attendant* or aide or aides or support* or person* or helper* or carer* or caregiver* or "care giver" or "care givers" or consultant* or advisor* or counselor* or counsellor* or assistant* or staff or nurse* or doctor* or physician* or therapist*) ) OR AB ( trained N3 (worker* or visitor* or attendant* or aide or aides or support* or person* or helper* or carer* or caregiver* or "care giver" or "care givers" or consultant* or advisor* or counselor* or counsellor* or assistant* or staff or nurse* or doctor* or physician* or therapist*) ) 
S14 TI ( untrained N3 (worker* or visitor* or attendant* or aide or aides or support* or person* or helper* or carer* or caregiver* or "care giver" or "care givers" or consultant* or advisor* or counselor* or counsellor* or assistant* or staff or nurse* or doctor* or physician* or therapist*) ) OR AB ( untrained N3 (worker* or visitor* or attendant* or aide or aides or support* or person* or helper* or carer* or caregiver* or "care giver" or "care givers" or consultant* or advisor* or counselor* or counsellor* or assistant* or staff or nurse* or doctor* or physician* or therapist*) )

S13 TI ( (voluntary or volunteer*) N3 (worker* or visitor* or attendant* or aide or aides or support* or person* or helper* or carer* or caregiver* or "care giver" or "care givers" or consultant* or advisor* or counselor* or counsellor* or assistant* or staff) ) OR AB ( (voluntary or volunteer*) N3 (worker* or visitor* or attendant* or aide or aides or support* or person* or helper* or carer* or caregiver* or "care giver" or "care givers" or consultant* or advisor* or counselor* or counsellor* or assistant* or staff) )

S12 TI ( lay N3 (worker* or visitor* or attendant* or aide or aides or support* or person* or helper* or carer* or caregiver* or "care giver" or "care givers" or consultant* or advisor* or counselor* or counsellor* or assistant* or staff) ) OR AB ( lay N3 (worker* or visitor* or attendant* ${ }^{*}$ or aide or aides or support* or person* or helper* or carer* or caregiver* or "care giver" or "care givers" or consultant* or advisor* or counselor* or counsellor* or assistant* or staff))

\begin{tabular}{|c|c|c|}
\hline S11 & (MH “Home Health Aides") & 892 \\
\hline S10 & (MH "Health Personnel, Unlicensed") & 2092 \\
\hline S9 & (MH “Personnel Staffing and Scheduling”) & 12,221 \\
\hline S8 & (MH “Health Manpower”) & 1106 \\
\hline S7 & (MH “Support Groups”) & 5721 \\
\hline S6 & (MH “Community Networks”) & 1069 \\
\hline S5 & (MH “Volunteer Workers”) & 7170 \\
\hline S4 & (MH “Caregivers”) & 13,761 \\
\hline S3 & (MH “Nursing Assistants”) & 4579 \\
\hline
\end{tabular}




\section{PsycINFO (OvidSP)}

\begin{tabular}{|c|c|c|}
\hline 1 & Nonprofessional Personnel/ & 150 \\
\hline 2 & Paraprofessional Personnel/ & 1351 \\
\hline 3 & Allied Health Personnel/ & 590 \\
\hline 4 & Psychiatric Aides/ & 122 \\
\hline 5 & Home Care Personnel/ & 259 \\
\hline 6 & Caregivers/ & 15,761 \\
\hline 7 & Volunteers/ & 3007 \\
\hline 8 & Support Groups/ & 3249 \\
\hline 9 & Social Support/ & 24,057 \\
\hline 10 & $\begin{array}{l}\text { (lay adj3 (worker? or visitor? or attendant? or aide or aides or } \\
\text { support* or person* or helper? or carer? or caregiver? or care } \\
\text { giver? or consultant? or advisor? or counselor? or counsellor? or } \\
\text { assistant? or staff)).ti,ab }\end{array}$ & 1051 \\
\hline 11 & $\begin{array}{l}\text { ((voluntary or volunteer?) adj3 (worker? or visitor? or atten- } \\
\text { dant? or aide or aides or support* or person* or helper? or carer? } \\
\text { or caregiver? or care giver? or consultant? or advisor? or coun- } \\
\text { selor? or counsellor? or assistant? or staff)).ti,ab }\end{array}$ & 1532 \\
\hline 12 & $\begin{array}{l}\text { (untrained adj3 (worker? or visitor? or attendant? or aide or } \\
\text { aides or support* or person* or helper? or carer? or caregiver? } \\
\text { or care giver? or consultant? or advisor? or counselor? or coun- } \\
\text { sellor? or assistant? or staff or nurse? or doctor? or physician? } \\
\text { or therapist?)).ti,ab }\end{array}$ & 213 \\
\hline 13 & $\begin{array}{l}\text { (trained adj3 (worker? or visitor? or attendant? or aide or aides } \\
\text { or support* or person* or helper? or carer? or caregiver? or care } \\
\text { giver? or consultant? or advisor? or counselor? or counsellor? or } \\
\text { assistant? or staff or nurse? or doctor? or physician? or therapist? } \\
\text { )).ti,ab }\end{array}$ & 4021 \\
\hline
\end{tabular}


14 (unlicensed adj3 (worker? or visitor? or attendant? or aide or aides or support* or person* or helper? or carer? or caregiver? or care giver? or consultant? or advisor? or counselor? or counsellor? or assistant? or staff or nurse? or doctor? or physician? or therapist?)).ti,ab

15 ((nonprofessional? or non professional?) adj3 (worker? or visitor? or attendant? or aide or aides or support* or person* or helper? or carer? or caregiver? or care giver? or consultant? or advisor? or counselor? or counsellor? or assistant? or staff)).ti, $\mathrm{ab}$

16 ((non medical or non health or non healthcare or non health care) adj3 (worker? or visitor? or attendant? or aide or aides or support* or person* or helper? or carer? or caregiver? or care giver? or consultant? or advisor? or counselor? or counsellor? or assistant? or staff)).ti,ab

17 (community adj3 (worker? or visitor? or attendant? or aide or aides or support* or person* or helper? or carer? or caregiver? or care giver? or consultant? or advisor? or counselor? or counsellor? or assistant? or staff)).ti,ab

18 (paraprofessional? or paramedic or paramedics or paramedical worker? or paramedical personnel or allied health personnel or allied health worker? or support worker? or non specialist? or specially trained or barefoot doctor? or nurs* aid* or psychiatric aide? or psychiatric attendant? or social worker? or teacher? or school staff or trainer?).ti,ab

19 ((health* or medical*) adj3 (auxiliary or auxiliaries)).ti,ab 28

20 (nurs* adj1 (auxiliary or auxiliaries)).ti,ab. 82

21 (informal adj (caregiver? or care giver? or carer?)).ti,ab. 987

22 (self help group? or support group?).ti,ab. 6342

23 ((social or psychosocial) adj (care or support)).ti,ab.

24 (village adj3 worker?).ti,ab. 37

25 community based.ti,ab. 15,516

26 (community adj3 intervention?).ti,ab. 3086

27 community network?.ti,ab. 
(Continued)

28 ((health or health care or healthcare) adj manpower).ti,ab. 60

29 human resources.ti,ab. $\quad 2764$

30 (task? adj3 shift $\left.^{*}\right)$.ti,ab. $\quad 848$

$31 \quad$ (staff* adj3 chang*).ti,ab. $\quad 576$

\begin{tabular}{ll}
\hline 32 or/1-31 & 229,404
\end{tabular}

33 Psychiatric Patients/ 26,383

34 exp Mental Disorders/ 388,263

35 exp Mental Retardation/ 37,021

36 exp Behavior Disorders/ $\quad 117,436$

37 exp Nervous System Disorders/ $\quad 178,225$

38 Epilepsy/ 14,888

39 Mental Health Services/ 23,413

40 Community Mental Health Services/ 6086

41 exp Crisis Intervention Services/ 2025

42 ((mentally or psycholog*) adj (ill or disabled or handicapped 26,193

or retarded or disturb* or traumati* or deficient)).ti,ab

43 (intellectually adj (disabled or handicapped or retarded or de- 531

ficient)).ti,ab

44 (mental adj (retardation or deficienc $\left.{ }^{*}\right)$ ).ti,ab. $\quad 14,880$

45 ((mental or behavioural or behavioral or anxiety or obsessive or 182,926 compulsive or panic or phobic or schizotypal or delusional or stress or cognitive or cognition or dissociative or personality or impulse control or mood or affective or bipolar or depressive or neurotic or paranoid or psychotic or somatoform or neurologic* or nervous or nervous system or eating) adj (disorder? or illness* or disease?)).ti,ab

46 ((substance related or alcohol or opioid or morphine or mari- 16,798 juana or heroin or cocaine) adj (disorder? or illness* or dependence or abuse or misuse)).ti,ab 
47 (depression or anxiety or schizophrenia or psychoses or stress 391,381 syndrome? or distress syndrome? or combat disorder? or war disorder? or pain disorder? or dementia or alzheimer or epilepsy or down syndrome or alcoholism or substance abuse or drug addict* or drug abus* or drug misuse or drug user?).ti,ab

48 (psychiatric adj (patient? or service? or care or assistance or help 20,821 or work)).ti,ab

49 (mental health service? or mental health care or mental health- 21234 care or mental care).ti,ab

50 ((psychiatric or psychosocial) adj (service? or care or assistance 9123 or help or work)).ti,ab

52 Developing Countries.sh.

53 (Africa or Asia or Caribbean or West Indies or South America or Latin America or Central America).hw,ti,ab

54 (Afghanistan or Albania or Algeria or Angola or Antigua or Barbuda or Argentina or Armenia or Armenian or Aruba or Azerbaijan or Bahrain or Bangladesh or Barbados or Benin or Byelarus or Byelorussian or Belarus or Belorussian or Belorussia or Belize or Bhutan or Bolivia or Bosnia or Herzegovina or Hercegovina or Botswana or Brazil or Brasil or Bulgaria or Burkina Faso or Burkina Fasso or Upper Volta or Burundi or Urundi or Cambodia or Khmer Republic or Kampuchea or Cameroon or Cameroons or Cameron or Camerons or Cape Verde or Central African Republic or Chad or Chile or China or Colombia or Comoros or Comoro Islands or Comores or Mayotte or Congo or Zaire or Costa Rica or Cote d'Ivoire or Ivory Coast or Croatia or Cuba or Cyprus or Czechoslovakia or Czech Republic or Slovakia or Slovak Republic or Djibouti or French Somaliland or Dominica or Dominican Republic or East Timor or East Timur or Timor Leste or Ecuador or Egypt or United Arab Republic or El Salvador or Eritrea or Estonia or Ethiopia or Fiji or Gabon or Gabonese Republic or Gambia or Gaza or Georgia Republic or Georgian Republic or Ghana or Gold Coast or Greece or Grenada or Guatemala or Guinea or Guam or Guiana or Guyana or Haiti or Honduras or Hungary or India or Maldives or Indonesia or Iran or Iraq or Isle of Man or Jamaica or Jordan or Kazakhstan or Kazakh or Kenya or Kiribati or Korea or Kosovo or Kyrgyzstan or Kirghizia or Kyrgyz Republic or Kirghiz or Kirgizstan or Lao PDR or Laos or Latvia or Lebanon or Lesotho or Basutoland or Liberia or Libya or 
Lithuania or Macedonia or Madagascar or Malagasy Republic or Malaysia or Malaya or Malay or Sabah or Sarawak or Malawi or Nyasaland or Mali or Malta or Marshall Islands or Mauritania or Mauritius or Agalega Islands or Mexico or Micronesia or Middle East or Moldova or Moldovia or Moldovian or Mongolia or Montenegro or Morocco or Ifni or Mozambique or Myanmar or Myanma or Burma or Namibia or Nepal or Netherlands Antilles or New Caledonia or Nicaragua or Niger or Nigeria or Northern Mariana Islands or Oman or Muscat or Pakistan or Palau or Palestine or Panama or Paraguay or Peru or Philippines or Philipines or Phillipines or Phillippines or Poland or Portugal or Puerto Rico or Romania or Rumania or Roumania or Russia or Russian or Rwanda or Ruanda or Saint Kitts or St Kitts or Nevis or Saint Lucia or St Lucia or Saint Vincent or St Vincent or Grenadines or Samoa or Samoan Islands or Navigator Island or Navigator Islands or Sao Tome or Saudi Arabia or Senegal or Serbia or Montenegro or Seychelles or Sierra Leone or Slovenia or Sri Lanka or Ceylon or Solomon Islands or Somalia or Sudan or Suriname or Surinam or Swaziland or Syria or Tajikistan or Tadzhikistan or Tadjikistan or Tadzhik or Tanzania or Thailand or Togo or Togolese Republic or Tonga or Trinidad or Tobago or Tunisia or Turkey or Turkmenistan or Turkmen or Uganda or Ukraine or Uruguay or USSR or Soviet Union or Union of Soviet Socialist Republics or Uzbekistan or Uzbek or Vanuatu or New Hebrides or Venezuela or Vietnam or Viet Nam or West Bank or Yemen or Yugoslavia or Zambia or Zimbabwe or Rhodesia) .hw,ti,ab

55 ((developing or less* developed or under developed or under- 7959 developed or middle income or low* income or underserved or under served or deprived or poor*) adj (countr* or nation? or population? or world)).ti,ab

56 ((developing or less* developed or under developed or underdeveloped or middle income or low* income) adj (economy or economies)).ti,ab

57 (low* adj (gdp or gnp or gross domestic or gross national)).ti, 17 $\mathrm{ab}$

58 (low adj3 middle adj3 countr*).ti,ab.

59 (lmic or lmics or third world or lami countr*).ti,ab.

60 transitional countr*.ti,ab. 35

61 or $/ 52-60$

125,331 
63 limit 62 to ("0400 empirical study" or "0410 experimental 1963 replication" or "0430 followup study" or "0451 prospective study" or 1800 quantitative study or "2000 treatment outcome/ randomized clinical trial")

64 (randomised or randomized or randomly allocated or random allocation or control* or evaluat* or effect? or impact or intervention* or time series or time points or quasi experiment* or quasiexperiment*).ti,ab

65 ((pretest or pre test) and (posttest or post test)).ti,ab.

66 ((multicenter or multicentre or multi center or multi centre) 1387 adj study).ti,ab

67 repeated measure*.ti,ab.

68 or $/ 64-67$

69 62 and 68 1451

$70 \quad 63$ or 69

71 (diagnos* or detect* or case finding?).ti,ab.

LILACS (VHL: regional.bvsalud.org/php/index.php?lang=en)

(mental* or psyc* or psiq*) AND (nurse or nurses or midwife or midwives or physician or physicians or clinician or clinicians or doctor or doctors or practitioner or practitioners or dentist or dentists or pharmacist or pharmacists or "health care staff" or "healthcare staff" or "medical staff" or "health personnel" or "health care personnel" or "healthcare personnel" or "medical personnel" or "health worker" or "health workers" or "health care worker" or "health care workers" or "healthcare worker" or "healthcare workers" or "medical worker" or "medical workers" or "health professional" or "health professionals" or "health care professional" or "health care professionals" or "healthcare professional" or "healthcare professionals" or "medical professional" or "medical professionals" or "health provider" or "health providers" or "health care provider" or "health care providers" or "healthcare provider" or "healthcare providers" or "medical provider" or "medical providers" or "health workforce" or "health care workforce" or "healthcare workforce" or "medical workforce" or "health manpower" or "human resources" or enfermer* or enfermeir* or medico* or odontologo* or farmaceutico* or partera* or parteira* or "equipo sanitario" or "trabajadores de salud" or "trabajadores de la salud" or "profissionais de saude" or "recursos humanos") AND (recruit* or retain* or retention or distribut* or "scale up" or "scaling up" or turnover or "turn over" or "brain drain" or maldistribut* or distribucion or retencion or distribuicao or fixacao or retencao) AND (randomised or randomized or "random allocation" or "randomly allocated" or "controlled trial" or "control group" or "control groups" or effect or evaluat* or intervention* or impact or "multicenter study" or "multi center study" or "multicentre study" or "multi centre study" or (pretest and posttest) or quasiexperiment* or (quasi and experiment*) or "time series" or "time point" or "time points" or "repeated measure" or "repeated measures" or "repeated measurement" or "repeated measurements" or "ensayo clinico controlado aleatorio" or "ensayo clinico controlado" or "ensaio clinico controlado 
aleatorio" or "ensaio clinico controlado" or aleatorios or azar or acaso or efecto or efectos or efeito or efeitos or evaluar or evaluacion or avaliacao or intervencion* or intervencao* or impacto or impactos or (estudio* and multicentrico*) or (estudo* and multicentrico*) or (ensaio* and multicentrico*) or (preteste and posteste) or ("pre teste" and "pos teste") or cuasiexperiment* or (cuasi and experiment*) or quaseexperiment* or (quase and experiment*) or "serie temporal" or "series temporal" or "serie temporales" or "series temporales" or "series temporais" or "puntos de tiempo" or "puntos temporales" or "pontos temporais" or "medida repetida" or "medida repetidas" or "medidas repetida" or "medidas repetidas" or "medicion repetida" or "medicion repetidas" or "mediciones repetida" or "mediciones repetidas")

\section{WHO Global Health Library}

\section{(AIM (AFRO), IMEMR (EMRO), IMSEAR (SEARO), WPRIM (WPRO), WHOLIS (KMS)}

((non and specialist* and health* and worker*) or (nonprofessional* and health* and worker*) or (non and professional* and health* and worker*) or (untrained and health* and worker*) or (unlicensed and health* and worker*) or (lay and health* and worker*) or (voluntary and health* and worker*) or (volunteer* and health* and worker*) or (community and health* and worker*) or (paraprofessional* and health* and worker*) or (informal and health* and worker*) or (village and health* and worker*) or (non and specialist* and health* and personnel) or (nonprofessional* and health* and personnel) or (non and professional* and health* and personnel) or (untrained and health* and personnel) or (unlicensed and health* and personnel) or (lay and health* and personnel) or (voluntary and health* and personnel) or (volunteer* and health* and personnel) or (community and health* and personnel) or (paraprofessional* and health* and personnel) or (informal and health* and personnel) or (village and health* and personnel) or (non and specialist* and health* and carer*) $^{*}$ or (nonprofessional* and health* and carer*) or (non and professional* and health* and carer*) or (untrained and health* and carer*) or (unlicensed and health* and carer*) or (lay and health* and carer*) or (voluntary and health* and carer*) or (volunteer* and health* and carer*) or (community and health* and carer*) or (paraprofessional* and health* and carer*) or (informal and health* and carer*) $^{*}$ or (village and health* and carer $^{*}$ ) or (non and specialist* and health* and caregiver*) or (nonprofessional* and health* and caregiver*) or (non and professional* and health* and caregiver*) or (untrained and health* and caregiver*) or (unlicensed and health* and caregiver*) or (lay and health* and caregiver*) or (voluntary and health* and caregiver*) or (volunteer* and health* and caregiver*) or (community and health* and caregiver*) or (paraprofessional* and health* and caregiver*) or (informal and health* and caregiver*) or (village and health* and caregiver*) or (non and specialist* and health* and (care and giver*)) or (nonprofessional* and health* and (care and giver*)) or (non and professional* and health* and (care and giver*)) or (untrained and health* and (care and giver*)) or (unlicensed and health* and (care and giver*)) or (lay and health* and (care and giver*)) or (voluntary and health* and (care and giver*)) or (volunteer* and health* and (care and giver*)) or (community and health* and (care and giver*)) or (paraprofessional* and health* and (care and giver*)) or (informal and health* and (care and giver*)) or (village and health* and (care and giver*)) or (non and specialist* and health* and provider*) or (nonprofessional* and health* and provider*) or (non and professional* and health* and provider*) or (untrained and health* and provider*) or (unlicensed and health* and provider*) or (lay and health* and provider*) or (voluntary and health* and provider*) or (volunteer* and health* and provider*) or (community and health* and provider*) or (paraprofessional* and health* and provider*) or (informal and health* and provider*) or (village and health* and provider*) or (social and worker*) or teacher* or (school and staff) or (self and help and group*) or (support and group*) or (task* and shift*) or taskshift* or (health* and manpower) or (human and resources)) AND ((mental ${ }^{*}$ and ill) or (mental ${ }^{*}$ and illness*) or (mental* and disorder $\left.{ }^{*}\right)$ or (mental ${ }^{*}$ and disabled) or (mental* and deficien*) or (mental and disease*) or (mental* and morbid*) or (mental* and handicapped) or (mental* and retarded) or (mental ${ }^{*}$ and traumati*) or (mental* and patient $\left.{ }^{*}\right)$ or $\left(\right.$ psych $^{*}$ and ill) or (psych* and illness $\left.{ }^{*}\right)$ or $\left(\right.$ psych $^{*}$ and disorder $\left.{ }^{*}\right)$ or $\left(\right.$ psych $^{*}$ and disabled) or (psych* and deficien*) or (psych* and disease*) or (psych* and morbid*) or (psych* and handicapped) or (psych* and retarded) or (psych* and traumati*) or (psych* and patient*) or (intellectually and disabled) or (intellectually and handicapped) or (intellectually and retarded) or (intellectually and deficien*) or (behavioural and disorder*) or (behavioral and disorder*) or anxiety or (obsessive and disorder*) or (compulsive and disorder*) or panic or phobic or schizotypal or delusional or (cognitive and disorder*) or (cognition and disorder*) or dissociative or (personality and disorder*) or (impulse and control and disorder*) or (mood and disorder ${ }^{*}$ ) or (affective and disorder) or bipolar or depressive or neurotic or paranoid or psychotic or somatoform or neurologic* or nervous or (eating and disorder*) or (substance and related and disorder*) or (substance and abuse) or (drug and addict ${ }^{*}$ ) or (drug and abuse) or (drug and misuse) or alcoholism or alcoholic* or (alcohol and abuse) or (alcohol and misuse) or (alcohol and dependenc*) or (drinking and behaviour) or (drinking and behaviour) or (opioid and abuse) or (opioid and misuse) or (opioid and dependenc*) or (opioid and addict $^{*}$ ) or (morphine and abuse) or (morphine and misuse) or (morphine and dependenc* ${ }^{*}$ or (morphine and addict*) or (marijuana and abuse) or (marijuana and misuse) or (marijuana and dependenc* $c^{*}$ or (marijuana and addict* ${ }^{*}$ or (heroin and abuse) or (heroin and misuse) or (heroin and dependenc* $c^{*}$ or (heroin and addict*) or (cocaine and abuse) or (cocaine and misuse) or (cocaine and dependenc ${ }^{*}$ ) or (cocaine and addict* ${ }^{*}$ or depression or anxiety or schizophrenia or psychoses or (stress and syndrome*) or (distress and syndrome*) or (combat and disorder*) or (pain and disorder*) or dementia or Alzheimer* or epilepsy or (down* and syndrome)) AND (randomiz* or randomis* or (controlled and trial) or (multicenter and study) or (multicentrer and study) or (cluster and trial) or (controlled and

Non-specialist health worker interventions for the care of mental, neurological and substance-abuse disorders in low- and middle- 
before and after) or pretest or (pre and test) or posttest or (post and test) or intervention* or evaluat* or effect or impact or (time and series) or (time and points) or (repeated and measure*))

\section{OpenGrey}

1 discipline:(05T - Health services, health administration, com- 0 munity care services) AND ("mental health") AND ("human resources")

2 discipline:(05T - Health services, health administration, com- 975 (MIC: 7) munity care services) AND ("mental health")

of which latvia: 7

3 discipline:(05T - Health services, health administration, com- 1 munity care services) AND ("mental health") and doctor

4 discipline:(05T - Health services, health administration, com- 9 munity care services) AND ("mental health") and nurse

5 discipline:(05T - Health services, health administration, com- 0 munity care services) AND ("mental health") and infirmiere

6 discipline:(05T - Health services, health administration, com- 23 munity care services) AND ("mental health") and social work

7 discipline:(05T - Health services, health administration, com- 1004 (MIC: 36) munity care services) AND ("mental health") OR "psych"

of which Latvia: 36

8 "mental" or "psych" AND "non-specialist" or "nonspecialist" 0 or "paramedic" or "paraprofessional" or "communit" or "nonprofessional" or "nonprofessional" or "carer" or "caregiver" or "teacher" or "school" or "task-shift" or "taskshift"

9 "mental" or "psych" 2124 (MIC: 81) Of which Latvia: 50; Russian: 14; Czech: 12; Portugal: 5

10 discipline:(05T - Health services, health administration, community care services) AND ("mental") OR "psych" And ("paramedic") (same number of hits came up substituting 'paramedic for :non-specialist" or "nonspecialist" or "paramedic" or "paraprofessional" or "non-professional" or "nonprofessional"

Of above search: categorised by language: excluded english and german and French. Checked Latvian (as only MIC listed) origin: "lv" and discipline:(05T - Health services, health administration, community care services) AND ("mental") OR "psych" And ("paramedic"')

11 ("mental”) OR "psych” And ("carer") From above: Latvian 21, Russian 14, Czech 12, Portugal 5 
12 "mental” and "doctor" (or nurse) 0 from MIC

13 "mental" and "school"

41

Latvian: 2; Czech: 2

14 "mental" and "teacher"

Latvian: 1; Czech: 1

Total screened from MIC (Middle Income Countries): 259

meta Register of Controlled Trials ( $m R C T$ )

Search 1: mental and health worker - 12 records

Search 2: psychiatr* and health worker - 6 records

Search 3: paramedic and mental - 1 record

Search 4: paramedic and psychiatr* -1 record

Search 5: paraprofessional and mental - 13 records

Search 6: paraprofessional and psychiatr* -8 records

Search 7: non-specialist and mental -2 records

Search 8: non-specialist and psychiatr* -0 records

Search 9: lay and worker and mental - 1 record

Search 10: lay and worker and psychiatr* - 0 records

Search 11: community and worker and mental -25 records

Search 12: community and worker and psychiatr* -13 records

Search 13: carer and mental - 27 records

Search 14: carer and psychiatr* -26 records

Search 15: caregiver and mental - 0 records

Search 16: caregiver and psychiatr* -0 records

Search 17: teacher and mental - 78 records

Search 18: teacher and psychiatr* -61 records

International Clinical Trials Registry Platform, (ICTRP)

Search 1: 119 records

mental or psych (in condition field) AND non-specialist or nonspecialist or paramedic or paraprofessional or communit or nonprofessional or nonprofessional or carer or caregiver or teacher or school or task-shift or taskshift (in intervention field)

Search 2: 10 records

mental or psych (in condition field) AND lay and worker (in intervention field)

Search 3: 0 records

mental or psych (in condition field) AND human and recourses (in intervention field)

Search 4: 1 record

mental or psych (in condition field) AND task and shift (in intervention field)

Search 5: 0 records

non-specialist and mental (in title field)

Search 6: 0 records

non-specialist and psych (in title field)

Search 7: 0 records

nonspecialist and mental (in title field)

Search 8: 0 records

nonspecialist and psych (in title field)

Search 9: 1 record

paramedic and mental (in title field)

Search 10: 0 records

Non-specialist health worker interventions for the care of mental, neurological and substance-abuse disorders in low- and middle- 
paramedic and psych (in title field)

Search 11: 0 records

paraprofessional and mental (in title field)

Search 12: 0 records

paraprofessional and psych (in title field)

Search 13: 1 record

community and worker and mental (in title field)

Search 14: 0 records

community and worker and psych (in title field)

Search 15: 1 record

lay and worker and mental (in title field)

Search 16: 1 record

lay and worker and psych (in title field)

Search 17: 0 records

non-professional and mental (in title field)

Search 18: 0 records

non-professional and psych (in title field)

Search 19: 0 records

nonprofessional and mental (in title field)

Search 20: 0 records

nonprofessional and psych (in title field)

Search 21: 2 records

carer and mental (in title field)

Search 22: 16 records

carer and psych (in title field)

Search 23: 6 records

caregiver and mental (in title field)

Search 24: 24 records

caregiver and psych (in title field)

Search 25: 3 records

teacher and mental (in title field)

Search 26: 1 record

teacher and psych (in title field)

Search 27: 18 records

school and mental (in title field)

Search 28: 16 records

school and psych (in title field)

Search 29: 0 records

task-shift and mental (in title field)

Search 30: 0 records

task-shift and psych (in title field)

Search 31: 0 records

taskshift and mental (in title field)

Search 32: 0 records

taskshift and psych (in title field)

Search 33: 0 records

task and shift and mental (in title field)

Search 34: 0 records

task and shift and psych (in title field)

Search 35: 0 records

human and resources and mental (in title field)

Search 36: 0 records

human and resources and psych (in title field)

Non-specialist health worker interventions for the care of mental, neurological and substance-abuse disorders in low- and middle- 
Recruitment status: ALL.

\section{Appendix 2. Adapted CHEC criteria list}

\section{yes no Not applicable Details}

1 Are competing alternatives clearly described?

2 Is a well defined economic question posed in an answerable form?

3 Is the economic study design appropriate to the stated objective?

4 Was there a comparison between 2 more groups receiving different interventions?

5 Is the chosen time horizon appropriate to include relevant costs and consequences?

6 Is the perspective/viewpoint** of the analysis explicitly stated? If yes, give details

7 Is the actual perspective chosen appropriate?

8 Are all important and relevant costs for each alternative identified?

9 Are costs measured? If yes, give details of costs measured.

10 Are all costs measured appropriately in physical units?

11 Are costs valued appropriately? 
12 Are all important and relevant outcomes for each alternative identified?

13 Were outcomes measured? If yes, give details of outcomes measured

14 Are all outcomes measured appropriately?

15 Are outcomes valued appropriately?

16 Is an incremental analysis of costs and outcomes of alternatives performed?

17 Are all future costs and outcomes discounted appropriately? *(where appropriate)

18 Were sensitivity analyses undertaken? If yes, give details of forms of sensitivity analyses.

19 Are all important variables, whose values are uncertain, appropriately subjected to sensitivity analysis?

20 Do the conclusions follow from the data reported?

21 Does the study discuss the generalizability of the results to other settings and patient/ client groups?

22 Does the article indicate that there is no po- 
tential conflict of interest of study researcher (s) and funder(s)?

23 Are ethical and distributional issues discussed appropriately?

\section{Appendix 3. Other economic studies of relevance but not included}

Thirteen economic studies did not meet our inclusion criteria, as they did not relate to one of the included studies. Their findings are presented and compared with those that are included in this review to enhance the usefulness and applicability of the Cochrane review for healthcare decision making. The economic questions addressed in excluded studies mainly fall into three broad categories in terms of cost analysis of specific disease conditions, carer and family burden, and comparison of improved or integrated mental health care with primary care with usual or no care.

The studies that looked at healthcare costs cannot be compared with those of included studies as they were from different settings, conditions and outcomes.

Health services costs: Chisholm 2000 dealt with integration of mental health services into primary health care in India and Pakistan and found that a significant category of healthcare costs were consultations with GPs. In Luengo-Fernandez 2011, primary care was costed in European middle-income countries as constituting 36\% (Portugal) and 9\% (Greece) of total healthcare costs. There is no costing specific to NSHWs. One review showed that collaborative care costs are no greater than usual care (Woltmann 2012). A community outreach intervention in rural India for untreated schizophrenia study found that the costs of informal care sector visits and family care giving costs considerably reduced during the follow-up period from USD10 to about USD2 (Murthy 2005). This study gives detailed costs of outreach clinic set up, unit costs per person accessing services and outcome data at intervention baseline and follow-up to 18 months. It shows that costs of services increase over time (the increase in costs is of the specialist outreach services, not of PHC services) and that overall costs remain stable (around USD34). This study also emphasises the need for early diagnosis and availability of services close to the affected populations helps in increased uptake of services and reduces associated costs. The most promising study on service changes and costs is from South Africa, where Petersen 2012 estimated that the costs of a primary healthcare staffing package (one post for a mental health counsellor or equivalent and 7.2 community mental health worker posts) would be offset by a reduction in the number of other specialist and non-specialist health personnel required to close service gaps at primary care level. The cost of these personnel amounts to GBP28,457 per 100,000 population.

Costs of specific interventions: Suh 2006 in their study on economic costs of dementia in Korea found that costs of care for dementia patients needing full-time care in community (USD44,121) were about 10 times higher than those who did not need long-term care (USD3986) and found that costs of informal care were very high, but it is unclear what the costs relating to NSHWs were. Another study dealt with societal costs of dementia (mainly informal costs) in both developed and LMICs, but does not explicitly state the costs of a NSHW-delivered service (Wimo 2007). The costs of providing epilepsy care through primary care in Zambia is estimated at under USD25 a day (Birbeck 2012).

Informal care costs: The high level of burden among family carers was also highlighted in other studies (Chisholm 2000; Murthy 2005; Papastavrou 2010; van Steenbergen-Weijenburg 2010; Woltmann 2012), and that was significantly related to the severity and frequency of the patients symptoms, gender and educational level of the carer.

Resource requirement analysis and resource use: Some studies described the status of resource use; Chisholm 2000 study showed low level of service utilisation in the government centres. Others attempt to calculate resource requirements. Scaling up specific interventions like the child and adolescent mental health services in their country context was done by modelling (Lund 2009), for different levels of coverage in South Africa. The model suggests most costs should be spent at primary care level with a range of NSHWs (occupational therapists, social workers, general nurses) and specialists (psychiatric nurses). However, this forecasted ideal situation is currently unrealistic due to budgetary constraints. Siskind 2010 estimated cost-effectiveness of usual care compared with improved primary care for depression in Chile using computer-based Markov cohort model. They found the incremental cost-effectiveness ratio (ICER) of usual care CLP113 per quality adjusted life year (QALY) gained versus no treatment, whereas stepped care had an ICER of CLP468 per 
QALY versus usual care. A sensitivity analysis was also performed and the results were sensitive to assumptions made about recurrent episodes coverage, cost of treatment and insensitive to changes in health state utility of depression and rate of recurrence.

We found one cost-effectiveness study on mental health intervention package in Nigeria (Gureje 2007), which estimated cost per DALYs averted for schizophrenia, depression, epilepsy and alcohol use. The most cost effective intervention for schizophrenia was a $70 \%$ coverage of antipsychotic drugs with either psychosocial treatment or case management with cost per DALY USD642 and USD680 respectively. Cost per DALY averted for depression was lowest for older antidepressant drug with psychotherapy at USD767. Similarly, for epilepsy older antiepileptic drugs in primary care implemented at $80 \%$ coverage offered the best cost per DALY at USD100 per DALY averted. Random road-side breath testing for alcohol had a cost per DALY averted at USD85 (Gureje 2007). A systematic review which included two cost-effectiveness studies in LMIC of costs of collaborative showed these to be cost-effective (van Steenbergen-Weijenburg 2010).

\section{Appendix 4. Description of studies not included in meta-analyses}

1. Non-specialist health workers versus usual care (life-skills training) in improving drug abuse outcomes (RCT)

Sutcliffe2009RCT Thailand peer-led education programme versus a best practice intervention (life skills building approach) probably improves index patients' recovery of depressive symptoms at 12 months (MD -2.20, 95\% CI -4.03 to -0.37), though this did not apply to reducing the prevalence of depression. However, this benefit did not filter to their network group (not involved in the intervention) (MD 0.00, 95\% CI -1.55 to 1.55). There was no significant effect on methamphetamine use (RR $1.01,95 \% 0.91$ to 1.13 ) at six months or at one year post intervention.

2. Non-specialist health workers versus usual care for treating schizophrenia (controlled before-and-after study)

A medical assistant-delivered psychoeducation programme for carers of people with schizophrenia in Malaysia reported slightly fewer cases of readmission rates (3/54 versus 5/55) and a better defaulter rate (6/54 versus 14/65) in the intervention versus the control group (Paranthaman2010CBAMalaysi). It may have little or no impact on carer burden, on activities of daily living, or on other outcomes (such as financial expenditure, reduction in worry, impact on daily routines and supervision).

\section{Non-specialist health workers versus specialist care in treating epilepsy (equivalence trial RCT)}

In China, Li's study shows that there is equivalence between NSHW (trained village doctors) and specialists (psychiatrists) in reducing how many of their patients had an $80 \%$ or more reduction in epileptic seizures after three-month treatment with phenobarbital $(60 \%$ versus 55\%) (Li 1989 RCT China). This also applied to patients with a 20\% to 79\% seizure rate reduction (30\% versus 35\%) or below $20 \%$ seizure rate reduction ( $5 \%$ versus $15 \%$ ).

However, there seems to be improvements in reported side effects in the NSHW versus specialist group, such as somnolence $(2 / 20$ versus 10/20) and drowsiness (6/20 versus 17/20). There was no difference in other reported side effects: dizziness, ataxia, nausea and vomiting, and return visits.

4. Other professionals with health roles versus usual care in delivering a psychosocial/activities intervention for parents of children with intellectual disabilities (RCT)

The Vietnamese RCT introduced a teacher-led Portage curriculum for parents of preschool children with intellectual disabilities versus wait-list control (Shin 2009 RCT Vietnam). The results are difficult to interpret, as often baseline data were different in both groups. This intervention may slightly improve behavioural changes (MD 1.10, 95\% CI -7.82 to 10.02), motor skills (MD -1.40, 95\% CI 12.93 to 10.13 ) and social skills (MD $0.80,95 \% \mathrm{CI}-11.51$ to 13.11 ) at six months (with similar scores at 12 months).

\section{WHAT'S NEW}

Last assessed as up-to-date: 2 October 2012.

\begin{tabular}{l|l|l}
\hline Date & Event & Description \\
\hline 30 October 2013 & Amended & addressed all copy editors' issues \\
\hline
\end{tabular}

Non-specialist health worker interventions for the care of mental, neurological and substance-abuse disorders in low- and middleincome countries (Review)

Copyright $\odot 2013$ The Cochrane Collaboration. Published by John Wiley \& Sons, Ltd. 


\section{CONTRIBUTIONSOFAUTHORS}

NvG conceived the review. All authors contributed to the development of the protocol. NvG, PT, GR, MSM, SC and JP extracted data and assessed risk of bias. NvG and PT checked these and did the statistical analysis and GRADE assessments. NvG wrote the final version. All authors commented on and approved the final version.

\section{DECLARATIONSOF INTEREST}

NvG, PT, GR, MSM, JP, SC: None known.

VP: A co-author on some of the papers that are included in this study.

SL: An editor for the Cochrane Effective Practice and Organisation of Care Group and the Consumers and Communication Review Group.

\section{SOURCES OF SUPPORT}

\section{Internal sources}

- Christian Medical College, Vellore, India.

Salary and logistic support for PT

\section{External sources}

- Indian Council of Medical Research, India.

Funding for the Prof. BV Moses \& ICMR Centre for Advanced Research and Training in Evidence-Informed Healthcare that hosts the South Asian Cochrane Network \& Centre

- Wellcome Trust, UK.

Clinical PhD Fellowship awarded to NvG.

- Wellcome Trust, UK.

Senior Research Fellowship awarded to VP

\section{DIFFERENCES BETWEEN PROTOCOLANDREVIEW}

\section{Authors}

Meera SM, Sudha Chandrashekar and Jessica Pian are new authors who helped with data extraction, analysis and writing (SC).

\section{Search strategy}

In the review protocol, we planned to search African Indexus Medicus, EurasiaHealth (Eastern European countries) and IndMED (Indian Medlars Centre). This was not done as we felt that the World Health Organization (WHO) trial registry, World Health Organization Library Information System (WHOLIS) and other databases would cover these sources.

We did not search the HEED database (as outlined in our protocol) as there were few identified studies. We will perform this search when conducting the next update of this review. 


\section{Data extraction and management}

- Settings: We narrowed down the options to workplace, school, community, PHC clinic and other.

- Results: We extracted more details pertaining to outcomes such as whether they were continuous our dichotomous and what the authors' conclusions were.

- Screening instruments: Removed citation details from data extraction.

\section{Assessment of risk of bias}

- All based on Cochrane Effective Practice and Organisation of Care (EPOC) criteria, not on the Cochrane Handbook for Systematic Reviews of Interventions.

- We added two extra categories for risk of bias assessment. The detection bias has been divided into two: that of assessing subjective and objective outcomes were assessed blindly. In addition, the attrition bias has been divided into how incomplete or not two types of outcomes are: efficacy outcomes and safety outcomes (e.g. adverse events).

- Consensus on Health Economic Criteria (CHEC) list criteria: This was adapted with more questions: 1. Was there a comparison between two more groups receiving different interventions? 2. Is the perspective/viewpoint** of the analysis explicitly stated? If yes, give detail; 3. Are costs measured? If yes, give details of costs measured; 4. Were outcomes measured? If yes, give details of outcomes measured; 5 . Were sensitivity analyses undertaken? If yes, give details of forms of sensitivity analyses.

\section{Data synthesis}

For NRCTs, we did not record whether the study restricted participant selection or demonstrated balance or matching between intervention and control groups on prognostic factors, or a combination of these. An imbalance of these may act as confounders (such as age, sex, socioeconomic status).

We also did not record whether the study adjusted for confounders or effect modifiers in statistical analyses to quantify the effect size (Reeves 2009). Therefore, we have not entered these into additional tables.

We did not transform ordinal outcomes (such as symptom severity, general psychosocial functioning, levels of dependency in disability and any other outcomes measured on a scale) into binary data (e.g. symptom improvement will become improvement or no improvement) or vice versa as it did not make clinical sense. There were very different scales and many studies that had binary data also pooled continuous data that could be pooled with other similar figures.

Pooling results: Though it is generally advised not to pool results if the $\mathrm{I}^{2}$ statistic is more than $50 \%$, we decided to pool outcomes and results that made clinical sense (based on settings, mental illnesses, types of interventions and outcomes measured), rather than rely only pooling those that had an $\mathrm{I}^{2}$ statistic less than $50 \%$.

Economic outcomes: There were too few studies to do any conversion of unit costs to 2010 International Dollars (Shemilt 2010), re-estimation of costs, adjustments for currency and price year or perform any further calculations of total costs, or resource use per patient, intervention or health provider.

Statistical analysis: We did not perform meta-regression to investigate both the effect of the intervention on the estimates of effects and to investigate the effect of multiple characteristics (regarding setting and the intervention) simultaneously (Deeks 2009), as there were never more than five studies per variable.

Sensitivity analyses: We did not perform additional sensitivity analyses that were listed as considered analyses in the protocol:

- based on specific decisions made during the review process, such as how ICCs are imputed for cluster trials;

- based on whether the included cluster RCTs found different estimates of effect to non-cluster trials for specific outcomes, but excluding cluster RCTs;

- based on whether the study reported a validated tool that confirmed the NSHWs diagnostic accuracy;

- if one or more studies reported outcomes using either a continuous scale or a dichotomous scale and in either scenario had been transformed (to dichotomous or continuous variable respectively);

- based on the effect.

For the economic analyses, we also did not perform additional sensitivity analyses, as there were too few studies to make this meaningful.

Non-specialist health worker interventions for the care of mental, neurological and substance-abuse disorders in low- and middle- 


\section{Subgroup analysis and investigation of heterogeneity}

We had initially planned to use non-overlapping CIs to indicate a statistically significant difference in treatment effect between the subgroups, acknowledging that the CIs can overlap to a small degree and the difference could still be statistically significant. However, the implementation in RevMan 2012 of the $\mathrm{Chi}^{2}$ test and $\mathrm{I}^{2}$ statistic for subgroup differences within random-effects meta-analyses meant that this approach was no longer needed.

\section{Definitions}

NSHW/OPHR: We excluded certain health workers that we classified as a specialist including those who were not traditionally thought of as specialists by the psychiatry/medical system: for example school counsellors who were trained to exclusively do that and who had a qualification, with or without extra experience and where their sole focus was on child psychology/counselling. We also excluded all healthcare providers within non-biomedical systems (e.g. a yoga master) as we had not searched for these specifically and it was difficult to judge, from our perspective, what constituted for them a mental health intervention.

MNS disorders: We relaxed our criteria for International Classification of Diseases (ICD)-10 diagnoses for inclusion criteria of participants. The reason we did this was that in some studies, the population studied did not have formal diagnoses administered (either because of lack of psychiatrist or because their aim was to look at reduction in symptoms and improvement in psychosocial functioning). Therefore, we included studies where the overwhelming majority of the participants (above 75\%) had significant mental health symptoms (such as high scores of depression symptoms or post-trauma symptoms, e.g. Jordans).

Clinical interventions: We decided not to include interventions delivered by people who were not within the medical paradigm (such as faith healers or yoga masters).

Social interventions: We did not include social interventions (initially defined as return to employment/school or general social support) if it was not part of a trial with a specific mental health intervention, as we discovered our search strategy did not address this completely and opened a whole array of studies that we had not considered at the protocol stage (such as income generating activities without a mental health intervention but that may look at mental health outcomes).

Non-specialist health worker interventions for the care of mental, neurological and substance-abuse disorders in low- and middle- 